\title{
Electric Power Monthly
}

\section{November 1997 Reosived With Data for August 1997

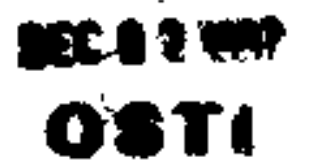

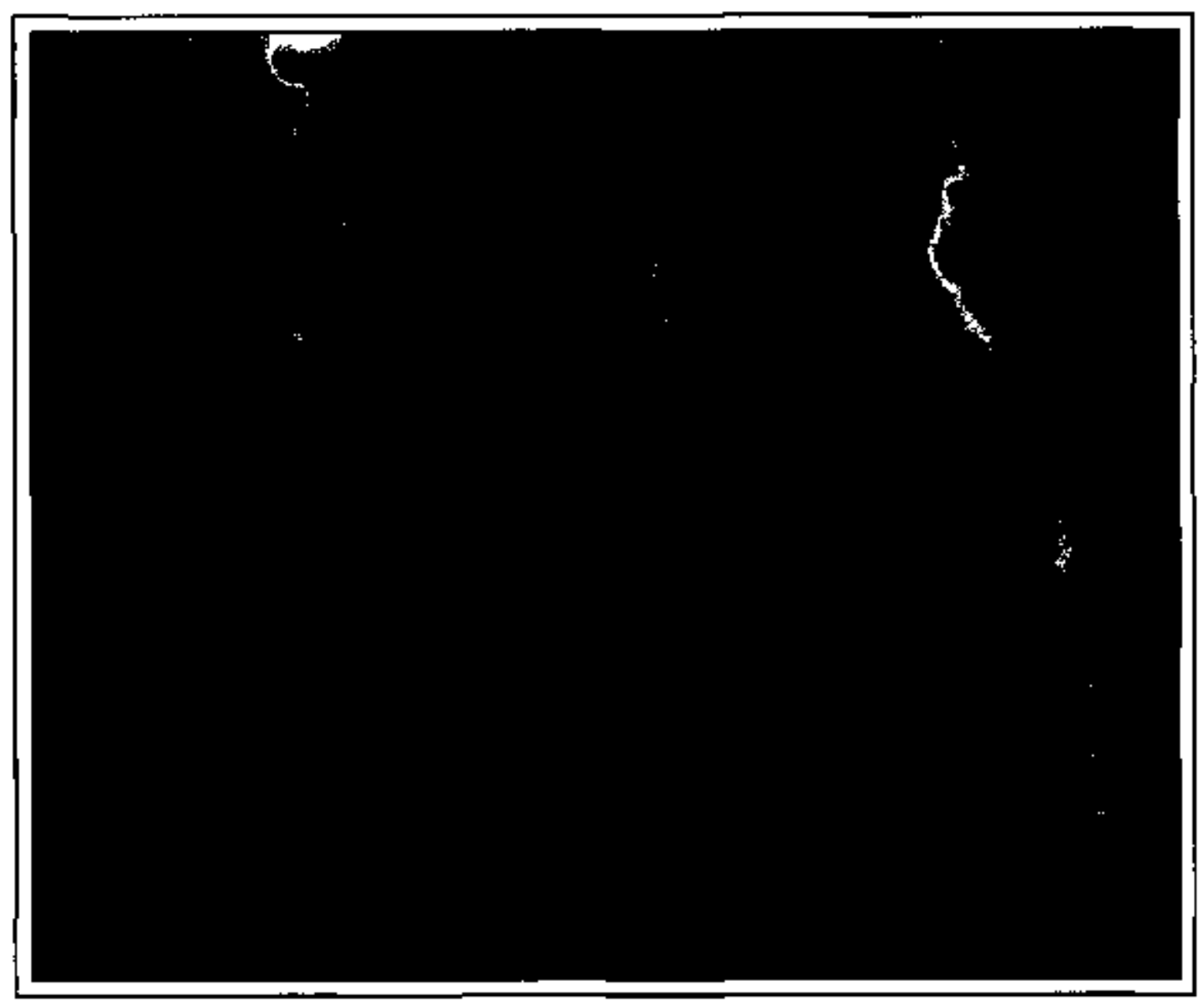

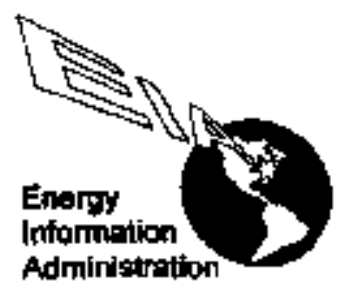




\section{EIA DATA ARE AVAILABLE IN ELECTRONIC AND PRINTED FORM}

For information on the following electrondc products and services, contact the National Energy Information Center on (202) 586-8800 or infoctr (巛eia.doe.gov.

Internet Site Services-offer nearly all ElA publications. Users can view and download selected pages or entire reports, search for information, download ElA data and analysis applications, and find out about wew ElA information products and services.

World Wide Web: http://wuw.eiactoe.gov

Gopher: gopher/gopher eia.doe.gov

FTP: ftp $/ /$ ftp.eia.doe.gov

ELA also offers a listserv service for EIA press releases and other short documents. Sign up on the EIA World Wide Web site.

ELA's CD-ROM, Energy InfoDisc, ptoduced quarterly, contains most EIA publications, several databases, and an energy forecasting model. It is available for a fee from STAT-USA, on 1-800-STAT-USA.

The Comprehensive Oll and Gas Information Source (COGIS), a bulletin boatd service, contains data files from most of ELA's oil- and gas-related reports. It is available for a fee from STAT-USA, on I-800-STAT-USA.

EIA's Electronic Publishing System (EPUB) bulletin board contains data files, directories, and forecasts from most EIA reports. It can be acoessed free of charge by dialing (202) 586-2557.

Recent pablications may be purchased from:

Superintendent of Documents

U.S. Government Printing Office

P.O. Box 371954

Pittsburgh, PA 15250-7954

(202) 512-1800

(202) 512-2250(fax)
Odder publications may be purchased from:

National Technical Information Service

U.S. Department of Commerce

5285 Port Royal Road

Springfield, VA 22161

(703) $487-4650$

(703) $321-8547$ (fax)

For further information on any of the above information sources, or for answers to questions on energy statistics, please contact EIA*'s National Energy Information Center:

National Energy lnformalion Center

Energy Information Administration

Forrestal Builcing, Room IF-048

Washington, DC 20585
(202) 586-8800

(202) 586-0727 (fax)

TTY: (202) 586-1181

Email: infoctr@etadoe gov

Cover Photo:

Lightning, the raw form of electricity, provides a backdrop for the harnessed form carried over transmission lines.

Feleased for Printing: November 18, 1997

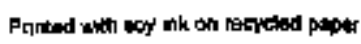

The Electris Power Monthy (ISSN 0732-2305) is publishod moxthly by the Energy Irformation Administration, 1000 Indiependence Avenue, SW, Washinglon, DC 20586, and sels lor \$29.00 per year (price subject to change without actrance notice). Second-class postage paid at Washington, DC 20066-9998, and additional malling ofices. POSTMASTER: Send adtiss changBs to Electric Power Monthy, Energy Information Adminisiration, E1-231, 1000 Independence Avenue, SW, Wasthington, DC 20585. 


\section{Electric Power Monthly November 1997}

With Data for August 1997

\section{Energy Informatlon Adminiatretlon}

Office of Coal, Nuclear, Electric and Altemate Fuels

U.S. Department of Energy

Washington, DC 20585

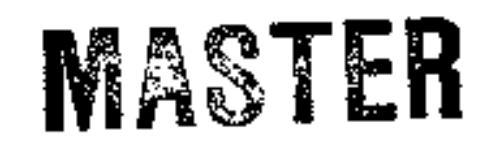

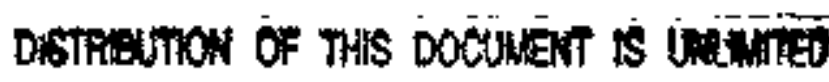




\section{Contacts}

The Elatic Power Monthly is prepared by the U.S. Depantment of Energy's Energy Information Aciministration. Questions and corranents concerning the contents of the Electric Power Manthy may be directed to:

Ms. Sandra Sinth, Project Leader

Energy Intormation Admintsiretion, Er-524

U.S. Department of Energy

Washington, 0C, 20585

Telephone number: (202)426-1173

Intermet E-Mail number: SANDAASMITHE EIA.DOE.GOV

or the lollowing subject speclalists:

Subioct

Electrielty Supply and Demand Forecast ..

industry Developments ..............

Now Elactric Generating Untts ..........

U.S. Electic Utity Net Generation .......

U.S. Electric Utility Consumption of Fuels .

U.S. Electric Utilly Stocks of Fueis. .......

U.S. Electric Utillty Fossi-Fuel Recelpts ...

U.S. Electric Uttity Fossit.Fuel Dellwered

Costs $, \ldots \ldots \ldots \ldots \ldots \ldots \ldots \ldots \ldots, \ldots$

U.S. Retall Sales of Electricty, Associated

Revewue and Average Revenue per

Kilowetthor $\ldots \ldots \ldots \ldots \ldots \ldots \ldots$.

U.S. Nonittlity Sales for Hesale . . . . . . . .

U.S. Nomutlity Net Generation ..........

Samoling and Estimation Methodoborios ..
Contad

Rebecce MeNamey

Kennath MaClevey

Karen McDarriel

Mitwin E. Johnson

Melvin E. Johnison

Metvin E. Jahnson

Kemeth MoClovey

Kennath MeClevey
Phone Number

202-426-1251

202-426-1144

202-426-1234

202-426-1172

$202-426-1172$

202-426-1172

202-426-1144

202-426-1144
Intemot E-Mlall

REBECCA.MCNEANEY EIA.DOE.GOV KENINETH,MCCLEVEY OEIA.DOE.GOV KAREN,MCDANIEL EIA,DOE,GOV MELVIN.JOHNSON EIADOE.GOV MELVIN.JOHNSONOELA.DOE.GOV MELVIN.JOHNSON EIA.DOE.BOV KENNETH.MCCLEVEY OEIA.DOE.GOV KENNETH+MCCLEVEYOEIA.DOE,GOV

Requests for additional information on other enerdy statistics gvailable from the Energy Information Adminlatration or culestions

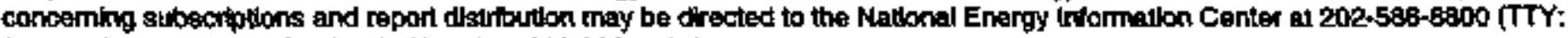
for people who are deaf or hand of heartis, 202-596+1131).

\section{To EIA's Customers}

To ensure that this report meets the highest standards for quality and customer satisfaction, we encourage our readers to contact Sandra Smith on (202) 426-1173(Internet:SANDRA.SMITH@EIA.DOE.GOV) with comments or suggestions to further improve the report. 


\section{DISCLAIMER}

This report was preported at en account of work sponsored by an agency of the United States Gowermaent. Neithet the Uailed Starte Govatmment nor any agency thereof, nof any of theit omployeer, makes apy workpty, expess of imptied, of

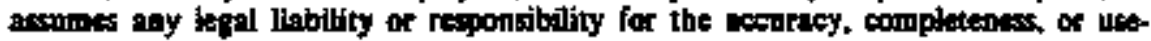

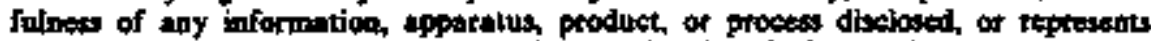
that its ute would not infrígte privately owned rights. Reference bestin to any apecific commercial prodoct, process, of wervice by trade nome, troderpork. monufac-

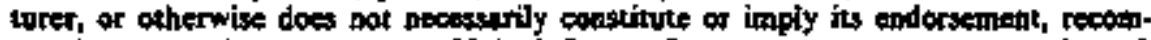

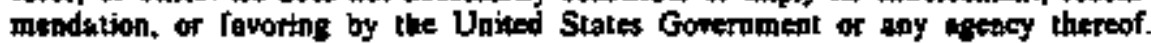
The vitws and opinioes of anthors expressed hereis do not necessarily slate or reflect those of the Uaited States Goverament of any asency theteof. 


\section{DECDANMER}

Partions of this docament may be fllegible in electranic inage prodinets. Impges are produed trom the best erriloble originel docement 


\section{Electronic Publishing System (EPUB) User Instructions}

EPUB is an electronic publishing system matntained by the Energy Information Admuntstration (EIA) of the U.S. Department of Energy. EPUB allows the general public to electronically access selected energy data from many of ELA's statistical reports. The system is a menu-driven, bulletin board type system with extensive online setp capabilities that can be accessed free-of-charge 24 hours a day by using a terminal or PC with an asynchronous moodem. (EPLIB will be taken down briefly at midnight for backup).

PC users must provide the following infonmation to their communications software in order to successfully access the EPUB system.

\section{Communtcations Parameters:}

\section{Batrd Rate: Up to 28,800 bps}

Data Bite: B; Stop Bitst 1

Parity: Nane; Duplex Full

Terminal Type: ANSI, ANSI-BBS, YTIO0, etc

Once your commurications software and/or hardware has been configured, EPUB can be accessed by dialing (202) 586-2557. Whan a connection to the system has been made, some users may find that the menu-driven instructions and the online help capabilities will provide enough inforrtation to effectively use EPUB. If needed, more extensive information may be found in the EPUB User's Guide, which is avatlable online from the EPUB system or from:

\footnotetext{
Nattonal Energy Information Center, El-231

Energy Information Adarinistretion.

Forrestal Building, Room 1F-048

Washington, DC 20585

(202) 586-8800

Internet E-Mail: ANFOCTRGEIA.DOE,GOV

TTY: For people who ate deal or hard of hearing:

(202) 586-1191

Hours: 9 a.m. to 5 p.rn., M-F, eastern time
}

For comumunication or technical assistance, call (202) 5868959,8 a.m. to 5 p.m. eastem time, Monday through Friday.

For questions about the content of EPUB reports andior data, call (202) 566-8800, 9 a.m. to 5 p.m. eastern time, Monday through Friday.
Following is a Iist of some of the data and reports that are provided on EPUB:

- Heating fuel data (April through September) Updated the 2nd week of the month.

- Oxygenate data Updated approximately the 25th of the month.

- Weekly Petroleum Status Regort Updated on Wednesdays (Thursdays in the event of a holiday) at 9 a.m.

- Petroletum Supply Monthity Updated between the 23rd and 26th of the month.

- Petroleum Marketing Monthly Updated on the 20th of the month.

- Natural Gass Monthiy

Updated on the 20th of the month.

- Weekly Corl Production

Updated on Fridays by noon.

- Quarkerly Coal Report

Updated 40 days after the end of the quarter.

- Electric Power Monthly

Updated during the first week of the month.

- Monthly Energy Reoiew

Updated the last week of the month.

- Shost-Term Energy Outloak

Updated 60 days after the end of the quarter.

- Winter Fuels Report (October through April) Prcpane inventory data updated Wednesdays at 5 p.m. All other data updated Thursdays (Friday in event of a holiday) at 5 p.m. 


\section{Otfice of Coal, Nuclear, Electric and Alternate Fuels Electrlc Power Industry Related Data: Avallabie In Electronic Form \\ (as of November 1997)}

\begin{tabular}{|c|c|c|c|c|c|c|}
\hline & \multicolumn{3}{|c|}{ Internet } & \multirow[b]{2}{*}{ CDROM } & \multirow[b]{2}{*}{ EPU: } & \multirow[b]{2}{*}{ Dlskentio } \\
\hline & $\begin{array}{l}\text { Portable } \\
\text { Document } \\
\text { Format } \\
\text { (PDF) }\end{array}$ & $\begin{array}{l}\text { Exucutable } \\
\text { Data FHes }\end{array}$ & $\begin{array}{l}\text { Hypentexi } \\
\text { Wharkup } \\
\text { Language } \\
\text { (HTML) }\end{array}$ & & & \\
\hline \multicolumn{7}{|l|}{ Surveys: } \\
\hline $\begin{array}{l}\text { Form ElA-412: Annual Report of } \\
\text { Putlle Electric Utilities }\end{array}$ & & $x$ & & & & $x$ \\
\hline $\begin{array}{l}\text { Form ElA-760: Monthly Power Flant } \\
\text { Foport }\end{array}$ & & $x$ & & $x$ & & $x$ \\
\hline $\begin{array}{l}\text { Form ElA-787: Steam-Electric } \\
\text { Operation and Design Repont }\end{array}$ & & $\mathrm{x}$ & & & & $x$ \\
\hline $\begin{array}{l}\text { Form ElA-92s: Monthy Electric } \\
\text { Ltillyy Sales and Fevenue Report } \\
\text { with State Distributions }\end{array}$ & & $x$ & & $x$ & & $\mathbf{x}$ \\
\hline $\begin{array}{l}\text { Form ElA-8G0: Annual Electalc } \\
\text { Generalor Report }\end{array}$ & & $x$ & & $\mathbf{x}$ & & $x$ \\
\hline $\begin{array}{l}\text { Form ElA-861: Annual Electric Utilty } \\
\text { Fioport }\end{array}$ & & $x$ & & $\mathbf{x}$ & & $x$ \\
\hline $\begin{array}{l}\text { FEAC Form 1: Antwad Report of } \\
\text { Major Electrio Utilitites, Lloonseas, } \\
\text { end Others }\end{array}$ & & $x$ & & & & $x$ \\
\hline $\begin{array}{l}\text { FERC Form 423: Monthy Peport of } \\
\text { Cosi and Ouality of Fuels for Electric } \\
\text { Plants }\end{array}$ & & $x$ & & & & $\mathbf{x}$ \\
\hline \multicolumn{7}{|l|}{ Publleation: } \\
\hline Electric Power Monthly & $x$ & & & $x$ & $x$ & \\
\hline $\begin{array}{l}\text { Data tables for Form ElA-759, } \\
\text { Form EIA-826, Fom EIA-860 (new } \\
\text { urits only), and FERC Fomin } 428 \text { a }\end{array}$ & $x$ & & $x$ & & & \\
\hline Electric Power Annual Vaktme I & $\mathrm{x}$ & & $x$ & $x$ & $x$ & \\
\hline Electric Powar Annual Volume II & $x$ & & $x$ & $\mathbf{x}$ & $x$ & \\
\hline $\begin{array}{l}\text { Inventory of Power Plants in the } \\
\text { United States }\end{array}$ & $x$ & & & $\mathbf{x}$ & & \\
\hline Eloctrle Sales and Revenup & $\mathrm{x}$ & & $x$ & $x$ & $x$ & \\
\hline $\begin{array}{l}\text { Financial Statistics of Malor U.S. } \\
\text { Imvestor Ownod Electric Uttilties }\end{array}$ & $x$ & & & $x$ & $x$ & \\
\hline 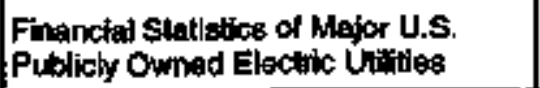 & $x$ & & & $x$ & $x$ & \\
\hline
\end{tabular}

Note: If you have any questions and/or need additionef information, please contact the National Energy Informelion Center at (202) 588-8600. 


\section{Preface}

The Electric Power Monthly (EPM) presents monthly electricity statistios for a wide audience including Congress, Federal and State agencies, the electric ubility industry, and the general public. The purpose of this publication is to provide energy decisionunakers with accurate and timely information that may be used in forming varkous perspectives on electric issues that lie ahead. The BJA collected the information in this report to fulfill its data collection and dissemination responsibilities as specifited in the Federal Energy Administration Act of 1974 (Public Law 93-275) as amended.

\section{Background}

The Cogl and Electric Data and Renewables Division; Office of Coal, Nuclear, Electitic and Alternate Fuels, Energy Information Admunistration (EIA), Department of Energy prepares the EPM. This publication provides monthly statistics at the State, Census division, and U.S. levels for net generation, fossil fuel consumption and stocks, quantity and quality of fossil fuels, cost of fossil fuels, electricity retail sales, associated revenue, and average revenue per kilowatthour of electricity sold. In addition, data on net generation, fuel consurnption, fuel stocks, quantity and cost of fossil fuels are also displayed for the North American Electric Reliability Council (NERC) regions.

The EIA publishes statistics in the EPM on net generation by energy sounce; consumption, stocks, quarntity, quality, and cost of fossil fuels; and capability of new generating units by company and plant.

\section{Date Sources}

The EPM contains information from seven data sources: Form EIA-759, "Monthly Power Plant Report"; Federal Energy Regulatory Commission (FERC) Form 423, "Monthly Report of Cost and Quality of Fuels for Electric Plants"; Form EIA-826, "Montfly Electric Utility Sales and Revenue Report with State Distributions"; Form EIA-900, "Monthly Nonutility Sales for Resale Report"; Form ElA-861, "Anmual Electric Utility Report"; Form ElA-860, "Annual Electric Generator Report," and Form EIA-867, "Annual Nonutility Power Producer Report" Coples of these forms and their instructions may be obtained from the National Energy Information Center. A detailed description of these forms is in Appendix B, "Technical Notes." 



\section{Contents}

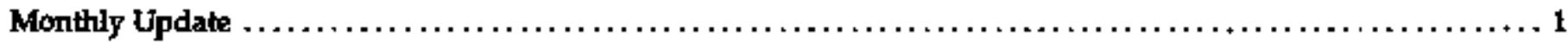

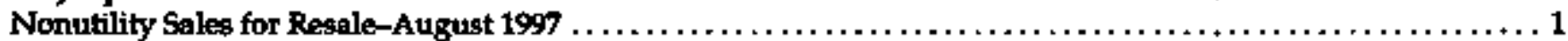

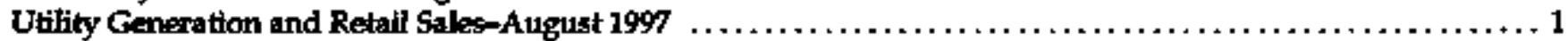

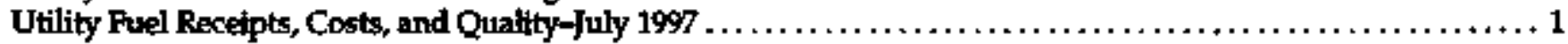

Industry Developments $\ldots \ldots \ldots \ldots \ldots \ldots \ldots \ldots \ldots \ldots \ldots \ldots \ldots \ldots \ldots \ldots \ldots, \ldots, \ldots \ldots, \ldots, \ldots, \ldots \ldots \ldots \ldots \ldots, 9$

Erron Batties PECO Energy for Phlladelphia Area Customers $\ldots \ldots \ldots \ldots \ldots \ldots \ldots \ldots \ldots \ldots \ldots, \ldots \ldots \ldots \ldots, 9$

PECO Energy Ct1stomers Show Heavy Interest in Pilot Program $\ldots \ldots \ldots \ldots \ldots \ldots \ldots \ldots \ldots \ldots \ldots \ldots \ldots \ldots \ldots, 9$

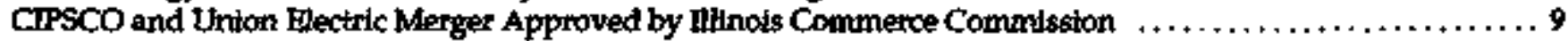

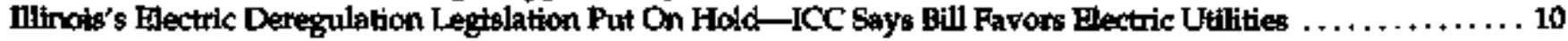

GPU To Sell Generation Assets, Set To Concentrate On Distribution Business $\ldots \ldots \ldots \ldots \ldots \ldots \ldots \ldots \ldots \ldots 11$

Coal Detiveries Via Union Pacific Railroad Behind Schedule $\ldots \ldots \ldots \ldots \ldots \ldots \ldots \ldots \ldots \ldots \ldots \ldots \ldots \ldots, 11$

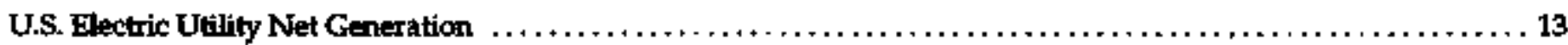

U.S. Electric Utility Consumption of Fossil Fuels $\ldots \ldots \ldots \ldots \ldots \ldots \ldots \ldots \ldots \ldots \ldots \ldots \ldots \ldots, \ldots \ldots \ldots \ldots \ldots \ldots, 25$

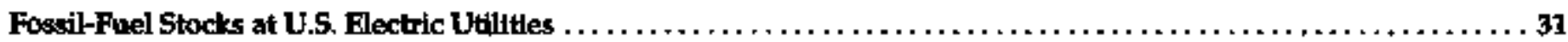

Receipts and Cost of Fossil Fuels at U.S. Electric Utilities $\ldots \ldots \ldots \ldots \ldots \ldots \ldots \ldots \ldots \ldots \ldots \ldots \ldots \ldots \ldots \ldots \ldots, 35$

US. Electric Utility Sales, Revenue, and Average Revenue per Kilowatthour $\ldots \ldots \ldots \ldots \ldots \ldots, \ldots, \ldots, \ldots \ldots \ldots, 53$

Monthly Plant Aggregates: U.S. Electric Utility Net Generation, Fuel Consumption, and Fuel Stocks . . . . . . . 67

Monthly Plant Aggregates: U.S. Electric Utility Receipts, Cost, and Quality of Fossit Fuels $\ldots \ldots \ldots \ldots \ldots \ldots \ldots \ldots 111$

Appendices

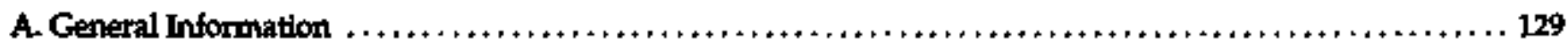

B. Technical Notes,$\ldots+\ldots+\ldots+, \ldots+\ldots+\ldots++\ldots+\ldots+\ldots+\ldots+\ldots+\ldots+\ldots+\ldots \ldots+\ldots \ldots+\ldots+\ldots \ldots+\ldots+\ldots+\ldots+\ldots \ldots 133$

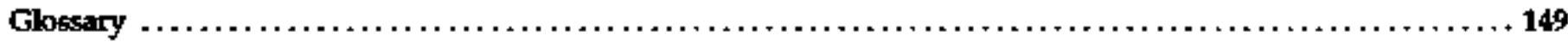




\section{Tables}

1. New Electric Generating Units by Operating Company, Plant, and State, and Retirements and Total Capability at U.S. Electric Utilities, $1997 \ldots \ldots \ldots \ldots \ldots \ldots \ldots \ldots \ldots \ldots \ldots \ldots+\ldots \ldots \ldots \ldots \ldots \ldots \ldots \ldots+\ldots$

2. U.S. Electric Power Summary Statistics $\ldots \ldots \ldots \ldots \ldots+\ldots+\ldots \ldots+\ldots \ldots+\ldots \ldots \ldots+\ldots \ldots \ldots \ldots \ldots \ldots, 7$

3. Ut.S. Electric Power Industry Net Generation, 1990 Through August $1997 \ldots \ldots \ldots \ldots \ldots \ldots \ldots \ldots \ldots \ldots$

4. U.S. Electric Utility Net Generation by Nonuenewable Energy Source, 1990 Through August $1997 \ldots \ldots \ldots+14$

5. U.S. Electric Utility Net Generation by Renewable Energy Source, 1990 Through August $1997 \ldots \ldots \ldots \ldots+15$

6. Electric Utility Net Generation by NERC Regjon and Hawaii $\ldots \ldots \ldots \ldots \ldots \ldots \ldots \ldots \ldots \ldots \ldots \ldots \ldots, 16$

7. Electric Utility Net Generation by Census Division and State $\ldots \ldots \ldots \ldots \ldots \ldots \ldots \ldots \ldots \ldots \ldots \ldots \ldots, \ldots \ldots \ldots$

8. Electric Utility Net Generation from Coal by Census Division and State $\ldots \ldots \ldots \ldots \ldots \ldots \ldots \ldots \ldots \ldots \ldots, 18$

9. Electric Utility Net Generation from Petroleum by Census Division and State.$\ldots \ldots \ldots \ldots \ldots \ldots \ldots \ldots \ldots$ 19

10. Electric Utility Net Generation from Gas by Census Division and State $\ldots \ldots \ldots \ldots \ldots \ldots \ldots \ldots \ldots \ldots \ldots \ldots$

11. Electric Utiflty Hydroelectrlc Net Generaton by Census Division and State $\ldots \ldots \ldots \ldots \ldots \ldots \ldots \ldots \ldots \ldots \ldots, 21$

12. Electric Utility Nuclear-Powered Net Generation by Census Division and State $\ldots \ldots \ldots \ldots \ldots \ldots \ldots \ldots \ldots 22$

13. Electric Utitity Net Generation from Other Energy Sources by Census Division and State $\ldots \ldots \ldots \ldots \ldots \ldots \ldots 23$

14. U.S. Electric Utility Cossumption of Eossil Fuels, 1987 Through August $1997 \ldots \ldots \ldots \ldots \ldots \ldots \ldots \ldots \ldots \ldots$

15. Electric Utilty Consum

16. Electric Utility Consumption of Petroleum by NERC Region and Hawain $\ldots \ldots \ldots \ldots \ldots \ldots \ldots \ldots \ldots \ldots \ldots \ldots, 26$

17. Electric Utility Consumption of Gas by NERC Reglon and Hawaii $\ldots \ldots \ldots \ldots \ldots \ldots \ldots \ldots \ldots \ldots \ldots \ldots \ldots, \ldots \ldots$

18. Eectric Utility Consumption of Coal by Census Division and State $\ldots \ldots \ldots \ldots \ldots \ldots \ldots \ldots \ldots \ldots \ldots, 28$

19. Electric Utility Consumption of Petroleum by Cersus Division and State $\ldots \ldots \ldots \ldots \ldots \ldots \ldots \ldots \ldots \ldots, 29$

20. Electric Utility Consumption of Gas by Censes Division and State $\ldots \ldots \ldots \ldots \ldots \ldots \ldots \ldots \ldots \ldots \ldots \ldots, \ldots \ldots$

21. U.S. Elextric Utility Stocks of Coal and Petroleum, 1987 Through August $1997 \ldots \ldots \ldots \ldots \ldots \ldots \ldots \ldots \ldots, \ldots 1$

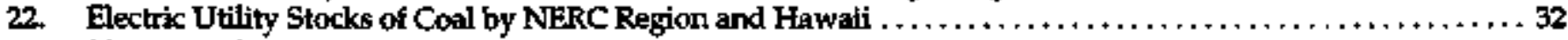

23. Electric Utility Stocks of Petroleum by NERC Region and Hawaii $\ldots \ldots \ldots \ldots \ldots \ldots \ldots \ldots, \ldots \ldots \ldots+\ldots, 32$

24. Electric Utility Stocks of Coal by Census Division and State $\ldots \ldots \ldots \ldots \ldots \ldots \ldots \ldots \ldots \ldots \ldots \ldots \ldots, 33$

25. Electric Utility Stocks of Petrotetum by Census Division and State $\ldots \ldots \ldots \ldots \ldots \ldots \ldots \ldots \ldots \ldots \ldots \ldots \ldots, 34$

26. U.S. Electric Utilty Recelpts of and Average Cost for Fossil Fuels, 1987 Through July $1997 \ldots \ldots \ldots \ldots \ldots \ldots 36$

27. Electric Utility Receipts of Coal by NERC Region and Hawaii $\ldots \ldots \ldots \ldots \ldots \ldots \ldots \ldots \ldots \ldots \ldots \ldots \ldots \ldots \ldots \ldots$

28. Average Cost of Coal Delivered to Electric Utilities by NERC Region and Hawaii $\ldots \ldots \ldots \ldots \ldots \ldots \ldots \ldots, \ldots, \ldots$

29. Electric Utility Receipts of Petroleum by NERC Region and Hawaih $\ldots \ldots \ldots \ldots \ldots \ldots \ldots \ldots \ldots \ldots \ldots \ldots, 38$

30. Average Cost of Petroleum Delivered to Electric Utiltities by NERC Region and Hawaii $\ldots \ldots \ldots \ldots \ldots \ldots, 38$

31. Electric Utility Recetpts of Gas by NERC Region and Hawail $, \ldots \ldots \ldots \ldots \ldots \ldots \ldots \ldots \ldots \ldots \ldots \ldots \ldots, 39$

32. Average Cost of Gas Delivered to Electric Utillites by NERC Region and Hawail $\ldots \ldots \ldots \ldots \ldots \ldots \ldots \ldots \ldots, \ldots 9$

33. Electric Utility Receipts of Coal by Type, Census Division, and State, July $1997 \ldots \ldots \ldots \ldots \ldots \ldots \ldots \ldots \ldots 40$

34. Recejpts and Average Cost of Coal Delivered to Electric Utilities by Census Division and State $\ldots \ldots \ldots \ldots 41$

35. Receipts and Average Cost of Coal Delivered to Electric Utilities by Type of Punctuase, Mining Method,

Census Division, and State, July $1997 \ldots \ldots \ldots \ldots \ldots \ldots \ldots \ldots \ldots \ldots \ldots \ldots \ldots \ldots \ldots \ldots \ldots \ldots \ldots \ldots \ldots 42$

36. Recelpts and Average Cost of Coal Dellvered to Electric Utilities by Stlfur Content, Census Division,

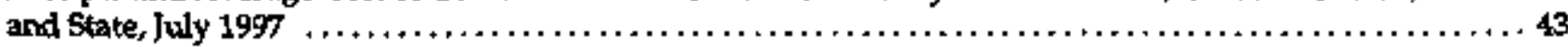

37. Electric Utilty Recelpts of Petrolenum by Type, Census Division, and State, July $1997 \ldots \ldots \ldots \ldots \ldots+\ldots \ldots 45$

38. Receipts and Average Cost of Petroleum Delivered to Electric Utilities by Census Division and State ......46

39. Receipts and Average Cost of Petroleum Delivered to Electric Utilities by Type of Purchase,

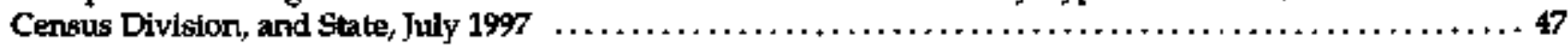

40. Receipts and Average Cost of Heavy Oil Detivered to Flectric Utitities by Sulfur Content, Census Division, and State, July $1997, \ldots \ldots \ldots \ldots \ldots \ldots \ldots \ldots \ldots \ldots \ldots \ldots \ldots \ldots \ldots \ldots \ldots \ldots \ldots \ldots+\ldots \ldots \ldots \ldots \ldots, 48$

41. Electric Utility Recejpts of Gas by Type, Census Division, and State, July $1997 \ldots \ldots \ldots \ldots \ldots \ldots \ldots \ldots \ldots$.

42. Receipts and Average Cost of Gas Delivered to Electric Utilities by Census Division and State, $\ldots \ldots \ldots \ldots 51$

4. Recespts and Average Cost of Gas Delivered to Electric Utilities by Type of Purchase, Census Diviston, and Stabe, July $1997 \ldots \ldots \ldots \ldots \ldots \ldots \ldots+\ldots \ldots+\ldots+\ldots \ldots \ldots \ldots+\ldots+\ldots+\ldots+\ldots \ldots+\ldots+\ldots \ldots \ldots, 52$

44. U.S. Electric Utility Retail Sales of Elextricity by Sector, 1987 Through August $1997 \ldots \ldots \ldots \ldots \ldots \ldots \ldots . \ldots 4$ 


\section{Tables, continued}

45. Estinuted Electric Utility Retail Sales of Electricity to Ultimate Constinters by Sector, Census Division, and State, Autgust 1997 and $1996 \ldots \ldots \ldots \ldots \ldots \ldots \ldots \ldots \ldots \ldots \ldots \ldots \ldots \ldots \ldots \ldots \ldots \ldots \ldots \ldots \ldots \ldots \ldots \ldots$

46. Estimated Coefficients of Variation for Electric Utility Retail Sales of Electricity by Sector, Census Division,

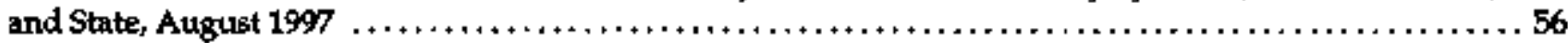

47. Estimabed Elextric Utitity Retail Sales of Electricty to Ultimate Consumers by Sector, Census Diviston, and

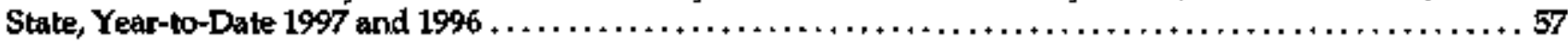

48. Revenue from U.S. Electric Utility Retail Sales of Electricity to Utimate Consumers by Sector, 1987 Through August $1997 \ldots \ldots \ldots \ldots \ldots \ldots \ldots \ldots \ldots+\ldots \ldots \ldots \ldots \ldots \ldots \ldots \ldots \ldots \ldots \ldots \ldots \ldots \ldots+58$

49. Estimated Revenue from Electric Utility Retail Sales of Electricity to Ultimate Consumers by Sector,

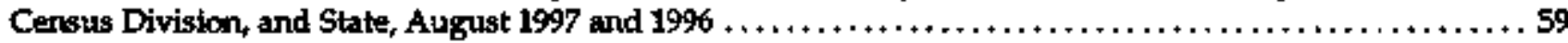

50. Estimated Coefficients of Variation for Revenue from Electric Utility Retail Sales of Electricity by Sector, Census Division, and State, August $1997 \ldots \ldots \ldots \ldots \ldots \ldots \ldots \ldots \ldots \ldots \ldots \ldots \ldots \ldots \ldots \ldots \ldots+\ldots \ldots+\ldots+60$

51. Estimated Revenue from Electric Utility Retail Sales to Ultimate Consumers by Sector, Census Division,

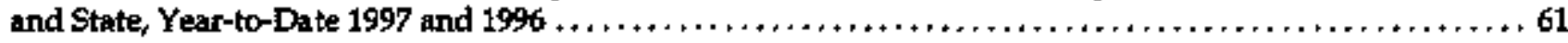

52. U.S. Flectric Utility Average Revenue per Kilowatthour by Sector, 1987 Through August $1997 \ldots \ldots \ldots \ldots \ldots$

53. Estimated Electric Utility Average Revenue per Kilowatthour by Sector, Census Division, and State, August 1997 and $1996 \ldots \ldots \ldots \ldots+\ldots \ldots \ldots+\ldots \ldots \ldots \ldots \ldots+\ldots \ldots \ldots+\ldots \ldots \ldots \ldots \ldots \ldots \ldots \ldots \ldots+63$

54. Estimated Coefficients of Variation for Electric Utility Average Revenue per Kilowatthour by Sector, Census Division, and State, August $1997 \ldots \ldots \ldots \ldots \ldots \ldots \ldots \ldots \ldots \ldots \ldots \ldots \ldots \ldots \ldots \ldots \ldots \ldots \ldots \ldots \ldots \ldots, 64$

55. Estimated Electric Utility Average Revenue per Kilowatthour by Sector, Census Division, and State, Year-to-Date 1997 and $1996 \ldots \ldots \ldots+\ldots \ldots+\ldots \ldots \ldots+\ldots \ldots \ldots \ldots+\ldots \ldots \ldots \ldots \ldots+\ldots \ldots \ldots \ldots \ldots \ldots 65$

56. U.S. Electric Utility Net Generation, Fuel Consumption, and Fuel Stocks by Company and Plant,

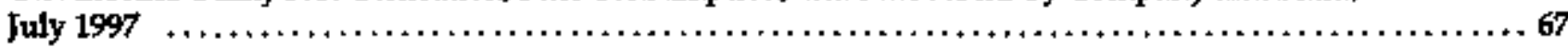

57. Receipts, Average Cost, and Quality of Fossil Fuels Delivered to U.S. Electric Utitities by Company

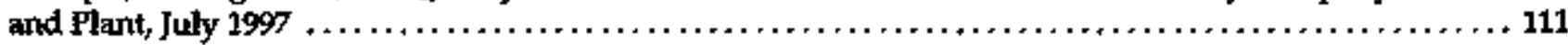

B1. Average Heat Content of Fossil-Fuel Receipts, July $1997 \ldots \ldots \ldots \ldots \ldots \ldots \ldots \ldots \ldots \ldots \ldots \ldots \ldots \ldots$

B2. Comparison of Estimated/Preliminary Versus Final Published Data at the U.S. Level, 1993 Through 1996 , 144

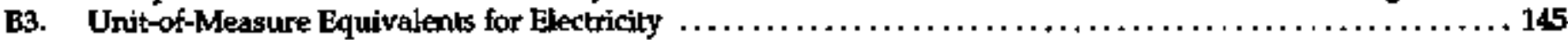
B4. Comparison of Sample Versus Census Published Data at the U.S. Level by End-Use Sector, 1995 and 1996.145 B5. Estimated Coefficients of Variation for Electric Utilty Net Generation by State, August $1997 \ldots \ldots \ldots \ldots .147$ B6. Estimated Coefficients of Variation of Electric Utility Fuel Consumption and Stocks by State, August 1997,148

\section{IIIustrations}

B1. North American Electric Reljablility Council Regions for the Contiguous United States and Alaska ....... 146 


\section{Monthly Update}

\section{Nonutility Sales for Fesale-August 1997}

Total estimated sales of elextricity for resale by nonutility power producers in the United States were 20 billion kilowatthours for August 1997. This reflected a level of sales for resale that was 7 percent higher than the level in August 1996, and a slight decrease from the prior month of July 1997 .

\section{Utfilty Generation and Retall Sales- August 1997}

Generation. U.S. net generation of electricity was 294 billion kilowatthours, 4 billion kilowatthours (1 percent) more than the amount reported in August 1996. The energy source with the largest kilowatithour increase in generation compared with August of last year was gas (higher by 2 billion kilowatthours. Electricity generated from petroleum and conventional hydroelectric power was also above the amount reported during the same period last year, higher by 20 and 3 percent, respectively.

Stles. Total sales of electricity to ultimate consumers in the United States during August 1997 were 292 billion kilowatthours, 3 billion kilowatthours (1 percent) higher than compared with a year ago at this time. Retail sales of electricity in all major end-use sectors during the month were higher conpared with August 1996. The only quantitative increase (3 bilition kilowatthours) in the sale of elestricity from july to Augtist cocurred in the industrial sector.

\section{Utility Fuet Receipts, Costs, and Quality- Juhy 1997}

Coal. July 1997 receipts of coal at electric utilities totaled 74 million short tors, down 1 million short tons from July
1996. While receipts of coal fell fron the prior year level, coal consumption for the month rose 4 million short tons to set an all-time monthly consumption record of 84 million short tons. The combination of these factors resulted in end-of-momth stooks of bituminous coal falling to the 101 millton short ton level. Sorme of this decrease can be traced to a decline in stocks of coal tn the West South Central Census Division. According to pubilshed reports, many electric vitilities that are located in this onstus division and served by the Union Pacific Railroad are not receiving all of their contracted coal deliveries. (See the "Industry Developments" section, page 11, for further detailk.)

Fetroleum. Receipts of petroleum totaled 12 million barrels, up slightly from July 1996. Consumption of fuel oil continues at a historically low rate. Competition from other fuels is a sigruficant factor in the low burn-rate. Year-to-date receipss of petroletm totaled 62 million barsels, down from 67 mtilion barrels in 1996. However, in the New England Census Division, year-to-date receipts were up 9 million barrels (85 percent) from 1996 levels as electric utitities truned petroleum (and gas) to corrpensate for several nuclear plants that have been out of servire during much of 1997. The Middle Atlantlc and the South Athantic Census Divisions posted large decreases in year-to-date receipts of petroleum due in part to an increase in the use of gas.

Gas. Receipts of gas in July 1997 totaled 374 bitlion cubtc feet (Bcf), up from 346 Bcf reported in July 1996. This increase in receipts was due in part to a rectuction in the cost of gas. Year-to-date receipts of gas totaled 1,516 billion cubic feet $(\mathrm{B} C \mathrm{C})$, as compared with 1 A80 Bof reported in 1996. 



\section{Electrlcity Supply and Demand Forecast for $1997^{\dagger}$}

The EIA prepares a short-term forecast for electricity that is published in the Short-Term Energy Outlook. This page provides that forecast for the current year along with explanations behind the forecast.

- In 1997 total electricity demand is expected to continue to grow, but at slower rates than the 2.7 percent seen in 1996. This is due partly to the expectation of scomewhat slower economic growth, as well as the assumption of normal weather, which means fewer cooling degree days than in 1996.

- Residential demand for electricity in 1997 is projected to decrease 2.8 percent from 1996 . Normal weather this year implies higher demand in the first quarter which will decrease in the sunumer, as is notmal.

- Commerial sector demand is projected to rise by 0.9 percent in 1997 due primarily to expanding exployrnent Industrial demand is projected to grow by 2.3 percent in 1997 reflecting the contrinuing growth in industrial output.

- U.S. utilities are expected to generate about 0.3 percent less electricity in 1997. Nonutlity generation is expected to increase by 5.1 percent in 1997, as a result of capacity additions.

- Hydropower generation by electric uttities is expected to increase by 4.0 pencent in 1997 due to the increased atailability of hydroelectric generation resulting from high runoff conditions in the Pacific Northwest, created by above-average rainfall in the latter half of 1996 .

- Nuclear power generation is expected to decrease by 7.9 percent from 1996 levels. This can be attributed mainiy to the recent shutuown of a substantial quantity of nuclear generating capactty, especially in the New England area.

- Net imports of electricity fron Canada are forecast to be 2.9 percent lower than in 1996, continuing a twoyear downward trend which is actually a retum to normal from the record high levels in 1994.

'Erergy Informaton Adminlstrabon, Short-Term Energy Outtook 4th Quarter 1997, DOE/EA-02(12 $97 / 40$ ] (Waskington, DC, October 1997).

"Further questlons on this section may be directed to Rekecca McNemry at 202-426-1251 or via Internet at rmmernedela.doe.gov.
Elestrlaty Supply and Domand (Billion Kiowatuthours)

\begin{tabular}{|c|c|c|c|c|c|}
\hline & \multicolumn{5}{|c|}{1807} \\
\hline & 19t & and & $3 \mathbf{v}$ & 4in & Ytar \\
\hline \multicolumn{6}{|l|}{ 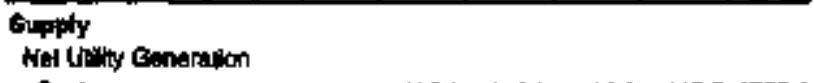 } \\
\hline Gow $\ldots, \ldots \ldots \ldots$ & $\$ 340$ & 4140 & $\$ 6.6$ & 44.5 & 1750.1 \\
\hline Petroleam $\ldots \ldots \ldots \ldots \ldots \ldots$ & $17 \%$ & 15.4 & $2 \times, 6$ & 14.0 & 84 \\
\hline 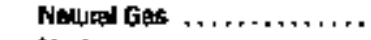 & 45.4 & 291 & 97.3 & 55.4 & 267,4 \\
\hline Mublest $\ldots \ldots \ldots \ldots \ldots \ldots$ & to0.9 & tw4t & 167.7 & 156.5 & 60 \\
\hline Hytoelectrilo $\ldots \ldots \ldots \ldots$ & oasts & $\$ 000$ & 78.6 & 72.3 & 341.2 \\
\hline Geothermel and Other " . . . . & 1.4 & 1.8 & 1.7 & 9.7 & 7.0 \\
\hline Suttoded $\ldots \ldots \ldots \ldots \ldots \ldots$ & rate & 740.0 & 827.6 & 748,3 & 3069.7 \\
\hline
\end{tabular}

Nomatily Gengration *

Con ................. 16. 16.510 .3 te. 66.4

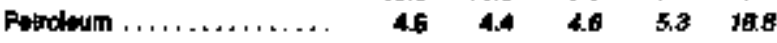

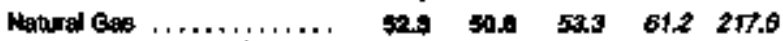

Olher Gasequa Fuets $5 \ldots, \quad 3.0 \quad 20 \quad 3.1 \quad 3.6 \quad 12.5$

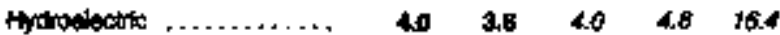

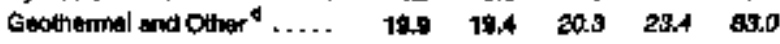

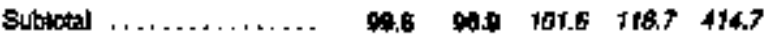

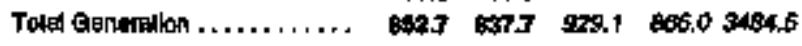

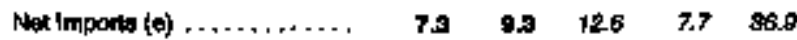

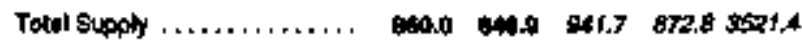

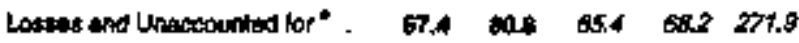

Doming

Eladido Lotity sulas

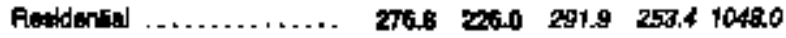

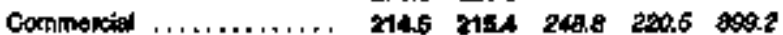

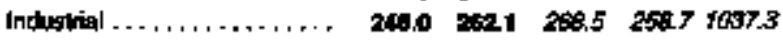

Other ................. 20.4 2010 20.6 25.5 58.3

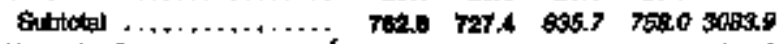

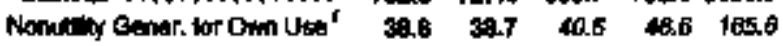

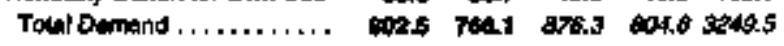

Mano:

Nonutilly Steve to

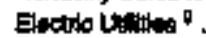

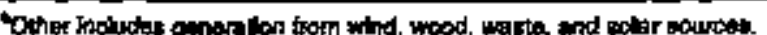

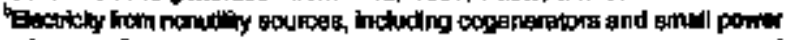

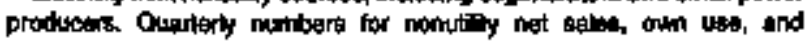

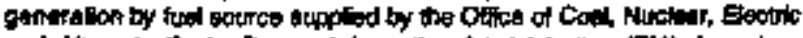

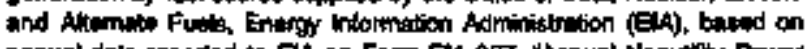

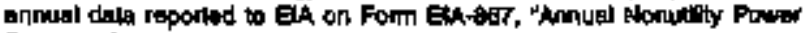
Producer Peport"

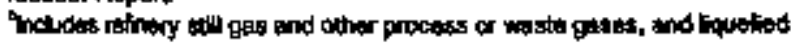
pofroloum gasin.

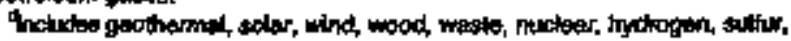

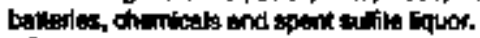

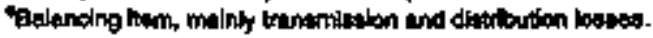

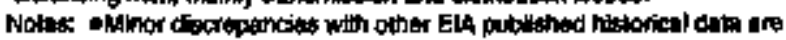

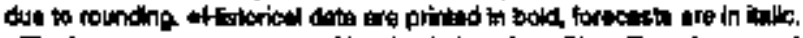

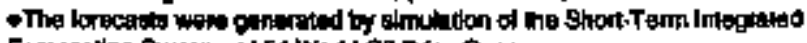

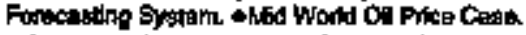

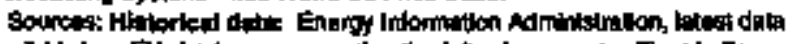

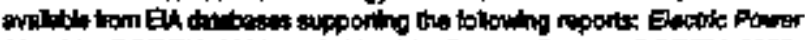

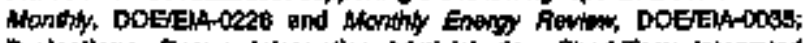

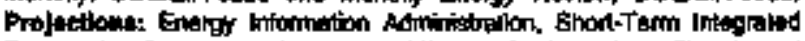

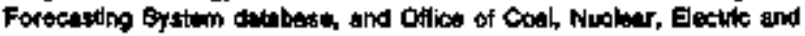
Alloming Funela. 


\section{Heating Degree-Days by Census Dlviston, August 1997}

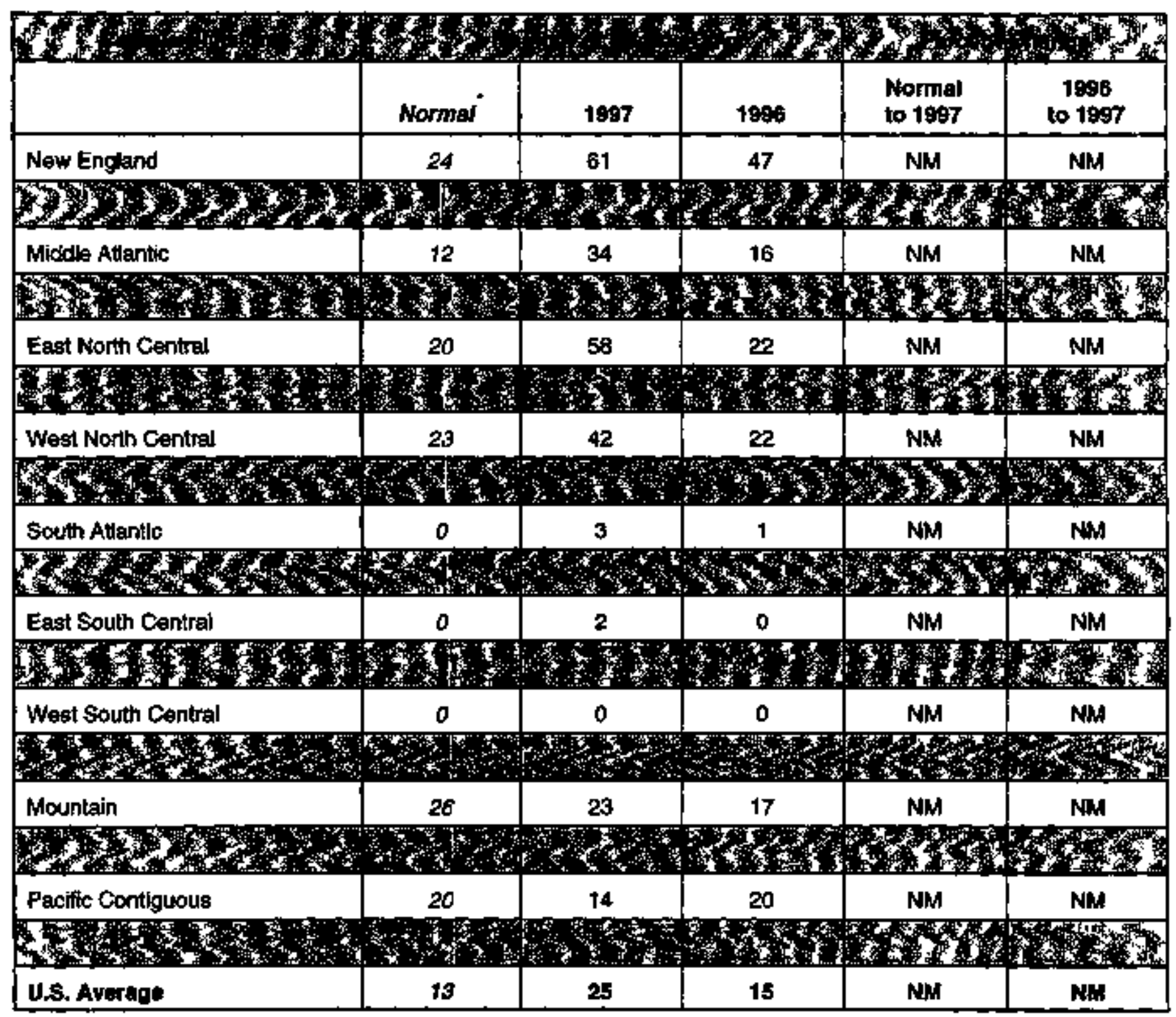

"Normal" is based on calculations using lemperature data from 1961 through 1990.

Nil = Not meaningitul (nomal is less than 100 or railo is incalculable).

Notes: * Heating Degree-days are relabve measures of outdoor air temperature used as indices of heating energy requsrements. - Heating degreedeys are the number of degrees per day that the daily average temperature falls below 65 dogrees Fahrenhert. The daihy average temperature is the mean of the minimum and maximum temperatures in a 24hour perked.

Source: National Oceanic and Atmospheric Administration's National Weather Service Clumate Analysis Center. 


\section{Coolting Degree-Days by Census Division, August 1997}

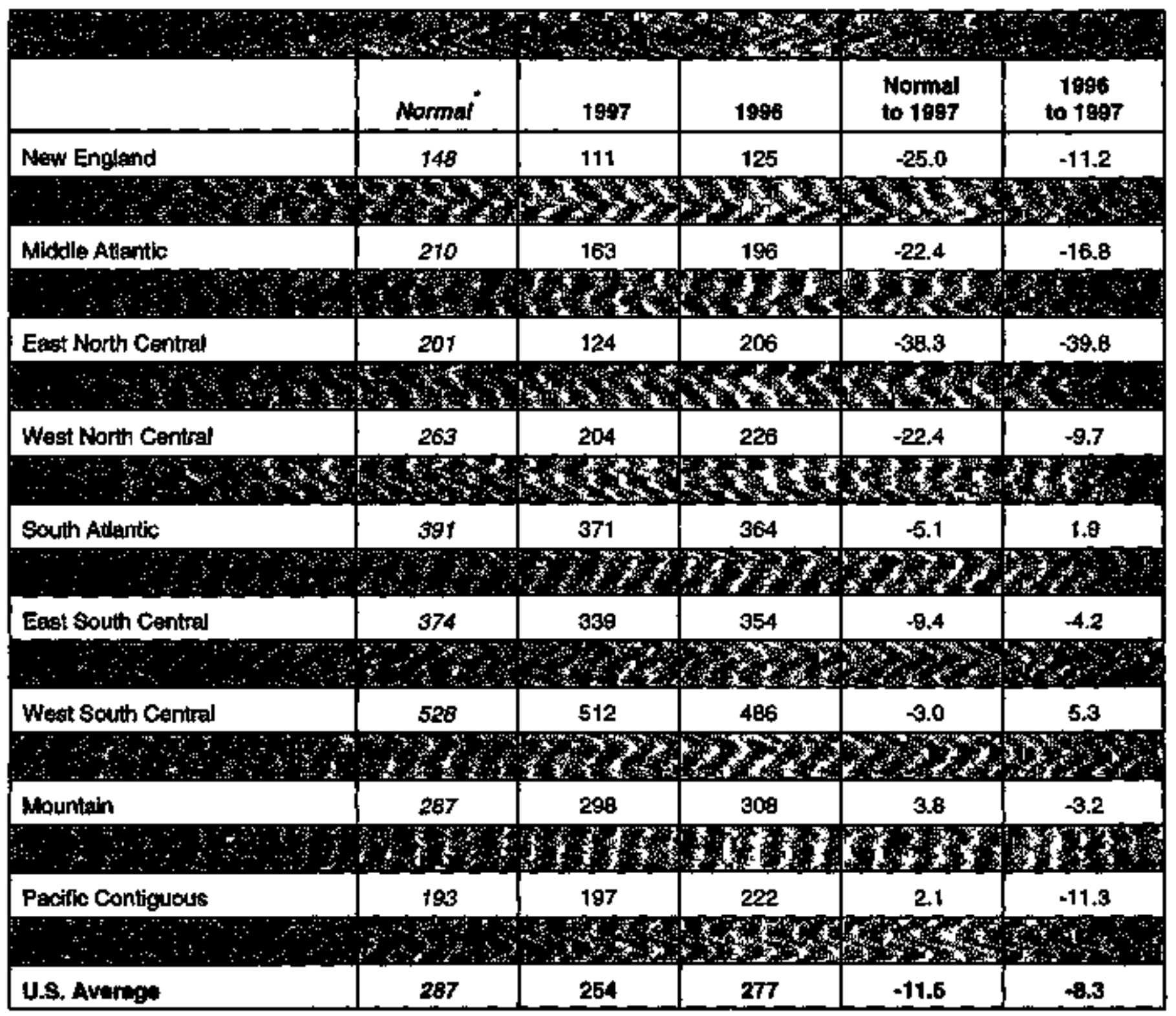

"Nomal" is based on calculations using temperature data for 1961 through 1990.

NM = Not meaningful (notmal Is less than 100 or ratio is incalculable).

Noles: - Cooling degree-days are relative measures of outdoor air temperature used as indices of cooling energy requirements. - Cooling degree-days are the number of degress per day that the daily average temperature falls above 65 degrees Fahrenthett. The delly average temperature is the mean of the minimum and meximum temperatures in a $24-$ hour period.

Source: National Oceanic and Atmospheric Administratlon's National Weather Service Cumate Analysis Center. 
Toble 1. New Blactric Generating Units by Openting Company, Plant, and Stote, and Retirements and Totol Capahtity at U.S. Electric Ut:"ities, 1997

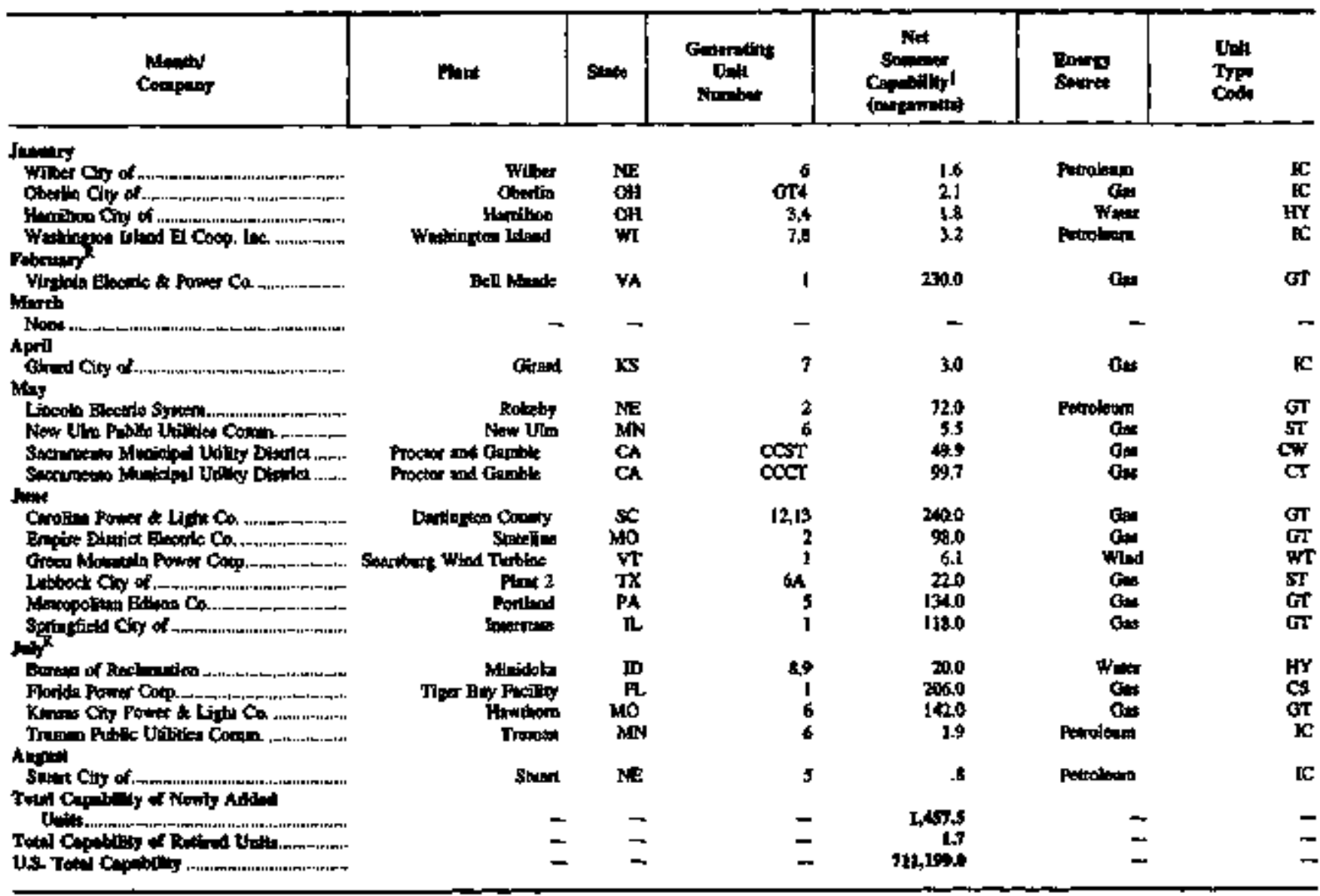

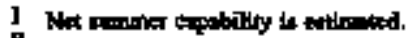

Revist.

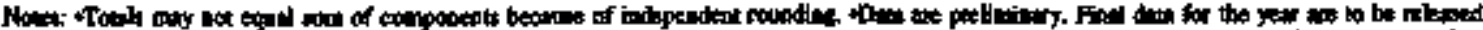

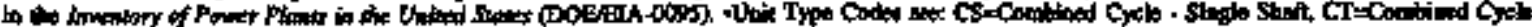

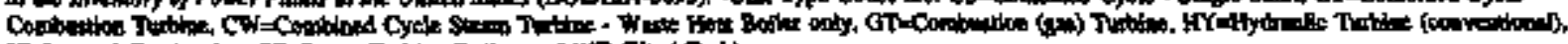

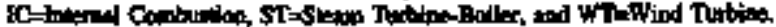

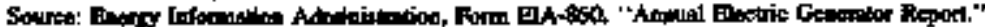


Tabte 2. U.S. Electre Power Summary Satistics

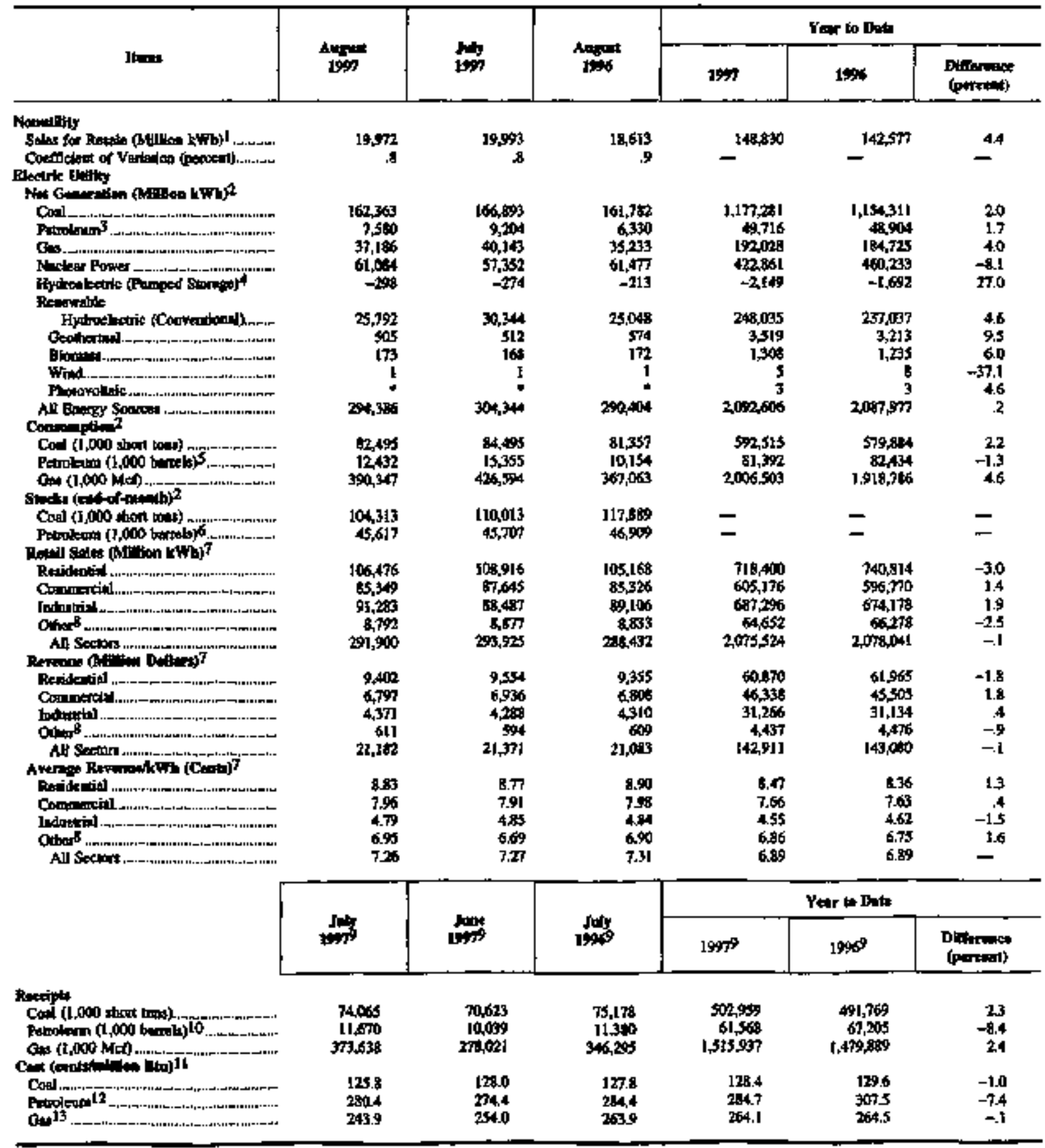

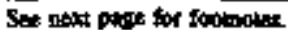




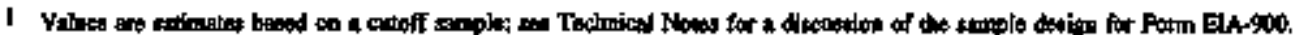

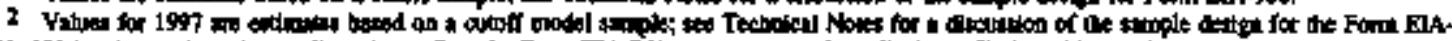

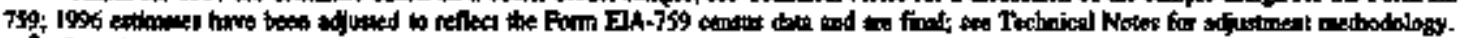

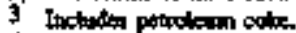

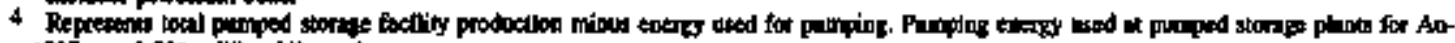

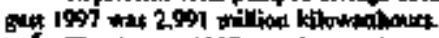

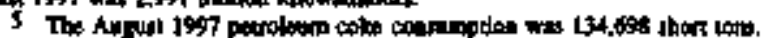

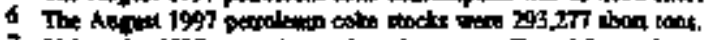

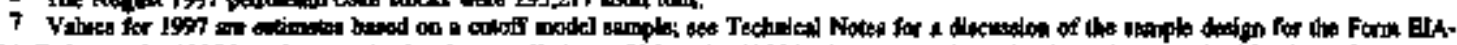

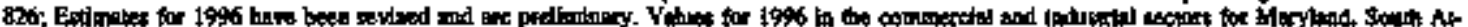

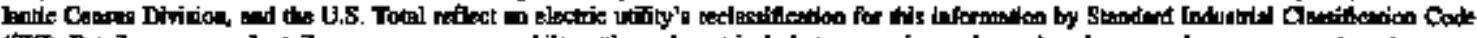

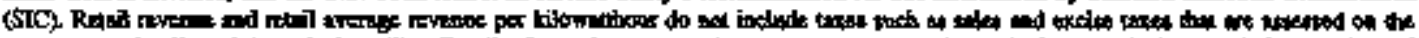

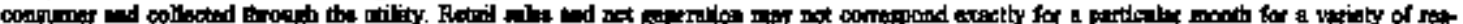

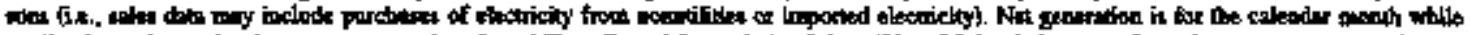

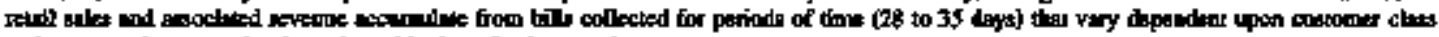

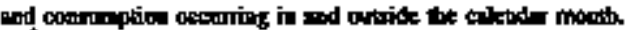

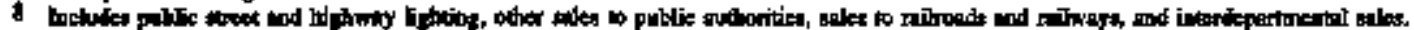

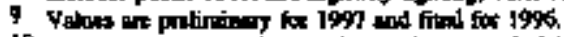

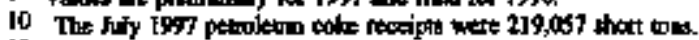

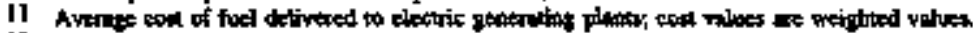

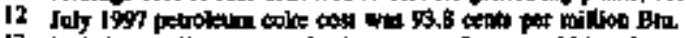

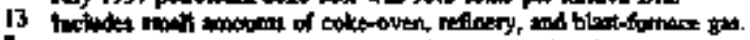

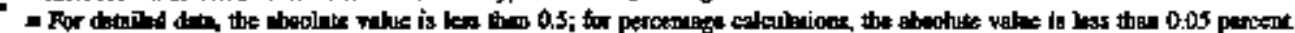

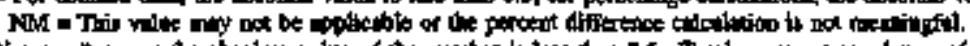

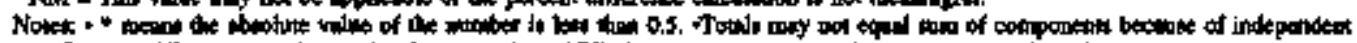

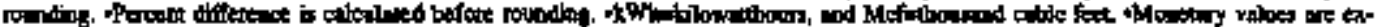
pressed in bornined bathe.

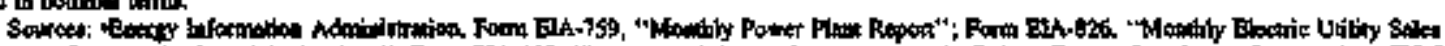

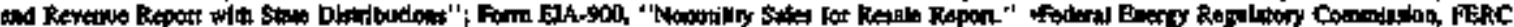

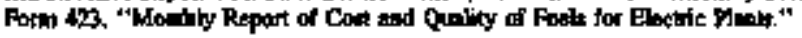




\section{Industry Developments}

\section{Enron Bettles PECO Energy for Phlladejphia Area Customers}

Enuron Corporation (Enron) has fled what it calls "The Choice Plan" with the Pennsylvania Public Utility Com* mission (PUC) in a bid to take Philadelphia area electric customers from PECO Finergy Company (PECO). According to the Enron proposal, all Philadelphia area electric customers would receive a 20-percent rate cut starting September 1, 1998 through 2000. Customers would then receive a 10-percent rate cut in 2001, and an additional 4-peroent cut in 2002. According to Enron, this doubles the rate reduction proposed by PECO in their rate restructuring filing with the PUC in August 1997. Enron also proposed that it pay PECO's $\$ 5.461$ billion in stranded costs. In return, Erron would become "the default service provider for customers not served by another conmany," and it would receive all revenues paid by customers to cover stranded cost charges. Enion expects that it would sign onntracts with $\mathrm{PECO}$ to generate electricity at local plants for distribution to its customers.

According to PECO, Enron has only "neconfigured" the transition charge to a later date. PECO explained that Enron has proposed a lower transition charge through 2003. However, PECO stated that its transition change will drop each year starting in 2004, while Erron's will tncrease each year. PECO also sited Exaron's request that the PUC arder PECO bo sell the \$5.461 bullion in transition bonds to Eruron "at par at a stated interest rate of 9.66 percent." That, PECO stated, would then allow Erron to resell the debt at a lower interest rate and "put $\$ 1.5$ to $\$ 2$ billion in Enron's pocket. ${ }^{.1}$

\section{PECO Energy Customers Show Heavy Interest in Pllot Program}

Over 400,000 of PECO Energy Company's (PECO) 1.5 million customers opted to enter a Penusylvania electric supplier customer choice pilot program, However, since the program is linited by law to 5 percent of PECO's customers, a lattery will be used to select 75,000 residential, commerial, and industrial customers. Participants of the program had until October 25, 1997, to select one of the energy suppliers that is licensed by the State
Public Utility Commission (PUC). Savings to customers selected for the program is expected to be about 10 percent. The plot is set to begin in November 1997 and run through December 31,1998 . If the PUC approves PECO's restructuring case, as presented in August 1997, all PECO customers will recelve a 10-percent reduction in electric costs beginning in September 1998 and fuil customer choice will start in January 1999. According to Permsylvania's Electric Competition Law, one third of all customers in the State will be able to choose their electric supplier beginning January 1, 1999, another third by January 1, 2000, and all customers by January 1, 2001.'

\section{CIPSCO and Union Electric Mergar Approved by Illinois Commerce Commlssion}

The merger of CIPSCO rncorporated (CIPSCO) and Union Electric Company of St. Louis, Missouri, has been approved by the Illinois Commence Conmission. First proposed by the two companies in 1995, the merger has already been approved by the Missourt Public Service Commission and by shareholders. However, approwal is still requiled by the Fedesal Engy Regulatory Commission (FERC). If completed, the merged companies intend o crease a public utility hulding company named Ameren Corporation, which will become the parent of both Union Electric and Central Illinois Public Service Company the principal operating company of CIPSCO Incorporated.) Arneren, fhrough these subsidiaries, will serve 1.4 million electric customers and 300,000 gas customers in Missouri and Dlinols. The combined company will have assets of $\$ 8$ billion.

The Minois Conturere Commission did set two conditions for the meger. Furst, Union Electric must not transfer its Illinois electric and gas service to Central Itinois Public Service Company. Second, both utlity comparies must "file a rate case or alternative regulatory plan" within 6 gronths after the merger is effective. The rate case will determine the distribution of merger savings between shareholders and ratepayers.

Additionally, on September 2, 1997, Union Electric propoeed a retail pilot program that will be used to

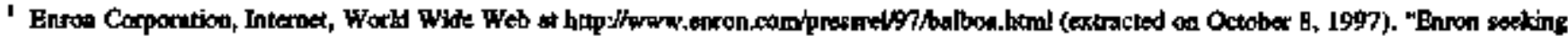

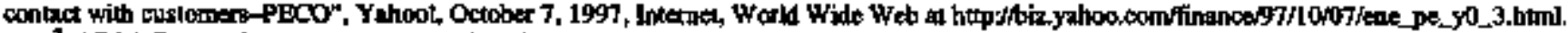

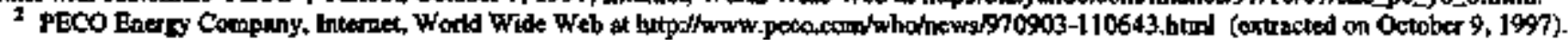


identify "potential effects of electric competition on the utility's customers" and to "test two market structures for implementing competition." The company wants to see how customers "react to different energy options, what kind of inforrution customers want, and what kind of infrastructure we need to support competition." The company expects to have about 5,000 residential and small business customers, and 200 large business customers in the pitot. Union Electric also expexts to open about 100 megawatts of the company's electric supply to competition. This pilot program is in response to a condition that the Mfissouri Public Service Commission set for their approval of the CIPSCO/ Union Elextric merger. ${ }^{3}$

\section{Illinois's Electrlc Deregulation Legislation Put On Hold-ICC Saye Bill Favors Electric Utilities}

The Minois State Senate has decided to delay a decision on Senate Bill 55 (SB 55) that will deregulate the electric utility industry in the State in order to review the complex details of the bill Supporters of SB 55, the lllinots Power Company and, in particular, the Commonwealth Edison Company (ComEd), were disappointed by the decision. ConnEd commented that it hoped the Senate would pass the bill during the fall veto session. The company noted that the bill offers Illinois consumers more than $\$ 11$ billion in savings on their electric bills. Residential customers would receive a 15 percent rate reduction starting in 1998. On May 30, 1997, the Dlincis House overwhelmingly passed the House version of the bill bited the Electric Service Customer Choice Rate Relief Law.

As currently drafted, SB 55 offers the following as a deregulation bill:

1. Choice for every industrial and commercial customer by the end of the year 2000; residential customers will be phased-in in thure stages: 10 percent by October 2000 , 40 percent by October 2001, and 100 percent by October 2002.

2. Electric utilities will necover $\mathbf{5 0}$ percent of stranded costs; utilities can securitize bonds, and transition fees end in 2008.

3. Local jurisdictions will not lose tax revenue due to changes in utilify tax rates.
4. \$1.6 billion in savings to Illinois electric customers over the next 3 years, and \$11 billion in savings over the next 10 years.

5. Low-income assistance of $\$ 76$ million per year; consumer protection to prevent redlining, slamming. and misleading marketing; consumer education; \$100 million over 10 years to support renewable energy resources; $\$ 30$ million over 10 years for energy efficiency.

On Augugt 15, 1997, the Illinois Commere Commission (ICC) released a scathing report on SB 55. The ICC declared that the Bill "as currently drafted, will not provide the benefits of competition to llimoris" It stated that the provisions of SB 55 "favor electric utilities at the expense of lower prices, economic development, and job creation" According to the ICC, "most small customers will find tit uneconomical to switch suppliets until after 2008." The ICC belteves that SB 55 will deregulate the electric utility industry, but it does mot have any provisions that will ensure that conpetition will develop in Illinois. The ICC lists 8 provisions that it considers bariers to competition including the nomlinking of stranded costs to transition charges; the collection of bjllions of dollars for electric utilities through securitization that could be used against competitors who want to enter the Illinois electric markets; and the continued operation of the transmission system by electric utilities.

To ensure faimess, the ICC believes that the following changes to the bill are necessary:

- an objective review of stranded costs and an approval through public proceedings;

- shortening the length of the transition period;

- separation of transmission services from utility power marketing activities;

- formaticn of an Independent System Operator (ISO) to operate the transmission system;

-

- provisions to prevent anti-competitive behavior."

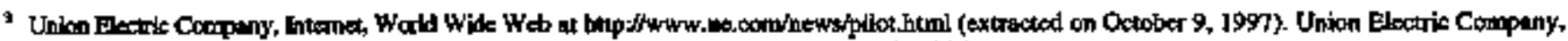

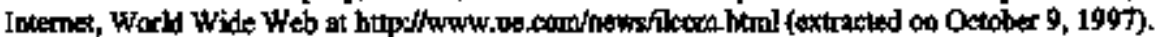

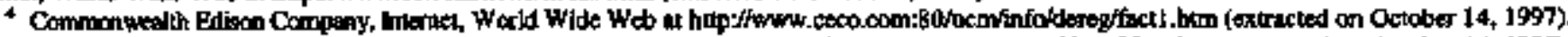

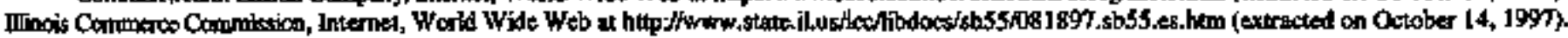




\section{GPU To Sell Generetion Aseets, Set To Concentrate On Dlstribution Business}

GPU, Inf., (GPU) the electric utility hoiding company of Jersey Central Power \& kight Company, Metropolitan Edison Company (MetEd), and Pennsylvaria Electíc Company (Penelec), ancounced that it interts to sell "possibly all" its nor-nuclear generating assets through an auction process and concentrate on its core electric distribution business. This is in addition to the announcement in April 1997 that the company is exploring the possibility of an early retirement or the sale of its Oyster Creek nuclear plant and the possible sale of the Three Mile Island nuclear plant. GPU's hydroelectric and fossil plants have a book value of $\$ 1.1$ billion and an electric generating capacity of 5,300 megawatts. The sale of the plants is expected to take about one year to complete and must be approved by State and federal regulatory agencles.

The planned auction of the plants will have an effect on the restructuring plans that were filed earlier this year with the Pennsylvania Public Utility Commission and New Jersey Boand of Public Litlities. In these filings, stranded costs related to power plants in New Jersey totaled about $\$ 200$ million, while in Pennsylvania stranded costs were estimated at about $\$ 600$ million for the MetEd and $\$ 400$ nillion for the Penelec. Sales of the power plants at prices above book value would reduce GPU's siranded costs. (These stranded costs figures inctude nuclear decommulssioning costs.)

The sale of the generating facilities follows a strategy set by GPU earlier this year when it announced that it intends to concentrate on its strengths, namely "to take aggressive actions to grow our electricity distribution business." The company also wants to expand into the natural gas and water distribution business. In 1996, GPU teaned with Cintigy Corposation, to purchese Mindlands Electricity plc, an electricity distribution company in the United Kingdom. On October 12, 1997, the company anrounced that the Australian State of Victoria had thosen GPU as the winner in bidding for PowerNet, the State's electric transmission company.

\section{Coal Dellveries Vla Union Pacifle Railroad Behind schedule}

Operational problems with the Union Pacific Rajtroad (UPR) have slowed the delivery of coal from Colorado, Utah, and Wyoming to industrial, cogeneration, and electric plants resulting in shortages at some fadifities.
Acooxding on some reports, the problems are related to the 1996 merger of the Union Pacific Corporation and the Sotuthern Paciflc Rail Corporation. Implementing the merger has rerulted in crew and locomotive shortages for moving coul and other commodities. Also, "computers that carnot talk to each other and inconsistent labor agreements" (remaining from the premerged companies) are blamed for track congestion that has slowed the Nation's largest ratlroad.

According to Corl Outlook, several UPR-served electric plants have had to reduce colat-burn in order to conserve coal stocks. Among these are the Arkansas Power \& Light Company's White Bluff and Independeroe Plants. According to Energy Information Administration (ELA) data collected on the Form EIA-759, "Monthly Power Plant Report," end-of-August stocks of coal at Whithe Bluff and Independence have fallen to 381,000 thousand short tons and 231,000 thousand short tons, respectively, as compared to 1,399 thousand short tons and 966 thousand short toms at the end of August 1996. Other electric utilities (plants) noted as having problems receiving all contracted coal deliveries are Houston Lighting \& Power Company (Parish), MidAmerican Energy (Neal), Oklahoma Gas \& Electric Company (Muscogee), Lower Colorado River Authority (Fayette), and City Public Service of San Antonio (Deely and Spruce).

On Octaber 1, 1997, Unton Pactific Corporation anmounced that the UPR had filed a Service Recovery Plan with the Surface Transportation Board that "is aimed at eliminating congestion and restoring normal service." It is hoped that the plan will "inove as many as 40,000 railcars off the railroad and generate the equivalent of 400-600 loocmotives for service necovery." According to the plan, the following actions will be implemented: scrme rail traffic will be kemporarily diverted to other railroads; the UPR will release selected traffic to other railroads (induding unit cosal trains originating in the Powder River Bastn in Wyoming and destined for Texas); it will stuspend some unit coal trains between the Powder Rjver Basin and Mexico, and reduce export coal shipments; some trains will be diverted from the heavily traveled Southern Corridor to other lines; and, the UPR will reposition up to 600 locomatives.

Based on the plan, the UPR expects that the railroad's Central Corridor (Chucago to Oakland) should return to "acceptable levels" within 30 days. The Southern Corridor ( from Memphis and New Orleans through Texas and into Southern California) is expected to be back to normal within 60 to 90 days. ${ }^{6}$

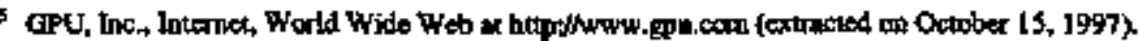

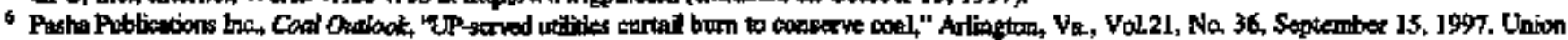

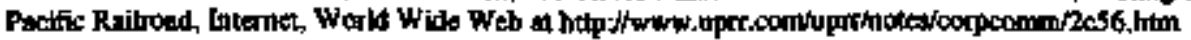





\section{U.S. Electric UtIIIty Net Generation}

Table 3. U.S. Electric Power Industry Net Generation, 1990 Through August 1997 (Mijlion Kilowathours)

\begin{tabular}{|c|c|c|c|c|c|c|c|c|c|c|}
\hline \multirow[b]{2}{*}{ Puid } & \multicolumn{8}{|c|}{ 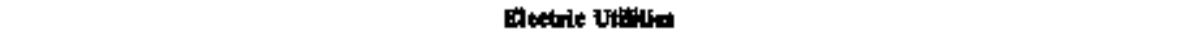 } & \multirow{2}{*}{ 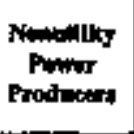 } & \multirow{2}{*}{ 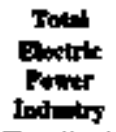 } \\
\hline & Conl & Putrols:onl & $\operatorname{\theta os} 2$ & Nediner & Figdro & Conthe & Other 3 & Tetrl & & \\
\hline 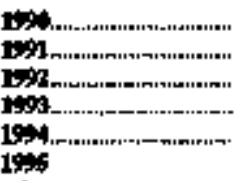 & 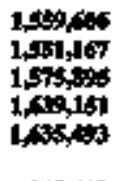 & 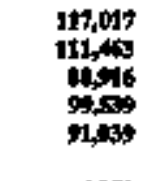 & 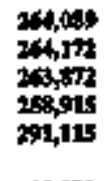 & 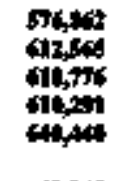 & 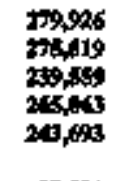 & 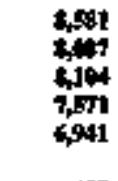 & $\begin{array}{l}1,07 \\
200 \\
1,004 \\
1,04\end{array}$ & 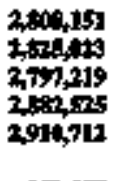 & 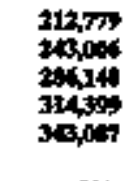 & 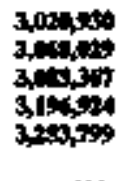 \\
\hline 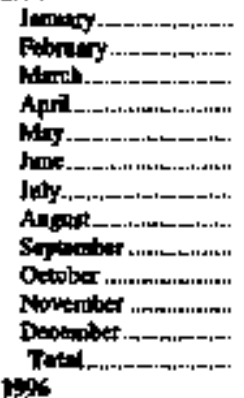 & $\begin{array}{l}142,412 \\
128,447 \\
126,970 \\
118,796 \\
126,013 \\
138,098 \\
138,378 \\
166,700 \\
135,241 \\
131,918 \\
133,899 \\
146,642 \\
1,40,914\end{array}$ & 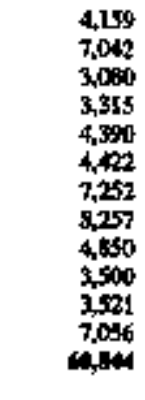 & $\begin{array}{r}19,339 \\
16,422 \\
23,844 \\
22,062 \\
24,662 \\
28,994 \\
38,756 \\
44,402 \\
30,479 \\
23,076 \\
19,261 \\
16,609 \\
3,7,306\end{array}$ & 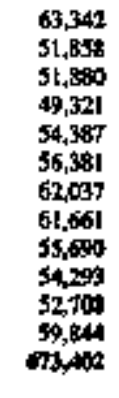 & $\begin{array}{l}23,291 \\
23,956 \\
27,458 \\
22,464 \\
26,570 \\
26,367 \\
28,942 \\
22,999 \\
19,298 \\
21,440 \\
24,019 \\
27,329 \\
293,653\end{array}$ & $\begin{array}{l}408 \\
296 \\
376 \\
282 \\
255 \\
281 \\
305 \\
524 \\
367 \\
615 \\
544 \\
528 \\
4745\end{array}$ & $\begin{array}{l}176 \\
166 \\
117 \\
151 \\
164 \\
129 \\
151 \\
165 \\
149 \\
163 \\
155 \\
143 \\
1.64\end{array}$ & 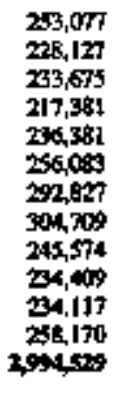 & $\begin{array}{r}\mathbf{N A} \\
\mathbf{M A} \\
\mathbf{M A} \\
\mathbf{M A} \\
\mathbf{M A} \\
\mathbf{M A} \\
\mathbf{N A} \\
\mathbf{N A} \\
\mathbf{N A}\end{array}$ & $\begin{array}{r}\text { NA } \\
\text { NA } \\
\text { NA } \\
\text { NA } \\
\text { NA } \\
\text { NA } \\
\text { MA } \\
\text { NA } \\
\text { Nh }\end{array}$ \\
\hline 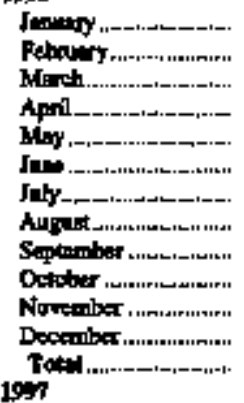 & 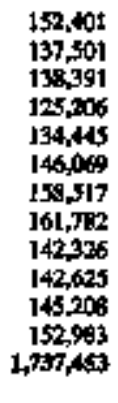 & $\begin{array}{l}7,872 \\
8,214 \\
6,101 \\
3,201 \\
3,997 \\
5,589 \\
7,583 \\
6,5144 \\
4,655 \\
3,359 \\
4,295 \\
5,939 \\
6,344\end{array}$ & $\begin{array}{l}16,053 \\
13,327 \\
15,214 \\
16,612 \\
25,424 \\
28,730 \\
34,129 \\
35,233 \\
27,254 \\
23,812 \\
16,525 \\
12,414 \\
20,734\end{array}$ & $\begin{array}{r}52,942 \\
55,928 \\
55,474 \\
50,325 \\
55,637 \\
57,494 \\
60,959 \\
61,477 \\
54,593 \\
50,612 \\
52,132 \\
57,159 \\
57,72\end{array}$ & $\begin{array}{l}20,831 \\
29,850 \\
32,221 \\
30,430 \\
31,645 \\
30,191 \\
27,951 \\
24,835 \\
20,706 \\
21,165 \\
21,956 \\
28,798 \\
327,970\end{array}$ & $\begin{array}{r}354 \\
361 \\
339 \\
385 \\
255 \\
389 \\
555 \\
574 \\
496 \\
591 \\
598 \\
456 \\
9,254\end{array}$ & $\begin{array}{l}149 \\
137 \\
160 \\
124 \\
141 \\
170 \\
190 \\
173 \\
167 \\
204 \\
190 \\
174 \\
1+4\end{array}$ & 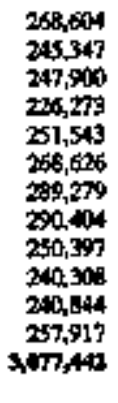 & $\begin{array}{l}N A \\
N A \\
N A \\
N A \\
N A \\
N A \\
N A \\
N A \\
N A \\
N A \\
N A \\
N A \\
N A\end{array}$ & $\begin{array}{l}\text { NA } \\
\text { NA } \\
\text { NA } \\
\text { NA } \\
\text { MA } \\
\text { NA } \\
\text { NA } \\
N A \\
N A \\
\text { NA } \\
\text { NA } \\
\text { NA } \\
\text { NA }\end{array}$ \\
\hline 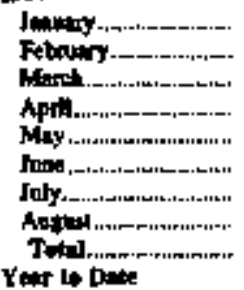 & $\begin{array}{r}161,276 \\
134,218 \\
137,554 \\
131,720 \\
136,185 \\
146,072 \\
166,893 \\
160,363 \\
1,17,, 401\end{array}$ & $\begin{array}{r}8,392 \\
4,644 \\
4,545 \\
4,094 \\
4,469 \\
6,789 \\
9,204 \\
7,590 \\
4,716\end{array}$ & $\begin{array}{r}13,927 \\
13,455 \\
18,1,101 \\
18,963 \\
22,094 \\
28,265 \\
40,143 \\
37,186 \\
13, \pm 21\end{array}$ & $\begin{array}{r}58,914 \\
50,651 \\
51,414 \\
45,359 \\
47,622 \\
52,095 \\
57,352 \\
61,094 \\
42,151\end{array}$ & $\begin{array}{r}31,090 \\
29,842 \\
39,313 \\
30,483 \\
32,757 \\
39,804 \\
30,070 \\
25,404 \\
24,415\end{array}$ & $\begin{array}{r}414 \\
310 \\
436 \\
494 \\
431 \\
385 \\
512 \\
505 \\
3,516\end{array}$ & $\begin{array}{r}162 \\
145 \\
136 \\
170 \\
178 \\
159 \\
169 \\
174 \\
1316\end{array}$ & $\begin{array}{r}274,177 \\
24,315 \\
246,369 \\
201,045 \\
243,206 \\
264,565 \\
304,344 \\
294,396 \\
2,000,446\end{array}$ & 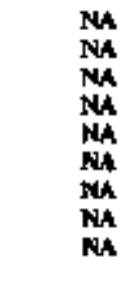 & $\begin{array}{l}\text { NA } \\
\text { MA } \\
\text { NA } \\
\text { Nh } \\
\text { NA } \\
\text { Nh } \\
\text { Nh } \\
\text { NA }\end{array}$ \\
\hline 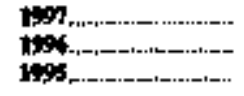 & $\begin{array}{l}1,177,2,11 \\
1,15,1311 \\
1,105795\end{array}$ & $\begin{array}{l}\text { ist16 } \\
\text { 4tiva } \\
\text { 4list }\end{array}$ & 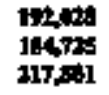 & 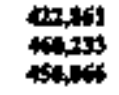 & 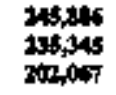 & $\begin{array}{l}3,519 \\
3,719 \\
2,479\end{array}$ & $\begin{array}{l}1,316 \\
1,2,46 \\
1,054\end{array}$ & $\begin{array}{l}2,0,6=1 \\
2,4,9,9 \pi \\
2,42,15\end{array}$ & $\begin{array}{l}\mathrm{Nh} \\
\mathrm{Nh}\end{array}$ & Nh \\
\hline
\end{tabular}

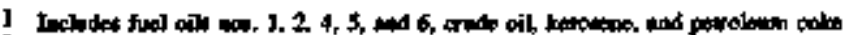

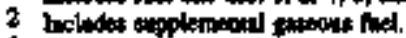

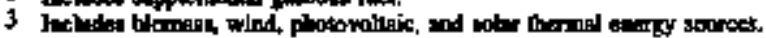

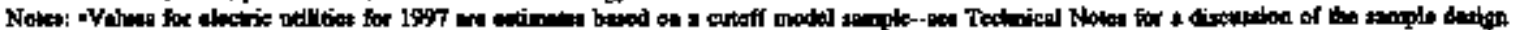

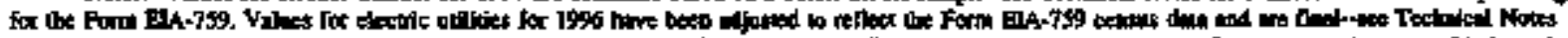

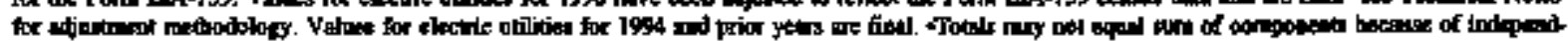
exe ronidint.

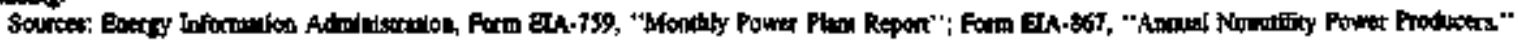


Tahle 4. U.S. Electric Utility Net Generation by Nonrenewable Enerzy \$onree, 19\%0 Through August 1997

(Million Kilowatthours)

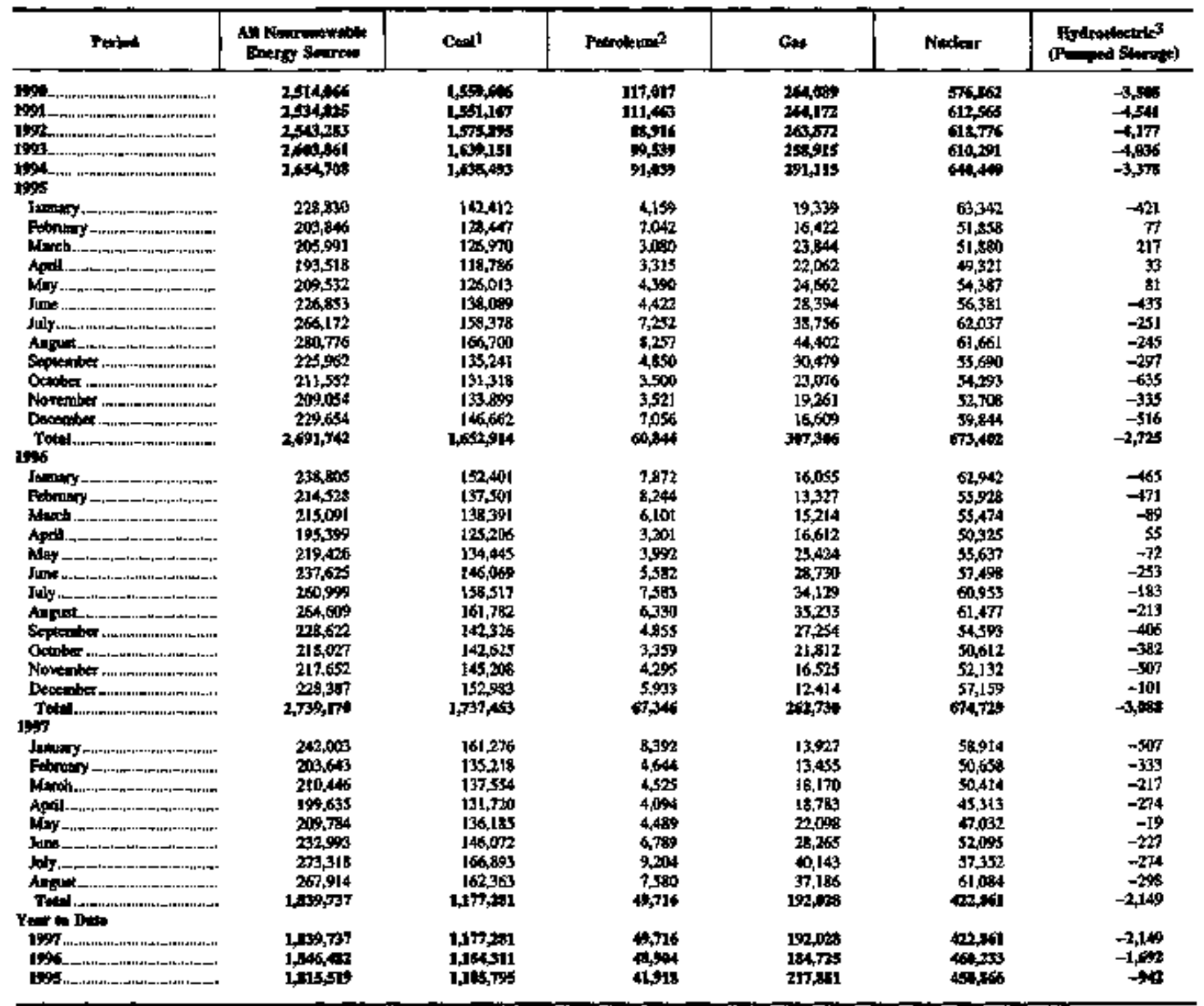

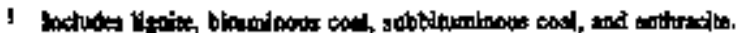

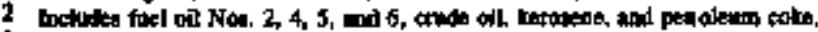

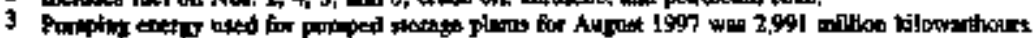

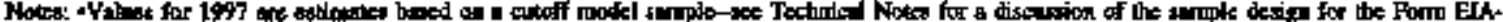

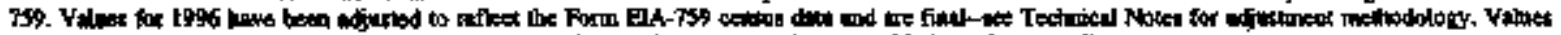

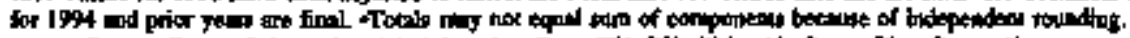

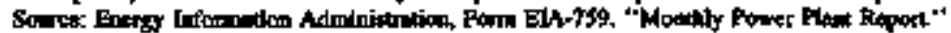


Table 5. U.S. Flectric Utllity Net Geweration by Renewable Eneray Source, 1990 Throngh Auguast 1997

(Thousand Kilowatthours)

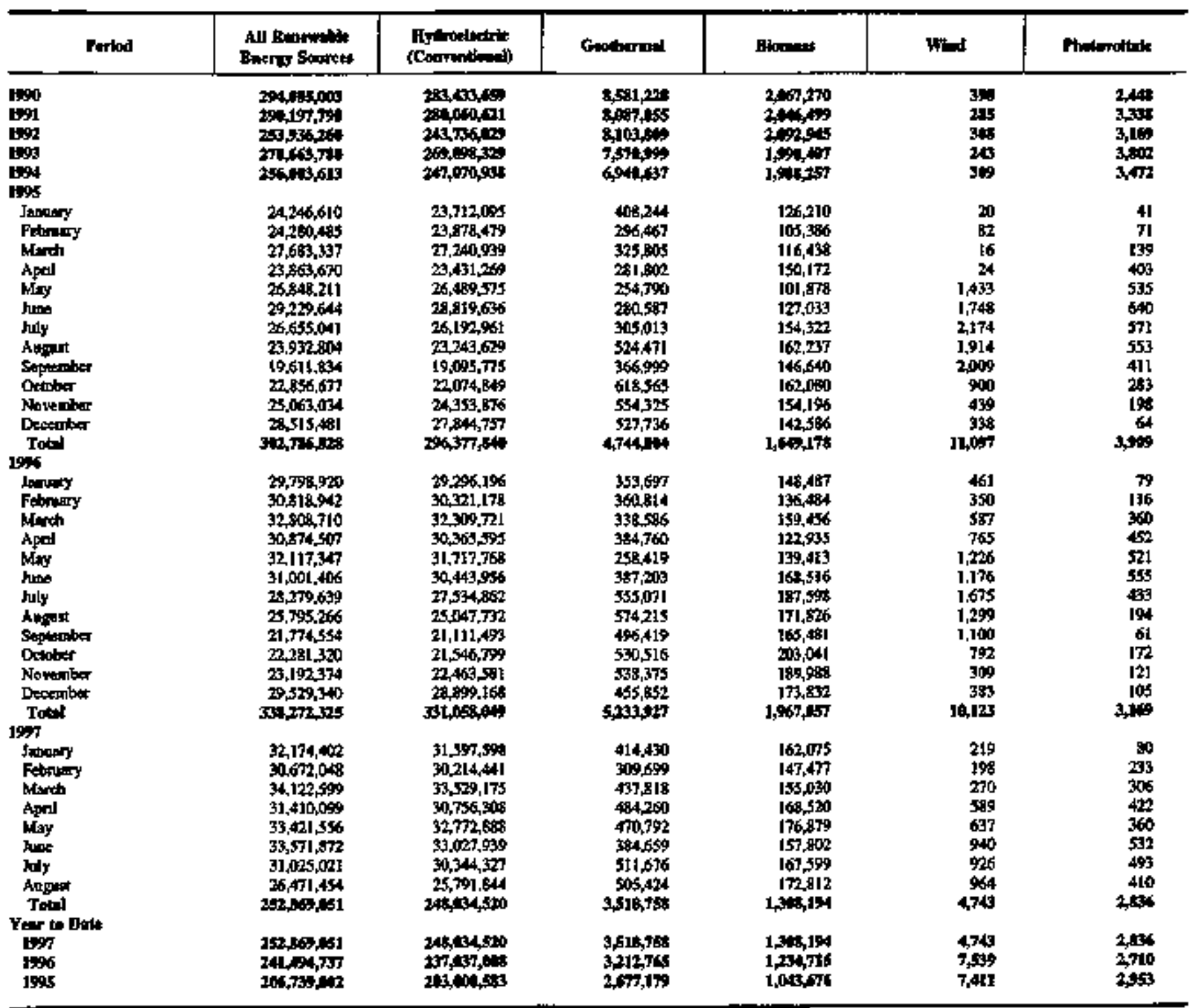

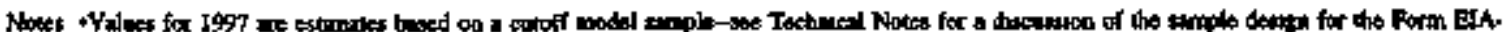

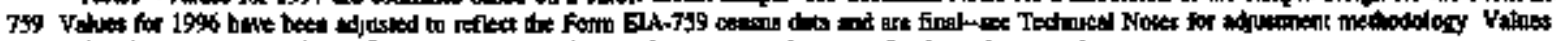

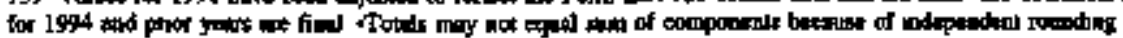

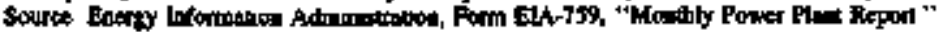


Table 6. Electric Utilty Net Geserntion by NERC Regiom and Hawait (Mftlion Kilowatthours)

\begin{tabular}{|c|c|c|c|c|c|c|}
\hline \multirow{2}{*}{ 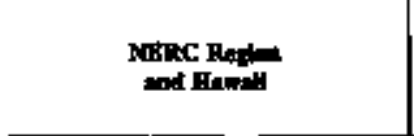 } & \multirow{2}{*}{ thent } & \multirow{2}{*}{ thy } & \multirow{2}{*}{ 19:4 } & \multicolumn{3}{|c|}{ 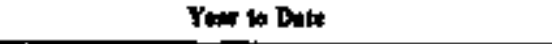 } \\
\hline & & & & $14 \pi$ & 1945 & 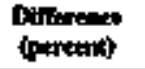 \\
\hline 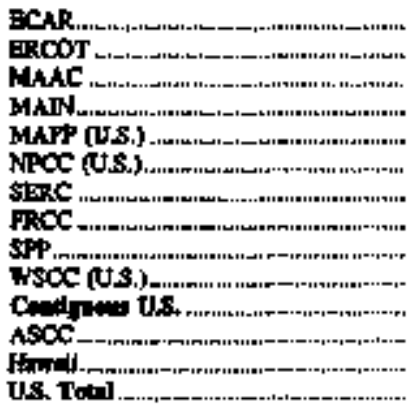 & 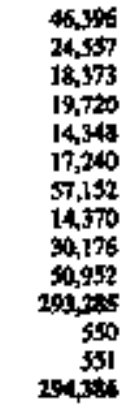 & 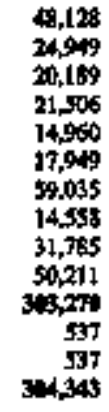 & 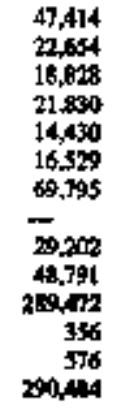 & $\begin{array}{r}354,278 \\
151,907 \\
135,180 \\
144,775 \\
104,394 \\
125,232 \\
401,746 \\
94,946 \\
199,945 \\
373,437 \\
2,04,114 \\
4,379 \\
4,113 \\
2,4,45\end{array}$ & 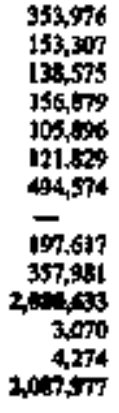 & $\begin{array}{r}-0.5 \\
-.9 \\
-1.7 \\
-7.7 \\
-4.2 \\
2.8 \\
-11.8 \\
1.4 \\
1.2 \\
4.0 \\
.2 \\
42.6 \\
-3.6 \\
1\end{array}$ \\
\hline
\end{tabular}

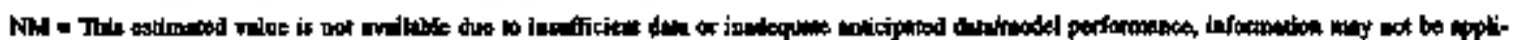

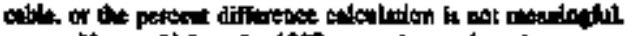

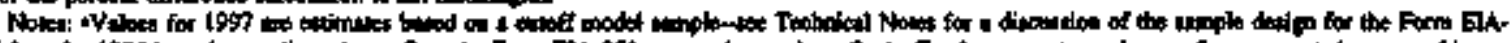

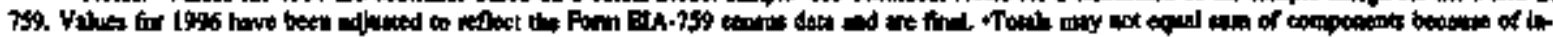

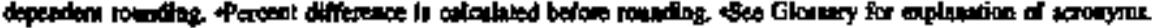

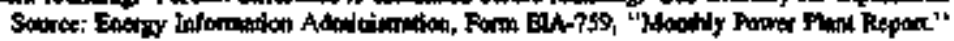


Table 7. Electric Utility Net Gentration by Census Division and State (Miltion Kilowatthours)

\begin{tabular}{|c|c|c|c|c|c|c|}
\hline \multirow{2}{*}{ Chtal Divison } & \multirow{2}{*}{ 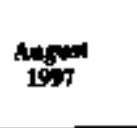 } & \multirow{2}{*}{ Joly } & \multirow{2}{*}{ Alines } & \multicolumn{3}{|c|}{ Yar to Dot } \\
\hline & & & & Itys & tos & 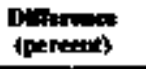 \\
\hline 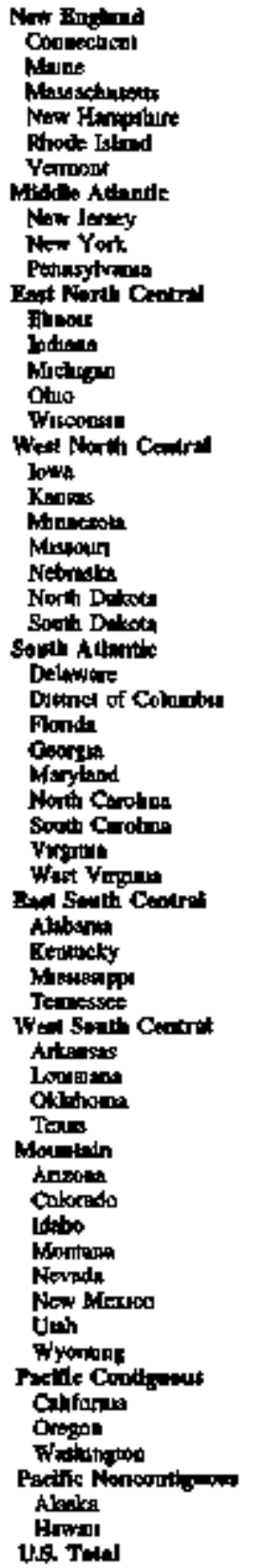 & 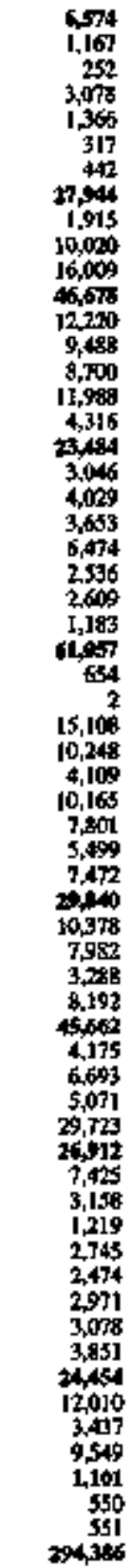 & 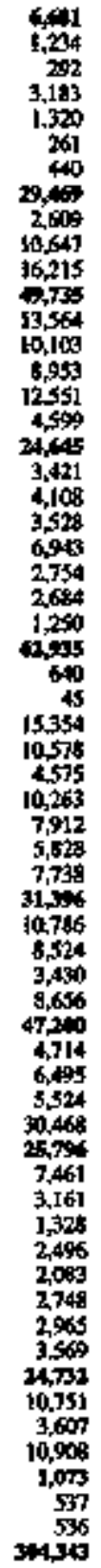 & 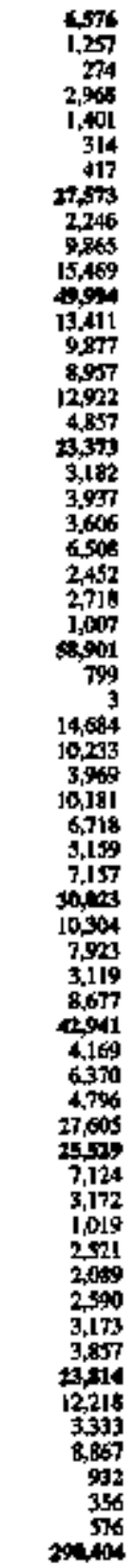 & 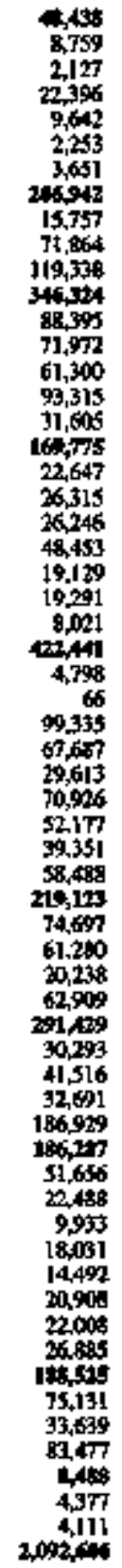 & 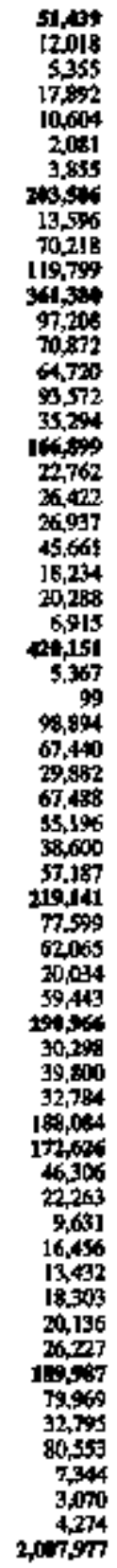 & 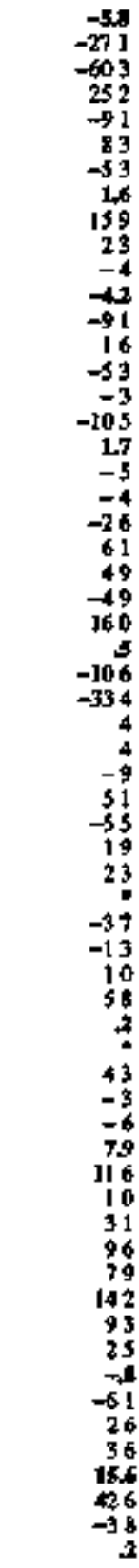 \\
\hline
\end{tabular}

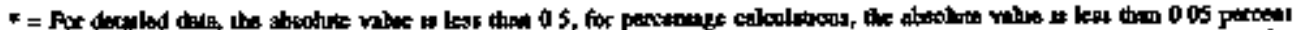

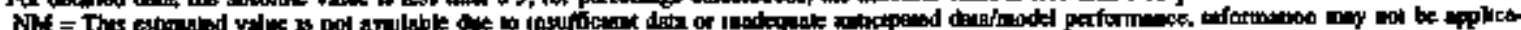

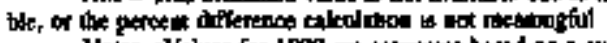

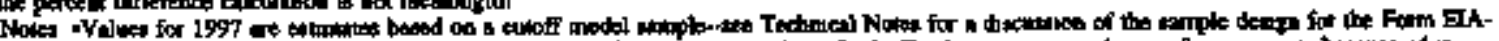

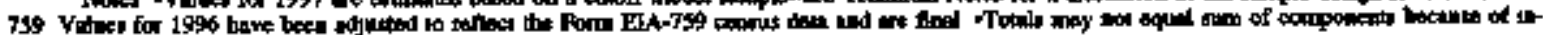

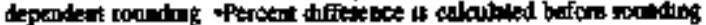

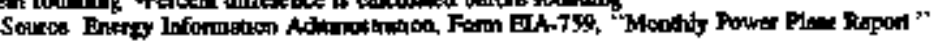


Table 8. Electric Utility Net Generation from Cool by Cenoul Divislow and State (Million Killowathours)

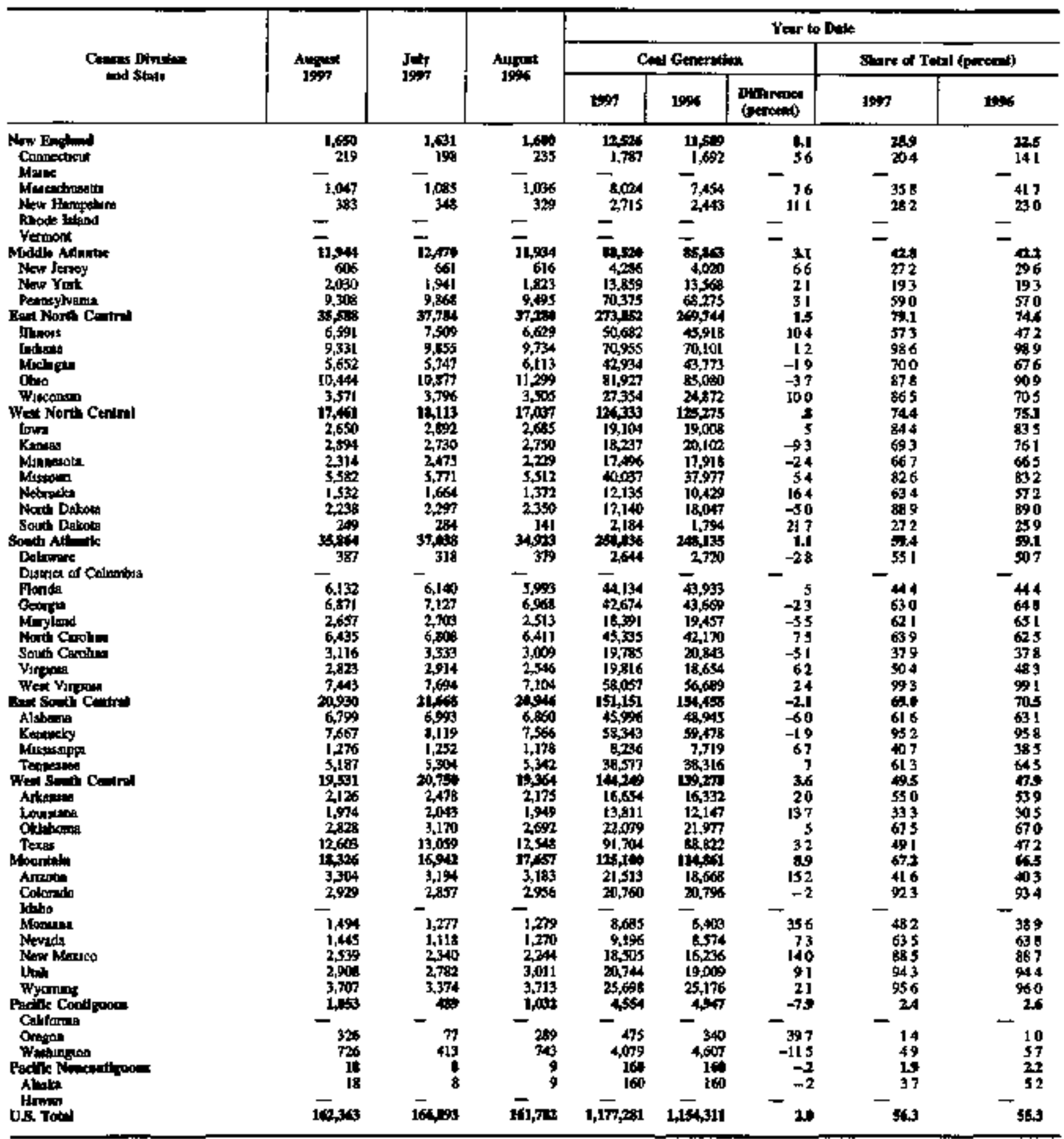

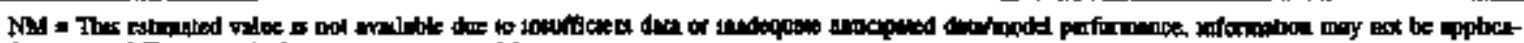

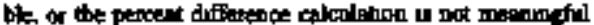

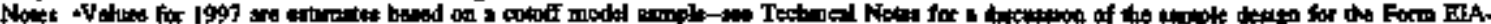

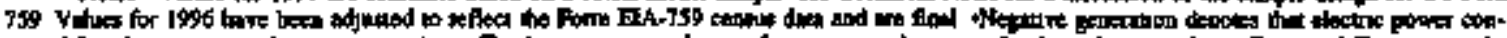

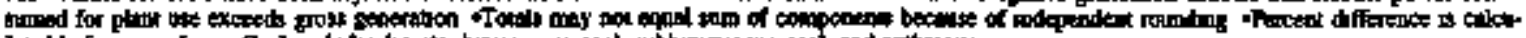

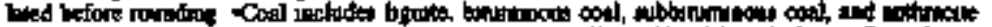

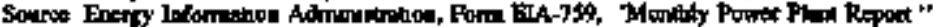


Table 9. Electric Utitity Net Generation from Petrolenm by Cencons Division and State (Milion Kelowatthours)

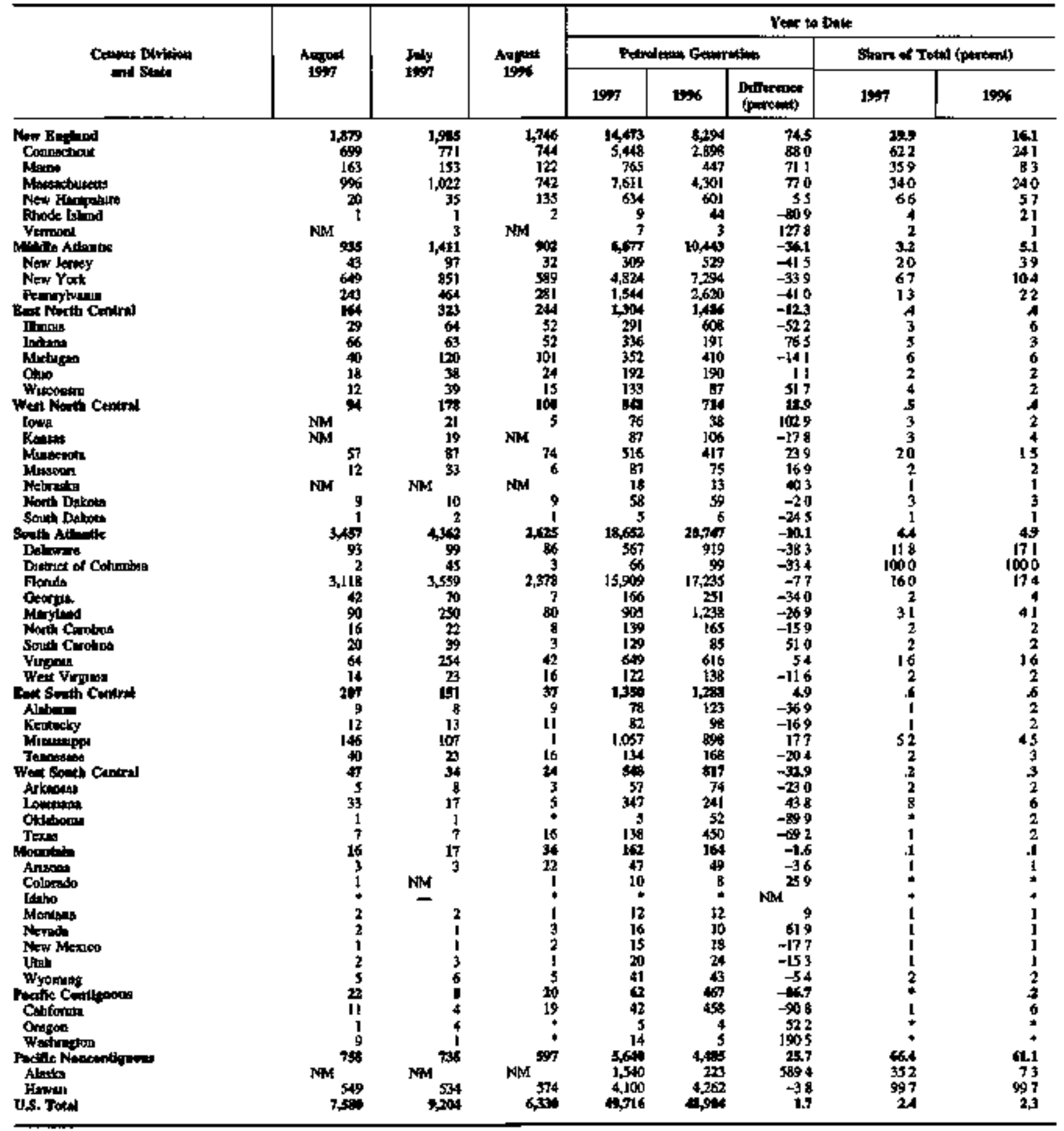

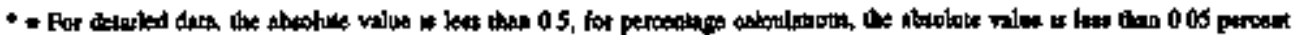

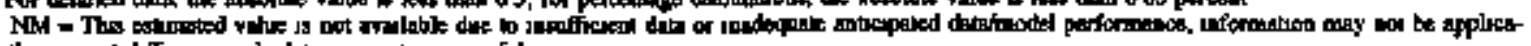

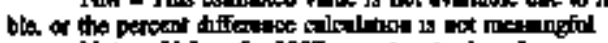

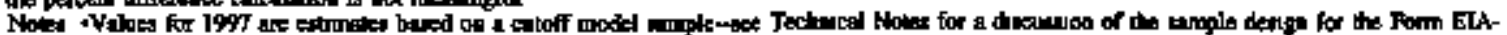

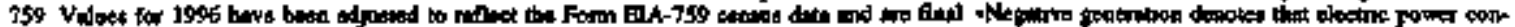

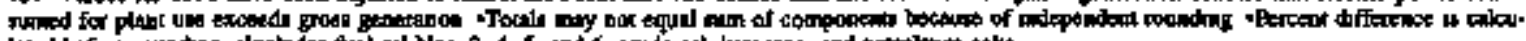

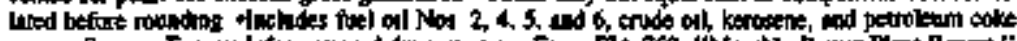

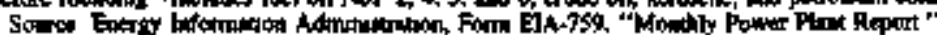


Table 10. Electric Utility Net Generation from Gas by Cenows Division and State (Mtllion Kilowatthours)

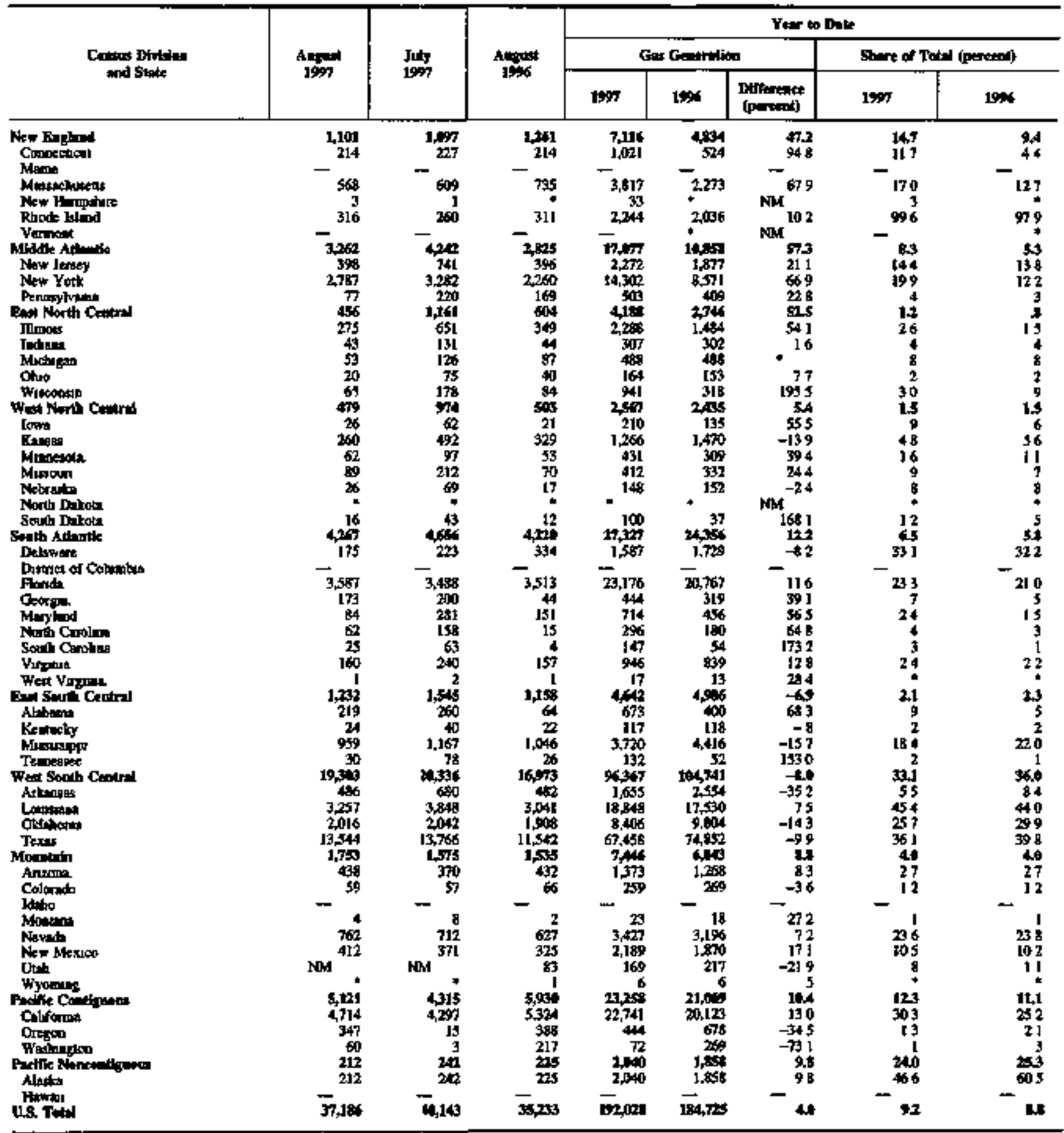

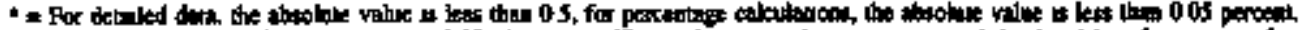

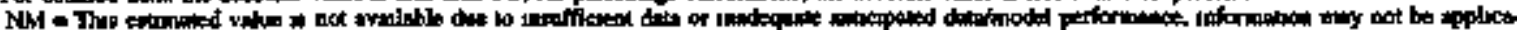

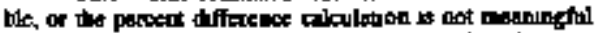

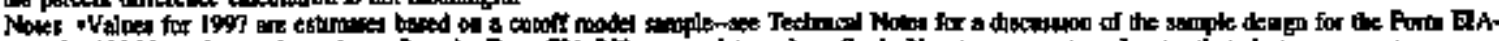
359 V w

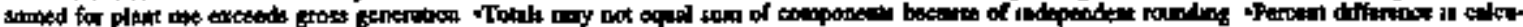
lined before rogedngt

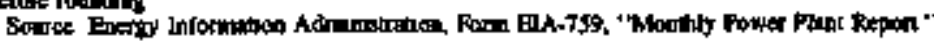


Tabbe 11. Electric Utility Hydroelectric Net Generation by Cenous Division and Stote (Million Kilowarthours)

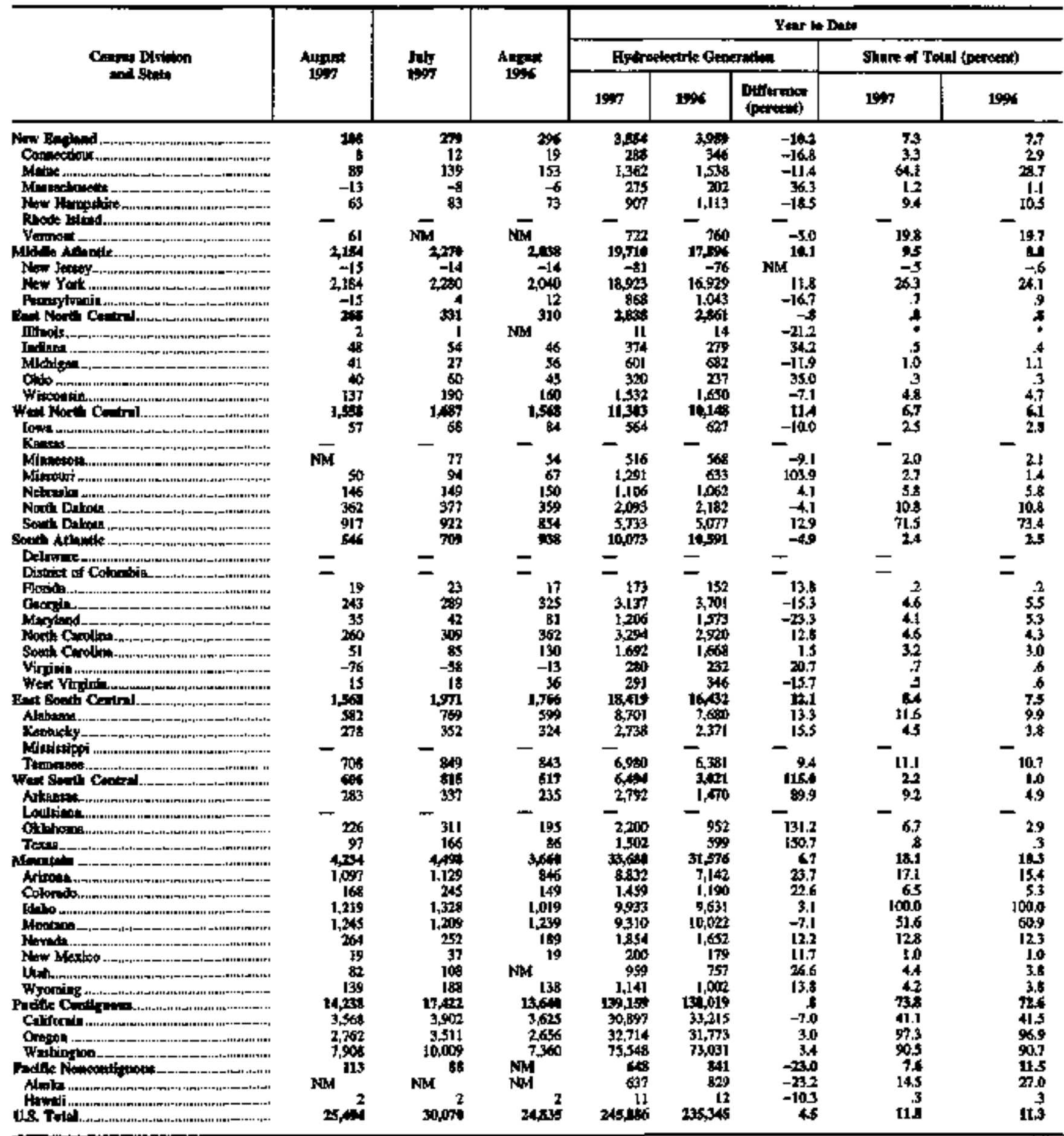

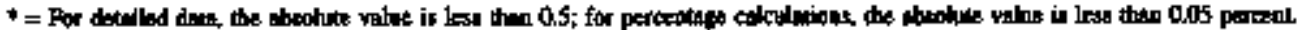

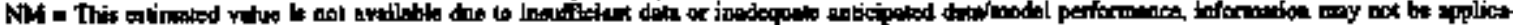

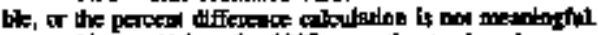

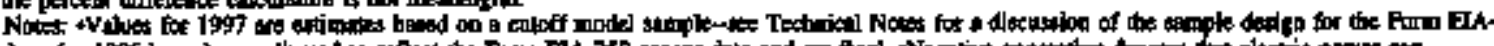

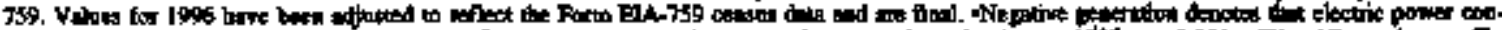

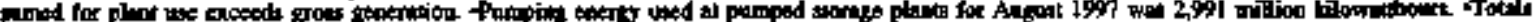

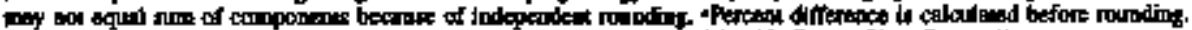

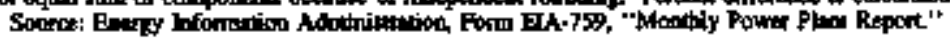


Tabłe 12. Electric Utility Nuclear-Powered Net Generation by Census Division and State (Miltıon Kilowathowirs)

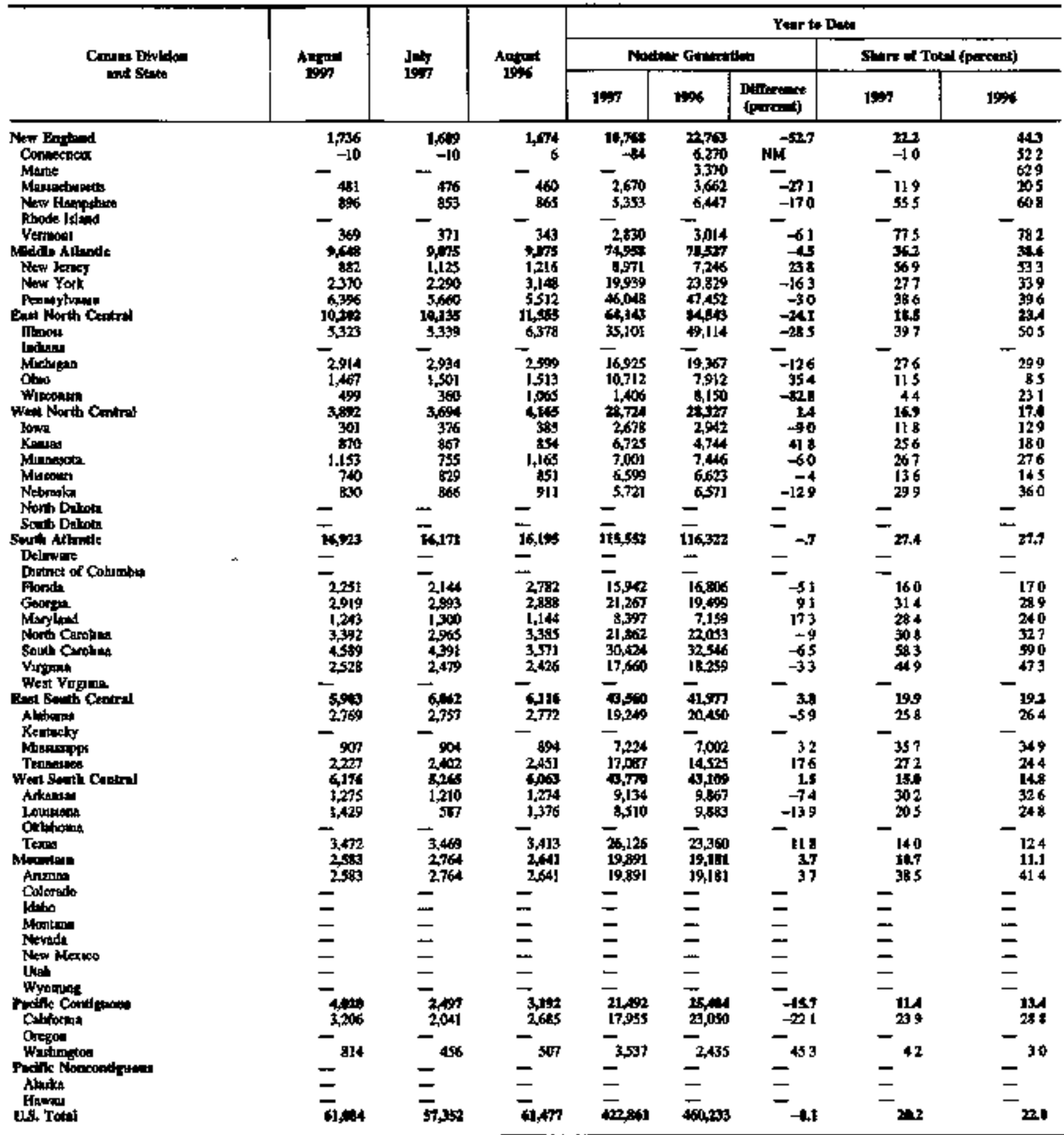

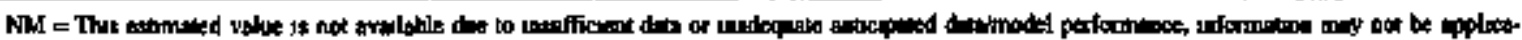

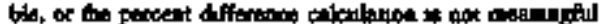

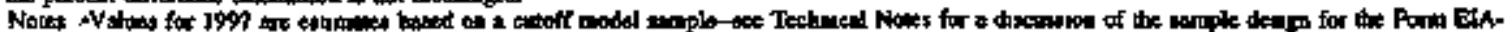

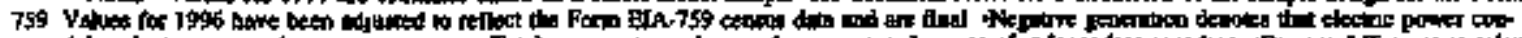

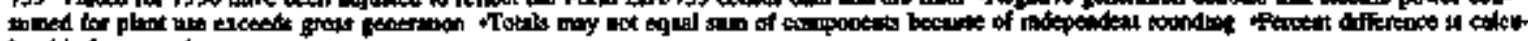

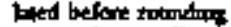

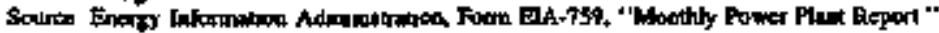


Table 13. Eteetric Utility Nel Generation from Other Enercy Soorces by Cengu Dividon and State (Million Kilowatthours)

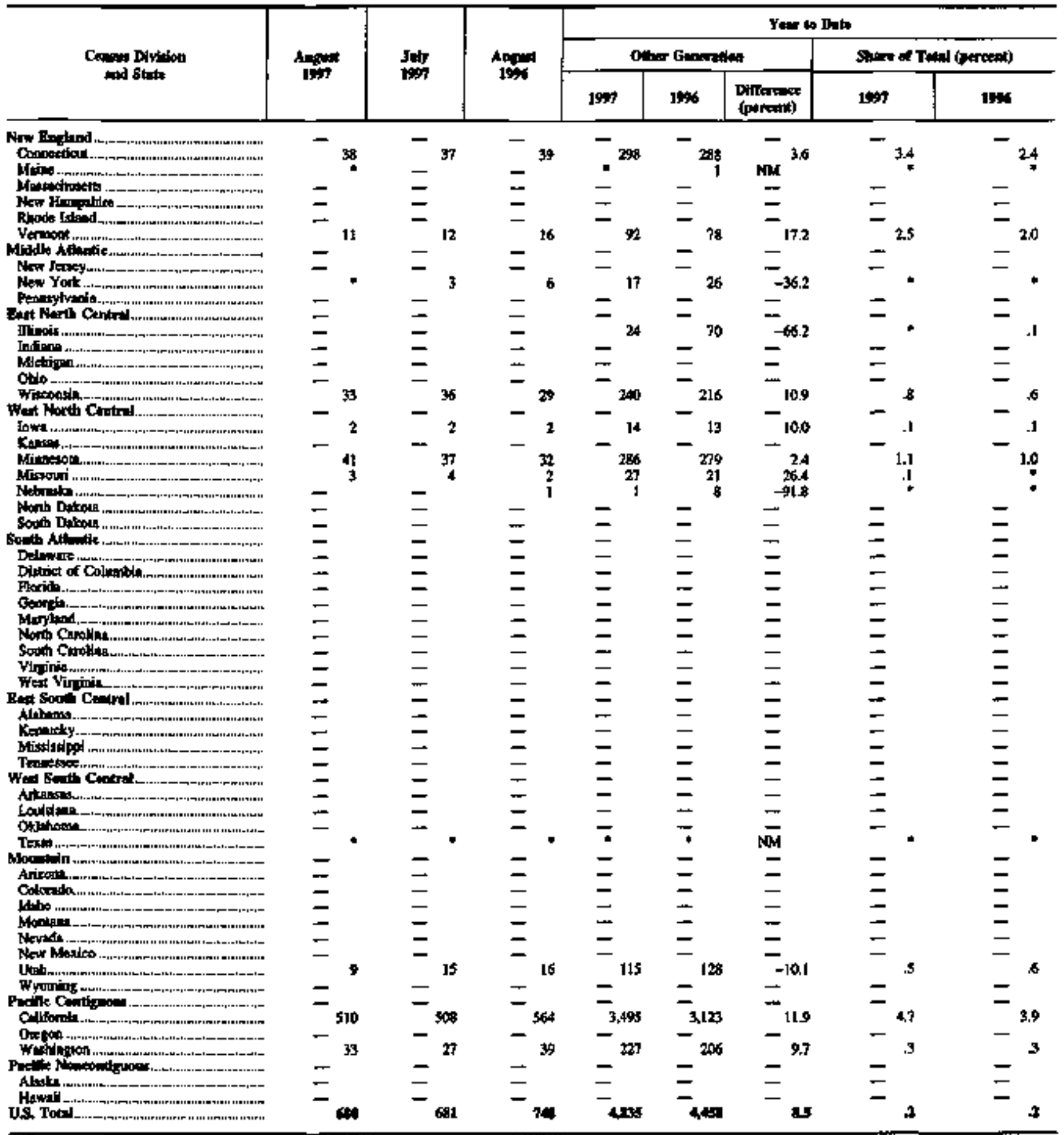

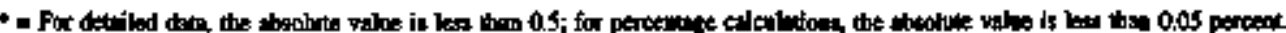

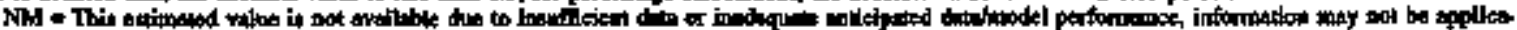

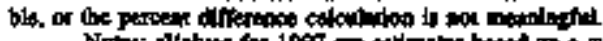

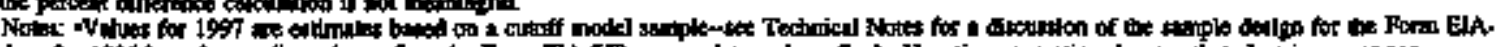

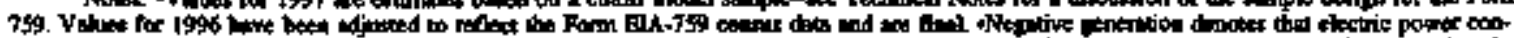

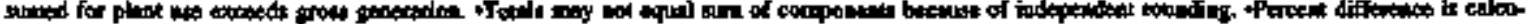

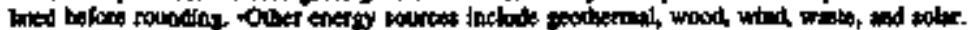

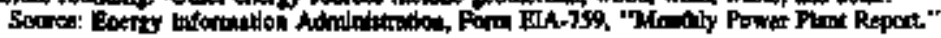





\section{U.S. Electric Utility Consumption of Fossil Fuels}

Table 14. US. Electric Utility Consmption of Fasdi Fuels, 1987 Through Augast 1997

\begin{tabular}{|c|c|c|c|c|c|c|c|c|c|}
\hline \multirow{2}{*}{ Finted } & \multicolumn{4}{|c|}{ fintind shoct cons } & \multicolumn{3}{|c|}{ 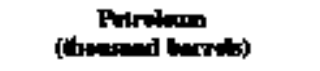 } & \multirow{2}{*}{ 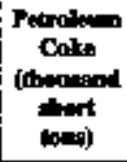 } & \multirow{2}{*}{ (t) } \\
\hline & Inthritulat & 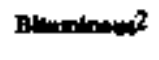 & Itiste & Total & 1:tt & Beany & Totel & & \\
\hline 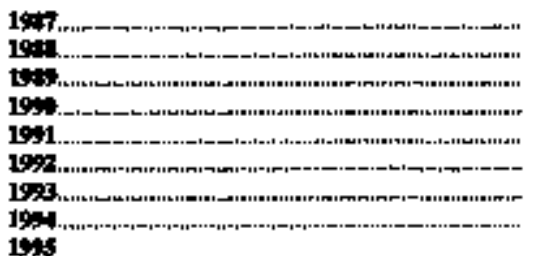 & $\begin{array}{r}972 \\
1,0 \times 5 \\
1,049 \\
1,001 \\
9,4 \\
94,41 \\
9,91 \\
1,125\end{array}$ & 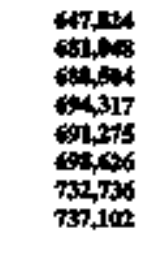 & 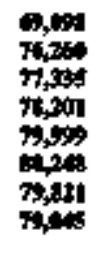 & 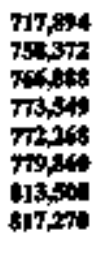 & 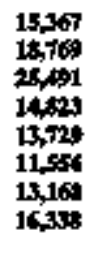 & 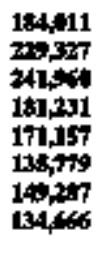 & 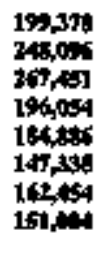 & 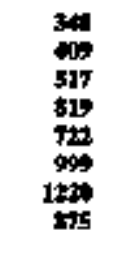 & 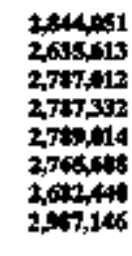 \\
\hline 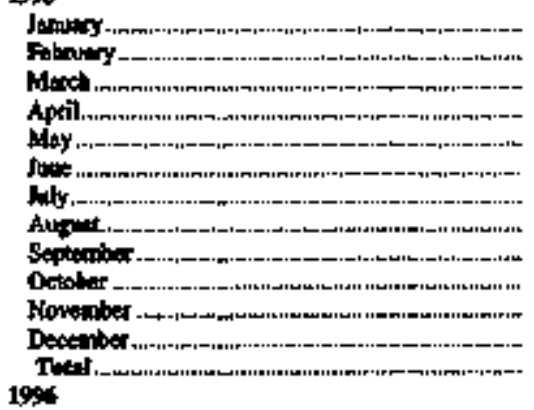 & $\begin{array}{l}75 \\
\mathbf{B 2} \\
83 \\
77 \\
86 \\
72 \\
67 \\
79 \\
87 \\
86 \\
93 \\
93 \\
975\end{array}$ & $\begin{array}{l}64,263 \\
57,970 \\
7,795 \\
53,899 \\
77,067 \\
62,442 \\
7,057 \\
76,043 \\
61,631 \\
59,747 \\
60,843 \\
66,216 \\
70,960\end{array}$ & $\begin{array}{l}7,103 \\
5,729 \\
5,092 \\
5,140 \\
5,502 \\
6,849 \\
7,539 \\
7,909 \\
6,906 \\
6,482 \\
6,249 \\
7,275 \\
7,075\end{array}$ & $\begin{array}{r}71,431 \\
63,752 \\
69,569 \\
59,110 \\
62,655 \\
69,342 \\
79,658 \\
83,720 \\
68,624 \\
66,326 \\
67,185 \\
73,574 \\
570,414\end{array}$ & $\begin{array}{r}1,057 \\
1,316 \\
907 \\
918 \\
1,133 \\
1,195 \\
1,879 \\
2,853 \\
903 \\
932 \\
1,051 \\
1,421 \\
t \leq, 4 x 5\end{array}$ & $\begin{array}{r}5,955 \\
10,457 \\
4,276 \\
4,673 \\
6,121 \\
6,267 \\
10,507 \\
21,446 \\
6,964 \\
4,747 \\
4,812 \\
10,364 \\
4,4,44\end{array}$ & $\begin{array}{r}7,012 \\
11,773 \\
5,183 \\
5,591 \\
7,255 \\
7,457 \\
12,385 \\
14,799 \\
7,867 \\
5,640 \\
5,863 \\
11,785 \\
10,490\end{array}$ & $\begin{array}{r}64 \\
61 \\
52 \\
36 \\
59 \\
68 \\
57 \\
80 \\
66 \\
74 \\
83 . \\
62 \\
741\end{array}$ & 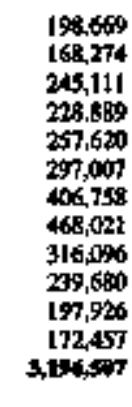 \\
\hline 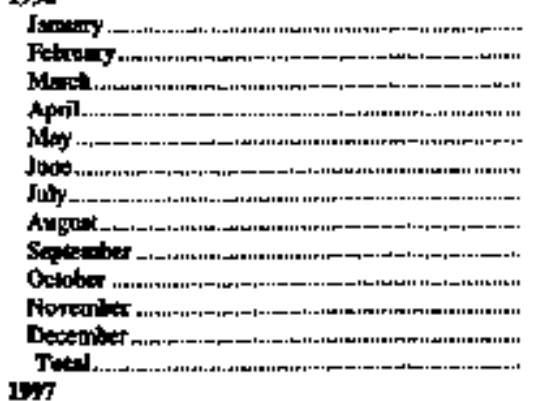 & $\begin{array}{l}87 \\
79 \\
88 \\
77 \\
67 \\
86 \\
89 \\
97 \\
97 \\
66 \\
63 \\
92 \\
10\end{array}$ & $\begin{array}{r}69,453 \\
62,553 \\
62,534 \\
57,224 \\
61,321 \\
66,642 \\
73,096 \\
74,140 \\
65,500 \\
65,199 \\
67,059 \\
70,586 \\
70,251\end{array}$ & $\begin{array}{l}7,289 \\
6,470 \\
6,439 \\
5,002 \\
5,981 \\
6,759 \\
7,201 \\
7,120 \\
6,325 \\
6,309 \\
6,49 \% \\
7,091 \\
7,010\end{array}$ & $\begin{array}{l}76,824 \\
69,109 \\
69,061 \\
60,334 \\
67,390 \\
73,467 \\
90,330 \\
\$ 1,359 \\
11,927 \\
71,575 \\
73,531 \\
77,769 \\
104,61\end{array}$ & $\begin{array}{r}1,967 \\
2,514 \\
1,593 \\
1,001 \\
1,354 \\
1,003 \\
1,322 \\
1,123 \\
1,193 \\
1,076 \\
1,113 \\
1,553 \\
16,852\end{array}$ & $\begin{array}{r}11,410 \\
11,857 \\
8,762 \\
4,344 \\
5,256 \\
8,353 \\
11,444 \\
9,041 \\
6,821 \\
4,509 \\
6,055 \\
8,520 \\
9,342\end{array}$ & $\begin{array}{r}13,376 \\
14,370 \\
10,775 \\
5,346 \\
6,610 \\
9,436 \\
12,766 \\
10,154 \\
8,014 \\
5,585 \\
7,167 \\
10,073 \\
113,474\end{array}$ & $\begin{array}{l}62 \\
47 \\
39 \\
44 \\
49 \\
49 \\
31 \\
86 \\
71 \\
39 \\
51 \\
55 \\
651\end{array}$ & $\begin{array}{r}168,406 \\
136,531 \\
136,076 \\
169,514 \\
264,160 \\
249,413 \\
357,600 \\
367,069 \\
284,744 \\
226,376 \\
169,829 \\
132,372 \\
2,732,147\end{array}$ \\
\hline 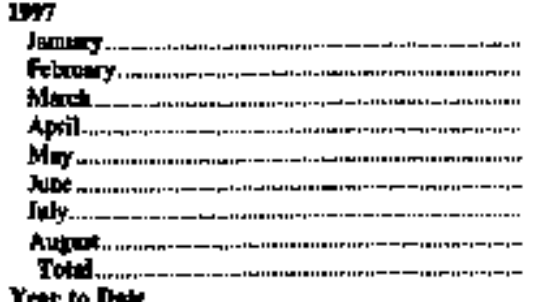 & $\begin{array}{l}97 \\
86 \\
89 \\
93 \\
72 \\
75 \\
91 \\
62 \\
685\end{array}$ & $\begin{array}{l}7,396 \\
61,630 \\
63,266 \\
60,258 \\
62,091 \\
66,939 \\
77,242 \\
73,266 \\
54,757\end{array}$ & $\begin{array}{r}7,0093 \\
6,204 \\
5,726 \\
4,811 \\
6,129 \\
6,852 \\
7,122 \\
7,146 \\
51,47\end{array}$ & $\begin{array}{l}81,175 \\
67,920 \\
69,091 \\
65,192 \\
68,292 \\
73,866 \\
84,495 \\
82,495 \\
97,515\end{array}$ & $\begin{array}{r}2,052 \\
1,195 \\
1,195 \\
1,362 \\
1,051 \\
1,559 \\
2,655 \\
1,626 \\
12,055\end{array}$ & $\begin{array}{r}11,935 \\
6,283 \\
6,055 \\
5,120 \\
6,123 \\
9,796 \\
12,500 \\
10,0160 \\
01,537\end{array}$ & $\begin{array}{r}13,987 \\
7,477 \\
7,260 \\
6,482 \\
7,174 \\
11,275 \\
15,155 \\
12,482 \\
81,392\end{array}$ & $\begin{array}{l}36 \\
35 \\
35 \\
100 \\
135 \\
144 \\
144 \\
160 \\
501\end{array}$ & $\begin{array}{r}139,104 \\
102,984 \\
199,131 \\
192,593 \\
230,637 \\
295,112 \\
426,594 \\
390,947 \\
200690\end{array}$ \\
\hline 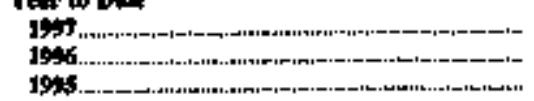 & $\begin{array}{l}485 \\
691 \\
619\end{array}$ & 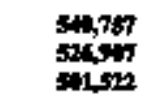 & 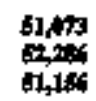 & 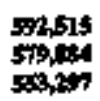 & $\begin{array}{l}12,50 \\
11,957 \\
11,15 \%\end{array}$ & 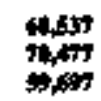 & 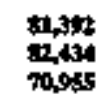 & 4h1 & 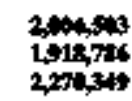 \\
\hline
\end{tabular}

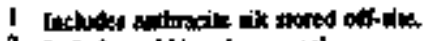

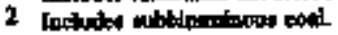

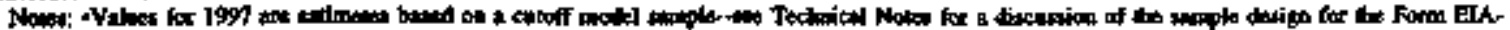

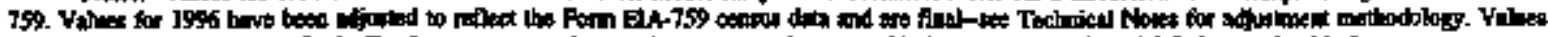

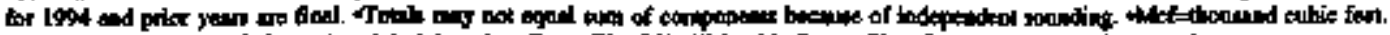

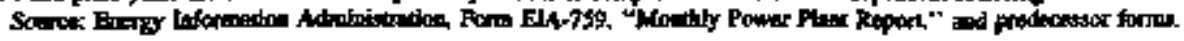


Table 15. Electric Utlity Consumption of Cood by NERC Region and Hawaif (Thousand Short Tons)

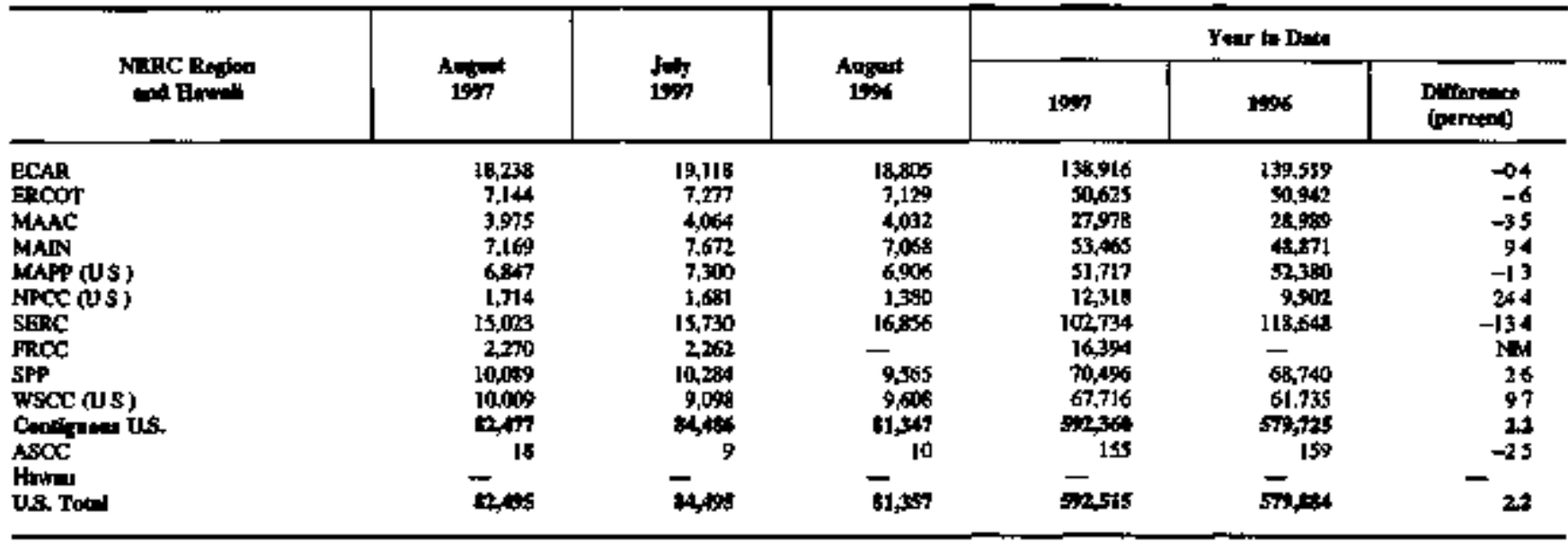

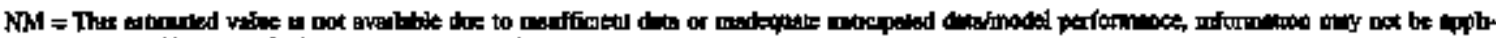

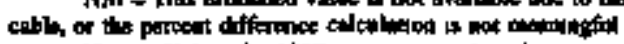

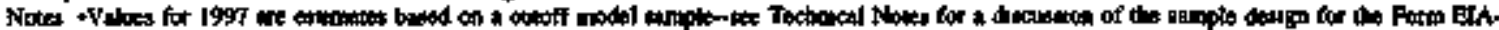

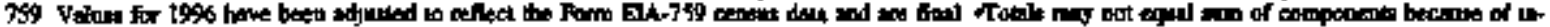

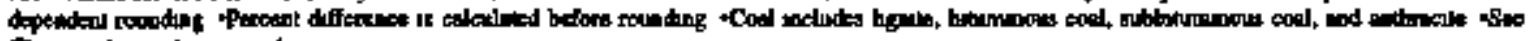
Ghatstoy fir explanen of actomins

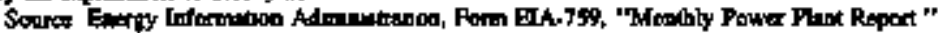

Table 16. Electric Utility Consumption of Petroteam by NERC Region and Hawati (Thousand Barrels)

\begin{tabular}{|c|c|c|c|c|c|c|}
\hline \multirow{2}{*}{ 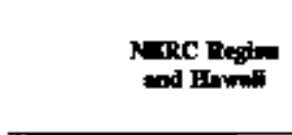 } & \multirow{2}{*}{ Aorist } & \multirow{2}{*}{ the } & \multirow{2}{*}{ An: } & \multicolumn{3}{|c|}{ Yam to Dote } \\
\hline & & & & 1997 & 1996 & $\begin{array}{l}\text { Ditherex } \\
\text { (pereat) }\end{array}$ \\
\hline ECAR & 204 & 45 & $\begin{array}{r}345 \\
26\end{array}$ & I & 2,184 & -160 \\
\hline MANC & 765 & 1,765 & gIT & 3.980 & 9,747 & -356 \\
\hline MALIN & 3 & 223 & 119 & 843 & $1,4,1$ & -380 \\
\hline MAPT (US) & 71 & 225 & 54 & 672 & 428 & 571 \\
\hline NPOC \{US ) & $4,0,4$ & 4,616 & 9.635 & $3 t, 0 \% 5$ & 26,157 & 189 \\
\hline SERC & 381 & 794 & 3,956 & 2,504 & 30,625 & -918 \\
\hline FROC & $5,10 \mathrm{~d}$ & 5,5945 & - & 25.043 & - & Nivi \\
\hline SPP & 335 & 321 & 35 & 2650 & 2,341 & 132 \\
\hline WSCC (Us) & 80 & II & $10 ?$ & 4as & $l_{106}$ & -597 \\
\hline Cantlyon 11.8 & $11,0,7$ & 14, & 9,10 & 71,363 & $x+2$ & -4 \\
\hline$A S C C$ & 380 & 370 & $\mathbf{4 5}$ & 2,903 & 413 & 699 \\
\hline Hrons & 95 & 936 & 999 & 7,159 & 7.259 & -14 \\
\hline C.S. Tot & 12,432 & 15,366 & 14134 & $\$ 1,79 t$ & $2 \pi, 43$ & -13 \\
\hline
\end{tabular}

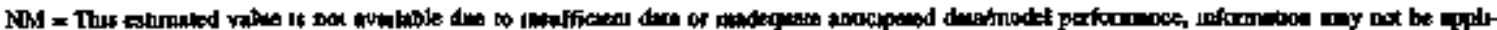

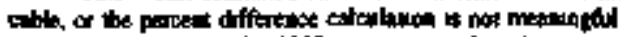

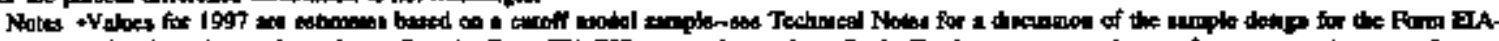

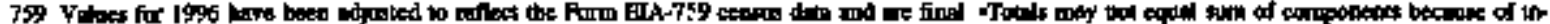

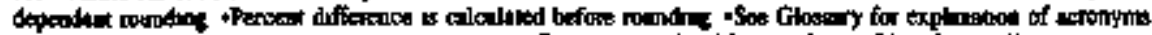

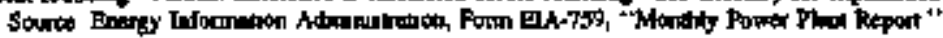


Tahle 17. Flectric Utility Consumption of Gas by NERC Region and Hawaï (Milion Cubic Feet)

\begin{tabular}{|c|c|c|c|c|c|c|}
\hline \multirow{2}{*}{$\begin{array}{l}\text { MERC Depion } \\
\text { and Howhin }\end{array}$} & \multirow{2}{*}{ Anit: } & \multirow{2}{*}{$\frac{15}{159}$} & \multirow{2}{*}{ 16: } & \multicolumn{3}{|c|}{ Yetr to Data } \\
\hline & & & & $19 \%$ & $19 \%$ & $\begin{array}{l}\text { Difrones } \\
\text { (percies) }\end{array}$ \\
\hline ECAR & 3,062 & $7,0,03$ & 4,136 & 28,816 & 27.456 & 50 \\
\hline ERCOT & 119.845 & 117,495 & 97.122 & 564,914 & 610,591 & -75 \\
\hline MAAC & $7,78 B$ & 16,283 & 10,145 & 52,869 & 44,821 & 180 \\
\hline MAIN & 4,838 & 10,346 & 5,602 & 43,887 & 25,042 & 753 \\
\hline MAPP (US) & 1.781 & 3,853 & 1,355 & 12,551 & 9,110 & 378 \\
\hline NPCD (U S ) & 39,261 & 44,666 & 35,965 & III,575 & 135,155 & 995 \\
\hline SERC & 12,655 & 17,053 & 40,074 & 56,303 & 235,549 & -761 \\
\hline FRCC & 33,121 & 32,708 & - & 210, ] 78 & - & NM \\
\hline SPP & 94,477 & 513,474 & 94,037 & 475,247 & 515,860 & -79 \\
\hline WSCE (Us) & N.18매 & 61,023 & 76,083 & 323,335 & 294,679 & 97 \\
\hline Condig. U.S. & 3r,ge: & 42,150 & 364510 & $1,513,675$ & 1, Actsu & 45 \\
\hline $\mathrm{ASCC}$ & 2,438 & 2,736 & 2,544 & 22,828 & 20,516 & 113 \\
\hline Hrita & $\overrightarrow{0}$ & - & - & - & - & - \\
\hline WS. Tom & $3943 \pi$ & Q86, 船4 & 34,0093 & 2,046,503 & $1,91 \%, 7 \%$ & 46 \\
\hline
\end{tabular}

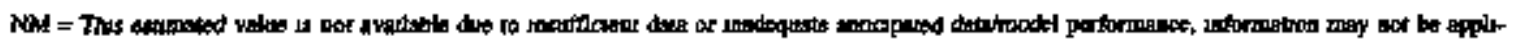

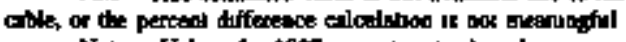

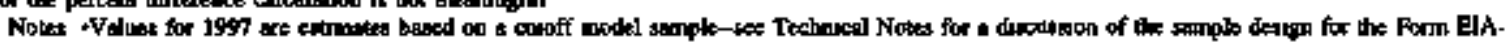

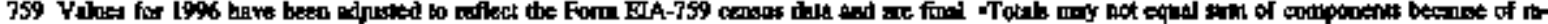

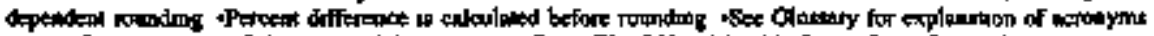

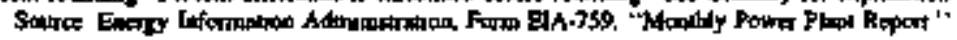


Table 18. Electric Utillty Consumption of Con by Census Division and State (Thousand Short Tons)

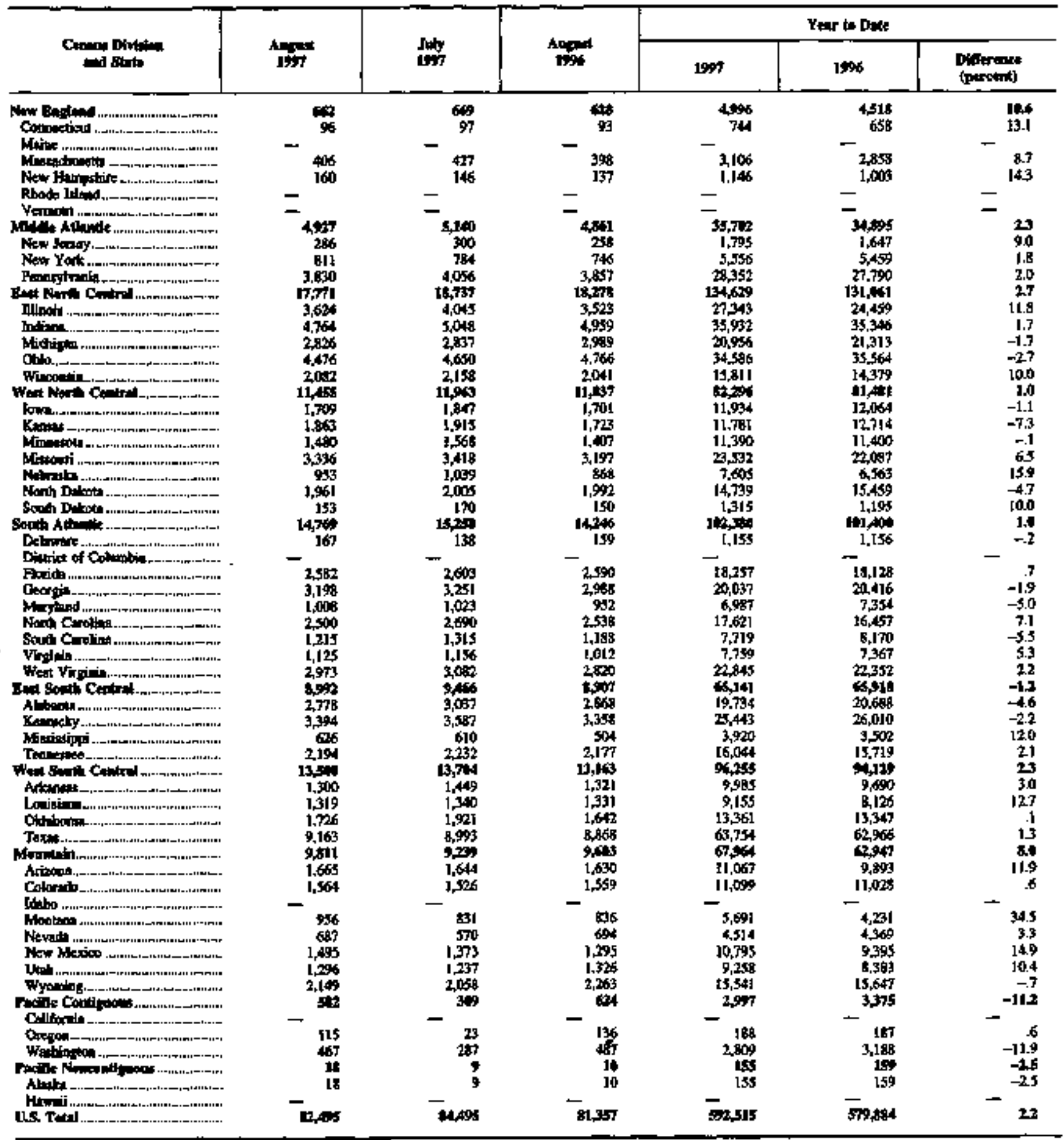

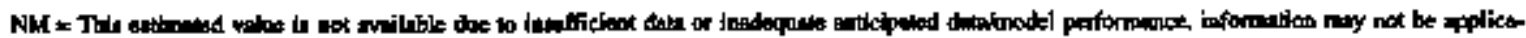

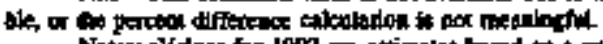

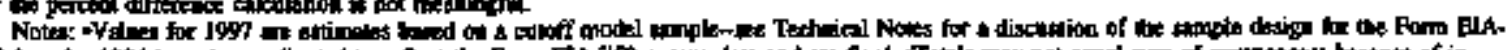

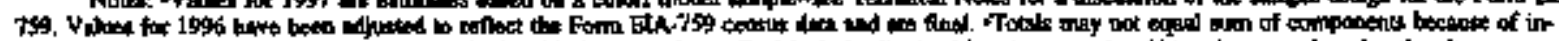

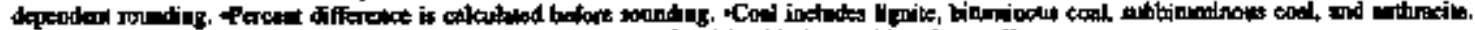

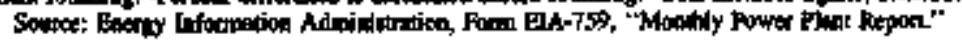


Table 19. Electric Utility Contumption of Petroleam by Census Dirision and Strte (Thousand Barrels)

\begin{tabular}{|c|c|c|c|c|c|c|}
\hline \multirow{2}{*}{ Cocis Drumin } & \multirow{2}{*}{ 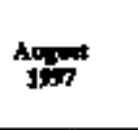 } & \multirow{2}{*}{ ty } & \multirow{2}{*}{ host } & \multicolumn{3}{|c|}{ Yur to but } \\
\hline & & & & $6 \%$ & tas & 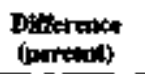 \\
\hline 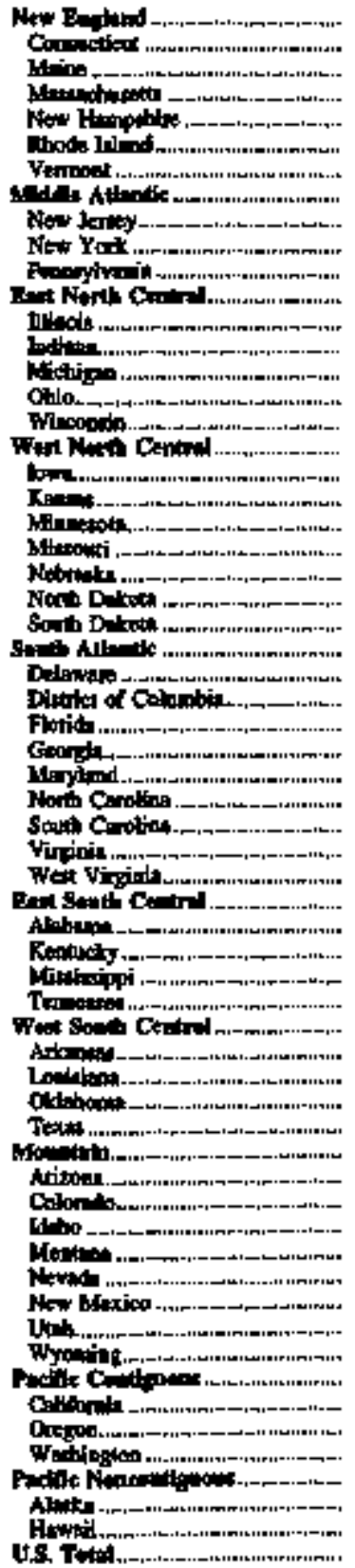 & 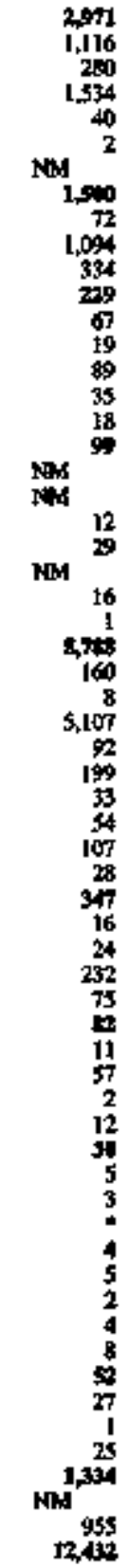 & 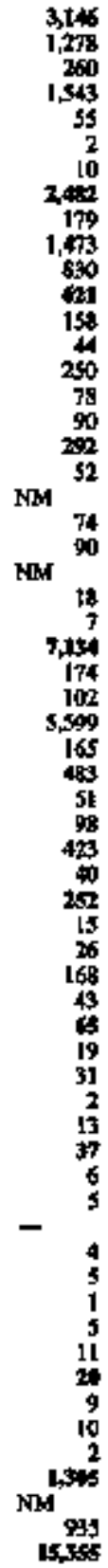 & 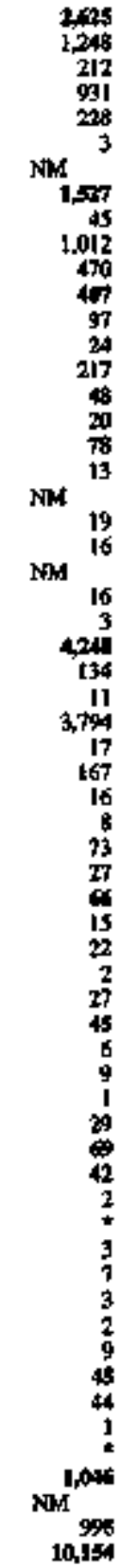 & 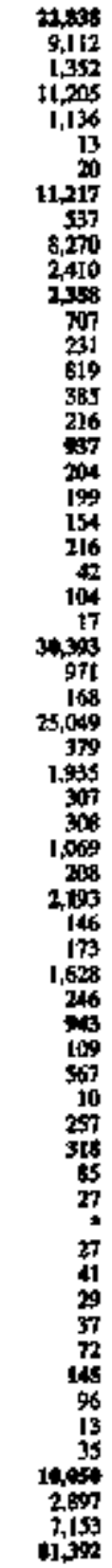 & 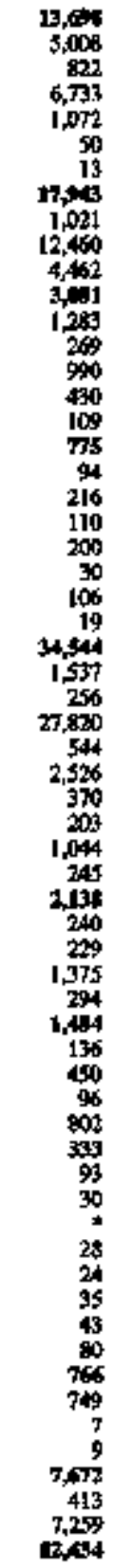 & 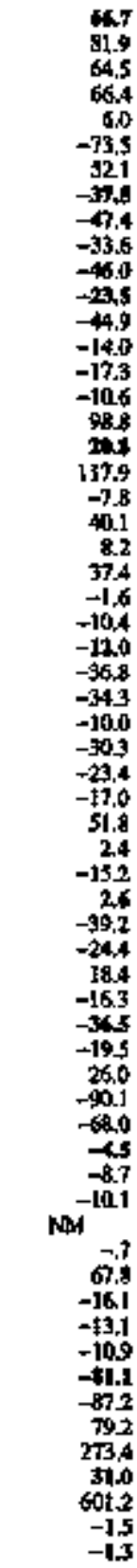 \\
\hline
\end{tabular}

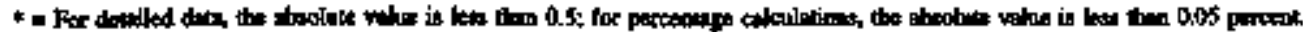

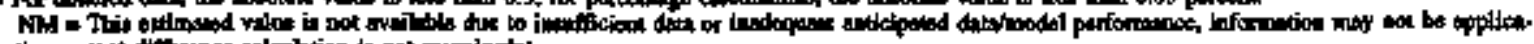

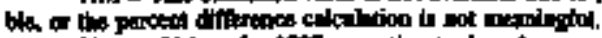

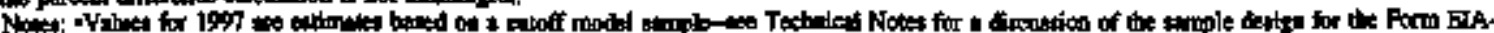

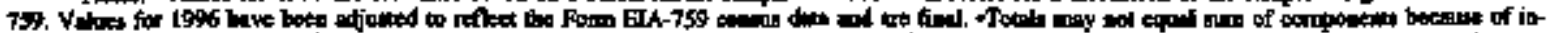

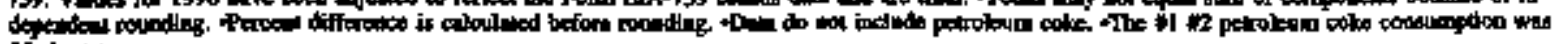
IS hint tom.

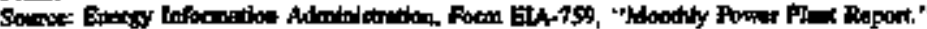


Table 20. Ebectric Utility Consunption of Gas by Census Division and State (Mijlion Cubic Feet)

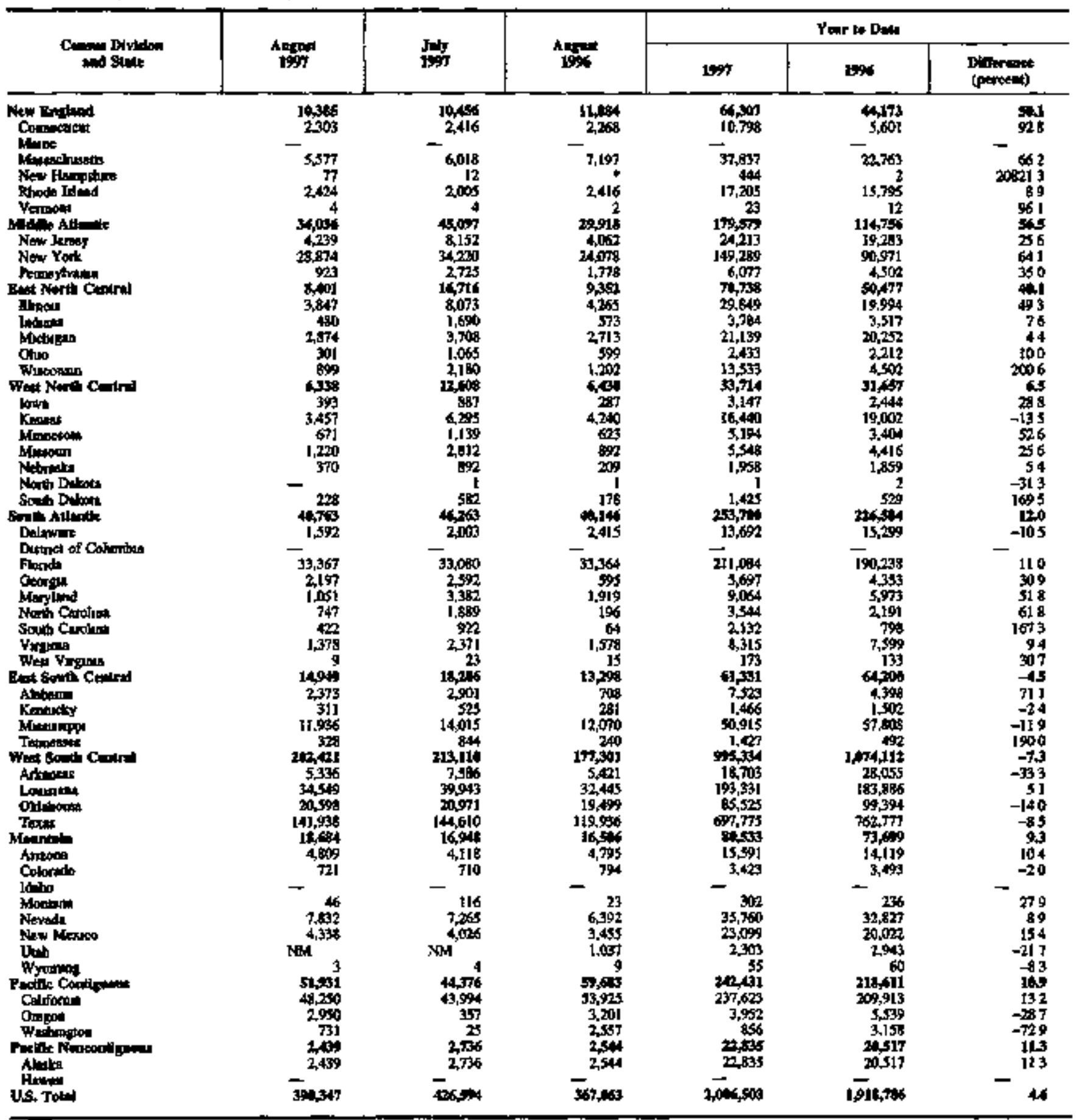

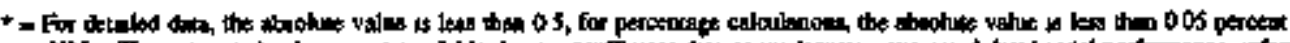

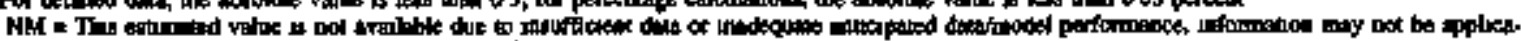

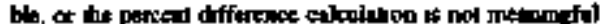

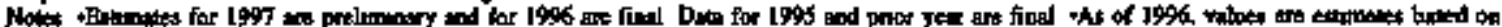

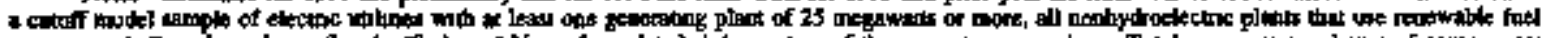

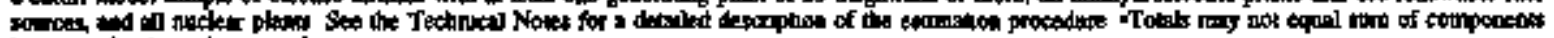

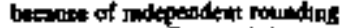

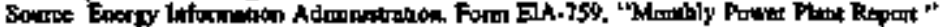




\section{Fossil-Fuel Stocks at U.S. Electric Utilities}

Table 21. US. Eleetric Dthlity Stocks of Cod and Petroleum, 1987 Through Aasust 1997

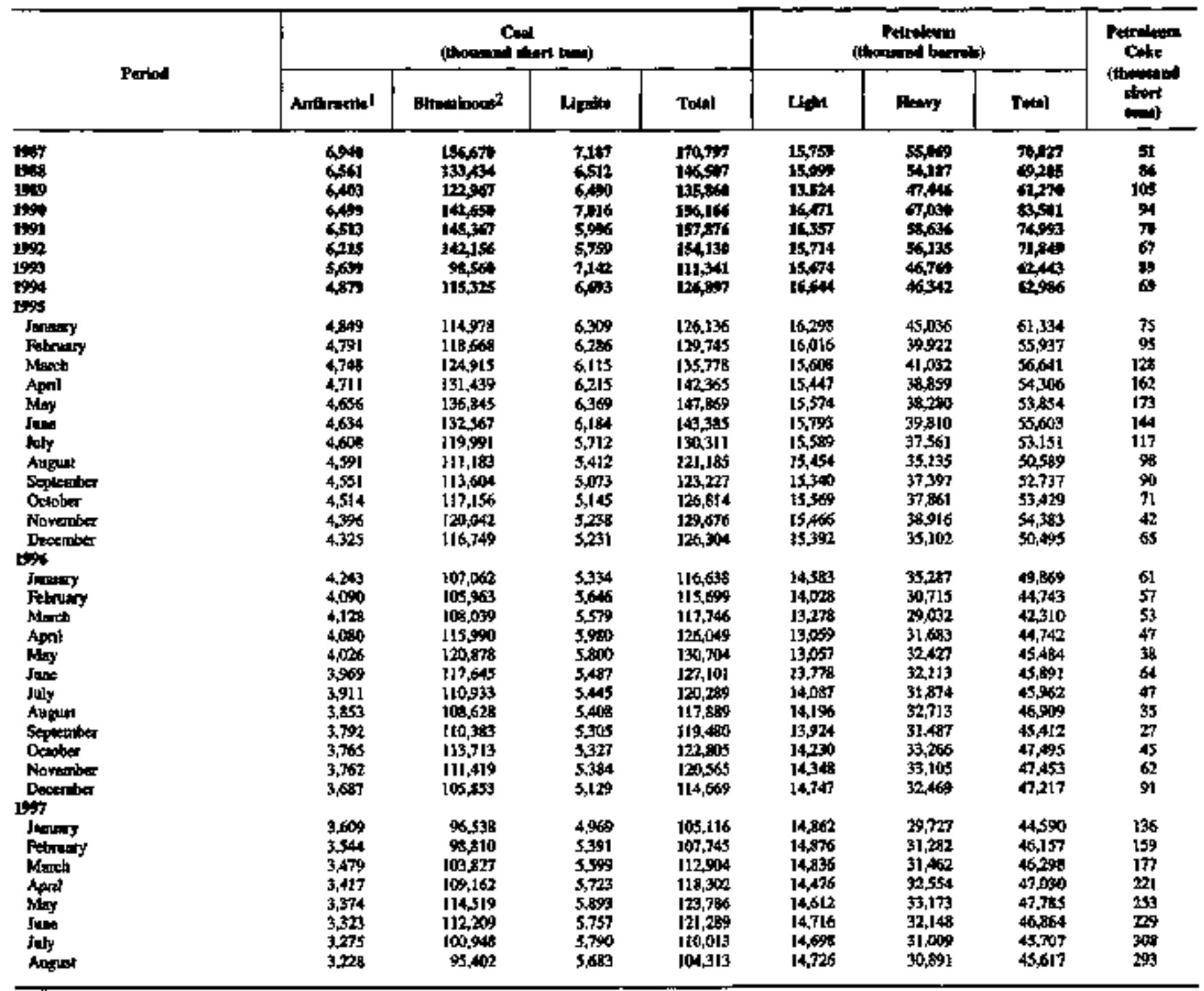

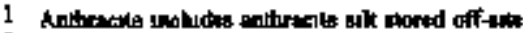

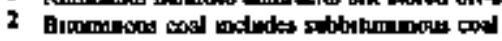

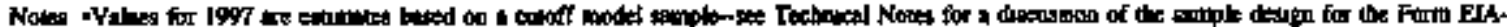

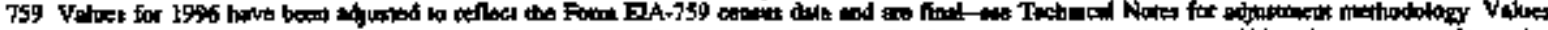

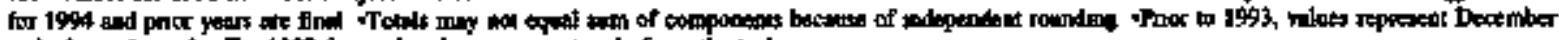

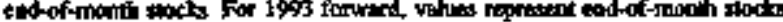

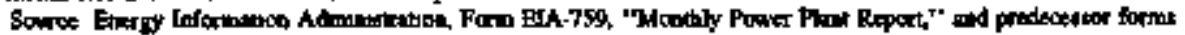


Table 22. Electric Utillty Stocks of Coel by NERC Reglon and Hawall (Thousand Short Tons)

\begin{tabular}{|c|c|c|c|c|c|}
\hline NoRC Reang & $\frac{\operatorname{Ant}}{t+\pi}$ & $\frac{1}{10 y}$ & 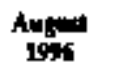 & 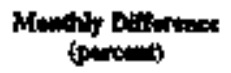 & Yariy butarnes \\
\hline 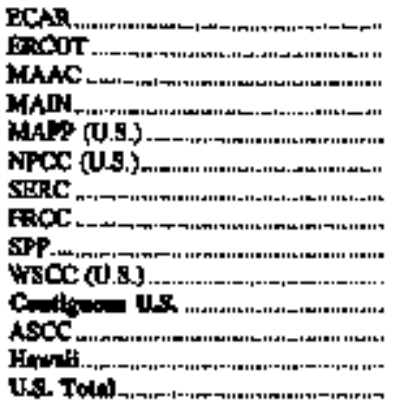 & $\begin{array}{r}20,355 \\
5,095 \\
7,890 \\
11,366 \\
10,174 \\
1,812 \\
14,875 \\
2,821 \\
128272 \\
11,108 \\
104311 \\
-\quad 1 \\
104013\end{array}$ & $\begin{array}{r}25,635 \\
5,772 \\
1,573 \\
11,789 \\
10,156 \\
2,008 \\
16,270 \\
3,095 \\
19,802 \\
11,961 \\
110,012 \\
-11 \\
110,013\end{array}$ & 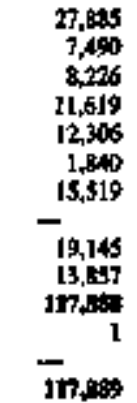 & 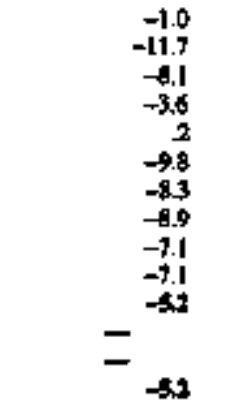 & $\begin{array}{r}-5.5 \\
-320 \\
-12 \\
-2.2 \\
-17.3 \\
-1.5 \\
-4.1 \\
-39.4 \\
-19.0 \\
-11.3 \\
-25.0 \\
- \\
-11.5\end{array}$ \\
\hline
\end{tabular}

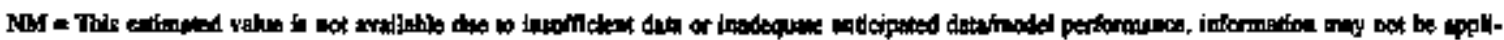

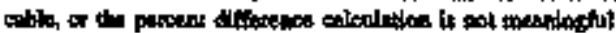

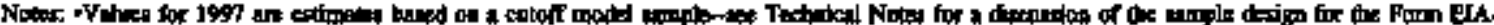

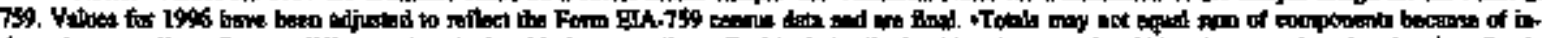

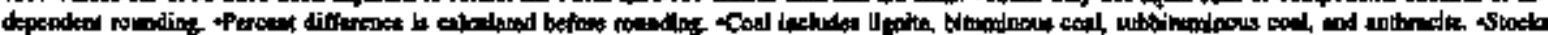

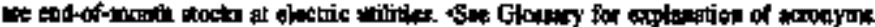

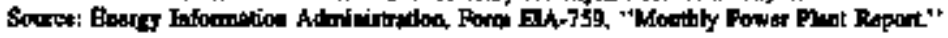

Table 23. Rlectric Utillky Stocks of Petrolfam by NERC Region and Hawati (Thousand Barrels)

\begin{tabular}{|c|c|c|c|c|c|}
\hline $\begin{array}{l}\text { NERC Rejon } \\
\text { and Fisura }\end{array}$ & Ariftit & $\operatorname{Ig}$ & timent & 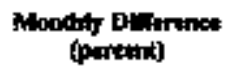 & 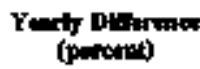 \\
\hline 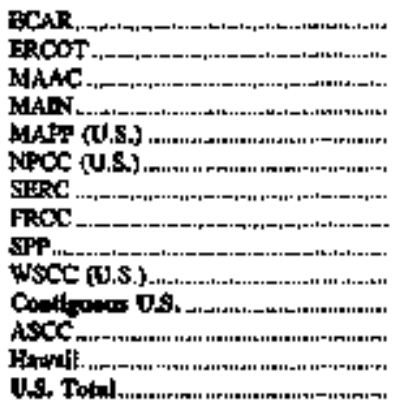 & $\begin{array}{r}1,530 \\
4,065 \\
5,460 \\
1,545 \\
715 \\
10,724 \\
3,181 \\
6,813 \\
3,292 \\
7,008 \\
4,144 \\
204 \\
999 \\
4,517\end{array}$ & $\begin{array}{r}1,521 \\
4,068 \\
5,512 \\
1,512 \\
692 \\
10,690 \\
3,012 \\
6,768 \\
3,462 \\
7,087 \\
44,345 \\
201 \\
1,163 \\
4,7,75\end{array}$ & 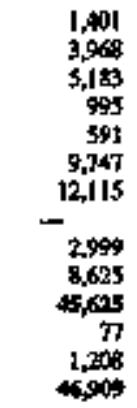 & $\begin{array}{r}0.6 \\
* \\
-1.0 \\
2.2 \\
3.3 \\
.3 \\
5.6 \\
.7 \\
-5.5 \\
6 \\
1.2 \\
1.2 \\
-1.1 \\
-7\end{array}$ & $\begin{array}{r}9.2 \\
2.5 \\
5.3 \\
55.3 \\
20.9 \\
14.6 \\
-73.7 \\
67.4 \\
9.8 \\
-17.8 \\
-7.7 \\
265.1 \\
-17.3 \\
-24\end{array}$ \\
\hline
\end{tabular}

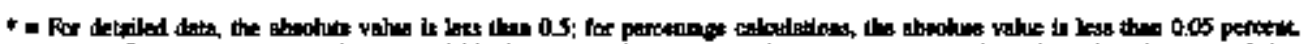

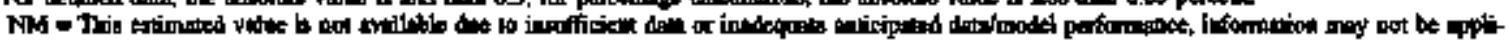

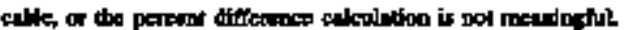

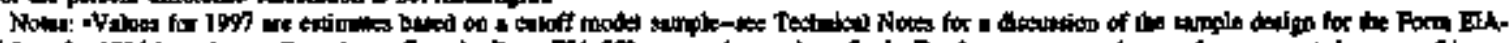

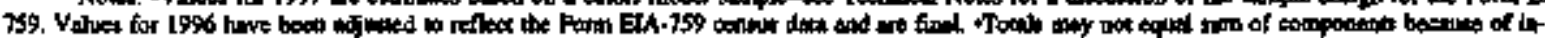

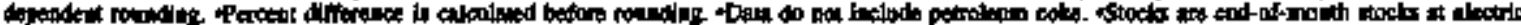

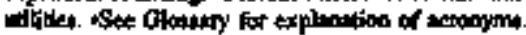

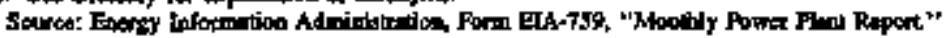


Table 24. Elettric Utility Stocks of Coel by Cetusus Division and State (Thousand Short Tons)

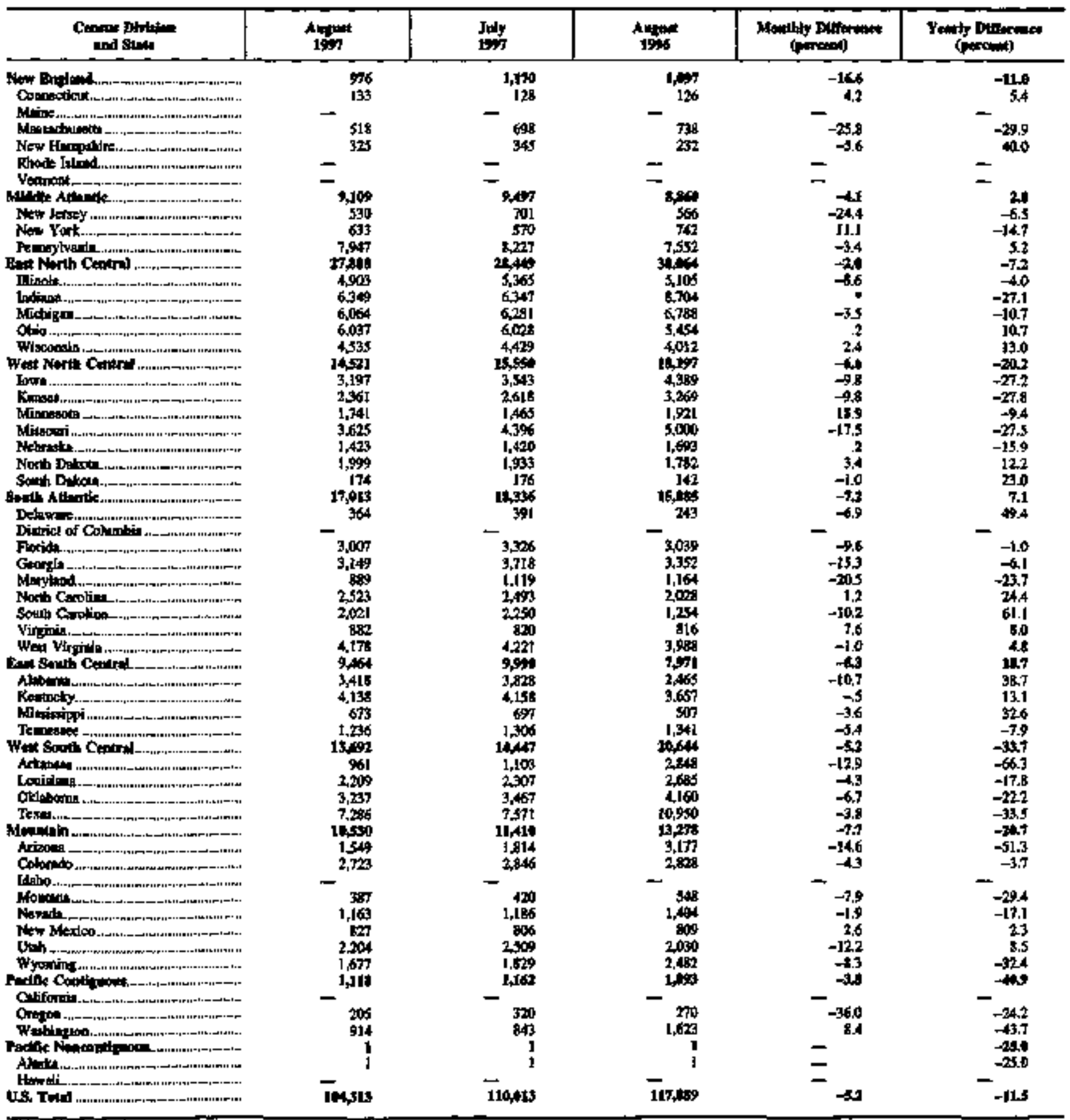

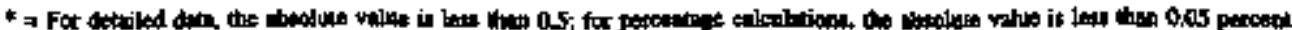

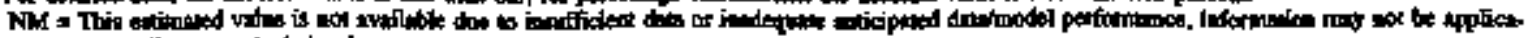

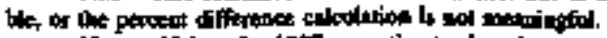

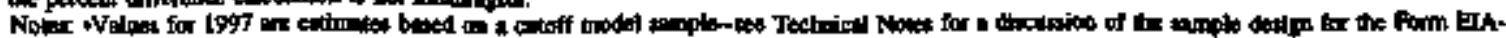

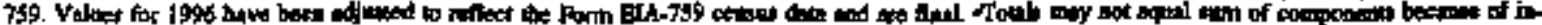

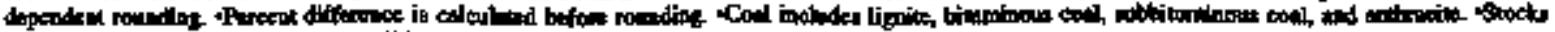

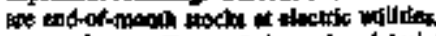

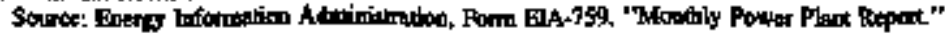


Table 25. Electric Utility Stocks of Petroleum by Census Divtsion and State (Thotsand Barreds)

\begin{tabular}{|c|c|c|c|c|c|}
\hline 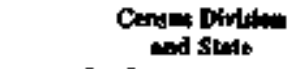 & Antist & hy & 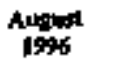 & 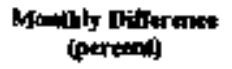 & Yourly bitunet \\
\hline 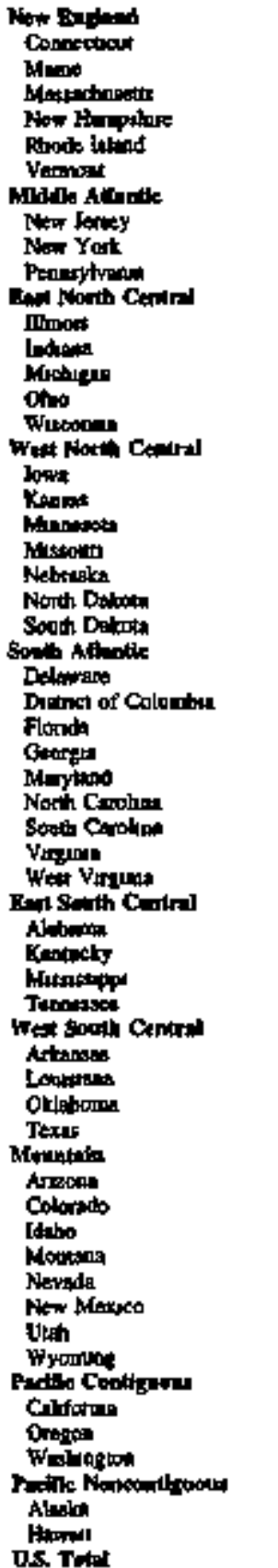 & 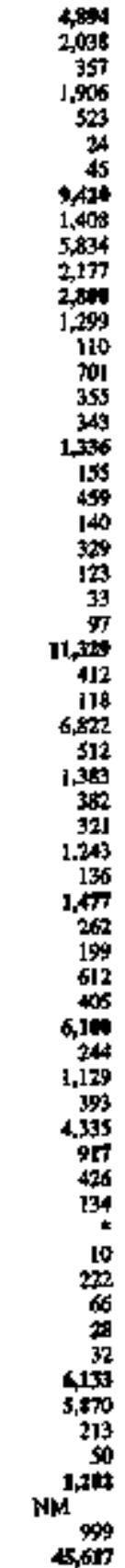 & 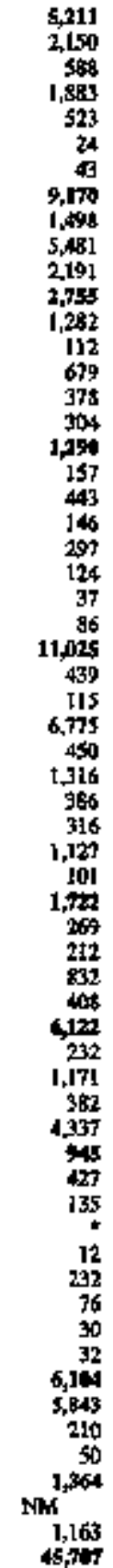 & 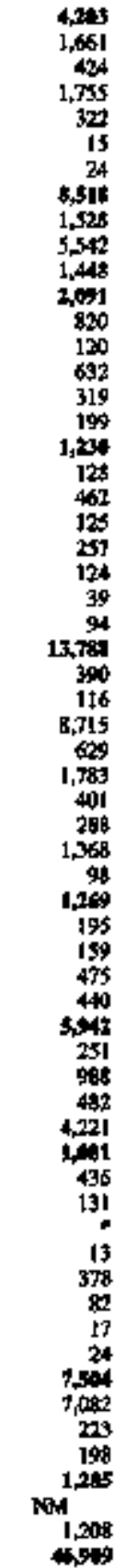 & 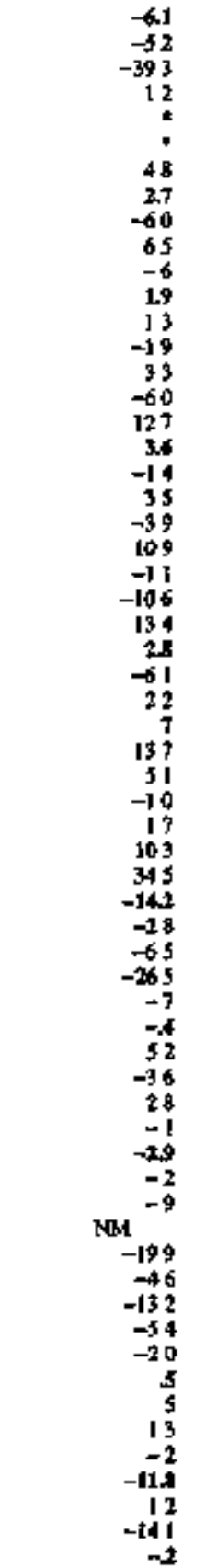 & 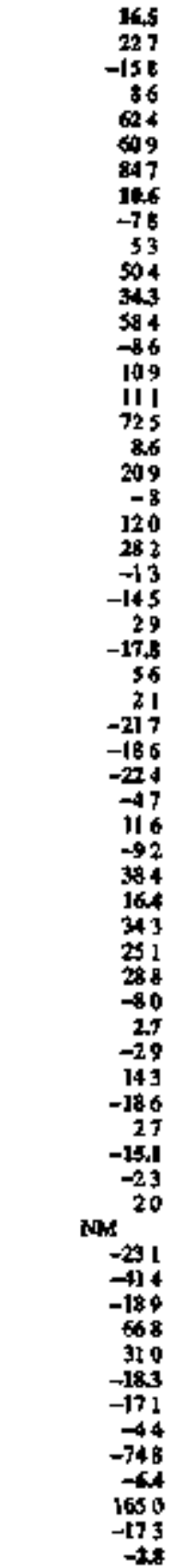 \\
\hline
\end{tabular}

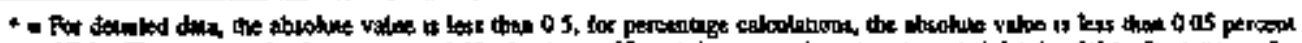

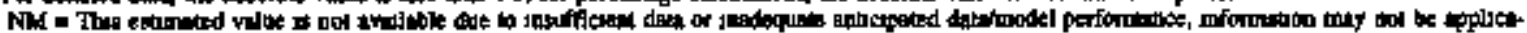

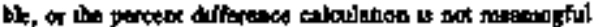

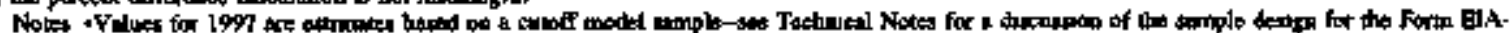

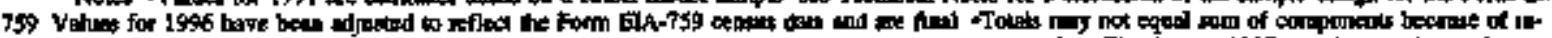

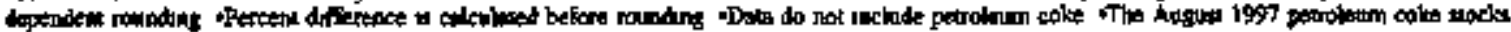

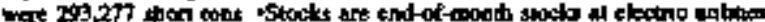

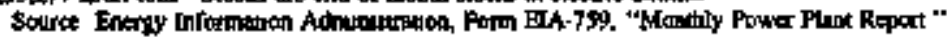




\section{Receipts and Cost of Fossil Fuels at U.S. Electric Utilities}

\section{July 1997 Receipts and Cost Data}

At the time of publication, the Western Farners Electric Cooperative (WFEC) and the City of Lafayette had not reported gas roceipt and cost data for the month of July 1997 on the FERC Form 423, "Monthly Report of Cost and Quality of Fuels at Electric Plants." Receipt data used in this report are based on July 1997 consumption data reported by each company on Form EIA-759, "Monthly Power Plant Report." Cost data shown in this report are based on costs reported by esch company for the month of June 1997. (Coal costs for WFEC are actual costs provided by the company).

The City of Los Angeles did not report gas receipt or cost data for July 1997 on the FERC Form 423. Thus, the cost data for gas receipts appeariog in this issue of the Electric Power Monthly includes estimates for this electric utility, calculated using a model-based statistical epproach. In addition. Form EIA-759 gas consumption data were used in place of receipts. 
Table 26. U.S. Electric Utility Receipts of and Average Cost for Fossil Pinels, 1987 Throut July 1997

\begin{tabular}{|c|c|c|c|c|c|c|c|c|c|}
\hline \multirow{3}{*}{ Puxlad } & \multicolumn{2}{|c|}{ Con 1} & \multicolumn{4}{|c|}{ Petrateinas } & \multicolumn{2}{|c|}{ Ga: } & \multirow{3}{*}{ 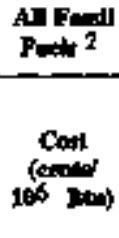 } \\
\hline & \multirow{2}{*}{ 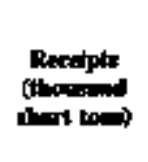 } & \multirow{2}{*}{ Const } & \multicolumn{2}{|c|}{ 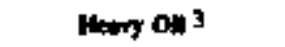 } & \multicolumn{2}{|c|}{ Thitid } & \multirow{2}{*}{ 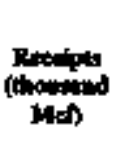 } & \multirow{2}{*}{ Cont } & \\
\hline & & & 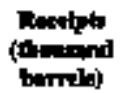 & 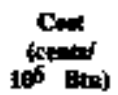 & 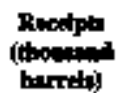 & inil & & & \\
\hline 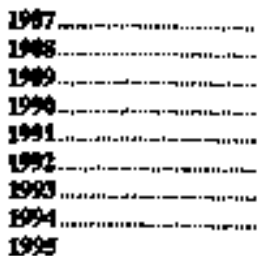 & 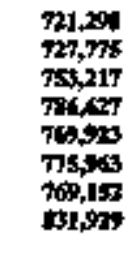 & $\begin{array}{l}1546 \\
1466 \\
1445 \\
1455 \\
1467 \\
1414 \\
1345 \\
1345\end{array}$ & 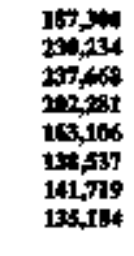 & $\begin{array}{l}2 \% 5 \\
2455 \\
2445 \\
335 \\
2465 \\
245 \\
2462 \\
2429\end{array}$ & 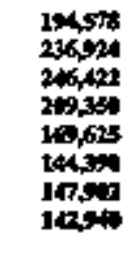 & 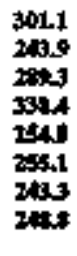 & 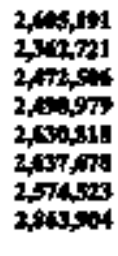 & 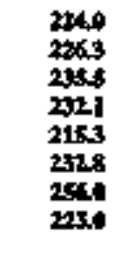 & 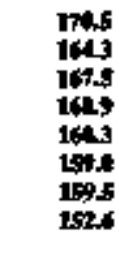 \\
\hline 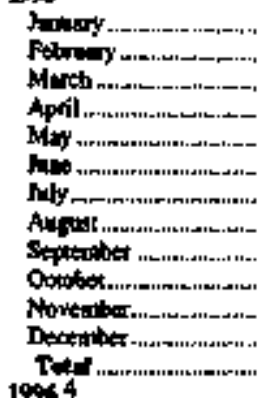 & $\begin{array}{l}70,206 \\
65,789 \\
69,059 \\
66,167 \\
60,564 \\
64,543 \\
67,734 \\
73,242 \\
70,938 \\
70,140 \\
70,195 \\
70,281 \\
96,560\end{array}$ & $\begin{array}{l}13,1.1 \\
139.5 \\
13.1 \\
133.7 \\
133.7 \\
133.3 \\
130.4 \\
130.5 \\
131.8 \\
129.6 \\
130.2 \\
113.7 \\
131.6\end{array}$ & $\begin{array}{r}5,560 \\
6,150 \\
5,040 \\
2,946 \\
5,964 \\
8,476 \\
8,367 \\
9,394 \\
9,0166 \\
5,553 \\
4,773 \\
7,259 \\
78,716\end{array}$ & $\begin{array}{l}273.2 \\
256.2 \\
258.9 \\
266.2 \\
279.0 \\
274.3 \\
250.8 \\
777.0 \\
234.7 \\
242.5 \\
250.5 \\
2958 \\
150.4\end{array}$ & 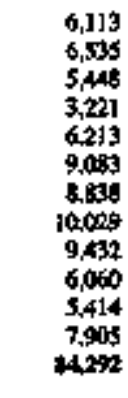 & 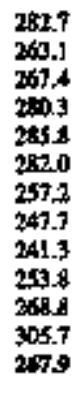 & 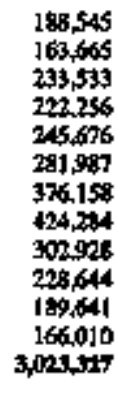 & $\begin{array}{l}209.2 \\
297.1 \\
189.1 \\
194.5 \\
202.1 \\
202.8 \\
186.1 \\
179.4 \\
189.5 \\
204.5 \\
218.9 \\
255.3 \\
15.4 .4\end{array}$ & $\begin{array}{l}145.4 \\
143.7 \\
144.3 \\
144.1 \\
147.3 \\
130.4 \\
145.1 \\
345.1 \\
145.1 \\
142.6 \\
249.3 \\
245.1 \\
144.1\end{array}$ \\
\hline 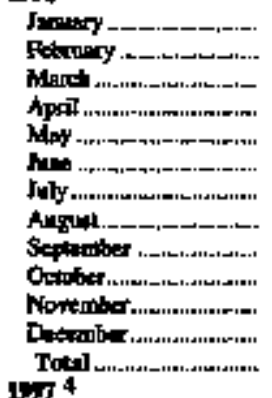 & $\begin{array}{l}67,852 \\
66,620 \\
69,921 \\
70,361 \\
72,158 \\
69,677 \\
75,178 \\
76,544 \\
72,730 \\
75,756 \\
71,375 \\
72,525 \\
862,74\end{array}$ & $\begin{array}{l}129.1 \\
129.3 \\
139.2 \\
130.8 \\
130.7 \\
129.2 \\
121.4 \\
123.9 \\
127.5 \\
128.9 \\
127.9 \\
127.6 \\
121 .\end{array}$ & $\begin{array}{r}13,255 \\
6,099 \\
9,031 \\
8,263 \\
5,882 \\
8,825 \\
10,799 \\
10,484 \\
5,138 \\
5,675 \\
6,382 \\
8,098 \\
98,926\end{array}$ & $\begin{array}{l}332.4 \\
252.5 \\
285.2 \\
309.7 \\
304.4 \\
277.0 \\
276.6 \\
202.5 \\
293.6 \\
331.9 \\
333.5 \\
398.1 \\
302.4\end{array}$ & $\begin{array}{r}14,540 \\
7,021 \\
9,595 \\
6,724 \\
6,473 \\
9,508 \\
11,390 \\
10,971 \\
5,925 \\
6,407 \\
7,159 \\
0,961 \\
14,465\end{array}$ & $\begin{array}{l}337.1 \\
360.6 \\
296.8 \\
319.0 \\
317.6 \\
288.2 \\
234.4 \\
294.6 \\
307.1 \\
34.7 \\
354.4 \\
355.2 \\
315.7\end{array}$ & 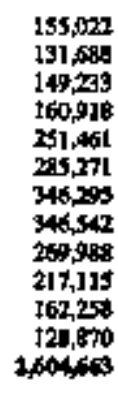 & $\begin{array}{l}291.0 \\
294.7 \\
268.4 \\
264.6 \\
247.6 \\
255.1 \\
265.9 \\
260.7 \\
219.4 \\
233.8 \\
301.9 \\
396.1 \\
244.1\end{array}$ & $\begin{array}{l}155.5 \\
148.5 \\
149.0 \\
130.0 \\
151.8 \\
155.1 \\
151.2 \\
154.6 \\
145.3 \\
146.6 \\
131.0 \\
156.1 \\
151.5\end{array}$ \\
\hline 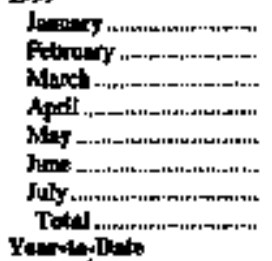 & $\begin{array}{l}71,900 \\
69,099 \\
72,678 \\
69,695 \\
74,509 \\
30,673 \\
74,065 \\
\text { sal, }\end{array}$ & $\begin{array}{l}128.0 \\
129.0 \\
129.8 \\
129.8 \\
128.0 \\
128.0 \\
125.8 \\
129.4\end{array}$ & $\begin{array}{r}8,811 \\
1,956 \\
6,736 \\
6,379 \\
6,476 \\
9,253 \\
10,800 \\
57,472\end{array}$ & $\begin{array}{l}305.7 \\
297.5 \\
267.2 \\
254.9 \\
257.1 \\
262.9 \\
269.3 \\
27.5\end{array}$ & $\begin{array}{r}9,682 \\
9,346 \\
7,164 \\
6,730 \\
6,967 \\
10,039 \\
11,600 \\
61,50\end{array}$ & $\begin{array}{l}321.0 \\
295.3 \\
276.3 \\
364.8 \\
270.5 \\
274.4 \\
260.4 \\
284.7\end{array}$ & $\begin{array}{r}133,193 \\
134,946 \\
185,394 \\
744,936 \\
215,499 \\
713,021 \\
373,618 \\
1,514,47\end{array}$ & $\begin{array}{l}45.8 \\
315.5 \\
237.1 \\
300.2 \\
246.9 \\
254.0 \\
243.9 \\
244.1\end{array}$ & $\begin{array}{l}157.5 \\
150.9 \\
14.5 .4 \\
144.5 \\
146.6 \\
153.2 \\
154.6 \\
150.6\end{array}$ \\
\hline 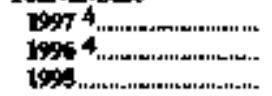 & $\begin{array}{l}\sec , 59 \\
401,709 \\
412,193\end{array}$ & $\begin{array}{l}1304 \\
1296 \\
\operatorname{cks} 1\end{array}$ & $\begin{array}{l}5747 \\
6>01 \\
4011\end{array}$ & $\begin{array}{l}2 \times 3.0 \\
207+7 \\
2 \times 5 . t\end{array}$ & $\begin{array}{l}61,84 \\
67,745 \\
45,451\end{array}$ & $\begin{array}{l}2,47 \\
3415 \\
2731\end{array}$ & 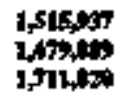 & $\begin{array}{l}2641 \\
2645 \\
2,402\end{array}$ & 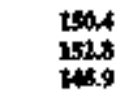 \\
\hline
\end{tabular}

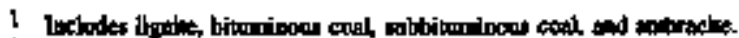

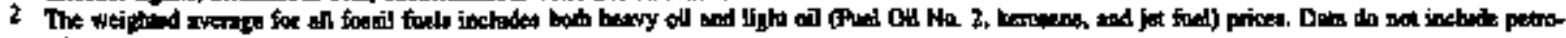
kim colke.

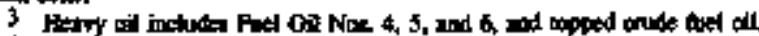

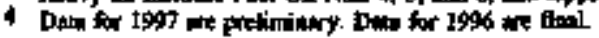

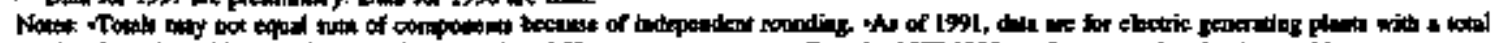

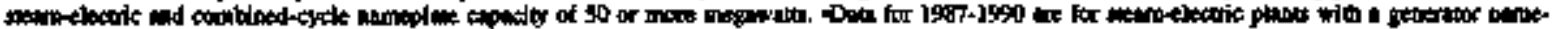

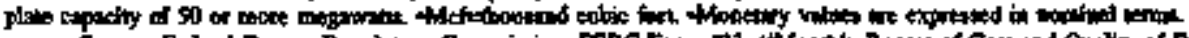
form.

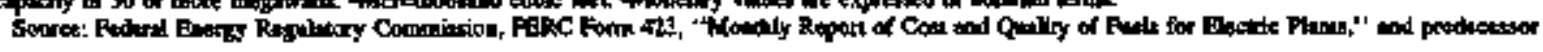


Table 27. Electric Utilicy Recelpts of Cool by NERC Reglon and Howail (Thousasd Short Tons)

\begin{tabular}{|c|c|c|c|c|c|c|}
\hline \multirow{2}{*}{ 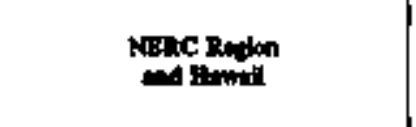 } & \multirow{2}{*}{ this } & \multirow{2}{*}{$\lim _{1 \rightarrow \infty}$} & \multirow{2}{*}{1} & \multicolumn{3}{|c|}{ Vear to Diek } \\
\hline & & & & t 1 & Ins I & $\begin{array}{l}\text { Dituntack } \\
\text { (pertind) }\end{array}$ \\
\hline 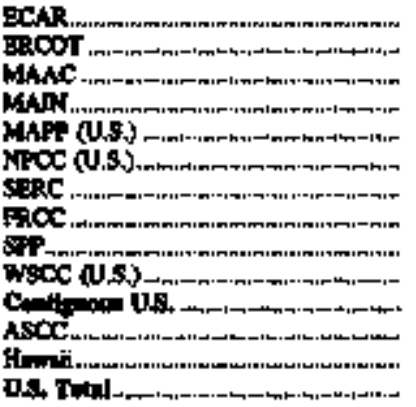 & 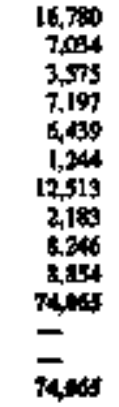 & 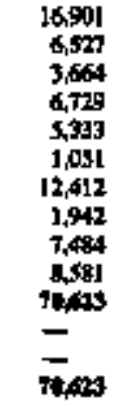 & $\begin{array}{r}17,457 \\
7,382 \\
3,213 \\
6,801 \\
6,461 \\
1,178 \\
15,014 \\
- \\
9,157 \\
8,371 \\
75,175 \\
- \\
-7,175\end{array}$ & $\begin{array}{r}117,052 \\
4,840 \\
24,858 \\
41,421 \\
41,450 \\
4,473 \\
89,066 \\
14,451 \\
53,638 \\
60,720 \\
502,48 \\
- \\
50,969\end{array}$ & $\begin{array}{r}116,410 \\
46,673 \\
24,554 \\
42,218 \\
42,056 \\
8,290 \\
98,035 \\
\overline{57,487} \\
55,477 \\
1,76 \\
- \\
\overline{1}\end{array}$ & $\begin{array}{r}0.6 \\
-3.9 \\
5.3 \\
52.3 \\
-1.4 \\
2.2 \\
-9.9 \\
2.4 \\
-6.4 \\
9.5 \\
2.3 \\
- \\
=\end{array}$ \\
\hline
\end{tabular}

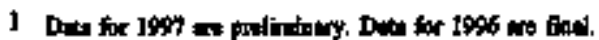

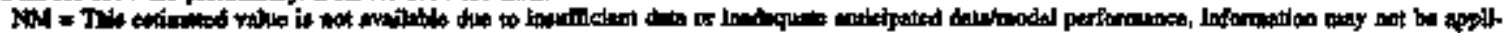

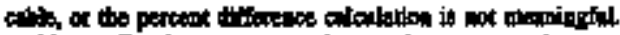

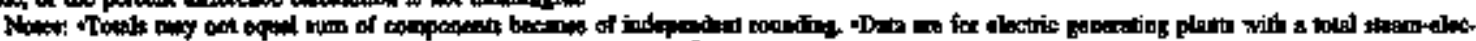

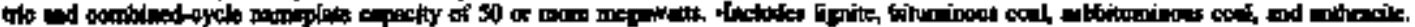

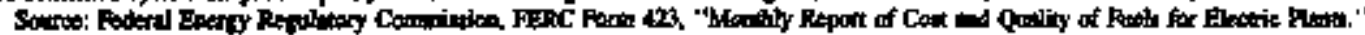

Table 28. Average Cost of Cosi Delivered to Flectric Utilities by NERC Region and Finwati (Cents/Million Btw)

\begin{tabular}{|c|c|c|c|c|c|c|}
\hline \multirow{2}{*}{ RERC nity } & \multirow{2}{*}{ J岁 } & \multirow{2}{*}{ | } & \multirow{2}{*}{$\underset{1964}{3}$} & \multicolumn{3}{|c|}{ Year ta Data } \\
\hline & & & & $\sin 1$ & $15 \% 1$ & 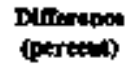 \\
\hline 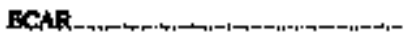 & 123.0 & 1244 & 125.9 & 124.6 & 127.0 & -1.9 \\
\hline 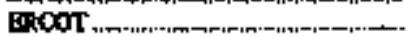 & 1048 & $10+3$ & 1320 & 113.9 & ]1] 1.8 & -4.2 \\
\hline MUNC _-n & 137.9 & 1344 & 142.2 & 140,4 & 1427 & -1.6 \\
\hline 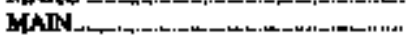 & 134.7 & 137.5 & 136,4 & 1390 & $\$ \$ 9.2$ & -.1 \\
\hline MAPP (US) & 900 & 86.9 & 8.2 & 80.9 & 90.2 & -15 \\
\hline FIPCC (U. & 156.1 & 1553 & 1970 & 156.3 & 135.6 & 5 \\
\hline EBRC & 1390 & 1394 & 146.0 & 540.4 & 546.4 & -4.1 \\
\hline FXCC & 1692 & 1732 & - & 271.6 & - & NB \\
\hline 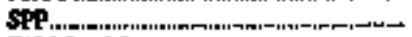 & 103 & 1202 & 120,0 & 125.3 & 123.6 & JA \\
\hline 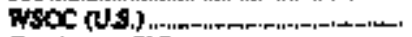 & 1140 & 118.5 & 114,1 & 215.2 & 116.2 & -9 \\
\hline 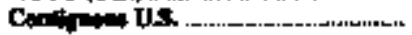 & 125:5 & 120 & 127 & 124.4 & 12,6 & $-\mathbf{L}$ \\
\hline 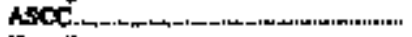 & - & - & - & - & - & 一 \\
\hline 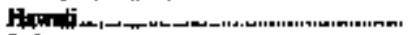 & - & $=$ & - & - & - & $\rightarrow$ \\
\hline 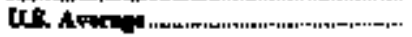 & 12:5: & I2to & tet: & $12 \pi A$ & $\operatorname{tra6}$ & -16 \\
\hline
\end{tabular}

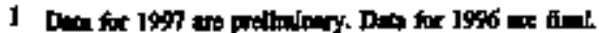

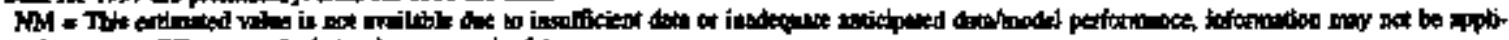

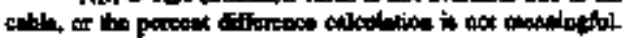

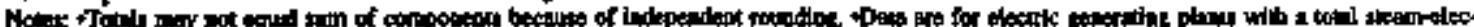

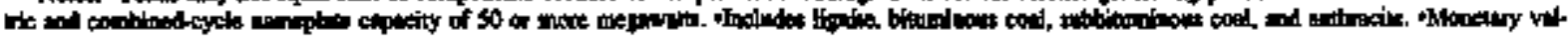

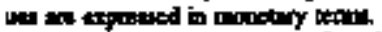

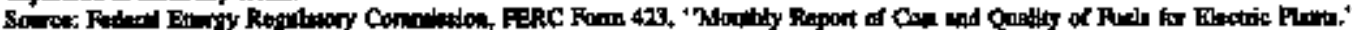


Table 29. Flectric Ut"lity Receipts of Peiroteom by NERC Region and Hawai (Thousand Barrels)

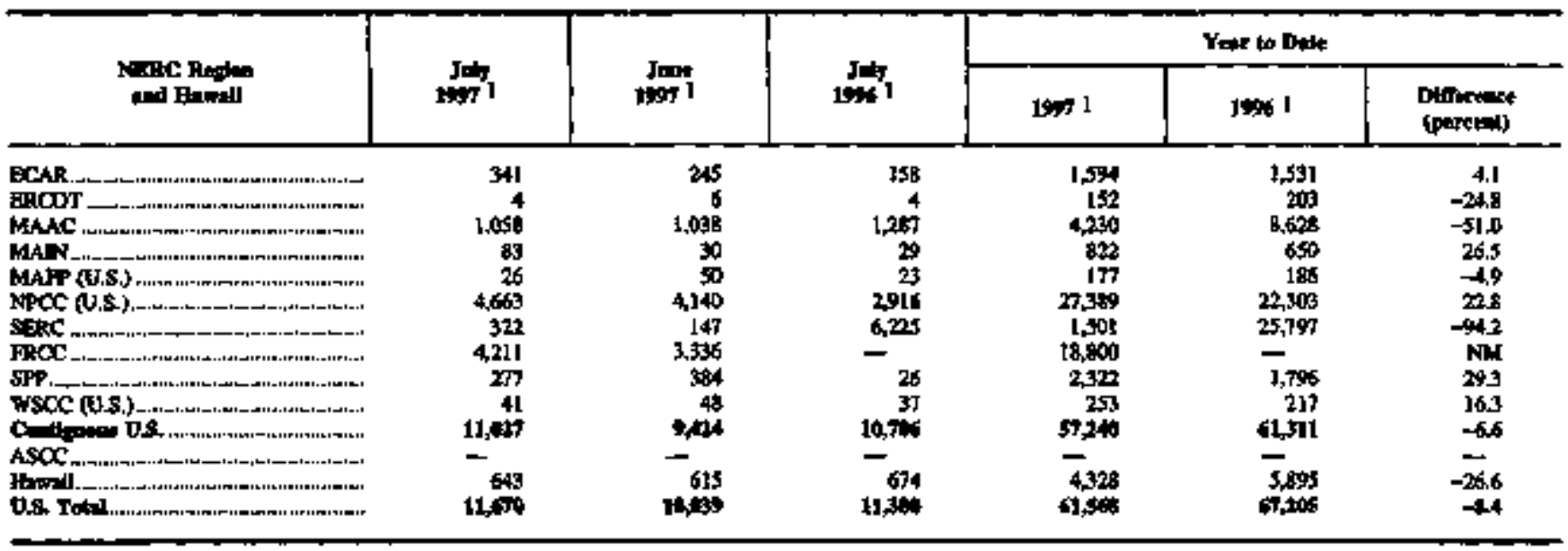

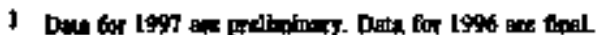

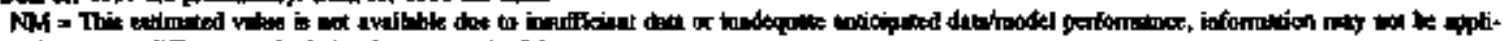

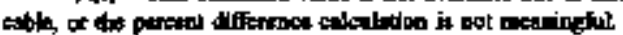

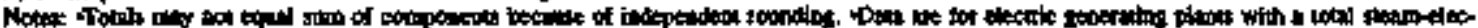

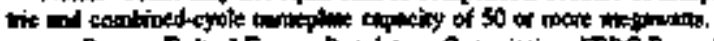

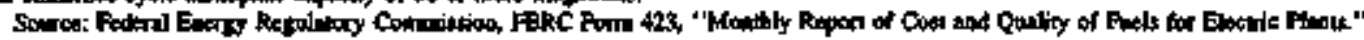

Table 30. Average Cost of Petroheum Delivered to Flectric Utilities by NERC Regars and Fawati (CentsilMillion Bit)

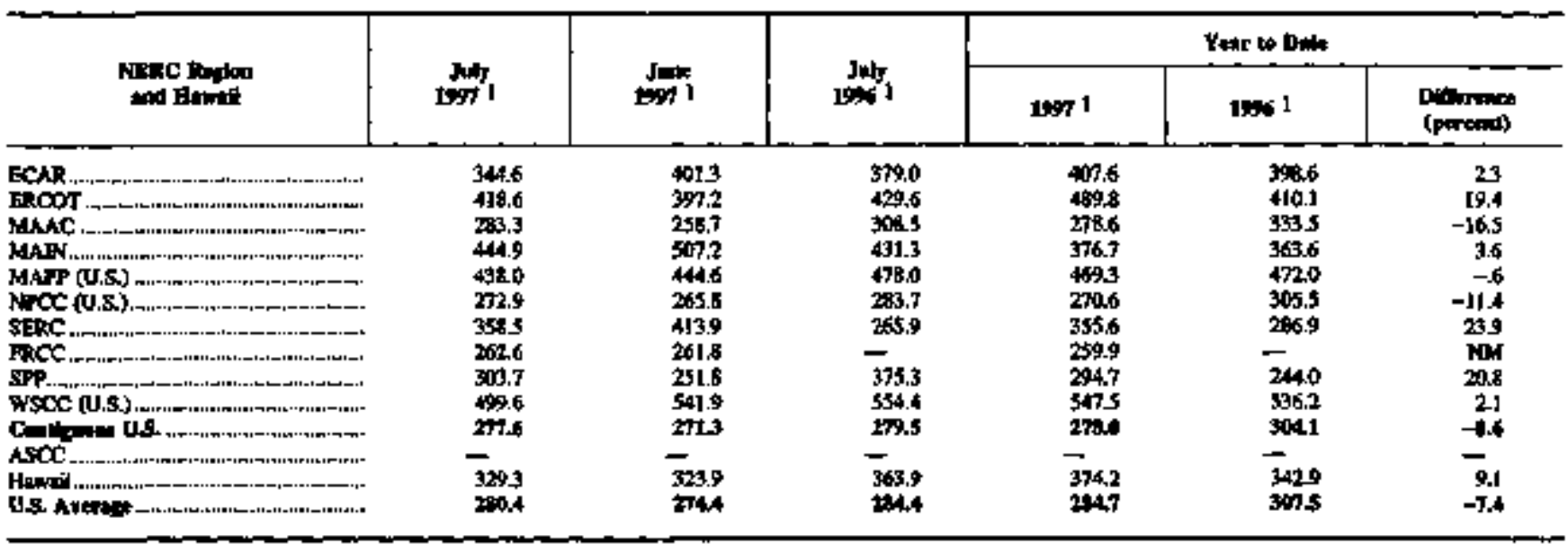

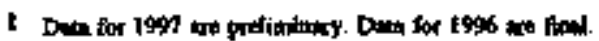

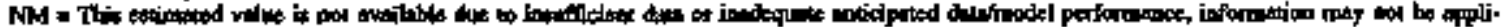

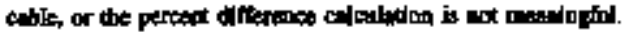

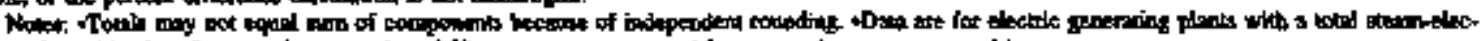

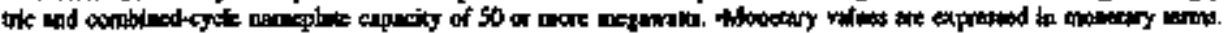

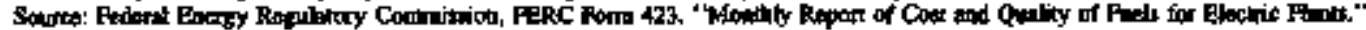


Table 31. Electric Dtilhy Recelpts of Gas by NERC Region and Hamaii (Million Cubic Feet)

\begin{tabular}{|c|c|c|c|c|c|c|}
\hline \multirow{2}{*}{ 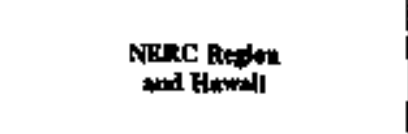 } & \multirow{2}{*}{ Joly } & \multirow{2}{*}{ 19.2 } & \multirow{2}{*}{ 1956 } & \multicolumn{3}{|c|}{ Yot to Dise } \\
\hline & & & & $\operatorname{teg} 1$ & toss I & 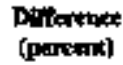 \\
\hline 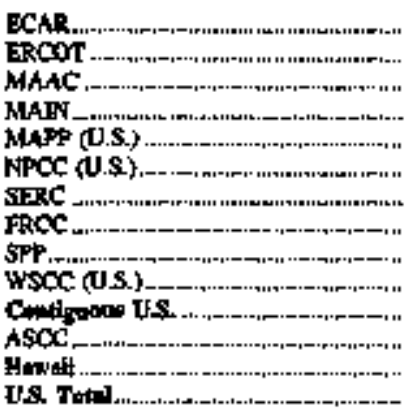 & 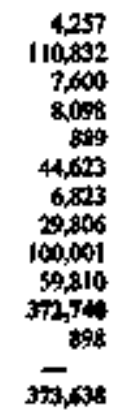 & 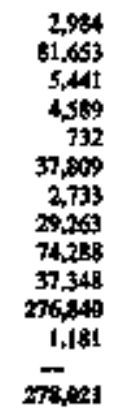 & 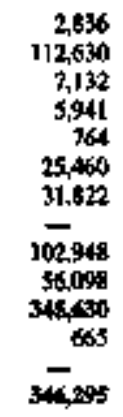 & 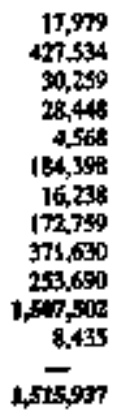 & 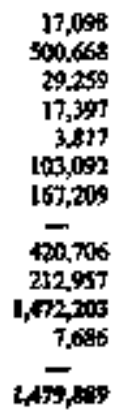 & $\begin{array}{r}5.2 \\
-14.6 \\
3.4 \\
63.5 \\
19.7 \\
78.9 \\
-90.3 \\
\mathrm{NM} \\
-11.7 \\
39.1 \\
2.4 \\
9.7 \\
- \\
2.4\end{array}$ \\
\hline
\end{tabular}

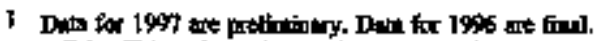

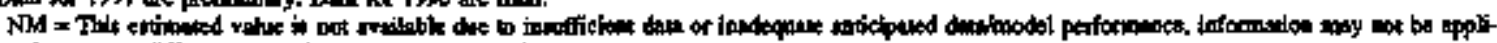

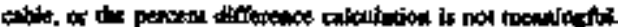

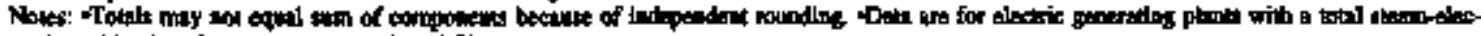

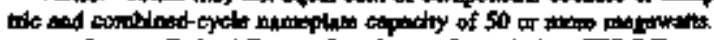

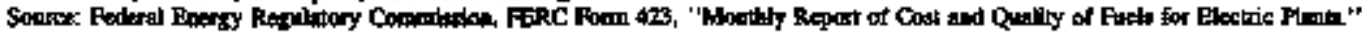

Table 32. A perage Cost of Gas Delivered to Electric Utilities by NERC Region and flowniti (CentsiMilion Bti)

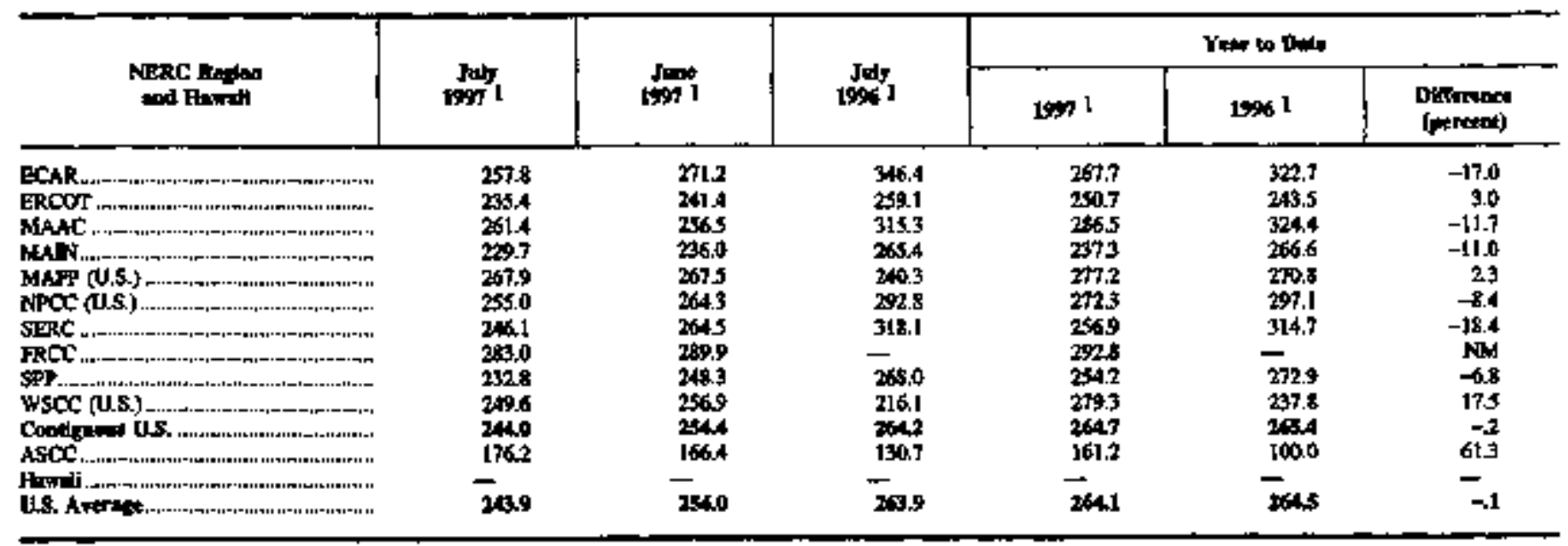

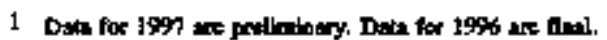

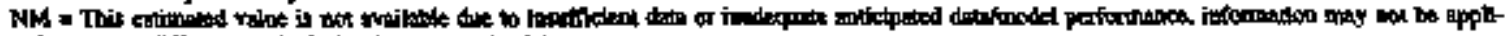

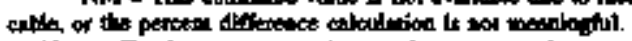

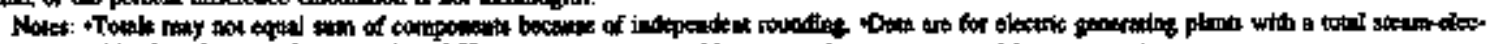

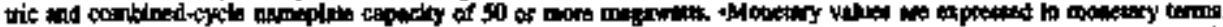

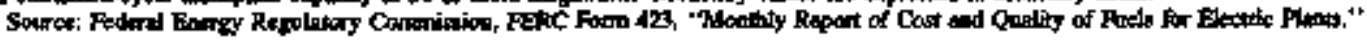


Tahle 33. Electric Utility Recelpts of Coel by Type, Confus Divisan, and Stote, Jo'y 1597

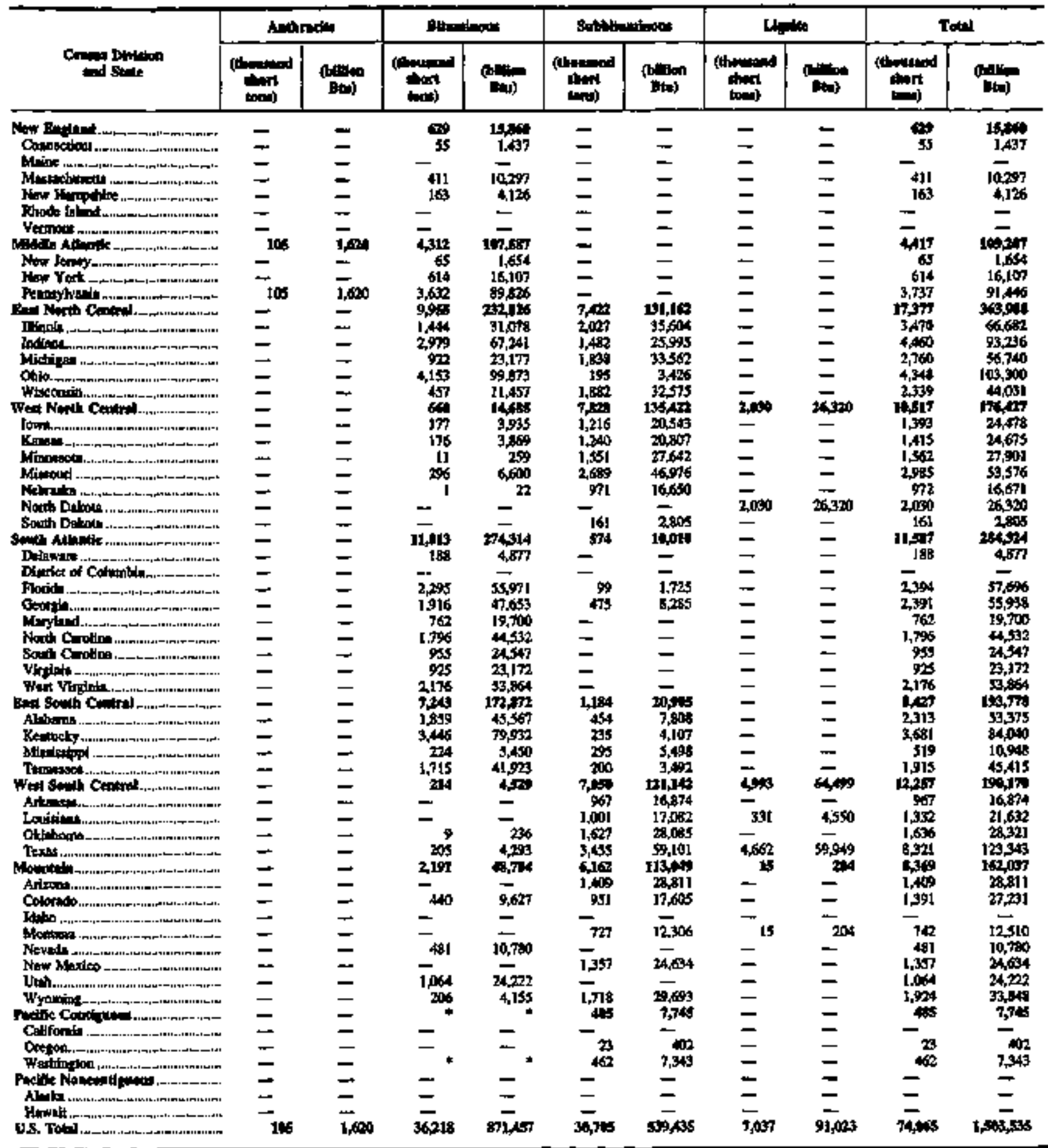

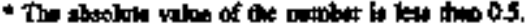

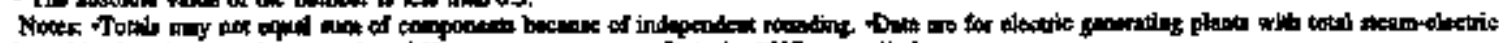

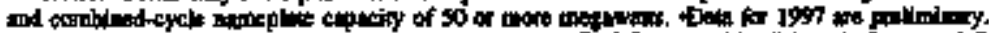

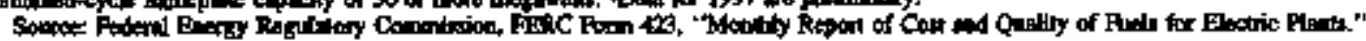


Tablo 34. Receipts and A rerige Cost of Cosl Delivered to Electric Utitities by Census Divblon and State

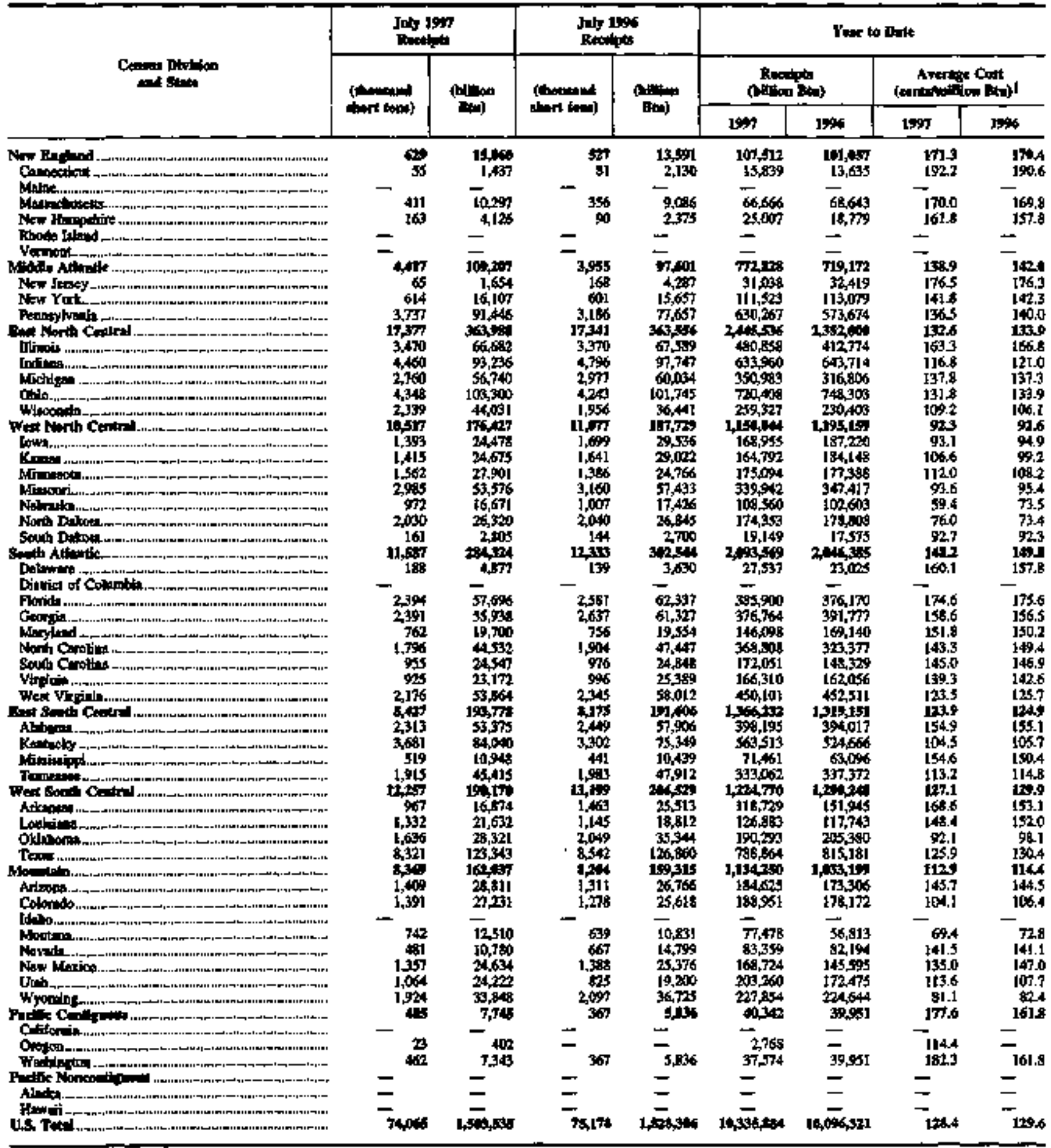

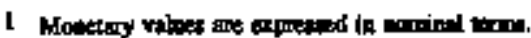

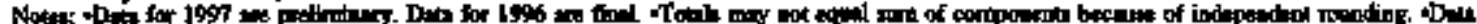

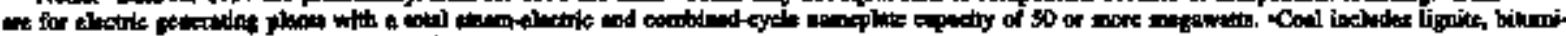

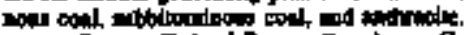

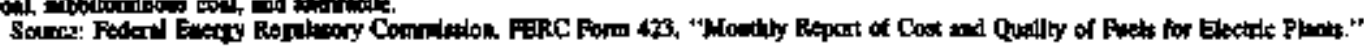


Table 35. Rexelpts and Average Cost of Coal Delivered to Klectric Utititles by Type of Punchase, Mining Method, Census Dirision, and State, Jnly 1997

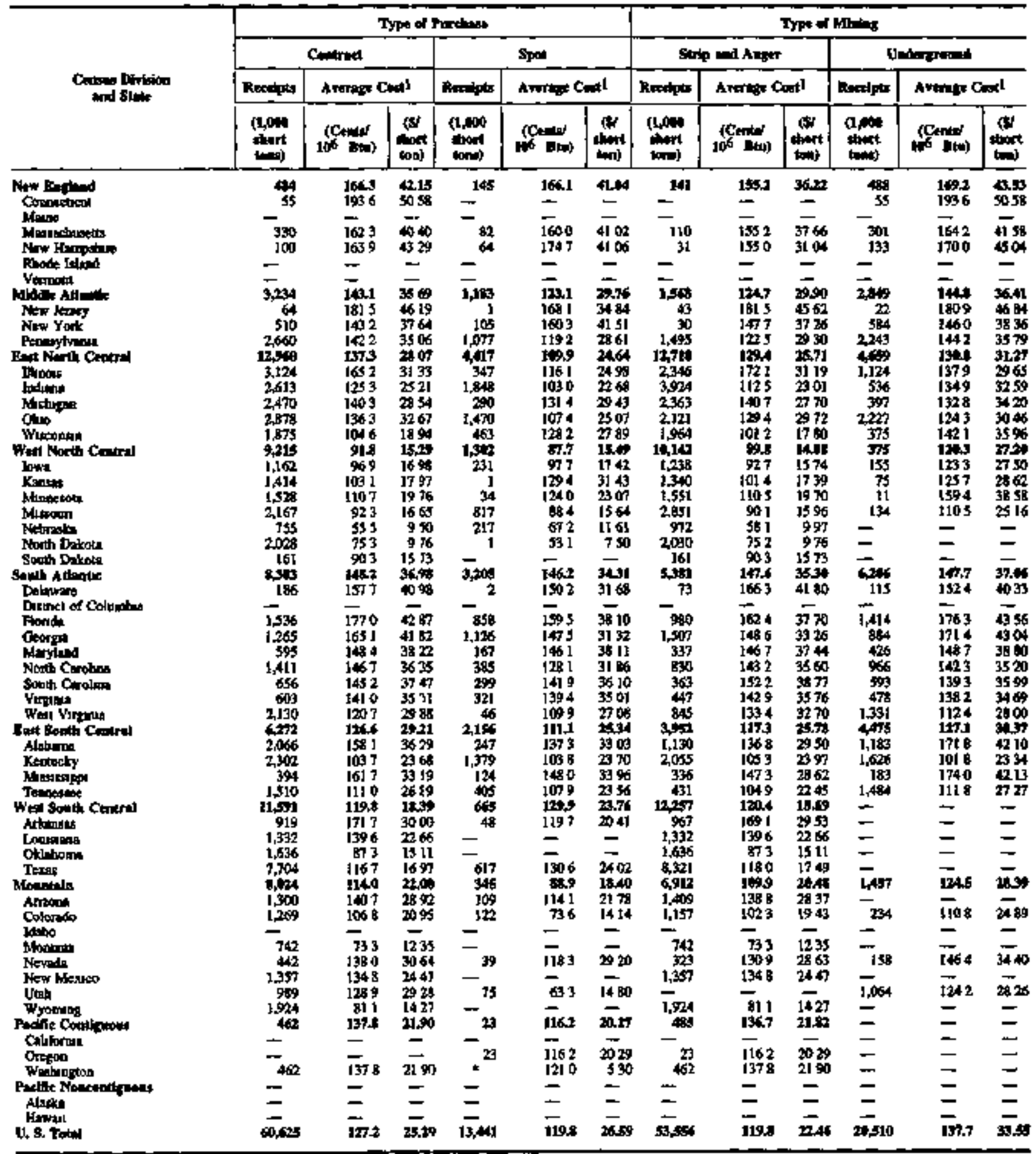

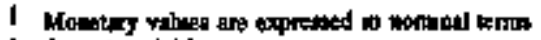

- Leit bain otos

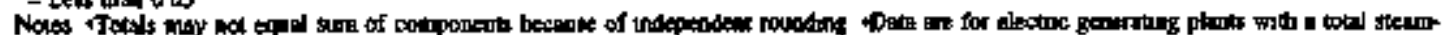

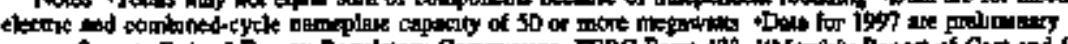

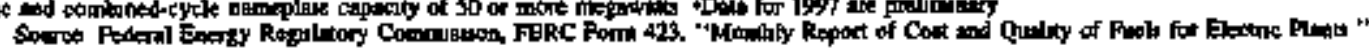


Table 36. Receipts and Average Cost of Coal Delivered to Electric Uthities by Sulfint Content, Census Division, and State, Jaly 1997

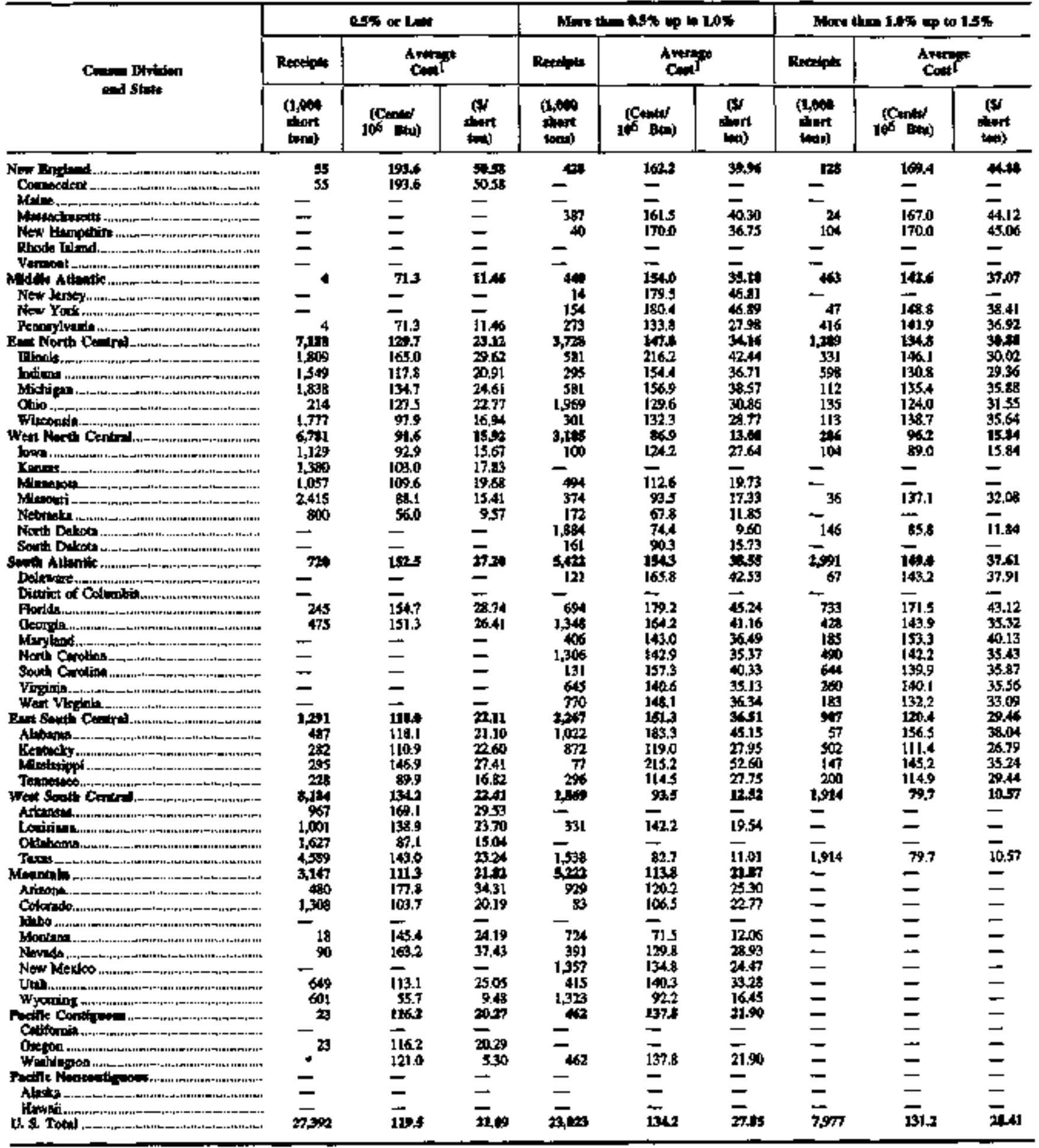

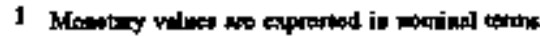

- Iasa pin o.04.

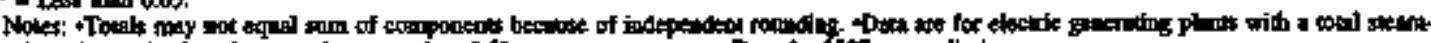

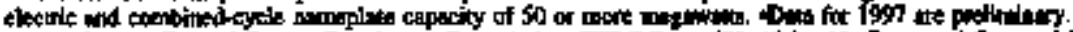

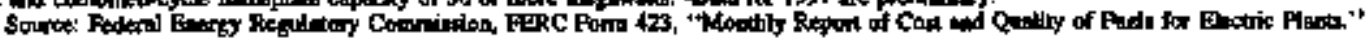


Table 36. Rexolps and Average Coot of Cogl Delivered to Bloxtrite Utilites by Sulfar Content, Cengus Divkion, and State, July 1997 (Conthaed)

\begin{tabular}{|c|c|c|c|c|c|c|c|c|c|c|c|}
\hline \multirow{3}{*}{ 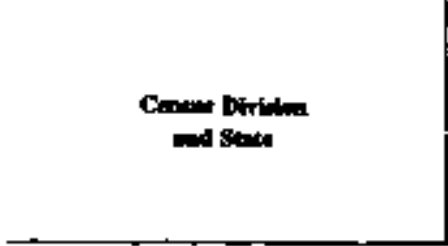 } & \multicolumn{3}{|c|}{ 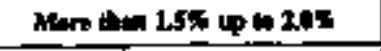 } & \multicolumn{3}{|c|}{ 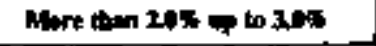 } & \multicolumn{3}{|c|}{ Mere than $3.0 x$} & \multicolumn{2}{|c|}{ An Pindien } \\
\hline & \multirow{2}{*}{ 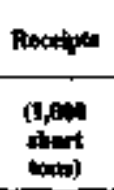 } & \multicolumn{2}{|c|}{ Creati } & \multirow{2}{*}{ 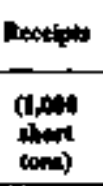 } & \multicolumn{2}{|c|}{ Aretio } & \multirow{2}{*}{ 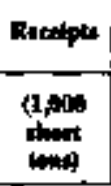 } & \multicolumn{4}{|c|}{ Cont } \\
\hline & & (C) & tif & & 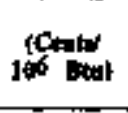 & $\underset{\operatorname{lin}}{(x)}$ & & $\begin{array}{l}\text { (Centw) } \\
\text { Hows) } \\
\text { (bin) }\end{array}$ & 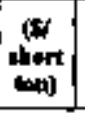 & $\underset{100}{(C)}$ & (\$nt \\
\hline 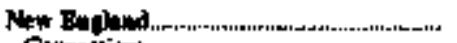 & - & - & - & $1 *$ & \$\$], & 4:- & - & - & - & 1063 & 41.5) \\
\hline 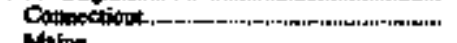 & - & 一 & - & - & $\longrightarrow$ & - & - & - & - & 193.6 & s0.5: \\
\hline Maline- & $\longrightarrow$ & - & - & - & - & - & - & - & - & & 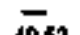 \\
\hline Chneth & - & - & - & - & - & - & - & - & - & 161.9 & 40.52 \\
\hline |. & - & - & - & 19 & 151.? & 40,05 & - & - & - & 1678 & 42.42 \\
\hline 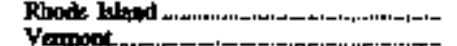 & $\bar{z}$ & $\bar{z}$ & - & $\overline{-}$ & $=$ & $\bar{z}$ & $\bar{z}$ & $\bar{z}$ & $\bar{z}$ & $\overline{-}$ & $\bar{z}$ \\
\hline 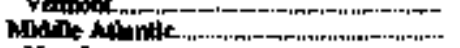 & $\overline{1}$ & $\overline{13} 62$ & $\overline{3,2,30}$ & $\overrightarrow{1 A 2}$ & $\overline{127,8}$ & $\overline{32}$ & 7 & $\overline{1521}$ & $\overline{360.30}$ & $\overline{13 n s}$ & $\overline{340}$ \\
\hline 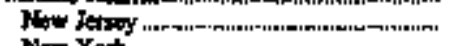 & - & $=$ & - & 52 & 181,8 & 45.84 & - & - & - & 191.3 & 16.04 \\
\hline 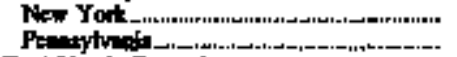 & $\begin{array}{l}139 \\
941\end{array}$ & 240.4 & $\begin{array}{l}36.76 \\
32.88\end{array}$ & $\begin{array}{r}275 \\
1,323\end{array}$ & 129.5 & 3.29 & - & $\overline{153,1}$ & $\overline{30.38}$ & $\begin{array}{l}146.1 \\
135.7\end{array}$ & $\begin{array}{l}38.30 \\
\$ 3.20\end{array}$ \\
\hline 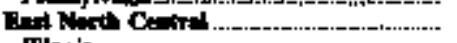 & 313 & 120 & $2 \pi$ & 1,90 & 1113 & 3.63 & $20 \%$ & 1167 & 269 & $32 \%$ & 29,14 \\
\hline 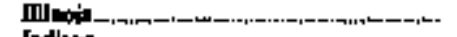 & 19 & $\$ 9$ & $1 \$ 97$ & 4hs & III.2 & 24.07 & 326 & 1203 & 25.25 & 159.7 & $\$ 0,69$ \\
\hline 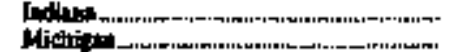 & $\begin{array}{l}455 \\
148\end{array}$ & $\begin{array}{l}111.2 \\
123.7\end{array}$ & 24,85 & 899 & 101,5 & 229 & 700 & lo2.1 & 27.74 & 115.6 & $\begin{array}{l}24.16 \\
28.64\end{array}$ \\
\hline 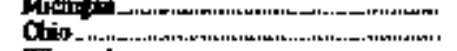 & 191 & 327.6 & 32,80 & 6ht & $\begin{array}{l}133.2 \\
12.4 .4\end{array}$ & 29,93 & 1,19 & is.7 & 20,66 & 139.3 & $\begin{array}{l}28.64 \\
30.10\end{array}$ \\
\hline 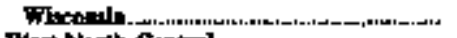 & 147 & 147.1 & 3838 & - & 4 & $=$ & - & - & - & 110,0 & 20.71 \\
\hline ath Ceatroll & 25 & $\begin{array}{l}103.6 \\
330.9\end{array}$ & 新:15 & 154 & $\begin{array}{l}104.6 \\
119.5\end{array}$ & $\begin{array}{l}23,12 \\
26,49\end{array}$ & & 1286 & 29.4 & $\begin{array}{l}\text { 913 } \\
\text { 9n.0 }\end{array}$ & $\begin{array}{l}\mathbf{1 6 3 1} \\
17.06\end{array}$ \\
\hline 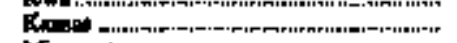 & - & $\div$ & - & 10 & 106.6 & 25.40 & 25 & 1065 & 24.00 & 103.1 & 17.98 \\
\hline 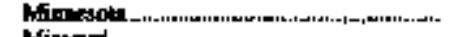 & II & 159.4 & 385: & - & - & -0 & - & $\bar{x}+2$ & - & 111.0 & 19.83 \\
\hline 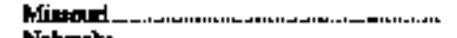 & - & $\rightarrow$ & - & 300 & 97.5 & 21.57 & 60 & 1375 & 317 & 91.2 & 16.34 \\
\hline ale & - & - & - & - & - & $\leftarrow$ & - & - & - & $\$$ & $9.9 ?$ \\
\hline 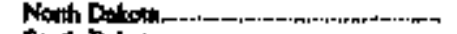 & - & - & - & - & - & - & - & - & - & 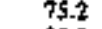 & 976 \\
\hline D. & - & - & $=$ & - & - & $\bar{\pi}$ & - & $=$ & 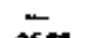 & 90.3 & 15.73 \\
\hline 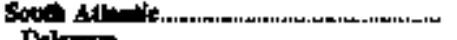 & 1,016 & 1326 & 33.15 & $\infty$ & IEKT & 37,32 & $8 \pi$ & $10 \%$ & 26)in & 18.7 & 36.24 \\
\hline 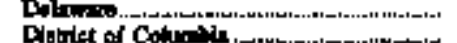 & $\bar{z}$ & $\bar{z}$ & 二 & $\bar{z}$ & $=$ & $\bar{z}$ & $\bar{z}$ & $\bar{z}$ & $\bar{z}$ & 157.6 & - 10.90 \\
\hline 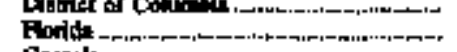 & 39 & $\overline{133.3}$ & $444^{2}$ & sos & 172.6 & 40.63 & 181 & 143.6 & 35.16 & 170.8 & $\$ 1.16$ \\
\hline 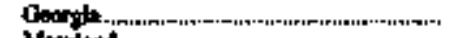 & 140 & 149.4 & 35.8S & - & $\overrightarrow{10}$ & $\bar{x}_{31}$ & - & - & - & 157.6 & 3680 \\
\hline (1) & -160 & 154.7 & $\$ 0.57$ & -11 & 129.4 & 3431 & - & $\bar{z}$ & - & 34.8 & $\begin{array}{ll}38,20 \\
35,38\end{array}$ \\
\hline 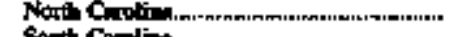 & -7 & $\overline{1488}$ & $\overline{3 B}_{86}$ & $z$ & $=$ & $\overrightarrow{-}$ & $\bar{z}$ & $\bar{z}$ & $\bar{z}$ & 142.7 & 35.38 \\
\hline 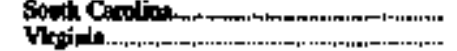 & $\begin{aligned} 181 \\
19\end{aligned}$ & 1414 & $\begin{array}{l}36,600 \\
3369\end{array}$ & 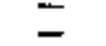 & $\overline{-}$ & $=$ & $=$ & $=$ & $\bar{z}$ & 140.5 & 35.21 \\
\hline 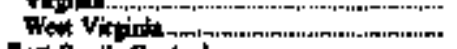 & $4 \pi$ & 106.6 & 3677 & 110 & 91.2 & 2260 & 柋 & 980 & 2453 & 120.5 & 29.69 \\
\hline 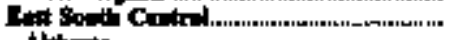 & 74 & 125.9 & \$1 & 1,321 & 1114 & 2665 & 1,66 & 930 & 20.75 & 132.7 & 26.4 \\
\hline 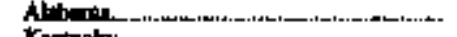 & 282 & IS26 & 3739 & 379 & 129.3 & 31,40 & 8 & 1062 & 2450 & 1558 & 3599 \\
\hline 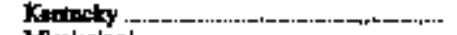 & 60 & 97.3 & 11.34 & H & IOIA & 24.16 & 1,516 & 915 & 2037 & I03.8 & 23.69 \\
\hline 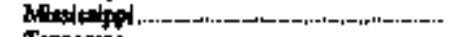 & - & 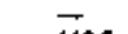 & - & - & - & - & - & $=$ & - & 158.1 & 33.37 \\
\hline 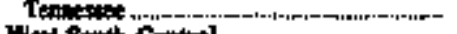 & 432 & 117.7 & 2960 & 694 & 102.0 & 25.6丁? & 65 & 107.5 & 24.55 & 110.4 & 26.18 \\
\hline 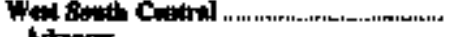 & 200 & $\mathbf{s t}$ & $\mathbf{6 7}$ & - & - & - & & Iods & 26.8 & $\mathbf{r a p 4}$ & 120 \\
\hline 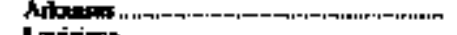 & $=$ & - & - & - & - & - & - & - & - & ]69.1 & 29.53 \\
\hline 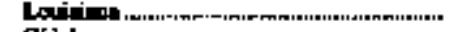 & - & - & - & - & - & - & - & - & - & 139.6 & 22.66 \\
\hline 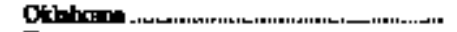 & - & - & - & - & - & - & 9 & $10: 0$ & 26.88 & 57.3 & 15.11 \\
\hline 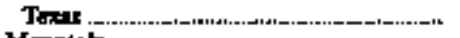 & 280 & 60:0 & 6.27 & - & - & - & - & - & - & 1180 & 17.9 \\
\hline 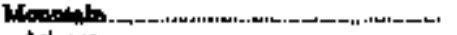 & - & $\rightarrow$ & - & - & - & - & - & - & - & 112.8 & 21.85 \\
\hline 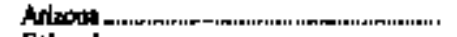 & - & - & - & - & - & - & - & - & - & 136.8 & 29,37 \\
\hline 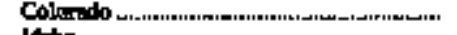 & - & - & - & - & - & - & - & - & - & 103.9 & 2035 \\
\hline 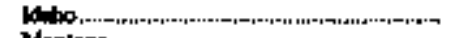 & - & - & - & - & - & - & - & - & - & $=$ & - \\
\hline 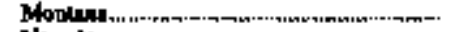 & - & 一 & - & - & - & - & - & - & - & 73.3 & 1233 \\
\hline 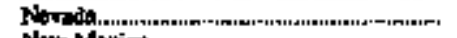 & - & - & - & - & - & - & - & - & - & 136.2 & 30.52 \\
\hline 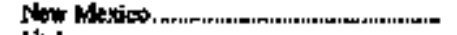 & - & - & - & - & - & - & - & - & - & 1348 & 24,47 \\
\hline 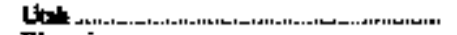 & - & - & $=$ & - & - & - & - & - & - & 124.2 & 28.26 \\
\hline 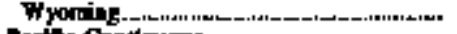 & - & - & - & - & - & - & - & - & - & 8l. & 14,27 \\
\hline 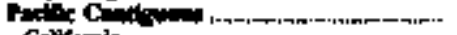 & - & - & - & 一 & - & - & $\pi$ & - & - & $13 \mathbf{4 . 7}$ & 21.52 \\
\hline Ik, & - & - & - & - & - & $\rightarrow$ & $=$ & - & - & $\overrightarrow{-}$ & - \\
\hline 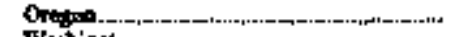 & - & - & - & 一 & - & - & - & - & - & 116.2 & 20.29 \\
\hline 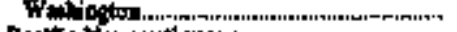 & - & $\rightarrow$ & - & - & - & - & $=$ & $=$ & - & 137,3 & 31.90 \\
\hline 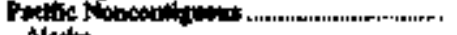 & - & - & - & - & - & 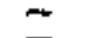 & 7 & - & - & - & - \\
\hline 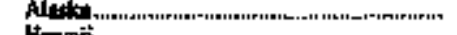 & - & 二 & 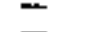 & $=$ & $=$ & $\bar{z}$ & $\bar{z}$ & $=$ & $\bar{z}$ & - & $\bar{z}$ \\
\hline 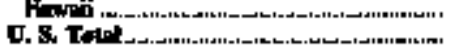 & $\overline{4}$ & $\overline{1+103}$ & $\overline{30} \mathbf{3}$ & $\overline{5,00}$ & $\overline{1}=$ & 2064 & $\overline{5,4616}$ & $\overline{11} 39$ & $\overline{20,03}$ & $\overline{12 \times 8}$ & 25.53 \\
\hline
\end{tabular}

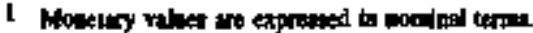

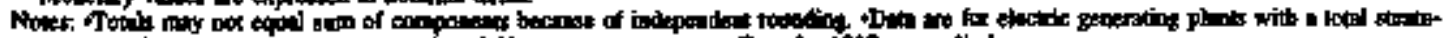

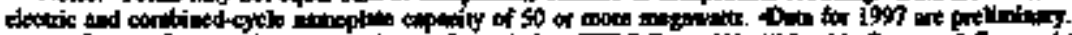

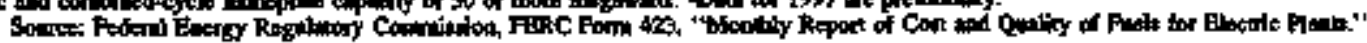


Tabłe 37. Flectrie Utility Receipts of Petroleutu by Type, Cowson Division, and State, July 199

\begin{tabular}{|c|c|c|c|c|c|c|c|c|c|c|}
\hline \multirow{2}{*}{ 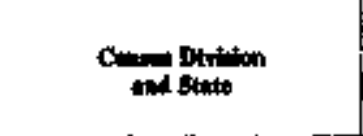 } & \multicolumn{2}{|c|}{ No. 2 Pent ol } & \multicolumn{2}{|c|}{ 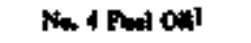 } & \multicolumn{2}{|c|}{ Nor 5 Find ond 1} & \multicolumn{2}{|c|}{ Nh. 6 Fent on } & \multicolumn{2}{|c|}{ Tat } \\
\hline & 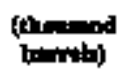 & (1) & 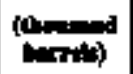 & (bialoa & fthoneded & (tolnima & 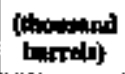 & (1) & (bound & (bilase \\
\hline 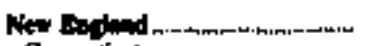 & $\mathbf{1 1}$ & 66 & - & - & - & - & 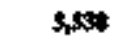 & 21, 304 & $3,3,40$ & 21,300 \\
\hline | & 2 & 9 & - & - & - & - & 1,299 & 8,004 & 1,261 & 8,069 \\
\hline 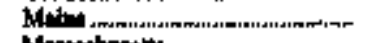 & $\mathbf{I}$ & 8 & $\longleftarrow$ & $=$ & - & - & 436 & 2780 & 437 & 2,788 \\
\hline 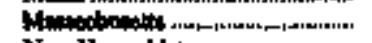 & 3 & 27 & - & - & - & - & 1,843 & 10,496 & thas & 10,514 \\
\hline 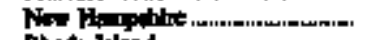 & 4 & $n$ & $\omega$ & - & - & - & - & - & 4 & 22 \\
\hline 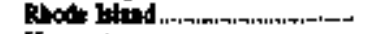 & - & - & - & - & $m$ & - & - & - & - & $=$ \\
\hline 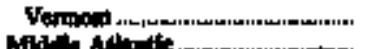 & - & - & - & 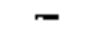 & - & - & - & - & - & - \\
\hline 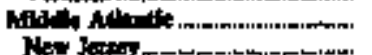 & 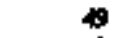 & 285 & $\rightarrow$ & $=$ & - & - & 1,736 & $t 1,30 \%$ & 1,046 & Ifjes \\
\hline New Jater & ! & $\mathrm{J}$ & - & - & - & - & - & $\leftarrow$ & 31 & 175 \\
\hline 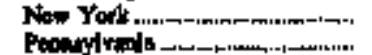 & 6 & 35 & - & - & - & - & 1,301 & 292 & 1,313 & 8,326 \\
\hline 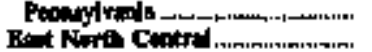 & 42 & 243 & - & - & - & - & 179 & $3,0 \mathrm{~s}$ & 521 & 3,323 \\
\hline 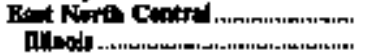 & 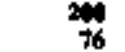 & 1,168 & $\Xi$ & $\ddot{z}$ & $\overline{-}$ & $\bar{E}$ & 151 & - & 361 & 2,1127 \\
\hline 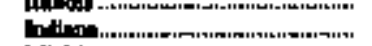 & 34 & 198 & - & $=$ & $=$ & $=$ & $=$ & $=$ & 34 & 198 \\
\hline Milethigna & 29 & 170 & $m$ & - & - & - & 151 & 955 & 180 & J, I35 \\
\hline Ohis & $\boldsymbol{9}$ & 340 & $\Leftrightarrow$ & $=$ & - & - & - & - & 59 & $3 \mathbf{4 0}$ \\
\hline 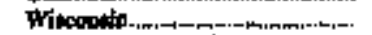 & 2 & 9 & - & - & - & - & - & - & 2 & $\boldsymbol{g}$ \\
\hline Wrat Neta Centril.................. & 榢 & s) & $\rightarrow$ & "ה & - & $m$ & 17 & 118 & $10 \%$ & (4) \\
\hline 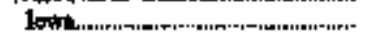 & 10 & sto & - & - & - & - & - & - & ID & 58 \\
\hline 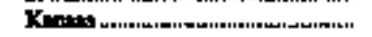 & 27 & เ59 & $\rightarrow$ & - & - & - & 15 & 102 & 42 & 201 \\
\hline 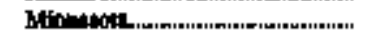 & 3 & 15 & - & - & - & - & - & - & 3 & $1 \mathrm{~B}$ \\
\hline 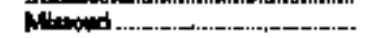 & 36 & zan & - & - & ـ & - & 2 & 13 & 38 & 218 \\
\hline 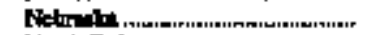 & $\cdot$ & 1 & ـ & - & - & - & - & - & * & ] \\
\hline North Dalmok & $\mathbf{1 a}$ & $7 \boldsymbol{s}$ & - & - & - & 一 & - & - & 13 & $\boldsymbol{m}$ \\
\hline 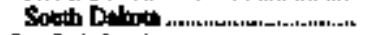 & - & - & $=$ & $=$ & - & - & - & - & - & - \\
\hline 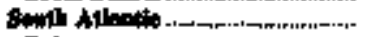 & 273 & 1, sid & 119 & m6 & - & - & 4,55 & 2,236 & $4 \mathbf{4 5}$ & 31,34 \\
\hline 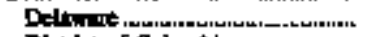 & : & 49 & - & $=$ & - & 一 & II2 & 716 & 120 & 765 \\
\hline Droukt of Colmmith & 3 & Is & 119 & n6 & - & - & - & - & 122 & 744 \\
\hline 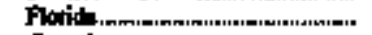 & 102 & $\operatorname{sos}$ & ـ & - & - & $\Rightarrow$ & 4,110 & 26,374 & $4,3+2$ & 26,972 \\
\hline 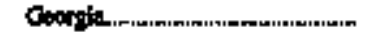 & 40 & 230 & - & - & - & - & 26 & 166 & 66 & 3 \\
\hline 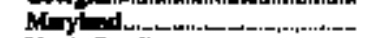 & $\$ 0$ & jot & - & - & ـ & - & $\mathbf{2 5}$ & 1,406 & 262 & 1,652 \\
\hline Nond Conotian. & 38 & 222 & - & - & - & - & - & - & 38 & $\mathbf{m}$ \\
\hline 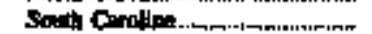 & 24 & 141 & - & - & - & - & - & - & 24 & j41 \\
\hline Yifpinit & $\mathrm{T}$ & 43 & - & - & - & - & 76 & 489 & 84 & 526 \\
\hline 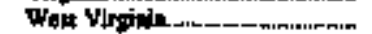 & 20 & 116 & - & سـ & - & - & - & - & 20 & 116 \\
\hline 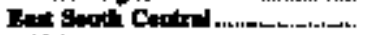 & 10 & $\$$ & $\Rightarrow$ & - & - & - & 14 & $1,0,5$ & 326 & 2,036 \\
\hline 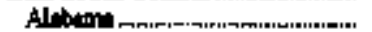 & 109 & 639 & - & - & - & - & - & - & 109 & 四束 \\
\hline 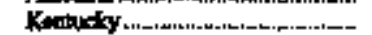 & 47 & $m$ & - & - & - & - & - & - & 47 & 27 \\
\hline Hista & $\mathbf{g}$ & 20 & - & - & - & - & 164 & 1,065 & 168 & $5,50 s$ \\
\hline 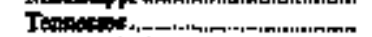 & 3 & 16 & - & - & - & - & - & - & 3 & 16 \\
\hline TeA Sod Conthl & is & 91 & - & - & - & - & 24 & $\mathbf{1 5 6}$ & 39 & $2 \pi$ \\
\hline 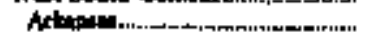 & $\$$ & 29 & - & $=$ & - & - & - & - & $\mathbf{s}$ & 29 \\
\hline 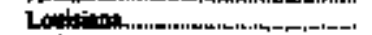 & $j$ & 39 & - & - & 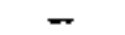 & - & 24 & 156 & 31 & 190 \\
\hline 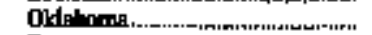 & - & - & - & - & - & - & - & - & - & - \\
\hline 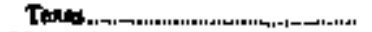 & 4 & $\mathbf{2 3}$ & - & - & - & - & $\sigma$ & - & 4 & $\mathbf{2}$ \\
\hline 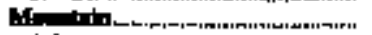 & 37 & 213 & - & - & - & - & - & - & 37 & 213 \\
\hline Arlizone & 4 & 21 & $\leftarrow$ & - & - & - & - & - & 4 & 21 \\
\hline 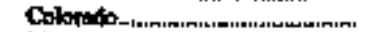 & - & - & - & - & - & - & - & - & - & - \\
\hline | & - & 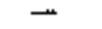 & $\rightarrow$ & - & - & - & - & - & - & - \\
\hline 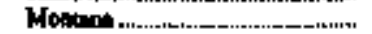 & 3 & 38 & $\rightarrow$ & - & - & - & - & - & $\mathbf{3}$ & 18 \\
\hline 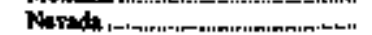 & 6 & 34 & - & - & - & - & - & - & 6 & 34 \\
\hline 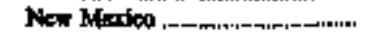 & 3 & 17 & - & - & - & - & - & - & 3 & l? \\
\hline | & 3 & 19 & - & - & - & - & - & - & 3 & 19 \\
\hline 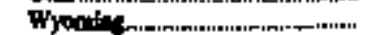 & 18 & $10 \%$ & $=$ & - & - & - & - & - & 18 & 104 \\
\hline 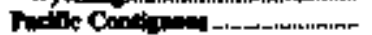 & 4 & 24 & $\rightarrow$ & 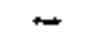 & - & - & - & 一 & 4 & 21 \\
\hline 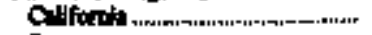 & - & - & - & - & - & - & - & - & & - \\
\hline 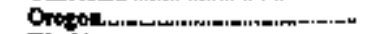 & $\mathbf{1}$ & 6 & - & - & - & - & - & - & I & 6 \\
\hline Phogod, & 3 & I\$ & $\rightarrow$ & - & - & $\sigma$ & - & - & 3 & 18 \\
\hline 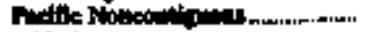 & - & - & - & - & - & - & 60.3 & 4,018 & $\cos$ & 4,011 \\
\hline Almba & سـ & - & - & - & - & - & - & $\overline{-}$ & - & - \\
\hline 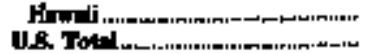 & $\overline{341}$ & $\overline{4 m}$ & - 119 & $-\overline{326}$ & $=$ & $\overline{-}$ & $\begin{array}{r}643 \\
10681\end{array}$ & $\begin{array}{r}4,018 \\
0 ;, 20 \%\end{array}$ & $\begin{array}{r}643 \\
11,690\end{array}$ & $\begin{array}{r}4,070 \\
74,400\end{array}$ \\
\hline
\end{tabular}

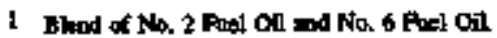

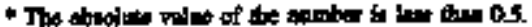

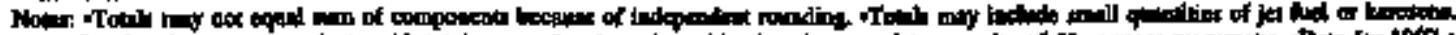

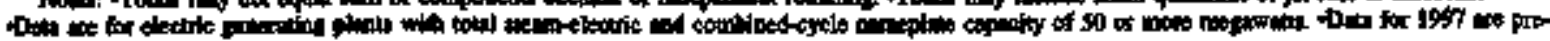
Fining.

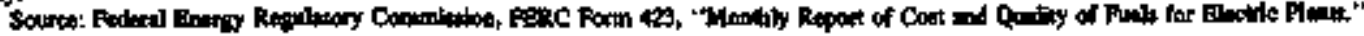


Table 38. Recejpts and Average Cost of Petroleum Delivered to Beatric Utatities by Census Divialon and State

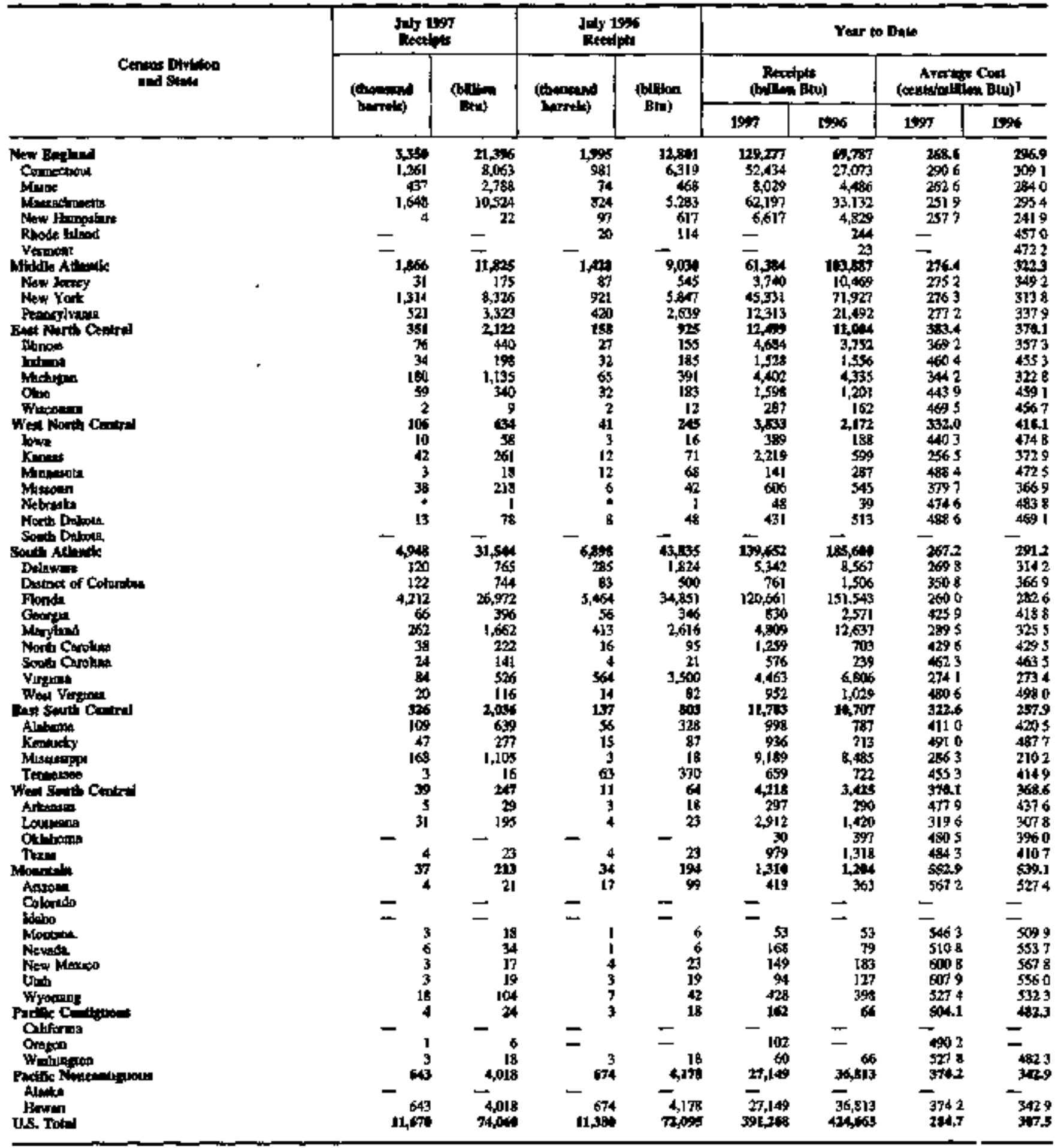

1 Monetiry valued are expreased m nomul terns

* Lew then os

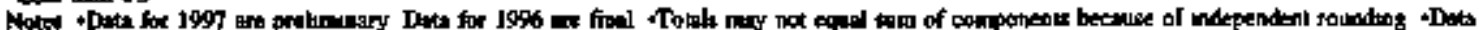

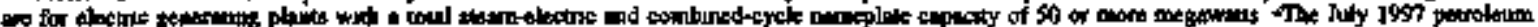

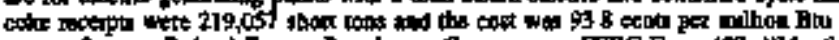

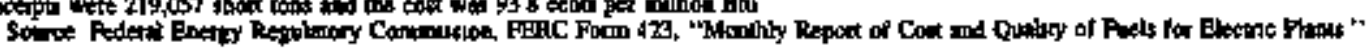


Table 39. Receipts and Average Cost of Petroleam Dellvered to Electric Utilities by Type of Purchase, Censas Division, and \$tate, July 1997

\begin{tabular}{|c|c|c|c|c|c|c|c|c|c|c|c|c|}
\hline \multirow{4}{*}{$\begin{array}{l}\text { Consus Dhioca } \\
\text { hind Stote }\end{array}$} & \multicolumn{6}{|c|}{ 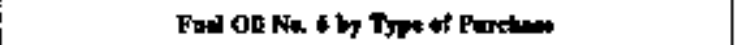 } & \multicolumn{6}{|c|}{ Awroged Coot of Fud Oln' } \\
\hline & \multicolumn{3}{|c|}{ Comiract } & \multicolumn{3}{|c|}{ Spet } & \multicolumn{2}{|c|}{$\operatorname{Ne} 2$} & \multicolumn{2}{|c|}{ Ne 4Nim 5} & \multicolumn{2}{|c|}{$\mathrm{Na}, \mathrm{G}$} \\
\hline & \multirow{2}{*}{$\begin{array}{c}\text { Rectipts } \\
1,100 \\
\text { bolis }\end{array}$} & \multicolumn{2}{|c|}{ Awrage Cunl } & \multirow{2}{*}{ 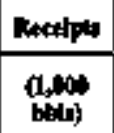 } & \multicolumn{2}{|c|}{ Awerapon Conl } & \multirow{2}{*}{ (C) Bat) } & \multirow{2}{*}{$\underset{6}{*}$} & \multirow{2}{*}{ (C) } & \multirow{2}{*}{ (5) } & \multirow{2}{*}{ 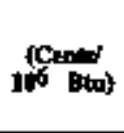 } & \\
\hline & & (C) & bist) & & (Chentit) & (st) & & & & & & (1) \\
\hline 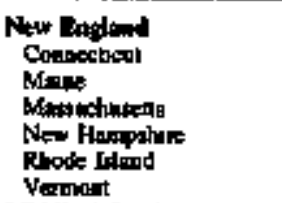 & $\begin{array}{l}1,768 \\
1,136 \\
- \\
= \\
=\end{array}$ & $\begin{array}{l}306 \\
3066 \\
- \\
2586 \\
= \\
=\end{array}$ & $\begin{array}{l}19.65 \\
1965 \\
165 \\
= \\
=\end{array}$ & $\begin{array}{l}1,57 \\
123 \\
436 \\
1,017 \\
= \\
=\end{array}$ & $\begin{array}{l}2565 \\
3021 \\
2452 \\
2561 \\
= \\
=\end{array}$ & $\begin{array}{l}1634 \\
1892 \\
1564 \\
1633 \\
= \\
=\end{array}$ & $\begin{array}{l}4103 \\
4704 \\
4010 \\
4110 \\
4156 \\
= \\
=\end{array}$ & $\begin{array}{l}24.44 \\
2125 \\
2339 \\
2413 \\
2407 \\
=\end{array}$ & $\begin{array}{l}= \\
= \\
= \\
=\end{array}$ & $\begin{array}{l}= \\
= \\
= \\
=\end{array}$ & $\begin{array}{l}2741 \\
3062 \\
2452 \\
2571 \\
= \\
=\end{array}$ & $\begin{array}{l}17.51 \\
1959 \\
1564 \\
1642 \\
= \\
=\end{array}$ \\
\hline $\begin{array}{l}\text { Mide diluntic } \\
\text { New letsy }\end{array}$ & & & IG.71 & & 26. & 1700 & 4320 & 2350 & - & $\overline{-}$ & 265 & 1690 \\
\hline 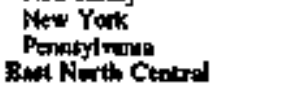 & $\begin{array}{r}941 \\
+7\end{array}$ & $\frac{2644}{3000}$ & $\frac{1678}{21 \pm 9}$ & $\begin{array}{l}36 \\
479 \\
124\end{array}$ & $\begin{array}{l}2767 \\
2593 \\
20,3\end{array}$ & $\begin{array}{l}1751 \\
1646 \\
148\end{array}$ & $\begin{array}{l}4425 \\
4042 \\
461\end{array}$ & $\begin{array}{l}2566 \\
2365 \\
24.76\end{array}$ & $\bar{z}$ & $=$ & $\begin{array}{l}2538 \\
2493 \\
2005\end{array}$ & $\begin{array}{l}1699 \\
1666 \\
1602\end{array}$ \\
\hline Illowis & $=$ & $=$ & - & $=$ & $=$ & $=$ & 445 & 26.07 & - & 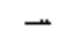 & - & - \\
\hline Intiana & $-n$ & $\overline{3600}$ & 2129 & 124 & $\overline{209}$ & 148 & $\begin{array}{rl}413 & 3 \\
3989 & 9\end{array}$ & $\begin{array}{l}23 \\
23 \\
2\end{array}$ & $\bar{z}$ & $\bar{Z}$ & $\overline{2509}$ & $\overrightarrow{1602}$ \\
\hline Oting & - & - & - & - & - & - & 4235 & 2445 & - & - & - & - \\
\hline Wed Noth Central & $=$ & $\bar{z}$ & $\bar{z}$ & - & $\overline{30}$ & $\overline{3} \mathbf{s}$ & 4067 & 219 & $\bar{z}$ & $\overline{-}$ & $\overrightarrow{2013}$ & $\overline{136}$ \\
\hline Igxa & $=$ & $\bar{z}$ & $=$ & - & - & - & 4301 & 28,72 & $\bar{z}$ & $\bar{z}$ & 213 & - \\
\hline Kanis & - & - & $m$ & is & 1995 & 1360 & 4322 & 2506 & - & - & 1999 & 1360 \\
\hline Mrtastan & - & - & - & - & - & - & 4672 & 26.92 & - & - & - & - \\
\hline Nebrestit & - & - & $=$ & 2 & 2128 & 1400 & 4117 & 281 & - & - & 2128 & 1400 \\
\hline $\begin{array}{l}\text { Nebrestra } \\
\text { Nanh Diken }\end{array}$ & $\bar{m}$ & $\bar{z}$ & $\bar{z}$ & $=$ & $\overline{-}$ & - & 4655 & 2701 & 二 & - & $\overline{-}$ & $\underline{-}$ \\
\hline 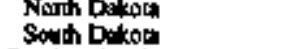 & - & $=$ & $=$ & $\overrightarrow{-}$ & $\overline{-}$ & $\bar{m}$ & 4368 & 256 & $\bar{z}$ & $\bar{z}$ & $\bar{z}$ & $=$ \\
\hline Sown Attiotic & $\begin{array}{r}2,144 \\
109\end{array}$ & 2001 & $\begin{array}{l}1641 \\
16+3\end{array}$ & 2,112 & 282.7 & is:ot & 4173 & 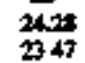 & 3464 & ritits & $\begin{array}{l}2090 \\
2583\end{array}$ & $\begin{array}{l}1604 \\
1636\end{array}$ \\
\hline Distere of Columbta & $\rightarrow$ & - & - & - & $=$ & - & 909 & 2320 & 3460 & 2112 & - & $=$ \\
\hline $\begin{array}{l}\text { Flondi } \\
\text { Georgen }\end{array}$ & $\underline{-}^{1,810}$ & $2 \sqrt{3} 4$ & 1641 & 2300 & $\begin{array}{l}2696 \\
279.3\end{array}$ & $\begin{array}{l}16 \$ 0 \\
1754\end{array}$ & $\begin{array}{l}4 \pi 99 \\
4359\end{array}$ & $\begin{array}{l}2459 \\
2536\end{array}$ & $=$ & $=$ & $\begin{array}{l}2991 \\
2793\end{array}$ & $\begin{array}{l}1663 \\
1354\end{array}$ \\
\hline Minjilind & 232 & 2535 & 1640 & - & - & $\ldots$ & 3993 & 2329 & $\overline{-}$ & - & 2555 & 1640 \\
\hline North Carollon & - & - & - & - & - & - & 405 & 2377 & - & - & - & - \\
\hline Soulh Carohnt & - & - & $\rightarrow$ & - & - & - & 1284 & 2426 & - & - & - & - \\
\hline Virganes & - & - & - & 36 & 2516 & $16 \$ 6$ & 4133 & 2421 & - & - & 2616 & 1656 \\
\hline war Vwown & - & - & $\rightarrow$ & - & 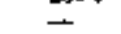 & - & 4036 & $23 \%$ & - & - & - & - \\
\hline 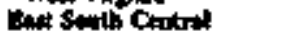 & - & - & - & 164 & $2 \pi 0.1$ & 17.83 & $\$ 12.8$ & 2420 & - & - & 270.1 & 1783 \\
\hline Alobsiom & - & - & - & - & - & - & 3900 & 2292 & - & - & - & - \\
\hline Kentracky & - & 二 & $=$ & $m$ & $=$ & - & 4653 & 2727 & 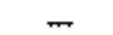 & - & - & - \\
\hline Mrsatosppe & - & - & - & 164 & 2701 & t383 & 492 & $2 \leqslant 54$ & - & - & $2 \times 1$ & 1789 \\
\hline Tenesses & - & - & $\rightarrow$ & - & ست & - & $\cos 1$ & 230 & - & - & - & - \\
\hline Weat South Central & - & - & $=$ & 24 & 20 & I9.06 & $\$ 21.5$ & 247 & ــ & - & 2931: & 19.06 \\
\hline Aknots & - & - & - & - & - & - & 406 & 2770 & - & - & - & - \\
\hline Lobutition & - & - & - & 24 & 2938 & 1906 & 3867 & 2274 & - & ـ & 2938 & 1906 \\
\hline Ottutitum & - & - & - & - & - & - & $=$ & - & - & - & - & - \\
\hline Texns & - & - & - & - & - & - & 4186 & 2426 & - & - & $-\pi$ & 一 \\
\hline Mountodn & - & - & - & - & - & - & sisks & 294 & - & - & - & - \\
\hline AnखDian & - & - & ــ & - & - & - & 5343 & 3102 & - & - & - & - \\
\hline Coldatida & - & - & - & - & - & - & - & - & - & عـ & - & - \\
\hline totho & - & - & 二 & - & - & - & - & - & - & - & - & - \\
\hline Momeng & - & - & - & - & - & - & 5104 & 3022 & - & - & - & - \\
\hline$N=\ln d a$ & - & - & - & - & - & - & $443 \mathrm{~B}$ & 259 & - & - & - & - \\
\hline New Mestos & - & - & - & - & - & - & 5610 & 3204 & - & - & - & - \\
\hline Unh & - & - & - & - & $m$ & - & 3679 & 3321 & - & - & - & - \\
\hline Wyow:15: & - & - & - & - & - & $\sim$ & 4094 & 2900 & - & - & ـ & - \\
\hline Puthe Cortisuon & - & - & - & - & - & - & 46.7 & 2689 & - & - & - & - \\
\hline Calberesich & - & - & - & - & - & - & - & - & - & - & - & 一 \\
\hline Oneton & - & 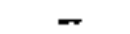 & - & - & - & - & 4577 & 2621 & - & - & - & - \\
\hline Wescuphot & - & - & - & - & $=$ & - & 4430 & 2605 & - & - & - & - \\
\hline 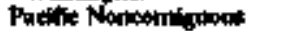 & $6 \mathbf{6 3}$ & 3009 & 20.57 & - & - & - & - & - & - & 一 & 329.3 & 245 \\
\hline Aluk & - & - & - & - & - & - & - & - & - & - & - & - \\
\hline $\begin{array}{l}\text { Krown } \\
\text { L. K. Tocel }\end{array}$ & $\begin{array}{r}643 \\
018\end{array}$ & 3259 & 20,53 & $\overline{s, 160}$ & $\overrightarrow{2614}$ & $\overline{1<0}$ & $\overline{m a t}$ & $\overline{246}$ & $\overline{3460}$ & $\overline{2 \pi .17}$ & $\begin{array}{l}3293 \\
29.0\end{array}$ & 2037 \\
\hline
\end{tabular}

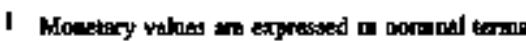

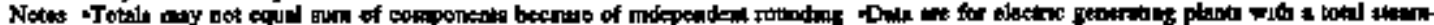

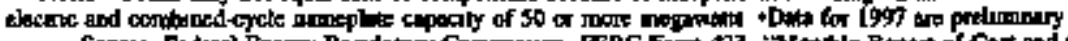

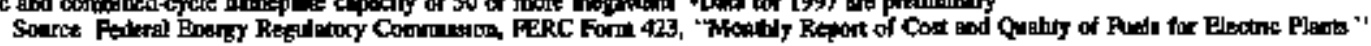


Toble 40. Recelpts and Average Cost of Heary Otl Delivered to Electric Utitities by Snlfur Content, Ceusm Division, and State, Jaly 199

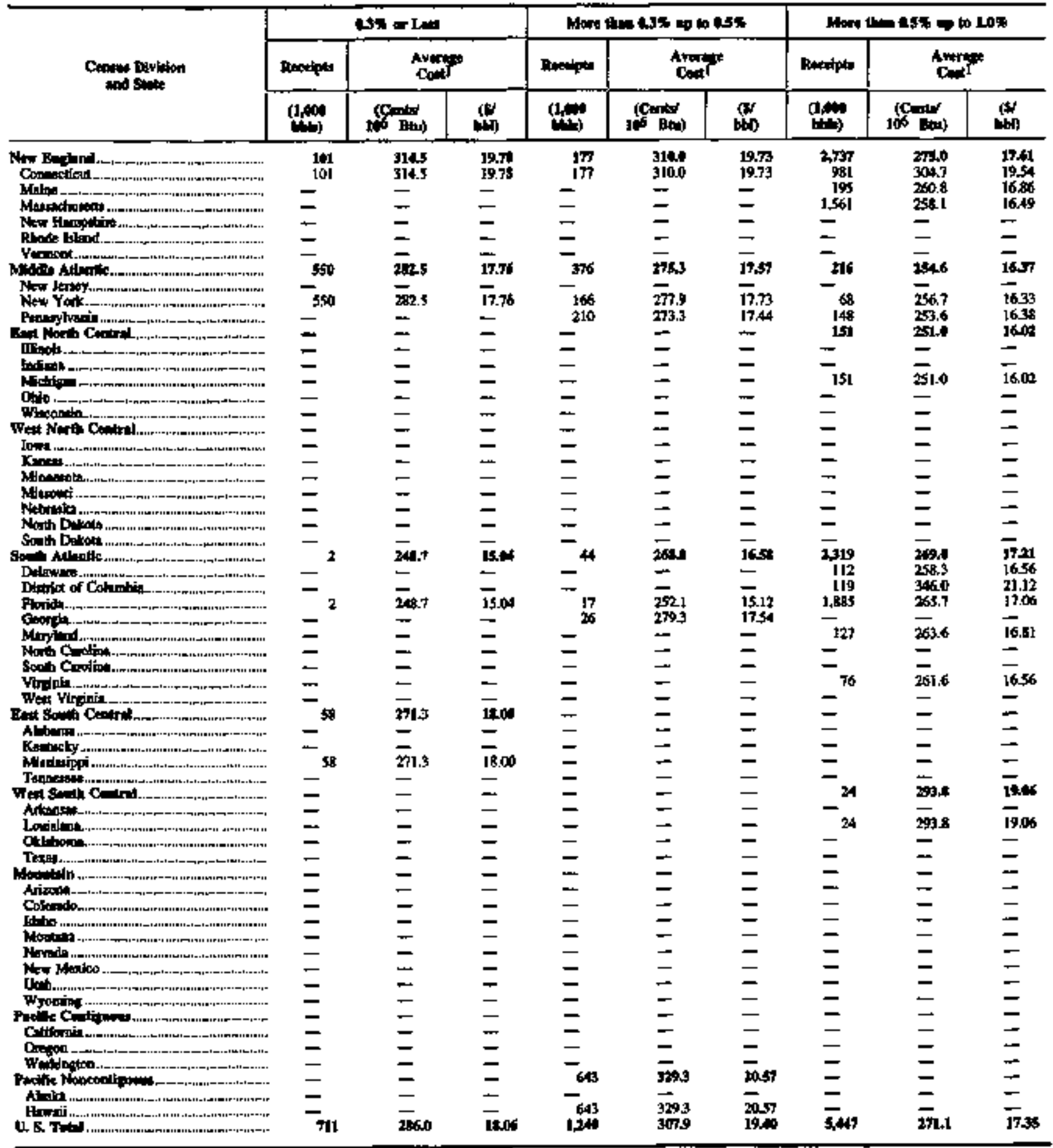

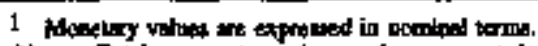

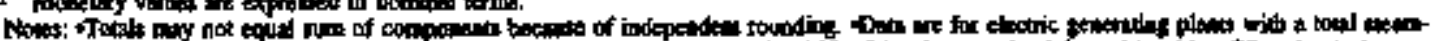

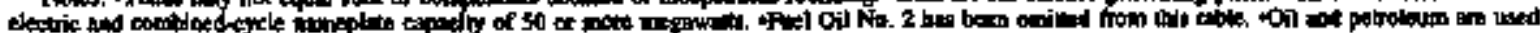

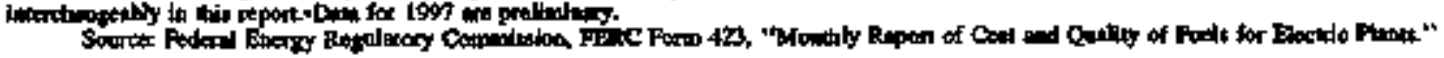


Table 40. Receipts and Averoge Coet of Heavy On Deliwered to Dlectric Utiltites by Sulfur Content, Censas Division, and State, Ju'y 1997 (Conthoed)

\begin{tabular}{|c|c|c|c|c|c|c|c|c|c|c|c|}
\hline \multirow{3}{*}{$\begin{array}{c}\text { Cunsod Divtabe } \\
\text { und Strote }\end{array}$} & \multicolumn{3}{|c|}{ Mare tand 106 p in 205 } & \multicolumn{3}{|c|}{ Mere the $2.0 \%$ ap to 346} & \multicolumn{3}{|c|}{$M a n$ the $30 \%$} & \multicolumn{2}{|c|}{ AB Puraten: } \\
\hline & \multirow{2}{*}{$\begin{array}{l}\text { Rerciptt } \\
\text { (1,4) }\end{array}$} & \multicolumn{2}{|c|}{ Cont } & \multirow{2}{*}{$\frac{\text { Rectipts }}{\text { (1) }}$} & \multicolumn{2}{|c|}{ Confl } & \multirow{2}{*}{$\frac{\text { Rectind }}{(1,010)}$} & \multicolumn{4}{|c|}{ Arents } \\
\hline & & (C) & (⿻) & & (1060) & $(\mathbf{s})$ & & 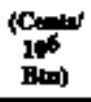 & (s) & $\begin{array}{c}\text { (Cencer } \\
10^{\circ} \\
\text { Bin) }\end{array}$ & $(\mathbf{p})$ \\
\hline 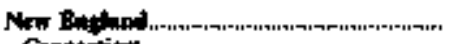 & - & - & - & 3 & $\mathbf{2 3 3 9}$ & 14.7t & س & - & - & 2741 & n.51 \\
\hline & 一 & - & - & 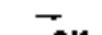 & - & - & - & - & - & 3062 & 19.59 \\
\hline 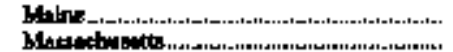 & - & - & - & 24 & $\mathbf{2} 2.3$ & 14.64 & - & - & - & 2452 & 15.64 \\
\hline 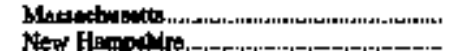 & - & - & ــ & & 20.5 & 15.18 & - & - & - & 257.1 & 16.42 \\
\hline 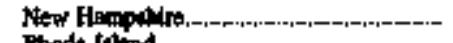 & - & - & - & - & - & - & - & - & - & - & - \\
\hline 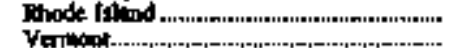 & - & - & - & - & 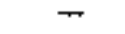 & - & - & - & - & - & - \\
\hline 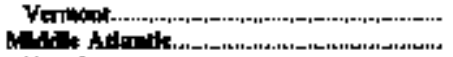 & -6 & $\overline{2}$ & $\overline{159}$ & $=$ & $\overline{-}$ & $\bar{z}$ & $\bar{z}$ & 二 & - & $\overline{245}$ & $\overrightarrow{\mathbf{1 x}}$ \\
\hline & - & - & - & $=$ & & - & $=$ & $=$ & $=$ & & - \\
\hline Yoot & 524 & 200,9 & 36.02 & - & - & - & - & - & - & 267.8 & 16.98 \\
\hline 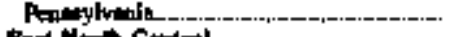 & & 2423 & 15.65 & & $=$ & $=$ & - & - & - & 259.3 & 16.66 \\
\hline 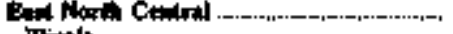 & - & - & - & - & 2390 & 14\$1 & - & - & $\boldsymbol{}$ & 23at & 16.9 \\
\hline 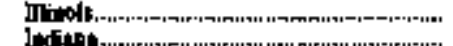 & $=$ & 二 & 二 & $\bar{z}$ & $=$ & $=$ & $\bar{z}$ & $\bar{z}$ & $=$ & $\bar{z}$ & $=$ \\
\hline 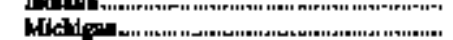 & - & $\overline{-}$ & - & 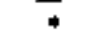 & 20.0 & 14.91 & - & - & - & 250.9 & 16.02 \\
\hline Oblo & - & - & - & - & $m$ & - & - & - & $\rightarrow$ & - & - \\
\hline 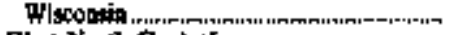 & - & $=$ & $\bar{\pi}$ & - & - & - & - & - & - & $\bar{z}+$ & - \\
\hline 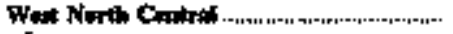 & 17 & 29t.3 & 1365 & - & - & - & - & - & - & 2013 & 13.65 \\
\hline 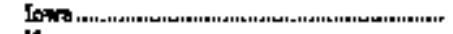 & - & $\bar{x}$ & 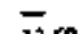 & - & - & - & - & - & - & $\overline{1 m a n}$ & $\overline{1} \times 0$ \\
\hline 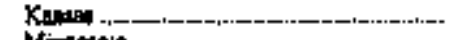 & I5 & 199.9 & 13,60 & - & - & - & - & - & - & 199.9 & 13.60 \\
\hline 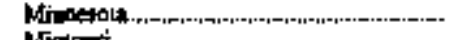 & $=$ & 7 & $\vec{u}$ & 一 & - & $=$ & $\bar{z}$ & $=$ & - & $\overline{a n k}$ & $\overline{1}$ \\
\hline 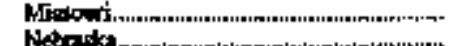 & 2 & 2128 & 14.00 & - & - & - & - & - & - & 212.6 & $14,0,00$ \\
\hline 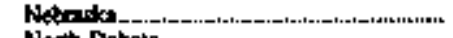 & - & - & - & - & - & - & - & - & - & $\rightarrow$ & - \\
\hline Nentu Dalkota, & - & - & - & - & - & - & $m$ & - & - & - & - \\
\hline 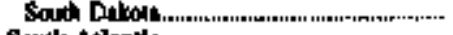 & $\overline{1}$ & $\overline{3426}$ & $\overline{ \pm}+$ & in1 & $\overline{7}+13$ & $\overline{ \pm 0}$ & - & - & - & $\bar{a}$ & 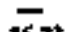 \\
\hline 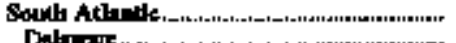 & 1,200 & 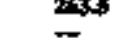 & 16,150 & L, & $\frac{2+13}{-2}$ & 15.3 .5 & 二 & $\bar{z}$ & 二 & 261.1 & 16.73 \\
\hline 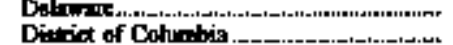 & $\overline{-}$ & $=$ & $\bar{z}$ & $\bar{z}$ & $\bar{z}$ & - & $\bar{z}$ & - & $=$ & 346.3 & 16.36 \\
\hline Flapin & 1,124 & 265.1 & 16.97 & 1,001 & 241.3 & 15.55 & - & $\overline{-}$ & $\overline{-}$ & 259.1 & $\begin{array}{lll}21.12 \\
16,63\end{array}$ \\
\hline 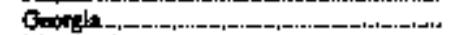 & - & - & - & $\ldots$ & - & - & - & - & - & 2793 & 3734 \\
\hline Matriand -, -, & 108 & $\mathbf{2 4 5 . 7}$ & 590 & - & - & - & - & - & - & 2553 & 16.60 \\
\hline 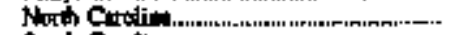 & 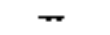 & - & - & - & - & - & - & - & - & - & - \\
\hline 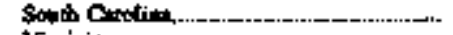 & - & - & - & - & - & - & - & - & - & $\bar{x}+c$ & $\overline{10}$ \\
\hline |' & - & - & 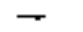 & - & سـ & - & - & - & - & 261.6 & 16.36 \\
\hline 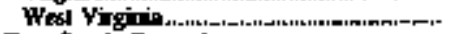 & - & - & - & - & - & $=$ & - & - & - & $=$ & $=$ \\
\hline 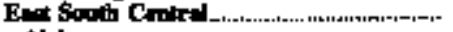 & - & - & - & $10 \mathrm{~s}$ & 2064 & $17+73$ & - & - & - & zTal & 1780 \\
\hline 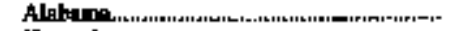 & - & - & - & - & - & - & - & - & - & - & - \\
\hline 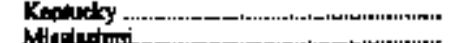 & $\bar{z}$ & 二 & - & -106 & $\overline{2694}$ & $\overline{17.73}$ & $\bar{z}$ & $\bar{z}$ & 二 & $\overline{270.1}$ & $\overline{7} .83$ \\
\hline 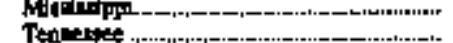 & $=$ & $\overline{-}$ & $\overline{-}$ & -100 & - & - & $\bar{z}$ & $=$ & - & - & - \\
\hline 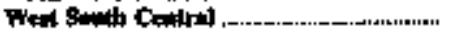 & - & - & - & - & - & - & - & - & - & 2938 & 10.6 \\
\hline 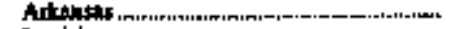 & - & - & - & - & - & - & $\longrightarrow$ & - & - & $m$ & - \\
\hline 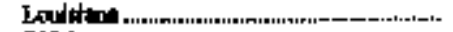 & - & - & - & - & - & $=$ & - & - & - & 2938 & 19.06 \\
\hline 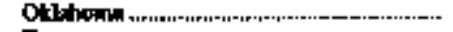 & - & - & - & - & - & - & - & - & - & - & - \\
\hline 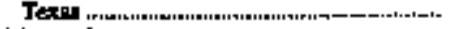 & - & - & - & - & $\rightarrow$ & - & - & - & - & 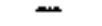 & - \\
\hline 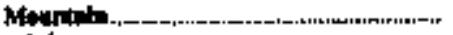 & - & - & $=$ & - & - & - & - & - & - & - & - \\
\hline 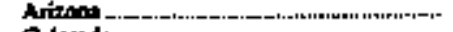 & - & - & - & - & - & - & - & - & - & - & - \\
\hline - & 一 & - & - & - & - & - & - & - & - & $m$ & - \\
\hline 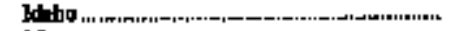 & - & - & - & - & - & - & - & 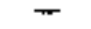 & - & - & - \\
\hline 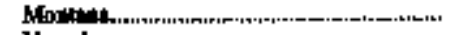 & - & - & - & - & - & - & - & - & - & - & - \\
\hline 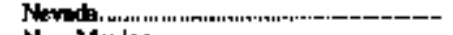 & - & - & - & - & - & - & & & - & - & - \\
\hline 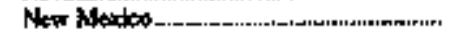 & - & - & - & - & - & - & - & - & - & - & - \\
\hline - & - & - & - & - & - & - & - & - & - & - & - \\
\hline 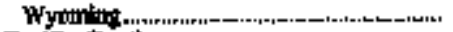 & - & 一 & - & - & - & - & — & - & - & - & - \\
\hline Conthour & - & - & 一 & - & - & $m$ & - & - & - & - & - \\
\hline 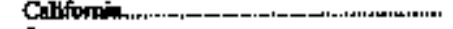 & - & - & - & - & - & - & - & - & - & - & - \\
\hline 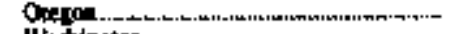 & - & - & - & - & - & - & - & - & - & - & - \\
\hline 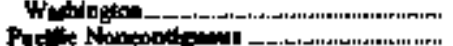 & 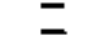 & $=$ & 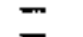 & $=$ & $\bar{z}$ & $=$ & $\bar{z}$ & $=$ & $=$ & $\overline{303} 3$ & $\overline{2}$ \\
\hline 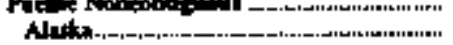 & 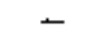 & - & - & - & - & - & - & - & - & - & - \\
\hline Hom it & - & - & - & - & - & 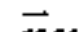 & - & - & - & 3293 & 20,57 \\
\hline ntol & IN: & t5to & 16.50 & 1,51t & 241. & IS:SA & - & - & - & 2498 & 1724 \\
\hline
\end{tabular}

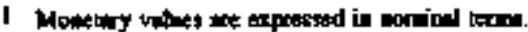

- Less and as.

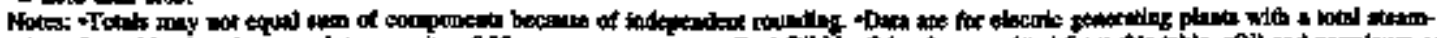

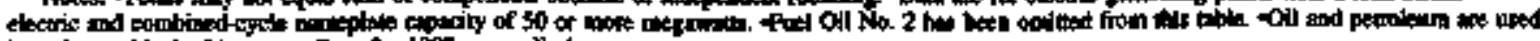

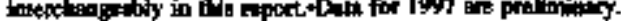

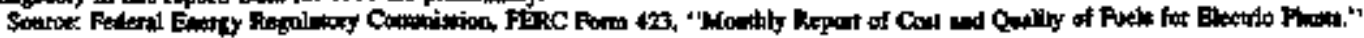


Table 41. Electric Utility Recelpts of Gas by Type, Census Diviston, and Statt, Juby 1997

\begin{tabular}{|c|c|c|c|c|c|c|c|c|}
\hline \multirow{2}{*}{ 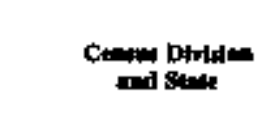 } & \multicolumn{2}{|c|}{ Numire } & \multicolumn{2}{|c|}{ 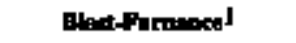 } & \multicolumn{2}{|c|}{ Refinery } & \multicolumn{2}{|c|}{ Telal } \\
\hline & $\begin{array}{l}\text { (then:end } \\
\text { Met) }\end{array}$ & (tifilon & (tthend & Bac) & (thended & 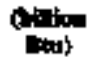 & (1k Mend & (Bta) \\
\hline New Fandand & Ins,345 & 14,405 & - & - & - & - & 10,346 & Indes \\
\hline Cosbecheal & 2,344 & 3,389 & - & - & - & - & 2,344 & 2,389 \\
\hline Mane & - & - & 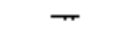 & - & - & - & $=$ & - \\
\hline Dessomingeth & 5,934 & 6116 & - & - & $\rightarrow$ & - & 5,939 & 6,116 \\
\hline Near Hampiphore & 57 & 58 & - & $m$ & - & 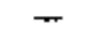 & 5 & 59 \\
\hline Thode Ialind & 3,006 & 2,068 & - & - & - & - & 2,006 & 2058 \\
\hline Yermonl & & & - & - & - & - & 4 & 4 \\
\hline 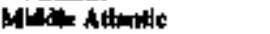 & 34,010 & 3900 & - & - & - & - & 38,610 & 30469 \\
\hline New lersey & 4004 & 4,257 & - & - & - & - & 4,096 & 4,257 \\
\hline Netr Yat & 34,278 & 35,176 & 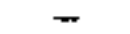 & $\rightarrow$ & - & - & 34,275 & 35,176 \\
\hline Pearivylutes & 248 & 256 & $=$ & - & - & - & 248 & 236 \\
\hline Ened North Centrol & 10,149 & 30,344 & 2,164 & 246 & - & - & 12,253 & Jo,kst \\
\hline Tunoss & 1,578 & 7,693 & - & - & - & - & 7,578 & 7,693 \\
\hline lodpatan & 275 & 093 & - & - & - & - & 875 & 893 \\
\hline Muctugan & 1,021 & 1.031 & 2,104 & 246 & - & - & 3,124 & $1,2 \pi$ \\
\hline O100 & 212 & 217 & $\rightarrow$ & - & - & - & 212 & 257 \\
\hline Wucandu & 463 & 47 & - & - & - & - & 463 & 470 \\
\hline Wex North Cantral & $5+560$ & $5,3 \pi$ & - & - & - & - & 6,506 & 5,370 \\
\hline lowa & 259 & 259 & - & - & - & - & 299 & 259 \\
\hline $\mathbf{Y n n a s s}_{10}$ & 4,520 & 3.59 & - & - & - & - & 4,020 & 3,799 \\
\hline Mumesol: & 406 & 408 & - & - & - & - & AB6 & 408 \\
\hline Sharown & 757 & 762 & - & - & - & - & 255 & 762 \\
\hline Netorekn & 145 & 143 & - & - & - & - & 145 & 149 \\
\hline Narth Doth & $*$ & $*$ & - & - & - & - & $\bullet$ & * \\
\hline Sonlh Ditots & - & - & - & - & - & - & - & - \\
\hline Solon Allatic & 36700 & $36,3,11$ & - & - & - & - & 36,730 & 30,311 \\
\hline Dedpwane & 1,993 & 2,064 & - & - & - & - & 1,993 & 2064 \\
\hline Dustict of Columbia & - & - & - & - & - & - & - & - \\
\hline Fanda & 30,168 & $31.40 \mathrm{I}$ & - & - & - & - & 30,168 & 31,401 \\
\hline 0 endin & 1,096 & 1.122 & - & - & - & - & 1,006 & 1,122 \\
\hline Marjimed & 1,303 & 1,359 & - & - & - & - & 1,305 & 1,359 \\
\hline Nowth Corotas & 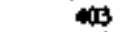 & 417 & - & - & س & - & 403 & 417 \\
\hline Sonb Camatra & 40 & 41 & - & - & - & - & 40 & 4t \\
\hline Vilonnos & 1,768 & 1,889 & - & - & - & - & 1,769 & 1,889 \\
\hline Wer Virguas & 18 & 18 & - & - & - & - & 18 & 18 \\
\hline 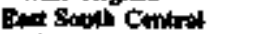 & 10 & 12,40 & - & $=$ & - & - & $11,9 \%$ & 12,40 \\
\hline Alobom & 6? & 69 & - & - & $m$ & - & 67 & 69 \\
\hline Sepancty & d1 & 42 & - & - & - & - & 41 & 42 \\
\hline Musessipg & 11,891 & 12,297 & - & - & $=$ & - & 12.891 & 12.29 \\
\hline Trunetate & - & - & - & - & - & - & - & - \\
\hline Wrat Sond Caniral & 197,174 & 202305 & - & - & - & - & 197,176 & 20,300 \\
\hline Arkemiat & 4.196 & 4,280 & - & - & - & - & 4,196 & 4,200 \\
\hline Lowetem & 38,370 & 39,725 & - & - & - & - & 38,370 & 39,78 \\
\hline Othromen & 22006 & 22,745 & - & - & - & - & 22,046 & 22745 \\
\hline Texta & 132364 & 135,590 & - & - & - & - & 132,564 & 135,550 \\
\hline Mourtian & 15,14 & 15,77 & - & - & - & - & $1 \mathrm{E}, 147$ & 15,717 \\
\hline Anzod & $\$ 989$ & 1,046 & - & - & - & - & 3.989 & 4,046 \\
\hline Colorendo & 325 & 324 & - & - & - & $\rightarrow$ & 385 & 324 \\
\hline Who & - & - & - & - & - & - & - & - \\
\hline Mhonano & 16 & 16 & - & - & - & - & 16 & 16 \\
\hline Nowis & 6.427 & 6,651 & - & - & - & - & 6,427 & 6,631 \\
\hline New Mexuco & 3,748 & 4,092 & - & - & - & - & 3,748 & 4,032 \\
\hline Uth & 639 & 663 & - & - & - & - & 639 & 663 \\
\hline Wromeng & & 4 & - & - & - & - & & 4 \\
\hline Pacilit Conilaun & $44 ; 39$ & $\mathbf{4}, \mathbf{1 6 1}$ & - & - & - & - & 4439 & 45,10 \\
\hline Colnorran & 44,1904 & 45,045 & - & - & - & - & 4,194 & 45,045 \\
\hline Orogod & 115 & 116 & - & - & - & - & 115 & 116 \\
\hline Wunngion & * & * & - & - & - & - & F & \\
\hline 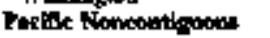 & 1,422 & 1,023 & - & - & - & - & 1,422 & $1,0+2$ \\
\hline Alasko & 1,122 & 1,422 & - & - & - & . & 1.482 & 1,42 \\
\hline Нтоны & $\rightarrow$ & $=$ & - & - & - & - & - & - \\
\hline Lis Toul & $5 \pi 1,634$ & 301,31t & 2,104 & $2 \omega$ & - & - & 373,$6 ; 6$ & 351,45 \\
\hline
\end{tabular}

1 Lackdes golse onep gut

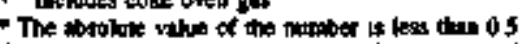

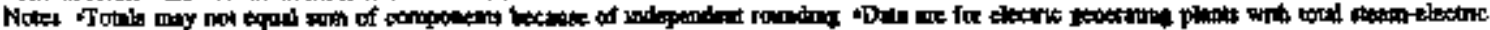

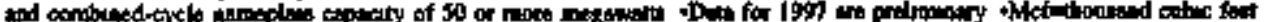

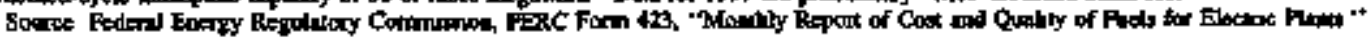


Table 42. Recelpts and Average Cost of Gas Delivered to Electric Utilties by Ceasus Division and State

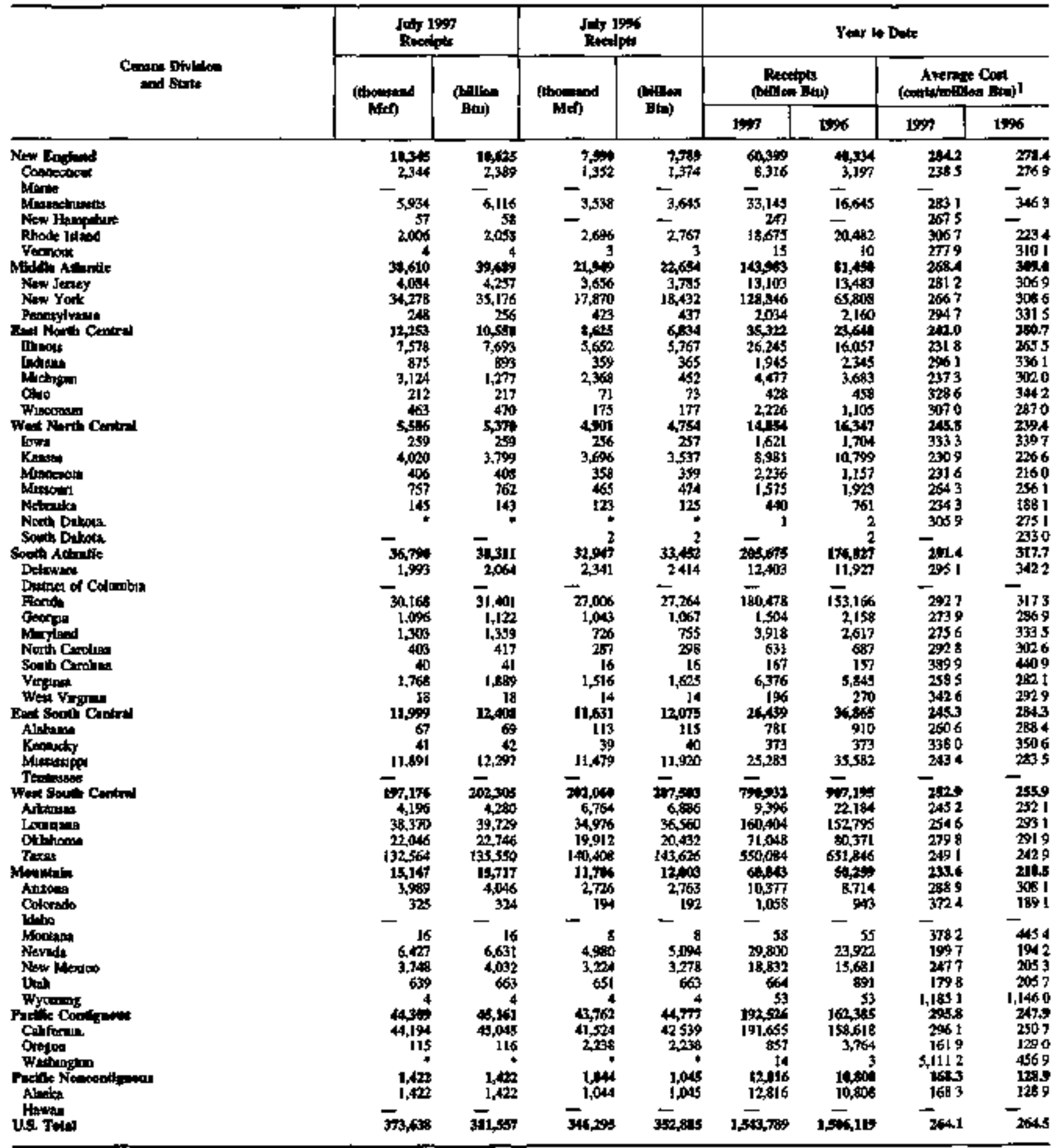

1 Kootary values we exproted un commal terms

* Last tin os

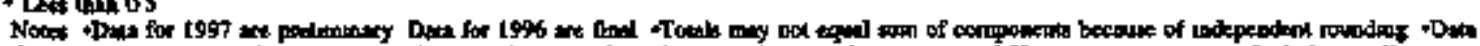

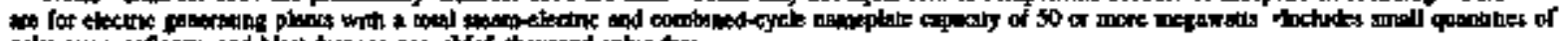

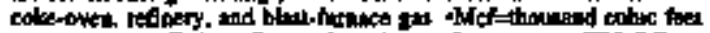

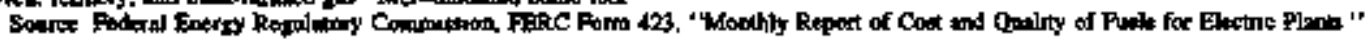


Table 43. Receipts and Average Cost of Gas Delivered to Electric Utilition by Type of Purcluase, Cenisus Division, and State, July 1997

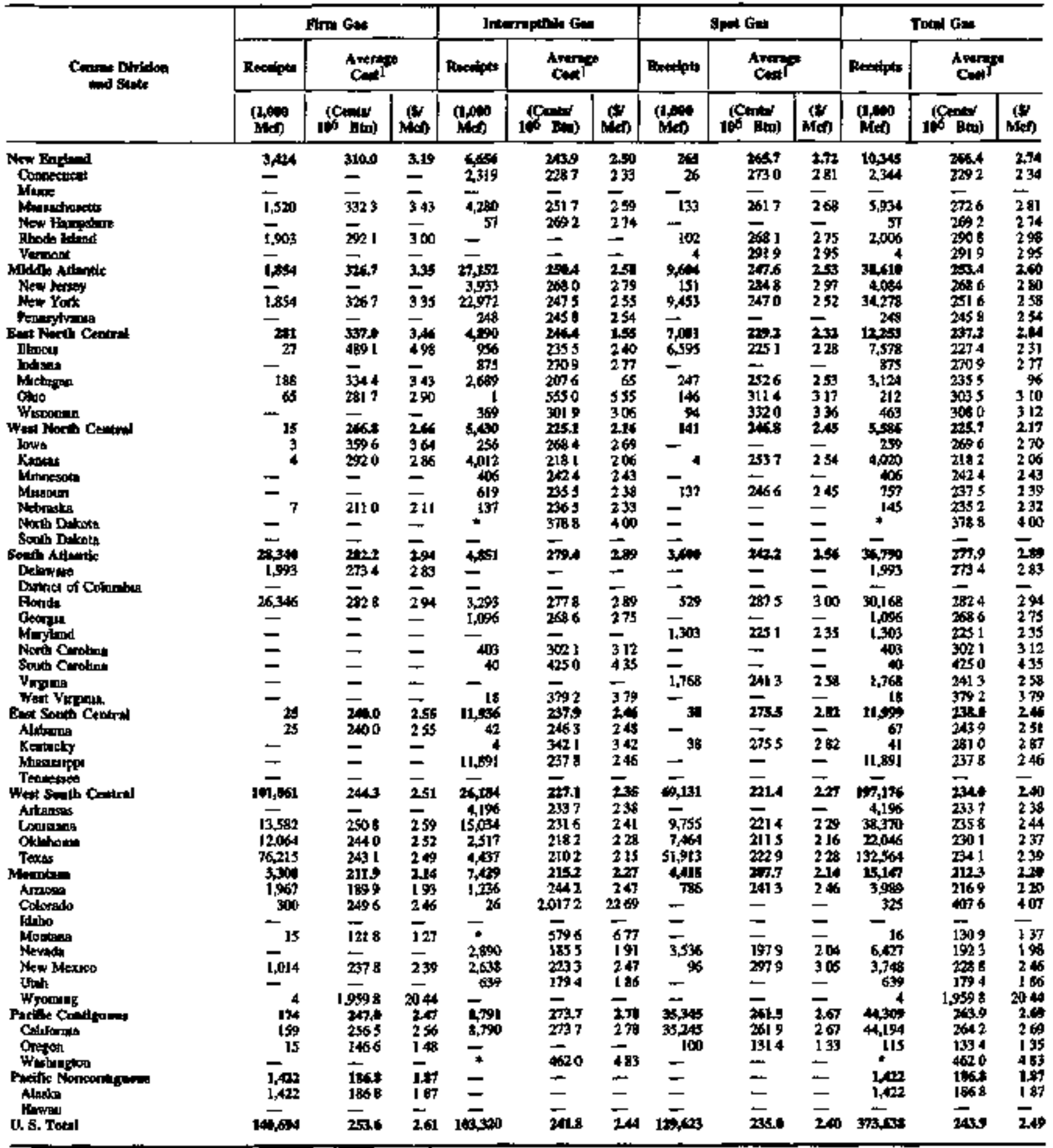

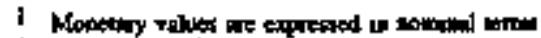

$=$ Leas then 000

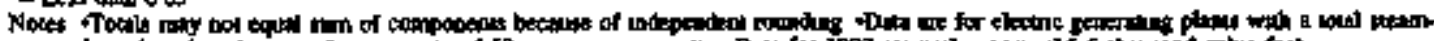

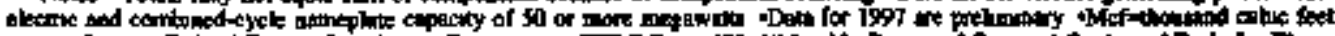

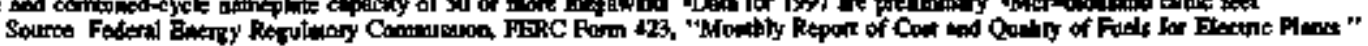


U.S. Electric Utility Sales, Revenue, and Average Revenue per Kilowatthour 
Tahle 44. U.S. Flectric Utilty Retoll Sales of Electriciny by Sector, 1987 Throngh Augnst 1997 (Million Kilowathours)

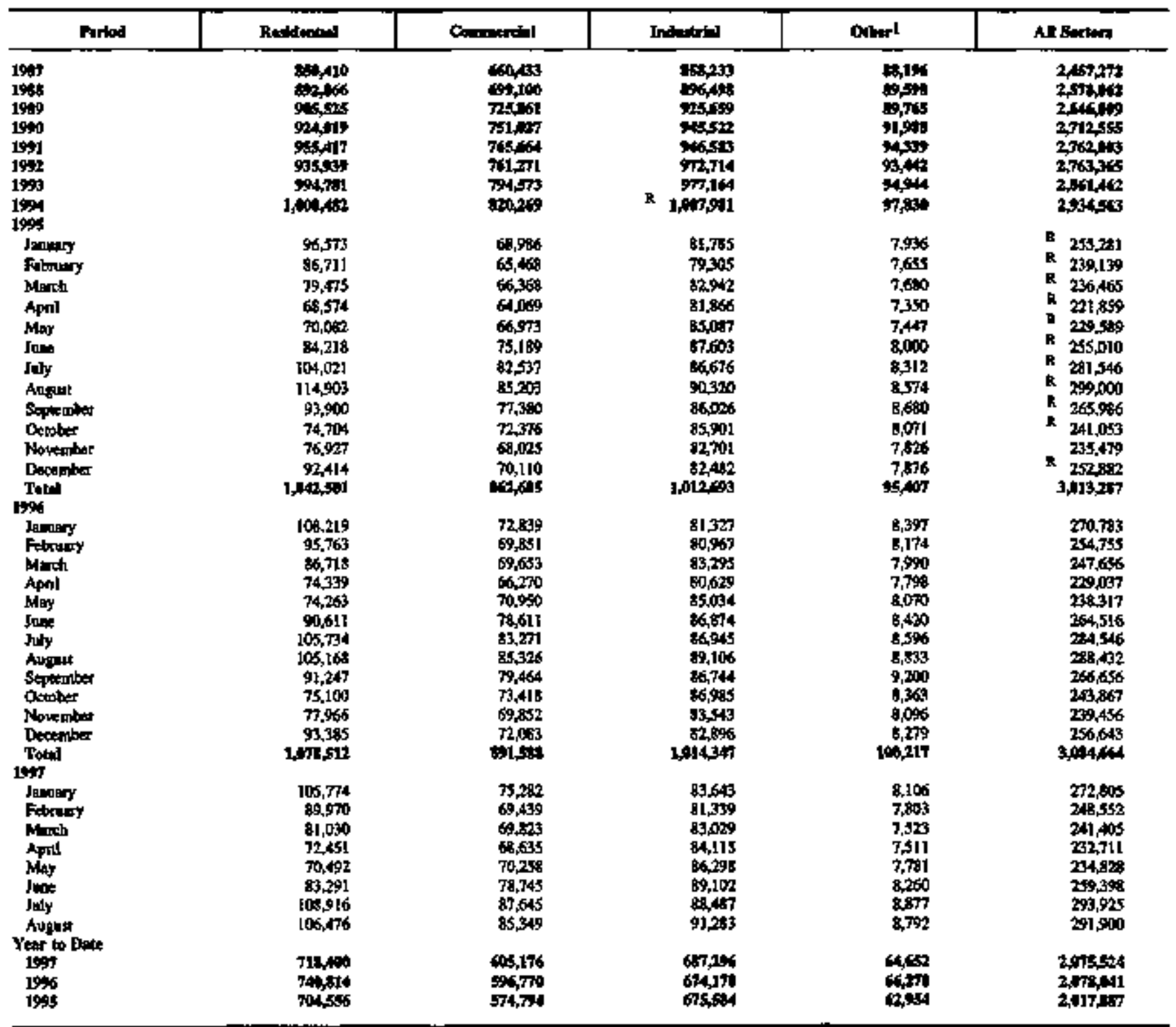

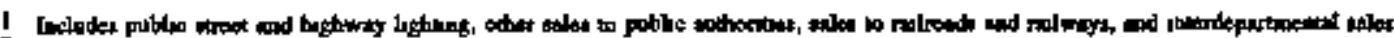

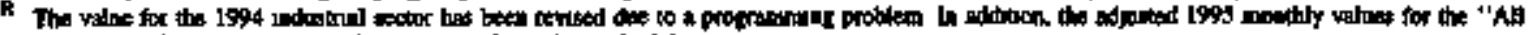

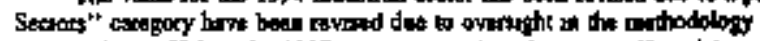

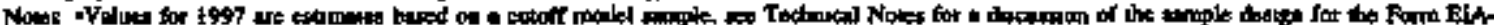

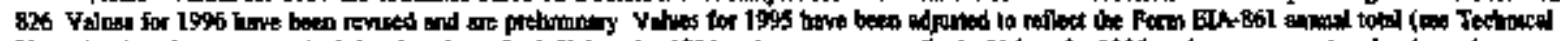

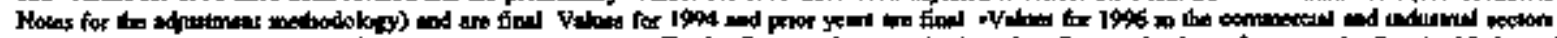

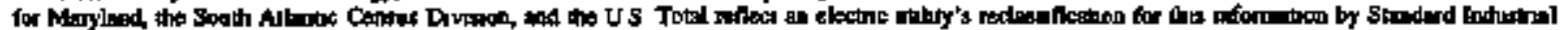

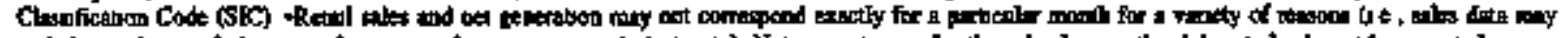

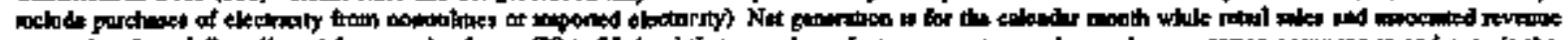

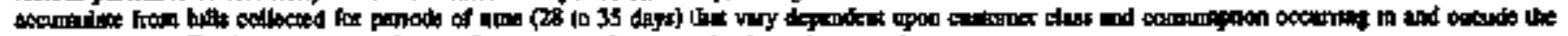

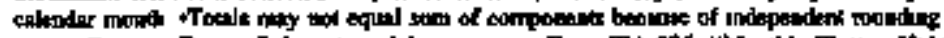

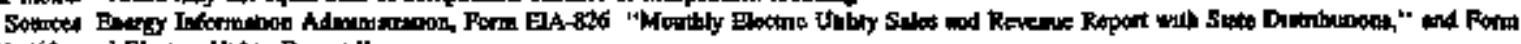

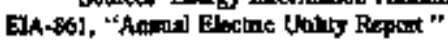


Tahfe 45. Estimated U.S. Electric Utility Retail Sales of Electricity to Ulimate Consangers by Sector, Census Divislon, and State, Augnest 1997 and 1996 (Million Kilowatchours)

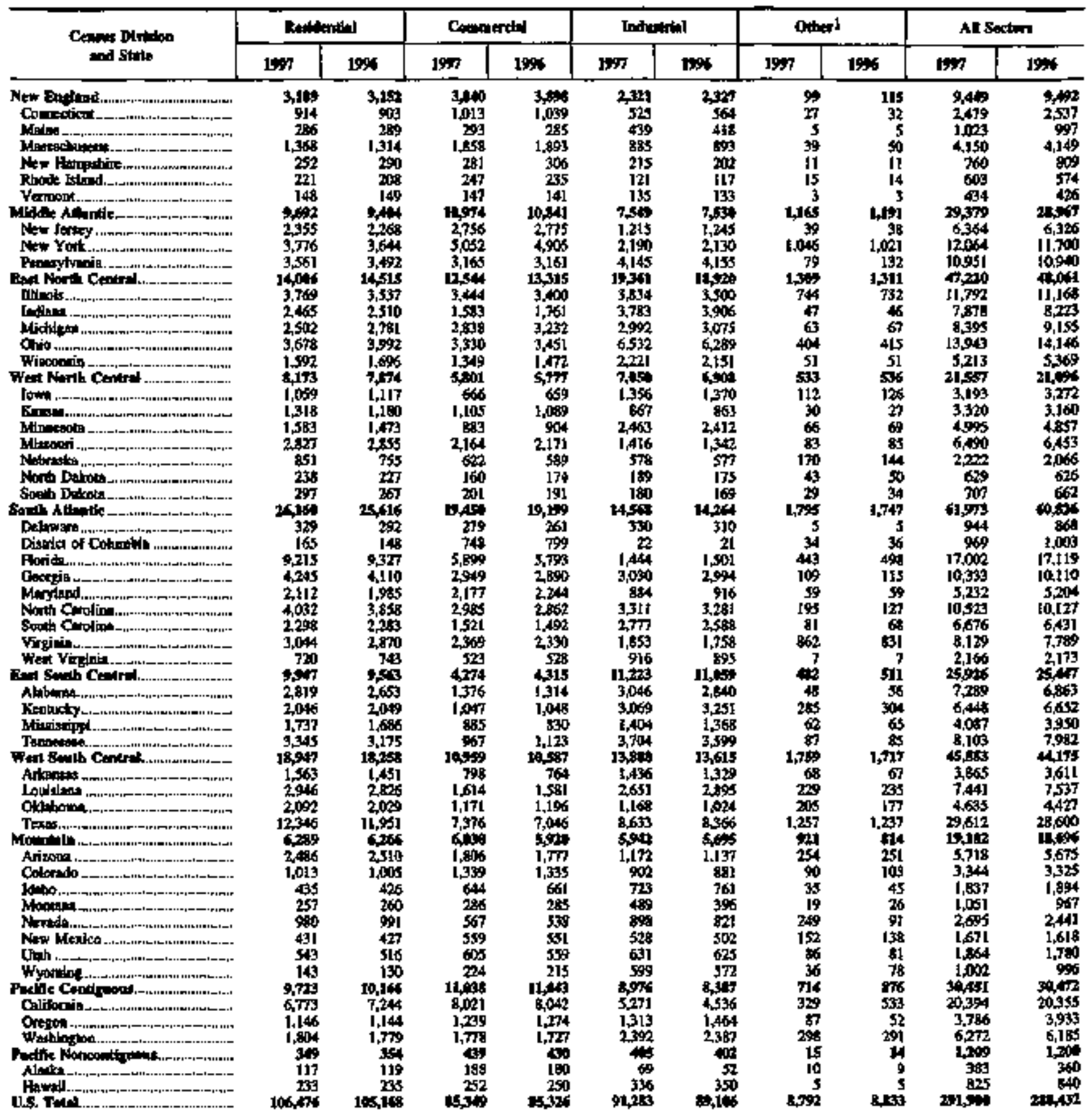

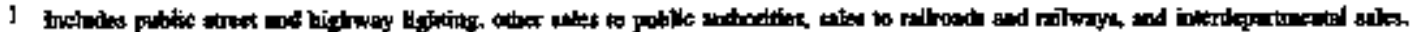

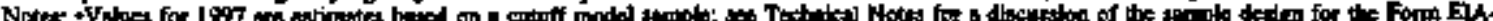

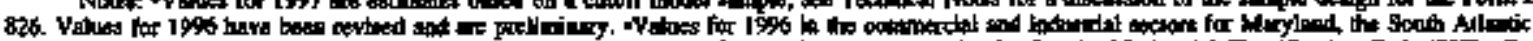

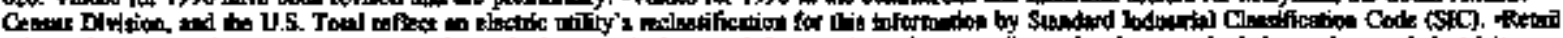

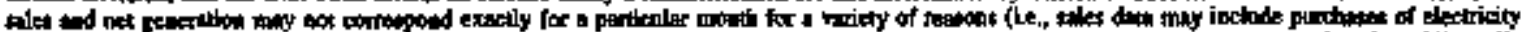

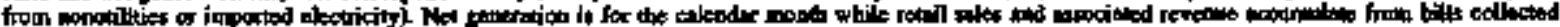

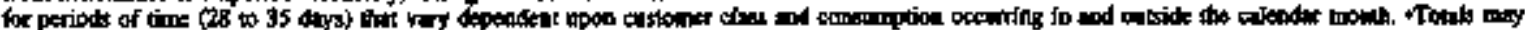

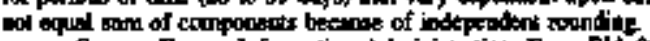

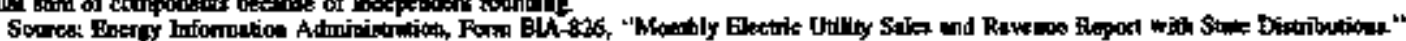


Twhle 46. Estimated Coefictents of Vartation for U.S. Electric Utilty Retall Sales of Electricity to Ultunate Consuners by Sector, Censos Division and State, Aaguat $19 \%$ (Percent)

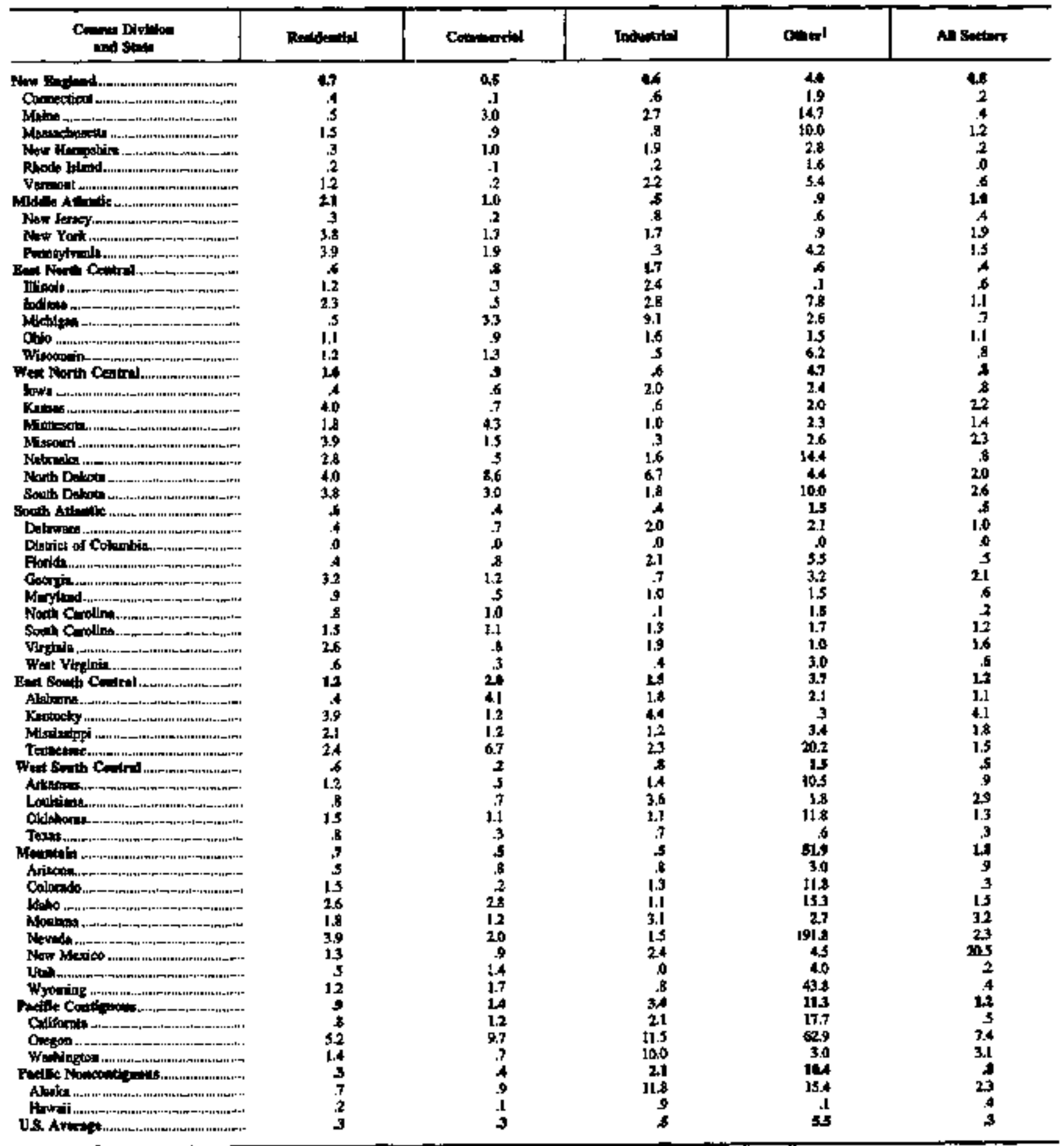

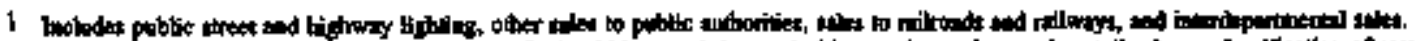

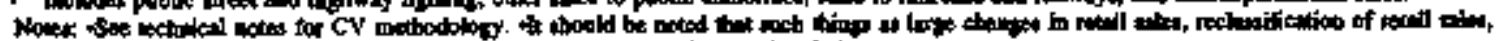

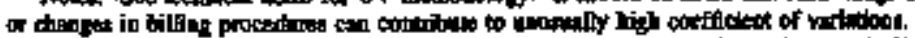

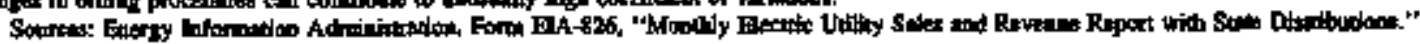


Table 47. Bstimated U.S. Bextric UGtity Retail Sales of Bectrietity to Ultimate Consumera by Sector, Census Division, and Sente, Year-to-Daid 1997 and 1996 (Million Kilowalthours)

\begin{tabular}{|c|c|c|c|c|c|c|c|c|c|c|}
\hline \multirow{2}{*}{ Cowned Dimition } & \multicolumn{2}{|c|}{ 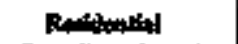 } & \multicolumn{2}{|c|}{ 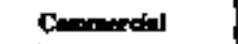 } & \multicolumn{2}{|c|}{ Inetatat } & \multicolumn{2}{|c|}{ Ouher' } & \multicolumn{2}{|c|}{ All setors } \\
\hline & $\mathrm{L} \% \mathrm{~T}$ & toss & 16 & $1 \%$ & $\cos$ & $19 \%$ & $19 *$ & bas & $16 \pi$ & $\cos$ \\
\hline 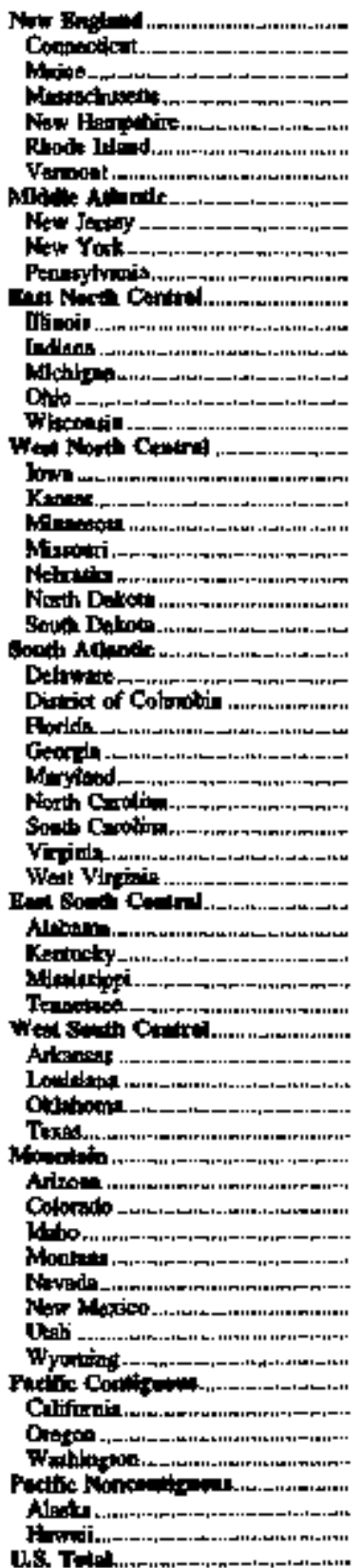 & 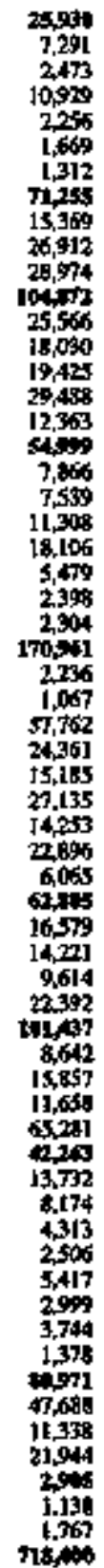 & 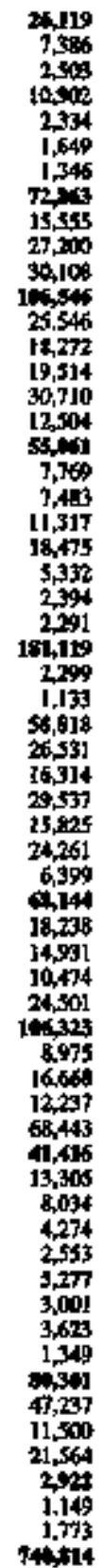 & 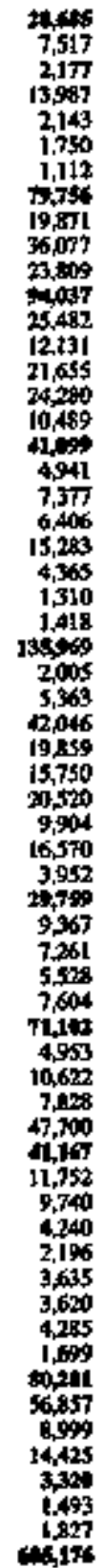 & 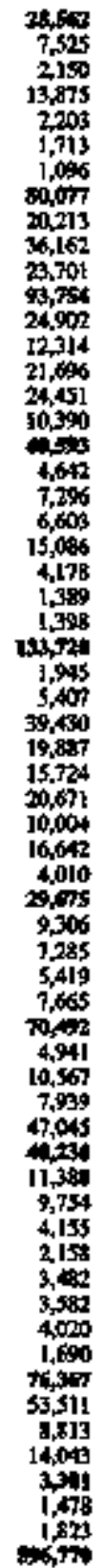 & 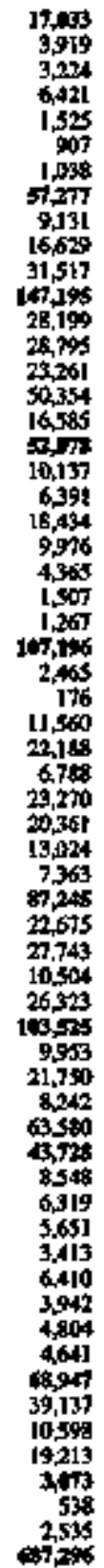 & 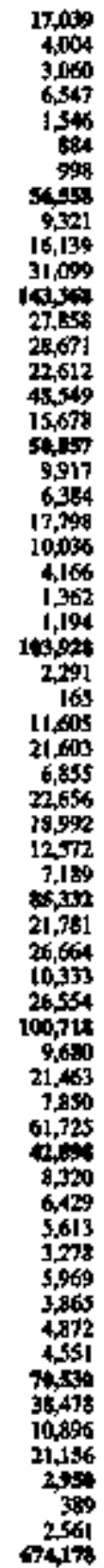 & 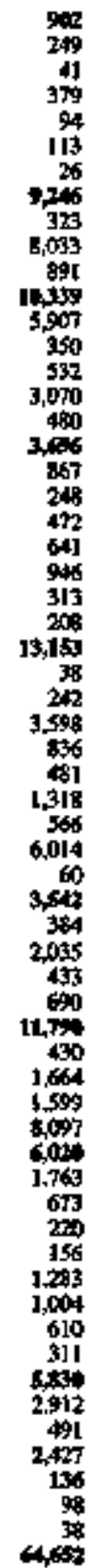 & 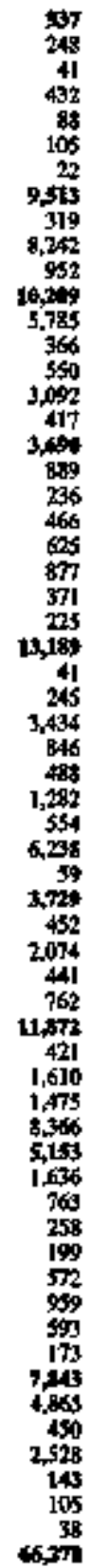 & 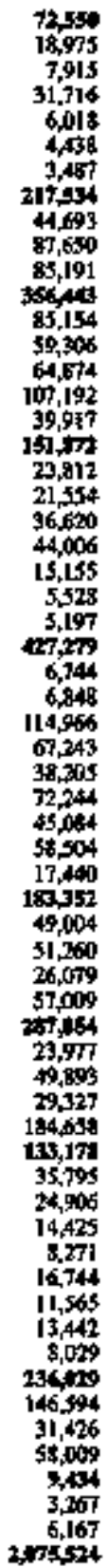 & 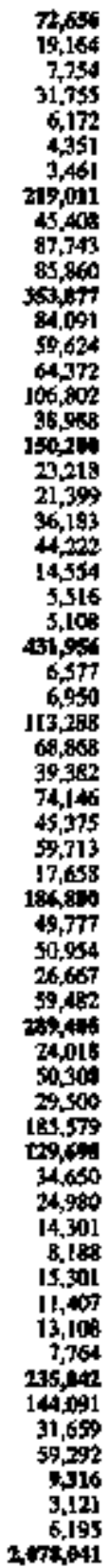 \\
\hline
\end{tabular}

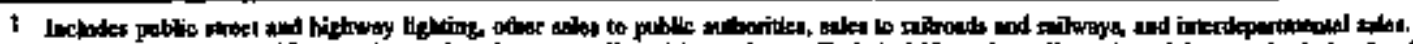

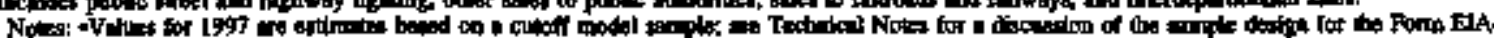

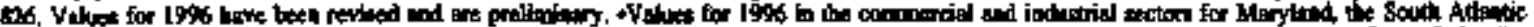

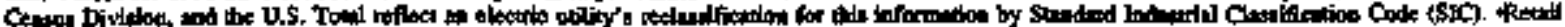

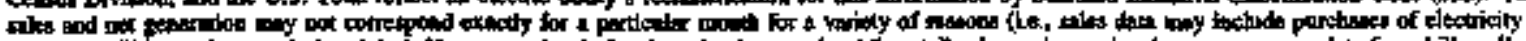

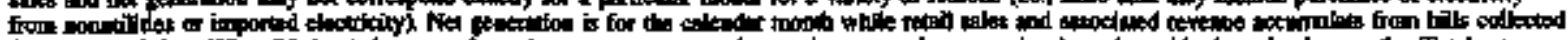

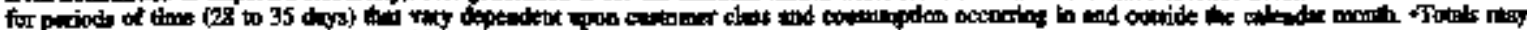

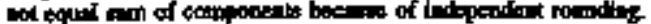

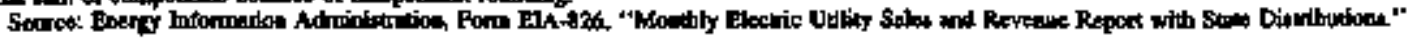


Table 48. Revenue from U.S. Fiectric Utility Retail Seles of Flectritity to Unimote Consumess by Sector, 1987 Through Angust 1997 (Millon Dollars)

\begin{tabular}{|c|c|c|c|c|c|}
\hline Puthod & Reldinotian & Conserdal & |lindinted & Oleal & All sexist \\
\hline 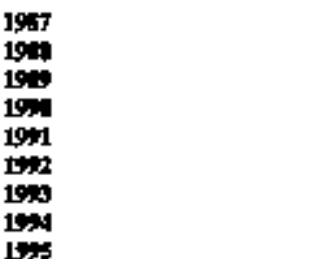 & 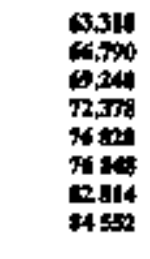 & $\begin{array}{l}45,7,7 \\
49,224 \\
52,2,5 \\
5,117 \\
57,05 \\
58,343 \\
61,521 \\
63,3 \% 4\end{array}$ & $\begin{array}{l}44,49 \\
4,144 \\
43,7,9 \\
44,57 \\
45,737 \\
46,93 \\
47,3 \times 7 \\
40,46\end{array}$ & 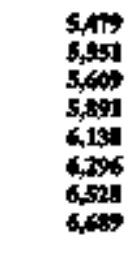 & 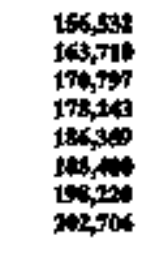 \\
\hline \multicolumn{6}{|l|}{ bes: } \\
\hline Inumby & $3 \$ 3$ & 5,059 & 3,667 & 528 & 16,937 \\
\hline Fetanesy & 6,945 & 4,9000 & $3,6,12$ & 517 & R Is, \\
\hline $\begin{array}{l}\text { Murch } \\
\text { Apsl! } \\
\text { Miny }\end{array}$ & $\begin{array}{l}6,469 \\
5,769 \\
5,979\end{array}$ & $\begin{array}{l}4999 \\
4,844 \\
5,119\end{array}$ & $\begin{array}{l}3,758 \\
3,699 \\
3,861\end{array}$ & $\begin{array}{l}521 \\
4 \$ 9 \\
518\end{array}$ & $\begin{array}{r}15,745 \\
14,755 \\
15,677\end{array}$ \\
\hline הוחו & 7,746 & 5,976 & 4,219 & 572 & $18,1 / 2$ \\
\hline Jaly & 9,155 & 6,655 & 4,290 & 593 & 20,893 \\
\hline Alotst & 10,008 & 6,373 & 4,49 & 601 & R 21,955 \\
\hline sepleniber & 8,048 & 6,067 & $\$ 1188$ & 397 & R 18.431 \\
\hline Oetober & 6,163 & 5,681 & 4,644 & 568 & R 16,755 \\
\hline $\begin{array}{l}\text { Nonember } \\
\text { Desention }\end{array}$ & $\begin{array}{l}6,356 \\
7,007\end{array}$ & $\begin{array}{l}5,167 \\
3,160\end{array}$ & $\begin{array}{l}3,731 \\
3,495\end{array}$ & $\begin{array}{l}535 \\
527\end{array}$ & $\begin{array}{l}15,789 \\
\text { R } 16,707\end{array}$ \\
\hline Totiol & anto & 66,35 & 47,175 & 6sen & R 207,717 \\
\hline \multicolumn{6}{|l|}{ 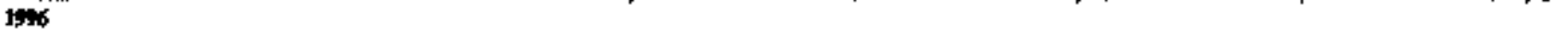 } \\
\hline Iomey & 8,423 & 5,321 & 3,637 & 545 & 17,926 \\
\hline Fanuary & 7,504 & 5,157 & 3,643 & 537 & 16,842 \\
\hline Minch & $7, \pi 37$ & S.18s & 3,738 & 532 & 26,495 \\
\hline Ant & 6,149 & 4.954 & 3,598 & 519 & 15,214 \\
\hline my & 6,163 & $5 \mathrm{AOO}$ & 3,856 & 550 & 16,169 \\
\hline cto & 7,H65 & 6,062 & 4,111 & 595 & {$[8,6,34$} \\
\hline wy & 9,268 & 6,614 & 4,241 & 594 & 20,718 \\
\hline Awine: & 9.155 & 6,800 & 4,310 & 609 & 21,083 \\
\hline Sepponater & 8051 & 6,320 & 4,147 & 614 & 19,132 \\
\hline Oatober & 6,137 & 5,753 & 4,011 & 577 & 16,578 \\
\hline Koveraber & 6,454 & 5,245 & 3,721 & 537 & 15,558 \\
\hline Dectaber & 7,490 & 5,200 & 9,633 & 534 & 16.9.8 \\
\hline Totil & Nyet & 6,073 & 46,644 & 6,7sil & $211, \$ 58$ \\
\hline \multicolumn{6}{|l|}{$19=7$} \\
\hline Janisty & B,746 & 5,505 & 3,712 & $\$ \$ 2$ & 318,115 \\
\hline Pubniagy & 7.2002 & 5,156 & 3,613 & $\$ 2 A$ & 16,496 \\
\hline Mereh & 6.706 & 5,231 & $3,69[$ & 525 & 16.14 \\
\hline Apd & 6,080 & 5109 & 3,659 & 517 & 15,374 \\
\hline limy & 6,120 & 5,357 & 3,812 & 525 & $15 \$ 23$ \\
\hline Jun & 7,449 & 6,247 & 4,131 & 578 & 18,405 \\
\hline Suly & 9,554 & 6,936 & 4,283 & 394 & $21,37 t$ \\
\hline Awout & 9.402 & 6,797 & 4,371 & 611 & 21,182 \\
\hline \multicolumn{6}{|l|}{ Your to Dith } \\
\hline ind & 64t7te & 46,35s & 31,206 & 4,47 & $14,4,1$ \\
\hline tow & $\begin{array}{l}1,365 \\
5,335\end{array}$ & $\begin{array}{l}4,505 \\
44300\end{array}$ & $\begin{array}{l}31,134 \\
31,58\end{array}$ & $\begin{array}{l}4,47 \\
4,311\end{array}$ & $\begin{array}{l}10,1,00 \\
13,585\end{array}$ \\
\hline
\end{tabular}

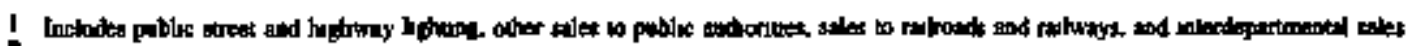

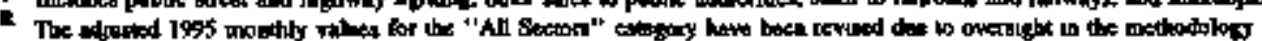

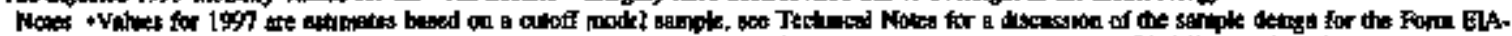

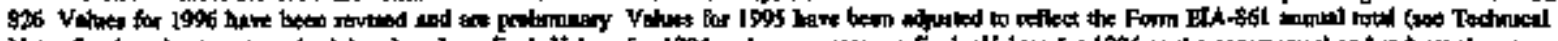

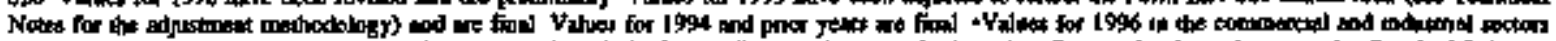

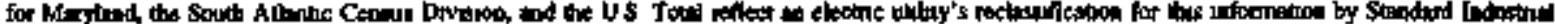

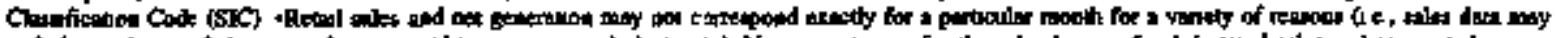

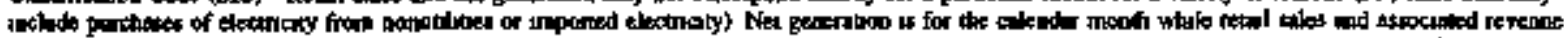

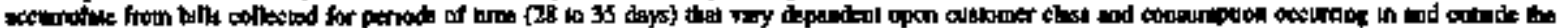

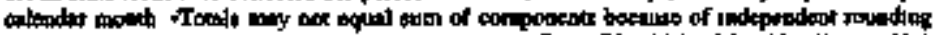

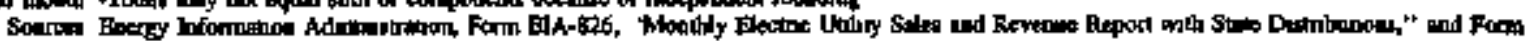

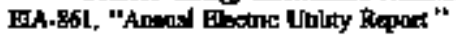


Table 49. Estrmated Revenue from U.S. Electric Uttity Retail Sales of Electricity to Eltimate Consumars by Sector, Census Divtsion, and State, August 1997 and 19\% (Miltion Doilars)

\begin{tabular}{|c|c|c|c|c|c|c|c|c|c|c|}
\hline \multirow{2}{*}{ 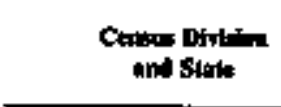 } & \multicolumn{2}{|c|}{ 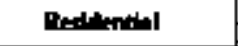 } & \multicolumn{2}{|c|}{ C::eredin } & \multicolumn{2}{|c|}{ 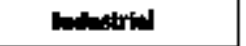 } & \multicolumn{2}{|c|}{ Othar } & \multicolumn{2}{|c|}{ Al Setwis } \\
\hline & 199 & $19 \%$ & 185 & L'6 & 197 & $15 \%$ & lost & ipos & $5>7$ & 1996 \\
\hline 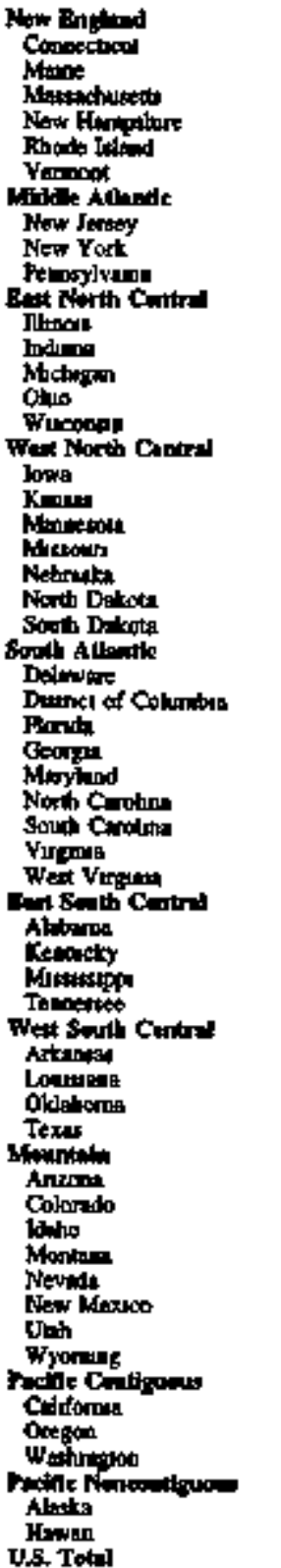 & 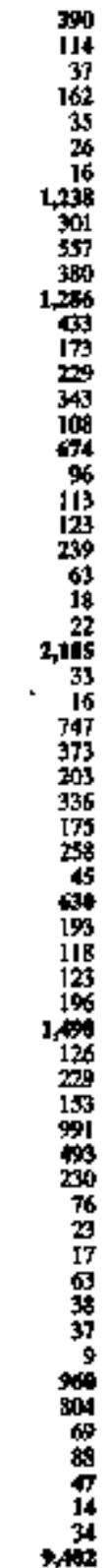 & 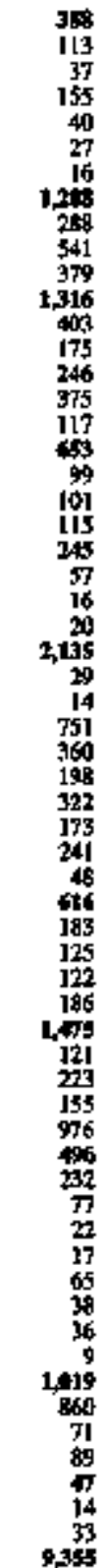 & 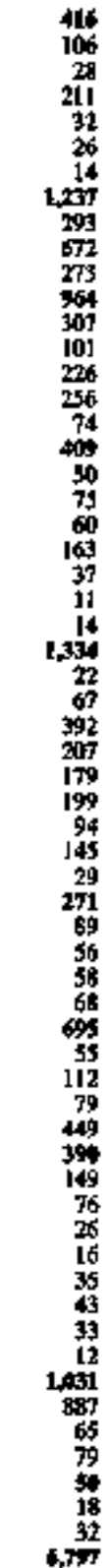 & 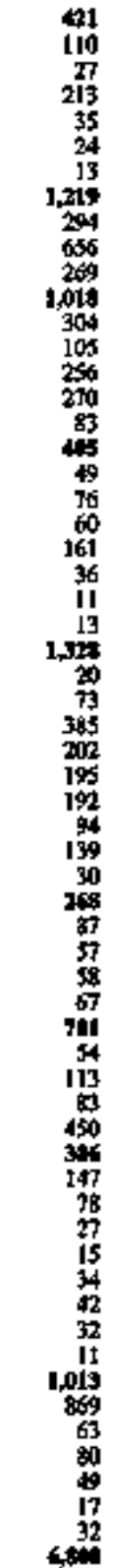 & 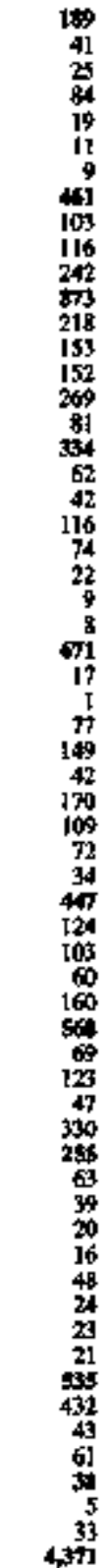 & 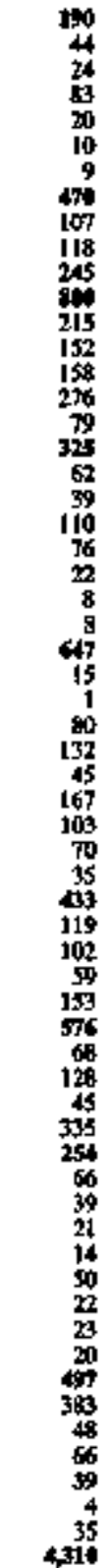 & 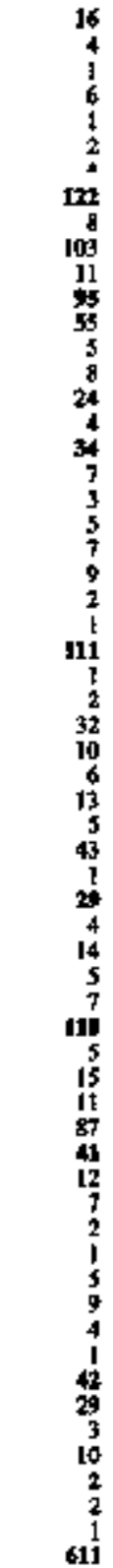 & 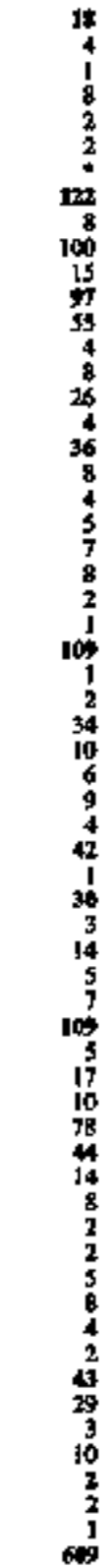 & 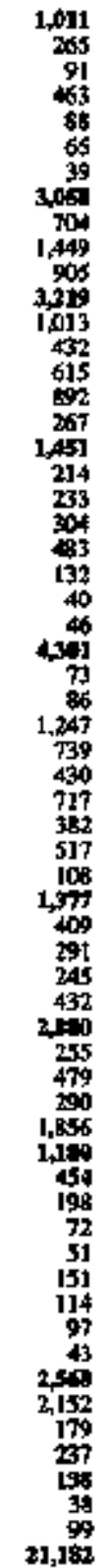 & 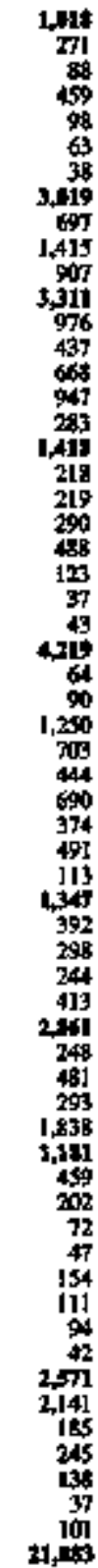 \\
\hline
\end{tabular}

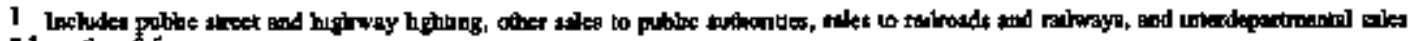

- Lon the of

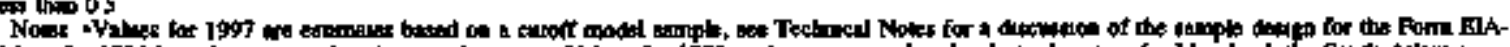

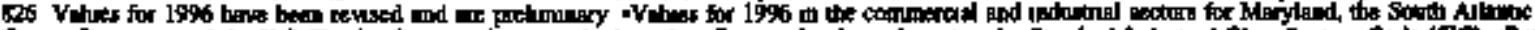

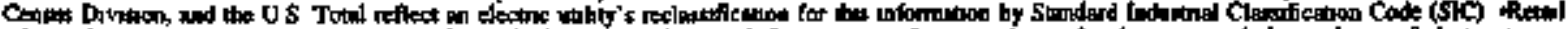

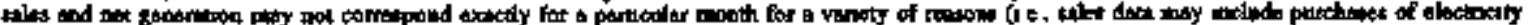

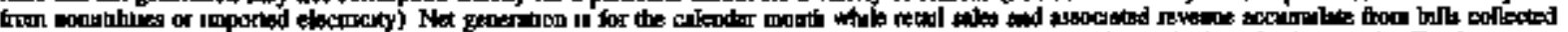

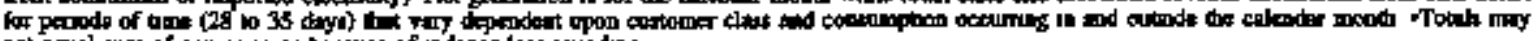

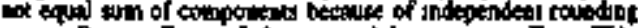

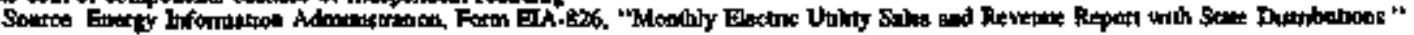


Table 50. Estimated Coefitetieate of Variation for Revenue from U.S. Electric Uthity Retall Seles of Electricity to Untimate Consuners by Sectior, Census Division, and State, August 19y (Percent)

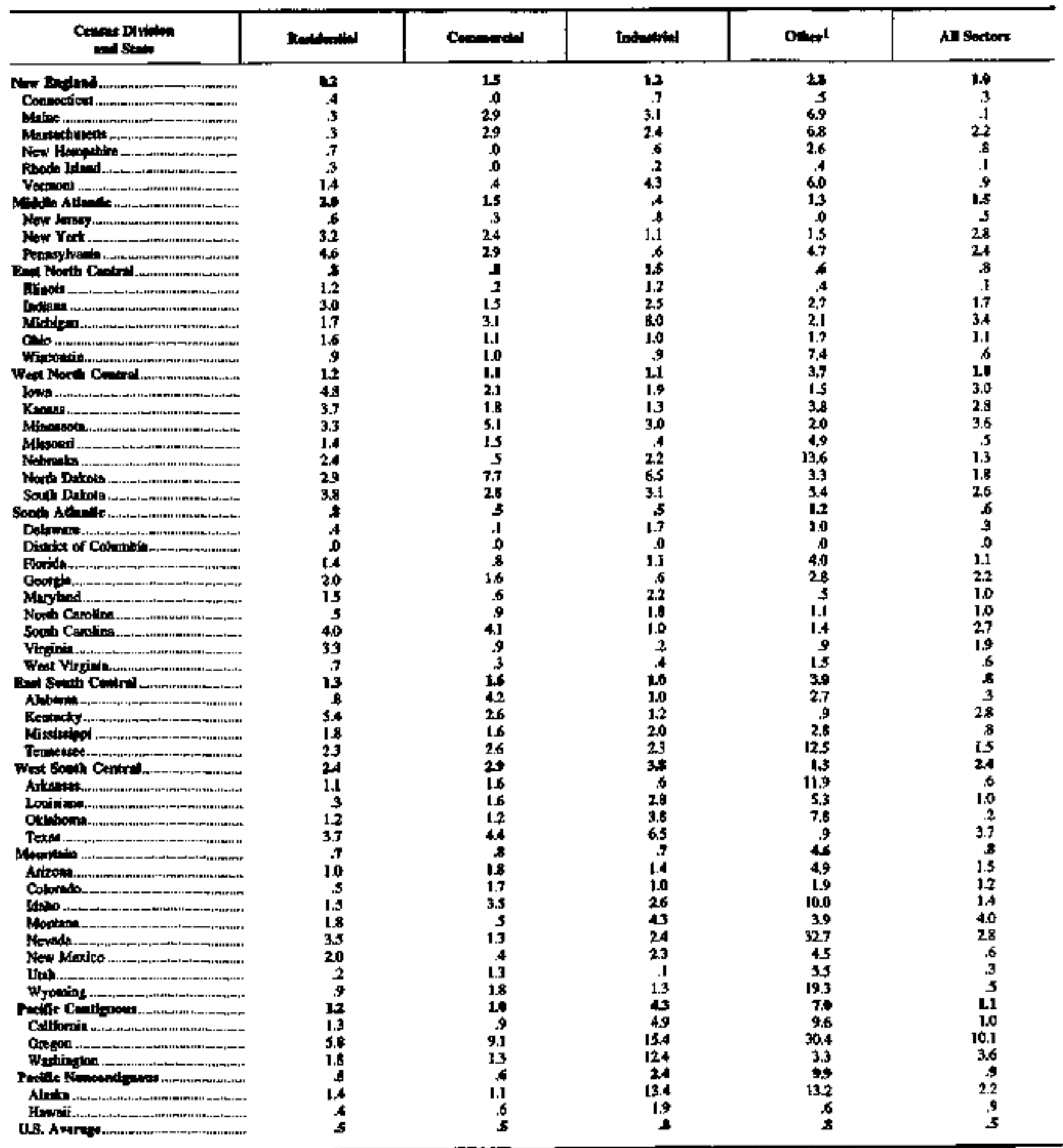

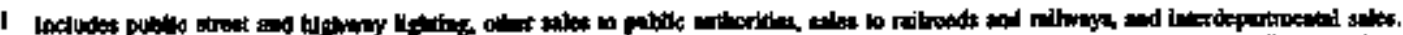

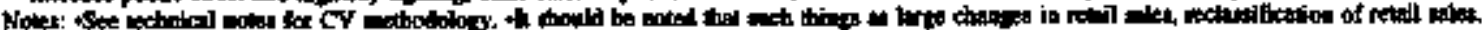

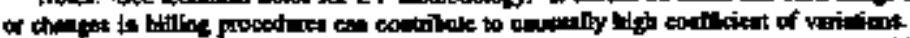

Soanes: Bnargy If 
Table 51. Estimated Revenue from U.S. Blectric Uthity Retail Sgles to Ulin?ote Consumers by Sector, Censes Division, and State, Year-to-Date 1997 and 1996 (Million Dollars)

\begin{tabular}{|c|c|c|c|c|c|c|c|c|c|c|}
\hline \multirow{2}{*}{ 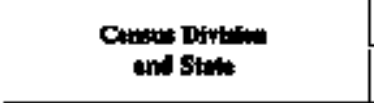 } & \multicolumn{2}{|c|}{ Redilendn } & \multicolumn{2}{|c|}{ Cocmerdal } & \multicolumn{2}{|c|}{ Imiduirle] } & \multicolumn{2}{|c|}{ obarl } & \multicolumn{2}{|c|}{ All Snctort } \\
\hline & $15 \%$ & $15 \%$ & 154 & toss & 19ק & I56 & 1507 & Las & 1997 & Les \\
\hline 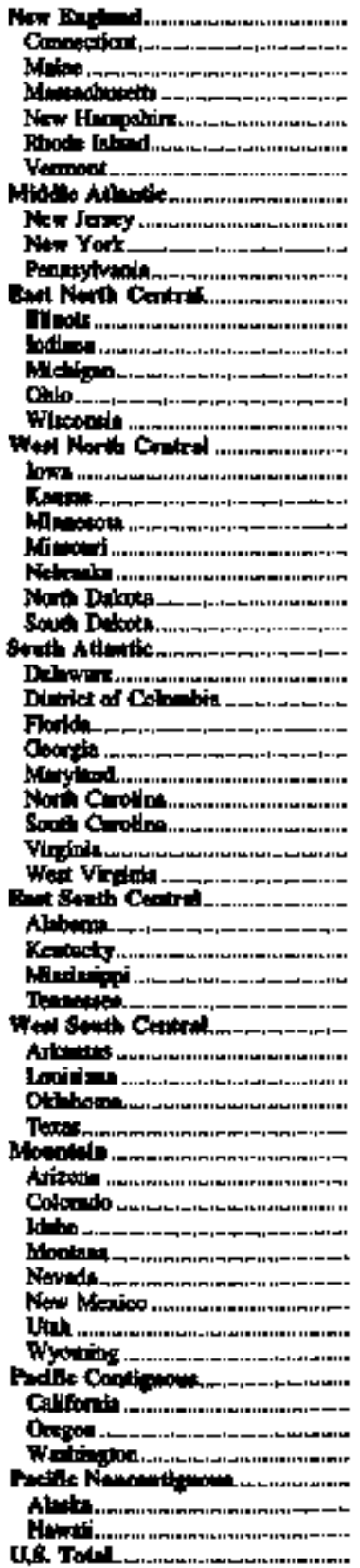 & 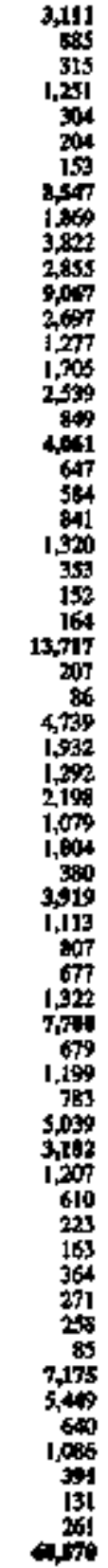 & 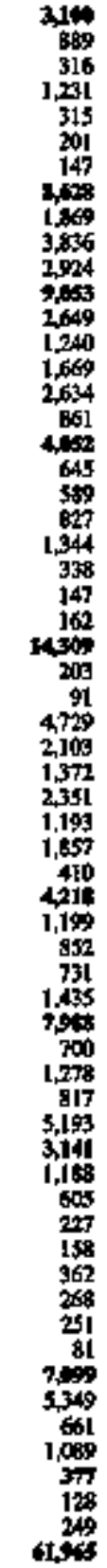 & 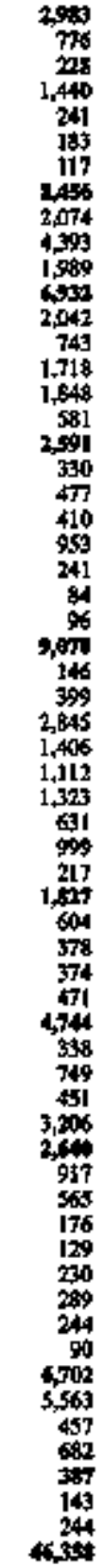 & 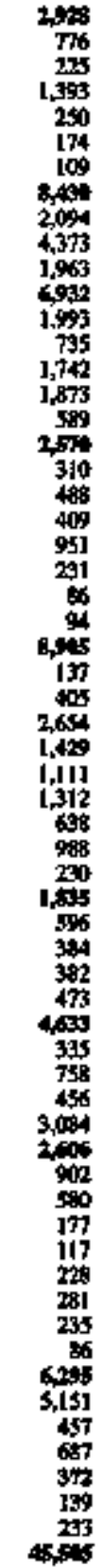 & 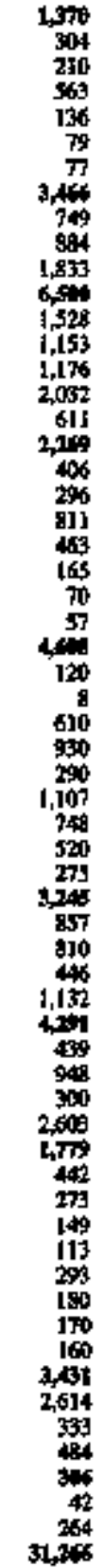 & 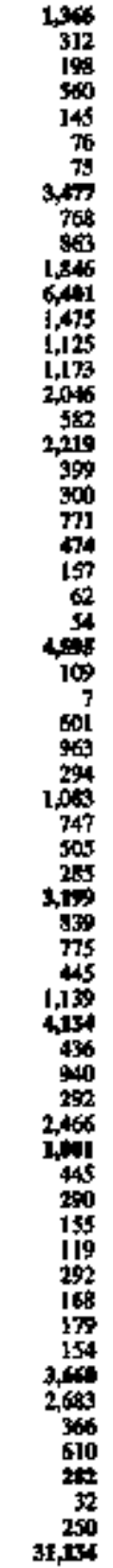 & 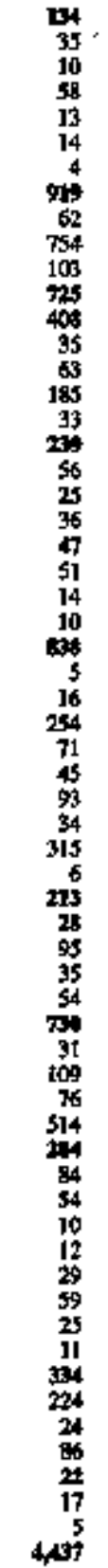 & 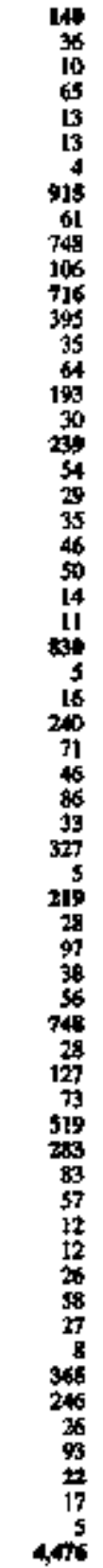 & 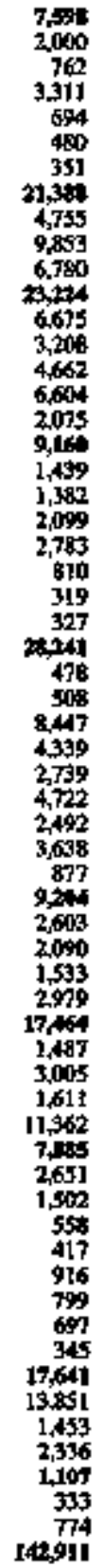 & 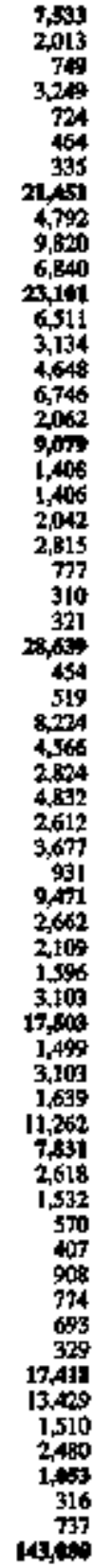 \\
\hline
\end{tabular}

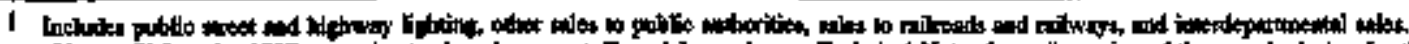

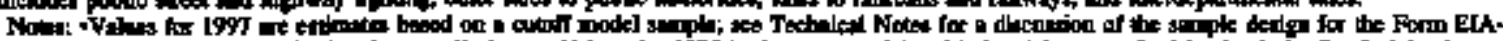

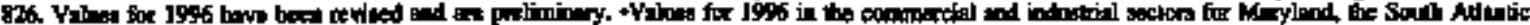

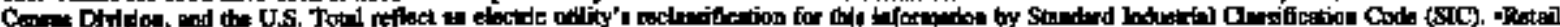

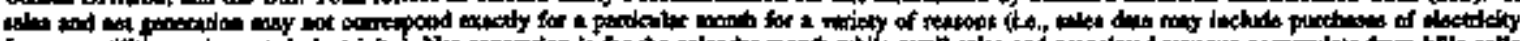

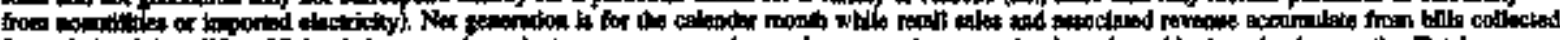

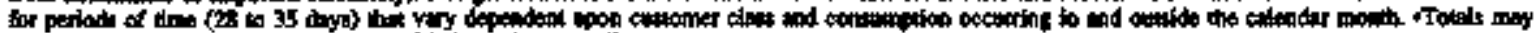

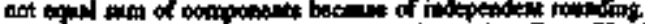

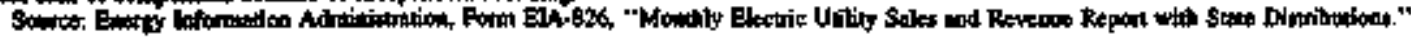


Table 52. US. Flectric Utilty Average Revemue per Kilowatthour by Sector, 1987 Through Augu; 1997 (Cents)

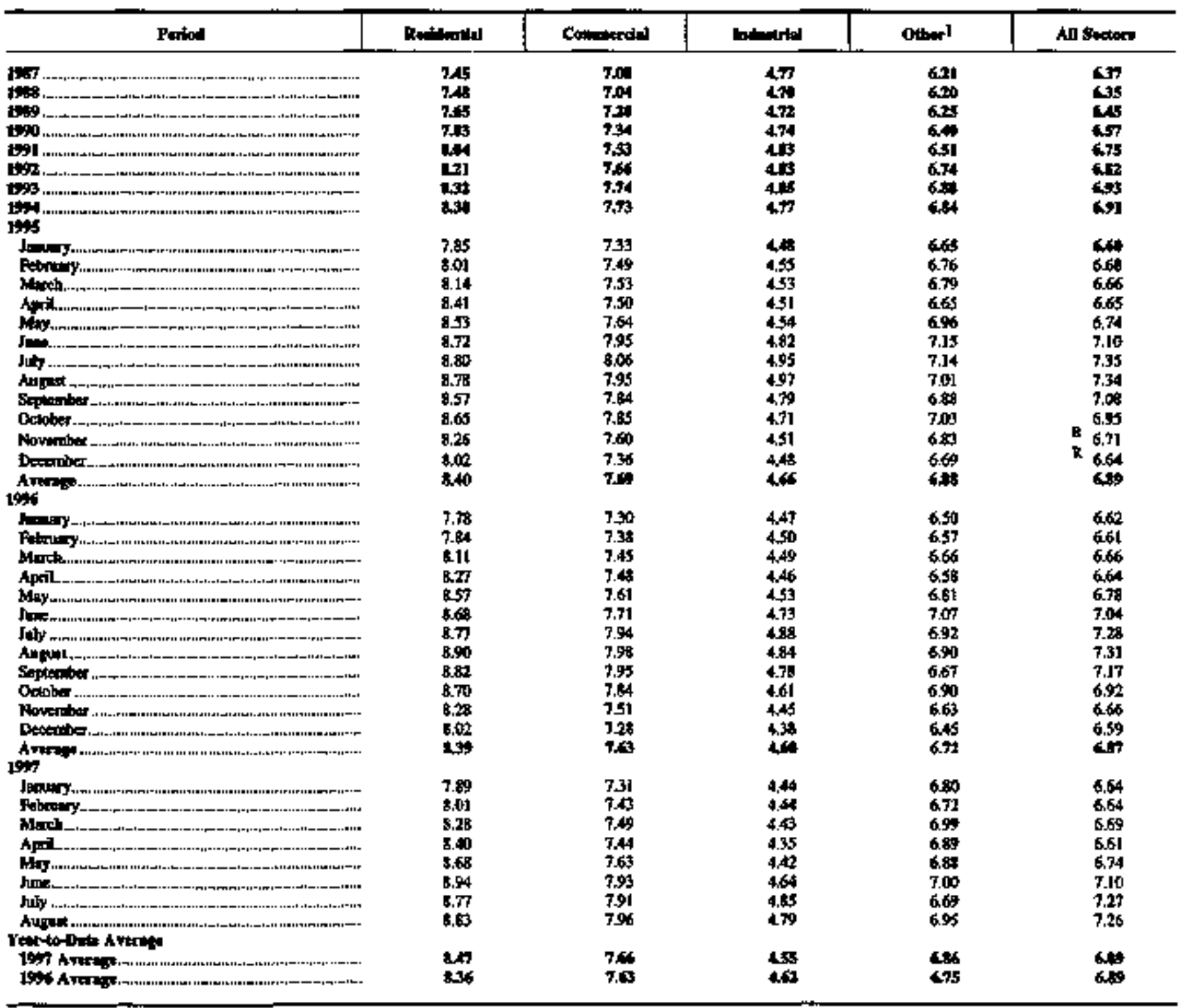

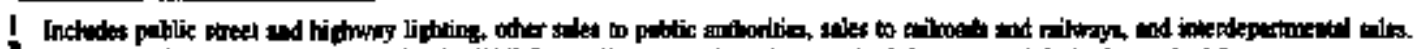

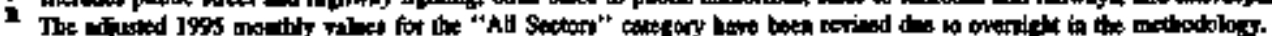

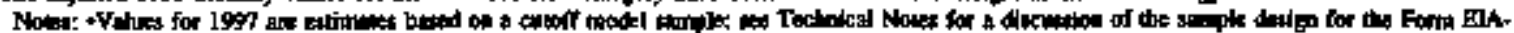

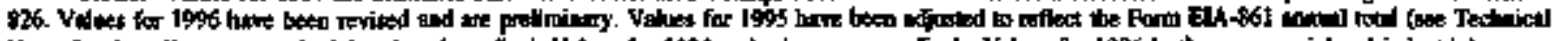

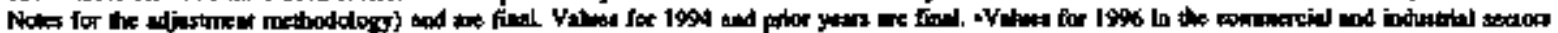

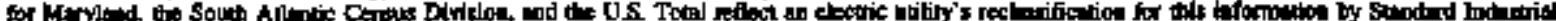

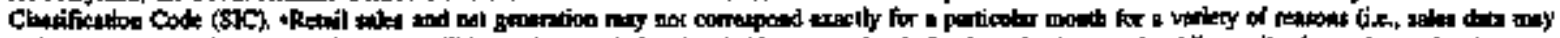

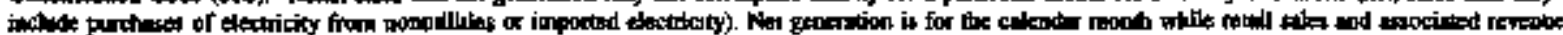

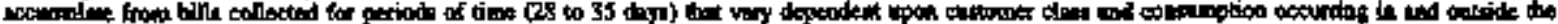

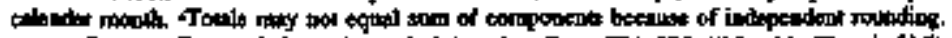

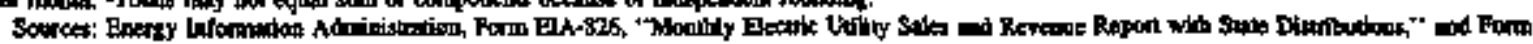

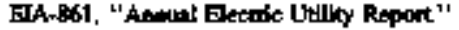


Toble 53. Estimated U.S. Mectric Utilly Avereme Revenue per KHowatthour to Ulimate Consunaers by Sector, Censas Division, and State, Angset 1997 and 1996 (Cents)

\begin{tabular}{|c|c|c|c|c|c|c|c|c|c|c|}
\hline \multirow{2}{*}{ 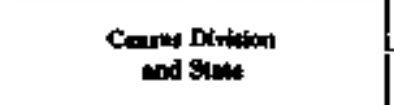 } & \multicolumn{2}{|c|}{ Enculdentiol } & \multicolumn{2}{|c|}{ Connotrile } & \multicolumn{2}{|c|}{ 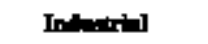 } & \multicolumn{2}{|c|}{ Ouher1 } & \multicolumn{2}{|c|}{ Al Sextor: } \\
\hline & $139 t$ & tos & 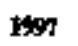 & LN4 & 19 & $19 \%$ & $1 \%$ & $15 * 1$ & $1 \% \%$ & Lat \\
\hline 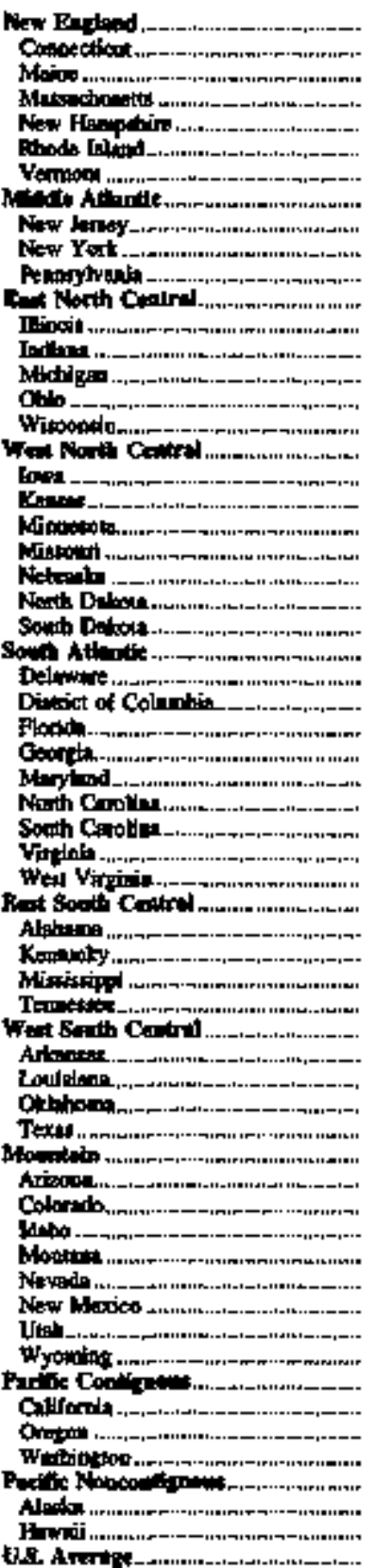 & 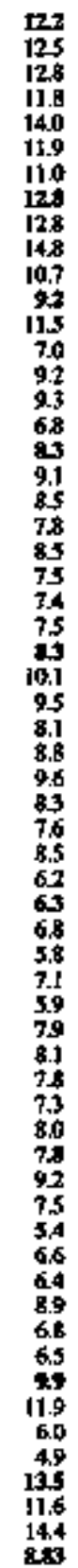 & 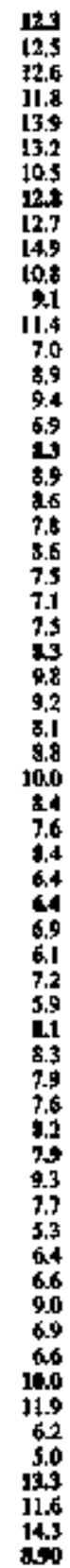 & 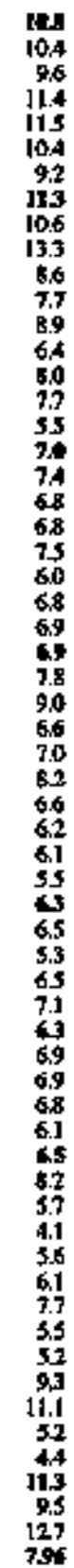 & 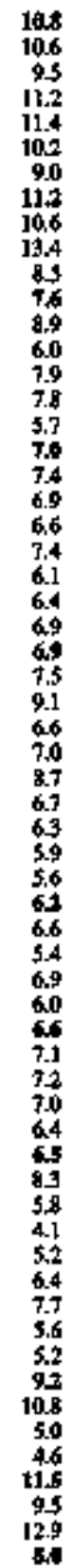 & 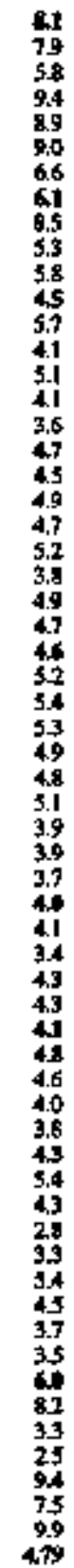 & 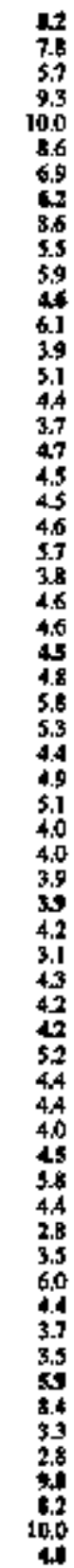 & 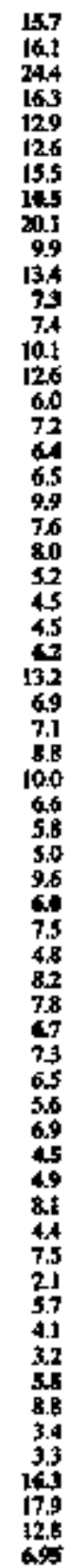 & 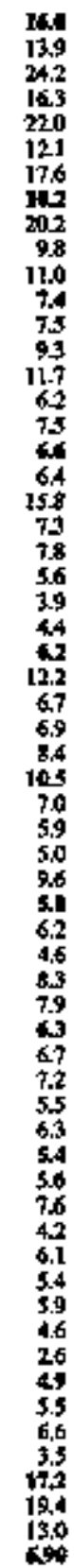 & 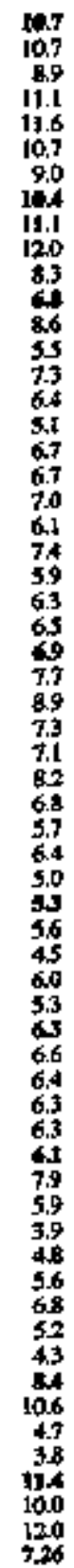 & 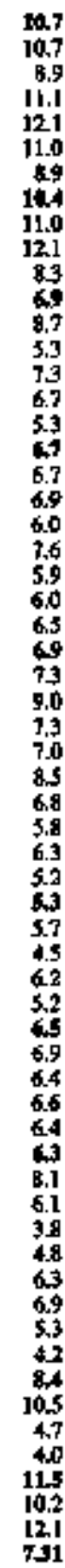 \\
\hline
\end{tabular}

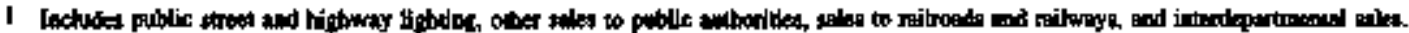

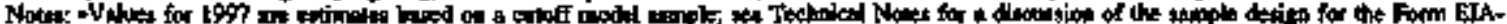

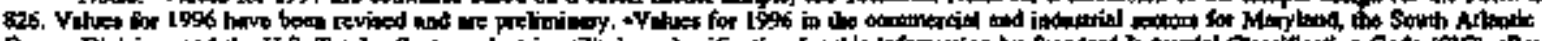

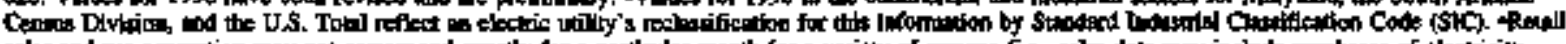

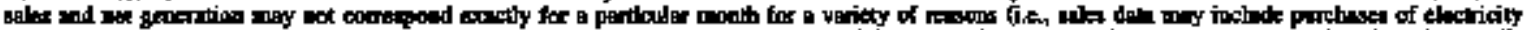

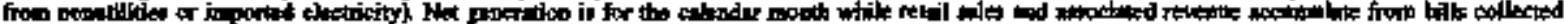

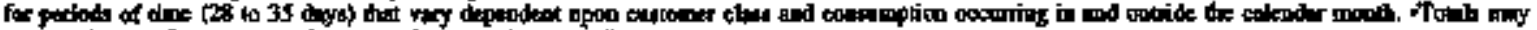

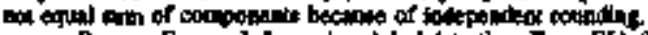

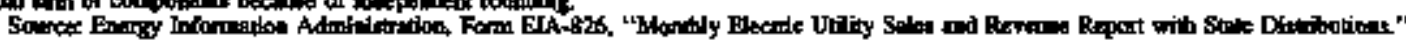


Table 34. Estimated Coeflleients of Variation tor US. Elextric Utility Average Revenue per Kilowatthoar to Unimate Consumers by Sector, Cenequs Division, and State, Aughst 1997 (Percent)

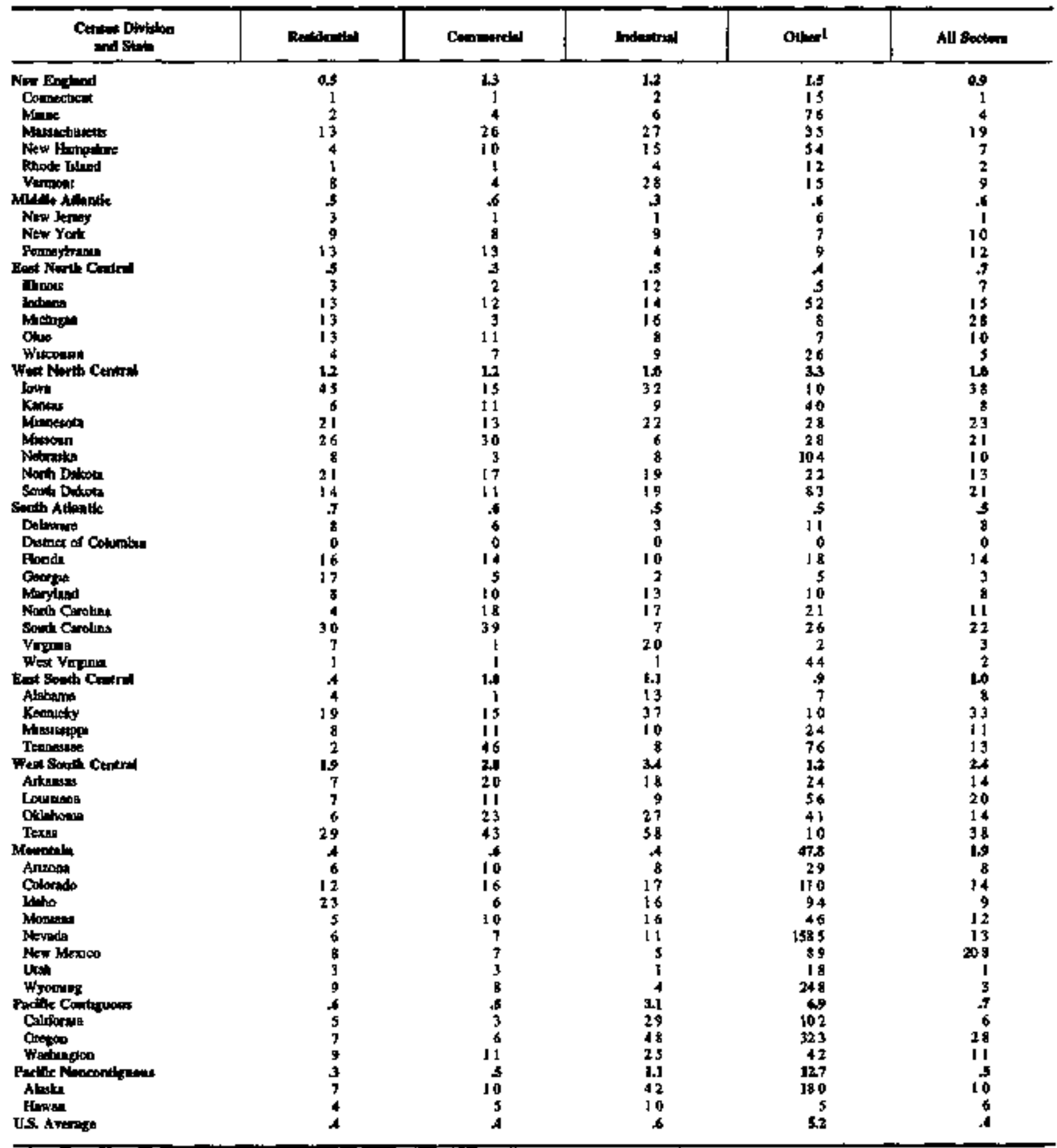

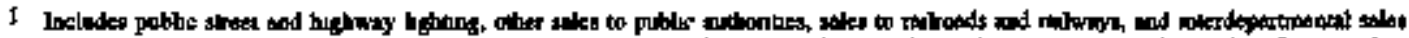

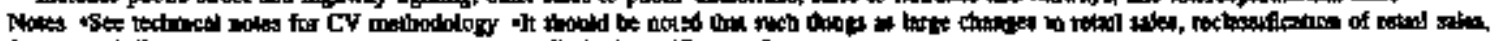

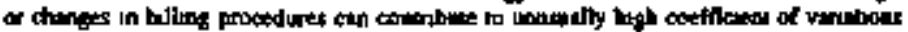

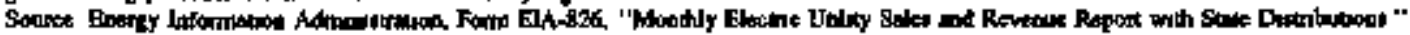




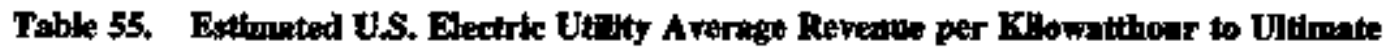
Consumers by Sector Census Divition, and State, Yearto-Date 1997 and 1996 (Cents)

\begin{tabular}{|c|c|c|c|c|c|c|c|c|c|c|}
\hline \multirow{2}{*}{ 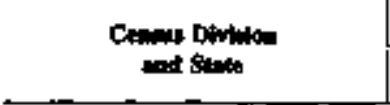 } & \multicolumn{2}{|c|}{ 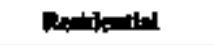 } & \multicolumn{2}{|c|}{ الלdי... } & \multicolumn{2}{|c|}{ 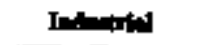 } & \multicolumn{2}{|c|}{ Oatarl } & \multicolumn{2}{|c|}{ Al sactart } \\
\hline & 16 & $1 \%$ & 197 & 1) & $\ln$ & 19N & $1 \geqslant 7$ & 19x & שת & bes \\
\hline 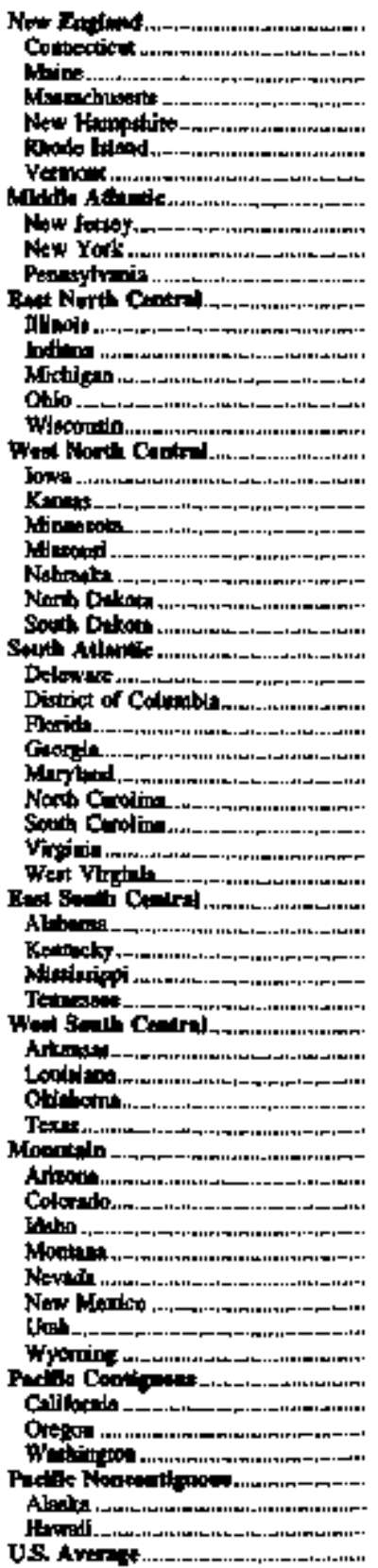 & $\begin{array}{r}12.0 \\
12.1 \\
12.7 \\
11.4 \\
13.5 \\
12.2 \\
11.7 \\
12 . \\
12.2 \\
14.2 \\
9.9 \\
10.4 \\
7.1 \\
8.8 \\
8.6 \\
6.9 \\
7.4 \\
7.2 \\
7.7 \\
7.4 \\
7.3 \\
6.4 \\
6.3 \\
7.1 \\
1.4\end{array}$ & 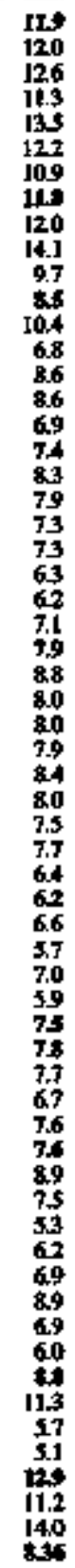 & 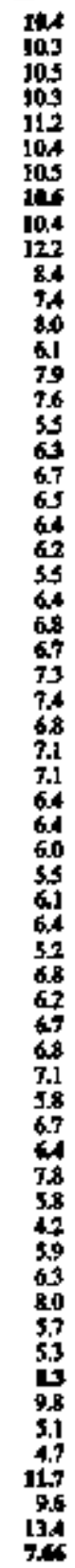 & 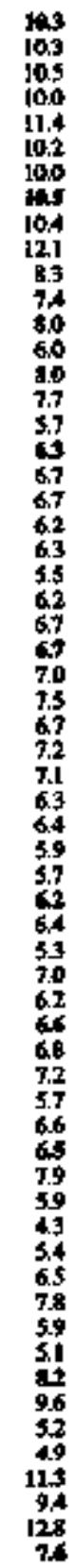 & 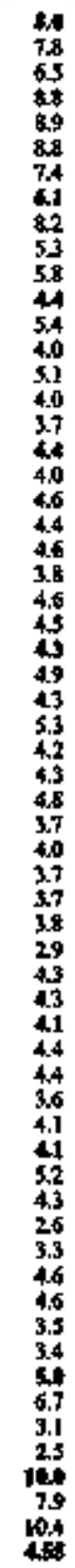 & 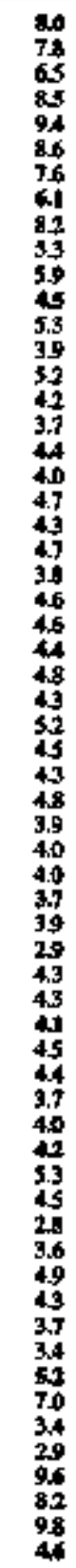 & 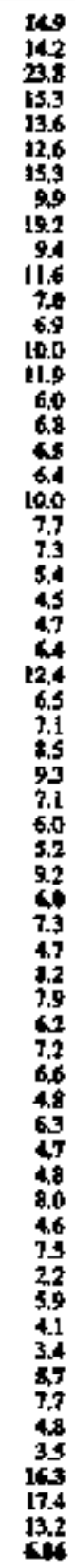 & 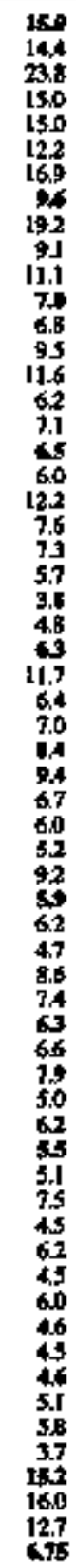 & 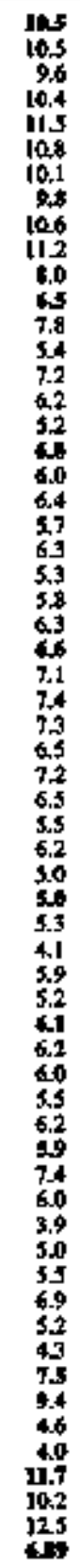 & 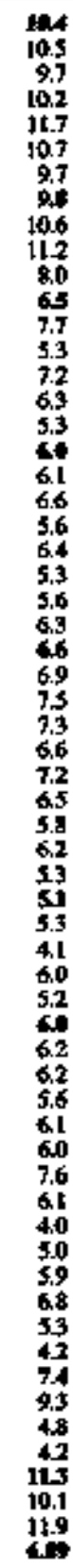 \\
\hline
\end{tabular}

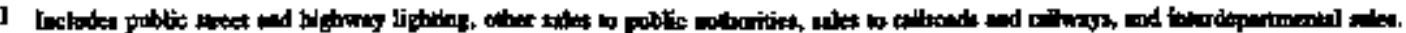

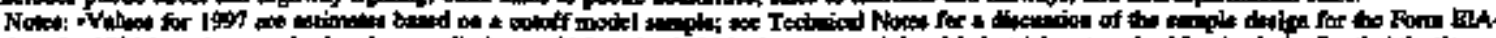

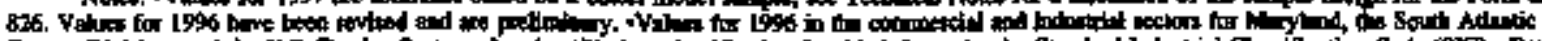

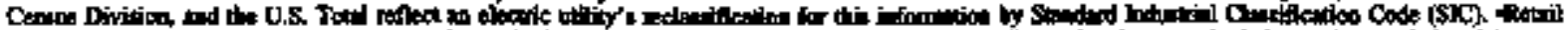

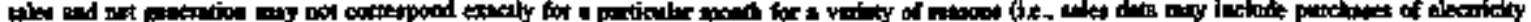

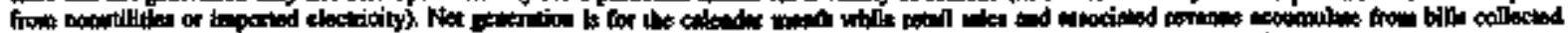

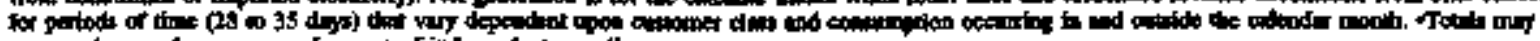

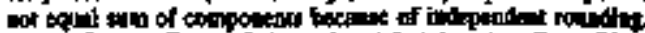

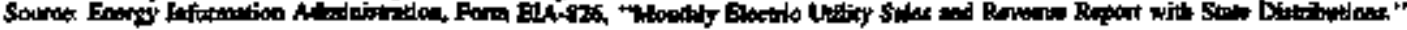





\section{Monthly Plant Aggregates: U.S. Electric Utility Net Generation, Fuel Consumption, and Fuel Stocks}

Table 56. U.S. Elextric Udlity Net Generation, Foel Consumption, and Fuel Stocks by Company and Pant, July 199

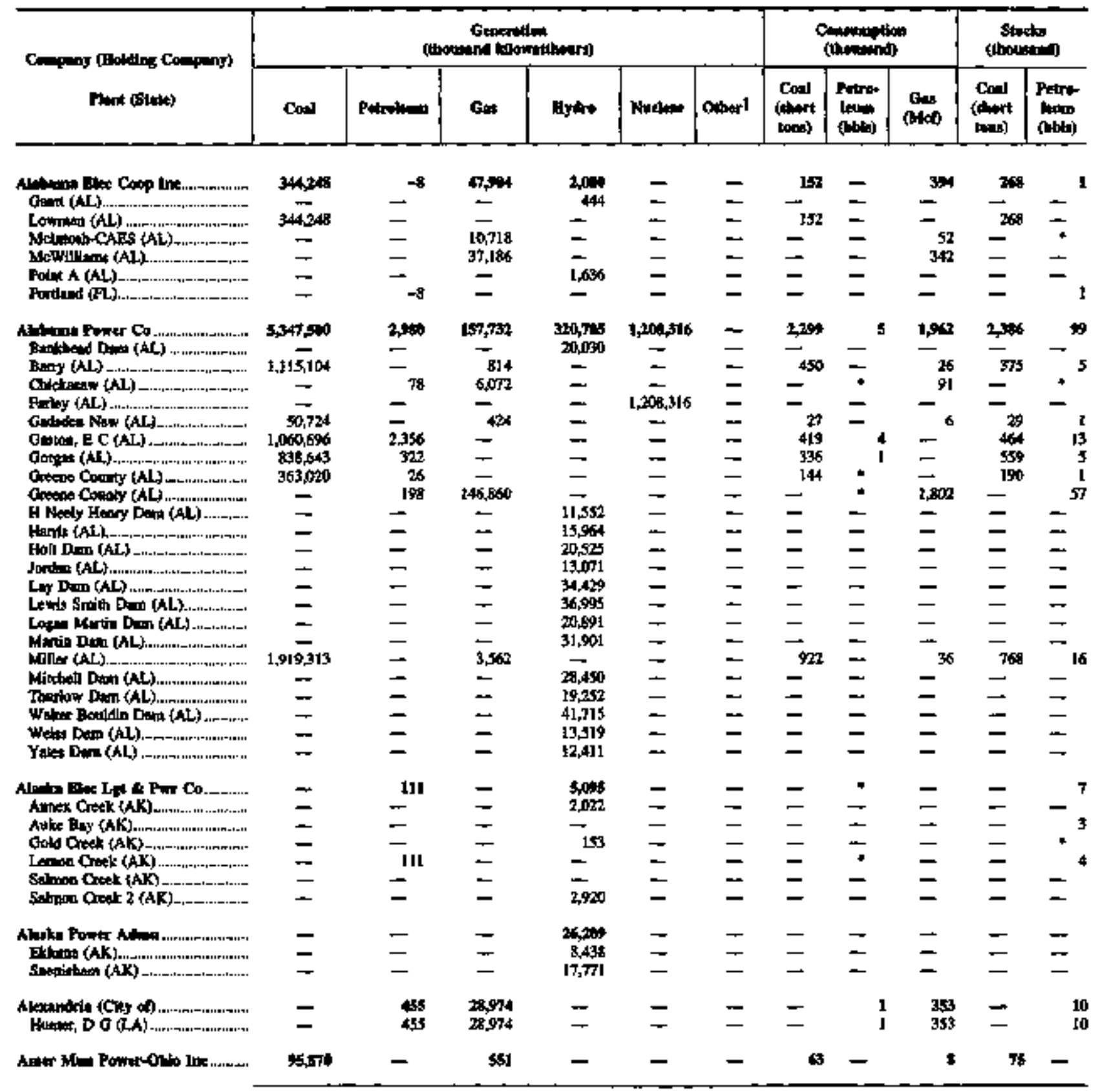

Soc footnowet at eod of toble 
Tohle 56. U.S. Electric Utility Net Generation, Foed Consamption, and Fuel Stocks by Company and Plant, July 19s' (Contimued)

\begin{tabular}{|c|c|c|c|c|c|c|c|c|c|c|c|}
\hline \multirow{2}{*}{ 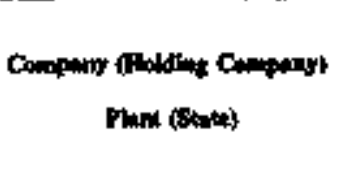 } & \multicolumn{6}{|c|}{ 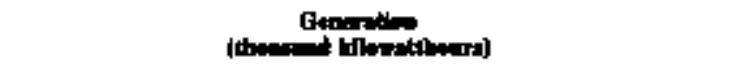 } & \multicolumn{3}{|c|}{ Cormanition } & \multicolumn{2}{|c|}{ (Atodistist) } \\
\hline & Cent & 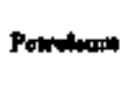 & Cen & Hybers & Nowenter & athed & 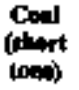 & Peint & Genten & 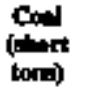 & Pitas \\
\hline
\end{tabular}

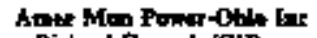
Richrid Gormith (OH)...

Anes (CXk ob

Anises (L).

Ano Ot (1)

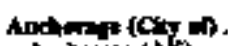

Anchotit: (Ak

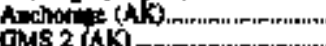

Appolectuten Poivt Cor.

Atonot Joth $B$ (WY)

Buck (VA)

Bytlesby 2 (VA).

Cinytrin (V)

Cin River (VA)

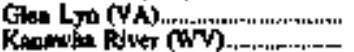

Lesritle (VA)

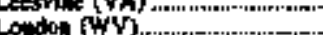

Merted inv?

Mountaines (WV)

Mowntginter (WV)....................."

Reusten (VA)................................

Sitin Monotid (VA)

Whated (WW)

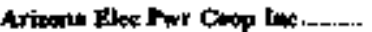
Aputit Station (AZ) _..............

Arbun Punle Servlet Ca .......... Collde (AZ).

Grdils (AZ)

Firvietw (A2).

Pour Conos (NM)

trims ( $\Omega$ T

Datho $(A D)$.

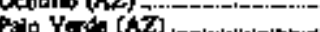

Pin (AT)

Saguro ( $/ 2)$

Yuact (AZ)

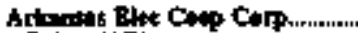
Btisy (AR)

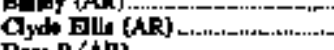

Dam 9 (AR)

se Cign (AE)

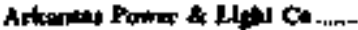

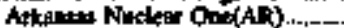

Bhythevild \{AR\}............-..-.

Capredta (AR).

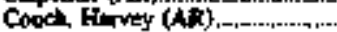

Iodopendence (AR)

L Conterlo (AR)

Lyod, Cecil (AR)

Menteraile (AB)

Mooks Ham (A)

Redined (AR).

Whatiof (AR)

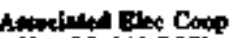

Now Modid QROY).

Thot 15 FEl (MO

Isiosillte (MAO)

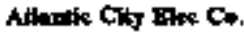

See footanoter at end of thbt

\begin{tabular}{|c|c|c|c|c|c|c|c|c|c|c|}
\hline 95,870 & - & 551 & - & - & - & 69 & - & 8 & 75 & - \\
\hline $\begin{array}{l}\text { 2,344 } \\
-1,304 \\
-\end{array}$ & $\begin{array}{l}301 \\
301\end{array}$ & $\bar{z}$ & $=$ & $\vec{z}$ & $=$ & $-^{\frac{21}{28}}$ & , 1 & $\bar{z}$ & $\begin{array}{r}19 \\
-\end{array}$ & $\begin{array}{l}4 \\
\mathbf{1}\end{array}$ \\
\hline$=$ & -6 & $\begin{array}{l}6,595 \\
2,257 \\
60,638\end{array}$ & $\bar{z}$ & $\bar{z}$ & $=$ & $=$ & $\dot{*}$ & 48 & $=$ & $\begin{array}{l}37 \\
3 \\
34\end{array}$ \\
\hline 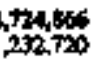 & $\begin{array}{l}15,011 \\
12,564\end{array}$ & $\overline{-}$ & $39,16 \%$ & $\bar{z}$ & $=$ & 1,46 & $\frac{4}{21}$ & $=$ & $\begin{array}{l}1,607 \\
1,104\end{array}$ & is \\
\hline $\bar{z}$ & $=$ & $\Xi$ & $\begin{array}{l}29300 \\
3,674\end{array}$ & $=$ & $=$ & $\bar{z}$ & $=$ & $=$ & $=$ & $=$ \\
\hline- & - & - & 10,553 & - & - & - & - & $=$ & $\bar{z}$ & $\overline{-}$ \\
\hline $\begin{array}{l}423,379 \\
146,510\end{array}$ & $m$ & $=$ & $=$ & $=$ & - & 199 & * & - & 158 & 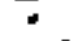 \\
\hline $\begin{array}{l}146,510 \\
206,64\}\end{array}$ & 99: & $\bar{z}$ & $\bar{z}$ & $\bar{z}$ & $=$ & $\begin{array}{l}63 \\
84\end{array}$ & $*^{2}$ & $=$ & $\begin{array}{l}68 \\
65\end{array}$ & 6 \\
\hline- & - & - & 3,041 & - & - & - & - & - & - & - \\
\hline$=$ & $=$ & $\overrightarrow{-}$ & 4357 & $=$ & $=$ & $=$ & $=$ & $=$ & $=$ & - \\
\hline 713,610 & 1,254 & - & $=$ & $=$ & $=$ & 278 & - & 二 & 20 & -11 \\
\hline - & - & - & 3 & - & - & - & 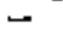 & - & - & 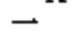 \\
\hline- & - & - & 1,763 & - & - & - & - & - & - & - \\
\hline $\bar{z}$ & $\bar{z}$ & $\overline{-}$ & 6,732 & $\bar{z}$ & $=$ & 단 & $=$ & $=$ & $=$ & $\bar{z}$ \\
\hline zasian & - & 1250 & - & - & - & 125 & $\rightarrow$ & $13 s$ & 132 & - \\
\hline 200,086 & 一 & 12,683 & - & $\leftarrow$ & $\rightarrow$ & 125 & - & I35 & 332 & - \\
\hline , & 60 & ISt,est & 2:460 & $2,74,927$ & - & 905 & 1 & 2,184 & $\mathbf{3 1 3}$ & 141 \\
\hline 550,353 & 495 & 394 & 1 & $\vec{z}$ & $\bar{z}$ & $\overline{3}_{34}$ & -1 & - & -236 & - \\
\hline 1059.609 & 18 & - & - & - & - & & $=$ & & & 6 \\
\hline $1,053,669$ & $=$ & 9.486 & $\overline{103}$ & - & - & 612 & - & 101 & 7 & - \\
\hline$=$ & $=$ & 5,218 & - & $\mp$ & $=$ & 二 & $\overrightarrow{\vec{\prime}}$ & -699 & Z & - \\
\hline- & $\rightarrow$ & - & - & $2,763,927$ & $\rightarrow$ & - & - & - & $\overline{-}$ & -30 \\
\hline 一 & $\pi$ & 7,486 & - & - & - & - & - & 754 & - & $\$ 2$ \\
\hline$\underline{-}$ & $\leftarrow$ & 28,667 & - & - & - & $\rightarrow$ & - & 349 & - & 34 \\
\hline & 20 & & 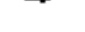 & 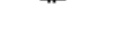 & 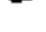 & - & & & - & 29 \\
\hline$=$ & $\mathbf{5 0 0}$ & ing & 3,120 & - & - & 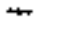 & 1 & 1,165 & - & 73 \\
\hline $\bar{z}$ & - & 33,862 & $\bar{x}$ & - & - & - & $\rightarrow$ & 397 & - & 28 \\
\hline- & 二 & $\overrightarrow{-}$ & 等 & $\vec{z}$ & $\vec{z}$ & $=$ & $=$ & $\bar{z}$ & $=$ & $=$ \\
\hline - & - & 20,592 & - & - & $\rightarrow$ & - & 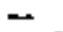 & 304 & $\longrightarrow$ & 15 \\
\hline- & Sold & 39,749 & - & $\rightarrow$ & $\rightarrow$ & - & ] & 425 & - & 29 \\
\hline $1,14,425$ & 6,011 & 871,674 & 12,413 & 1,100605 & - & 1,24 & 16 & 6,327 & 764 & 154 \\
\hline$\vec{z}$ & $\overline{3}$ & - & - & $1,209,645$ & $=$ & 二 & - & 二 & 二 & ${ }^{n} n$ \\
\hline$\rightarrow$ & - & $=$ & 8,108 & $=$ & 二 & $=$ & - & $=$ & $=$ & -4 \\
\hline$\overline{-}$ & - & 0,363 & - & - & - & - & - & 476 & - & - \\
\hline $1,346,930$ & 1,210 & -7 & - & - & - & 625 & 2 & - & $\mathbf{3 3 8}$ & 4 \\
\hline$=$ & 二 & - & $=$ & $\bar{z}$ & $=$ & $=$ & $=$ & 2,322 & $=$ & $=$ \\
\hline - & 229 & - & - & - & - & - & I & $\rightarrow$ & - & l \\
\hline$=$ & - & - & $\vec{a}$ & $\rightarrow$ & - & - & - & - & 一 & - \\
\hline E & $=$ & 294561 & מה, & $\bar{z}$ & $\bar{z}$ & $=$ & $=$ & 5,504 & 二 & - 95 \\
\hline 995379s & 1,609 & - & - & - & - & $60 \%$ & 3 & - & 416 & 22 \\
\hline $1,5065 \%$ & 1,73 & - & - & - & - & 300 & 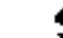 & - & 64 & 12 \\
\hline $\begin{array}{r}750,914 \\
751,956\end{array}$ & 312 & $=$ & $=$ & $\overline{-}$ & 二 & $\begin{array}{l}459 \\
4 \$ 1\end{array}$ & 1 & $\bar{z}$ & $\begin{array}{l}341 \\
353\end{array}$ & 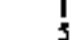 \\
\hline- & 기이 & ت & - & - & - & - & 2 & - & - & 6 \\
\hline $176,3 \geqslant 0$ & $21, \mathrm{~ns}$ & $39,4 \pi$ & - & - & - & 79 & 4 & SM & 100 & 354 \\
\hline
\end{tabular}


Trbłe 56. U.S. Bloctric Utility Net Geanstion, Fuel Cossomption, and Fuel Stoeks by Conpany and Fint, Jaty 1997 (Continued)

\begin{tabular}{|c|c|c|c|c|c|c|c|c|c|c|c|}
\hline \multirow{2}{*}{ 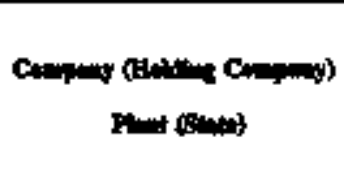 } & \multicolumn{6}{|c|}{ 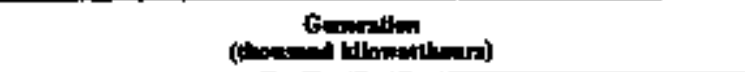 } & \multicolumn{3}{|c|}{ (thernations) } & \multicolumn{2}{|c|}{ 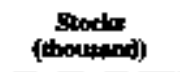 } \\
\hline & Cand & Retralka: & daty & Hyth & Mandenen & ontert & coll & 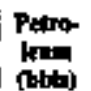 & Gist) & Cont & Petro. \\
\hline
\end{tabular}

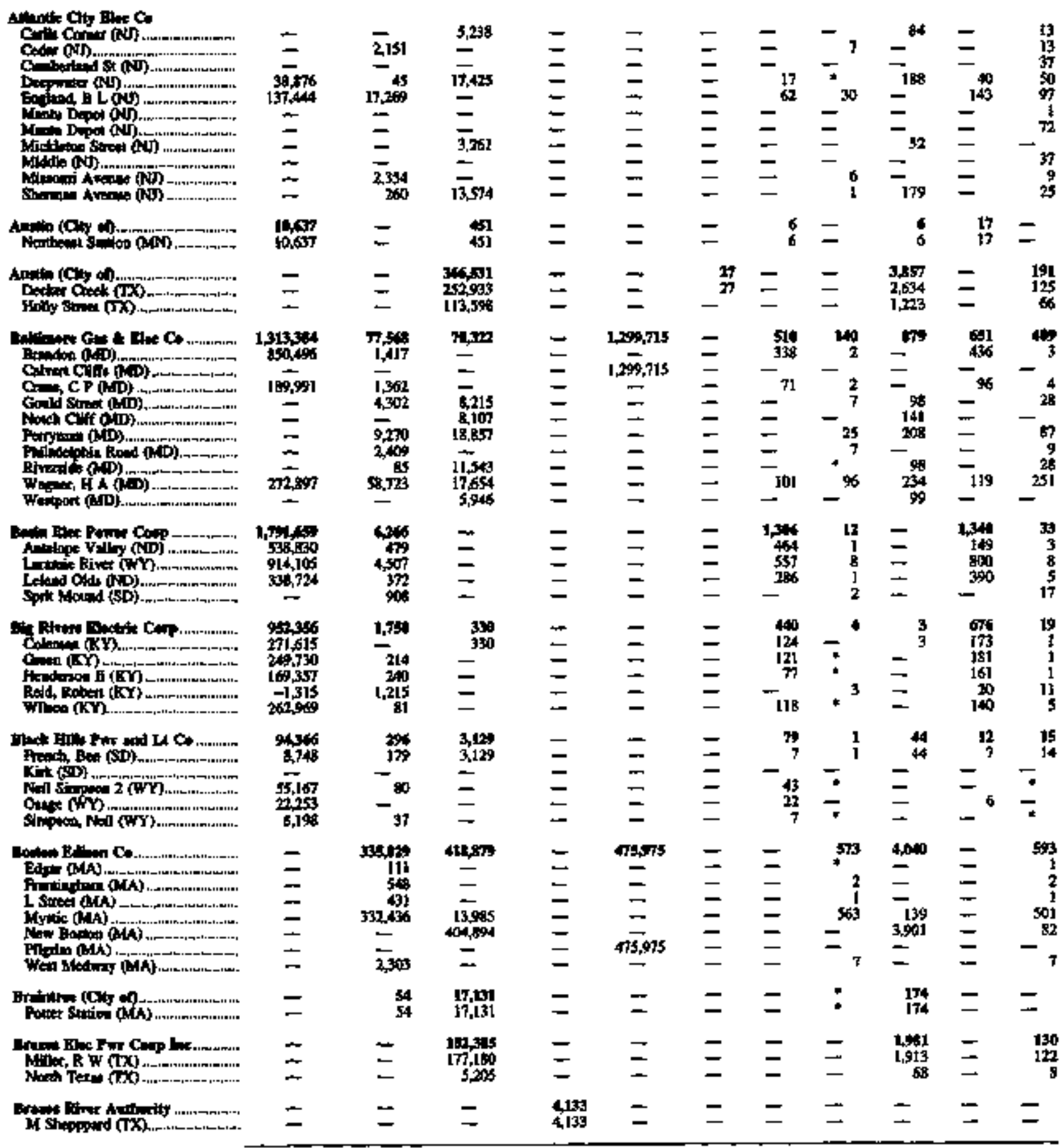

got foptoots a ad of bible 
Tabile S6. US. Electric Utaty Net Generation, Fuel Consumptiow, and Fuel Stocks by Conpany and Plant, Juky 1997 (Contioned)

\begin{tabular}{|c|c|c|c|c|c|c|c|c|c|c|c|}
\hline \multirow{2}{*}{ 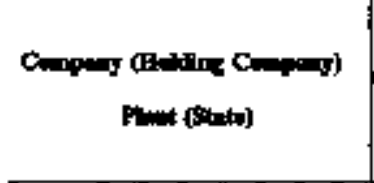 } & \multicolumn{6}{|c|}{ 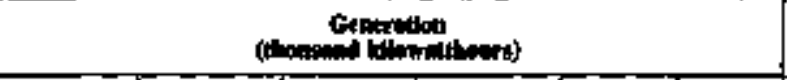 } & \multicolumn{3}{|c|}{ Conantion } & \multicolumn{2}{|c|}{ 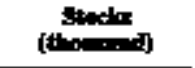 } \\
\hline & $C=1$ & صatrits & Gax & Egdro & Madeor & [ & 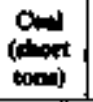 & $\begin{array}{l}\text { Putro } \\
\text { inistas }\end{array}$ & $\stackrel{(N)}{(N)}$ & 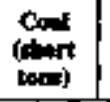 & (bon \\
\hline 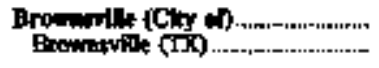 & $\overline{-}$ & $=$ & 45,463 & $\bar{z}$ & $=$ & $\bar{z}$ & $=$ & $\bar{z}$ & $\begin{array}{l}468 \\
488\end{array}$ & $=$ & 15 \\
\hline 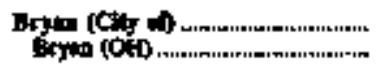 & $\bar{z}$ & $\bar{z}$ & 1,162 & $\bar{z}$ & $\bar{z}$ & $\overline{-}$ & $\overline{-}$ & $\overline{-}$ & $\begin{array}{l}\text { i1 } \\
\text { il }\end{array}$ & $\overline{-}$ & $\begin{array}{l}6 \\
6\end{array}$ \\
\hline 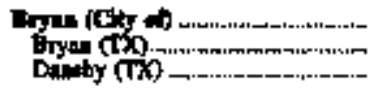 & $=$ & $=$ & $\begin{array}{l}61,411 \\
1,174 \\
47,337\end{array}$ & $\bar{z}$ & $\bar{z}$ & $\vec{z}$ & $\bar{z}$ & $=$ & $\begin{array}{l}731 \\
178\end{array}$ & $\bar{z}$ & $\begin{array}{l}\mathbf{5 6} \\
\mathbf{3 2} \\
24\end{array}$ \\
\hline 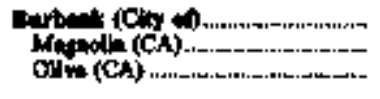 & $\bar{z}$ & $\bar{z}$ & $\begin{array}{r}19,9,00 \\
-32 \\
19,992\end{array}$ & $\bar{z}$ & $\bar{z}$ & $\bar{z}$ & $\bar{z}$ & $\bar{z}$ & $\begin{array}{l}754 \\
258\end{array}$ & $\bar{z}$ & $\begin{array}{r}23 \\
21 \\
2\end{array}$ \\
\hline 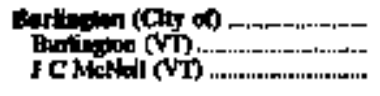 & $\bar{\Xi}$ & $\begin{array}{c}67 \\
-\end{array}$ & $\vec{z}$ & $\bar{z}$ & $\bar{z}$ & $\frac{11,976}{11,976}$ & $\simeq$ & $\begin{array}{r}2 \\
+2\end{array}$ & -4 & $=$ & $\begin{array}{l}\mathbf{4} \\
\mathbf{3}\end{array}$ \\
\hline 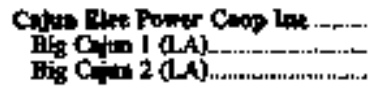 & $\frac{246,063}{24,0000}$ & $\frac{2,855}{2+855}$ & $\begin{array}{l}89,451 \\
83,481 \\
-\end{array}$ & $\bar{\Xi}$ & $=$ & $=$ & -50 & -5 & $\stackrel{902}{902}$ & $\frac{1,43}{t, 408}$ & $\begin{array}{l}24 \\
12 \\
12\end{array}$ \\
\hline 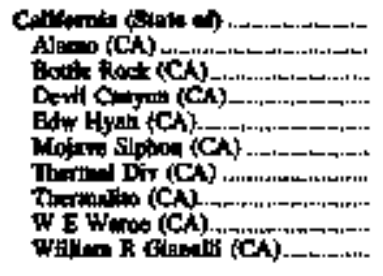 & $\begin{array}{l}= \\
= \\
= \\
= \\
=\end{array}$ & $\begin{array}{l}= \\
= \\
= \\
= \\
=\end{array}$ & $\begin{array}{l}= \\
= \\
= \\
= \\
=\end{array}$ & $\begin{array}{r}46,512 \\
1,937 \\
\frac{16,446}{3+8,290} \\
6,929 \\
2010 \\
49,564 \\
1,168 \\
1,86\end{array}$ & $\begin{array}{l}= \\
= \\
= \\
\bar{z} \\
=\end{array}$ & $\begin{array}{l}= \\
=30 \\
= \\
= \\
= \\
=\end{array}$ & $\begin{array}{l}\bar{z} \\
\bar{z} \\
\bar{z} \\
\bar{z}\end{array}$ & $\begin{array}{l}= \\
= \\
= \\
= \\
=\end{array}$ & $\begin{array}{l}= \\
= \\
= \\
= \\
=\end{array}$ & $\begin{array}{l}= \\
\bar{z} \\
= \\
= \\
=\end{array}$ & $\begin{array}{l}= \\
= \\
= \\
= \\
=\end{array}$ \\
\hline 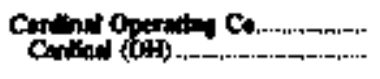 & $\begin{array}{l}\text { o11,999 } \\
\text { s11,919 }\end{array}$ & $\frac{2,416}{2,410}$ & 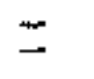 & $\underline{-}$ & $=$ & $\bar{z}$ & $\begin{array}{l}331 \\
331\end{array}$ & 4 & $\overline{-}$ & 494 & $\begin{array}{l}17 \\
17\end{array}$ \\
\hline 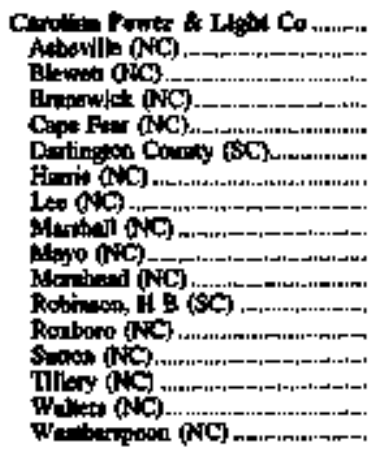 & 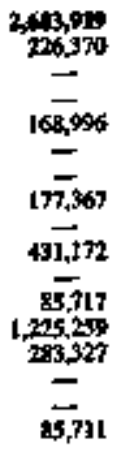 & 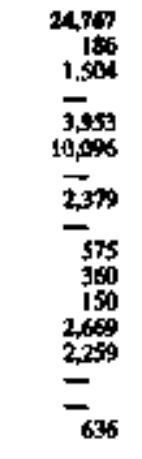 & $\begin{array}{l}30,925 \\
= \\
\bar{z} \\
= \\
= \\
= \\
= \\
= \\
\overline{1} \\
\overline{1}, 6 \pi\end{array}$ & $\begin{array}{l}50,43 \\
8.371 \\
= \\
= \\
= \\
2,401 \\
= \\
= \\
= \\
15,713 \\
=\end{array}$ & 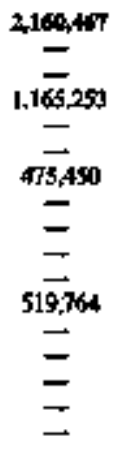 & $\begin{array}{l}\bar{z} \\
\bar{z} \\
\bar{z} \\
= \\
= \\
= \\
= \\
=\end{array}$ & $\begin{array}{l}1,101 \\
-^{92} \\
\overline{-}^{79} \\
{ }^{7} \\
{ }^{181} \\
{ }^{36} \\
{ }^{497}\end{array}$ & 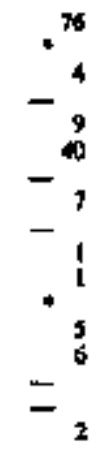 & $\begin{array}{l}{ }^{40} \\
= \\
= \\
= \\
= \\
= \\
= \\
= \\
= \\
\end{array}$ & 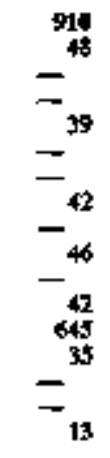 & $\begin{array}{r}147 \\
1 \\
-6 \\
8 \\
-\quad 8 \\
-8 \\
6 \\
1 \\
3 \\
9 \\
-9 \\
-9\end{array}$ \\
\hline 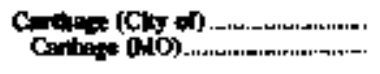 & 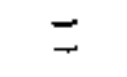 & $\begin{array}{l}28 \\
28\end{array}$ & $\begin{array}{l}256 \\
256\end{array}$ & $=$ & $\because$ & $\ddot{-}$ & $=$ & : & 3 & $\overline{-}$ & $\begin{array}{l}2 \\
2\end{array}$ \\
\hline 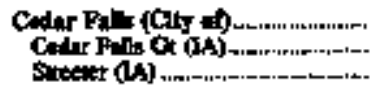 & $\frac{9400}{9,40}$ & $-{ }_{27}^{27}$ & $\begin{array}{r}107 \\
2076 \\
551\end{array}$ & $\bar{z}$ & $\bar{z}$ & $\bar{\Xi}$ & $-\mathbf{5}$ & $\stackrel{*}{*}$ & $\begin{array}{l}57 \\
36 \\
11\end{array}$ & ${ }_{-}^{12}$ & $-{ }^{2}$ \\
\hline 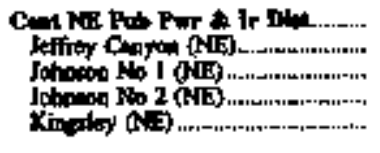 & $\begin{array}{l}= \\
= \\
=\end{array}$ & $\begin{array}{l}\bar{z} \\
\bar{z}\end{array}$ & $\begin{array}{l}= \\
=\end{array}$ & $\begin{array}{r}1,7,70 \\
11,741 \\
5,093 \\
5,962 \\
22,168\end{array}$ & $\begin{array}{l}\bar{z} \\
\bar{z}\end{array}$ & $\begin{array}{l}\bar{z} \\
\bar{z}\end{array}$ & $\begin{array}{l}\bar{z} \\
\bar{z}\end{array}$ & $\begin{array}{l}= \\
= \\
=\end{array}$ & $\begin{array}{l}= \\
=\end{array}$ & $\begin{array}{l}= \\
z\end{array}$ & $\begin{array}{l}z \\
z \\
=\end{array}$ \\
\hline Condred Dinc Pent Coop & $\begin{array}{l}\text { d1,71t: } \\
41,718\end{array}$ & $=$ & $\overline{-}$ & $=$ & $\overline{-}$ & $\overline{-}$ & 나 & $=$ & $\bar{z}$ & $\begin{array}{l}76 \\
26\end{array}$ & 5 \\
\hline
\end{tabular}

See footions an end of tolb. 
Table 56. US. Electole Udilty Net Generotion, Fued Cousumption, and Fnel Stoefos by Compang and Fiant, July 1997 (Continned)

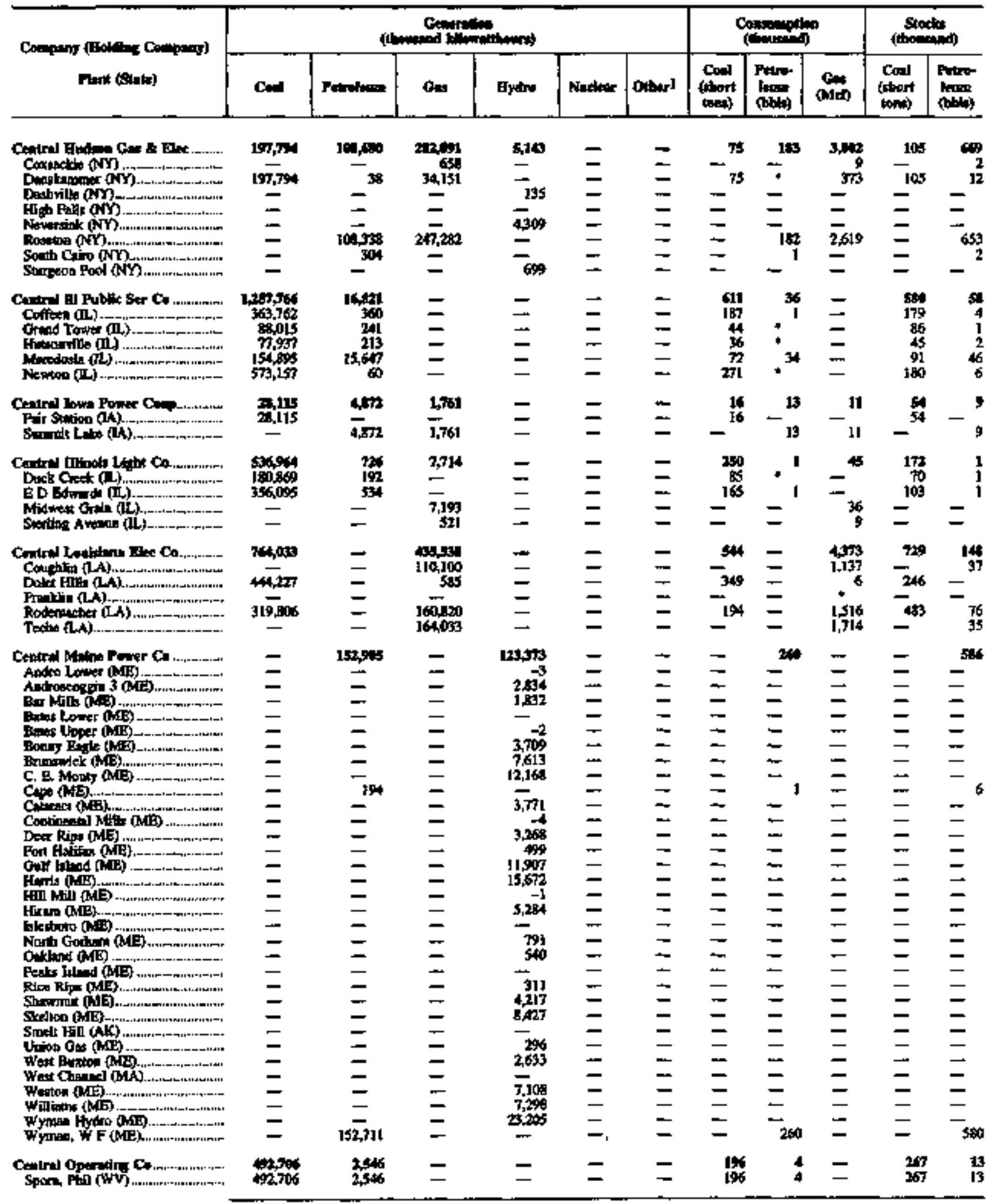

See fooknowed at end of loble. 
Table 56. U.S. Electrie Utility Nat Geoeratton, Foul Consumption, and Fuel Stocks by Company and Fant, July 1997 (Continoed)

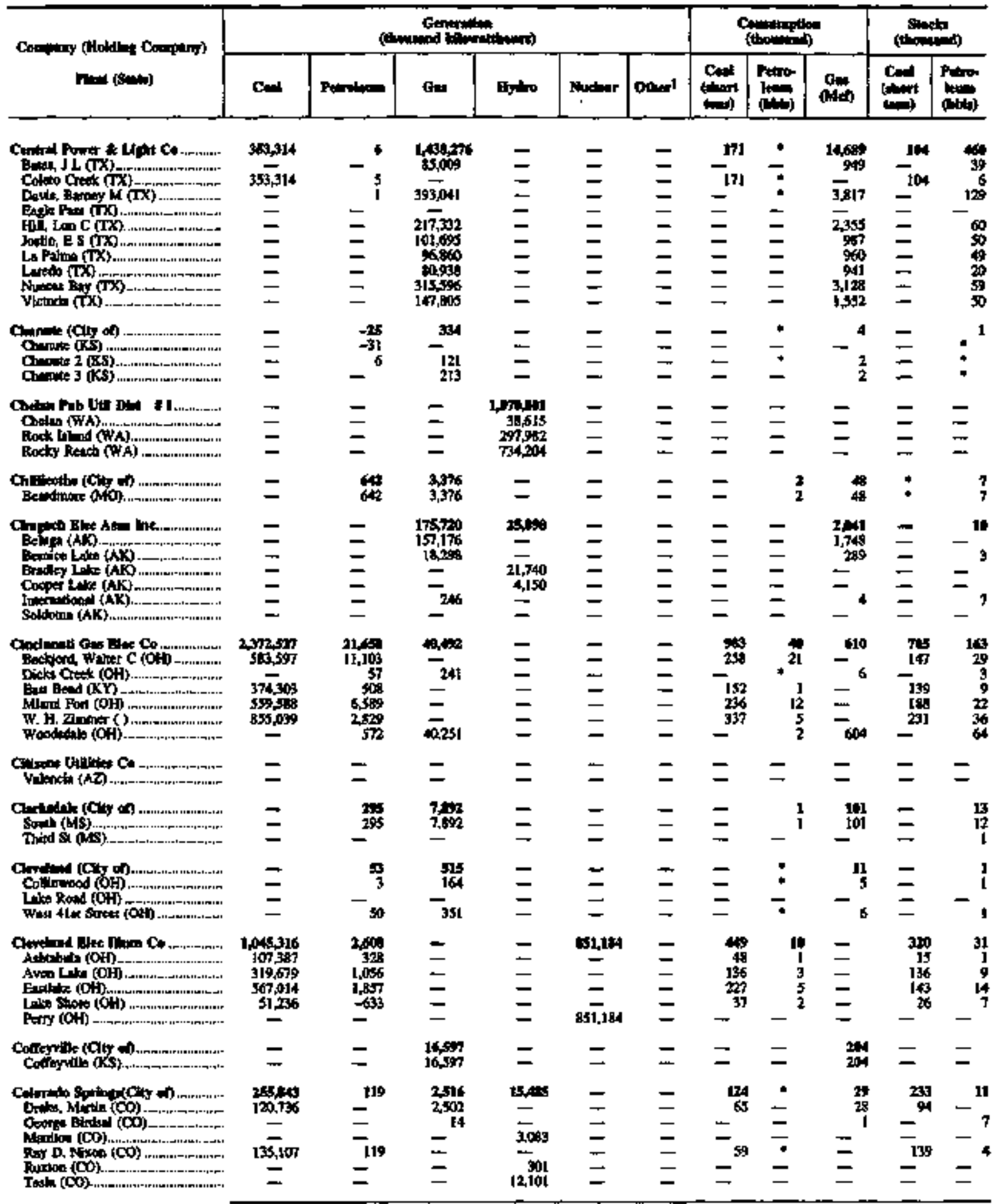

Soc foutioles of end of toble. 
Table 56. U.S. Rlectric Utilly Net Generation, Feed Confumption, and Paed Stocks by Conapany and Plant, Jaty 19\% (Continoded)

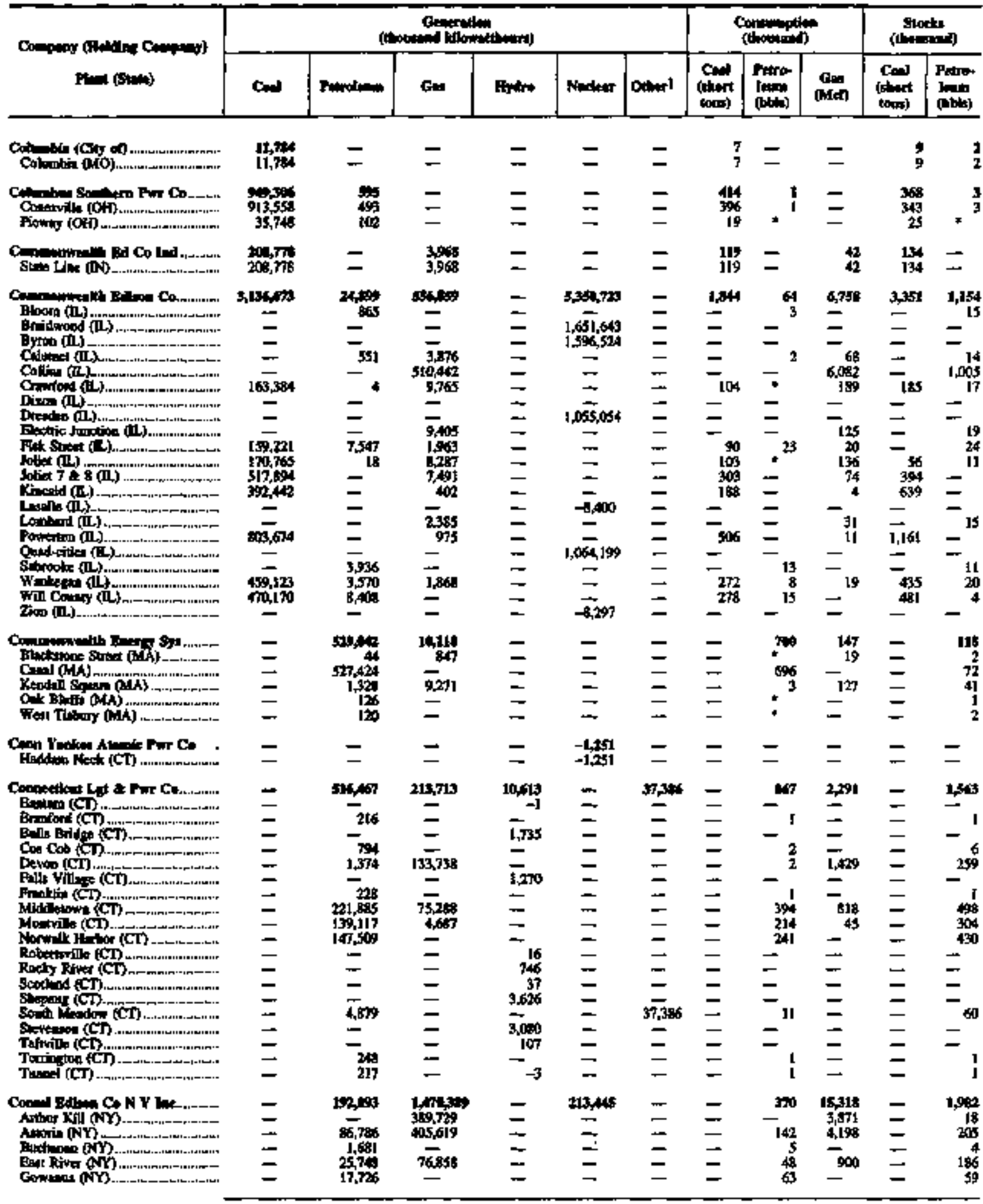

See foolmones at at of uble. 


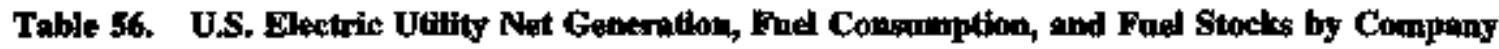
and Phant, Juty 1997 (Continned)

\begin{tabular}{|c|c|c|c|c|c|c|c|c|c|c|c|}
\hline \multirow{2}{*}{ 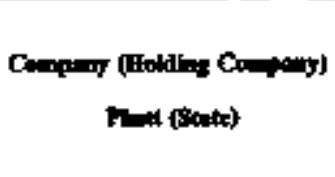 } & \multicolumn{6}{|c|}{ 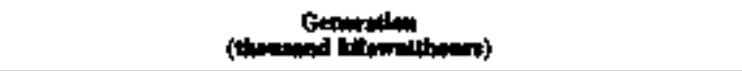 } & \multicolumn{3}{|c|}{ 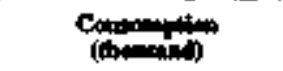 } & \multicolumn{2}{|c|}{ (t) } \\
\hline & Cod & atroltate & C*: & Brdis & Nedimor & Othor 1 & $\begin{array}{c}\text { Cand } \\
\text { (dbott } \\
\text { tonel) }\end{array}$ & 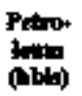 & Cart & $\begin{array}{l}\text { Conl } \\
\text { (atierd } \\
\text { inne) }\end{array}$ & 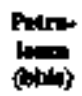 \\
\hline
\end{tabular}

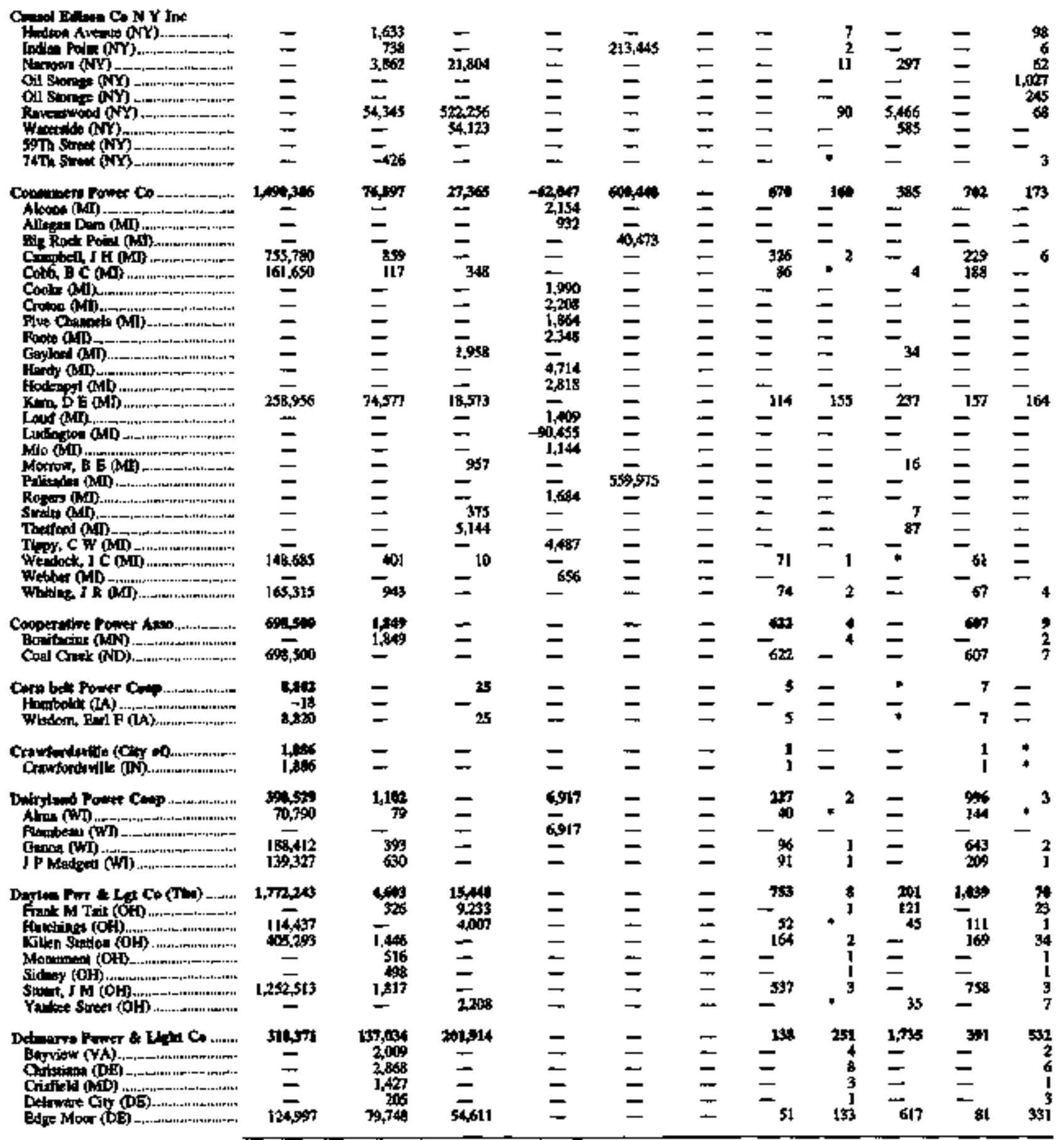

Soct footroles an end of toble. 
Table 56. U.S. Blectric Utility Net Generation, Fuel Consumption, and Fuel Stocks by Conpany and Plont, Juby 1997 (Conthued)

\begin{tabular}{|c|c|c|c|c|c|c|c|c|c|c|c|}
\hline \multirow{2}{*}{ 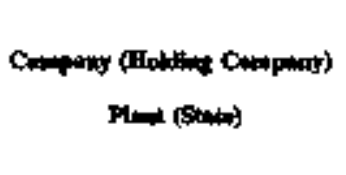 } & \multicolumn{6}{|c|}{ (the Gentitisen } & \multicolumn{3}{|c|}{ Cononintien } & \multicolumn{2}{|c|}{ (toods } \\
\hline & Cine & 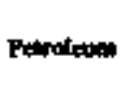 & ta & Fylo & Nodian & Oan:1 & 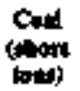 & Paro- & (Moset) & 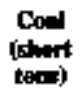 & $\begin{array}{l}\text { Pitro: } \\
\text { louls } \\
\text { (bobs) }\end{array}$ \\
\hline
\end{tabular}

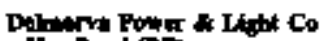

Fint Rond (DE)

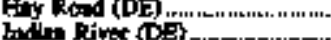

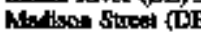

Tastey (WA)

What Sobution the

Derto (Cl) of)

Emitale (TX)

Robertu (IX)

Spente (TX)

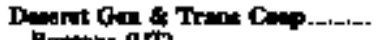

Bontind (WT)

Deitrak fCKy of .........................

Wistits (AII)

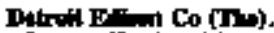

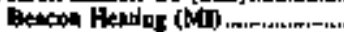

iplit River (bit)

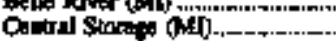

Colfix (ND)

Conters Coet ont

Dayton (Ail)

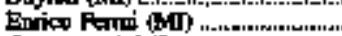

Creernood (Mil)

Fracoct onis

phot Beth

Mervorit

Monrow (ML)

Norlxent (Mr

Plecid (MT)

Purom (A1)

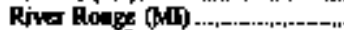

Sloalit (III).

S. Clir aMiD.

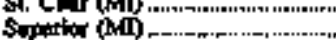

Trabter Cramel (MII) ..............-

Wilnot (MI).

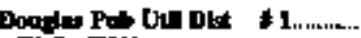
Wels $[W h]$

Dower (C) an)

Mclee Rom fOE

Viat Stot (DE).

Dowt (Cty of)

Dowt (C.1)

Dile Proer 0

Nlan (NK) -.

Bud Oret. (SC).

Belewt Creek (NC)

Biditomater of

Bazzind Roous (5C)

Catopila (NC)

Cod Ot Ote (SC)

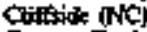

Cown' Fond NIC

Den Rivar INC

Destonen (SC)

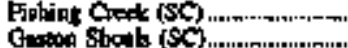

Geotod Shoth (SC)

Jocentat (JC)

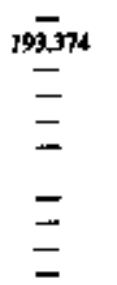

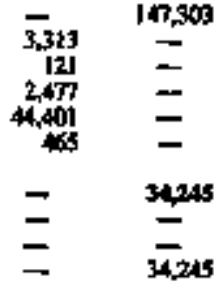

272,272

278,272

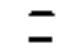

3953

船29?

-

-

-

$\overline{-}$

15,217

13, 100

$1.714,504$

-

ב

279.155

547,634

302,390

-

$=$

$=$

6.944

6,664

4 tapt

53,46

$1,428,044$

205 583

-

-

138,556

-

=

14 -

15 int 14,35

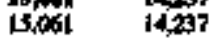

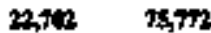

$7,470 \quad 1,875$

2, 470

344

152

169

7,493

7

$\overrightarrow{5,005}$

5,005

630

291

37

41

1,259

1,183

-

=

41,261

2289

853

$\overline{1,662}$

=

26,153

$\overline{1,679}$

$\bar{z}$

\section{$=$}

l1수 24:01

tI, 4 t IB, 922

1.947

II

35

6,71 12406

$1, \mathbf{2 3 9}$

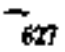

763

845

$=$

40.

二

$\vec{z}$

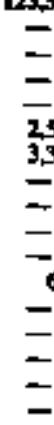

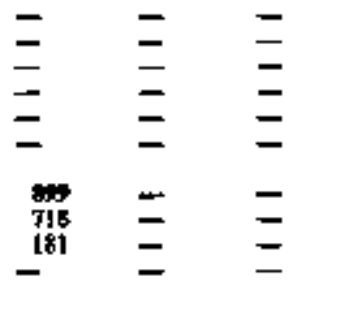

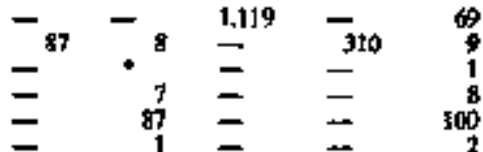

$=$

$=\bar{z}_{379}^{379}=\Sigma^{25}$
$= \pm$

$= \pm$

$\bar{z}$
$z$
$z$
$z$
$z$
$z$
$z$
$z$
$z$
$z$
$z$

$\operatorname{mos}$

$=$
$=$
$=$
505
$=$
$=$
$=$
$=$
$=$
$=$
$=$
$=$

Exack

532948

$\Xi \equiv \equiv \equiv$

$=$

40854040

$-54, \overline{1} 2$

$-54,120$

3,253

3,958

icos

$\frac{8,602}{12555}$

i7n

11.761

12789

4,771

4,099
-32894

Sec foocolas at eod of table. 
Table 56. US. Etetric Uthlity Net Generotion, Fued Cosammption, and Fuel Stocks by Company and Ptant, Jubl 1997 (Continned)

\begin{tabular}{|c|c|c|c|c|c|c|c|c|c|c|c|}
\hline \multirow{2}{*}{ 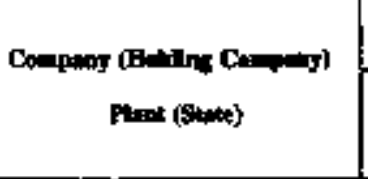 } & \multicolumn{6}{|c|}{ 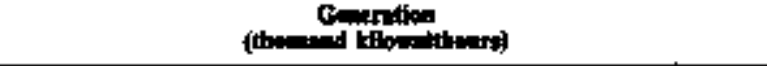 } & \multicolumn{3}{|c|}{ Contmating } & \multicolumn{2}{|c|}{ 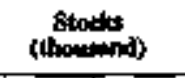 } \\
\hline & Can & Potrulewen & CN & Kpjrt & Nodur & Otherl & (imind & 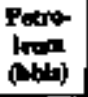 & (Ment) & 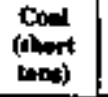 & 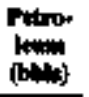 \\
\hline 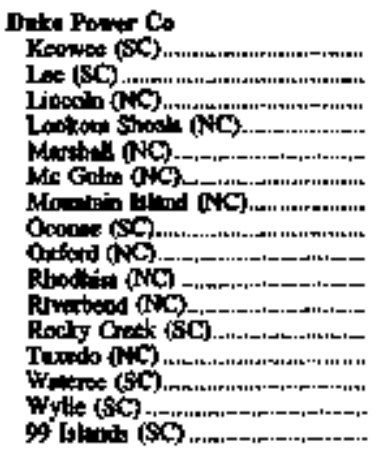 & $\begin{array}{c}158,993 \\
\bar{z} \\
1,293,332 \\
\bar{z} \\
\bar{z} \\
\overline{-} \\
240,906 \\
\bar{z} \\
\bar{z} \\
=\end{array}$ & $\begin{array}{l}\overline{T .59]} \\
1.150 \\
\overline{2,038} \\
= \\
= \\
= \\
= \\
= \\
= \\
= \\
=\end{array}$ & $\begin{array}{l}\bar{y} \\
114,453 \\
= \\
= \\
= \\
= \\
\overline{2,264} \\
= \\
= \\
=\end{array}$ & 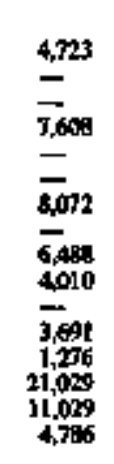 & $\begin{array}{c}\bar{z} \\
\bar{z} \\
1,920, n 10 \\
1,525,210 \\
= \\
= \\
= \\
=\end{array}$ & $\begin{array}{l}= \\
= \\
= \\
= \\
= \\
= \\
= \\
=\end{array}$ & $\begin{array}{l}-71 \\
= \\
= \\
= \\
= \\
= \\
= \\
= \\
= \\
=\end{array}$ & $\begin{array}{l}={ }^{6} \\
= \\
= \\
= \\
= \\
= \\
= \\
=\end{array}$ & $\begin{array}{l}={ }^{3} \\
1.386 \\
= \\
= \\
= \\
= \\
= \\
= \\
= \\
=\end{array}$ & $\begin{array}{l}- \\
= \\
= \\
= \\
= \\
= \\
= \\
= \\
=\end{array}$ & $\begin{aligned} & 12 \\
& 197 \\
&= 9 \\
&= \\
&= \\
&= \\
&= \\
&= \\
&=\end{aligned}$ \\
\hline 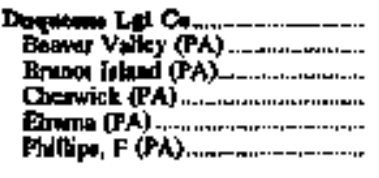 & $\begin{array}{l}\text { stostr } \\
\frac{-}{20,276} \\
20,231 \\
-\end{array}$ & $\begin{array}{l}2,43 \\
\frac{1,438}{6} \\
\frac{925}{6}\end{array}$ & $\begin{array}{l}2,353 \\
- \\
2333 \\
-\end{array}$ & $\begin{array}{l} \pm \\
z \\
z\end{array}$ & $\begin{array}{l}32654 \\
= \\
=\end{array}$ & $\begin{array}{l}- \\
= \\
= \\
=\end{array}$ & $\begin{array}{l}218 \\
- \\
- \\
111 \\
-\end{array}$ & $\begin{array}{l}-10 \\
-2 \\
-\end{array}$ & $z^{2}$ & $\begin{array}{l}304 \\
\frac{-}{247} \\
-\end{array}$ & $-^{3}$ \\
\hline 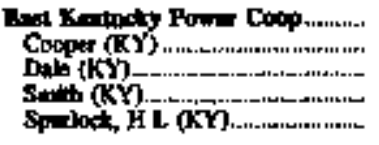 & $\begin{array}{l}261,191 \\
153,660 \\
56,943 \\
510,280\end{array}$ & $\begin{array}{r}34 \\
121 \\
180 \\
29 \\
184\end{array}$ & $\frac{\pi}{20,393}$ & $\begin{array}{l}\bar{z} \\
\bar{z}\end{array}$ & $\begin{array}{l}\bar{z} \\
\bar{z}\end{array}$ & $\begin{array}{l}\bar{z} \\
\bar{z}\end{array}$ & $\begin{array}{r}313 \\
63 \\
45 \\
-\quad 205\end{array}$ & $:$ & $\begin{array}{l}265 \\
= \\
z 65\end{array}$ & $\begin{array}{r}404 \\
43 \\
-301\end{array}$ & $\begin{array}{r}61 \\
* \\
57 \\
3\end{array}$ \\
\hline 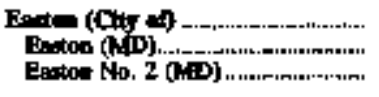 & $=$ & $\begin{array}{l}\frac{1}{2,005} \\
2,376 \\
3,029\end{array}$ & 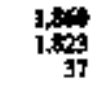 & $\bar{z}$ & $\bar{z}$ & $\bar{z}$ & $\bar{z}$ & $\begin{array}{r}11 \\
6\end{array}$ & $\begin{array}{r}18 \\
+17\end{array}$ & $\bar{z}$ & \\
\hline 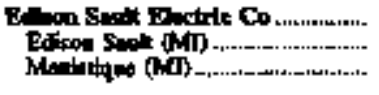 & $\bar{z}$ & $-{ }^{16}$ & $\bar{z}$ & 19,241 & $\bar{z}$ & $=$ & $\bar{z}$ & $\ddot{*}$ & $\bar{z}$ & $=$ & $\div$ \\
\hline 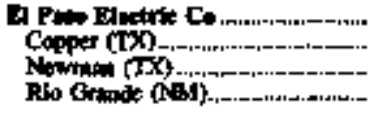 & $\bar{z}$ & $\begin{array}{l}\bar{z} \\
\overline{-}\end{array}$ & $\begin{array}{r}324780 \\
5,401 \\
21,190 \\
95,189\end{array}$ & $\begin{array}{l}z \\
=\end{array}$ & $\begin{array}{l}\bar{z} \\
\bar{z}\end{array}$ & $\begin{array}{l}\bar{z} \\
\bar{z}\end{array}$ & $\bar{z}$ & $\begin{array}{l}\bar{z} \\
\bar{z}\end{array}$ & $\begin{array}{l}3,446 \\
11,256 \\
1,069\end{array}$ & $\begin{array}{l}z \\
=\end{array}$ & $\begin{array}{c}7 \\
6 \\
31\end{array}$ \\
\hline 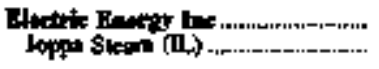 & 710034 & 65 & 1 & $\overline{-}$ & $\overline{-}$ & $\overline{-}$ & 43! & : & $*$ & 300 & * \\
\hline 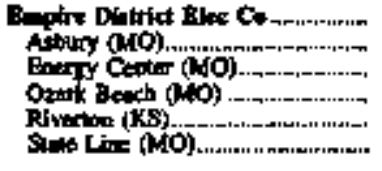 & $\begin{array}{l}14406 \\
\frac{14,382}{2}\end{array}$ & $\begin{array}{l}r_{11}^{21} \\
= \\
= \\
704\end{array}$ & $\begin{array}{l}\overrightarrow{141,693} \\
\overrightarrow{39,74} \\
\overrightarrow{16,742} \\
4,603\end{array}$ & $\begin{array}{l}\frac{8,377}{2} \\
\frac{8,397}{2}\end{array}$ & $\begin{array}{l}= \\
= \\
=\end{array}$ & $\begin{array}{l}\bar{z} \\
\bar{z} \\
z\end{array}$ & $z^{-{ }^{15}}$ & $\dot{-}^{2}$ & $\begin{array}{c}1,614 \\
-583 \\
- \\
548 \\
540\end{array}$ & $-^{47}$ & $\begin{array}{r}+3 \\
-\quad 8 \\
24\end{array}$ \\
\hline 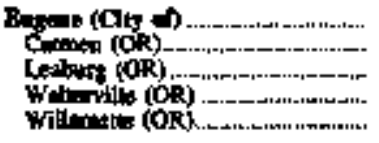 & $\begin{array}{l}\bar{z} \\
\bar{z}\end{array}$ & $\begin{array}{l}\bar{z} \\
\bar{z}\end{array}$ & $\begin{array}{l}= \\
\overline{=}\end{array}$ & $\begin{array}{l}3,409 \\
2,431 \\
5,170 \\
5,290 \\
-\end{array}$ & $\begin{array}{l}z \\
z\end{array}$ & $\begin{array}{l}\bar{z} \\
\bar{z}\end{array}$ & $\begin{array}{l}= \\
=\end{array}$ & $\begin{array}{l}\bar{z} \\
\bar{z}\end{array}$ & $\begin{array}{l}\bar{z} \\
\bar{z}\end{array}$ & $\begin{array}{l}\bar{z} \\
\bar{z}\end{array}$ & $\begin{array}{l}\bar{E} \\
\bar{E} \\
-\end{array}$ \\
\hline 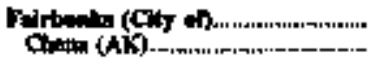 & 2,507 & $\mathbf{3}$ & $\overline{-}$ & $\overline{-}$ & $\bar{z}$ & $\bar{z}$ & 4 & : & $\bar{z}$ & $\begin{array}{l}\mathbf{1} \\
\mathbf{1}\end{array}$ & 1 \\
\hline 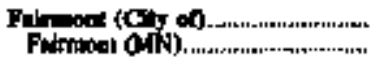 & $\bar{z}$ & 6 & 702 & $\bar{z}$ & $\bar{z}$ & $\bar{z}$ & $\overline{-}$ & 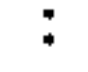 & 10 & $\overline{-}$ & I \\
\hline 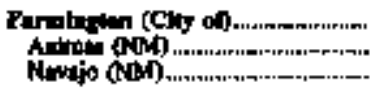 & $\bar{z}$ & $\bar{z}$ & $\begin{array}{l}15 \% 5 \\
-1505\end{array}$ & $\frac{15,161}{\mid 5,161}$ & $\bar{z}$ & $\bar{z}$ & $\bar{z}$ & $\bar{z}$ & $\begin{array}{l}148 \\
148 \\
-\end{array}$ & $\bar{z}$ & $\bar{z}$ \\
\hline
\end{tabular}

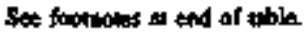




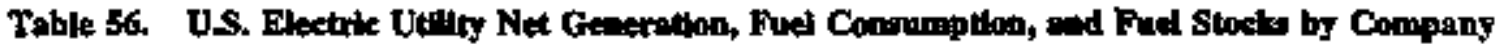
and Plant, Jub 1 WM (Contimed)

\begin{tabular}{|c|c|c|c|c|c|c|c|c|c|c|c|}
\hline \multirow{2}{*}{ 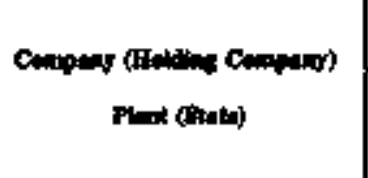 } & \multicolumn{6}{|c|}{ 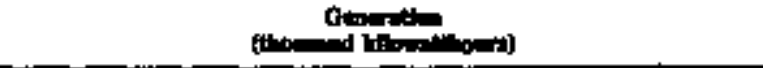 } & \multicolumn{3}{|c|}{ 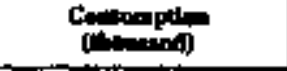 } & \multicolumn{2}{|c|}{ Stoods } \\
\hline & Conl & PAtrel-a & Gn & Hy. & Nodhar | & Bonetl & $\begin{array}{l}\text { Cad } \\
\text { thend } \\
\text { thin) }\end{array}$ & (16) & Gents) & $\begin{array}{l}\text { Cod } \\
\text { (otert } \\
\text { tong) }\end{array}$ & $\lim _{(b \rightarrow 0)}$ \\
\hline 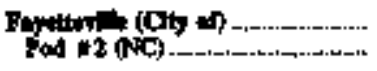 & 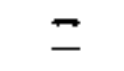 & 17 & thaty & $\overline{-}$ & $\overline{-}$ & $\stackrel{-}{-}$ & $=$ & $\because$ & 404 & $\overrightarrow{-}$ & $\pi_{i}$ \\
\hline 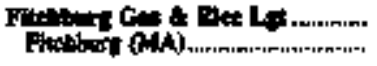 & $=$ & 247 & $=$ & $\bar{z}$ & $\vec{z}$ & $=$ & $\overline{-}$ & $\mathbf{1}$ & $=$ & $\because$ & $\frac{2}{2}$ \\
\hline 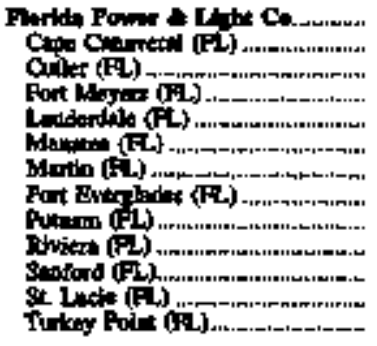 & $\begin{array}{l}= \\
= \\
= \\
= \\
= \\
=\end{array}$ & 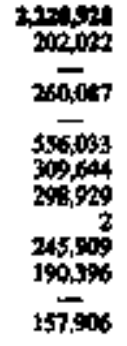 & 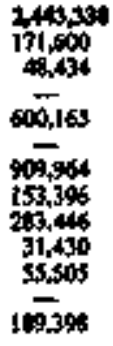 & $\begin{array}{l}= \\
= \\
= \\
= \\
= \\
= \\
=\end{array}$ & 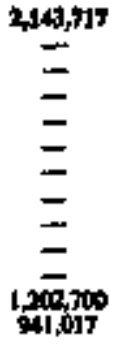 & $\begin{array}{l}= \\
= \\
= \\
= \\
= \\
= \\
=\end{array}$ & $\begin{array}{l}= \\
= \\
= \\
= \\
= \\
=\end{array}$ & 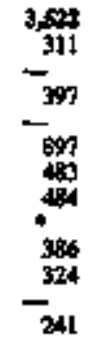 & $\begin{array}{c}21,816 \\
1,311 \\
637 \\
\overline{4,505} \\
\overline{7,416} \\
1,747 \\
2,67 \\
329 \\
677 \\
\overline{1,756}\end{array}$ & $\begin{array}{l}= \\
= \\
= \\
= \\
= \\
= \\
=\end{array}$ & $\begin{array}{r}3,810 \\
398 \\
-358 \\
69 \\
765 \\
574 \\
448 \\
40 \\
325 \\
542 \\
-382\end{array}$ \\
\hline 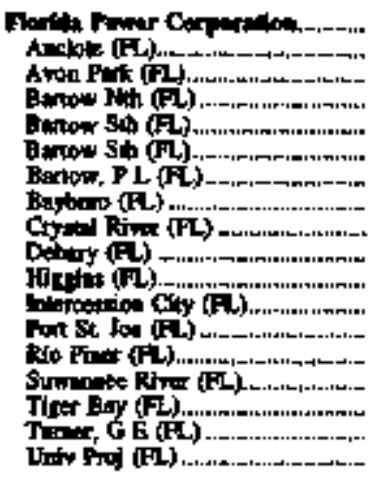 & $\begin{array}{l}= \\
= \\
= \\
= \\
\bar{z} \\
= \\
= \\
= \\
= \\
= \\
=\end{array}$ & 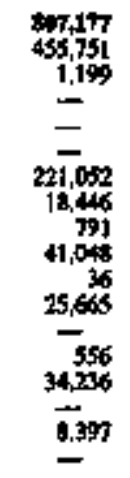 & 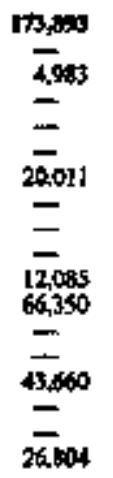 & $\begin{array}{l}= \\
= \\
= \\
= \\
= \\
= \\
= \\
= \\
=\end{array}$ & $\begin{array}{l}= \\
= \\
= \\
= \\
= \\
= \\
= \\
=\end{array}$ & $\begin{array}{l}= \\
= \\
= \\
= \\
= \\
= \\
= \\
= \\
=\end{array}$ & $\begin{array}{l}= \\
= \\
= \\
= \\
= \\
= \\
= \\
= \\
= \\
=\end{array}$ & 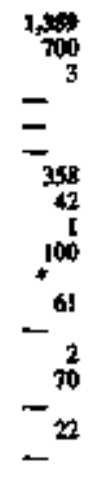 & $\begin{array}{l}2,604 \\
=76 \\
= \\
= \\
= \\
= \\
= \\
= \\
= \\
= \\
= \\
256\end{array}$ & $\begin{array}{l}= \\
= \\
= \\
= \\
= \\
= \\
= \\
= \\
= \\
= \\
= \\
= \\
=\end{array}$ & $\begin{array}{r}1540 \\
352 \\
5 \\
123 \\
233 \\
217 \\
23 \\
16 \\
215 \\
11 \\
136 \\
2 \\
136 \\
136 \\
-37 \\
13\end{array}$ \\
\hline 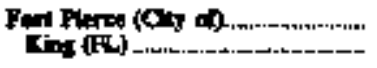 & $\bar{z}$ & 哲 & 22,99 & $=$ & 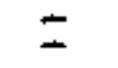 & $\overline{-}$ & $\overline{-}$ & 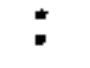 & 302 & $=$ & 21 \\
\hline 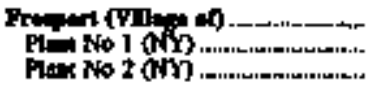 & $\bar{z}$ & $\frac{140}{166}$ & $=$ & $\bar{z}$ & $\bar{z}$ & $=$ & $\bar{z}$ & i & \pm & $\bar{z}$ & $\begin{array}{l}7 \\
1 \\
6\end{array}$ \\
\hline 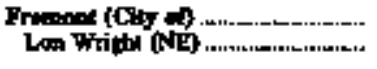 & 40,117 & $\overline{-}$ & 8 & $=$ & $\overline{-}$ & $\ddot{z}$ & 29 & $=$ & $\begin{array}{l}7 \\
7\end{array}$ & 30 & $\begin{array}{l}1 \\
1\end{array}$ \\
\hline 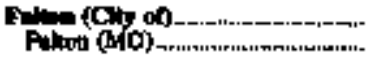 & $\bar{z}$ & 20 & 4 & $=$ & $\overline{-}$ & 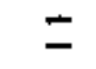 & $\vec{z}$ & : & 2 & $\overline{-}$ & $\mathbf{1}$ \\
\hline 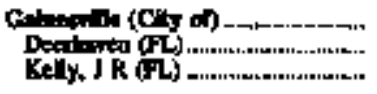 & $\frac{133,409}{133,940}$ & 1,099 & $\begin{array}{l}\$ 3,974 \\
48,019 \\
15,955\end{array}$ & $\bar{z}$ & $\bar{z}$ & $=$ & -5 & $-\quad \begin{array}{r}\mathbf{3} \\
-\end{array}$ & $\begin{array}{l}765 \\
565 \\
191\end{array}$ & $\begin{array}{l}99 \\
-99\end{array}$ & 51 \\
\hline 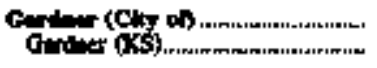 & 二 & $=$ & 4397 & $\bar{z}$ & $\vec{z}$ & 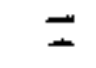 & $=$ & $\overline{-}$ & 73 & $\overline{-}$ & $\Xi$ \\
\hline 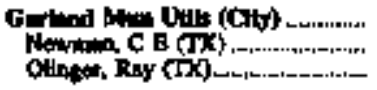 & $\bar{z}$ & $\bar{z}$ & $\begin{array}{l}10+901 \\
7,6001\end{array}$ & $\ddot{-}$ & $=$ & $=$ & $=$ & 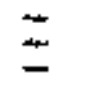 & $\begin{array}{l}1,777 \\
1,693\end{array}$ & $\vec{z}$ & $\begin{array}{l}96 \\
19 \\
78\end{array}$ \\
\hline 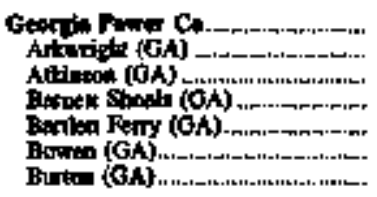 & 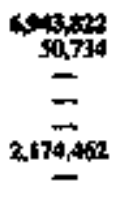 & $\begin{array}{l}139 \\
= \\
= \\
2004\end{array}$ & 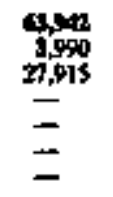 & 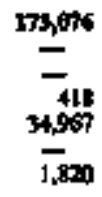 & $\begin{array}{l}2107=7 \\
= \\
= \\
=\end{array}$ & $\begin{array}{l}= \\
= \\
=\end{array}$ & $\begin{array}{l}3,101 \\
= \\
= \\
=\end{array}$ & $\begin{array}{l}0^{10} \\
=\end{array}$ & $\begin{array}{l}104 \\
124 \\
= \\
= \\
=\end{array}$ & $\begin{array}{l}3,625 \\
= \\
= \\
= \\
=\end{array}$ & 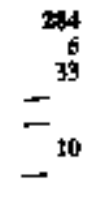 \\
\hline
\end{tabular}

See footuons at end of able. 
Table 56. U.S. Electric Utity Nat Gearatow, Fuet Consumpitim, and Foel Stocks by Company and Ponk, Jaly 1997 (Contioued)

\begin{tabular}{|c|c|c|c|c|c|c|c|c|c|c|c|}
\hline \multirow{2}{*}{ 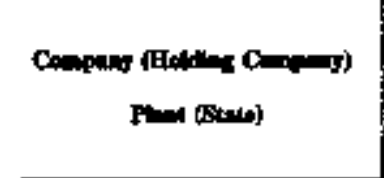 } & \multicolumn{6}{|c|}{ 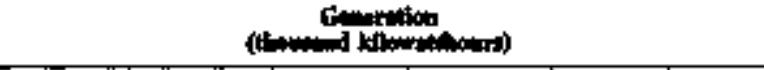 } & \multicolumn{3}{|c|}{ Conchithen } & \multicolumn{2}{|c|}{ 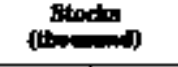 } \\
\hline & Cow & Petrolecen & Ea & Pypo & Nerinerg & Qther 1 & 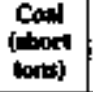 & $\begin{array}{l}\text { Petro- } \\
\text { lans) }\end{array}$ & (a) & (i) & $\begin{array}{l}\text { Petro } \\
\text { letis } \\
\text { (bita) }\end{array}$ \\
\hline \multicolumn{12}{|l|}{ Gong Pan Co } \\
\hline 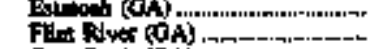 & $=$ & $=$ & $=$ & $\overline{3.124}$ & $=$ & $\vec{a}$ & - & - & - & - & - \\
\hline Pot Rock (GA) & $\overline{7}$ & $=$ & $=$ & 14,033 & $\bar{\Xi}$ & $\bar{z}$ & $=$ & - & $\approx$ & $\bar{z}$ & 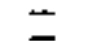 \\
\hline Heminged (GA) & 437,960 & 127 & - & - & - & - & 176 & 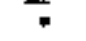 & $=$ & 101 & $\bullet$ \\
\hline 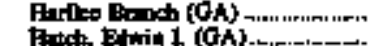 & 900,340 & $1^{158}$ & $=$ & $=$ & $1, \overline{7}, 991$ & $\Xi$ & 358 & * & - & 414 & 1 \\
\hline 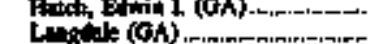 & $\overline{-}$ & $\bar{z}$ & $\ddot{z}$ & $\overline{211}$ & - & $=$ & $\vec{z}$ & 二 & 二 & - & - \\
\hline Lend shoth (GA) & 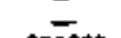 & - & - & 5,432 & - & - & $=$ & $=$ & $=$ & $=$ & Z \\
\hline 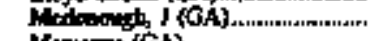 & 324,323 & 244 & 10.311 & - & - & - & 12 & - & 63 & 8 & - \\
\hline 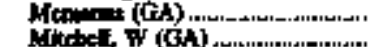 & $\overrightarrow{s i n}$ & $\begin{array}{l}29,715 \\
10361\end{array}$ & $\bar{z}$ & $\bar{z}$ & $=$ & $\overline{-}$ & -3 & 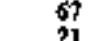 & - & - & s) \\
\hline 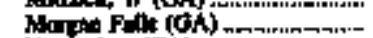 & - & $\Rightarrow$ & $=$ & $\overline{4,097}$ & $=$ & $\bar{z}$ & $\pi^{x}$ & $-^{21}$ & 二 & $n^{31}$ & 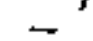 \\
\hline Nhodacte $[(\mathrm{N})$ & - & - & - & 1,106 & - & 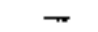 & - & - & - & - & - \\
\hline North liphod $(\sigma \alpha)$ & - & - & - & jogents & - & - & $\rightarrow$ & - & - & - & - \\
\hline Other Dim (CA) & - & - & - & 18806 & - & - & - & - & - & - & - \\
\hline 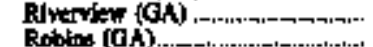 & $=$ & $\bar{z}$ & $\overrightarrow{16,7 x}$ & 132 & $=$ & $\bar{z}$ & $\vec{z}$ & $=$ & $-{ }_{10}$ & $\bar{z}$ & - \\
\hline 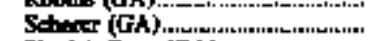 & $1,418,551$ & 9,185 & 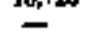 & $=$ & $=$ & $=$ & 961 & 23 & - & $\overline{1.443}$ & 3 \\
\hline 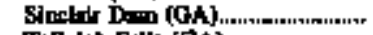 & - & - & - & 7,351 & - & - & - & - & $\rightarrow$ & $\rightarrow$ & $=$ \\
\hline 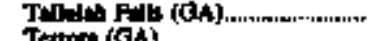 & - & - & - & 12,648 & $=$ & - & $=$ & - & - & - & - \\
\hline 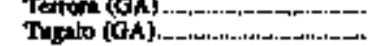 & $=$ & z & $=$ & $\begin{array}{l}3,602 \\
7 \% 81\end{array}$ & $\vec{z}$ & $\bar{z}$ & $\vec{z}$ & $=$ & $=$ & $=$ & $=$ \\
\hline Voglk $(\mathrm{O} A)$ - & - & $=$ & $=$ & - & $1,774,960$ & $\bar{m}$ & - & - & $\overline{-}$ & $=$ & $=$ \\
\hline Ho $\mathrm{Dm}(\mathrm{OA})$ & - & $=$ & 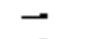 & 42,052 & $\rightarrow$ & - & - & - & - & & - \\
\hline 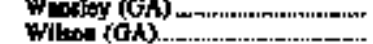 & $1,0] 1,037$ & $\begin{array}{l}3,005 \\
13,062\end{array}$ & $\vec{z}$ & 二 & $=$ & $\bar{z}$ & -387 & 38 & 二 & -320 & 25 \\
\hline 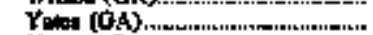 & 575,312 & 40 & - & - & - & $=$ & 241 & i & - & 464 & \\
\hline 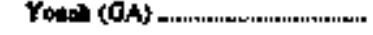 & - & $\rightarrow$ & - & 4,122 & - & - & $m$ & $\rightarrow$ & - & - & - \\
\hline 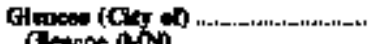 & - & min & $m$ & - & - & $\boldsymbol{-}$ & - & 1 & 9 & - & 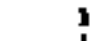 \\
\hline & & & & & & & & & & & \\
\hline 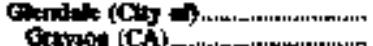 & $=$ & 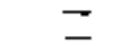 & 7,99 & $\bar{Z}$ & 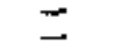 & 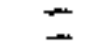 & $z$ & $=$ & 118 & - & 50 \\
\hline & & & & & & & & & & & \\
\hline 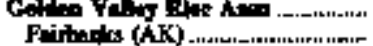 & 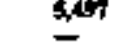 & 3), & $\bar{z}$ & 二 & $\bar{z}$ & $\overline{-}$ & $-\mathrm{s}$ & $\underset{2}{\mathbf{d}}$ & $\bar{z}$ & $=$ & 3 \\
\hline Henly (AK) & 5,497 & 240 & - & - & - & - & $s$ & 1 & - & - & j \\
\hline Norti Pole $(A K)_{n}$ & - & 32,751 & - & - & - & - & - & st & - & - & 2 \\
\hline 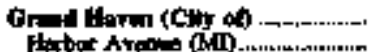 & 30,192 & 18 & 28 & - & - & - & 17 & - & $*$ & & to \\
\hline 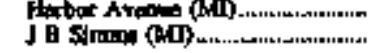 & $\overline{33,852}$ & $-^{18}$ & $-^{28}$ & $\vec{Z}$ & $\bar{z}$ & $\bar{z}$ & $-{ }_{17}$ & * & 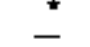 & 一船 & 10 \\
\hline 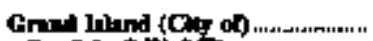 & 95,79 & - & 2871 & - & - & $\Rightarrow$ & $\mathbf{3 5}$ & $\bullet$ & 110 & a & 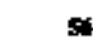 \\
\hline Bardick, $\mathrm{C}$ W (N) & - & - & 8,87 & - & - & - & - & - & 110 & & so \\
\hline P(w & 55,749 & - & - & - & 一 & - & $\$ 5$ & - & - & 64 & - \\
\hline 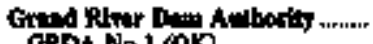 & sints & - & 1,79 & stits: & - & - & 34 & - & $\mathbf{s}$ & 736 & 3 \\
\hline $\begin{array}{l}\text { GROA No } 1(\mathrm{OK}) \\
\text { Matimn }(\mathrm{OK})\end{array}$ & $597 \$ 10$ & $=$ & $2^{1.79}$ & $2 \overline{2}, 3 \times 0$ & $=$ & $\ddot{z}$ & $-^{383}$ & 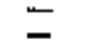 & -19 & $Z^{736}$ & $-{ }^{2}$ \\
\hline Femonods (OK) & - & - & - & 44,694 & - & - & 二 & $=$ & - & $\overline{-}$ & $\overline{-}$ \\
\hline Strat (OK) & - & - & - & $-10,0$ BS & - & - & - & - & - & - & - \\
\hline 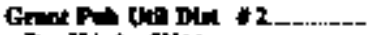 & $\longrightarrow$ & - & $=$ & $1,112,724$ & - & - & - & - & - & $=$ & - \\
\hline 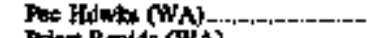 & - & - & - & 3,352 & - & - & - & - & - & - & - \\
\hline Fie: R pdid (WA) & $\bar{z}$ & $=$ & $\bar{z}$ & 494,899 & $\bar{z}$ & $\bar{z}$ & $\bar{z}$ & $\bar{z}$ & $=$ & $=$ & 二 \\
\hline 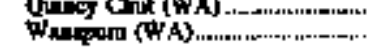 & Z & $=$ & $=$ & 614,992 & $\overline{-}$ & $\rightarrow$ & - & - & - & - & $\overline{-}$ \\
\hline 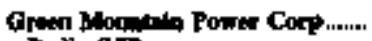 & - & L, st & - & 9206 & - & - & $m$ & 4 & $=$ & $\rightarrow$ & It \\
\hline Badin $(\sqrt{D}-$ & - & 1,339 & - & 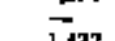 & - & $\rightarrow$ & - & 3 & - & - & IS \\
\hline Bolkon Hit (VT) & 二 & $\bar{z}$ & $\bar{z}$ & 1,432 & $\bar{z}$ & $\Xi$ & $\bar{z}$ & $=$ & $\bar{z}$ & $\bar{z}$ & $\bar{z}$ \\
\hline 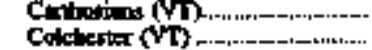 & 二 & 85 & 二 & $=$ & $=$ & - & $=$ & 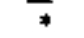 & $=$ & $\bar{z}$ & -1 \\
\hline Gatal Jnoctios 19 (YT) & - & 66 & - & $2 \times 67$ & - & - & - & • & - & - & " \\
\hline G & $\bar{z}$ & $\bar{z}$ & $=$ & 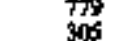 & $=$ & $=$ & 二 & $=$ & $\bar{z}$ & $\bar{\square}$ & 二 \\
\hline 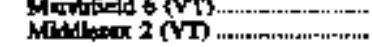 & - & $=$ & $=$ & 360 & - & $=$ & $=$ & 二 & 二 & - & - \\
\hline
\end{tabular}

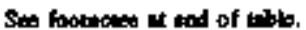


Table 56. U.S. Electric Utilty Net Generation, Fuel Consumption, and Fuel Stocles by Company and Plant, July 1997 (Continaed)

\begin{tabular}{|c|c|c|c|c|c|c|c|c|c|c|c|}
\hline \multirow{2}{*}{ 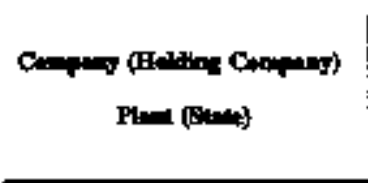 } & \multicolumn{6}{|c|}{ 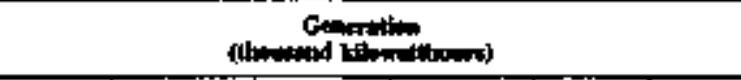 } & \multicolumn{3}{|c|}{ Cocopoption } & \multicolumn{2}{|c|}{ Strods } \\
\hline & Cin & Puratione & Gat & Fyimes & Nedear & On:1 & Coid & Putro- & (Ma) & $\begin{array}{c}\text { Cond } \\
\text { (diont } \\
\text { tont }\end{array}$ & Ration \\
\hline 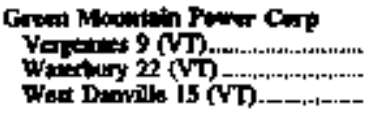 & $\bar{z}$ & ${ }_{-}^{164}$ & $\bar{z}$ & $\begin{array}{l}\mathbf{4 8} \\
\mathbf{8 5 2} \\
273\end{array}$ & $=$ & $\bar{z}$ & $\bar{z}$ & $\stackrel{*}{=}$ & $\bar{z}$ & $\bar{z}$ & $\stackrel{*}{=}$ \\
\hline 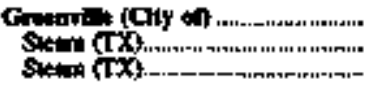 & $\bar{z}$ & $\bar{z}$ & $\bar{z}$ & $\bar{z}$ & $=$ & $\bar{z}$ & $=$ & $\bar{z}$ & $\bar{z}$ & $\bar{z}$ & $\bar{z}$ \\
\hline 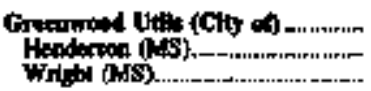 & $\bar{z}$ & $\bar{z}$ & $\begin{array}{l}1402 \\
0.988 \\
1.414\end{array}$ & $\bar{z}$ & $\bar{z}$ & $\bar{z}$ & $\bar{z}$ & $\underline{z}$ & $\begin{array}{r}169 \\
136 \\
13\end{array}$ & .9 & \\
\hline 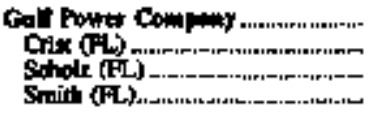 & $\begin{array}{l}74,117 \\
40,792 \\
31,694 \\
120,651\end{array}$ & $\begin{array}{r}2408 \\
258 \\
1,768\end{array}$ & $\begin{array}{l}31,921 \\
31,921 \\
-\end{array}$ & $\bar{z}$ & $\begin{array}{l}\bar{z} \\
\bar{z}\end{array}$ & $\bar{z}$ & $\begin{array}{r}341 \\
227 \\
17 \\
97\end{array}$ & . 1 & $\begin{array}{l}367 \\
362 \\
=\end{array}$ & $\begin{array}{r}230 \\
144 \\
24\end{array}$ & : \\
\hline 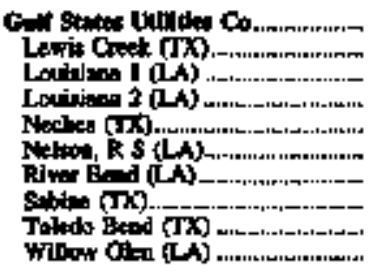 & $\begin{array}{l}30,207 \\
= \\
= \\
390,207 \\
= \\
=\end{array}$ & $\begin{array}{l}= \\
= \\
= \\
= \\
=\end{array}$ & 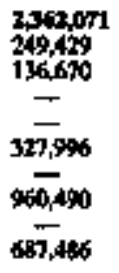 & $\begin{array}{l}15,67 \\
= \\
= \\
= \\
\overline{15607}\end{array}$ & $\begin{array}{l}\text { s\%,15 } \\
= \\
= \\
= \\
= \\
=\end{array}$ & $\begin{array}{l}\bar{z} \\
\bar{z} \\
\bar{z} \\
\bar{z}\end{array}$ & $\begin{array}{l}z^{20} \\
= \\
= \\
= \\
=\end{array}$ & $\begin{array}{l}z^{1} \\
= \\
= \\
=\end{array}$ & $\begin{array}{l}25,300 \\
2,652 \\
1,183 \\
\frac{-}{3,438} \\
\frac{10,371}{7,526}\end{array}$ & $\begin{array}{l}= \\
= \\
= \\
= \\
=\end{array}$ & $\begin{array}{l}360 \\
= \\
= \\
-{ }_{108}^{34} \\
-{ }_{184}^{43}\end{array}$ \\
\hline 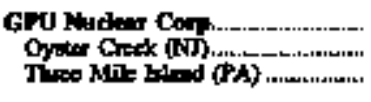 & $\bar{z}$ & $\bar{z}$ & $\bar{z}$ & $\bar{z}$ & $\begin{array}{l}1,0,4,564 \\
43,092 \\
590,430\end{array}$ & $\bar{z}$ & $\bar{z}$ & $\bar{z}$ & $\bar{z}$ & $\bar{z}$ & $\bar{z}$ \\
\hline 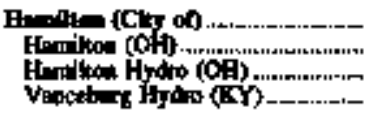 & $\begin{array}{l}31340 \\
= \\
=\end{array}$ & $=^{\frac{2}{2}}$ & $\begin{array}{l}6,76 \\
= \\
6786\end{array}$ & $\underset{135}{32,400}$ & $=$ & $\bar{z}$ & $\overline{-}^{\mathbf{6}}$ & $\stackrel{*}{*}$ & $\begin{array}{l}\mathbf{y 1} \\
- \\
-\end{array}$ & $\begin{array}{r}5 \\
-5\end{array}$ & $\begin{array}{r}3 \\
-\quad 3 \\
-\end{array}$ \\
\hline 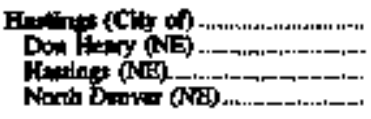 & $\frac{44,92}{14,392}$ & -5 & $\begin{array}{l}743 \\
\$ 45\end{array}$ & $\bar{z}$ & $\begin{array}{l}= \\
=\end{array}$ & $\begin{array}{l}\bar{z} \\
\bar{z}\end{array}$ & -79 & $\ddot{*}$ & $\begin{array}{r}\mathbf{1 3} \\
-\quad 9\end{array}$ & -64 & \\
\hline 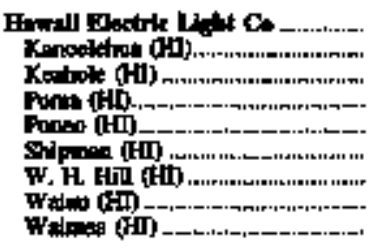 & $\begin{array}{l}\bar{z} \\
= \\
= \\
\bar{z}\end{array}$ & $\begin{array}{c}0,051 \\
2,106 \\
6,797 \\
17,090 \\
- \\
3,667 \\
22,230 \\
-200\end{array}$ & $\begin{array}{l}= \\
= \\
= \\
= \\
=\end{array}$ & $\begin{array}{l}2,014 \\
= \\
\overline{1} \\
= \\
= \\
-6468\end{array}$ & $\begin{array}{l}= \\
= \\
= \\
= \\
=\end{array}$ & $\begin{array}{l}= \\
= \\
= \\
= \\
=\end{array}$ & $\begin{array}{l}= \\
= \\
= \\
= \\
=\end{array}$ & $\begin{array}{r}116 \\
45 \\
15 \\
-40 \\
-46\end{array}$ & $\begin{array}{l}= \\
= \\
= \\
= \\
=\end{array}$ & $\begin{array}{l}= \\
= \\
= \\
= \\
=\end{array}$ & $\begin{array}{r}66 \\
4 \\
5 \\
-\quad 16 \\
-\quad 3 \\
-\quad 3\end{array}$ \\
\hline 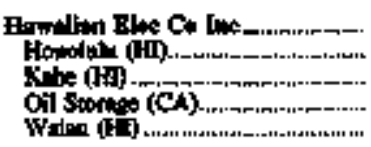 & $\begin{array}{l}\bar{z} \\
\bar{z}\end{array}$ & $\begin{array}{l}39,74 \\
20,275 \\
205,519 \\
63,949\end{array}$ & $\begin{array}{l}\bar{z} \\
\bar{z}\end{array}$ & $\begin{array}{l}\bar{z} \\
\bar{z}\end{array}$ & $\begin{array}{l}z \\
z \\
z\end{array}$ & $\begin{array}{l}\bar{z} \\
\bar{z}\end{array}$ & $\begin{array}{l}\bar{z} \\
\bar{\Xi}\end{array}$ & $\begin{array}{r}601 \\
42 \\
461 \\
-117\end{array}$ & $\begin{array}{l}\bar{z} \\
\bar{z}\end{array}$ & $\begin{array}{l}= \\
= \\
=\end{array}$ & $\begin{array}{l}125 \\
36 \\
266 \\
135 \\
138\end{array}$ \\
\hline 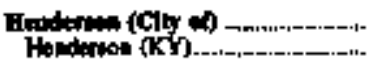 & 6,546 & i & $\overline{-}$ & $\overline{-}$ & $\Rightarrow$ & E & 4 & 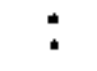 & - & $*$ & * \\
\hline 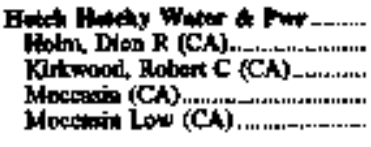 & $\begin{array}{l}\bar{z} \\
\bar{z}\end{array}$ & $\bar{z}$ & $\begin{array}{l}\bar{z} \\
\bar{E}\end{array}$ & $\begin{array}{l}10,476 \\
89,777 \\
57,997 \\
41,234 \\
458\end{array}$ & $\begin{array}{l}\bar{z} \\
z\end{array}$ & $\begin{array}{l}\bar{z} \\
=\end{array}$ & $\begin{array}{l}\bar{z} \\
\bar{z}\end{array}$ & $\begin{array}{l}\bar{z} \\
\bar{z}\end{array}$ & $\begin{array}{l}\bar{z} \\
\bar{z}\end{array}$ & $\begin{array}{l}= \\
=\end{array}$ & $\begin{array}{l}\bar{z} \\
\bar{z}\end{array}$ \\
\hline 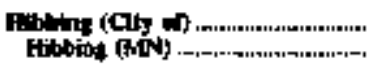 & $j_{\text {jon }}$ & $\overline{-}$ & \pm & $\overline{-}$ & $=$ & $\bar{z}$ & i & $\overline{-}$ & $\bar{z}$ & : & $\overline{-}$ \\
\hline
\end{tabular}

Sec toomons at and of wie. 


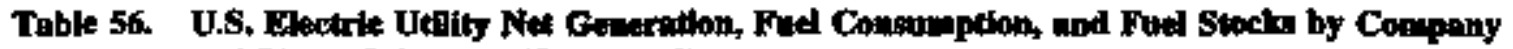
and Ftant, Jaly 1957 (Conenared)

\begin{tabular}{|c|c|c|c|c|c|c|c|c|c|c|c|}
\hline \multirow{2}{*}{ 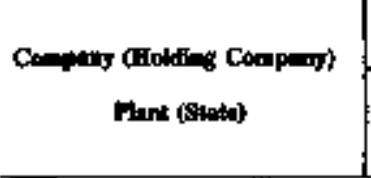 } & \multicolumn{6}{|c|}{ 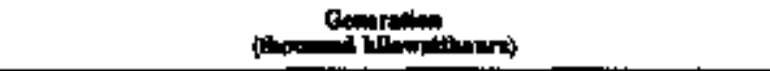 } & \multicolumn{3}{|c|}{ 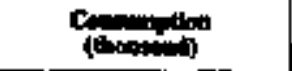 } & \multicolumn{2}{|c|}{ 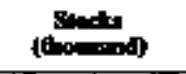 } \\
\hline & Can & 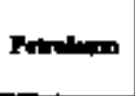 & Ev & Fighos & Made: & | & 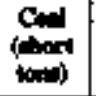 & Fino- & (Metef) & Cont & $\underset{\text { amblat }}{\text { Rut }}$ \\
\hline 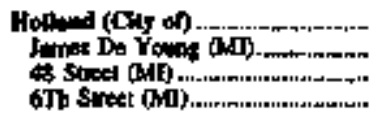 & $\begin{array}{l}m, 104 \\
= \\
27,104\end{array}$ & - & $\begin{array}{r}1,909 \\
1 \\
1,908 \\
-\end{array}$ & $\bar{z}$ & $\begin{array}{l}\bar{z} \\
\bar{z}\end{array}$ & $\begin{array}{l}= \\
z\end{array}$ & $=^{14}$ & $\begin{array}{r}1 \\
-1\end{array}$ & $-^{24}$ & $=^{5}$ & *6 \\
\hline 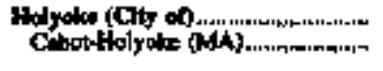 & $=$ & 19. & 614 & 211 & $\bar{z}$ & $\bar{z}$ & $=$ & : & & $\overline{-}$ & 16 \\
\hline 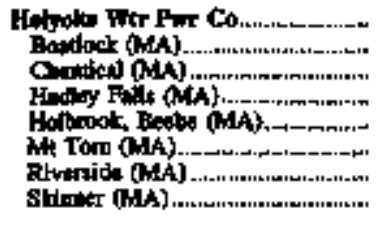 & $\begin{array}{l}\operatorname{tis,791} \\
= \\
= \\
\frac{=}{103,791} \\
=\end{array}$ & $\begin{array}{l}-2 \\
= \\
= \\
=\end{array}$ & $\begin{array}{l}\bar{z} \\
\bar{z} \\
\bar{z}\end{array}$ & $\begin{array}{r}13,051 \\
197 \\
65 \\
12066 \\
31 \\
-\quad 665 \\
37\end{array}$ & $\begin{array}{l}\bar{z} \\
\bar{z} \\
\bar{z}\end{array}$ & $\begin{array}{l}= \\
= \\
= \\
=\end{array}$ & $\begin{array}{l}= \\
= \\
=\end{array}$ & $\begin{array}{l}\ddot{z} \\
\bar{z} \\
\bar{z}\end{array}$ & $\begin{array}{l}z \\
= \\
z \\
=\end{array}$ & $\begin{array}{l}= \\
= \\
= \\
=\end{array}$ & $\begin{array}{l}= \\
\bar{z} \\
\bar{z} \\
=\end{array}$ \\
\hline 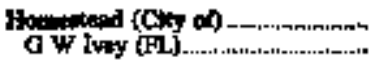 & $\bar{z}$ & istis & 3,906 & $=$ & $\overline{-}$ & $=$ & $\bar{z}$ & i & 39 & $\bar{z}$ & \\
\hline 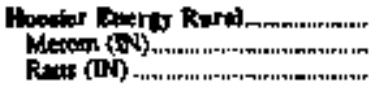 & $\begin{array}{l}\text { mint } \\
6753 \\
112,999\end{array}$ & $\begin{array}{l}214 \\
146\end{array}$ & $\bar{z}$ & $\bar{z}$ & $\bar{z}$ & $\bar{z}$ & $\begin{array}{r}301 \\
\mathbf{3 0 0} \\
53\end{array}$ & 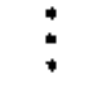 & $\bar{z}$ & 45 & ${ }^{10}$ \\
\hline 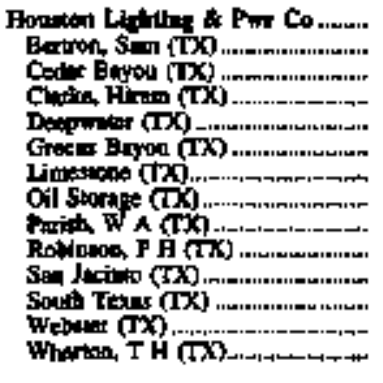 & 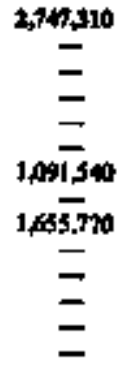 & $\begin{array}{l}\bar{z} \\
\bar{m} \\
\bar{z} \\
\bar{m} \\
\bar{z}\end{array}$ & 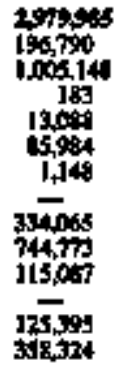 & $\begin{array}{l}= \\
= \\
= \\
= \\
= \\
=\end{array}$ & $\begin{array}{l}= \\
= \\
= \\
= \\
= \\
= \\
=\end{array}$ & $\begin{array}{l}= \\
= \\
= \\
= \\
= \\
= \\
=\end{array}$ & $\begin{array}{l}= \\
= \\
= \\
\overline{1} \\
= \\
= \\
=\end{array}$ & $\begin{array}{l}= \\
= \\
= \\
= \\
= \\
= \\
=\end{array}$ & 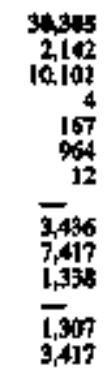 & $\begin{array}{l}= \\
= \\
= \\
= \\
5360 \\
= \\
= \\
= \\
=\end{array}$ & $\begin{array}{l}{ }^{13} \\
= \\
= \\
= \\
= \\
= \\
= \\
=\end{array}$ \\
\hline 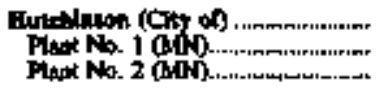 & $=$ & $\begin{array}{l}149 \\
1,367\end{array}$ & $\begin{array}{l}34,25 t \\
5999 \\
2053\end{array}$ & $\bar{z}$ & $=$ & $\bar{z}$ & $\bar{z}$ & $\cdot 3$ & $\begin{array}{l}345 \\
244\end{array}$ & $\bar{z}$ & \\
\hline 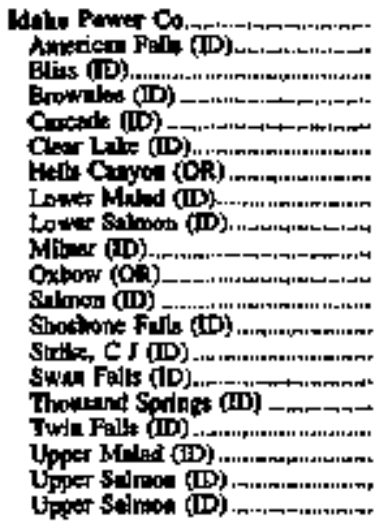 & $\begin{array}{l}= \\
= \\
= \\
= \\
= \\
= \\
= \\
= \\
= \\
=\end{array}$ & $\begin{array}{l}= \\
= \\
= \\
= \\
= \\
= \\
= \\
= \\
=\end{array}$ & $\begin{array}{l}= \\
= \\
= \\
= \\
= \\
= \\
= \\
= \\
= \\
=\end{array}$ & 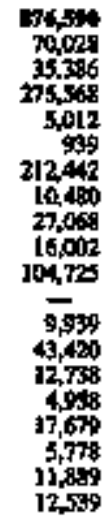 & $\begin{array}{l}\bar{z} \\
\bar{z} \\
= \\
= \\
\bar{z} \\
= \\
\bar{z} \\
= \\
=\end{array}$ & $\begin{array}{l}= \\
= \\
= \\
= \\
= \\
= \\
= \\
= \\
= \\
= \\
=\end{array}$ & $\begin{array}{l}= \\
= \\
= \\
= \\
= \\
= \\
= \\
= \\
= \\
= \\
= \\
=\end{array}$ & $\begin{array}{l}= \\
= \\
= \\
= \\
= \\
= \\
= \\
= \\
= \\
= \\
= \\
=\end{array}$ & 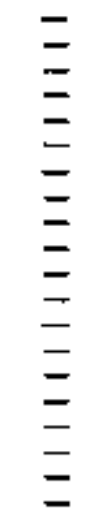 & $\begin{array}{l}= \\
= \\
= \\
= \\
= \\
= \\
= \\
= \\
= \\
= \\
=\end{array}$ & $\begin{array}{l}= \\
= \\
= \\
= \\
= \\
= \\
= \\
= \\
= \\
= \\
=\end{array}$ \\
\hline 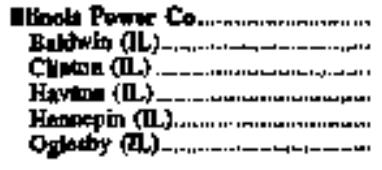 & 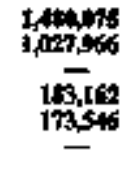 & $\begin{array}{l}2,191 \\
7 \% 5 \\
511 \\
1,000\end{array}$ & $\begin{array}{l}37,273 \\
= \\
78 \\
3,540 \\
1,540\end{array}$ & $\begin{array}{l}\bar{z} \\
\bar{z} \\
=\end{array}$ & $\begin{array}{c}-11,90 \\
-11,59 \\
=\end{array}$ & $\begin{array}{l}= \\
\Rightarrow \\
=\end{array}$ & $\begin{array}{r}47 \\
-\quad 86 \\
-\quad \frac{76}{7}\end{array}$ & $\begin{array}{r}\mathbf{1} \\
-\quad 1 \\
-\end{array}$ & $\underbrace{515}_{\substack{1 \\
3 \\
75}}$ & $\begin{array}{r}103 \\
201 \\
-\begin{array}{r}124 \\
34\end{array} \\
-\end{array}$ & $\begin{array}{r}12 \\
-\quad 2 \\
-9\end{array}$ \\
\hline
\end{tabular}

Set botinoke an end of thb. 
Table 56. U.S. Electric Utilty Net Generation, Foel Consurption, and Fand Stocks by Company and Ptant, Juty 1997 (Contlaned)

\begin{tabular}{|c|c|c|c|c|c|c|c|c|c|c|c|}
\hline \multirow{2}{*}{ 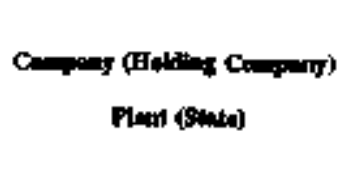 } & \multicolumn{6}{|c|}{ 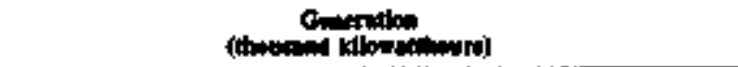 } & \multicolumn{3}{|c|}{ Compingn } & \multicolumn{2}{|c|}{ (timects } \\
\hline & Cent & Petrilief & Go: & Fylo & Nechar & الוئ10 & timit & 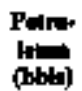 & AMen & 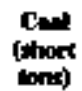 & Petin. \\
\hline
\end{tabular}

Pon' Ponm Co

Verrition (II.)

Wood Riva (II)

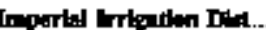

Briwley (C).

Concheln (CH)

Douth Wotr [CA

Dup No I [Ch].

Desp No. 5 (CA)

Drep 2 (C)

Drep 3 (C).

Depo 4 (CA).

E Highto $\mathrm{CH}$

El Centin (CA).

Pilod Knob fChk

Tuthin

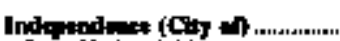

Broviley (Mo)

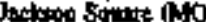

Mintoud dy (MO)

Station H aco)

Stotion I (MNO)

Pan Pontr Co.--n

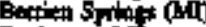

Bachengin (M)

Cometonatn

Coxk, Domid C omm

Elhte (tN)

Fourt Strat (IN)

Martinte (MI)

(10.

Boctwort (TN)

Trencer

Twin Brich (1)

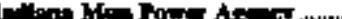

Anderco (IN) Cliny Creck (IIN)

Ldinnapots Purr a Let Co.._...

Patry $R$ (IN).

Perty W (IN)

Pextopary (IN).

Pattenged H T (IN)

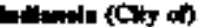

Indiolale (L)

Incerntionet Loond 8 Wang

Con

Avitum (TX)

Fiten (TX)

Encentule Pawer Co

Dutougue (IA)

Fox Lale OMD

Hit: (M)

Kap, M L (IA)

Lnetug (A).L.

Line Creek (L)

Monigorary an

Nen fith (L)

Puptortor (MN)

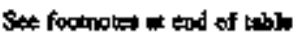

\begin{tabular}{|c|c|c|c|c|c|c|c|c|c|c|}
\hline 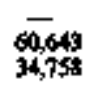 & $-_{-}^{622}$ & $\begin{array}{r}7,972 \\
11,406 \\
18,921\end{array}$ & $\bar{z}$ & $\bar{z}$ & $=$ & 年 & -1 & $\begin{array}{l}224 \\
128\end{array}$ & $-{ }^{11}$ & $\overline{-}$ \\
\hline- & -9 & 0,15 & $3,0 \leq 1$ & $=$ & - & $=$ & - & 68 & $\bar{z}$ & \\
\hline $\bar{z}$ & $\Xi$ & $-{ }^{-3}$ & 二 & 二 & - & $\bar{z}$ & $\bar{z}$ & -1 & $\bar{z}$ & \\
\hline 二 & $=$ & $\approx$ & - & - & - & - & - & - & - & - \\
\hline 二 & $\bar{z}$ & $=$ & $2,4 \pi$ & $\bar{z}$ & $\bar{Z}$ & 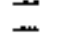 & $\bar{z}$ & z & z & 二 \\
\hline$=$ & $=$ & $=$ & $6,6.63$ & $\bar{z}$ & $=$ & $\bar{z}$ & $\bar{z}$ & $\bar{z}$ & $=$ & 二 \\
\hline$=$ & $=$ & $\bar{z}$ & $12,70\}$ & - & - & - & - & - & - & - \\
\hline 二 & 二 & 9,099 & $\underline{-}$ & $\bar{z}$ & Z & $\bar{z}$ & $\bar{z}$ & ${ }_{676}$ & $=$ & \\
\hline- & - & - & 6,846 & $=$ & $=$ & $=$ & 7 & - & 二 & - \\
\hline $\bar{z}$ & - & - & 325 & - & - & $=$ & - & - & - & - \\
\hline 36440 & 34 & 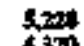 & - & $=$ & 二 & 23 & $4^{2}$ & 74 & 34 & \\
\hline- & 2 & $=$ & - & - & - & - & - & - & - & \\
\hline $\bar{z}$ & $\begin{array}{r}-101 \\
|14|\end{array}$ & $\overline{B 58}$ & $\vec{z}$ & $=$ & - & $\bar{z}$ & 7 & -15 & $-{ }^{26}$ & \\
\hline - & 268 & - & - & - & - & - & I & - & - & \\
\hline $16, m$ & 2,313 & - & int & $1,528: A n$ & - & 1,1677 & 4 & - & $1,7 n$ & \\
\hline $\bar{z}$ & $=$ & 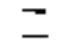 & $1,2,000$ & $=$ & 二 & $\Xi$ & 二 & 二 & 二 & 二 \\
\hline$=$ & $=$ & $\bar{z}$ & 304 & - & 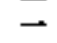 & - & $=$ & 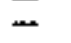 & - & - \\
\hline $\bar{z}$ & z & $\bar{z}$ & $T_{403}$ & $1,528,0402$ & $=$ & z & $\bar{z}$ & $\bar{z}$ & $\bar{z}$ & z \\
\hline z & Z & $\bar{z}$ & $\underline{-},+\infty$ & $\bar{z}$ & $\overline{-}$ & 二 & $\bar{z}$ & $\bar{z}$ & $\bar{z}$ & 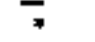 \\
\hline$x=\overline{0}$ & 7 & $\approx$ & 46. & $\bar{z}$ & $\approx$ & ${ }_{0.065}$ & - & $\bar{z}$ & $\overline{1}$ & - \\
\hline 502.522 & 579 & $=$ & $\overline{-}$ & $=$ & $=$ & 2012 & 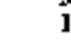 & $=$ & 221 & \\
\hline- & - & - & 2704 & - & - & - & - & - & - & - \\
\hline- & 17 & 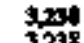 & 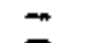 & z & z & $=$ & ; & 52 & 二 & \\
\hline $68 t=9$ & 230 & - & - & - & - & $\mathbf{3 6 3}$ & • & - & sit & \\
\hline 655,060 & 230 & - & - & - & - & 363 & - & - & 730 & \\
\hline${ }_{1,469}^{37,69}$ &, 0023 & 11,99 & $\bar{z}$ & $\bar{z}$ & $=$ & $\frac{162}{2}$ & $-^{15}$ & $\mathbf{1 3}_{2}$ & $1,1 \%$ & 38 \\
\hline-1 & -40 & - & - & - & - & - & $=$ & - & - & \\
\hline $\begin{array}{l}91,1009 \\
113,591\end{array}$ & $\begin{array}{r}207 \\
\mathbf{2 8 8 8 6}\end{array}$ & $\bar{z}$ & 二 & 二 & 二 & $\begin{array}{r}r, 8 \\
62\end{array}$ & & $\bar{z}$ & 61 & \\
\hline 265581 & 5910 & 19,57 & - & - & - & 128 & & 135 & 262 & \\
\hline$=$ & 779 & 4 & $\underline{z}$ & $\bar{z}$ & $=$ & $=$ & 1 & : & $\bar{z}$ & \\
\hline - & - & -- & 12,776 & - & - & - & - & - & - & - \\
\hline $\bar{z}$ & 二 & $=$ & $\begin{array}{l}6,630 \\
6,246\end{array}$ & $\ddot{z}$ & - & $\bar{z}$ & $\bar{z}$ & $z$ & $=$ & 二 \\
\hline $203, \pi / 2$ & 2,075 & 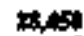 & - & - & - & 125 & 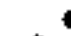 & 315 & 314 & \\
\hline 23,024 & $1 \propto 5$ & & - & - & & & $"$ & 313 & 19 & \\
\hline$=$ & $\begin{array}{r}195 \\
-2\end{array}$ & $2,2,1$ & $\bar{z}$ & 二 & $\overline{-}$ & $=$ & • & $-^{313}$ & $\bar{z}$ & $\star^{+}$ \\
\hline 80084 & - & 212 & - & - & - & 41 & $\because$ & 2 & $\mathbf{1 4 5}$ & \\
\hline 20,364 & 1,564 & - & 二 & $=$ & $\overline{-}$ & - & 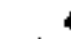 & 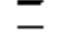 & - & \\
\hline$=$ & 119 & $=$ & $z$ & $\bar{z}$ & - & z & 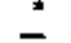 & 二 & z & . \\
\hline$=$ & - & - & - & - & - & - & - & - & - & - \\
\hline
\end{tabular}


Table 56. U.S. Electric Utility Net Generotion, Foel Concunption, and Foel Stocks by Company and Plant, Jaly 1997 (Continned)

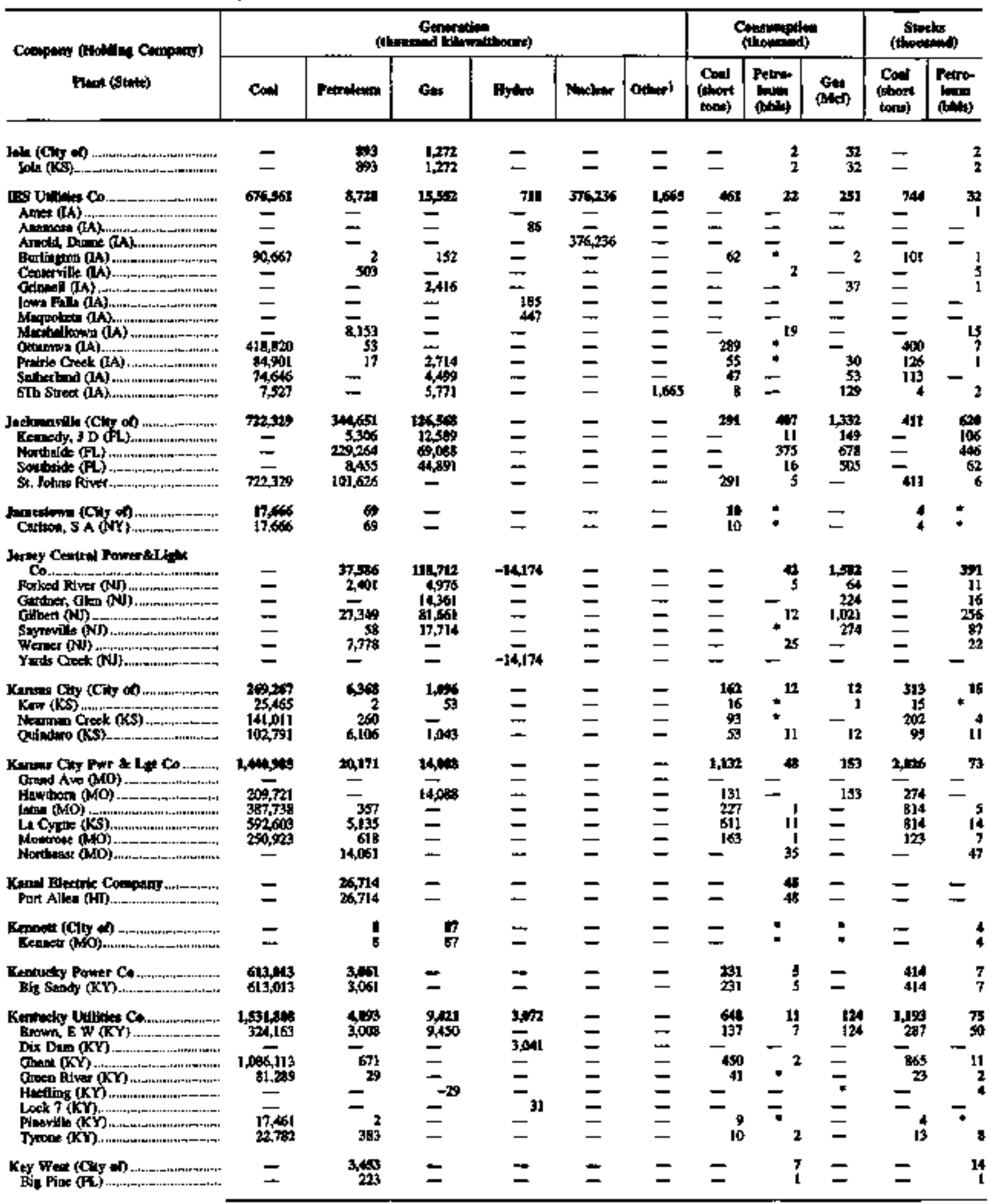

Seet fortand of and of whe 
Table 56. U.S. Electrie Utfity Net Generatton, Fut Conamuption, end Fud Stacks by Conpany and Fiant, July 1597 (Continued)

\begin{tabular}{|c|c|c|c|c|c|c|c|c|c|c|c|}
\hline \multirow{2}{*}{ 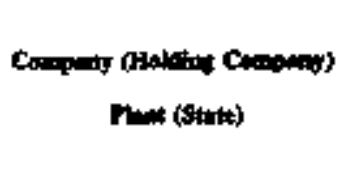 } & \multicolumn{6}{|c|}{ 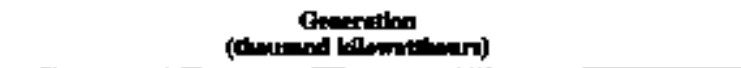 } & \multicolumn{3}{|c|}{ 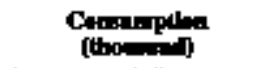 } & \multicolumn{2}{|c|}{ Sthockin } \\
\hline & col & Partite & Gm & Hying & Nodlear & Otherl & 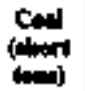 & $\begin{array}{l}\text { Patro: } \\
\text { (bit; }\end{array}$ & Garth & $\underset{\text { (nimit }}{(\cos )}$ & $\begin{array}{l}\text { Petro- } \\
\text { kath } \\
\text { abbin) }\end{array}$ \\
\hline
\end{tabular}

\begin{tabular}{|c|c|c|c|c|c|c|c|c|c|c|c|}
\hline 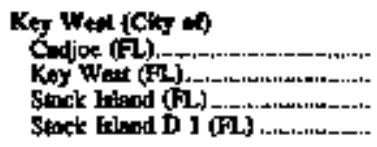 & $\bar{z}$ & $\begin{array}{r}506 \\
168 \\
366 \\
2,190\end{array}$ & $\bar{z}$ & $\bar{z}$ & $\begin{array}{l}\bar{z} \\
\bar{z}\end{array}$ & $\bar{z}$ & $\bar{z}$ & 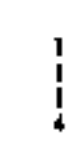 & $\bar{z}$ & $\begin{array}{l}\bar{z} \\
\bar{z}\end{array}$ & $-{ }^{1}$ \\
\hline 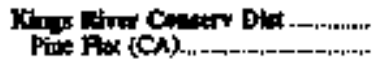 & $=$ & $=$ & $=$ & $\begin{array}{l}114287 \\
116 ; 287\end{array}$ & $=$ & $z$ & 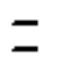 & $\bar{z}$ & $=$ & $=$ & $=$ \\
\hline 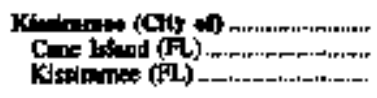 & $\bar{z}$ & $-{ }_{-3}^{-3}$ & 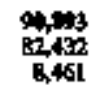 & $\bar{z}$ & $\bar{z}$ & $\bar{z}$ & $\underline{-}$ & $\ddot{*}$ & $\begin{array}{l}734 \\
629 \\
105\end{array}$ & $\bar{z}$ & 24 \\
\hline 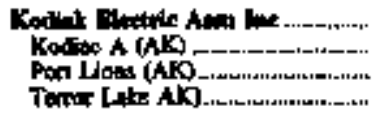 & $\begin{array}{l}\bar{z} \\
\bar{z}\end{array}$ & $\begin{array}{l}2,911 \\
2,914 \\
-3\end{array}$ & $\begin{array}{l}\bar{z} \\
\bar{z}\end{array}$ & $\begin{array}{l}1215 \\
\frac{1}{8295}\end{array}$ & $\Xi$ & $\bar{z}$ & $\bar{z}$ & $z^{5}$ & $\bar{z}$ & $\begin{array}{l}z \\
\Xi\end{array}$ & \\
\hline 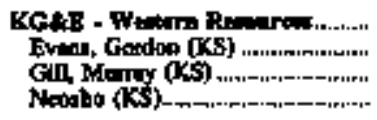 & $\bar{z}$ & $\begin{array}{r}29 \\
29 \\
179 \\
-\end{array}$ & 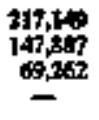 & $\begin{array}{l}\bar{z} \\
\overline{-}\end{array}$ & $\bar{z}$ & $\begin{array}{l}\bar{z} \\
\bar{z}\end{array}$ & $\begin{array}{l}\bar{z} \\
\bar{z}\end{array}$ & $\vdots$ & $\begin{array}{l}1,606 \\
1,091 \\
-919\end{array}$ & $\begin{array}{l}\bar{z} \\
z\end{array}$ & $\begin{array}{l}204 \\
199 \\
-\end{array}$ \\
\hline 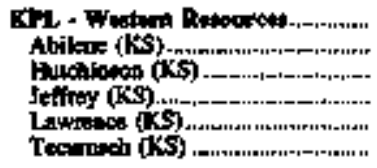 & 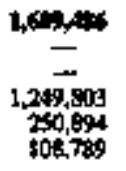 & $\begin{array}{l}2,605 \\
308 \\
2,205 \\
=\end{array}$ & 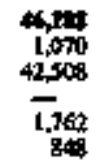 & $\begin{array}{l}\bar{z} \\
\bar{z}\end{array}$ & $\begin{array}{l}\bar{z} \\
\bar{z}\end{array}$ & $\begin{array}{l}\bar{z} \\
\bar{z}\end{array}$ & $=$ & $\begin{array}{l}-5 \\
=\end{array}$ & 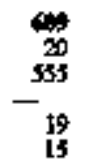 & 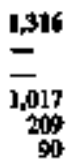 & $\begin{array}{r}136 \\
15 \\
95 \\
24 \\
2\end{array}$ \\
\hline 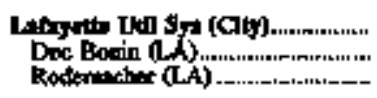 & $\bar{z}$ & $\bar{z}$ & $\underset{-16}{8,400}$ & $\bar{z}$ & $\Xi$ & $\bar{z}$ & $\bar{z}$ & $\bar{z}$ & - & $\bar{z}$ & - \\
\hline 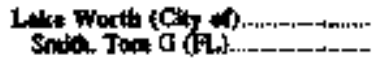 & $=$ & 66 & $\begin{array}{l}24,0904 \\
24,090\end{array}$ & $=$ & $=$ & $=$ & 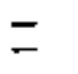 & $*$ & 209 & $=$ & 8 \\
\hline 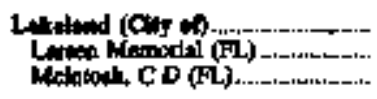 & $\frac{177,129}{177,129}$ & $\begin{aligned} \mathbf{3 1 , 1 1 7} \\
\mathbf{7 , 0 2 4} \\
26,093\end{aligned}$ & $\begin{aligned} 100,035 \\
43,365 \\
36,65 \%\end{aligned}$ & $\bar{z}$ & $\Xi$ & $\bar{z}$ & $-{ }^{n}$ & $\begin{array}{l}33 \\
16 \\
14\end{array}$ & $\begin{array}{l}1,644 \\
435 \\
609\end{array}$ & - & $\begin{array}{l}111 \\
25 \\
89\end{array}$ \\
\hline tom & $=$ & $=$ & $\begin{array}{l}10,00 \% \\
10,005\end{array}$ & $\bar{z}$ & $\bar{z}$ & $\overline{-}$ & $=$ & $=$ & $\begin{array}{l}136 \\
136\end{array}$ & $\bar{z}$ & 6 \\
\hline 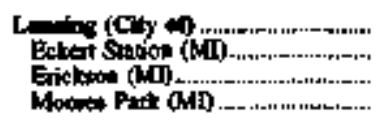 & $\begin{array}{l}154006 \\
74,806 \\
93,069 \\
-\end{array}$ & $\begin{array}{r}540 \\
-476 \\
-44\end{array}$ & $\begin{array}{l}\bar{z} \\
=\end{array}$ & $={ }^{64}$ & $\bar{z}$ & $\begin{array}{l}\bar{z} \\
\overline{-}\end{array}$ & $\begin{array}{r}3 \\
4 \\
-3 \\
-\end{array}$ & $\stackrel{1}{1}^{1}$ & $\begin{array}{l}\bar{z} \\
\bar{z}\end{array}$ & $\begin{array}{r}15 \\
15 \\
-\quad \\
-\quad\end{array}$ & * \\
\hline 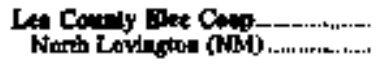 & $\bar{z}$ & $\bar{z}$ & $\bar{z}$ & $=$ & $\overline{-}$ & $=$ & $\bar{z}$ & $\bar{z}$ & $\bar{z}$ & $\bar{z}$ & $\bar{z}$ \\
\hline 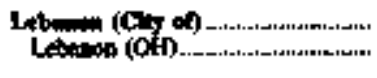 & $=$ & $\frac{228}{228}$ & $\overline{-}$ & $\overline{-}$ & $=$ & $=$ & $\bar{z}$ & * & $\bar{z}$ & $\overline{-}$ & : \\
\hline 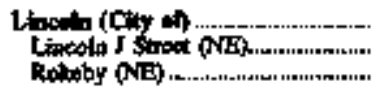 & $\bar{z}$ & $\bar{z}$ & $\begin{array}{l}1,291 \\
9,718 \\
9,716\end{array}$ & $\bar{z} \quad$ & $\Xi$ & $\Xi$ & $\bar{z}$ & $\bar{z}$ & $\begin{array}{r}137 \\
8 \\
139\end{array}$ & $\bar{z}$ & $\begin{array}{r}\mathbf{1} \\
2 \\
9\end{array}$ \\
\hline 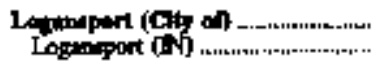 & 105 & $\bar{z}$ & $=$ & $\overline{-}$ & $=$ & $\overline{-}$ & $\frac{12}{12}$ & $=$ & $=$ & ? & $\frac{2}{2}$ \\
\hline 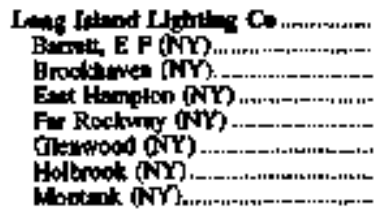 & $\begin{array}{l}\bar{z} \\
\bar{z} \\
=\end{array}$ & 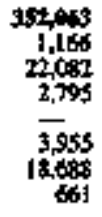 & $\begin{array}{l}76,397 \\
217,331 \\
= \\
36,347 \\
90,453 \\
=\end{array}$ & $\begin{array}{l}= \\
= \\
= \\
=\end{array}$ & $\begin{array}{l}= \\
= \\
= \\
=\end{array}$ & $\begin{array}{l}= \\
= \\
= \\
=\end{array}$ & $\begin{array}{l}\bar{z} \\
\bar{z} \\
\bar{z} \\
\bar{z}\end{array}$ & $\begin{array}{r}2 \\
20 \\
-6 \\
-\quad 10 \\
42 \\
4\end{array}$ & $\begin{array}{l}\frac{1,003}{2,409} \\
= \\
= \\
1,0100 \\
=\end{array}$ & $\begin{array}{l}= \\
= \\
= \\
=\end{array}$ & $\begin{array}{r}1,475 \\
184 \\
35 \\
3 \\
1 \\
25 \\
109 \\
0\end{array}$ \\
\hline
\end{tabular}

Soe foctmoted $x \mathrm{kAd}$ of uble. 
Table 56. US. Electric Ut:?ty Net Generotion, Fuel Cousumption, wed Ded Stocks by Company and Plant, July 1997 (Conthacei)

\begin{tabular}{|c|c|c|c|c|c|c|c|c|c|c|c|}
\hline \multirow{2}{*}{ 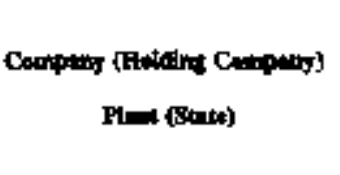 } & \multicolumn{6}{|c|}{ 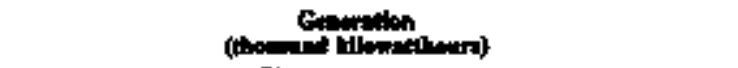 } & \multicolumn{3}{|c|}{ 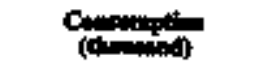 } & \multicolumn{2}{|c|}{ (thenden } \\
\hline & Coed & ש:Fodrole & G* & Fjolv & Nucle:m & Other] & (inent & 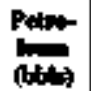 & Ge: & 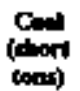 & mation \\
\hline
\end{tabular}

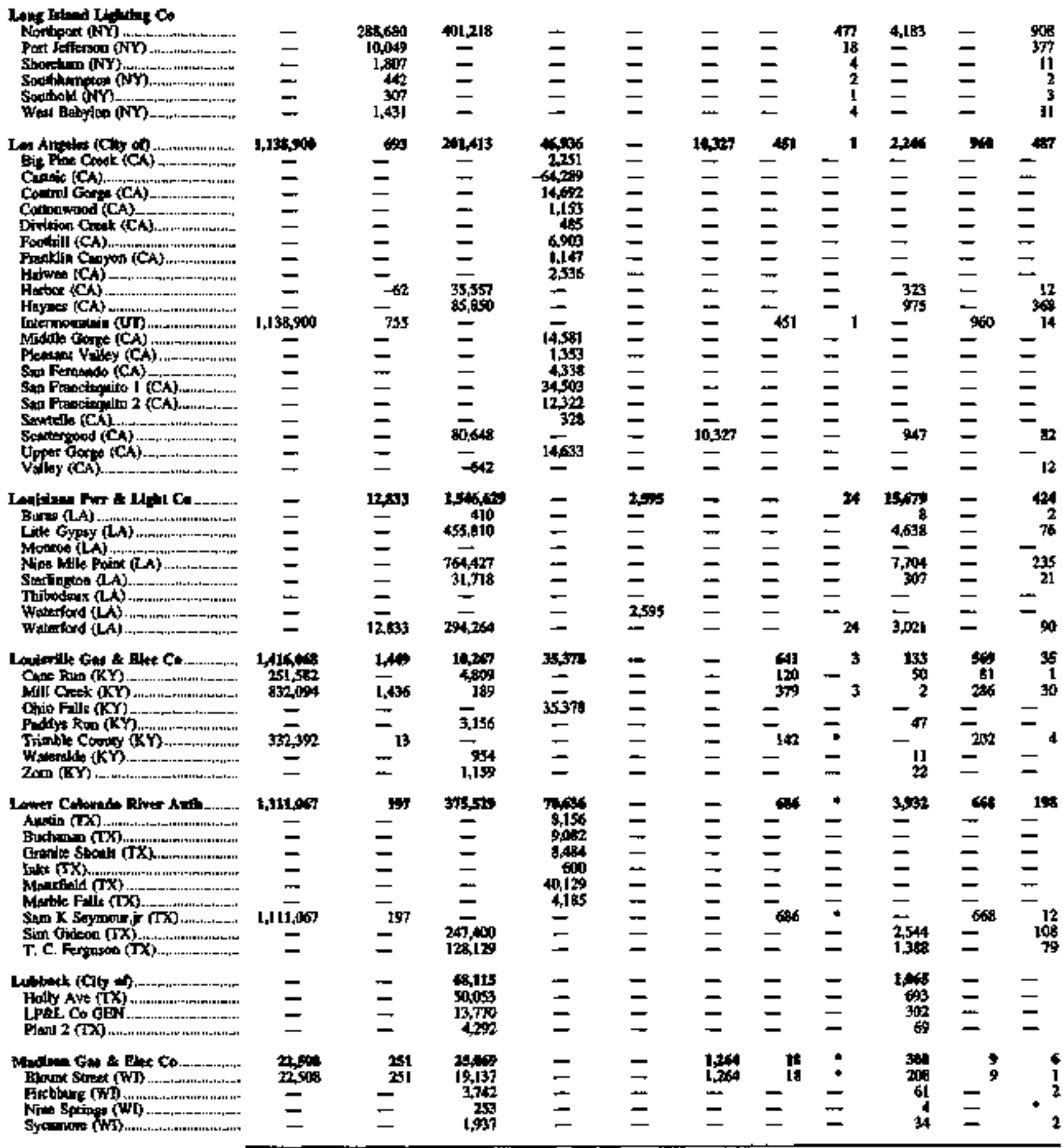

Set fooldookes an exd of tables. 
Table 56. US. Electric Utility Net Generation, Fuel Comsumpthon, and Fuel Stocks by Compatay and Plant, July 1997 (Contimasd)

\begin{tabular}{|c|c|c|c|c|c|c|c|c|c|c|c|}
\hline \multirow{2}{*}{ 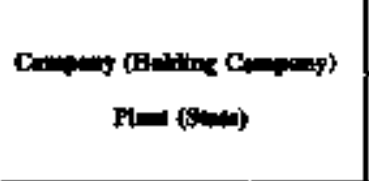 } & \multicolumn{6}{|c|}{ 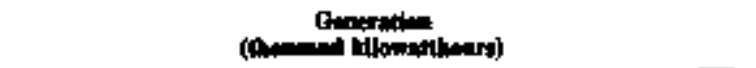 } & \multicolumn{3}{|c|}{ 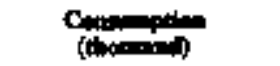 } & \multicolumn{2}{|c|}{ Shedrs } \\
\hline & البn & Pestoln & G & Bydn & Nuclener & Other] & Coal & Pen: & Giten & 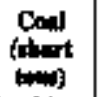 & 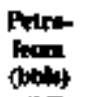 \\
\hline 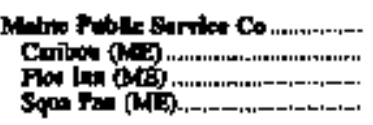 & $\begin{array}{l}\bar{z} \\
\overline{-}\end{array}$ & $\begin{array}{l}-4 \\
-44 \\
-20\end{array}$ & $\begin{array}{l}\bar{z} \\
\bar{z}\end{array}$ & $\begin{array}{r}352 \\
337 \\
-\quad 15\end{array}$ & $\begin{array}{l}\beth \\
\bar{z}\end{array}$ & $\begin{array}{l}\bar{z} \\
\bar{z}\end{array}$ & $\bar{z}$ & $\bar{z}$ & $\bar{z}$ & $\begin{array}{l}\Sigma \\
=\end{array}$ & $\begin{array}{r}1 \\
+\quad 1 \\
-\end{array}$ \\
\hline 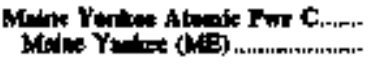 & $\overline{-}$ & $\bar{m}$ & $=$ & $=$ & 二 & $\overline{-}$ & $=$ & $\overline{-}$ & $\overline{-}$ & $\Xi$ & - \\
\hline 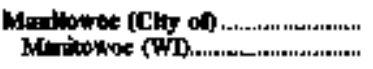 & $\begin{array}{l}20,003 \\
20,903\end{array}$ & 6,154 & 304 & $\bar{z}$ & $\overline{-}$ & $\bar{m}$ & 11 & * & t & 37 & j \\
\hline 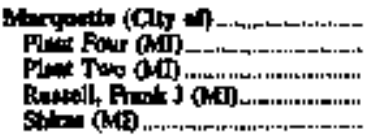 & $\frac{\bar{Z}}{23,760}$ & $\begin{array}{r}601 \\
- \\
- \\
22\end{array}$ & $\begin{array}{l}\bar{z} \\
\bar{z}\end{array}$ & $\begin{array}{l}155 \\
-115 \\
-\end{array}$ & $\begin{array}{l}\bar{z} \\
\bar{z}\end{array}$ & $\begin{array}{l}\bar{z} \\
\bar{z}\end{array}$ & $=^{17}$ & $=$ & $\begin{array}{l}= \\
=\end{array}$ & $\bar{z}^{25}$ & $z^{3}$ \\
\hline 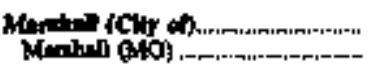 & 809 & 5 & $2, \pi$ & 二 & $\bar{z}$ & 二 & 5 & $*$ & 39 & i & t \\
\hline 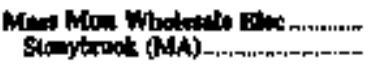 & $\overline{-}$ & $\frac{13,115}{13,285}$ & $\frac{85,43}{85,033}$ & 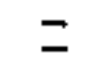 & $\bar{z}$ & 二 & $\overline{-}$ & 24 & 758 & $\Sigma$ & 243 \\
\hline 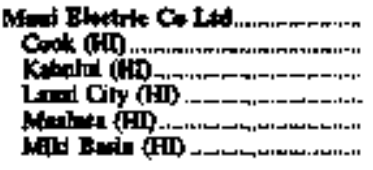 & $\begin{array}{l}= \\
= \\
= \\
=\end{array}$ & $\begin{array}{l}2,0,04 \\
3,214 \\
19,211 \\
6,1,140 \\
2,469\end{array}$ & $\begin{array}{l}\bar{z} \\
\bar{z}\end{array}$ & $\begin{array}{l}= \\
= \\
=\end{array}$ & $\begin{array}{l}= \\
\bar{z} \\
=\end{array}$ & $\begin{array}{l}= \\
= \\
=\end{array}$ & $\begin{array}{l}\bar{z} \\
\bar{z} \\
\bar{z}\end{array}$ & $\begin{array}{r}5 \\
5 \\
-4 \\
9 \\
4\end{array}$ & $\begin{array}{l}= \\
= \\
=\end{array}$ & $\begin{array}{l}\bar{z} \\
\bar{z} \\
\bar{E}\end{array}$ & $\begin{array}{r}172 \\
10 \\
16 \\
203 \\
2\end{array}$ \\
\hline 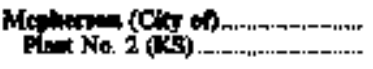 & $\bar{z}$ & 641 & 6,463 & $\overline{-}$ & $=$ & $\overline{-}$ & $=$ & $\frac{\mathbf{2}}{2}$ & s0 & $\overline{-}$ & 13 \\
\hline 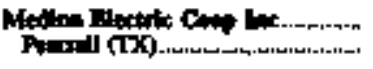 & 二 & $\overline{-}$ & 3,897 & $=$ & $\overline{-}$ & $\overline{-}$ & $\overline{-}$ & $\overline{-}$ & 45 & $\bar{z}$ & is \\
\hline 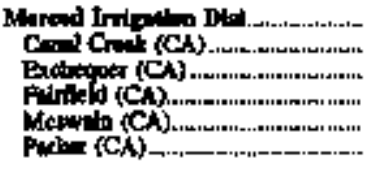 & $\begin{array}{l}\bar{z} \\
\pm \\
=\end{array}$ & $\begin{array}{l}\bar{z} \\
\bar{z} \\
\bar{z}\end{array}$ & $\begin{array}{l}\bar{z} \\
\bar{z} \\
=\end{array}$ & $\begin{array}{l}-907 \\
42,231 \\
643 \\
5,029 \\
1,194\end{array}$ & $\begin{array}{l}\bar{z} \\
\bar{z} \\
\bar{z}\end{array}$ & $\begin{array}{l}\bar{z} \\
\bar{z} \\
=\end{array}$ & $\begin{array}{l}\bar{z} \\
\bar{z} \\
\bar{z}\end{array}$ & $\begin{array}{l}\bar{z} \\
\bar{z}\end{array}$ & $\begin{array}{l}\bar{z} \\
\bar{z} \\
\bar{z}\end{array}$ & $\begin{array}{l}\bar{z} \\
\bar{z} \\
=\end{array}$ & $\begin{array}{l}= \\
\bar{z} \\
=\end{array}$ \\
\hline 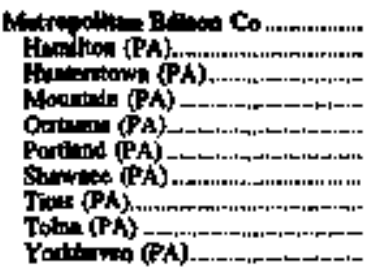 & $\begin{array}{l}30,44 \\
= \\
\bar{z} \\
221.932 \\
12 \overline{1}= \\
=\end{array}$ & 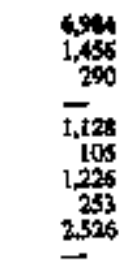 & 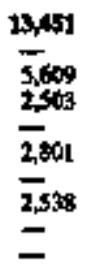 & $\begin{array}{l}2,37 \\
= \\
= \\
= \\
=\end{array}$ & $\begin{array}{l}\bar{z} \\
\bar{z} \\
\bar{z} \\
\bar{z}\end{array}$ & $\begin{array}{l}\bar{z} \\
= \\
= \\
= \\
=\end{array}$ & $\begin{array}{l}= \\
= \\
= \\
=\end{array}$ & $\begin{array}{r}3 \\
-3 \\
-3 \\
-3 \\
-3\end{array}$ & $\begin{array}{l}-78 \\
-78 \\
-77 \\
-27 \\
=\end{array}$ & $\begin{array}{l}{ }^{186} \\
= \\
= \\
= \\
=\end{array}$ & $\begin{array}{r}76 \\
3 \\
6 \\
6 \\
3 \\
47 \\
2 \\
5 \\
-5\end{array}$ \\
\hline 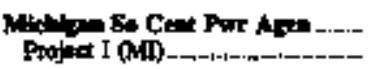 & 2,069 & 227 & $=$ & $\overline{-}$ & $\overline{-}$ & $\overline{-}$ & 5 & $\mathbf{1}$ & $\overline{-}$ & 20 & s \\
\hline 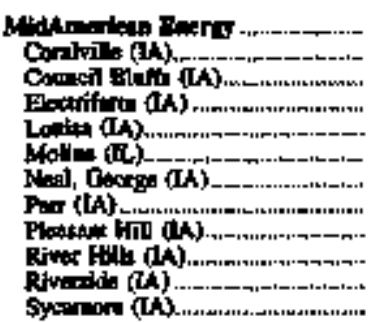 & 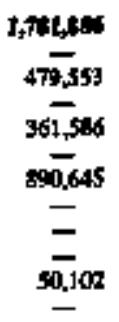 & $\begin{array}{l}1,730 \\
E_{332} \\
= \\
=75 \\
= \\
=\end{array}$ & 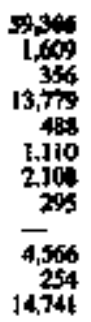 & $\begin{array}{l}= \\
= \\
= \\
= \\
= \\
=\end{array}$ & $\begin{array}{l}= \\
= \\
= \\
= \\
= \\
=\end{array}$ & $\begin{array}{l}= \\
= \\
= \\
= \\
= \\
=\end{array}$ & $\begin{array}{l}1,118 \\
-313 \\
-2046 \\
-535 \\
= \\
- \\
{ }_{34}\end{array}$ & $\begin{array}{l}=4 \\
=1 \\
= \\
=1 \\
=\end{array}$ & $\begin{array}{r}568 \\
24 \\
4 \\
185 \\
5 \\
20 \\
25 \\
-5 \\
70 \\
3 \\
218\end{array}$ & $\begin{array}{l}2013 \\
-5965 \\
- \\
\overline{10015} \\
= \\
= \\
=\end{array}$ & $\begin{array}{r}60 \\
13 \\
10 \\
10 \\
8 \\
2 \\
6 \\
2 \\
9 \\
-\quad 4 \\
\end{array}$ \\
\hline
\end{tabular}

Sop foomous as and of uble. 


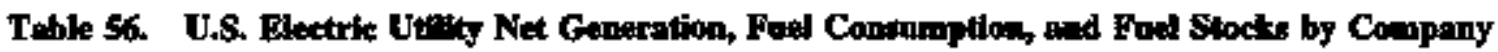
and Plant, July 1999 (Conftioned)

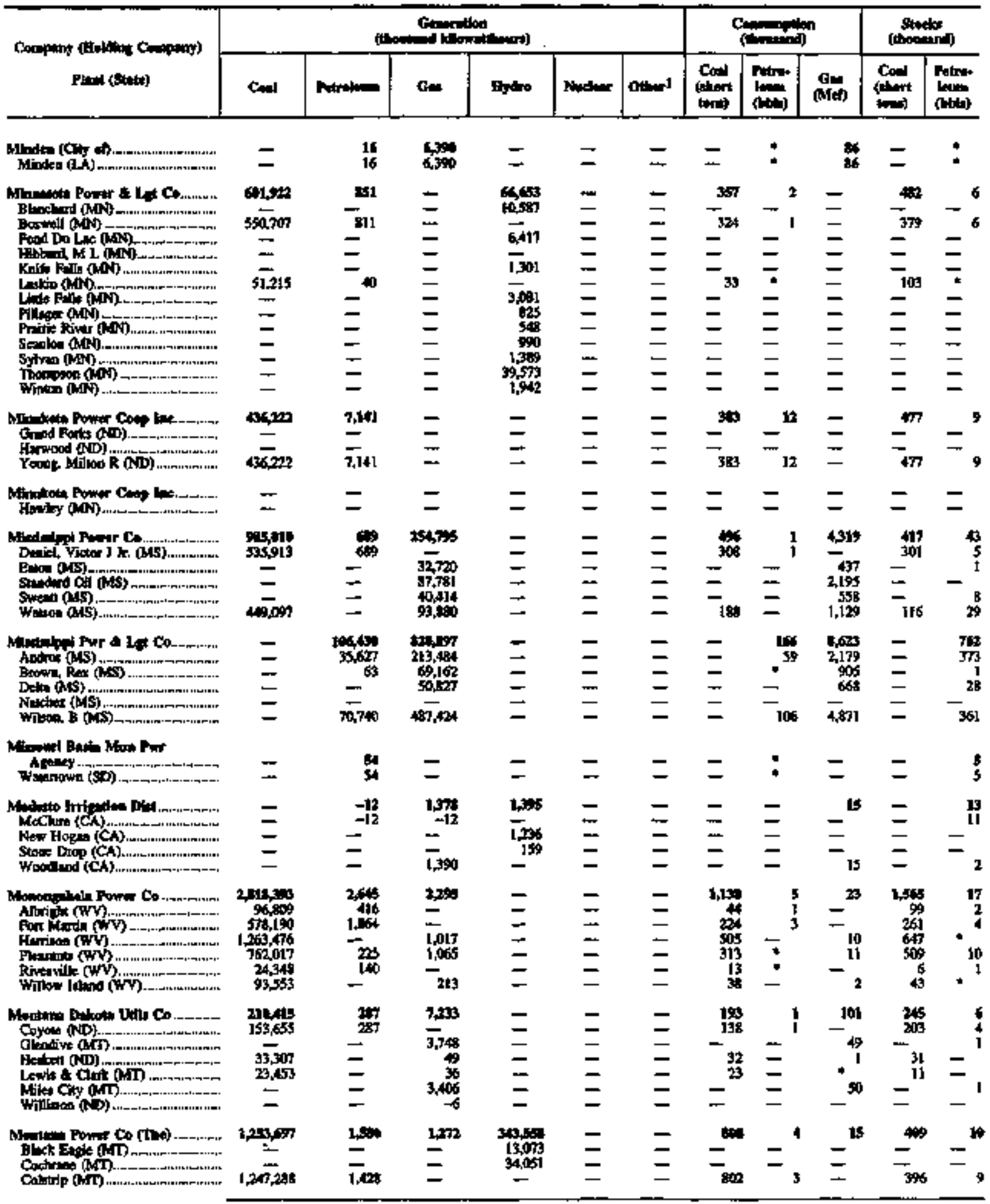

San frotnotes at and of the. 
Tahle S6. U.S. Fleetric traliky Net Gearotion, Fuel Consunption, and Foef Stocks by Compong and Plant, Joly 1997 (Continned)

\begin{tabular}{|c|c|c|c|c|c|c|c|c|c|c|c|}
\hline \multirow{2}{*}{ 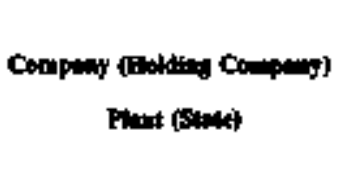 } & \multicolumn{6}{|c|}{ 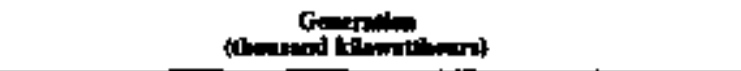 } & \multicolumn{3}{|c|}{ Consing } & \multicolumn{2}{|c|}{ 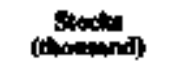 } \\
\hline & Cod & Potridean & Ga: & Aydre & Ninelwer & 1] & $\begin{array}{l}\text { Ced } \\
\text { (thert } \\
\text { toon) }\end{array}$ & Patren. & Gald & $\underset{\substack{\text { Cond } \\
\text { loont }}}{\log )}$ & (imitis \\
\hline
\end{tabular}

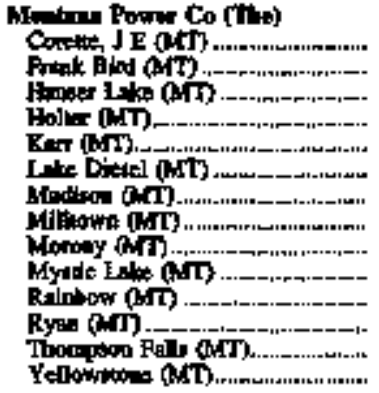

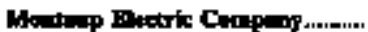
Sopmenox (MA)

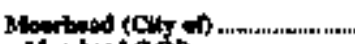

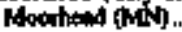

Moritit (Cits of

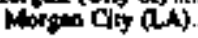

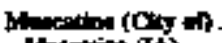
Masction: (T).

N Y Suan riet 4 Gan Cons ---Catrvilk (NY)

Goudey (NY)

Greer

Geen to (Ny)

Fiotilie (ONY)

Fintivitiv

Jamion (NY)

Kaits Paid (NY)

CaY

Mitiks ONY

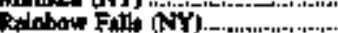

Solper Fetio (NV)

sowerset (NQY)

Wotrló (NY)

$=$
$=$
$=$
$=$
$=$
$=$
$=$
$=$

$=$
$=$
$=$
$=$
$=$
$=$
$=$

1,272
$=$
$=$
$=$
$=$
$=$
$=$

$\frac{-}{11,727}$

$7 \times$

77326

$1483=$

二 $=$

$=\quad=7,02$

군도

I2T,436

711:45

70550

$60 \%$

2i, 11,

$\overline{17,850}$

-

-

18982

18982

$\overline{-}$

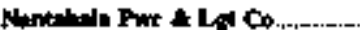

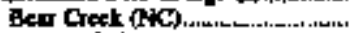
Bryson (x)

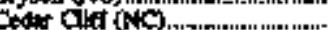

Dillbors (NC)

Pranifion (NC).

Mision (NC)

Nabinta (ANC)

Qument Crat (NC)

Tenouse Creck (NC)

Therpe (NK)

Tuckoroce (NT)

Noninglite Elac Co

Ninnacket (MA)

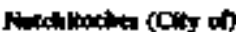

Namchitocibe (Li)

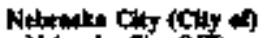

Nurask City (N.F).

Syration No 2 (NE)

Nebrendt Puld Rawer Dibet

Sex loaloous in tod of thite

1,7

I

$-$

$--^{654}=$

$=$

$=\quad \bar{z}$

$\bar{z}$

$=\quad \bar{z}$

$\bar{z}=$

$\bar{z}=$

ב

- $\quad$ 11

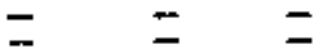

$-$

-

ascing

$18 x$

465

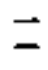

$=$

\begin{tabular}{|c|c|c|}
\hline- & - & $=$ \\
\hline$[\overline{1,727}$ & - & \\
\hline 33,787 & - & - \\
\hline 115.222 & $\bar{z}$ & - \\
\hline,+ 384 & - & - \\
\hline 3917 & - & 7 \\
\hline 7,952 & - & - \\
\hline 42602 & $=$ & 2 \\
\hline 32,6425 & 二 & 7 \\
\hline & & 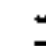 \\
\hline
\end{tabular}

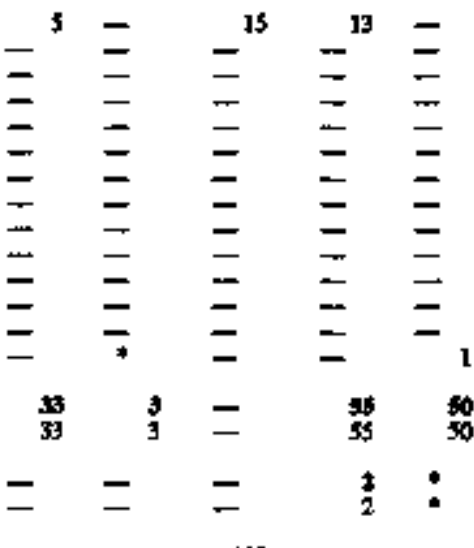

二 二 ニ ニ

二 $=$ ニ $79=176$

\section{tos}

15

= $=$

$\overline{6} \times$

$\overline{4}_{116}$

$\overline{3054}$

2025

$\overline{1,145}$

37,462
2,962
428

2,177

9.

20,599

3,375

6,17

$\rightarrow$

-

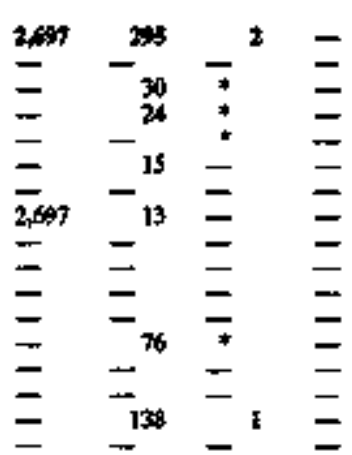

$16-1$
$-{ }_{13}^{13}-1$
$-{ }^{12}=$
$-{ }^{8}=$
$= \pm$
$=11=$
$={ }_{92}=$

$-$

= $=$

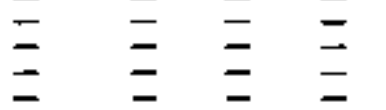

$\bar{z}=$

$=\quad=$

$\bar{z}$

1 
Table 56. U.S. Fleetric Utility Net Generation, Fuel Comsuraption, and Fuel Stocks by Canpang and Plont, July 1997 (Condinued)

\begin{tabular}{|c|c|c|c|c|c|c|c|c|c|c|c|}
\hline \multirow{2}{*}{ 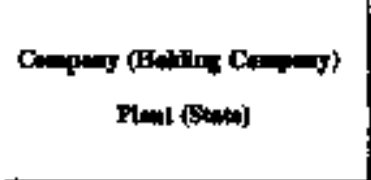 } & \multicolumn{6}{|c|}{ 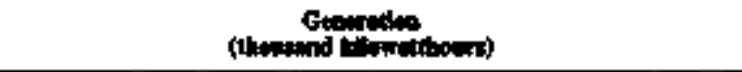 } & \multicolumn{3}{|c|}{ Cot } & \multicolumn{2}{|c|}{ (t) } \\
\hline & Cod & Pranolen & An & Ptrte & Natele: & اعي & $\underset{i=1}{\left(\lim _{i=1}\right.}$ & (1) & (iven & Cont & 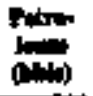 \\
\hline 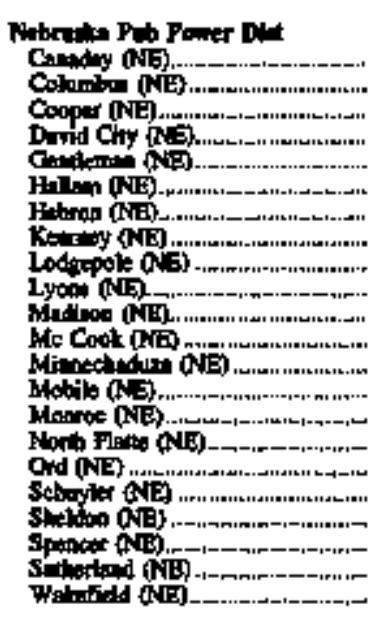 & 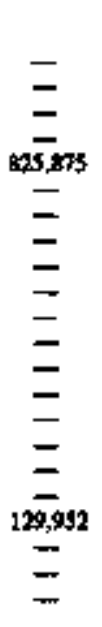 & $\begin{array}{l}= \\
= \\
= \\
-613 \\
-16 \\
91 \\
= \\
= \\
= \\
= \\
= \\
210 \\
165\end{array}$ & 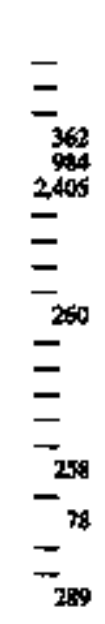 & $\begin{array}{l}\overline{7,49} \\
= \\
= \\
\overline{13} \\
= \\
= \\
\overline{1} \\
1,94 \\
\bar{z} \\
\overline{1,253} \\
=\end{array}$ & $\begin{array}{l}\overline{=} \\
= \\
= \\
= \\
= \\
= \\
= \\
= \\
= \\
= \\
=\end{array}$ & $\begin{array}{l}\bar{z} \\
\bar{z} \\
\bar{z} \\
\bar{z} \\
\bar{z} \\
\bar{z} \\
\bar{z} \\
\bar{z}\end{array}$ & $\begin{array}{l}= \\
= \\
= \\
= \\
= \\
= \\
= \\
= \\
= \\
=\end{array}$ & $\begin{array}{l}= \\
= \\
=1 \\
: \\
* \\
= \\
= \\
= \\
= \\
=\end{array}$ & $\begin{array}{l}= \\
= \\
\\
= \\
= \\
=3 \\
= \\
= \\
=3 \\
= \\
= \\
3\end{array}$ & $\begin{array}{l}= \\
= \\
= \\
= \\
= \\
= \\
= \\
= \\
= \\
= \\
=\end{array}$ & $\begin{array}{l}\overline{7} \\
\quad 6 \\
3 \\
3 \\
\vdots \\
= \\
= \\
= \\
= \\
= \\
* \\
*\end{array}$ \\
\hline 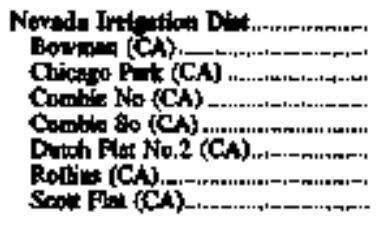 & $\begin{array}{l}\bar{z} \\
= \\
= \\
\bar{z}\end{array}$ & $\begin{array}{l}= \\
= \\
= \\
=\end{array}$ & $\begin{array}{l}\bar{z} \\
\bar{z} \\
\bar{z} \\
=\end{array}$ & 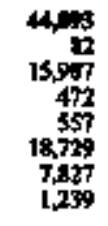 & $\begin{array}{l}\bar{z} \\
\bar{z} \\
\bar{z} \\
\bar{z}\end{array}$ & $\begin{array}{l}\bar{z} \\
\bar{z} \\
\bar{z}\end{array}$ & $\begin{array}{l}= \\
= \\
= \\
=\end{array}$ & $\begin{array}{l}= \\
= \\
\bar{z} \\
=\end{array}$ & $\begin{array}{l}= \\
= \\
= \\
=\end{array}$ & $\begin{array}{l}= \\
= \\
= \\
=\end{array}$ & $\begin{array}{l}\bar{z} \\
\bar{z} \\
\bar{z}\end{array}$ \\
\hline 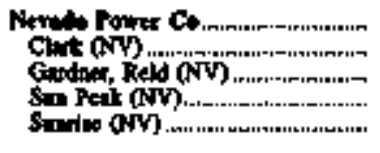 & 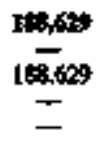 & $\begin{array}{l}206 \\
-296\end{array}$ & $\begin{array}{l}21,45 \\
34,343 \\
36,460 \\
39,1 \% 0\end{array}$ & $\begin{array}{l}= \\
=\end{array}$ & $\begin{array}{l}= \\
= \\
=\end{array}$ & $\begin{array}{l}\bar{z} \\
\bar{z}\end{array}$ & $\begin{array}{l}\text { 10 } \\
-\end{array}$ & $-^{4}$ & $\begin{array}{l}\text { 3,045 } \\
\frac{595}{417}\end{array}$ & $\begin{array}{l}567 \\
5 \\
=\end{array}$ & $\begin{array}{r}67 \\
-11 \\
19\end{array}$ \\
\hline 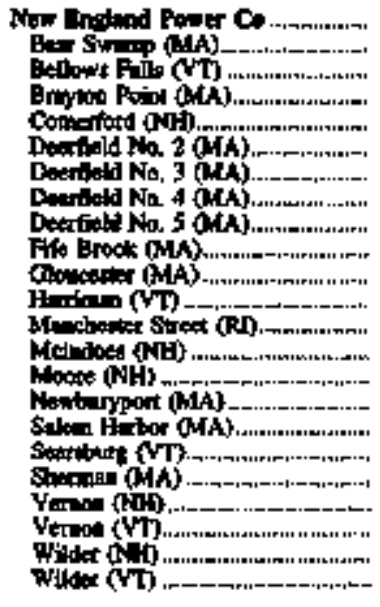 & 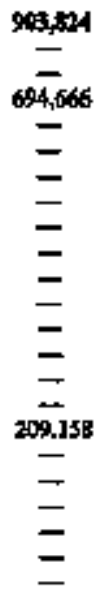 & $\begin{array}{l}121,136 \\
= \\
= \\
= \\
= \\
= \\
\overline{25 n} \\
= \\
= \\
= \\
124,507 \\
= \\
= \\
= \\
=\end{array}$ & 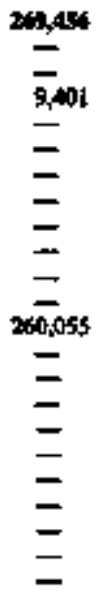 & 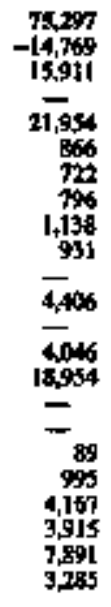 & $\begin{array}{l}= \\
= \\
= \\
= \\
= \\
= \\
= \\
= \\
= \\
= \\
= \\
=\end{array}$ & $\begin{array}{l}\bar{z} \\
\bar{z} \\
= \\
\bar{z} \\
\bar{z} \\
= \\
= \\
\bar{z} \\
= \\
= \\
\bar{z}\end{array}$ & $\begin{array}{l}Z^{201} \\
= \\
= \\
= \\
= \\
= \\
= \\
= \\
= \\
= \\
=\end{array}$ & $\begin{array}{l}z^{21} \\
= \\
= \\
= \\
= \\
= \\
= \\
= \\
= \\
= \\
= \\
=\end{array}$ & $\begin{array}{l}2130 \\
= \\
= \\
= \\
= \\
= \\
= \\
2005 \\
= \\
= \\
= \\
= \\
= \\
=\end{array}$ & $\begin{array}{l}= \\
= \\
= \\
= \\
= \\
= \\
= \\
= \\
= \\
= \\
= \\
= \\
=\end{array}$ & $\begin{array}{l}= \\
=397 \\
= \\
= \\
= \\
=3 \\
= \\
= \\
= \\
= \\
=\end{array}$ \\
\hline 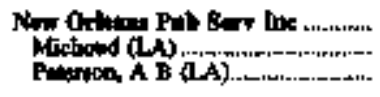 & $=$ & $\frac{254}{254}$ & $\frac{25,53}{357,532}$ & $\bar{z}$ & $\bar{z}$ & $\bar{z}$ & $\bar{z}$ & -1 & $\frac{3,100}{3,600}$ & $=$ & $\frac{130}{30}$ \\
\hline 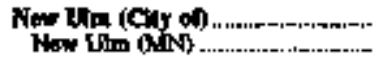 & $\overline{-}$ & 1.049 & 20003 & $\overline{-}$ & $\overline{-}$ & $\bar{z}$ & $=$ & $\mathbf{t}$ & 39 & 3 & 3 \\
\hline
\end{tabular}

Sed footronat at and of tobl. 
Twble S6. U.S. Elactric Utilty Net Generation, Fod Consumption, and Fetl Stocks by Company and Pant, July 1997 (Contheed)

\begin{tabular}{|c|c|c|c|c|c|c|c|c|c|c|c|}
\hline \multirow{2}{*}{ 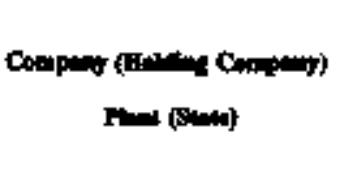 } & \multicolumn{6}{|c|}{ 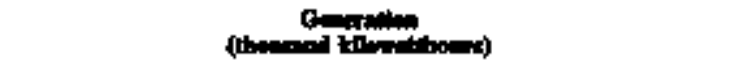 } & \multicolumn{3}{|c|}{ 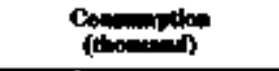 } & \multicolumn{2}{|c|}{ Stactir } \\
\hline & Curt & 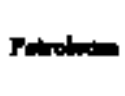 & An: & fitro & Norer & onar & $\underset{\text { (imit }}{\text { Cond }}$ & Patrop & Ames & 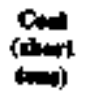 & $\begin{array}{l}\text { Purte } \\
\text { peinge) }\end{array}$ \\
\hline
\end{tabular}

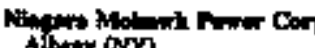

Albut (NY)

Alliw Ents (N)

Bandion ONY

bevont at.

Betwete Bdipe oNY

Bloct Bur (NY)

Bation (AFY).

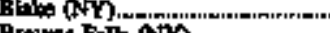

Chan (NY)

Coling (NY)

befint (NY)

burist (NY)

Enive (MY) -

E.r. Woth ovr

EA 매영 (NY)

Ei,isy (MY)

Bon (NY) -

Equats (NY).

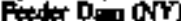

Hue Fill: (NY)

Plat Roct (NY)

Pritif (NN

Putuan (Nin)

Glownood QNY

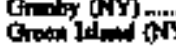

Herring $\mathrm{AYY}$

itovelow otr

Fot Dind (NY)

It th Fin (NY)

Fister (NT)

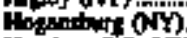

Elithy, CR (NY)

Iejum ONY

Kantas (N)'

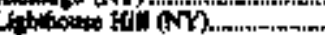

Maxanb (NY).

Machriente (NY).

Minfto (NY)

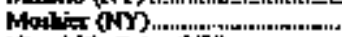

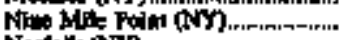

Norfolk (NY)

Norwood (NY)

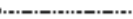

(1)

Osmepthie (NY) ...................

Dowsto (NY)

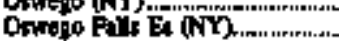

Orwoto Eill W/ aN

Putshill oNY

Profeld (NY)

Porpecd (NY)

Eutanow an

(a)

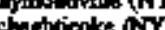

school sitet (NYY

Scturylarvile (NY)

Sewol (WY)

so Glen PIh ont

Sot Mape (NY).

Sour Colon (N)

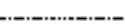

Sowit Bdwand (NY)

Spen Folh WNY

sudt an?

\begin{tabular}{|c|c|c|}
\hline ست CS1,101 & 2,161 & $\begin{array}{l}1311 \\
6 \times 71\end{array}$ \\
\hline- & - & - \\
\hline - & - & - \\
\hline - & 一 & - \\
\hline - & - & - \\
\hline - & 一 & - \\
\hline - & $\bar{z}$ & 二 \\
\hline E & - & $=$ \\
\hline- & $=$ & $=$ \\
\hline 一 & - & - \\
\hline & - & - \\
\hline - & - & 一 \\
\hline 2388898 & 1,539 & - \\
\hline - & - & عب \\
\hline - & - & 一 \\
\hline - & - & - \\
\hline - & - & $\overline{-}$ \\
\hline - & - & - \\
\hline 一 & - & - \\
\hline 一 & - & - \\
\hline - & 一 & 一 \\
\hline- & - & - \\
\hline- & 一 & - \\
\hline$\rightarrow$ & - & - \\
\hline$\longrightarrow$ & - & - \\
\hline - & $\vec{z}$ & - \\
\hline- & ב. & - \\
\hline 一 & - & $=$ \\
\hline- & - & 一 \\
\hline - & - & 一 \\
\hline$=$ & 一 & - \\
\hline$\overline{t a t}$ & - & - \\
\hline $3 T 2,205$ & 35 & - \\
\hline - & - & - \\
\hline$\sigma$ & - & - \\
\hline - & - & - \\
\hline 一 & - & - \\
\hline - & - & 一 \\
\hline - & - & $\boldsymbol{\omega}$ \\
\hline - & - & - \\
\hline 二 & - & - \\
\hline - & $\boldsymbol{T}$ & - \\
\hline 二 & $=$ & - \\
\hline - & - & - \\
\hline$\rightarrow$ & ع & - \\
\hline 一 & 38,667 & 8,521 \\
\hline - & - & - \\
\hline 一 & - & - \\
\hline 一 & - & - \\
\hline - & 一 & - \\
\hline 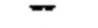 & $\rightarrow$ & - \\
\hline$\rightarrow$ & - & - \\
\hline 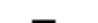 & - & عب \\
\hline - & $=$ & - \\
\hline - & - & - \\
\hline 一 & 一 & 一 \\
\hline - & 一 & عـ \\
\hline & - & - \\
\hline & - & 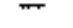 \\
\hline - & z & $m$ \\
\hline & & - \\
\hline & - & - \\
\hline
\end{tabular}

Becton Letud (Nin)

tranowion (N)

Jolneomile (NY)

Bvern Ind aNy

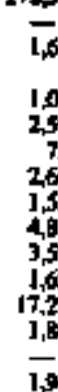

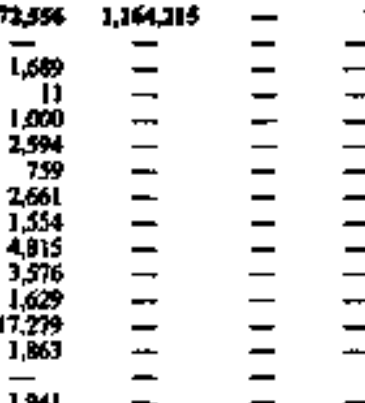

1341

2391

286

$\infty 0$

1.354

7,494

854

$=$

5:532

2,34

5,180

5,180

1,367

2,492

19

ist

629

132

319

286

$-277$

1,154

2612

2763

1,04

20.7

二

$\overline{1.29}$

$1,-2$

1534

2901

796

1,364

1,344

7,701

51

9,689

1,900

6,271

1,405

13,005
$73 \% 5$

$\bar{z}$

$\bar{z}$ $\begin{array}{lllll}204 & 404 & 16 & 394\end{array}$

$=$

-

=

-

-

$=$

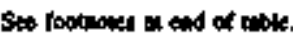


Table 56. U.S. Flectric Utility Net Generation, Foel Conganeption, and Fuel Stocks by Company and Plant, Jutity 1997 (Comtinaned)

\begin{tabular}{|c|c|c|c|c|c|c|c|c|c|c|c|}
\hline \multirow{2}{*}{ 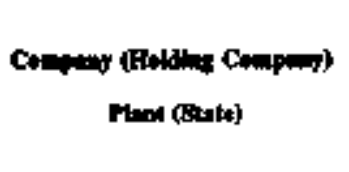 } & \multicolumn{6}{|c|}{ 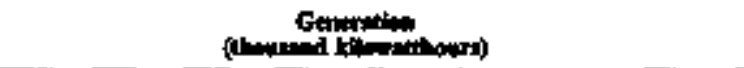 } & \multicolumn{3}{|c|}{ Cimpotion } & \multicolumn{2}{|c|}{ (thincts } \\
\hline & Con & Potroluten & G.: & Ejyto & Nochan & Otwerl & 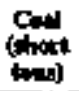 & Pars & Gad & 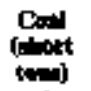 & (beta) \\
\hline
\end{tabular}

Ninow Matow Puower Corp

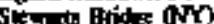

Snomitat Fifil (NY)

Sury band ANY

Tarinavile ory

Tretion (NY)

$\checkmark$ eick (AT)

Wampen (N)

We-t $\mathrm{B} J$ (NY)

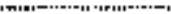

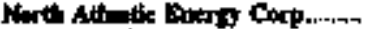

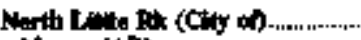
Merny (AR)

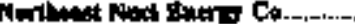
Millito (CT)

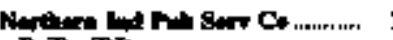
Binly (IN).

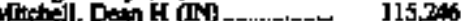

Norinty (II).

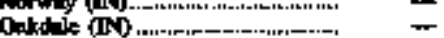

schiniter, R M- (T)

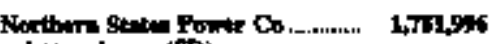

Atipu Aneon (SD)

Apile Rive (WT).

Bay Poot (WP)

Bie Fills (WT)

Bteck Doi ( Min)

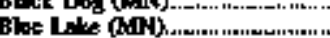

Cod- Ralls (WT)

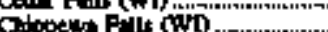

Corten (WD

Dells (MT)

Panch

Ga: Cin QMOS)

Hinsid (WT)

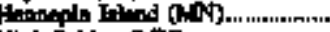

Heb Bidga an(N)

Holerarbe (w)

tover Hits (MAN)

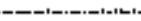

Soy Cin MN

King (kon)

Lady:mith (WT)

(1)

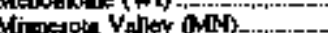

slocicelb (N)

Publifider (SD)

Pritila laland (MDN)

Redist (AN)

Divetide aMo

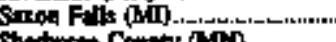

Shenbutw Cowny (MND)

Ft Credr Fill (WD).

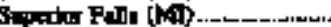

Thate gite (WI) ...

Thets [W]

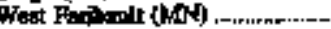

Wherbon (WD.

winte Erter (N)

Wianta (Wt).

13.67

13,67

|II,

=

$=$

二

二

$\overline{128,002}$

$=$

-

28471

2847

-

-

$=$

200,62

1,042,85?

-

-

E

-

E

$=$
$=$
$=$
$=$

9,740

-
437
101
1.356
5,975
492
794
5675
534

$-$

2674

26,747

$=$

4hass

$-$

-

-

10,466

51766

-

$=$

$-$

6,591

-

$=$

-7ms

$\underline{-}$

-

-

10,349

-

41,635

-

$-13$

-

-

17850

1.065

E

-

$\sqrt{6,27 s}$

-

$+$

$=$

206s

2589

-

61,597

工

4,995

4,238

-

-

$\overline{1}, 13 ?$

6.685

-

2499

-

6,165

-

276

551

170

109

$6+3$

4870

-

3.60

7,435

S.5.3

4,765

-

-

139

6.716

9,399

13,015

…

1. 353

2,521

-

=

$=$

29

551

10,694

1.010

-
- 1040

$\begin{array}{llll}= & = & = & = \\ = & = & = & = \\ = & = & = & = \\ = & = & = & = \\ = & = & = & = \\ = & = & = & = \\ & & & \end{array}$

$\begin{array}{lll}= & = & = \\ = & = & = \\ = & = \\ = & = \\ = & =\end{array}$

832,93: - $=$ - $=$

二 $\quad$ - $\quad$ - $\quad$ - $\quad$ - $\quad$ -

$\begin{array}{llllll}\rightarrow, 135 & - & - & - & - & -\end{array}$

4607 -

$=$
$=$
$=$
$=$

natis 7x,183

$\frac{414}{54,125}$
$=$
$=$
$=$
$=$
$=$
$=$
$=$
$=$
$=$
$=$

$-2,500$

$7 \pi$

$=$

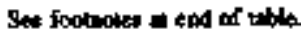


Table 56. U.S. Electric Utilty Net Geaeration, Fued Consumptiou, and Fuel Stocks by Company and Pant, July 1997 (Continned)

\begin{tabular}{|c|c|c|c|c|c|c|c|c|c|c|c|}
\hline \multirow{2}{*}{ 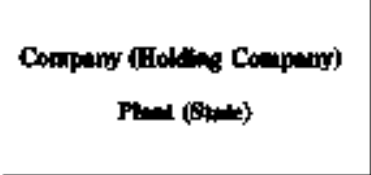 } & \multicolumn{6}{|c|}{ (thenrind titition } & \multicolumn{3}{|c|}{ 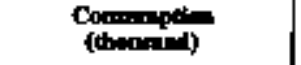 } & \multicolumn{2}{|c|}{ 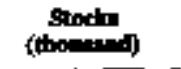 } \\
\hline & cm: & Petroleum & Gat & Kagho & Now & 10ther & Cind & Pots & Gent) & Cost & $\begin{array}{l}\text { Pitto: } \\
\text { lewis } \\
\text { (bbis) }\end{array}$ \\
\hline 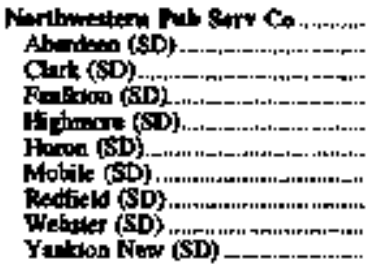 & $\begin{array}{l}\bar{z} \\
\bar{z} \\
\bar{z} \\
\bar{z}\end{array}$ & $\begin{array}{r}283 \\
-\quad 36 \\
-\quad 46 \\
- \\
31\end{array}$ & $\begin{array}{l}5,34 \\
= \\
= \\
3,994 \\
-{ }_{310}\end{array}$ & $\begin{array}{l}\bar{z} \\
= \\
= \\
= \\
=\end{array}$ & $\begin{array}{l}\bar{z} \\
= \\
= \\
= \\
= \\
=\end{array}$ & $\begin{array}{l}= \\
= \\
= \\
= \\
=\end{array}$ & $\begin{array}{l}\bar{z} \\
\bar{z} \\
\bar{z} \\
\bar{z}\end{array}$ & $\dot{:}^{1}$ & $\begin{array}{l}= \\
= \\
= \\
= \\
-1\end{array}$ & $\begin{array}{l}\bar{z} \\
\bar{z} \\
\bar{z} \\
\bar{z}\end{array}$ & ${ }^{+4}$ \\
\hline 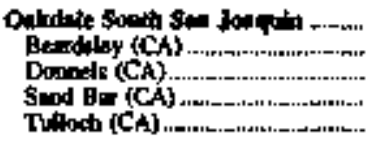 & $\begin{array}{l}\bar{z} \\
\bar{z}\end{array}$ & $\begin{array}{l}\vec{z} \\
=\end{array}$ & $\begin{array}{l}\bar{z} \\
\bar{z}\end{array}$ & $\begin{array}{r}9,84 \\
8,119 \\
39,731 \\
9,827 \\
13,129\end{array}$ & $\begin{array}{l}= \\
\bar{z}\end{array}$ & $\begin{array}{l}\bar{z} \\
\bar{z}\end{array}$ & $\begin{array}{l}\overline{-} \\
\bar{z}\end{array}$ & $\begin{array}{l}\bar{z} \\
\bar{z}\end{array}$ & $\begin{array}{l}= \\
= \\
=\end{array}$ & $\begin{array}{l}\bar{z} \\
\bar{z}\end{array}$ & $\begin{array}{l}\bar{z} \\
\bar{z}\end{array}$ \\
\hline 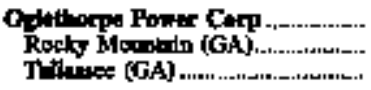 & $\overrightarrow{-}$ & $\bar{z}$ & $\bar{z}$ & $\begin{array}{r}-56905 \\
899\end{array}$ & $=$ & $\bar{z}$ & $\bar{z}$ & $\bar{z}$ & 二 & $\bar{z}$ & $\bar{z}$ \\
\hline 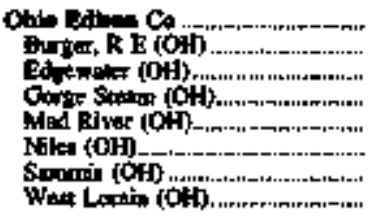 & $\begin{array}{c}1,591,254 \\
207,509 \\
= \\
\overline{1} \\
1,24,564,181 \\
-\end{array}$ & $\begin{array}{r}1,214 \\
191 \\
-\quad 85 \\
16 \\
65 \\
913 \\
-\end{array}$ & $\begin{array}{l}\frac{9,24}{92 * 6} \\
= \\
= \\
=\end{array}$ & $\begin{array}{l}= \\
= \\
= \\
=\end{array}$ & $\begin{array}{l}\bar{z} \\
\bar{z} \\
= \\
=\end{array}$ & $\begin{array}{l}\bar{z} \\
\bar{z} \\
\bar{z}\end{array}$ & 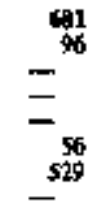 & $i^{3}$ & $\begin{array}{l}=10 \\
= \\
= \\
=\end{array}$ & $\begin{array}{l}110 \\
= \\
= \\
-531 \\
031\end{array}$ & $\begin{array}{r}34 \\
2 \\
-\quad 6 \\
15 \\
6 \\
-3\end{array}$ \\
\hline 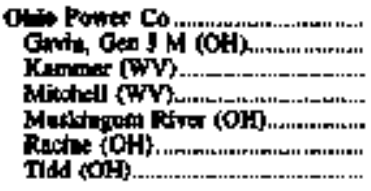 & $\begin{array}{c}2,85,59 \\
916,051 \\
452,794 \\
307,734 \\
689,020 \\
= \\
-\end{array}$ & $\begin{array}{l}1,403 \\
1,200 \\
1,733 \\
1,508 \\
-\end{array}$ & $\begin{array}{l}= \\
= \\
= \\
=\end{array}$ & $\begin{array}{l}27,100 \\
= \\
\bar{z} \\
2 \pi, 500\end{array}$ & $\begin{array}{l}\bar{z} \\
\bar{z} \\
\overline{-}\end{array}$ & $\begin{array}{l}= \\
\bar{z} \\
= \\
=\end{array}$ & $\begin{array}{l}1,19 \\
404 \\
183 \\
327 \\
290 \\
- \\
-\end{array}$ & $\begin{array}{r}3 \\
-3 \\
-\end{array}$ & $\begin{array}{l}= \\
= \\
= \\
=\end{array}$ & $\begin{array}{l}2,109 \\
1,31 \\
149 \\
326 \\
260 \\
-\end{array}$ & $\begin{array}{r}75 \\
38 \\
1 \\
26 \\
-\quad\end{array}$ \\
\hline 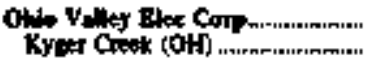 & 65,76 & 230 & $\overline{-}$ & $=$ & $\bar{z}$ & $\bar{z}$ & 2288 & * & $\bar{z}$ & 411 & 1 \\
\hline 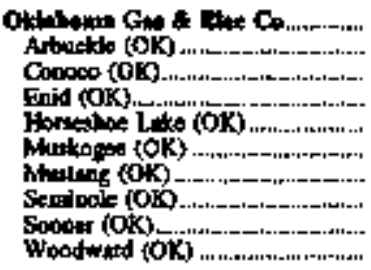 & $\begin{array}{c}1,67,15 \\
\bar{z} \\
\bar{z} \\
96,639 \\
\overline{-} \\
71,129\end{array}$ & $\begin{array}{l}{ }^{60 x} \\
= \\
= \\
= \\
= \\
-\end{array}$ & 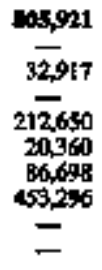 & $\begin{array}{l}= \\
= \\
= \\
= \\
= \\
=\end{array}$ & $\begin{array}{l}= \\
= \\
= \\
= \\
= \\
=\end{array}$ & $\begin{array}{l}= \\
= \\
= \\
= \\
=\end{array}$ & $\begin{array}{l}\frac{1,13}{E} \\
= \\
-585 \\
= \\
{ }^{4288}\end{array}$ & $\begin{array}{l}{ }^{1} \\
= \\
= \\
-\end{array}$ & 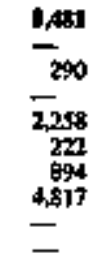 & $\begin{array}{l}2,126 \\
= \\
= \\
\overline{1} \\
\frac{7}{7} \\
= \\
=\end{array}$ & 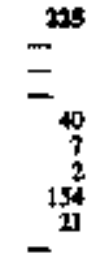 \\
\hline 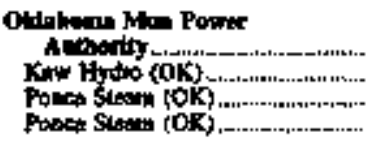 & $\begin{array}{l}\bar{z} \\
\bar{z}\end{array}$ & $z_{13}^{15}$ & $\frac{13,3,06}{13,267}$ & $\begin{array}{l}\text { 19.455 } \\
\text { 19,9.95 } \\
= \\
=\end{array}$ & $\bar{z}$ & $\begin{array}{l}\bar{z} \\
\bar{z}\end{array}$ & $\bar{z}$ & $\stackrel{\bullet}{*}$ & $\begin{aligned} 116 \\
15\end{aligned}$ & $\bar{z}$ & $\overline{-}_{1}^{1}$ \\
\hline 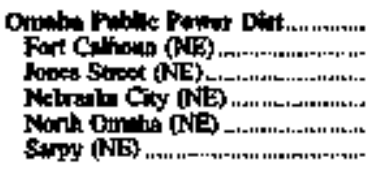 & $\begin{array}{l}564602 \\
\bar{z} \\
315,650 \\
248,952 \\
-\end{array}$ & $\begin{array}{l}1,34 \\
= \\
= \\
=\end{array}$ & $\begin{array}{l}34733 \\
\overline{-} \\
\overline{-} \\
10774 \\
25,959\end{array}$ & $\begin{array}{l}\bar{z} \\
\bar{z} \\
=\end{array}$ & $\begin{array}{c}351, m 7 \\
351,07 \\
= \\
= \\
=\end{array}$ & $\begin{array}{l}z \\
z \\
=\end{array}$ & $\begin{array}{l}-^{361} \\
{ }^{199} \\
-^{162}\end{array}$ & $\begin{array}{l}-2 \\
-1 \\
-\end{array}$ & $\begin{array}{l}\frac{470}{-} \\
\frac{123}{355}\end{array}$ & $\begin{array}{l}- \\
- \\
397 \\
-26]\end{array}$ & $\begin{array}{r}24 \\
-\quad 4 \\
6\end{array}$ \\
\hline 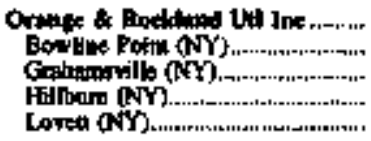 & $\begin{array}{l}197,306 \\
= \\
197,398\end{array}$ & $\begin{array}{l}102,532 \\
102,528 \\
=\end{array}$ & $\begin{array}{c}321,114 \\
274,362 \\
\overline{1,176} \\
42,314\end{array}$ & $\frac{11,706}{9,738}$ & $\begin{array}{l}\vec{z} \\
\bar{z}\end{array}$ & $\begin{array}{l}= \\
=\end{array}$ & $=$ & $=$ & $\begin{array}{l}3,734 \\
2,719 \\
-\underset{40}{20}\end{array}$ & $=^{3}$ & $\begin{array}{r}300 \\
-\quad 2 \\
300 \\
40\end{array}$ \\
\hline
\end{tabular}

Sos tooinotes at ead of ubla. 
Table 56. US. Electric Utilly Net Generation, Foel Coammption, and Bnel Stocks by Company and Flont, Jaly 19 in (Contianod)

\begin{tabular}{|c|c|c|c|c|c|c|c|c|c|c|c|}
\hline \multirow{2}{*}{ 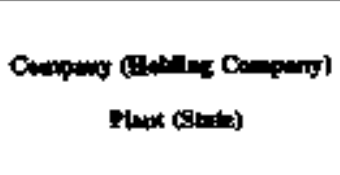 } & \multicolumn{6}{|c|}{ (D) } & \multicolumn{3}{|c|}{ 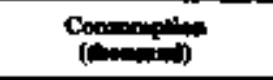 } & \multicolumn{2}{|c|}{ (itherists } \\
\hline & Cov & Publen & Ge. & Ey & 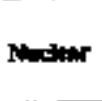 & 1 & $\lim _{i=1}^{\infty}$ & pingt & Gos & (bont & 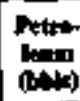 \\
\hline
\end{tabular}

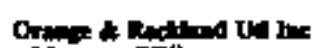

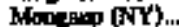

Rio (NT)

Storing (NV)

Swintins Btion I (NY)

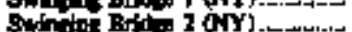

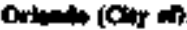

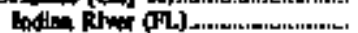

SClod thi

Stand (FL)

Orow Myadm I but

Futolitio (CA)

Kelly bidot (c)

Sty Crote (CA)

Orrite (Cing of )

Orevilie (OH)

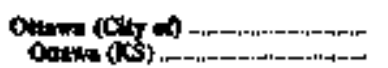

Othe Th rum Co

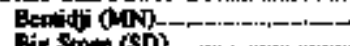

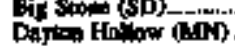

Hoot Lily (M)

Inontion (ND)

.......................

Puge dar)

Fon IA QNiN)

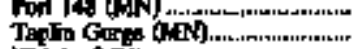

Wripl (MN)

Ombien (Cty of .....................

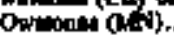

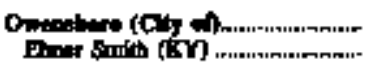

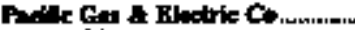
Alis (C) .

Anotb (C)

inch 1 (C)

Balp 2 (CH)

Betden (CA)

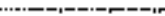

and chen $\mathrm{B}$ (C)

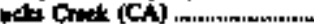

Bea Veloy (C)

Carions 1 (CA)

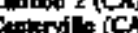

Chil Bar (CA)

Coll Crmpan (CA)

Colanta (CA)

Cone (CN)

Contio Crin [CA)

Crith (C)

$D=$ sible (CA)

Dowe Orik (CA)

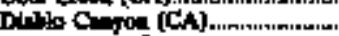

Domitrille cChl.

1 (C)

Dutw 2 (CAl)

Duth Fin (CA)

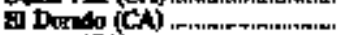

Exiti (C).

$\begin{array}{ll}\bar{z} & \bar{z} \\ z & =\end{array}$

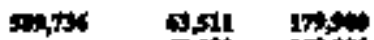

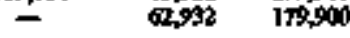

$59.73 x$

$=\quad=$

$=$

7fse:

T.840

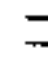

329

275,106

s434

-

7

$=$

$=$

= $\quad$

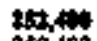

25240

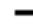

च

$\bar{z}$

$=$

$=$

=

=

$\bar{z}$

$=$

$\bar{z}$

$=$

z

$=$

$\bar{z}$

$=$

$\bar{z}$

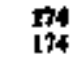

$=\quad \overline{5}$

$=\quad=$

$=$

$=\quad=$

-\$0

$-59$

-

$\begin{array}{cccc}393 & - & - \\ 746 & = & = \\ -93 & = & =\end{array}$

$=\quad \underline{-}$

$\begin{array}{lll}- & = & - \\ -55 & = & -\end{array}$

$=$

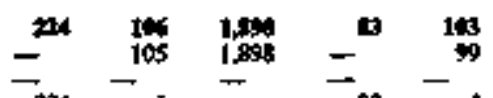

\section{Axt?}

17,164

7,942

4,358

2800

-

$=\quad=$

144

-

600

121

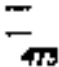

350

$$
\begin{aligned}
& = \\
& = \\
& = \\
& = \\
& = \\
& =
\end{aligned}
$$

-

-

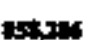

$\begin{array}{ll}= & = \\ = & = \\ & =\end{array}$

6s,

7,69

392.2

32, 4 .

T.414

7241

15,977

52.72:

15

237

\$\$!

6.195

亡
-

$m$

เ5.

irs

2255

=

$=$

$\rightarrow$

36,29

Ste footnotes al ead of thes. 
Tuble 56. U.S. Electric Utility Net Generation, Fued Consunption, ind Foel Stocks by Company and Pitant, Johy 1997 (Continued)

\begin{tabular}{|c|c|c|c|c|c|c|c|c|c|c|c|}
\hline \multirow{2}{*}{ 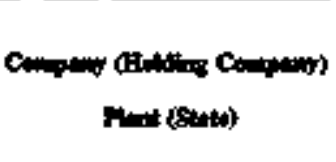 } & \multicolumn{6}{|c|}{ 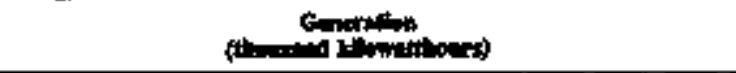 } & \multicolumn{3}{|c|}{ 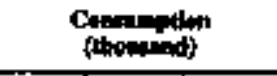 } & \multicolumn{2}{|c|}{ Suth: } \\
\hline & Call & Futrolimina & C: & Eyira & 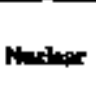 & I & coal & 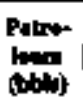 & Ges) & cint & Pintor \\
\hline
\end{tabular}

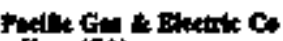

Fin (CA).

Halion (CA)

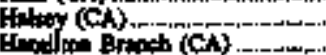

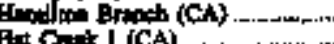

Afr Cn: (CA)

16. 2 (CA)

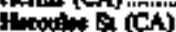

(C) 1 )

Honpoik Bays (CA)

thomente

hith (C)

Kaxinf (CA)

rot

Kllote (C)

Kinot Rtver (C)

1 in Sondin (CA)

Mexil Fill (C)

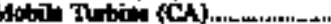

Mors Buy (Ch)

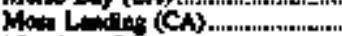

Mophin (CA)

Pt पOW: (CA)

Nowerats (CA)

Oil Fin (CA)

ouklod (CA)

Ploctix (C)

Pik 1 (C)

it (ck)

it: 4 (ch)

Pit 5 (C).

Pis 6 (C)

Pit $t$ ich

riting (c)

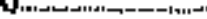

Panto (CA)

Pater VDler (CA)

PVUSA $1\left(\hat{s}^{\prime}\right)$

Rodx Orek fCs

Sin spting (CS)

Sth lont

St Jonedo No. 1 (CA) _.........

$\sin$ losen 3 (CA)

sonth (CA).

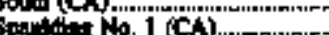

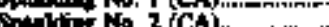

Spuntin Ho, 3 (CA)..............

Spro 0 , f

stodinin (CA)

The Earen fCA).

Tras Cond (CA)

Thentom (CA)

Tuto Buter (CA)

Volin (CA)

Yoth 2 ich

Wen Palor (CN)

Win (CK)

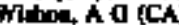

Pacilkerp.

Ancriver Feet (UT)

Anitot (D)

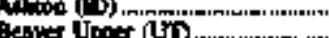

Band (o k

Big For (MT)

BindkI (fT)

Cutoon (UT)

Cearall (W/

Carime 1 (OR)

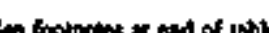

\begin{tabular}{|c|c|c|c|c|c|c|c|c|c|c|}
\hline & & & & & & & & & & \\
\hline$=$ & $=$ & $=$ & 42,206 & $=$ & $=$ & $=$ & $\vec{z}$ & $=$ & 三 & $=$ \\
\hline z & 二 & $\bar{z}$ & 1,756 & $\bar{z}$ & $\bar{z}$ & $=$ & $=$ & $=$ & 二 & $=$ \\
\hline$=$ & $=$ & $=$ & 3,037 & $=$ & $=$ & $=$ & $=$ & $=$ & $=$ & $=$ \\
\hline 二 & 二 & $=$ & $-2,066$ & $\bar{z}$ & $=$ & $=$ & 二 & $=$ & $=$ & $=$ \\
\hline 二 & $-\infty$ & $\overline{0}$ & $=$ & $=$ & $=$ & $=$ & - & $\tau_{145}$ & $=$ & \\
\hline - & 31 & \$צח & $=$ & 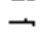 & $=$ & - & 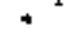 & 1,161 & $=$ & I5 \\
\hline $\bar{z}$ & $=$ & $\bar{z}$ & 4,59 & $=$ & 二 & $=$ & $\bar{z}$ & $=$ & $=$ & $z$ \\
\hline $\bar{\Xi}$ & $\bar{z}$ & 二 & 50,497 & $\bar{z}$ & च & $\bar{\Xi}$ & $=$ & $\bar{z}$ & $=$ & $=$ \\
\hline$=$ & $=$ & - & 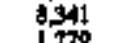 & $=$ & - & $\approx$ & $=$ & $=$ & - & - \\
\hline 二 & $=$ & $=$ & $\begin{array}{l}1,779 \\
13,97\end{array}$ & $\bar{z}$ & $=$ & $=$ & $\overline{7}$ & $=$ & $=$ & 二 \\
\hline 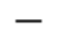 & $=$ & - & 791 & $=$ & $=$ & - & - & $=$ & - & - \\
\hline$m$ & - & - & 2,198 & - & $=$ & $=$ & $=$ & $=$ & $=$ & $\bar{\square}$ \\
\hline $\bar{z}$ & $\vec{z}$ & $\mid \overrightarrow{55,118}$ & $=$ & $\Xi$ & $=$ & $\Xi$ & $\Xi$ & $\overline{1,550}$ & $=$ & - \\
\hline$=$ & - & 659,319 & - & $=$ & - & $=$ & - & 6,096 & - & \\
\hline $\bar{z}$ & $\bar{z}$ & $\bar{z}$ & 1,493 & $=$ & $=$ & 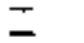 & Z & $=$ & 二 & $=$ \\
\hline- & - & - & $=$ & - & - & - & - & - & - & - \\
\hline 二 & -11 & 二 & -8 & 二 & 二 & $=$ & $\bar{*}$ & $\bar{z}$ & 二 & -19 \\
\hline عـ & - & - & 859 & - & - & $=$ & - & $=$ & $=$ & - \\
\hline$=$ & $z$ & $=$ & $\begin{array}{l}26,577 \\
28,376\end{array}$ & Z & $=$ & $=$ & $\bar{z}$ & $=$ & $\bar{z}$ & $=$ \\
\hline & - & - & 38,008 & 二 & 二 & - & - & $=$ & - & - \\
\hline & - & - & 64260 & $\bar{z}$ & $=$ & $=$ & $=$ & $=$ & $=$ & $=$ \\
\hline$=$ & $=$ & $=$ & 32,170 & $=$ & $\bar{z}$ & $=$ & $\bar{z}$ & $=$ & $=$ & $=$ \\
\hline- & - & 439,566 & - & - & - & - & - & 4,528 & - & 769 \\
\hline - & $\overline{9}_{911}$ & $\overline{8}, \bar{x}$ & 31,765 & $\Rightarrow$ & $\bar{z}$ & 二 & ${ }_{2}$ & $\overline{903}$ & $=$ & $\bar{z}_{314}$ \\
\hline & - & - & 3,609 & - & - & - & - & - & - & - \\
\hline- & $=$ & 二 & 5 & $\bar{z}$ & -10 & - & - & - & - & $=$ \\
\hline Z & Z & $\bar{z}$ & 20,972 & $\bar{z}$ & $=$ & $=$ & $=$ & $=$ & 二 & $=$ \\
\hline - & - & - & 123 & - & - & - & - & - & - & - \\
\hline & $\bar{z}$ & $\bar{z}$ & $\begin{array}{r}9,99 \\
1279\end{array}$ & 二 & 二 & $=$ & $=$ & $=$ & $=$ & $=$ \\
\hline- & 2 & $=$ & 4.551 & - & - & - & - & - & - & - \\
\hline$\pi$ & - & - & 5,255 & - & - & - & - & - & - & 二 \\
\hline $\bar{z}$ & $=$ & $=$ & $\begin{array}{l}2,136 \\
2>90\end{array}$ & $=$ & 二 & $=$ & $=$ & $\bar{z}$ & $\bar{z}$ & $\bar{z}$ \\
\hline 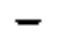 & - & - & 274 & - & - & - & - & - & $\overline{-}$ & - \\
\hline- & $=$ & $=$ & 41,234 & $=$ & 48713 & $=$ & $=$ & $=$ & 二 & $=$ \\
\hline 二 & $\bar{z}$ & $=$ & $\overline{32110}$ & 二 & 457,113 & $=$ & $=$ & $=$ & $\bar{z}$ & $\bar{z}$ \\
\hline - & - & - & 506 & - & - & - & - & - & - & - \\
\hline- & $=$ & $=$ & $\begin{array}{l}1,674 \\
1613\end{array}$ & $\bar{z}$ & $\bar{z}$ & $\bar{z}$ & 二 & $\ddot{z}$ & 二 & $=$ \\
\hline- & $\vec{z}$ & 二 & 5 & - & 二 & $=$ & - & $=$ & - & $=$ \\
\hline & $=$ & 二 & 8,356 & $=$ & $=$ & $=$ & $=$ & $\bar{z}$ & $=$ & $=$ \\
\hline$=$ & $=$ & ב & 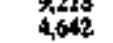 & $=$ & $\Xi$ & $=$ & $=$ & $=$ & $\overline{-}$ & $=$ \\
\hline $151, \% 1$ & $4 \leq 13$ & sen+85 & silker & - & $15,4+1$ & 2,301 & & $\infty$ & 3061 & 34 \\
\hline & & - & -7 & - & $\tau$ & - & 二 & - & & \\
\hline Z & Z & 二 & $4,1,086$ & E & 二 & $\bar{z}$ & $=$ & $=$ & $=$ & $=$ \\
\hline- & $=$ & 二 & 630 & - & - & - & - & - & - & - \\
\hline $\overrightarrow{0}$ & $=$ & $=$ & & & 15,441 & $=$ & $=$ & $=$ & & \\
\hline 176,489 & 1,063 & $=$ & $=$ & $=$ & $\bar{z}$ & 670 & $*^{2}$ & $=$ & $\begin{array}{r}350 \\
70\end{array}$ & .17 \\
\hline 412,125 & $1,0 \mathrm{it}$ & $=$ & $\overline{1}$ & $\bar{z}$ & $\bar{z}$ & 286 & 2 & $=$ & 841 & 3 \\
\hline & & - & 4,300 & & & & & & & \\
\hline
\end{tabular}




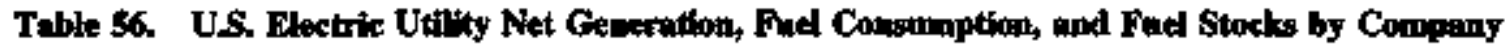
and Plank, July 1997 (Continued)

\begin{tabular}{|c|c|c|c|c|c|c|c|c|c|c|c|}
\hline \multirow{2}{*}{ 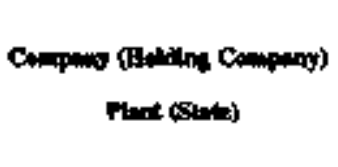 } & \multicolumn{6}{|c|}{ 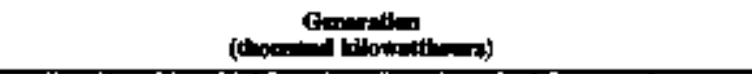 } & \multicolumn{3}{|c|}{ 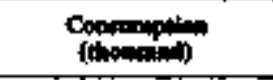 } & \multicolumn{2}{|c|}{ Stocts } \\
\hline & C.1) & Pelroluten & Ger: & Ayom & Nuclear & Onerr & $\underset{t \rightarrow d}{\text { Cad }}$ & Ftor & tats & Cad & $\underset{(x+1)}{\operatorname{lath}}$ \\
\hline
\end{tabular}

\section{Focilowp}

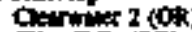

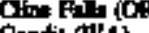

Copoo 1 (CA)

Copto 2 (c)

cono (1.0).

Covar UIm.

E.jl Point (O)

Pon Side (OR)

Fir Cuet (c)

Fh Conk (OR)

Fur Croco (UT)

Gediby (UT)

Grax (II)

Crate (T)

Howner (ancy) (UT)

inger

(1) 2 (UT)

(n)

Ion oba (C)

Iot: C Boyile (OR)

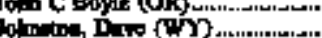

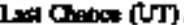

Lomala 1 (OR)

1 :molo 2 CORh

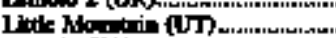

Maril (WA)

Norbet (Wh)

Ned hex Drop (WA)

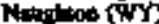

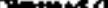

Oidis $(1 . D)$

Fide (10)

Ploneir (UT)

Ponerale (OR)

Tromet 1 (OR)

Prapect 2 (Cot)

rivepect 3 (COR)

Slocin netret (WAK.

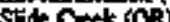

Sthork (UT)

sodia (I.D)

Sod Spina (OR)

SA Anthey (D)

Stim (t)

Swith 2 (Wh)

Swit I (WA)

Tolin: (OR)

Whome Fill (OR

Weter (UT).

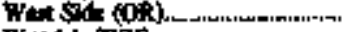

Wyod (WV)

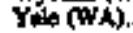

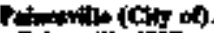

Pale rothle (OH)

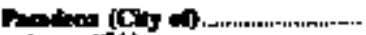
Antar (CA)

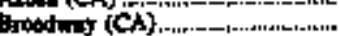

ctenger (C)

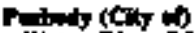

Winte Rhet aLt)

Fin (C) 1 ).

Fetle (I)

$\begin{array}{lll}= & = & = \\ = & = & = \\ = & = \\ = & = \\ = & =\end{array}$

- $\quad-\quad 52030$

7

704,568

$5+1,200$

-

$\overline{-}$

$\overline{-}$

$=$

=

$\overline{-}$

$\overline{-1}$

=

-

$=$

-

$=$

$=$

E

E

$=$

$=$

-

-

347

ton

LS045

$=$

3,238

-

$=$

-

4

-

$=$

$=$

=

$=$

$=$

二

二

=

$=$

$=$

$-$

$\overline{=}$

14,94

$=\quad \overline{14.600}$

6,946

E

8.490

4901

5,73 :

4,

7.866

1, Tho

-

$\underline{-}$

$\bar{z}$

$=$

$=$

$=$

-10\%

$=$

434

$=$

$=$

$=$

$=$

$\bar{z}$

$=$

$=$

$=$

$=$

$=$

$=$

$\underline{\Xi}$

-

$-$

$-98$

-

4,045

-

6ils

二

14

5,34

ro, 42

TSO

16,898

20369

21.4A

2,967

$\div$

2619

8.096

20033

3,300

13,300

$-3$

$\overrightarrow{9} 403$

9.409

4.90

6,399

848

89

34,379

2.39\%

\%7

2,348

212

25551

$=\quad= \pm$

$=\quad= \pm$

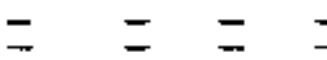

$=\quad=$

$=\quad=$

$= \pm$

$= \pm$

$\Xi$
$\vdots$
$\vdots$
$\vdots$
$\vdots$
$\vdots$
$\vdots$
$\vdots$

$= \pm=$

z $=$ =

$=$

$=$

=

$\bar{z}$

二

$=$

639 $= \pm$

E $= \pm$

$=\quad=$

$\bar{z}=\bar{z}$

$=$

$=\quad$ s35

$=\quad=$

$= \pm \bar{z}$

$\bar{\Xi} \bar{z}$

$\bar{z}=\overline{20}_{236}$

$= \pm=$

$= \pm=$

$=\quad \Xi$

$= \pm=$

$\approx= \pm$

$=z=$

$=\quad=$

$\bar{z}=$

$=z=$

$=$

$=\quad=$

= $=$

$\bar{z}$

$\underline{E}$

$=$

$=$

$\bar{z}=$

=

$\bar{z}$

$\overline{-}$

$-180$

34

$-$

z $= \pm \bar{z}$

$=\bar{z}$

$=$

-

357 -

- $=$ -

-

$=$

$\Xi^{17}=$

$+514-$

$=\quad$ =

See toondes an of of blo 
Table 56. US. Electric Utilikg Net Generstion, Fuel Consunaption, and Foel Stocks by Company and Plant, Jaby 1997 (Contimot)

\begin{tabular}{|c|c|c|c|c|c|c|c|c|c|c|c|}
\hline \multirow{2}{*}{ 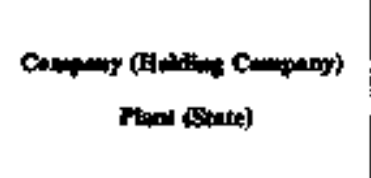 } & \multicolumn{6}{|c|}{ 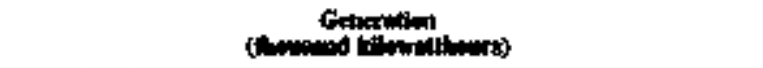 } & \multicolumn{3}{|c|}{ (0) } & \multicolumn{2}{|c|}{ (Stokts } \\
\hline & Cid & Petrolne & an & Mptot & Numper & oulsel & 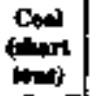 & Purst & (Misf) & 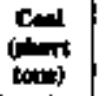 & tonth \\
\hline 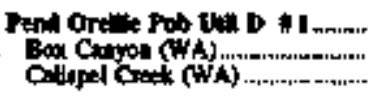 & $\bar{z}$ & $\bar{z}$ & $\bar{z}$ & $\begin{array}{l}42,197 \\
41,0505 \\
292\end{array}$ & $\underline{z}$ & $=$ & $=$ & $\bar{F}$ & $\vec{z}$ & $\bar{二}$ & $\bar{z}$ \\
\hline 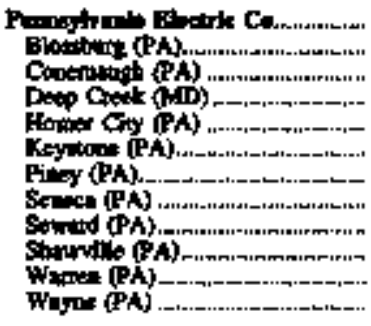 & 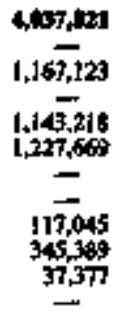 & 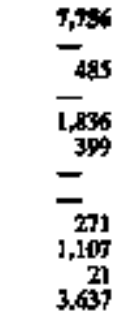 & $\begin{array}{l}8000 \\
1,296 \\
= \\
= \\
= \\
= \\
=\end{array}$ & $\begin{array}{l}-4600 \\
\overline{1,390} \\
= \\
\overline{2,8300} \\
-9,0,0 \\
= \\
=\end{array}$ & $\begin{array}{l}= \\
= \\
= \\
= \\
= \\
=\end{array}$ & $\begin{array}{l}= \\
= \\
= \\
= \\
=\end{array}$ & 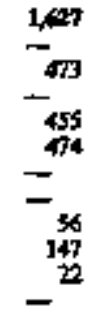 & $\begin{array}{l}-16 \\
-1 \\
= \\
=1 \\
=\frac{1}{2}\end{array}$ & $\begin{aligned} & 74 \\
&= 13 \\
&= \\
&= \\
&= \\
&-\end{aligned}$ & 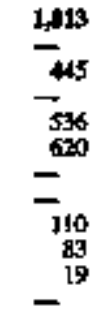 & $\begin{array}{c}-56 \\
-5 \\
-5 \\
9 \\
9 \\
10 \\
9 \\
17\end{array}$ \\
\hline 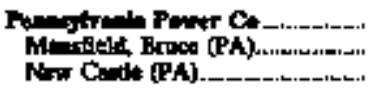 & $\begin{array}{l}1,4,2,2 \pi \\
1,325,788 \\
163,270\end{array}$ & $\begin{array}{l}1,3 * 3 \\
1,213 \\
110\end{array}$ & $\bar{z}$ & $\bar{z}$ & $\bar{z}$ & $\bar{z}$ & \$5 & $\begin{array}{r}2 \\
+2\end{array}$ & $\bar{z}$ & $\underset{19}{671}$ & 24 \\
\hline 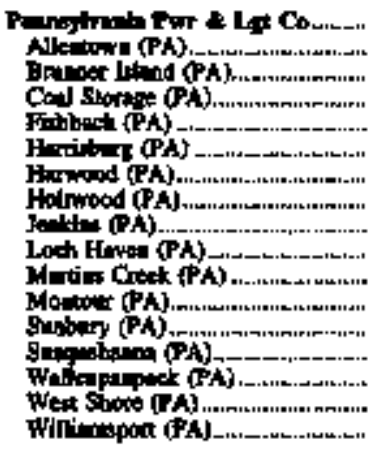 & 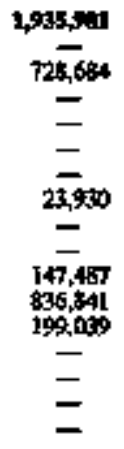 & 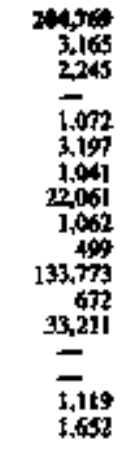 & $\begin{array}{l}180,340 \\
= \\
= \\
= \\
155,300 \\
= \\
= \\
=\end{array}$ & $\begin{array}{l}26,309 \\
= \\
= \\
= \\
21,963 \\
= \\
= \\
\overline{4} \\
=\end{array}$ & $\begin{array}{l}= \\
= \\
= \\
= \\
= \\
= \\
= \\
= \\
=\end{array}$ & $\begin{array}{l}= \\
= \\
= \\
= \\
= \\
= \\
= \\
=\end{array}$ & $\begin{array}{l}-111 \\
282 \\
= \\
= \\
= \\
= \\
21 \\
= \\
= \\
=\end{array}$ & 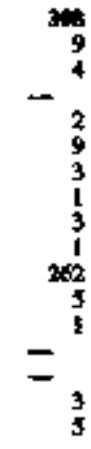 & $\begin{array}{l}1, \text { sss } \\
= \\
= \\
= \\
= \\
= \\
\bar{z} \\
= \\
= \\
= \\
= \\
=\end{array}$ & $\begin{array}{l}47 \\
254 \\
258 \\
= \\
= \\
= \\
182 \\
745 \\
= \\
= \\
=\end{array}$ & $\begin{array}{r}1,43 \\
4 \\
-\quad 2 \\
4 \\
2 \\
1 \\
2 \\
2 \\
1,588 \\
7 \\
-\quad 1 \\
-\quad 2 \\
2\end{array}$ \\
\hline 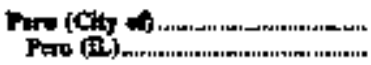 & $\bar{z}$ & 347 & $\begin{array}{l}-116 \\
-115\end{array}$ & $\overline{-}$ & $\bar{z}$ & $=$ & $=$ & $\mathbf{1}$ & $\bar{F}$ & $=$ & $\mathbf{1}$ \\
\hline 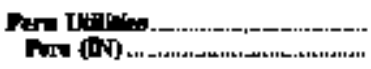 & $2,2,138$ & 43 & $\overline{-}$ & $\bar{z}$ & $\bar{z}$ & $\bar{z}$ & $\frac{2}{2}$ & $*$ & $\overline{-}$ & 1 & : \\
\hline 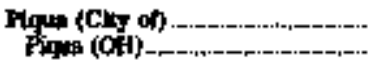 & $\begin{array}{l}-151 \\
-131\end{array}$ & $\begin{array}{l}353 \\
353\end{array}$ & $\overline{-}$ & $\overline{-}$ & $\bar{z}$ & $=$ & $\bar{z}$ & t & $\bar{z}$ & $=$ & $\frac{2}{2}$ \\
\hline 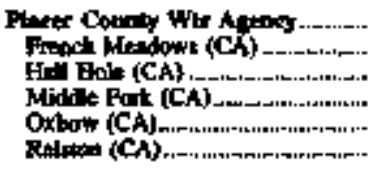 & $\begin{array}{l}\bar{z} \\
\bar{z}\end{array}$ & $\begin{array}{l}\bar{z} \\
\bar{z}\end{array}$ & $\begin{array}{l}\bar{z} \\
\bar{z}\end{array}$ & $\begin{array}{r}15, \pi 4 \\
6,603 \\
469 \\
76,322 \\
3,430 \\
50,610\end{array}$ & $\begin{array}{l}\bar{z} \\
\bar{z} \\
\bar{z}\end{array}$ & $\begin{array}{l}= \\
= \\
= \\
=\end{array}$ & $\begin{array}{l}= \\
= \\
=\end{array}$ & $\begin{array}{l}\bar{z} \\
\bar{E} \\
\bar{z}\end{array}$ & $\begin{array}{l}= \\
= \\
=\end{array}$ & $\begin{array}{l}= \\
\vec{z} \\
=\end{array}$ & $\begin{array}{l}= \\
\ddot{z} \\
\ddot{z}\end{array}$ \\
\hline 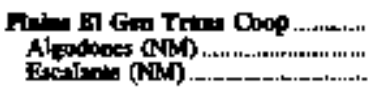 & $\frac{15435 t}{[54,35 t}$ & $\bar{z}$ & $\bar{z}$ & $\bar{z}$ & $\bar{z}$ & $\bar{z}$ & $-{ }^{* 2}$ & $\bar{z}$ & $=$ & -6 & -9 \\
\hline 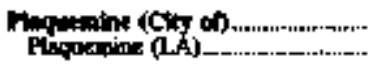 & $\bar{z}$ & $\overline{-}$ & $\overline{-}$ & $\overline{-}$ & $\overline{-}$ & $\overline{-}$ & $\bar{z}$ & $\overline{-}$ & $\overline{-}$ & $\bar{z}$ & $\bar{z}$ \\
\hline 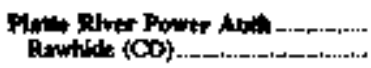 & $\begin{array}{l}121,646 \\
121,635\end{array}$ & $\operatorname{lin}_{182}$ & $\bar{z}$ & $\bar{z}$ & $\bar{z}$ & $z$ & $\begin{array}{l}73 \\
73\end{array}$ & * & $=$ & 荎 & 3 \\
\hline 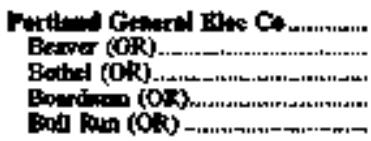 & $\begin{array}{l}76,776 \\
\frac{-76878}{-}\end{array}$ & $\begin{array}{l}\frac{3,700}{5} \\
\frac{3,100}{2}\end{array}$ & $\begin{array}{l}18,19 \\
15019 \\
= \\
-\end{array}$ & $\begin{array}{l}107,807 \\
= \\
0,696\end{array}$ & $\begin{array}{l}\bar{z} \\
\bar{z}\end{array}$ & $\begin{array}{l}\bar{z} \\
\bar{z}\end{array}$ & $z^{25}$ & $-^{10}$ & $\begin{array}{l}357 \\
= \\
=\end{array}$ & $\begin{array}{l}3 \\
= \\
-320\end{array}$ & $\begin{array}{l}111 \\
197 \\
- \\
-\end{array}$ \\
\hline
\end{tabular}

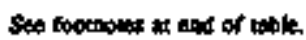


Table 56. U.S. Electric Uulity Net Generotion, Feel Conanuption, and Fuel Sibcts by Conpany and Plant, July 1997 (Continued)

\begin{tabular}{|c|c|c|c|c|c|c|c|c|c|c|c|}
\hline \multirow{2}{*}{ 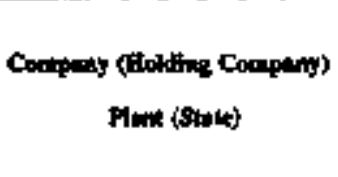 } & \multicolumn{6}{|c|}{ 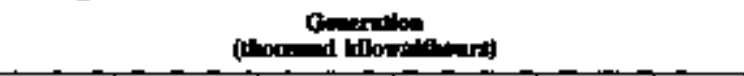 } & \multicolumn{3}{|c|}{ 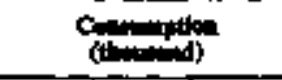 } & \multicolumn{2}{|c|}{ (tivistist) } \\
\hline & Cw & Putroter & ten & 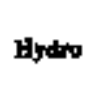 & Nedur & Other 1 & 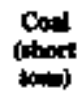 & 隹) & Gat & limel & 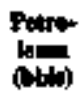 \\
\hline
\end{tabular}

Partind Qtenerid Rle C.

Conots Spingt (On)

Fand (OR) (Ond.........................

Mocth firt (OR)

Orl Orowe (OR)

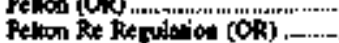

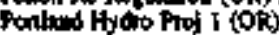

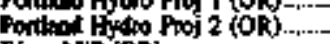

pher MD $[\mathrm{OR}]$

Rourd Bon (OA)

Saring (OR)

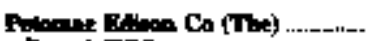

Dam 4 (WV)

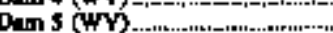

Latry (Y)

Hill

Nempot (NA)

Nempot (VA) ....

Shoridioll (NA)

(1)

Wath (WA).

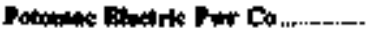

Bearidn (DC).

Burzand Pole (DC)

Qurk Foivt (ND)

Dickrason (MID)

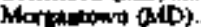

$\begin{array}{ll}= & = \\ = & = \\ = & = \\ = & =\end{array}$

$=$

- $\quad \overline{8,24}$

- $\quad 9698$

- $\quad 35,991$

4,200

5.210

81,781

- 9,60

35,m 281 - 2,21

-

- $\quad-$

$=$

3597

-

281

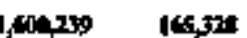

I4,37:

-

64504

36,9s5

95,336

5ग

246,258

20,427

2,59

42

410

245

-190

20.77\%

Panter Anthy of S4 A N V.

Achokan (NY)

Whatm (NY)

Frowitik (NY)

Flyon (NY)......

hation Foint arr

Restico (NY).

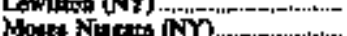

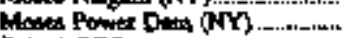

Pot=1 (NY).

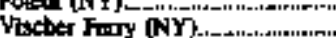

Prtactoon (C)y of

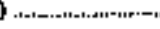

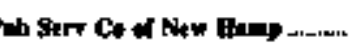

Amodkets (N)

A vem bland 0

Cotsen (VT)

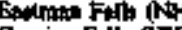

Carvins Fall (NT)

Gortom (NH)

tookects (IN)

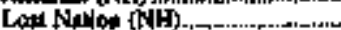

Merirnask (NH)

Newiogton (NH)

sching ant

thith

$-$

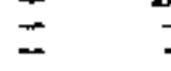

$=\quad \bar{z}$

$\vec{z} \quad \underline{z}$

$\bar{z} \quad \bar{z}$

$=\quad=$

$=\quad=$

$=\quad 29,801$

$2,0,9,173$
2,413
$-35,46$

- $\quad-3 \$, 19$

$=$

95,739

$=$

$\overrightarrow{1,3}$

1,156

$-23,596$

I.48 1 , 30

67,382

$\overline{1,62 t}$

$\begin{array}{ll}= & = \\ = & = \\ = & = \\ = & = \\ = & = \\ = & =\end{array}$

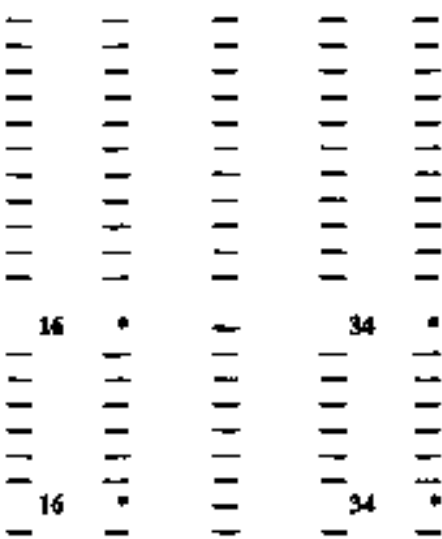

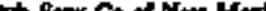

Lan Yeras (NA)

Revies (ND).

Sin Juen (hith)

$\begin{array}{lll}- & - & - \\ - & 191 & 134\end{array}$

$\begin{array}{lll}- & 191 & 1,356 \\ - & 191 & 1,376\end{array}$

35302

1,017

$=$

$=\quad=$

$=\quad=$

$=\quad=$

27009

$\overline{75,144}$

$\rightarrow$

25
576
3250

32,09
1,691

$\frac{1,00}{305}$

-

-

$=$

$\bar{z}$

=

-

$\bar{i}, 01]$

-

$\begin{array}{lll}= & = & \\ = & = & - \\ = & = & = \\ = & =\end{array}$

$-$

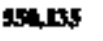

-

-

$=\quad=$

$\because \quad=$

$=\quad-$

$=\quad \bar{z}$

二

27,0

$5,75:$

590

2,002

2,660

1,133

$-^{12}$

see footmones at end of table.

1,16,004

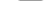

$1,119,300$

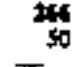

t.45

$\bar{B}, 495$

$=$
$=$
$=$
$=$
$=$
$=$
$=$

$=$

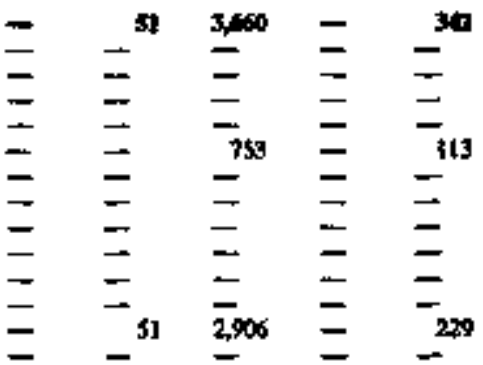

二 : 14 $14=1$

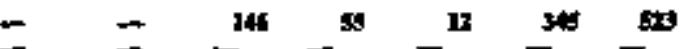

$\exists= \pm \quad$ z $=$ =

$\Xi \Xi \Xi \Xi \pm \equiv=$

$\exists=$

$=\quad=$

こ こ こ

三 $\equiv \equiv \equiv$

${ }_{109} \quad=255$

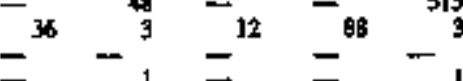

- 216

$-$

$=\quad=$

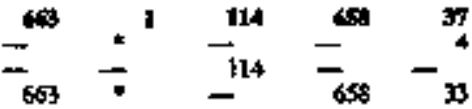


Table 56. US. Eractric Utilty Net Ganerntion, Fuel Consmption, and Fuel Stocka by Conpany and Ploms, July 1997 (Continued)

\begin{tabular}{|c|c|c|c|c|c|c|c|c|c|c|c|}
\hline \multirow{2}{*}{ 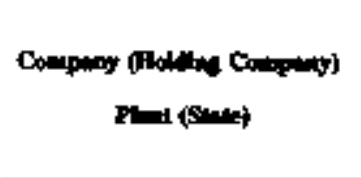 } & \multicolumn{6}{|c|}{ 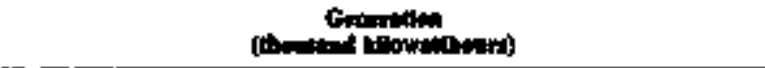 } & \multicolumn{3}{|c|}{ (d) } & \multicolumn{2}{|c|}{ (Stactat } \\
\hline & Con & 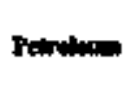 & Ga: & Byitro & Naclener & Otherl & (did & Pato- & (NA) & $\begin{array}{l}\text { Conl } \\
(\text { intirt } \\
\text { ind })\end{array}$ & 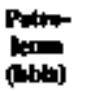 \\
\hline 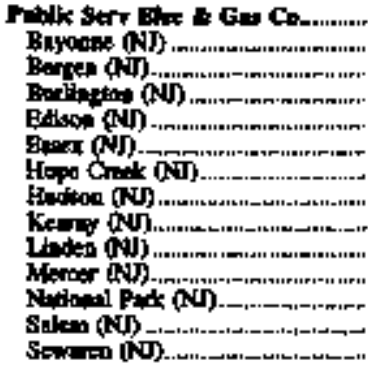 & 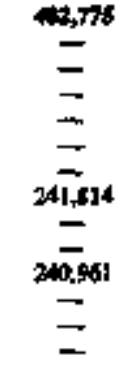 & 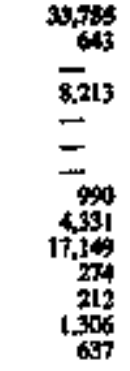 & 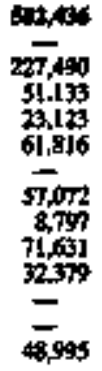 & $\begin{array}{l}= \\
= \\
= \\
= \\
= \\
=\end{array}$ & 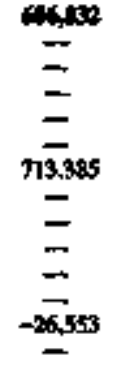 & $\begin{array}{l}= \\
\bar{z} \\
\bar{z} \\
\bar{z} \\
= \\
=\end{array}$ & $\begin{array}{l}= \\
= \\
= \\
= \\
=127 \\
=93 \\
= \\
=\end{array}$ & 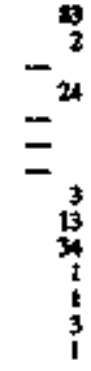 & 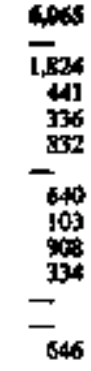 & $\begin{array}{l}= \\
= \\
= \\
= \\
= \\
z \\
= \\
z 298 \\
=\end{array}$ & $\begin{array}{r}50 \\
-\quad 2 \\
-\quad 69 \\
6\end{array}$ \\
\hline 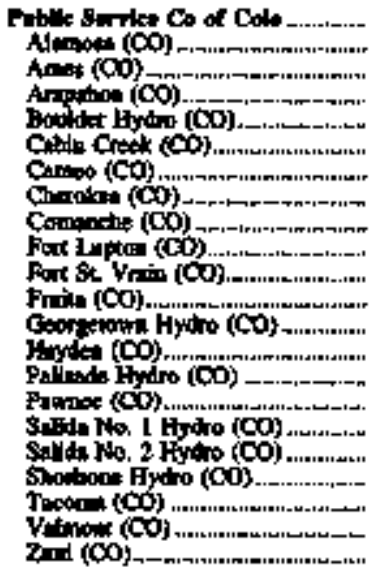 & 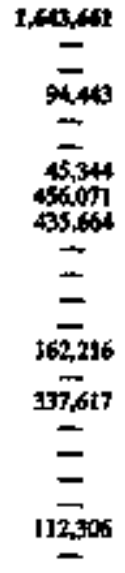 & $\begin{array}{l}= \\
= \\
= \\
= \\
= \\
= \\
= \\
= \\
= \\
= \\
= \\
=\end{array}$ & 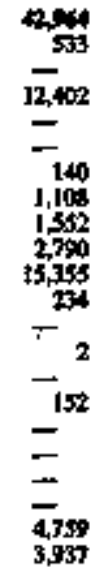 & 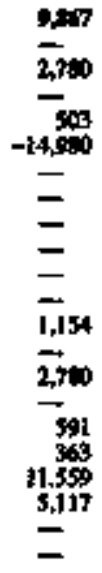 & $\begin{array}{l}= \\
= \\
= \\
= \\
= \\
= \\
= \\
= \\
= \\
= \\
=\end{array}$ & $\begin{array}{l}\bar{z} \\
\bar{z} \\
\bar{z} \\
\bar{z} \\
= \\
= \\
= \\
= \\
= \\
= \\
= \\
=\end{array}$ & $\begin{array}{l}z^{5} \\
= \\
= \\
= \\
= \\
= \\
= \\
= \\
= \\
= \\
=\end{array}$ & $\begin{array}{l}= \\
= \\
= \\
= \\
= \\
= \\
= \\
= \\
= \\
= \\
= \\
= \\
=\end{array}$ & 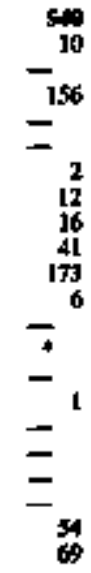 & 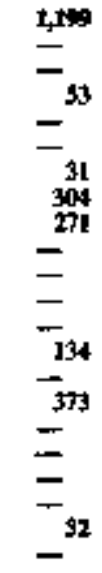 & $\begin{array}{l}= \\
= \\
=1 \\
-14 \\
-2 \\
-1 \\
= \\
= \\
4\end{array}$ \\
\hline 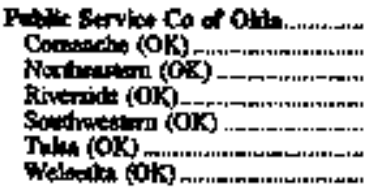 & $\begin{array}{l}60.95 \\
= \\
= \\
=\end{array}$ & $\begin{aligned} & 15 \\
= & \\
- & 9\end{aligned}$ & 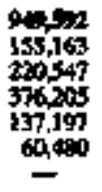 & $\begin{array}{l}= \\
= \\
= \\
=\end{array}$ & $\begin{array}{l}\bar{z} \\
\bar{z} \\
\bar{z}\end{array}$ & $\begin{array}{l}= \\
= \\
=\end{array}$ & $\begin{array}{l}3 \\
= \\
= \\
=\end{array}$ & $\begin{array}{l}* \\
= \\
= \\
-\end{array}$ & $\begin{array}{l}3,94 \\
1,313 \\
2,316 \\
3,970 \\
1,676 \\
673 \\
-\end{array}$ & $\begin{array}{l}-40 \\
= \\
= \\
=\end{array}$ & $\begin{array}{c}145 \\
- \\
- \\
\$ 3 \\
49\end{array}$ \\
\hline 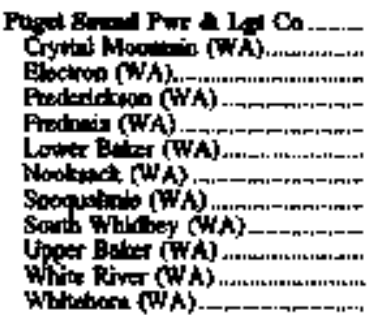 & $\begin{array}{l}\vec{z} \\
= \\
= \\
= \\
= \\
=\end{array}$ & $\begin{array}{l} \\
= \\
= \\
= \\
= \\
= \\
=\end{array}$ & $\begin{array}{l}\bar{z} \\
\bar{z} \\
\bar{z} \\
\bar{z} \\
\bar{z}\end{array}$ & 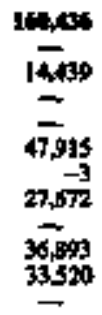 & $\begin{array}{l}= \\
= \\
= \\
= \\
= \\
=\end{array}$ & $\begin{array}{l}= \\
= \\
= \\
= \\
= \\
=\end{array}$ & $\begin{array}{l}= \\
\bar{z} \\
\bar{z} \\
\bar{z} \\
\bar{z}\end{array}$ & $\begin{array}{l}: \\
= \\
= \\
= \\
= \\
=\end{array}$ & $\begin{array}{l}= \\
= \\
= \\
= \\
= \\
=\end{array}$ & $\begin{array}{l}\bar{z} \\
\bar{z} \\
\bar{z} \\
\bar{z} \\
=\end{array}$ & $\begin{array}{l}=1 \\
=22 \\
=2 \\
=2\end{array}$ \\
\hline 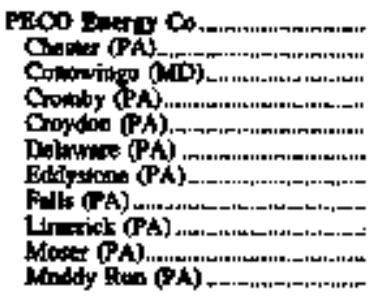 & $\begin{array}{l}\bar{z} \\
\overline{24310} \\
\bar{z} \\
= \\
= \\
=\end{array}$ & 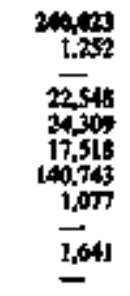 & 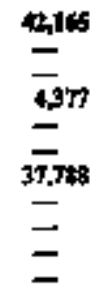 & $\begin{array}{l}-12,409 \\
= \\
= \\
= \\
= \\
=-20,6 \times 0\end{array}$ & $\begin{array}{c}3151405 \\
= \\
= \\
= \\
= \\
= \\
=\end{array}$ & $\begin{array}{l}= \\
= \\
= \\
= \\
= \\
=\end{array}$ & $\begin{array}{l}= \\
= \\
= \\
= \\
= \\
=\end{array}$ & 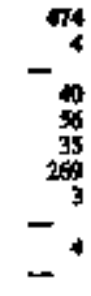 & $\begin{array}{l}= \\
= \\
= \\
= \\
=\end{array}$ & $\begin{array}{l}2 \\
= \\
= \\
= \\
= \\
=\end{array}$ & $\begin{array}{r}50 \\
-\quad 40 \\
40 \\
43 \\
204 \\
-7 \\
-7\end{array}$ \\
\hline
\end{tabular}

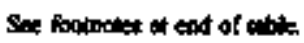


Table 56. DS. Electric Utility Net Generation, Foat Consunption, wnd Foel Stocks by Company and Plant, July 1997 (Coatinned)

\begin{tabular}{|c|c|c|c|c|c|c|c|c|c|c|c|}
\hline \multirow{2}{*}{ 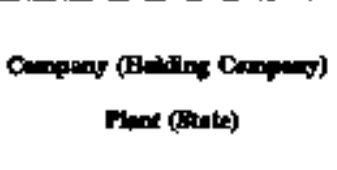 } & \multicolumn{6}{|c|}{ 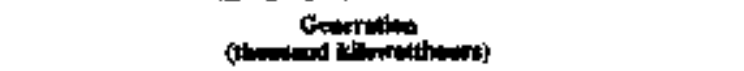 } & \multicolumn{3}{|c|}{ 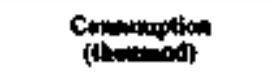 } & \multicolumn{2}{|c|}{ (dithends) } \\
\hline & Cad & Fetrolenes & Ge: & Biture & 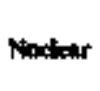 & otind & $\underset{\text { cond }}{\text { tant }}$ & 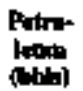 & Getes & $\underset{\substack{\text { Cool } \\
\text { thingt }}}{\operatorname{lint}}$ & מitip \\
\hline
\end{tabular}

Foco rung to

Dil Suthy (PA)

Fon' Bototid (PA)

Rithroed (PA).

Scturylinil (EA)

Solibwet (P)

rsi Eners, lat

Coyous (11)

Collarivilu (IN)

Bdiatipat (an)

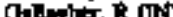

atboe (IN)

Mattind (IN).

MTori Whath (PN)

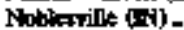

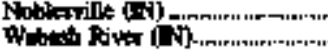

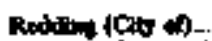

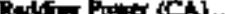

whltejtim (C)

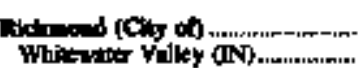

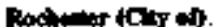

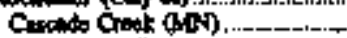

Rocbetar (MT)

Bitut litr (NiN).

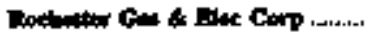
Crow (ANY)

Strition 160 (Niv)

stition 170 दूर

Station 2 (NY)

Stition 26 (NY)

Sominat 3 (NY)

(a) 3 (N)

Sadoo 7 (NY)

strion o sn

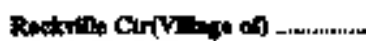
Rockvilim (NY)

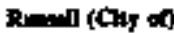

Rnpapin (XS)

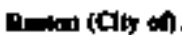

Rinticos (LA).

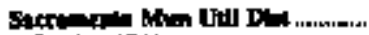

Conits (CA).

Cr. F-W (CA)

Cnow (CA).

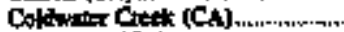

Hede FY (CA) ..........................

Iayoird (CA)...

Jowe Forlt (CH)..............

Lootso (CA)

McCKIn (CA)

Robbs Fotk (C)

slab Cotat (Ch)

Findyon (CA)

Solmo [CH]

Solm (CA)......

Ution Valloy (CA)

Wht kack (C)

Sop soxtendes at end of table.

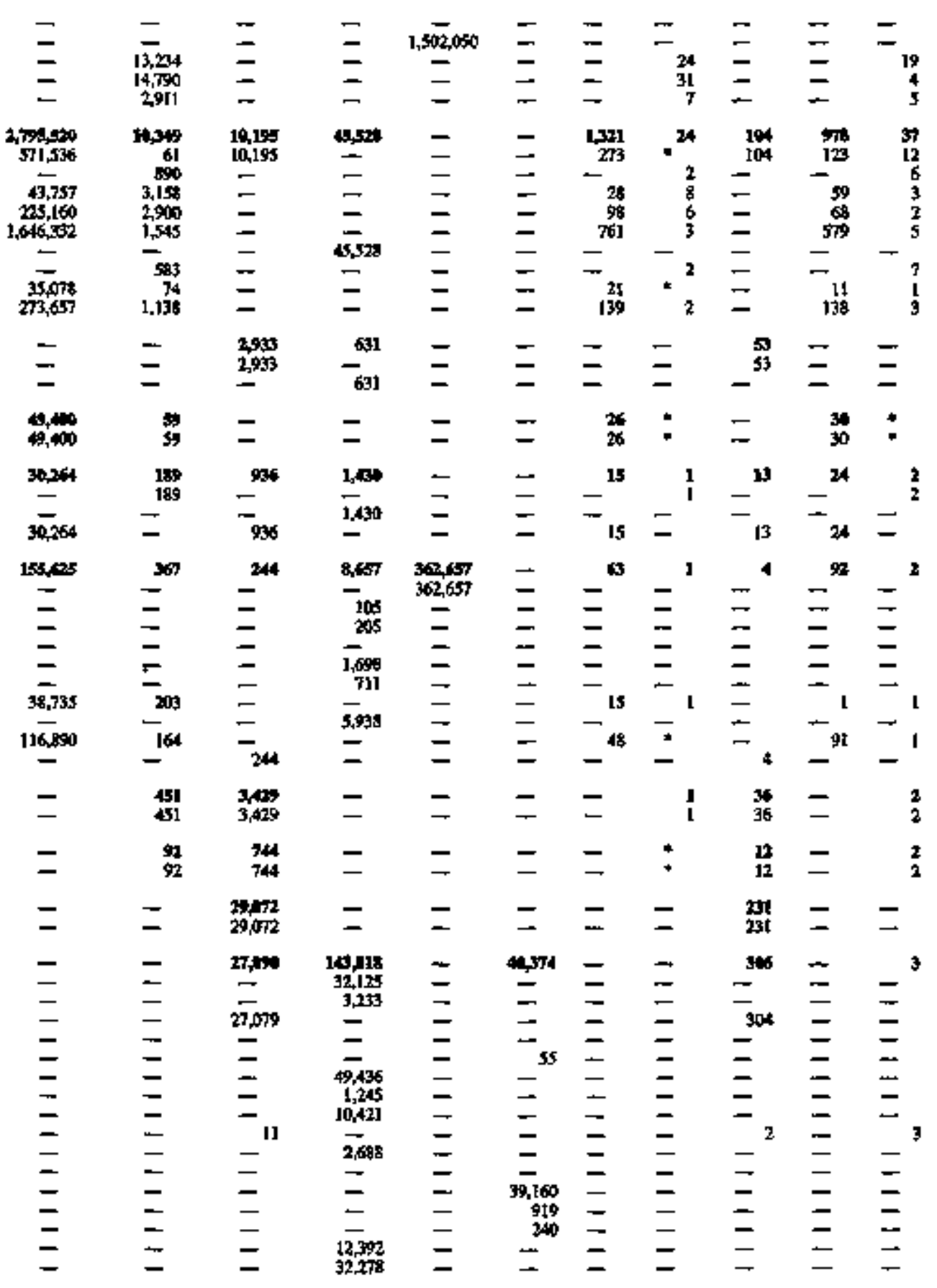

571.536

4375

125,160

646,235

15.075

273,657 
Table 56. U.S. Electric Uthlity Net Generation, Foel Consumption, and Fud Stocks by Conapany and Plant, Joly 1997 (Continaed)

\begin{tabular}{|c|c|c|c|c|c|c|c|c|c|c|c|}
\hline \multirow{2}{*}{ 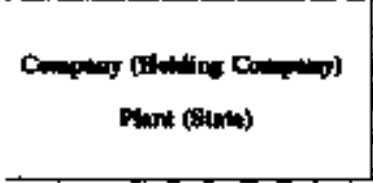 } & \multicolumn{6}{|c|}{ (thenased vlownthours) } & \multicolumn{3}{|c|}{ (thosentid) } & \multicolumn{2}{|c|}{ 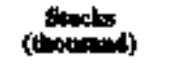 } \\
\hline & Dond & Petriken & Gas & Flpito & Nedent & Onturl & 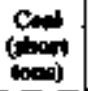 & Patro- & (hat & 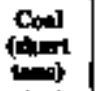 & $\begin{array}{l}\text { Putre- } \\
\text { (bila) }\end{array}$ \\
\hline 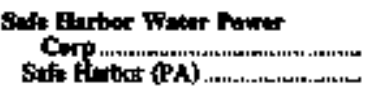 & $\overline{-}$ & $\overline{-}$ & $\overline{-}$ & $\frac{22,26}{22,269}$ & $\overline{-}$ & $\overline{-}$ & $\overline{-}$ & $\overline{-}$ & $\overline{-}$ & $\overline{-}$ & $\Sigma$ \\
\hline 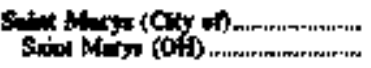 & 4,900 & 64 & $\overline{-}$ & $\bar{z}$ & $\Xi$ & $\bar{z}$ & 3 & * & $\bar{z}$ & 1 & $*$ \\
\hline 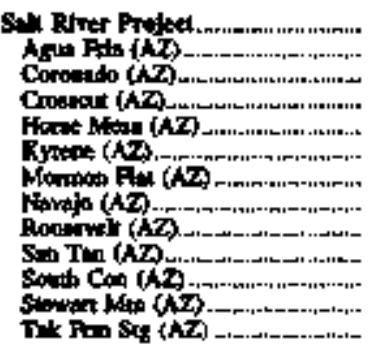 & 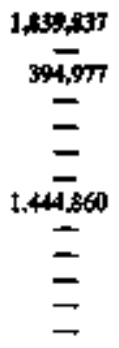 & $\begin{array}{l}1,540 \\
1,369 \\
= \\
= \\
=6 \\
= \\
=\end{array}$ & $\begin{array}{l}113,364 \\
61,515 \\
= \\
= \\
= \\
= \\
= \\
=\end{array}$ & $\begin{array}{l}7,536 \\
\overline{-} \\
1,547 \\
35,176 \\
17,150 \\
\overline{16,106} \\
\overline{565} \\
9,393\end{array}$ & $\begin{array}{l}= \\
= \\
= \\
= \\
= \\
=\end{array}$ & $\begin{array}{l}= \\
= \\
= \\
= \\
= \\
=\end{array}$ & $\begin{array}{l}{ }^{60} \\
= \\
= \\
= \\
= \\
= \\
=\end{array}$ & $\begin{array}{l}- \\
= \\
= \\
= \\
= \\
=\end{array}$ & $\begin{array}{l}1,241 \\
- \\
= \\
- \\
= \\
= \\
= \\
-534 \\
-\end{array}$ & $\begin{array}{l}1,00: \\
=2 \\
= \\
= \\
= \\
= \\
= \\
=\end{array}$ & 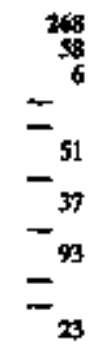 \\
\hline 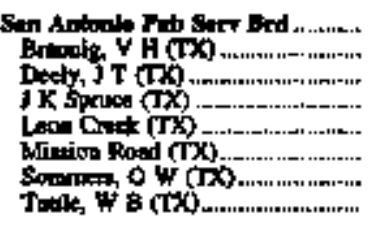 & $\begin{array}{l}164,536 \\
573,408 \\
391,128 \\
= \\
= \\
=\end{array}$ & $\begin{array}{l}369 \\
295 \\
= \\
= \\
=\end{array}$ & 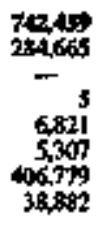 & $\begin{array}{l}\bar{z} \\
\bar{z} \\
\bar{z}\end{array}$ & $\begin{array}{l}= \\
= \\
= \\
=\end{array}$ & $\begin{array}{l}= \\
= \\
= \\
=\end{array}$ & $\begin{array}{l}3 \\
-364 \\
= \\
= \\
=\end{array}$ & $\begin{aligned} & 1 \\
\pm & 1 \\
\pm & \end{aligned}$ & 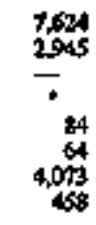 & $\begin{array}{l}{ }^{703} \\
= \\
= \\
=\end{array}$ & $\begin{aligned} & 313 \\
& 193 \\
& 120 \\
&= \\
&= \\
&= \\
&=\end{aligned}$ \\
\hline 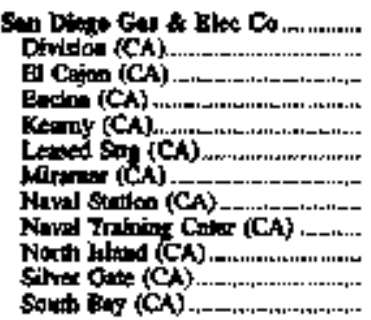 & $\begin{array}{l}= \\
= \\
= \\
= \\
= \\
=\end{array}$ & $\begin{array}{l}= \\
= \\
= \\
= \\
=22 \\
-25\end{array}$ & $\begin{array}{r}535,951 \\
-60 \\
262661 \\
525 \\
-821 \\
313 \\
68 \\
156 \\
-6324\end{array}$ & $\begin{array}{l}\bar{z} \\
\bar{z} \\
\bar{z} \\
\bar{z} \\
\bar{z}\end{array}$ & $\begin{array}{l}\bar{z} \\
\bar{z} \\
= \\
\bar{z} \\
=\end{array}$ & $\begin{array}{l}\bar{z} \\
= \\
= \\
= \\
\bar{z}\end{array}$ & $\begin{array}{l}= \\
= \\
= \\
= \\
= \\
=\end{array}$ & $\begin{array}{l}* \\
* \\
\dot{*} \\
= \\
= \\
*\end{array}$ & 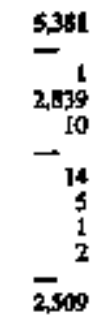 & $\begin{array}{l}= \\
\bar{z} \\
\bar{z} \\
\bar{z} \\
=\end{array}$ & $\begin{array}{r}-1 \\
-19 \\
319 \\
36 \\
1 \\
4 \\
12 \\
1 \\
3 \\
-\quad 322\end{array}$ \\
\hline 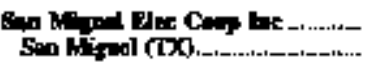 & $\frac{284,443}{285,449}$ & $\frac{\mathbf{2 2}}{22}$ & $\overline{-}$ & $\overline{-}$ & $\overline{-}$ & $\overline{-}$ & $\begin{array}{l}\mathbf{5 3 8} \\
\mathbf{3 3 8}\end{array}$ & $*$ & $\overline{-}$ & $\begin{array}{l}145 \\
145\end{array}$ & 4 \\
\hline 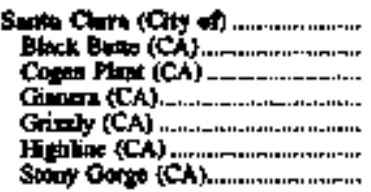 & $\begin{array}{l}\bar{z} \\
= \\
=\end{array}$ & $\begin{array}{l}\vec{z} \\
= \\
= \\
=\end{array}$ & $\begin{array}{l}\frac{\$ 119}{4569} \\
650 \\
= \\
=\end{array}$ & $\begin{array}{l}\$, 264 \\
= \\
= \\
1,607 \\
247 \\
1,410\end{array}$ & $\begin{array}{l}\bar{z} \\
= \\
\bar{z}\end{array}$ & $\begin{array}{l}= \\
= \\
\bar{z}\end{array}$ & $\begin{array}{l}= \\
= \\
= \\
=\end{array}$ & $\begin{array}{l}= \\
= \\
=\end{array}$ & $\begin{array}{l}-74 \\
= \\
=\end{array}$ & $\begin{array}{l}= \\
\bar{z} \\
=\end{array}$ & $\begin{array}{l}\bar{z} \\
\bar{z} \\
=\end{array}$ \\
\hline 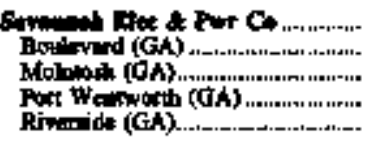 & 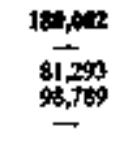 & $\begin{array}{l}\frac{60}{600} \\
=\end{array}$ & $\begin{array}{r}135,941 \\
1,799 \\
12,022 \\
35,761 \\
16,379\end{array}$ & $\begin{array}{l}\vec{z} \\
=\end{array}$ & $\begin{array}{l}= \\
=\end{array}$ & $\begin{array}{l}= \\
=\end{array}$ & $\begin{array}{r}59 \\
-47 \\
-\end{array}$ & $\begin{array}{l}-1 \\
=\end{array}$ & $\begin{array}{r}1,777 \\
32 \\
1,002 \\
396 \\
260\end{array}$ & $\begin{array}{l}31 \\
-\quad 60 \\
-3 I \\
-\end{array}$ & $\begin{array}{r}167 \\
9 \\
129 \\
-\quad 26\end{array}$ \\
\hline 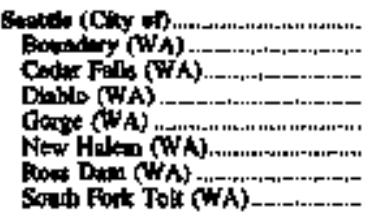 & $\begin{array}{l}= \\
= \\
= \\
=\end{array}$ & $\begin{array}{l}= \\
= \\
= \\
=\end{array}$ & $\begin{array}{l}\bar{z} \\
\bar{z} \\
\bar{z}\end{array}$ & $\begin{array}{r}17,345 \\
500,250 \\
7,680 \\
114,397 \\
120,765 \\
-5 \\
139276 \\
5,1068\end{array}$ & $\begin{array}{l}\bar{z} \\
z \\
= \\
z\end{array}$ & $\begin{array}{l}= \\
= \\
= \\
=\end{array}$ & $\begin{array}{l}= \\
= \\
= \\
=\end{array}$ & $\begin{array}{l}= \\
= \\
= \\
=\end{array}$ & $\begin{array}{l}\bar{z} \\
\bar{z} \\
\bar{z}\end{array}$ & $\begin{array}{l}\bar{z} \\
\bar{z} \\
\bar{z}\end{array}$ & $\begin{array}{l}\bar{z} \\
= \\
\bar{z} \\
=\end{array}$ \\
\hline 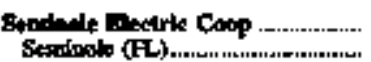 & $206,97 \%$ & $\begin{array}{l}\$, 516 \\
9,916\end{array}$ & $\vec{a}$ & $\bar{z}$ & $\approx$ & $=$ & 3 & 3 & $\bar{z}$ & *4: & 6 \\
\hline
\end{tabular}

Son tooknolas al and of tabla. 


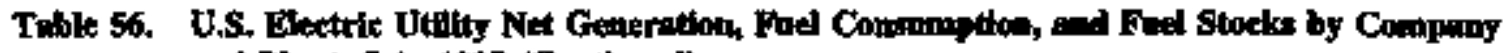
and Phan, Joly 19\% (Contioned)

\begin{tabular}{|c|c|c|c|c|c|c|c|c|c|c|c|}
\hline \multirow{2}{*}{ 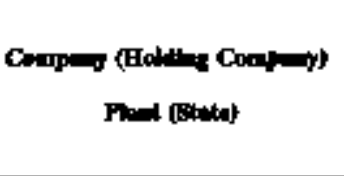 } & \multicolumn{6}{|c|}{ 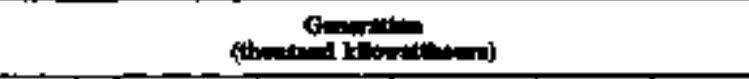 } & \multicolumn{3}{|c|}{ Consing } & \multicolumn{2}{|c|}{ 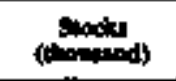 } \\
\hline & لוanc & 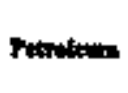 & M & Eyon: & Nodear & Othel & $\underset{\operatorname{col}}{\text { Cod }}$ & Tatror & ams & $\underset{\text { (ithert }}{\operatorname{col}}$ & $\lim _{0 \rightarrow 4}$ \\
\hline
\end{tabular}

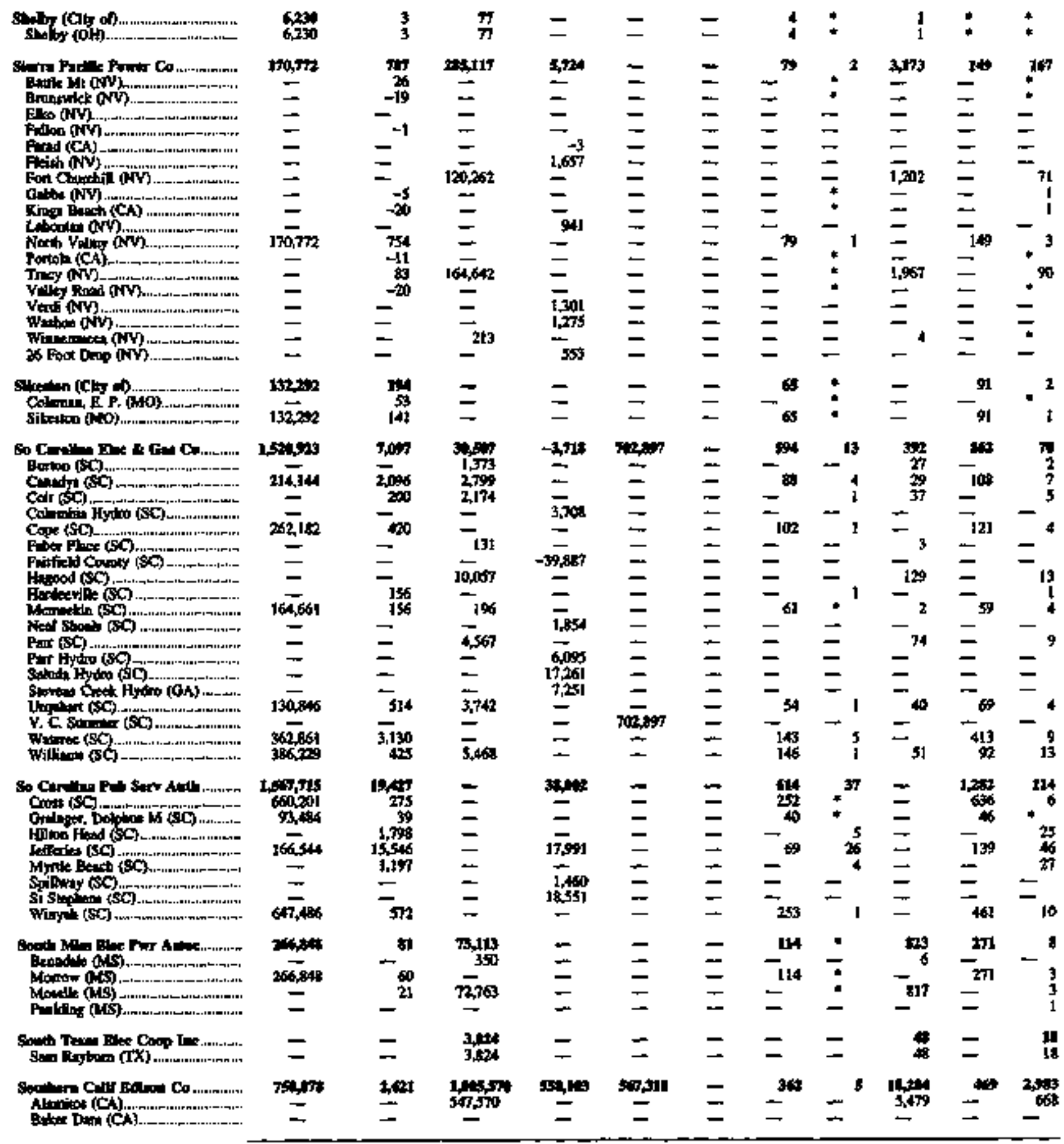

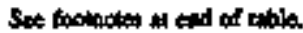


Tuble 56. US. Electric Utiliy Net Geaeration, Fael Consangption, and Fuel Stacks by Company and Plant, July 1997 (Contineed)

\begin{tabular}{|c|c|c|c|c|c|c|c|c|c|c|c|}
\hline \multirow{2}{*}{ 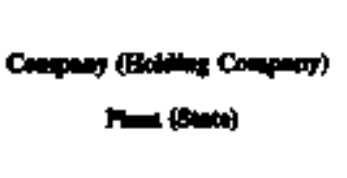 } & \multicolumn{6}{|c|}{ 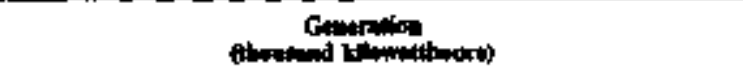 } & \multicolumn{3}{|c|}{ 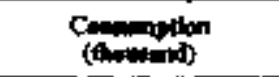 } & \multicolumn{2}{|c|}{ (itimenty } \\
\hline & cen & 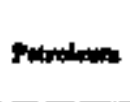 & Giw & Hydrw & Ninktan & 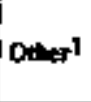 & Cont & $\begin{array}{l}\text { Potro } \\
\text { letion } \\
\text { fibla) }\end{array}$ & (Man) & $\begin{array}{c}\text { Ond } \\
\text { (titort } \\
\text { tones) }\end{array}$ & Petro- \\
\hline
\end{tabular}

\begin{tabular}{|c|c|c|c|c|c|c|c|c|c|c|c|}
\hline & & & & & & & & & & & \\
\hline 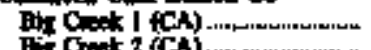 & 二 & - & - & 59,965 & - & $\bar{z}+(x+1+1+1$ & - & - & $=$ & - & - \\
\hline 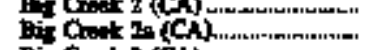 & 二 & $\vec{z}$ & $\bar{z}$ & 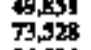 & $\Xi$ & $=$ & $z$ & 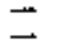 & $\overline{-}$ & 二 & $z$ \\
\hline Bit Com 3 (CA) & - & - & - & 94,526 & - & - & - & - & - & - & \\
\hline $\mathrm{Crot}_{4}(\mathrm{CA})$ & - & - & - & 4,54 & - & - & - & - & - & - & - \\
\hline 5 Chock $2(\mathrm{CA})$ & $\rightarrow$ & 二 & 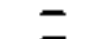 & \$6,110 & $=$ & $=$ & $=$ & 二 & $=$ & $=$ & - \\
\hline pindep Cen 2 (CA) & $=$ & $=$ & $=$ & 5.716 & $\bar{z}$ & $=$ & $\bar{z}+r+r+1$ & $\bar{z}$ & $=$ & $\bar{z}$ & - \\
\hline 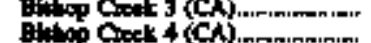 & $\Xi$ & $\bar{z}$ & $\Xi$ & $\begin{array}{ll}5,793 \\
5,899\end{array}$ & $\bar{z}$ & 二 & $\Xi$ & $=$ & $=$ & 二 & $=$ \\
\hline Bupe ows $S(C A)$ & $\bar{z}$ & - & - & 2160 & $=$ & ב & $=$ & 二 & - & $\vec{z}$ & $=$ \\
\hline Gopop Det 6 (CA) & - & $=$ & - & $\$ 12$ & $=$ & - & - & - & 二 & - & - \\
\hline 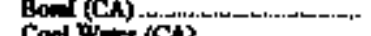 & $=$ & - & $\overline{0}_{0,0}$ & 7,192 & - & $=$ & $=$ & $=$ & Fou & $=$ & - \\
\hline & $\bar{z}$ & $\bar{z}$ & 95,007 & $\bar{z}$ & $\bar{z}$ & $\bar{z}+r+r+1+1$ & $\bar{z}+\mathrm{r}+\mathrm{x}+\mathrm{x}$ & $\bar{z}$ & hon & $\bar{z}$ & 355 \\
\hline Dominowa $(\mathrm{E} A)$ (CA) & $\Xi$ & $=$ & $=$ & $3 \overline{3,567}$ & $=$ & $\Xi$ & $\Xi$ & 二 & $=$ & $=$ & 616 \\
\hline G songundo $(\mathrm{C})$ & $=$ & - & 95,569 & - & - & ב & $=$ & $\Xi$ & 1,034 & 二 & \\
\hline 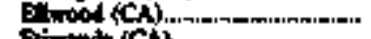 & - & - & 34 & $\overrightarrow{-}$ & - & - & - & - & ' & - & \\
\hline 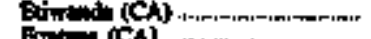 & 二 & 二 & 110,500 & - & $\bar{z}$ & $=$ & $=$ & $=$ & 1,200 & 二 & 283 \\
\hline Fin & 二 & $=$ & $\overline{-1}_{-16}$ & - & $=$ & $\Xi$ & $z$ & $=$ & 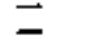 & $=$ & $=$ \\
\hline 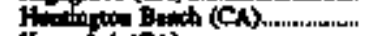 & - & - & 99,696 & $\rightarrow$ & - & - & - & - & 1,055 & - & 162 \\
\hline 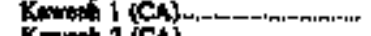 & - & - & - & 1,195 & - & $=$ & - & - & - & - & $=$ \\
\hline 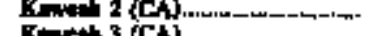 & - & - & $=$ & 1397 & $=$ & - & - & 二 & - & - & - \\
\hline Chet 3 (C) & $=$ & $\bar{z}$ & $\bar{z}$ & 10208 & z & च & - & $\vec{z}$ & $\bar{z}$ & $\bar{z}$ & $=$ \\
\hline Son River 3 (CA & $z$ & $\vec{z}$ & $\Xi$ & 27,459 & $\bar{z}$ & $\bar{z}$ & 二 & - & 二 & $\bar{z}$ & $\bar{z}$ \\
\hline Looi Beach (C) & - & - & $14,9 n$ & - & $=$ & - & - & - & 174 & - & 710 \\
\hline $\operatorname{Lan}_{2},(\mathrm{C} N)_{-}$ & - & - & - & $2 m$ & - & - & - & - & - & - & - \\
\hline Lrike Get (CA) & - & 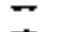 & - & 239 & 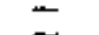 & 二 & $\bar{z}$ & - & 二 & - & - \\
\hline 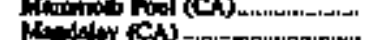 & & & 130.310 & $5,75 s$ & & $\bar{z}$ & E & 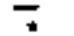 & T242 & $\bar{z}+r+r+r+1$ & - \\
\hline 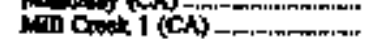 & $\bar{z}$ & - & - & -11 & - & $\overline{-}$ & 二 & - & $-1,2+2$ & 二 & 239 \\
\hline Wip Qeek $2+3$ (CA).............. & - & - & - & - & - & - & - & - & - & - & - \\
\hline $\min$ Gesk $3(C A)$ - & - & - & -5 & $83 \mathrm{~B}$ & $\bar{z}+1+r+1+1$ & $=$ & - & 二 $+r+r+r+1$ & - & - & $=$ \\
\hline tio [ [CA & 735,678 & & 3,342 & $-{ }_{2 \mathrm{st}}$ & - & 二 & & $\Xi$ & & 469 & \\
\hline 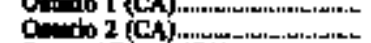 & $=$ & $=$ & $=$ & is & $=$ & $=$ & 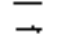 & 二 & $=$ & $\vec{z}$ & \\
\hline Onot Bthth (CA)............... & - & - & 197.366 & - & - & - & - & - & 3,536 & - & \\
\hline Pothly Bowith (CA) & - & 2601 & 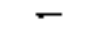 & - & - & - & - & 5 & - & - & \\
\hline Foolo (CA) & - & - & - & 7,452 & - & - & - & - & - & - & - \\
\hline & $\overline{-}$ & - & & -10 & - & 二 & $\bar{z}$ & $=$ & & & \\
\hline 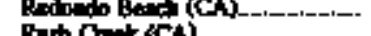 & - & $\bar{z}$ & 34,001 & $\overline{T e x}$ & - & $=$ & - & - & 3,412 & $=$ & 75 \\
\hline 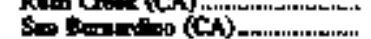 & - & $=$ & 2000 & 1, & $\bar{Z}$ & 二 & - & $=$ & & $=$ & 15 \\
\hline Gortontio (CA)-man & - & - & & 92 & - & $=$ & $=$ & $=$ & $\bar{z}+x+1+x+10$ & $=$ & $=$ \\
\hline 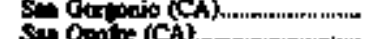 & 二 & $\bar{z}+\mathrm{r}+\mathrm{r}+\mathrm{r}+\mathrm{r}$ & 二 & $=$ & 507318 & 二 & $\bar{z}+r+1+x$ & $\bar{z}+r+1+$ & $\bar{z}$ & $=$ & $=$ \\
\hline 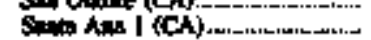 & - & - & - & 3 & - & - & - & - & - & - & 2 \\
\hline$A=2(\mathrm{C} N)$ & $\rightarrow$ & - & - & 210 & - & - & - & - & - & - & - \\
\hline$\{\mathrm{CA}\}_{\text {, }}$ & 二 & $=$ & 二 & -3 & 二 & $=$ & $\vec{z}$ & $=$ & $\bar{z}$ & $\bar{z}$ & $=$ \\
\hline 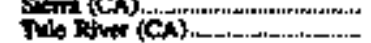 & $=$ & 二 & $=$ & {$[, 68]$} & - & - & - & $\bar{z}+r+1+x+1+3$ & $=$ & $=$ & $\bar{z}+r+r+1+x$ \\
\hline Q & Kr & 301 & - & - & - & - & & ! & & 37 & 1 \\
\hline (2) & 9 & 381 & - & - & - & - & & & - & $\$ 27$ & $\mathbf{i}$ \\
\hline 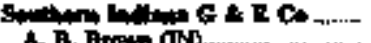 & teren & $=$ & 17,46 & 二 & $=$ & 二 & $\operatorname{mon}_{132}$ & $=$ & 24 & $\begin{array}{l}396 \\
156\end{array}$ & $\frac{7}{3}$ \\
\hline 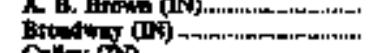 & $=$ & $\bar{z}$ & 6,912 & $\bar{z}$ & 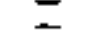 & - & - & $\bar{z}$ & 97 & - & \\
\hline 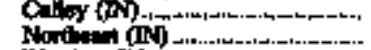 & 240.223 & $z$ & $\begin{array}{l}2005 \\
320\end{array}$ & $\bar{z}$ & $z$ & $\Xi$ & $\rightarrow$ & 二 & 67 & -22 & $=$ \\
\hline W & 0.487 & - & & - & - & - & 32 & - & & 118 & - \\
\hline 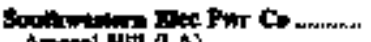 & 1, & 1054 & $m_{10}$ & - & - & - & 1,319 & & 5,488 & 1,264 & 91 \\
\hline 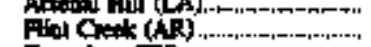 & 336.291 & $1,0,3$ & 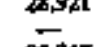 & z & & $\bar{z}$ & 715 & 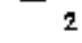 & -3 & $\overline{348}$ & \\
\hline 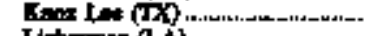 & $+\sigma_{0}$ & - & 89,217 & - & - & - & - & - & 886 & - & 43 \\
\hline 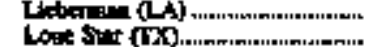 & $=$ & こ & $\frac{17,064}{6,024}$ & $=$ & $=$ & $=$ & $=$ & $\bar{z}$ & $\begin{array}{c}1088 \\
85\end{array}$ & $=$ & $\begin{array}{c}200 \\
3\end{array}$ \\
\hline
\end{tabular}

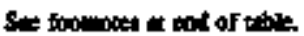


Tabte 56. U.S. Moctric Ut"ity Net Generntton, Foed Consimption, and Fued Stocks by Company and Plant, Jaig 1997 (Conthrared)

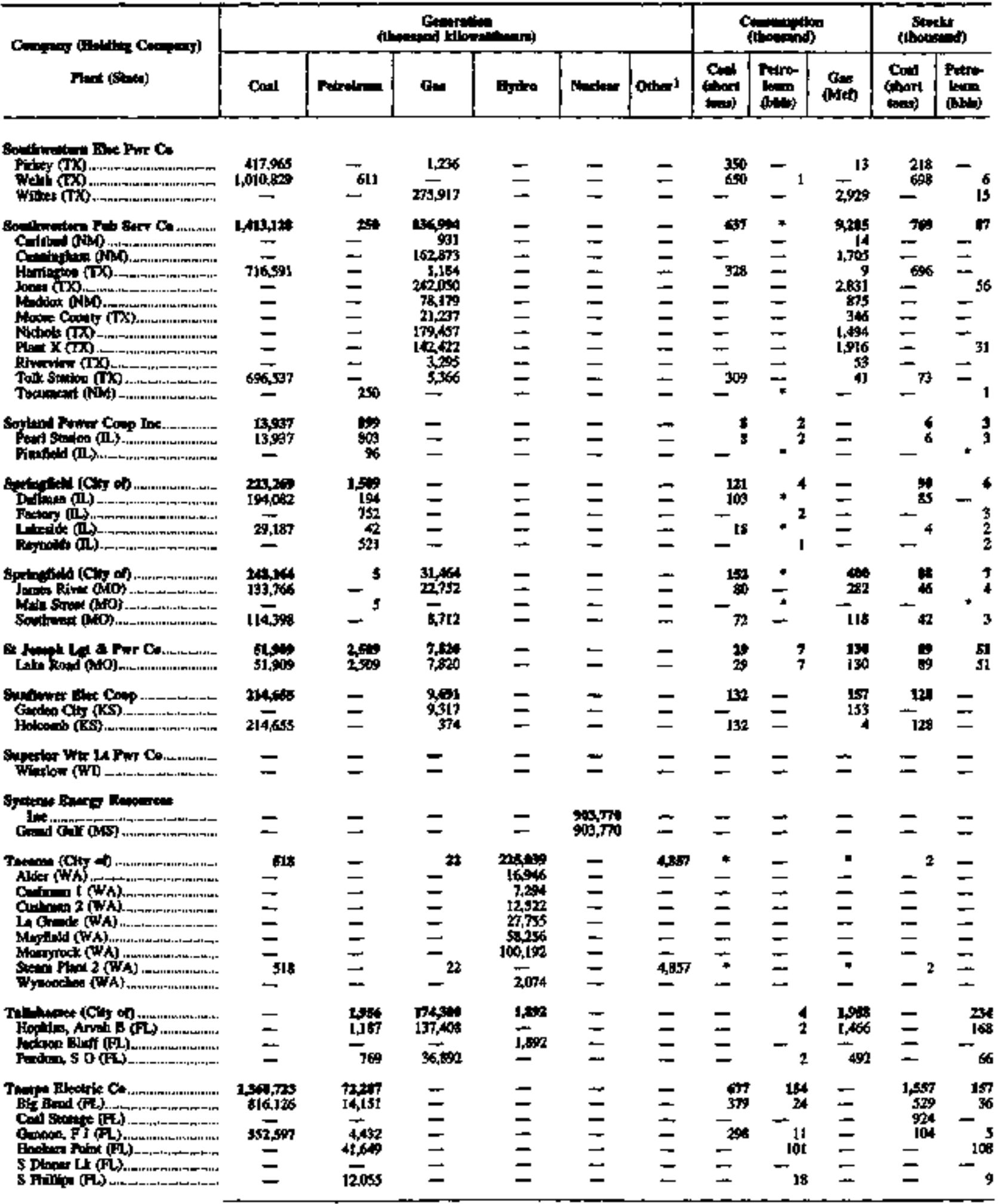

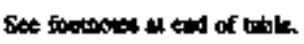


Twble 56. U.S. Blectric Utilty Net Generation, Fuel Consumption, and Fuel Stocks by Company and Fiant, July 19\%7 (Contioued)

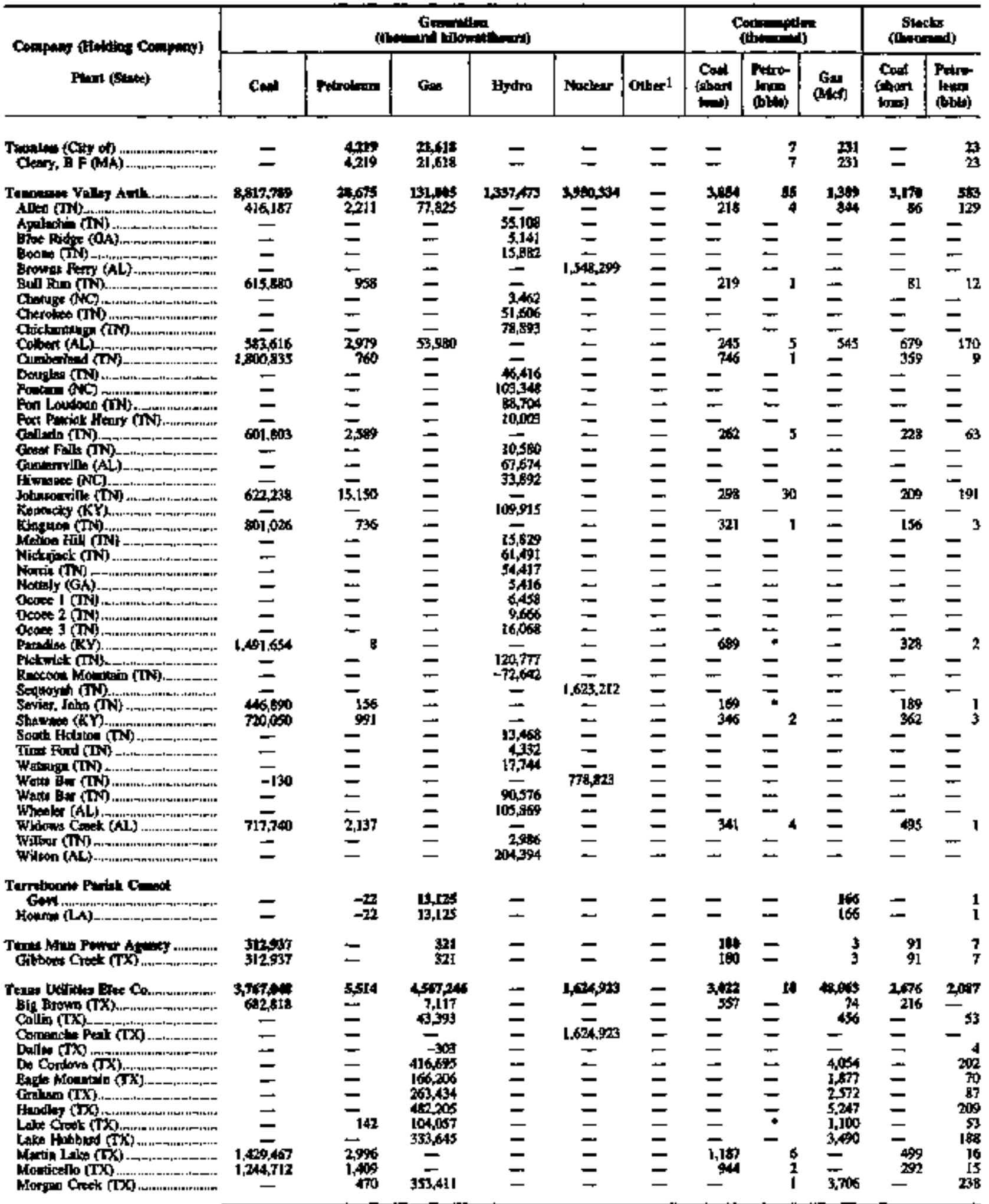

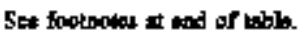


Tabłe 56. US. Electric Utilly Net Generatlow, Fuel Comsamption, and Fod Stocks by Company and Plont, Jinly 1997 (Contlnoed)

\begin{tabular}{|c|c|c|c|c|c|c|c|c|c|c|c|}
\hline \multirow{2}{*}{ 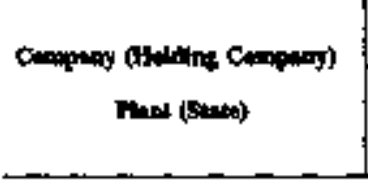 } & \multicolumn{6}{|c|}{ 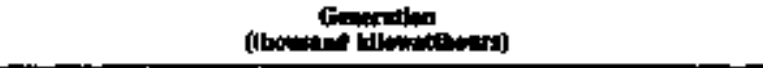 } & \multicolumn{3}{|c|}{ 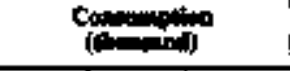 } & \multicolumn{2}{|c|}{ (ה) } \\
\hline & Conl & Fetroloin & Car & 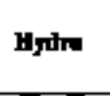 & Stoctaser & Oher 1 & $\underset{\text { Cond }}{(\lim }$ & $\begin{array}{l}\text { Pans } \\
\text { lothen }\end{array}$ & (Min) & Cwed & קוm \\
\hline 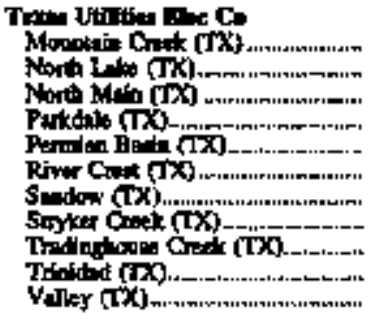 & $\begin{array}{l}\bar{z} \\
\bar{z} \\
\overline{=} \\
\overline{=} \\
=\end{array}$ & $\begin{array}{l}= \\
= \\
-14 \\
26 \\
-12 \\
-12\end{array}$ & $\begin{array}{r}327,176 \\
262,730 \\
102,140 \\
342,333 \\
-39 \\
291,529 \\
60,352 \\
78,471 \\
3 \% 4,140\end{array}$ & $\begin{array}{l}= \\
= \\
= \\
= \\
= \\
=\end{array}$ & $\begin{array}{l}= \\
= \\
= \\
= \\
=\end{array}$ & $\begin{array}{l}= \\
= \\
= \\
= \\
= \\
=\end{array}$ & $\begin{array}{l}= \\
= \\
= \\
= \\
= \\
=\end{array}$ & $\begin{array}{l}= \\
= \\
= \\
= \\
=\end{array}$ & $\begin{array}{l}3,501 \\
2,338 \\
\frac{1}{1,256} \\
3,580 \\
= \\
2,46 \\
6,273 \\
818 \\
4,0 \% 6\end{array}$ & $\begin{array}{l}= \\
= \\
= \\
= \\
= \\
=\end{array}$ & $\begin{array}{r}146 \\
125 \\
-\quad 30 \\
218 \\
-\quad 84 \\
154 \\
140\end{array}$ \\
\hline 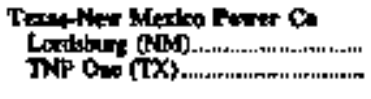 & $\frac{207,700}{207,700}$ & $\bar{z}$ & $\frac{T}{67}$ & $\bar{z}$ & $\bar{z}$ & $\bar{z}$ & - tit: & $\stackrel{\sim}{-}$ & -1 & $-_{\mathbf{3}}^{\mathbf{3}}$ & $\vec{z}$ \\
\hline 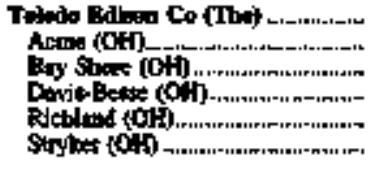 & $\begin{array}{c}271,003 \\
\frac{-}{27,003} \\
=\end{array}$ & $\frac{1,775}{1,196}$ & $\begin{array}{l}-143 \\
= \\
=\end{array}$ & $\begin{array}{l}= \\
= \\
=\end{array}$ & $\frac{\bar{z}}{201866}$ & $\begin{array}{l}z \\
z \\
=\end{array}$ & $\begin{array}{l}197 \\
- \\
=\end{array}$ & ${ }^{2}{ }^{2}$ & $=^{4}$ & $\begin{array}{l}104 \\
= \\
=\end{array}$ & $\begin{array}{r}-3 \\
-1 \\
-2\end{array}$ \\
\hline 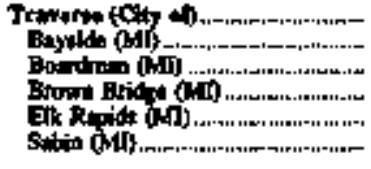 & $\begin{array}{l}1,716 \\
1,710 \\
= \\
=\end{array}$ & $\begin{array}{l}= \\
= \\
=\end{array}$ & $\begin{array}{l}= \\
= \\
=\end{array}$ & $\begin{array}{l}1,191 \\
-445 \\
246 \\
114 \\
194\end{array}$ & $\begin{array}{l}= \\
\bar{z} \\
=\end{array}$ & $\begin{array}{l}= \\
= \\
=\end{array}$ & $\begin{array}{l}{ }^{1} \\
=\end{array}$ & $\begin{array}{l}= \\
\bar{z}\end{array}$ & $\begin{array}{l}= \\
= \\
=\end{array}$ & $=\frac{12}{=}$ & $\begin{array}{l}= \\
z \\
=\end{array}$ \\
\hline 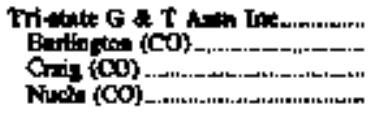 & 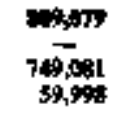 & $\begin{array}{l}1,805 \\
-\quad 167\end{array}$ & ${ }_{-}^{8+3}$ & $\begin{array}{l}\Xi \\
\bar{z}\end{array}$ & $\begin{array}{l}\bar{z} \\
\bar{z}\end{array}$ & $=$ & $\begin{array}{r}\mathbf{4 5} \\
\mathbf{3 8 3} \\
\mathbf{3 2}\end{array}$ & $\begin{array}{r}2 \\
-\quad 1\end{array}$ & -5 & $\frac{1,240}{1,246}$ & $\begin{array}{r}21 \\
13 \\
3 \\
1\end{array}$ \\
\hline 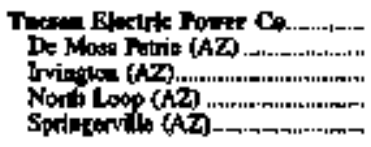 & $\begin{array}{l}57,160 \\
52,924 \\
51 \overline{7,435}\end{array}$ & $\begin{array}{l}= \\
=\end{array}$ & $\begin{array}{l}5,368 \\
2,030 \\
50,197 \\
1,122 \\
-\end{array}$ & $\begin{array}{l}= \\
=\end{array}$ & $\begin{array}{l}= \\
=\end{array}$ & $\begin{array}{l}= \\
=\end{array}$ & $\begin{array}{l}-36 \\
-29 \\
296\end{array}$ & $\begin{array}{l}= \\
= \\
=\end{array}$ & $\begin{array}{r}45 \\
28 \\
-\quad 20 \\
-11 \\
\end{array}$ & $\begin{array}{r}408 \\
-461\end{array}$ & $\begin{array}{r}\mathbf{5} \\
\mathbf{5} \\
7 \\
3\end{array}$ \\
\hline 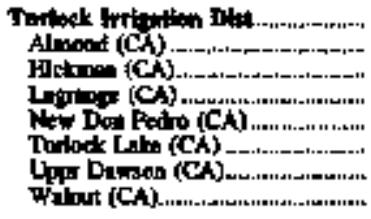 & $\begin{array}{l}\bar{z} \\
\bar{z} \\
=\end{array}$ & $\begin{array}{l}= \\
= \\
z \\
=\end{array}$ & $\begin{array}{l}11,7 \in 5 \\
11,6 \pm 4 \\
= \\
= \\
=\end{array}$ & 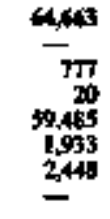 & $\begin{array}{l}= \\
= \\
= \\
=\end{array}$ & $\begin{array}{l}= \\
= \\
= \\
=\end{array}$ & $\begin{array}{l}= \\
= \\
= \\
=\end{array}$ & $\begin{array}{l}= \\
= \\
= \\
=\end{array}$ & $\begin{array}{l}111 \\
= \\
= \\
=\end{array}$ & $\begin{array}{l}= \\
= \\
= \\
=\end{array}$ & $\begin{array}{l}z^{3} \\
= \\
=\end{array}$ \\
\hline 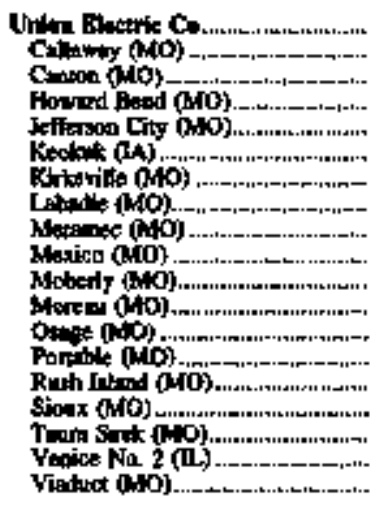 & 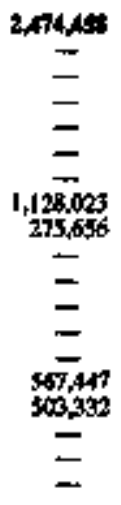 & 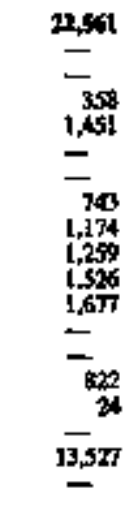 & 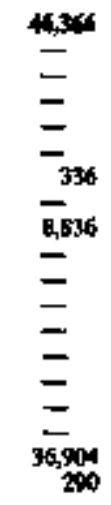 & $\begin{array}{l}7,061 \\
= \\
= \\
= \\
= \\
= \\
= \\
36,685 \\
= \\
-25,480 \\
=\end{array}$ & $\begin{array}{l}\text { sians } \\
= \\
= \\
= \\
= \\
= \\
= \\
= \\
= \\
= \\
=\end{array}$ & $\begin{array}{l}= \\
= \\
= \\
= \\
= \\
= \\
= \\
= \\
= \\
= \\
=\end{array}$ & $\begin{array}{l}1 / 60 \\
= \\
= \\
= \\
= \\
= \\
= \\
= \\
= \\
350 \\
= \\
= \\
=\end{array}$ & $\begin{array}{l}=^{6} \\
=5 \\
={ }^{1} \\
3 \\
5 \\
{ }^{5} \\
{ }^{6} \\
{ }^{3}\end{array}$ & $\begin{array}{l}= \\
= \\
= \\
=6 \\
=96 \\
= \\
= \\
= \\
= \\
{ }^{98}\end{array}$ & $\begin{array}{l}1= \\
= \\
= \\
= \\
= \\
= \\
= \\
= \\
= \\
597 \\
= \\
=\end{array}$ & 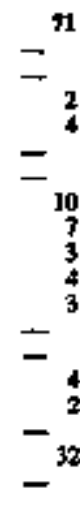 \\
\hline
\end{tabular}

Ste frotionotat al and of whle. 
Table 56. U.S. Electric Utilty Net Goweration, Foel Conounption, and Fuel Stocks by Company had Plant, Joly I9's (Conthored)

\begin{tabular}{|c|c|c|c|c|c|c|c|c|c|c|c|}
\hline \multirow{2}{*}{ 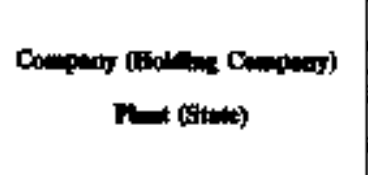 } & \multicolumn{6}{|c|}{ 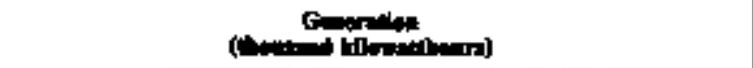 } & \multicolumn{3}{|c|}{ Conimilon } & \multicolumn{2}{|c|}{ 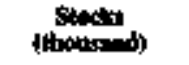 } \\
\hline & Cen & 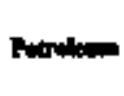 & Gew & Ry,leo & Nodisar & Otherl & $\underset{\text { (thoct }}{\text { Cond }}$ & $\lim _{n \rightarrow \infty}$ & (Avat) & $\begin{array}{c}\text { Cod } \\
(\text { thend }\end{array}$ & $\begin{array}{l}\text { Petro } \\
\text { leven } \\
\text { (bils) }\end{array}$ \\
\hline 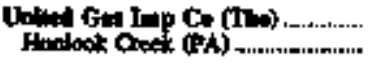 & $\begin{array}{l}\text { 30,6s } \\
\text { 30,6s's }\end{array}$ & $\$$ & $\overline{-}$ & $\bar{z}$ & $\bar{z}$ & $\overline{-}$ & $\begin{array}{l}21 \\
21\end{array}$ & : & $=$ & 17 & * \\
\hline 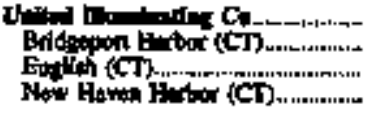 & $\begin{array}{c}191,345 \\
198,348 \\
-\end{array}$ & 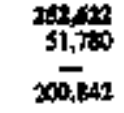 & $\frac{7}{12899}$ & $\bar{z}$ & $\begin{array}{l}z \\
z\end{array}$ & $\begin{array}{l}\bar{z} \\
\end{array}$ & $\begin{array}{l}\boldsymbol{9} \\
- \\
-\end{array}$ & $\begin{array}{r}100 \\
-\quad 306\end{array}$ & $\bar{z}^{124}$ & $\begin{array}{l}128 \\
=\end{array}$ & $\begin{array}{r}578 \\
136 \\
-\quad 43\end{array}$ \\
\hline 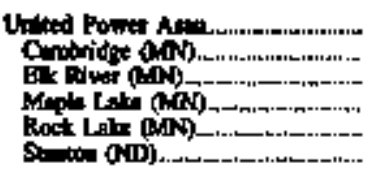 & 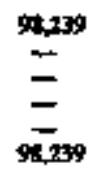 & $\begin{array}{r}736 \\
296 \\
205 \\
102 \\
102\end{array}$ & $\begin{array}{l}2 n \\
2 m \\
=\end{array}$ & $\begin{array}{l}\bar{z} \\
\bar{z}\end{array}$ & $\begin{array}{l}= \\
\bar{z} \\
=\end{array}$ & $\begin{array}{l}15,200 \\
15,206 \\
= \\
=\end{array}$ & $\begin{array}{l} \\
= \\
= \\
=\end{array}$ & $\begin{array}{r}2 \\
-\quad 1 \\
*\end{array}$ & $\begin{array}{l}=5 \\
= \\
=\end{array}$ & $\begin{array}{l}{ }^{76} \\
= \\
76\end{array}$ & $\begin{array}{l}\mathbf{5} \\
\mathbf{1} \\
\mathbf{1} \\
\mathbf{1}\end{array}$ \\
\hline 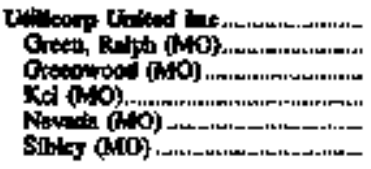 & $\begin{array}{l}26406 \\
= \\
=\end{array}$ & $\begin{array}{l}= \\
= \\
203 \\
153\end{array}$ & $\begin{array}{l}0,48 \\
8,187 \\
39,477 \\
1,991 \\
-\end{array}$ & $\begin{array}{l}\bar{z} \\
\bar{z}\end{array}$ & $\begin{array}{l}z \\
z \\
z \\
=\end{array}$ & $\begin{array}{l}\bar{z} \\
\bar{z}\end{array}$ & $\begin{array}{l}14 \\
\ddot{2} \\
= \\
142\end{array}$ & $\begin{array}{ll} & 1 \\
= & 1 \\
& 1\end{array}$ & $\begin{array}{r}64 \\
106 \\
531 \\
37 \\
-\end{array}$ & $\begin{array}{l}{ }^{2} \\
= \\
=\end{array}$ & $\begin{array}{r}33 \\
-\quad 4 \\
1\end{array}$ \\
\hline 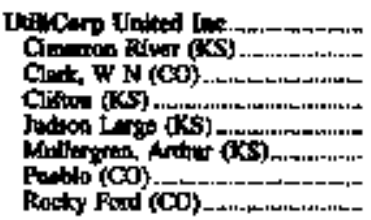 & $\begin{array}{l}n 1,99 \\
21,929 \\
= \\
= \\
=\end{array}$ & $\begin{array}{l}2 \boldsymbol{y} \\
= \\
= \\
= \\
= \\
\mathbf{1 7}\end{array}$ & 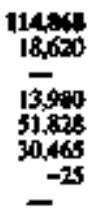 & $\begin{array}{l}= \\
= \\
= \\
=\end{array}$ & $\begin{array}{l}= \\
= \\
= \\
=\end{array}$ & $\begin{array}{l}= \\
\bar{z} \\
\bar{z} \\
=\end{array}$ & $\begin{array}{l}-12 \\
= \\
= \\
=\end{array}$ & $\begin{array}{l}= \\
= \\
= \\
=\end{array}$ & $\begin{array}{l}1+41 \\
- \\
261 \\
211 \\
- \\
-\end{array}$ & $\begin{array}{l}-8 \\
= \\
= \\
=\end{array}$ & $\begin{array}{l}= \\
= \\
1 \\
1\end{array}$ \\
\hline 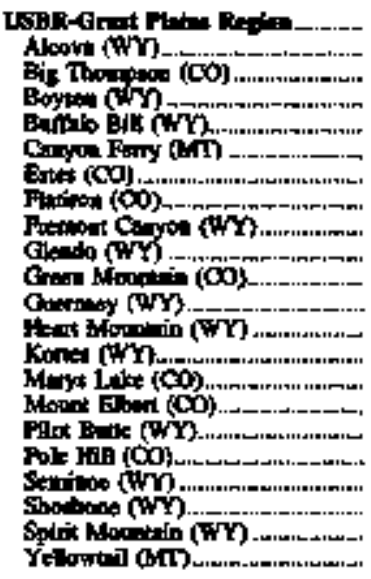 & $\begin{array}{l}= \\
= \\
= \\
= \\
= \\
= \\
= \\
= \\
= \\
= \\
=\end{array}$ & $\begin{array}{l}= \\
= \\
= \\
= \\
= \\
= \\
= \\
= \\
= \\
=\end{array}$ & $\begin{array}{l}= \\
= \\
= \\
= \\
= \\
= \\
= \\
= \\
= \\
= \\
=\end{array}$ & 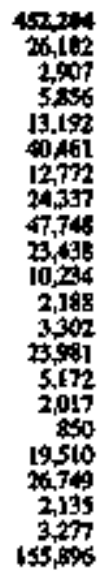 & $\begin{array}{l}= \\
= \\
= \\
= \\
= \\
= \\
= \\
= \\
= \\
= \\
= \\
=\end{array}$ & $\begin{array}{l}\bar{z} \\
\bar{z} \\
z \\
z \\
= \\
\bar{z} \\
= \\
= \\
= \\
\bar{z} \\
= \\
=\end{array}$ & $\begin{array}{l}\bar{z} \\
= \\
= \\
\bar{z} \\
= \\
\bar{z} \\
\bar{z} \\
= \\
= \\
= \\
\bar{z}\end{array}$ & $\begin{array}{l}= \\
= \\
= \\
z \\
= \\
= \\
= \\
= \\
= \\
= \\
= \\
=\end{array}$ & $\begin{array}{l}\bar{z} \\
= \\
= \\
\bar{z} \\
\bar{z} \\
\bar{z} \\
\bar{z} \\
= \\
= \\
= \\
= \\
=\end{array}$ & $\begin{array}{l}= \\
= \\
= \\
= \\
= \\
= \\
= \\
= \\
= \\
= \\
= \\
= \\
=\end{array}$ & $\begin{array}{l}= \\
= \\
= \\
= \\
= \\
= \\
= \\
= \\
= \\
= \\
= \\
=\end{array}$ \\
\hline 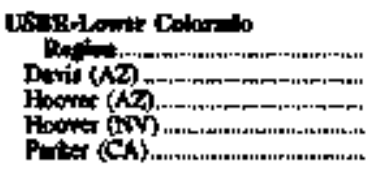 & $\begin{array}{l}\bar{z} \\
\bar{z}\end{array}$ & $\begin{array}{l}\bar{z} \\
\bar{z}\end{array}$ & $\begin{array}{l}\bar{z} \\
\bar{z}\end{array}$ & $\begin{array}{r}16,532 \\
131,360 \\
259,567 \\
246,539 \\
58,066\end{array}$ & $\begin{array}{l}= \\
= \\
=\end{array}$ & $\begin{array}{l}= \\
= \\
=\end{array}$ & $\begin{array}{l}= \\
= \\
=\end{array}$ & $\begin{array}{l}\bar{z} \\
\bar{z}\end{array}$ & $\begin{array}{l}\bar{z} \\
\bar{z}\end{array}$ & $\begin{array}{l}= \\
= \\
=\end{array}$ & $\begin{array}{l}= \\
= \\
=\end{array}$ \\
\hline 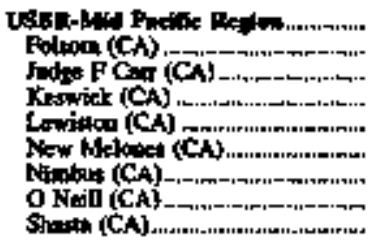 & $\begin{array}{l}= \\
= \\
= \\
=\end{array}$ & $\begin{array}{l}\bar{z} \\
= \\
= \\
\bar{z}\end{array}$ & $\begin{array}{l}\bar{z} \\
\bar{z} \\
\bar{z} \\
\bar{z}\end{array}$ & 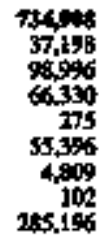 & $\begin{array}{l}= \\
= \\
= \\
= \\
=\end{array}$ & $\begin{array}{l}= \\
= \\
= \\
=\end{array}$ & $\begin{array}{l}= \\
= \\
= \\
= \\
=\end{array}$ & $\begin{array}{l}= \\
= \\
= \\
= \\
=\end{array}$ & $\begin{array}{l}= \\
= \\
= \\
= \\
=\end{array}$ & $\begin{array}{l}= \\
= \\
= \\
=\end{array}$ & $\begin{array}{l}\bar{z} \\
\bar{z} \\
\bar{z} \\
=\end{array}$ \\
\hline
\end{tabular}

Sro footrount al and of coblh. 
Table S6. U.S. Glectric Utlity Net Generation, Dod Consumption, and Fued Stocks by Co:pany and Phant, Jaly 1997 (Continued)

\begin{tabular}{|c|c|c|c|c|c|c|c|c|c|c|c|}
\hline \multirow{2}{*}{ 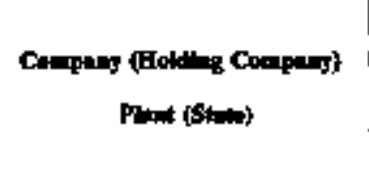 } & \multicolumn{6}{|c|}{ Gocketin: } & \multicolumn{3}{|c|}{ Condingente } & \multicolumn{2}{|c|}{ Btodt } \\
\hline & Conl & Pitrolt:- & Fa: & HFt. & Mudety & Ounerl & Cint & $\begin{array}{l}\text { Potro } \\
\text { leatia) }\end{array}$ & (ANt) & 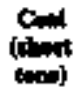 & Pator \\
\hline 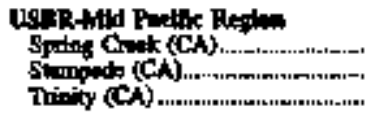 & $\bar{z}$ & $\bar{z}$ & $\bar{z}$ & $\begin{array}{r}1,02,083 \\
1,641 \\
12,802\end{array}$ & $\overline{-}$ & $\overline{-}$ & $\bar{z}$ & $\bar{z}$ & $\underline{z}$ & $\overline{-}$ & $\bar{\Xi}$ \\
\hline 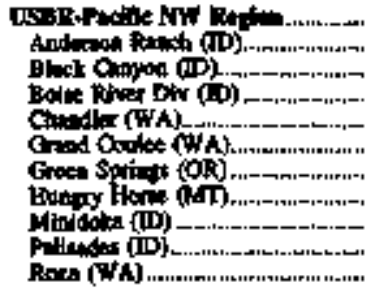 & $\begin{array}{l}= \\
= \\
= \\
= \\
=\end{array}$ & $\begin{array}{l}= \\
= \\
= \\
= \\
= \\
=\end{array}$ & $\begin{array}{l}= \\
= \\
= \\
= \\
=\end{array}$ & 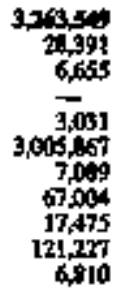 & $\begin{array}{l}= \\
= \\
= \\
= \\
=\end{array}$ & $\begin{array}{l}= \\
= \\
= \\
= \\
=\end{array}$ & $\begin{array}{l}= \\
= \\
= \\
= \\
=\end{array}$ & $\begin{array}{l}= \\
= \\
= \\
= \\
= \\
=\end{array}$ & $\begin{array}{l}\bar{z} \\
= \\
= \\
=\end{array}$ & $\begin{array}{l}= \\
= \\
= \\
= \\
=\end{array}$ & $\begin{array}{l}= \\
= \\
= \\
= \\
= \\
=\end{array}$ \\
\hline 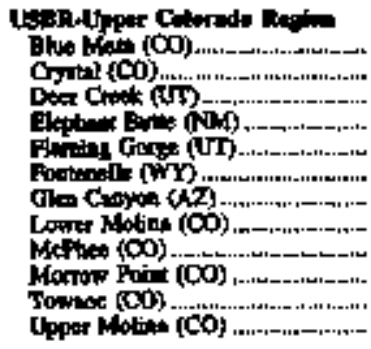 & $\begin{array}{l}= \\
= \\
= \\
= \\
= \\
=\end{array}$ & $\begin{array}{l}= \\
= \\
= \\
= \\
= \\
=\end{array}$ & $\begin{array}{l}= \\
= \\
= \\
= \\
= \\
= \\
= \\
=\end{array}$ & 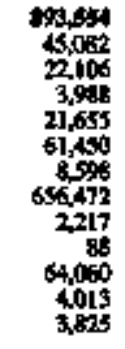 & $\begin{array}{l}= \\
= \\
= \\
= \\
= \\
= \\
=\end{array}$ & $\begin{array}{l}= \\
= \\
= \\
= \\
= \\
=\end{array}$ & $\begin{array}{l}= \\
= \\
= \\
= \\
= \\
=\end{array}$ & $\begin{array}{l}= \\
= \\
= \\
= \\
= \\
=\end{array}$ & $\begin{array}{l}= \\
= \\
\bar{E} \\
\bar{E} \\
= \\
= \\
= \\
=\end{array}$ & $\begin{array}{l}\bar{E} \\
\bar{E} \\
\bar{z} \\
\bar{z} \\
\bar{z} \\
\bar{E}\end{array}$ & $\begin{array}{l}= \\
= \\
= \\
= \\
= \\
=\end{array}$ \\
\hline 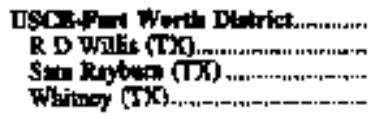 & $\bar{z}$ & $\begin{array}{l}z \\
=\end{array}$ & $\begin{array}{l}\bar{z} \\
=\end{array}$ & $\begin{array}{r}24,44 \\
4,464 \\
10,464 \\
9,552\end{array}$ & $\begin{array}{l}\bar{z} \\
\bar{z}\end{array}$ & $=$ & $=$ & $=$ & $=$ & $=$ & $=$ \\
\hline 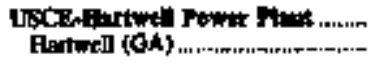 & $\bar{z}$ & $\bar{z}$ & $=$ & $\begin{array}{l}39,204 \\
39,200\end{array}$ & $\overline{-}$ & 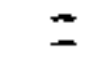 & $=$ & $\bar{z}$ & $=$ & $=$ & $\ddot{z}$ \\
\hline 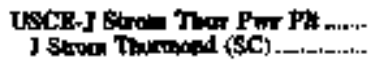 & $\overline{-}$ & $\Xi$ & $=$ & $\begin{array}{l}48,345 \\
48,345\end{array}$ & $\bar{z}$ & $=$ & $\Xi$ & 二 & $=$ & $=$ & $\bar{z}$ \\
\hline 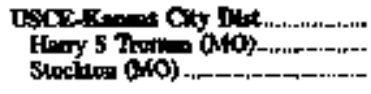 & $\bar{z}$ & $\bar{z}$ & $\bar{z}$ & $\begin{array}{r}18,64 \\
13,617 \\
4,877\end{array}$ & $\bar{z}$ & $\bar{z}$ & $\bar{z}$ & $\bar{z}$ & 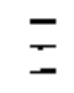 & $\bar{z}$ & $\bar{z}$ \\
\hline 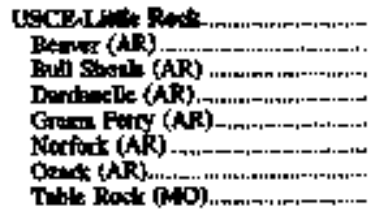 & $\begin{array}{l}\vec{z} \\
= \\
=\end{array}$ & $\begin{array}{l}= \\
= \\
= \\
=\end{array}$ & $\begin{array}{l}= \\
= \\
=\end{array}$ & $\begin{array}{r}23,80.5 \\
14,093 \\
77,891 \\
72,221 \\
12,013 \\
17,553 \\
43,586 \\
46,500\end{array}$ & $\begin{array}{l}\bar{z} \\
= \\
=\end{array}$ & $\begin{array}{l}= \\
= \\
= \\
=\end{array}$ & $\begin{array}{l}= \\
= \\
= \\
=\end{array}$ & $\begin{array}{l}= \\
= \\
\Rightarrow \\
=\end{array}$ & $\begin{array}{l}= \\
\bar{z} \\
=\end{array}$ & $\begin{array}{l}= \\
= \\
=\end{array}$ & $\begin{array}{l}= \\
= \\
= \\
=\end{array}$ \\
\hline 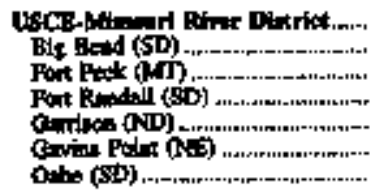 & $\begin{array}{l}= \\
= \\
=\end{array}$ & $\begin{array}{l}\bar{z} \\
\bar{z} \\
\bar{z}\end{array}$ & $\begin{array}{l}= \\
= \\
= \\
=\end{array}$ & 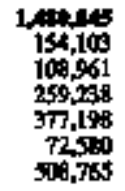 & $\begin{array}{l}= \\
= \\
=\end{array}$ & $\begin{array}{l}= \\
= \\
=\end{array}$ & $\begin{array}{l}= \\
= \\
=\end{array}$ & $\begin{array}{l}= \\
= \\
=\end{array}$ & $\begin{array}{l}= \\
= \\
=\end{array}$ & $\begin{array}{l}= \\
\bar{z} \\
\bar{z}\end{array}$ & $\begin{array}{l}= \\
= \\
=\end{array}$ \\
\hline 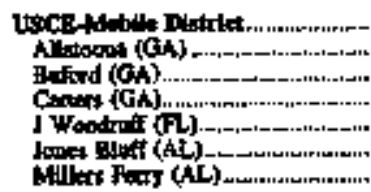 & $\begin{array}{l}\bar{z} \\
= \\
\bar{z}\end{array}$ & $\begin{array}{l}= \\
= \\
= \\
\Rightarrow\end{array}$ & $\begin{array}{l}= \\
= \\
= \\
=\end{array}$ & 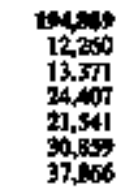 & $\begin{array}{l}= \\
= \\
=\end{array}$ & $\begin{array}{l}= \\
= \\
=\end{array}$ & $\begin{array}{l}= \\
= \\
=\end{array}$ & $\begin{array}{l}= \\
= \\
= \\
=\end{array}$ & $\begin{array}{l}= \\
= \\
=\end{array}$ & $\begin{array}{l}z \\
= \\
= \\
=\end{array}$ & $\begin{array}{l}\bar{z} \\
\bar{z} \\
\bar{z}\end{array}$ \\
\hline
\end{tabular}

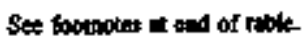


Twble 56. U.S. Fleetric Utilty Net Generation, Fuel Comsumption, and Fuel Stocks by Compang and Plant, Jaly 1997 (Contílued)

\begin{tabular}{|c|c|c|c|c|c|c|c|c|c|c|c|}
\hline \multirow{2}{*}{$\begin{array}{c}\text { Corpan (Holthis Cocipory) } \\
\text { Finel (Stan) }\end{array}$} & \multicolumn{6}{|c|}{ 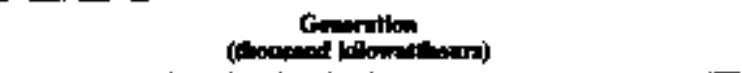 } & \multicolumn{3}{|c|}{ Dorition } & \multicolumn{2}{|c|}{$\begin{array}{l}\text { Strodx } \\
\text { (ttivesedd) }\end{array}$} \\
\hline & Conl & Ph⿻三丨日: & Gm & Arsio & Kinder & ] & $\underset{1 \rightarrow \infty}{c}$ & $\begin{array}{l}\text { Petro- } \\
\text { latin) }\end{array}$ & $\begin{array}{l}\text { thes } \\
\text { (MAls }\end{array}$ & 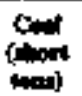 & $\underset{\text { lein }}{\text { Pathe }}$ \\
\hline
\end{tabular}

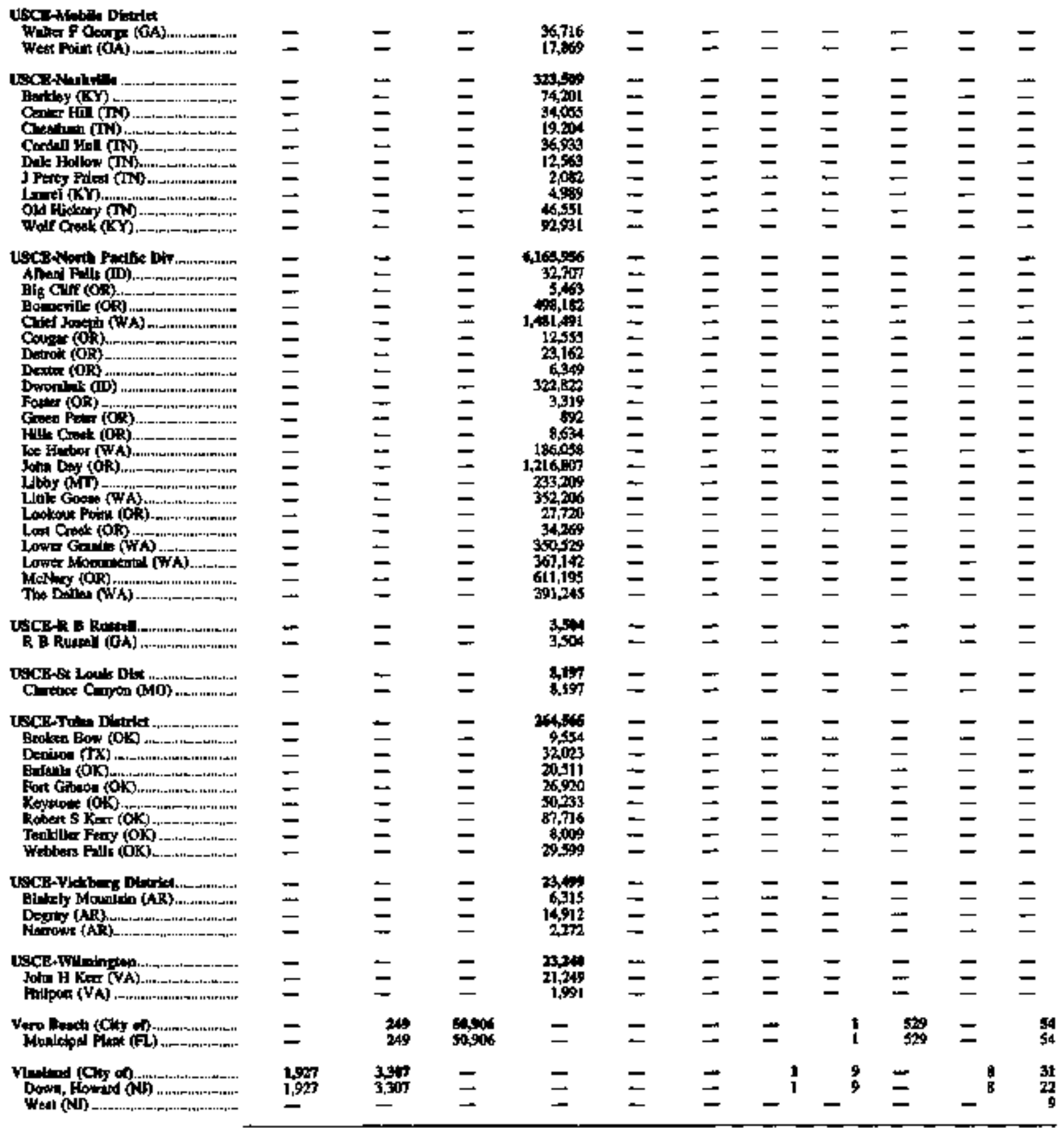

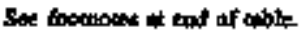


Table 56. US. Blactric Utlity Net Generation, Fuel Canoumption, and Fuel Stocls by Conpany and Ptont, July 1997 (Contioued)

\begin{tabular}{|c|c|c|c|c|c|c|c|c|c|c|c|}
\hline \multirow{2}{*}{ 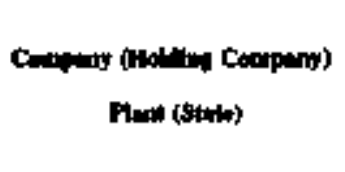 } & \multicolumn{6}{|c|}{ 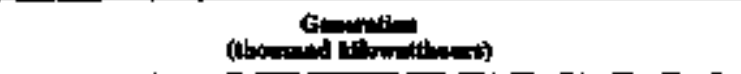 } & \multicolumn{3}{|c|}{ 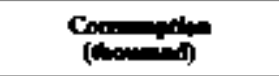 } & \multicolumn{2}{|c|}{ Stacts } \\
\hline & Conl & 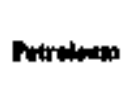 & C.* & Bytro & Nhinerer & Onenes & $\underset{\operatorname{tandi}}{\text { Cond }}$ & pintive & (Mtef & $\begin{array}{l}\text { Cool } \\
\text { (thint } \\
\text { toris) }\end{array}$ & Puto \\
\hline
\end{tabular}

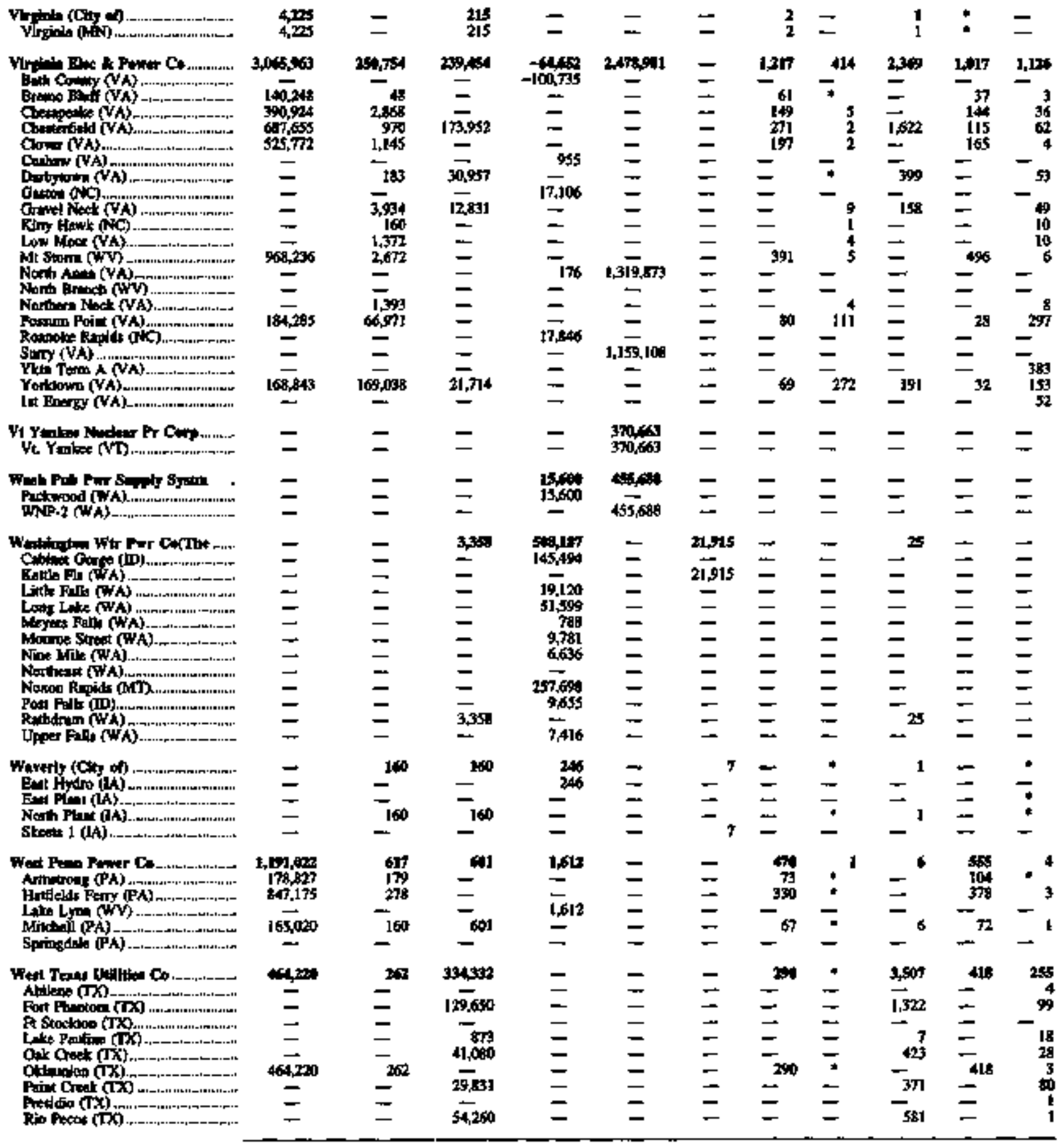

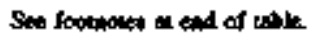




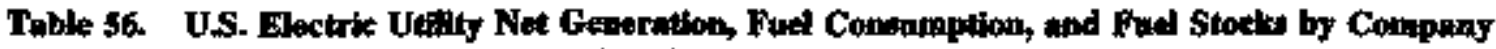
and Plant, Juily Ler/ (Cowtiuned)

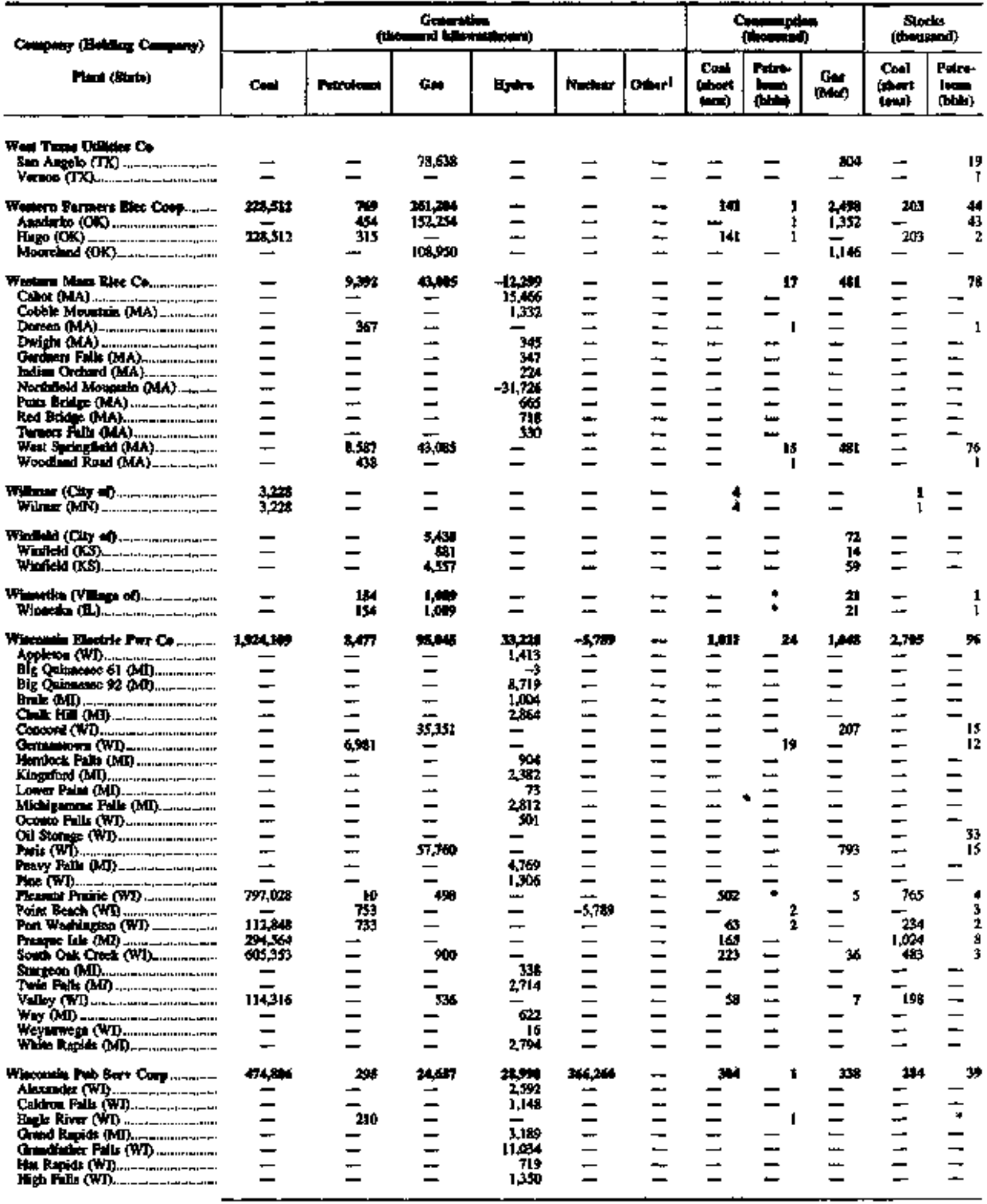

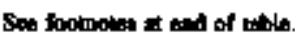


Table 56. U.S. Electric Utility Net Geperatton, Fuel Consmmption, and Fost Stocks by Company and Plont, July 1997 (Contimued)

\begin{tabular}{|c|c|c|c|c|c|c|c|c|c|c|c|}
\hline \multirow{2}{*}{ 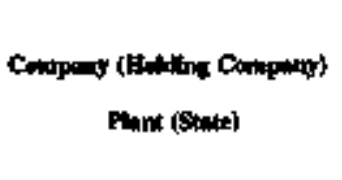 } & \multicolumn{6}{|c|}{ Gemartho } & \multicolumn{3}{|c|}{ Cantinention } & \multicolumn{2}{|c|}{ 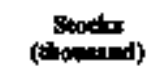 } \\
\hline & Co:d & Patrelena & ㅂy & Bydo & Nhidar & Othar1 & $\begin{array}{c}\text { Cond } \\
\text { (chert } \\
\text { ton: })\end{array}$ & Ptant & Ges & $\underset{(\lim )}{c i n}$ & inting \\
\hline
\end{tabular}

\section{Winon:lin Pub Sert Cor}

Javey fith

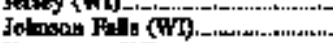

Kewrinoe (WT)

Hoinl (Whi)

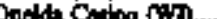

Other Roridn (WT

pestiog (t).

Poto Rivido (WT)

Polion (tit)

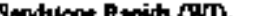

Tomahing (WT)

Widan (Wh).

West Mringete (WM)

Weoton (WT)

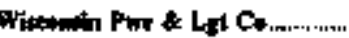
Blackhowt (WT)

Cohutha (Wh)

Dawey, Jislow (Wh)

Bdoptint (WT)

Juknile (WT)

Giloon (WT)

NA 1 (TNT)

Partable (WT)

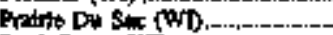

Rock Rfivo (w)

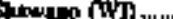

Shecpnkin ofith.

Walf Cumla Nucker Corp...... walf Citek [KS)

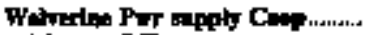
Adrates (MI).

Bawat lond (MI).

thomo frane (MID.

Kletwe (NA)

Sctinide (MI)

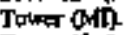

Towa Houro thil.

A

Vexteors (MAl).

Whoder, CA (AII)

Wrandowe (CSty of )

Wyandate (MID.

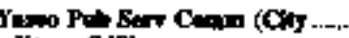

Yow QNS?

Yula Coenty Fitar Agonky......... Fich Rutro (CA)

Now Colpt (CN)

Nkw Narowd (C)

$\bar{z}$
$=$
$\bar{z}$
$\bar{z}$
192,563
$=$
$\overline{282,233}$

$=$
$=$
$=$
$=$
$=$
$=$
$=$

$=$
$=$
$=$
$=$
$\overline{2 n}$
$\bar{z}$
$\overline{18,003}$
3,081

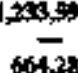

94,7d1

414033

-

ב

$=$

60,534

-

-

$=$

$-345$

$-346$

-

-

-

- $\quad 46$

二.

二.

2서하

21.221

$\begin{array}{lll}- & - & - \\ - & - & - \\ - & - & - \\ - & - & -\end{array}$

$15 x$ 2548:

I33

632

6.161

-

-

$\overline{-}$

-

$\overline{5,662}$

-920

-

2.15

799

\begin{tabular}{|c|c|c|c|}
\hline 322 & - & - & $=$ \\
\hline 021 & 356266 & I & Z \\
\hline 1,019 & - & - & טـ \\
\hline- & & $\rightarrow$ & - \\
\hline 236 & - & - & - \\
\hline & 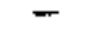 & - & - \\
\hline 451 & - & - & - \\
\hline- & - & $\rightarrow$ & 133 \\
\hline 84 & - & - & - \\
\hline & - & - & - \\
\hline 3.635 & - & - & - \\
\hline$\rightarrow$ & & & \\
\hline & & & 171 \\
\hline
\end{tabular}

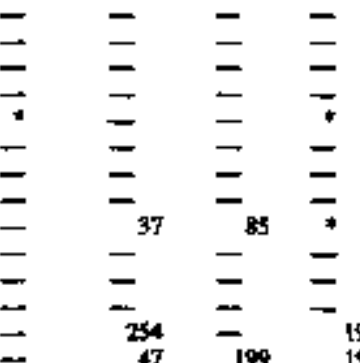

19,0r는

$-7$

-

-

intit

$\overline{12819}$

391

-

-

560
-
-
$=$
-169
$=$
$=$

$\bar{\Xi}$
$\bar{\Xi}$
$\bar{\Xi}$
$\bar{\Xi}$
$\bar{\Xi}$
$\bar{\Xi}$

- $\quad-\quad-\quad-12$

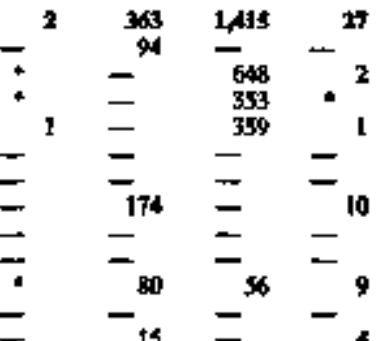

$\rightarrow$

$=$

$z=z=$

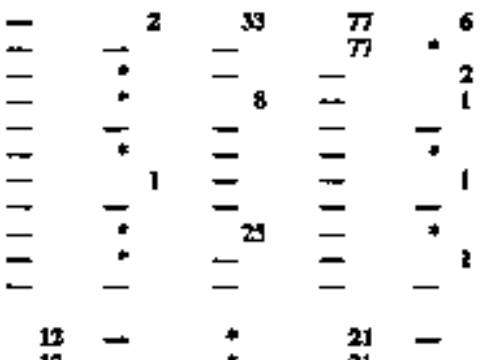

- $\quad-\quad-\quad-1 \quad-\quad-$

$\begin{array}{rlllllll}161,920 & - & - & - & - & - & - & = \\ 108 & - & - & - & - & - & = & = \\ 136,780 & - & - & - & - & - & - & =\end{array}$

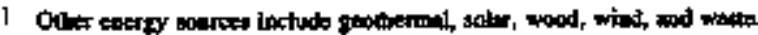

- Lesa tha DO

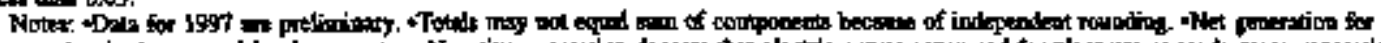

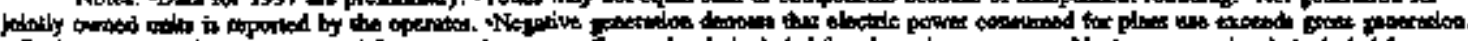

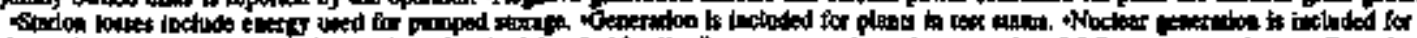

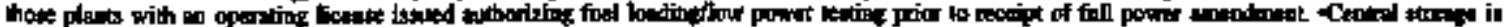

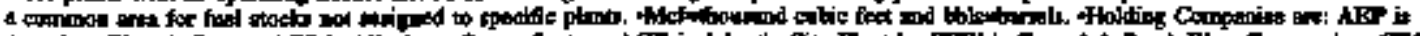

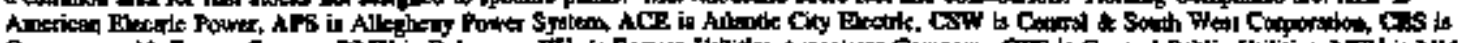

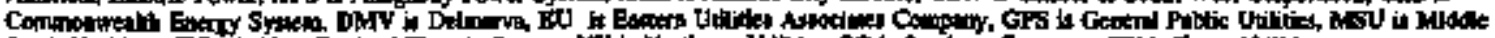

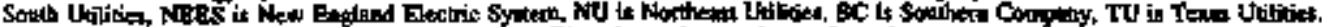

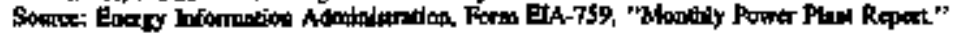




\section{Monthly Plant Aggregates: U.S. Electric Utility Receipts, Cost, and Quality of Fossil Fuels}

Tabte 57. Receipts, A wroge Cost, and Quolity of Fossil Fuets Delfived to US. Electric Utilities by Company and Pant, Jury 1997

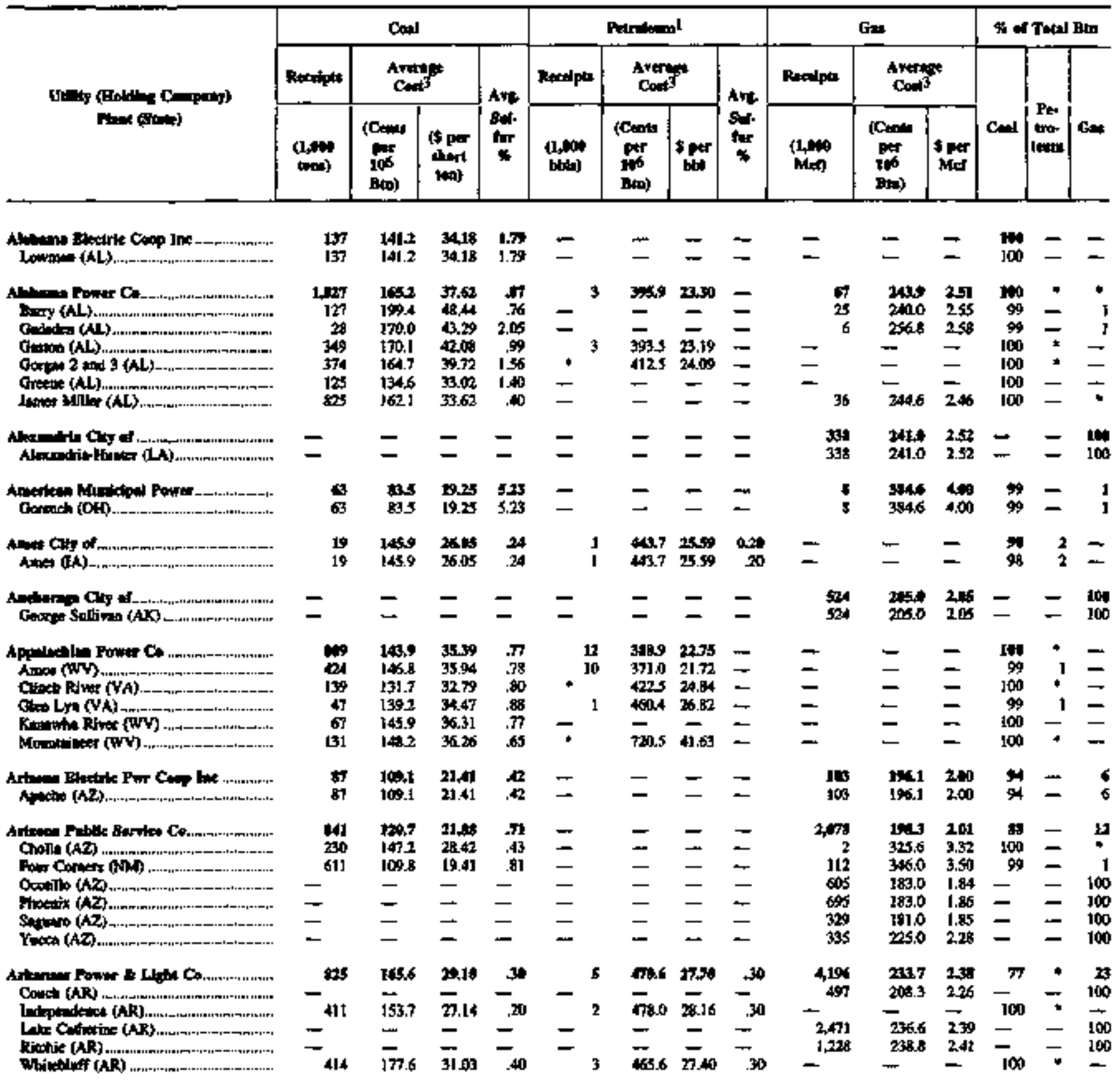

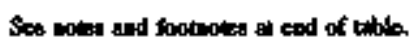




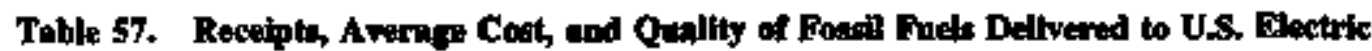
Utiltitea by Conpany and Plant, Joly 1997 (Conatinued)

\begin{tabular}{|c|c|c|c|c|c|c|c|c|c|c|c|c|c|c|}
\hline \multirow{3}{*}{ 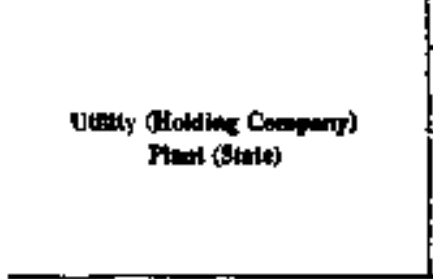 } & \multicolumn{4}{|c|}{ Cond } & \multicolumn{4}{|c|}{ Potran. } & \multicolumn{3}{|c|}{ Cos } & \multicolumn{3}{|c|}{ F t t Twel } \\
\hline & \multirow{2}{*}{ 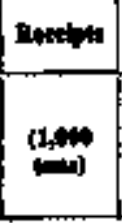 } & \multicolumn{2}{|c|}{ cons } & \multirow{2}{*}{$\begin{array}{c}\text { ave } \\
\text { sit } \\
6 \\
5\end{array}$} & \multirow{2}{*}{ | } & \multicolumn{2}{|c|}{ Averpit } & \multirow{2}{*}{$\sum_{n=1}^{\ln }$} & \multirow{2}{*}{ Ratpt: } & \multicolumn{2}{|c|}{ Conts } & \multirow[b]{2}{*}{ Cons. } & \multirow[b]{2}{*}{ into } & \multirow[b]{2}{*}{ G*: } \\
\hline & & (Cind & inger & & & (C) & 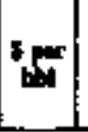 & & & 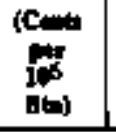 & Hat & & & \\
\hline 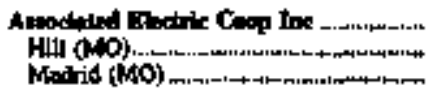 & $\underset{385}{305}$ & $\begin{array}{l}87.2 \\
740 \\
98.3\end{array}$ & $\begin{array}{l}\tan 29 \\
12,93 \\
1+25\end{array}$ & $\frac{021}{25}$ & $=$ & $\bar{z}$ & $\bar{z}$ & $\bar{z}$ & $\bar{z}$ & $\bar{z}$ & $\underline{z}$ & $\begin{array}{l}100 \\
100\end{array}$ & $\bar{z}$ & $\bar{z}$ \\
\hline 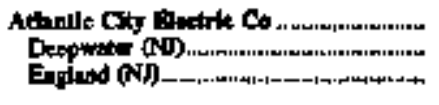 & $\begin{array}{l}4 \\
3 \\
32\end{array}$ & $\begin{array}{l}151.5 \\
181.6 \\
185.8\end{array}$ & $\begin{array}{l}\mathbf{4} .94 \\
45.90 \\
45.84\end{array}$ & $\frac{2 \pi}{27}$ & -1 & $\frac{4320}{4320}$ & $\frac{25.6}{2 \times 30}$ & $\frac{10}{10}$ & $-\quad 151$ & $\begin{array}{l}2948 \\
2848 \\
-\end{array}$ & $\begin{array}{l}297 \\
297 \\
\cdots\end{array}$ & 9 & $\vec{*}$ & $\begin{array}{l}\mathbf{4} \\
-\end{array}$ \\
\hline 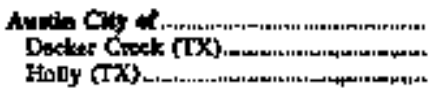 & $\bar{z}$ & $\bar{\Xi}$ & $\bar{z}$ & $\bar{z}$ & $=$ & $\bar{z}$ & $=$ & $\bar{z}$ & $\begin{array}{l}3,402 \\
2,372 \\
t, 120\end{array}$ & $\begin{array}{l}207.5 \\
235 \\
20.3\end{array}$ & $\begin{array}{l}2.41 \\
2.18 \\
2.46\end{array}$ & $\bar{z}$ & $\bar{z}$ & 100 \\
\hline 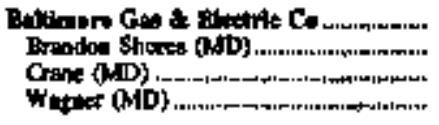 & $\begin{array}{c}47 \\
\mathbf{3 0} \\
\mathbf{2 0} \\
68\end{array}$ & $\begin{array}{l}14.1 \\
141.4 \\
147.4 \\
141.4\end{array}$ & $\begin{array}{l}36.15 \\
33.97 \\
36,66 \\
36.90\end{array}$ & $\underset{6}{m}$ & $\bar{z}$ & $\begin{array}{l}3904 \\
3904 \\
-\end{array}$ & $\begin{array}{l}\mathbf{2 3 . 2 9} \\
\mathbf{2 3 . 2 9} \\
=\end{array}$ & $\begin{array}{l}\text { 19: } \\
-19 \\
-\end{array}$ & $\begin{array}{l}= \\
=\end{array}$ & $=$ & $\bar{z}$ & $\begin{array}{l}100 \\
100 \\
100 \\
100\end{array}$ & $\stackrel{*}{-}$ & $\bar{z}$ \\
\hline 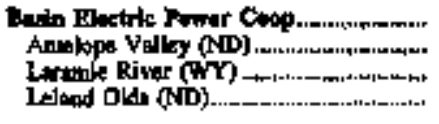 & 29 & $\begin{array}{l}694 \\
84.9 \\
93.4 \\
81.7\end{array}$ & 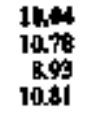 & $\begin{array}{l}.99 \\
.76 \\
.93 \\
.68\end{array}$ & $\begin{array}{r}15 \\
14\end{array}$ & $\begin{array}{l}91.3 \\
453.9 \\
49.2 \\
45.2\end{array}$ & $\begin{array}{l}24.45 \\
25.29 \\
26.62 \\
25.78\end{array}$ & $\begin{array}{l}34 \\
34 \\
.34 \\
.34\end{array}$ & $=$ & $\bar{z}$ & $\bar{\Xi}$ & $\begin{array}{c}100 \\
100 \\
100\end{array}$ & 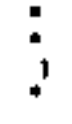 & $\begin{array}{l}\bar{z} \\
\bar{z}\end{array}$ \\
\hline 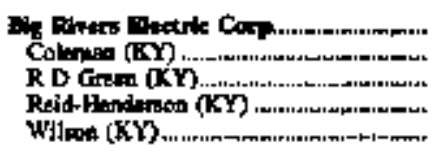 & $\begin{array}{l}404 \\
141 \\
610 \\
110\end{array}$ & $\begin{array}{l}1049 \\
1020 \\
928 \\
101.5 \\
964\end{array}$ & $\begin{array}{l}2140 \\
2+50 \\
2003 \\
21+10 \\
2 \pi+0\end{array}$ & $\begin{array}{l}2.79 \\
1.06 \\
3.05 \\
2.44 \\
1.44\end{array}$ & ${ }^{-1}$ & $\frac{-}{416.1}$ & $\frac{2412}{24.12}$ & $\begin{array}{l}= \\
=\end{array}$ & $\bar{z}$ & $\begin{array}{l}3 \times 1.1 \\
3 \times 2.1 \\
= \\
=\end{array}$ & $\begin{array}{l}3,42 \\
3,42 \\
= \\
=\end{array}$ & $\begin{array}{c}100 \\
100 \\
100 \\
98 \\
100\end{array}$ & $\frac{-}{2}$ & $\begin{array}{l}* \\
z\end{array}$ \\
\hline 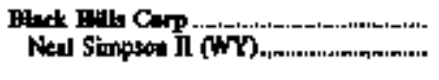 & 4 & stas & $\begin{array}{l}\mathbf{5} 12 \\
\mathbf{1 1 2}\end{array}$ & $\pi$ & * & $\lim _{490}$ & 27.74 & it.4 & $\overline{-}$ & $\overline{-}$ & $\bar{m}$ & ${ }_{100}^{100}$ & : & $\overline{-}$ \\
\hline 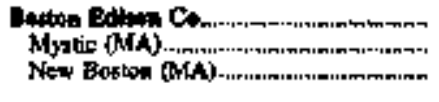 & $\overline{-}$ & $=$ & $\bar{z}$ & $=$ & $-{ }^{\mathbf{7 4 4}}$ & $\frac{20.4}{2080}$ & $\frac{1643}{16.43}$ & $\stackrel{49}{-4}$ & $\begin{array}{l}3,998 \\
129 \\
3,106\end{array}$ & $\begin{array}{l}2746 \\
2359 \\
2760\end{array}$ & $\begin{array}{l}214 \\
255 \\
285\end{array}$ & $\ddot{z}$ & 97 & $\begin{array}{r}4 \\
100\end{array}$ \\
\hline 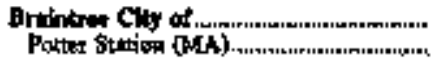 & $\overline{-}$ & $\bar{z}$ & $\bar{z}$ & $\bar{Z}$ & $\overline{-}$ & $\bar{Z}$ & $\bar{Z}$ & 二 & 174 & 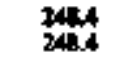 & $\frac{254}{256}$ & $\bar{z}$ & $\bar{z}$ & 100 \\
\hline 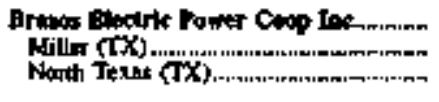 & $\bar{z}$ & $\bar{z}$ & $\bar{z}$ & $\bar{z}$ & $=$ & $\bar{z}$ & $\bar{z}$ & $\bar{z}$ & $\underset{88}{2,43}$ & $\frac{2013}{2213}$ & $\begin{array}{l}2.24 \\
2.44 \\
2.4 !\end{array}$ & $\bar{z}$ & $\bar{z}$ & $\begin{array}{l}100 \\
100 \\
100\end{array}$ \\
\hline 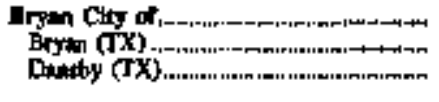 & $\overline{-}$ & $=$ & $\vec{m}$ & $=$ & $=$ & $\underline{-}$ & $\bar{z}$ & $=$ & $\begin{array}{l}63 \\
123 \\
536\end{array}$ & $\begin{array}{l}2390 \\
2433 \\
2045\end{array}$ & $\begin{array}{l}2.6 \\
3,33 \\
2,30\end{array}$ & $=$ & $\bar{z}$ & 100 \\
\hline 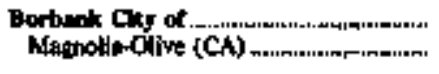 & $\bar{z}$ & $\overline{-}$ & $\overline{-}$ & $\overline{-}$ & $\bar{z}$ & $\overline{-}$ & $\overline{-}$ & $=$ & 265 & $\begin{array}{l}3 \times 0 \\
3250\end{array}$ & $\frac{3 x}{32 x}$ & $\overline{-}$ & $\overline{-}$ & 100 \\
\hline 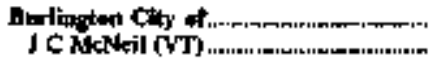 & $\bar{z}$ & $\bar{z}$ & $\bar{z}$ & $\overline{-}$ & $\overline{-}$ & $\bar{z}$ & $\bar{z}$ & $\overline{-}$ & $\begin{array}{l}4 \\
4\end{array}$ & $\begin{array}{l}2019 \\
2919\end{array}$ & $\begin{array}{l}295 \\
295\end{array}$ & $\bar{z}$ & $\overline{-}$ & 100 \\
\hline 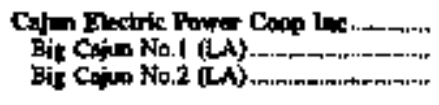 & - & $\frac{1 m 2}{147.2}$ & $\frac{24.76}{2 \pi .78}$ & $\underset{10}{\infty}$ & T & $\frac{3 \times 47}{3 \times 67}$ & 22,74 & $\overline{-}$ & $-\quad$ & $\begin{array}{l}\text { mal. } \\
2.1 \\
-\end{array}$ & $\frac{2.35}{2.35}$ & $\frac{91}{100}$ & $\stackrel{-}{\bullet}$ & $\underline{100}$ \\
\hline 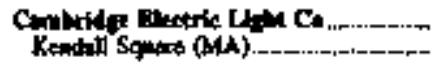 & $=$ & $\bar{\sigma}$ & $\check{-}$ & $=$ & $\vec{F}$ & 二 & $=$ & $m$ & $\frac{127}{127}$ & $\begin{array}{l}\cos 8 \\
\operatorname{2ts} 8\end{array}$ & 279 & $\overline{-}$ & $\overline{-}$ & 200 \\
\hline Cand Eloctrik Co & $\overline{-}$ & 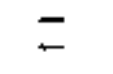 & $\bar{z}$ & $\bar{z}$ & 62 & 250.4 & $\begin{array}{l}1646 \\
1656\end{array}$ & 99 & $\bar{z}$ & $\bar{z}$ & $\underline{-}$ & $\bar{z}$ & 100 & $\bar{z}$ \\
\hline 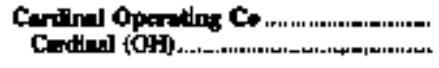 & 29 & 147.0 & 35.50 & 1.4 & $\bar{z}$ & $=$ & $\overline{-}$ & $\bar{z}$ & $\overrightarrow{-}$ & $\vec{\square}$ & ت ع & 100 & $\overline{-}$ & $m$ \\
\hline
\end{tabular}

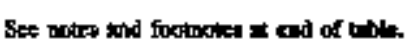


Table 57. Recelpts, Avernge Cost, and Qunlity of Pout Foels Delivered to U.S. Electric Utilities by Company and Fient, July 1997 (Contoued)

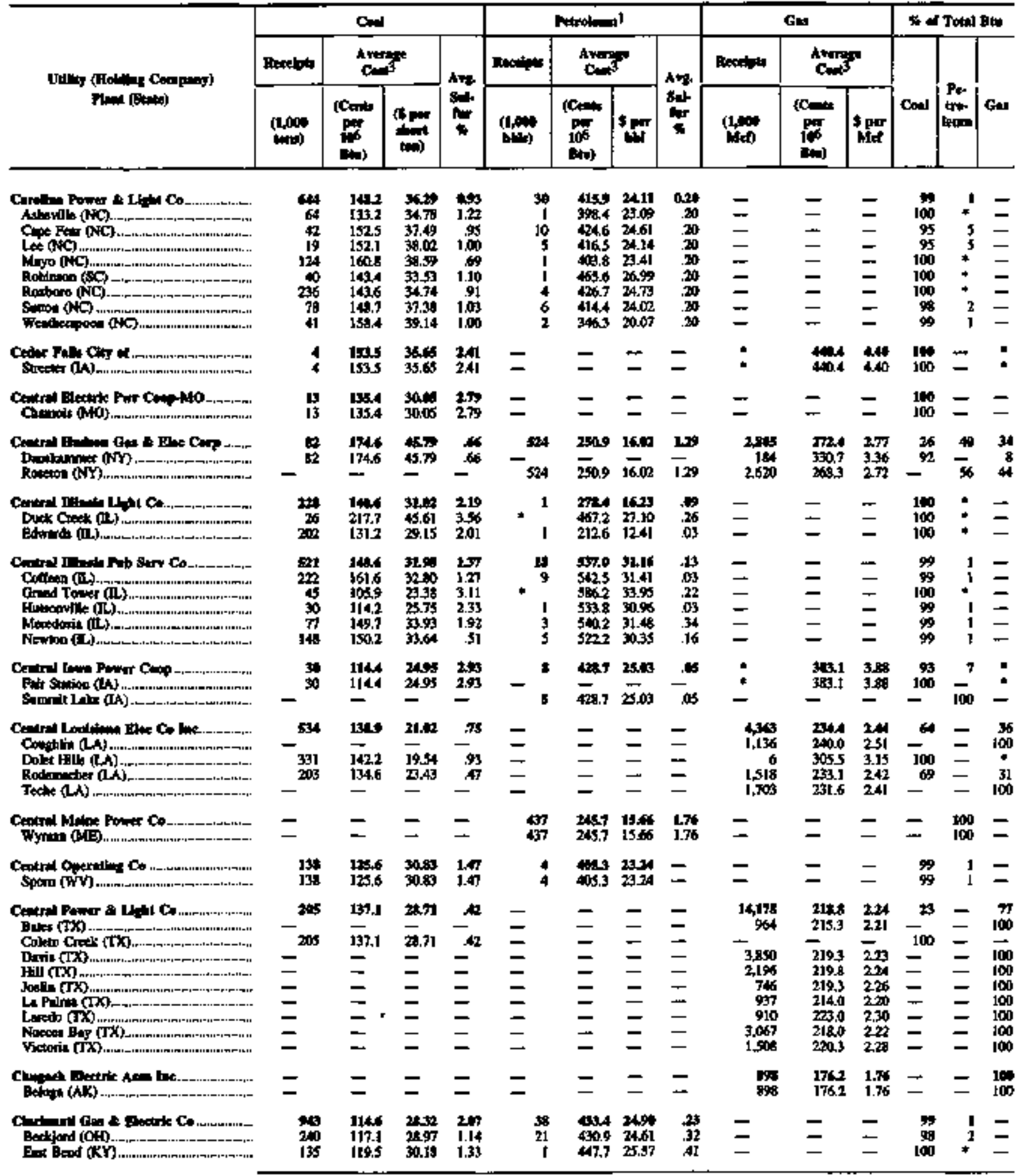

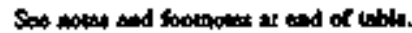


Thble 57. Recelpts, Avernge Cost, and Quality of Fossil Fuels Dellvered to U.S. Dlectric Utilities by Condpany and Piant, Jub 1997 (Coatinned)

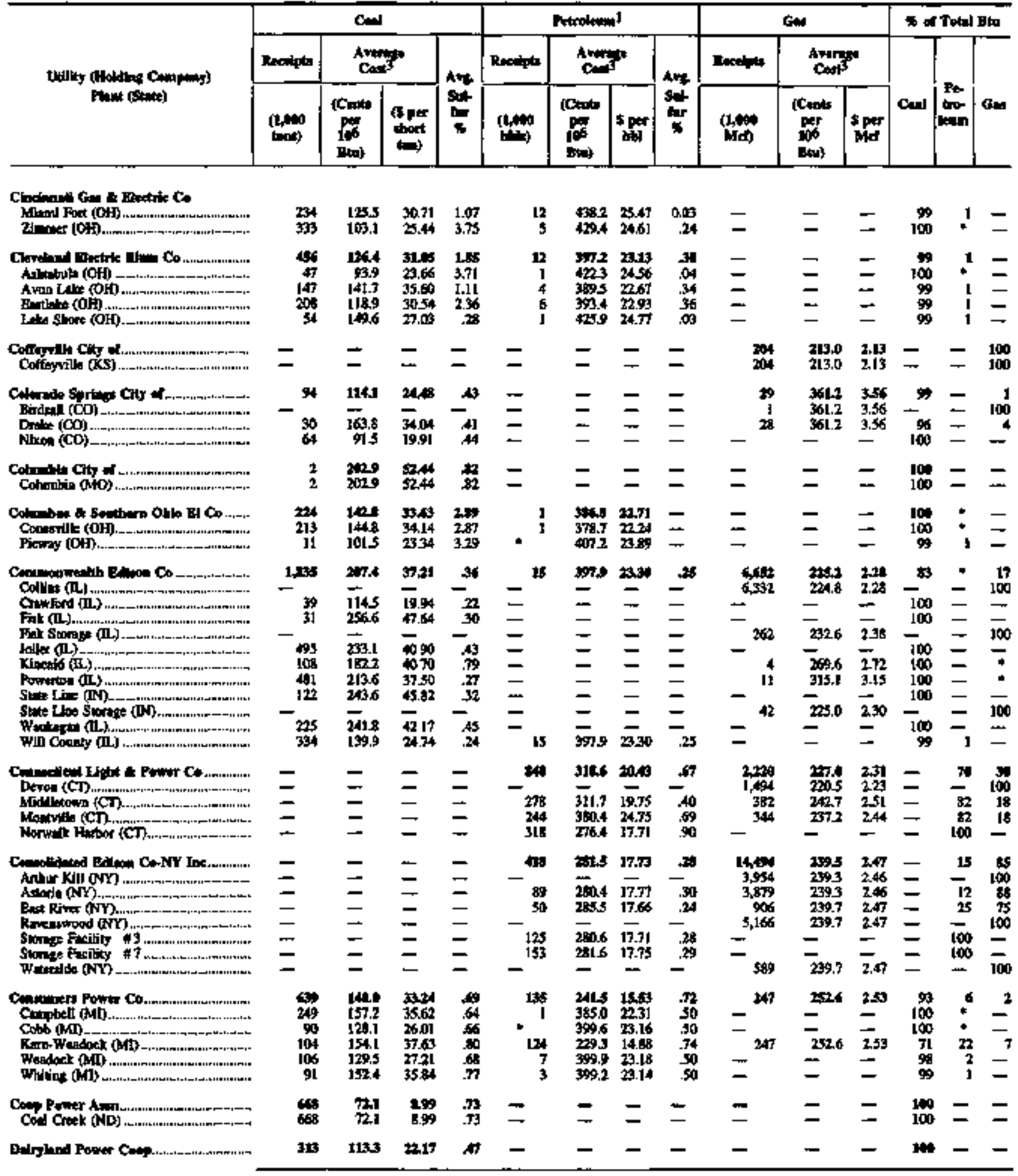

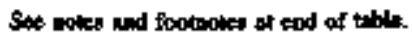


Table 57. Receipts, A Termge Cost, and Quallity of Foss: Fuels Delivered to US. Electric Utitites by Company and Finot, July $19 \%$ (Constimued)

\begin{tabular}{|c|c|c|c|c|c|c|c|c|c|c|c|c|c|c|}
\hline \multirow{3}{*}{ (5) } & \multicolumn{4}{|c|}{ Cod } & \multicolumn{4}{|c|}{ Putrolmen' } & \multicolumn{3}{|c|}{ Gall } & \multicolumn{3}{|c|}{ to oTotd Bib } \\
\hline & \multirow{2}{*}{ Pestall } & \multicolumn{2}{|c|}{ 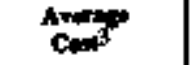 } & \multirow{2}{*}{ 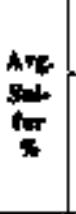 } & \multirow{2}{*}{ 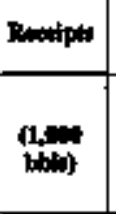 } & \multicolumn{2}{|c|}{ Arerot } & \multirow{2}{*}{ sin. } & \multirow{2}{*}{ 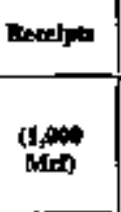 } & \multicolumn{2}{|c|}{ compos } & \multirow[b]{2}{*}{ Con } & \multirow{2}{*}{$\operatorname{tim}_{\substack{\infty \\
\infty}}^{\infty}$} & \multirow[b]{2}{*}{ Ga } \\
\hline & & $\underset{100}{(c+i s}$ & tort & & & (Conts) & $\$$ & & & 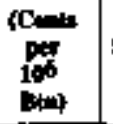 & iper & & & \\
\hline 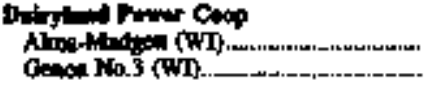 & 139 & $\begin{array}{l}1058 \\
1220\end{array}$ & $\begin{array}{l}19,95 \\
24,94\end{array}$ & $\begin{array}{r}0.40 \\
.56\end{array}$ & $\overline{-}$ & $\overline{-}$ & $\check{-}$ & $\overrightarrow{-}$ & $\overline{-}$ & $\bar{z}$ & $z$ & $\begin{array}{l}100 \\
100\end{array}$ & $\overline{-}$ & $\overline{-}$ \\
\hline 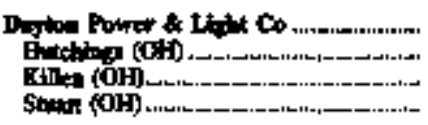 & $\begin{array}{r}70 \\
\$ 9 \\
143 \\
511\end{array}$ & $\begin{array}{l}1759 \\
138.5 \\
127.5 \\
125.5\end{array}$ & $\begin{array}{l}2 \rightarrow 40 \\
34.82 \\
30.57 \\
6.69\end{array}$ & $\begin{array}{l}m \\
.72 \\
.01 \\
\$ 11\end{array}$ & ${ }^{2}$ & $\frac{\pi}{422.7}$ & $\frac{245}{2}$ & $\frac{0}{-}$ & $-\quad 45$ & $\begin{array}{l}4446 \\
444.6 \\
=\end{array}$ & $\begin{array}{l}4.5 \\
4.53 \\
-\end{array}$ & $\begin{array}{l}\mathbf{1 0} \\
\stackrel{9}{100} \\
100\end{array}$ & $\stackrel{*}{*}$ & $\stackrel{*}{*}$ \\
\hline 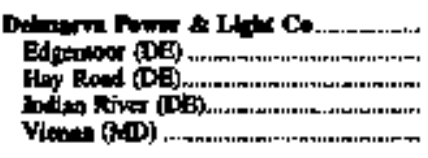 & $\begin{array}{r}158 \\
-\quad 32 \\
-\quad 136\end{array}$ & $\begin{array}{l}\text { thr.s } \\
158.9 \\
13.1 \\
-\end{array}$ & $\begin{array}{l}46.9 \\
4.75 \\
-\end{array}$ & $\begin{array}{l}.94 \\
\frac{904}{1.01} \\
-\end{array}$ & $\begin{array}{r}204 \\
-\quad 7 \\
119\end{array}$ & $\begin{array}{l}264.5 \\
2 \times 14 \\
\frac{704.7}{264.6}\end{array}$ & $\begin{array}{l}16.94 \\
16.55 \\
\overline{20.54} \\
16,90\end{array}$ & $\begin{array}{l}1.15 \\
.55 \\
-.21 \\
1.76\end{array}$ & $\begin{array}{r}1,75 \\
617 \\
1,119 \\
-\end{array}$ & $\begin{array}{l}\operatorname{lng} \\
2142 \\
3125 \\
-\end{array}$ & $\begin{array}{l}2.7 \\
2.22 \\
3.23 \\
- \\
-\end{array}$ & $\begin{array}{l}\text { 4 } \\
-9 \\
-9\end{array}$ & $\frac{10}{2}$ & $\begin{array}{r}27 \\
24 \\
100 \\
= \\
=\end{array}$ \\
\hline Donten Cty & $\overline{-}$ & $=$ & $=$ & $\overline{-}$ & $\overline{-}$ & $\bar{z}$ & 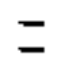 & 二 & 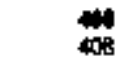 & $\underset{253.6}{2356}$ & 2.45 & $=$ & 二 & $\begin{array}{l}104 \\
100\end{array}$ \\
\hline 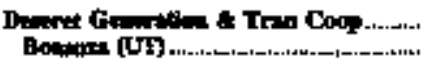 & $\begin{array}{l}154 \\
154\end{array}$ & $\begin{array}{l}1963 \\
189.3\end{array}$ & 39.22 & 4 & $\begin{array}{l}1 \\
1\end{array}$ & 675.9 & 3906 & $z$ & $\because$ & $\bar{z}$ & $\bar{z}$ & 100 & $*$ & $=$ \\
\hline 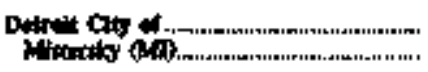 & $\overline{-}$ & $\overline{-}$ & $=$ & $\overline{-}$ & $\frac{77}{27}$ & 360.0 & $\begin{array}{l}21.29 \\
21.29\end{array}$ & 6 & 185 & $\begin{array}{l}\mathbf{3 3 4 0} \\
\mathbf{3 3 4 . 0}\end{array}$ & 3.42 & $=$ & $\begin{array}{l}45 \\
45\end{array}$ & 50 \\
\hline 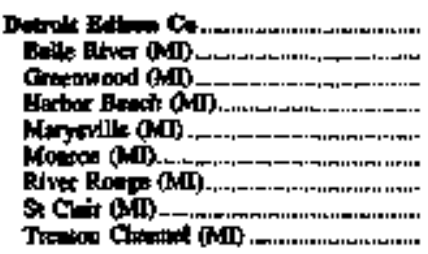 & 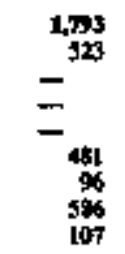 & $\begin{array}{l}135.4 \\
148.0 \\
= \\
\overline{113.0} \\
117.8 \\
115.0 \\
117.4\end{array}$ & $\begin{array}{l}\text { H.19 } \\
27.54 \\
= \\
= \\
2.77 \\
27.03 \\
27.3 \\
23.90\end{array}$ & $\begin{array}{l}\$ 4 \\
34 \\
= \\
- \\
.74 \\
49 \\
59\end{array}$ & $\begin{array}{r}\mathbf{1 3} \\
-\quad \mathbf{3} \\
-\quad 1 \\
-\quad 3 \\
-\quad 3\end{array}$ & $\begin{array}{l}303.3 \\
402.5 \\
\frac{1038}{403} \\
393.5 \\
312.7 \\
-\end{array}$ & $\begin{array}{l}23.72 \\
23.14 \\
23.23 \\
\frac{1}{22.74} \\
21.61 \\
-\end{array}$ & $\begin{array}{r}24 \\
-18 \\
-30 \\
-.26 \\
-24 \\
-\end{array}$ & $\begin{array}{l}-260 \\
-\quad 167 \\
-\quad 13 \\
-\quad 128\end{array}$ & 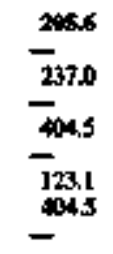 & $\begin{array}{l}\frac{-60}{240} \\
\frac{-104}{4} \\
\frac{.16}{-106}\end{array}$ & $\begin{array}{l}100 \\
= \\
= \\
100 \\
98 \\
100 \\
100\end{array}$ & $\begin{array}{l}* \\
\ddot{100} \\
\ddot{*} \\
\div\end{array}$ & $\begin{array}{l}\frac{2}{100} \\
\frac{2}{100} \\
\frac{12}{12} \\
-\end{array}$ \\
\hline Dover Chy of & $=$ & $=$ & $=$ & $\overline{-}$ & $\begin{array}{l}9 \\
9\end{array}$ & $\frac{1124}{2824}$ & 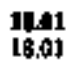 & $\frac{83}{13}$ & $\begin{array}{l}25 \\
258\end{array}$ & $\begin{array}{l}24588 \\
245.8\end{array}$ & $\begin{array}{l}255 \\
255\end{array}$ & $=$ & $\begin{array}{l}\mathbf{1 8} \\
18\end{array}$ & 82 \\
\hline 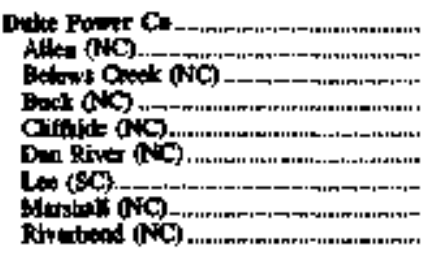 & $\begin{array}{r}1,2046 \\
204 \\
390 \\
27 \\
02 \\
48 \\
34 \\
396 \\
63\end{array}$ & $\begin{array}{l}141.6 \\
130.7 \\
144.1 \\
126.6 \\
193.2 \\
123.4 \\
180.8 \\
129.7 \\
179.1\end{array}$ & $\begin{array}{l}18.14 \\
32.92 \\
35.99 \\
31.78 \\
47.27 \\
31.03 \\
45.92 \\
31.73 \\
45.16\end{array}$ & $\begin{array}{l}92 \\
.78 \\
.78 \\
1.05 \\
1.10 \\
.95 \\
1.43 \\
1.05 \\
1.06\end{array}$ & $\begin{array}{r}14 \\
2 \\
-\quad 1 \\
-\quad 2 \\
5 \\
4\end{array}$ & $\begin{array}{l}394.7 \\
394.7 \\
399.8 \\
-599.3 \\
-\end{array}$ & $\begin{array}{l}22.95 \\
23.22 \\
1950 \\
-13 \\
23.35 \\
23.20 \\
23.19\end{array}$ & $\begin{array}{l}.30 \\
.30 \\
-30 \\
-30 \\
.30 \\
-.30\end{array}$ & $\begin{array}{l}\bar{z} \\
\bar{z} \\
\bar{z} \\
\bar{z}\end{array}$ & $\begin{array}{l}z \\
z \\
z \\
z \\
z\end{array}$ & $\begin{array}{l}z \\
z \\
z \\
z \\
z\end{array}$ & $\begin{array}{c}100 \\
100 \\
100 \\
100 \\
99 \\
100 \\
97 \\
100 \\
104\end{array}$ & $\begin{array}{l}: \\
\stackrel{*}{*} \\
-1 \\
-{ }^{3} \\
-\end{array}$ & $\begin{array}{l}\bar{z} \\
= \\
= \\
= \\
=\end{array}$ \\
\hline 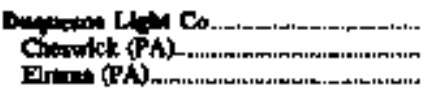 & $\begin{array}{l}158 \\
\text { I11 } \\
7 \pi\end{array}$ & $\begin{array}{l}112.8 \\
114.5 \\
110.1\end{array}$ & $\begin{array}{l}29.43 \\
30,39 \\
2 B .30\end{array}$ & $\begin{array}{l}157 \\
1.81 \\
2.20\end{array}$ & - & $\frac{379}{397.9}$ & $\frac{23.19}{2.19}$ & -.34 & $\begin{array}{r}\mathbf{n} \\
-\end{array}$ & 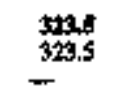 & $\begin{array}{l}3.34 \\
3.36 \\
-\end{array}$ & 99 & - & $\begin{array}{r}\cdot \\
-\end{array}$ \\
\hline 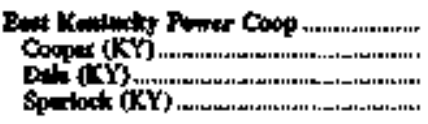 & $\begin{array}{r}215 \\
56 \\
43 \\
193\end{array}$ & $\begin{array}{l}11 \$ 5 \\
111.9 \\
114.2 \\
115.0\end{array}$ & $\begin{array}{l}20.26 \\
27.44 \\
28.57\end{array}$ & 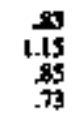 & $\begin{array}{r}\mathbf{1} \\
-\quad 1 \\
-\quad 1\end{array}$ & $\begin{array}{l}41.5 \\
415.7 \\
4102 \\
-\end{array}$ & $\begin{array}{l}21.66 \\
24.20 \\
23.48 \\
-\end{array}$ & $\begin{array}{l}.14 \\
.20 \\
.12 \\
-\end{array}$ & $\begin{array}{l}z \\
=\end{array}$ & $\begin{array}{l}\overline{-} \\
\overline{-}\end{array}$ & $\begin{array}{l}z \\
\bar{z}\end{array}$ & $\begin{array}{l}100 \\
100 \\
100 \\
100\end{array}$ & : & $\begin{array}{l}= \\
= \\
=\end{array}$ \\
\hline 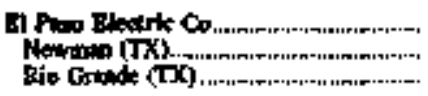 & $\bar{z}$ & $\bar{z}$ & $=$ & $\ddot{z}$ & $\bar{z}$ & $\bar{z}$ & $=$ & $\ddot{-}$ & $\begin{array}{l}3, \pi 24 \\
2,257 \\
1,068\end{array}$ & $\begin{array}{l}208.1 \\
207.1 \\
210.0\end{array}$ & $\begin{array}{l}2.13 \\
2.12 \\
2.15\end{array}$ & $\vec{z}$ & $=$ & $\begin{array}{l}100 \\
100 \\
100\end{array}$ \\
\hline 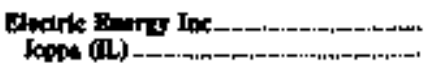 & as & 923 & $\begin{array}{l}16.24 \\
66.20\end{array}$ & $\frac{3}{33}$ & $:$ & 4030.6 & $\underset{26.96}{269}$ & .6 & is & 2780 & $\begin{array}{l}249 \\
289\end{array}$ & intor & $\dot{*}$ & : \\
\hline 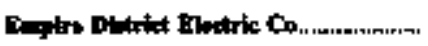 & $\mathbf{s}$ & 1166 & N2.16 & $s$ & - & - & - & - & 4 & 233.3 & 24 & $\mathbf{m}$ & - & - \\
\hline
\end{tabular}

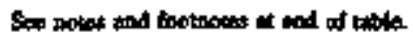


Table 57. Recelpts, Average Cost, and Quility of Fossal Fucis Delivered to U.S. Electrik Utitities by Company and Plant, Jibl 1997 (Conthined)

\begin{tabular}{|c|c|c|c|c|c|c|c|c|c|c|c|c|c|c|}
\hline \multirow{3}{*}{ 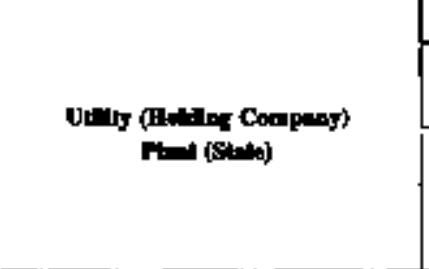 } & \multicolumn{4}{|c|}{ Con } & \multicolumn{4}{|c|}{ 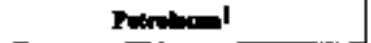 } & \multicolumn{3}{|c|}{$\mathrm{G}=\mathrm{A}$} & \multicolumn{3}{|c|}{ * a Total Bam } \\
\hline & \multirow{2}{*}{ 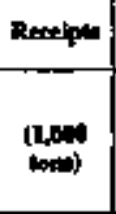 } & \multicolumn{2}{|c|}{ Aristis } & \multirow{2}{*}{$\begin{array}{c}\text { Ant } \\
\text { sol- } \\
4 \\
5\end{array}$} & \multirow{2}{*}{ 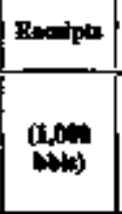 } & \multicolumn{2}{|c|}{ 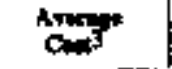 } & \multirow{2}{*}{$\mid \begin{array}{c}n+2 \\
s+2 \\
n \\
y\end{array}$} & \multirow{2}{*}{ 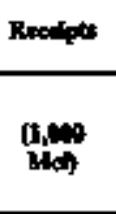 } & \multicolumn{2}{|c|}{ Axtent } & \multirow[b]{2}{*}{ Cost } & \multirow{2}{*}{ Po- } & \multirow[b]{2}{*}{ Gas } \\
\hline & & 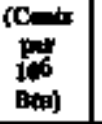 & that & & & 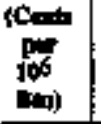 & per & & & 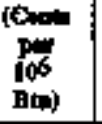 & \$per & & & \\
\hline 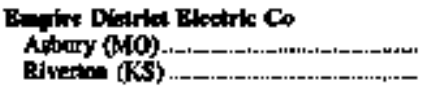 & $\begin{array}{l}37 \\
21\end{array}$ & $\begin{array}{l}111.7 \\
125.3\end{array}$ & $\begin{array}{l}21.12 \\
21.18\end{array}$ & $\begin{array}{r}086 \\
.97\end{array}$ & $\overline{-}$ & $\bar{z}$ & $=$ & - & - & 201.7 & 254 & $\operatorname{lig}_{99}$ & - & - \\
\hline 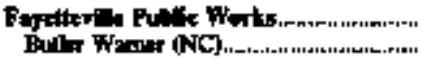 & $=$ & $\overline{-}$ & $\overline{-}$ & $\overline{-}$ & $\overline{-}$ & $\overline{-}$ & $\overline{-}$ & $\overline{-}$ & 4 & $\begin{array}{l}30.1 \\
302.1\end{array}$ & $\begin{array}{l}3.12 \\
3.12\end{array}$ & $\overline{-}$ & $\overline{-}$ & 100 \\
\hline 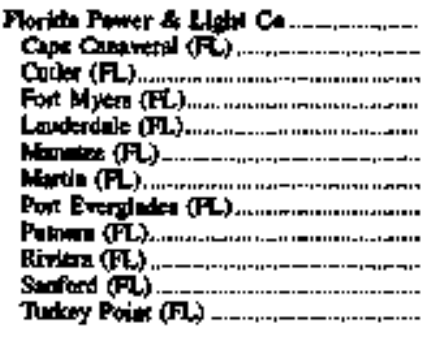 & $\begin{array}{l}\bar{z} \\
\bar{z} \\
\bar{z} \\
\bar{z} \\
\bar{z}\end{array}$ & $\begin{array}{l}= \\
= \\
= \\
= \\
= \\
=\end{array}$ & $\begin{array}{l}\bar{z} \\
\bar{z} \\
\bar{z} \\
\bar{z} \\
\bar{z}\end{array}$ & $\begin{array}{l}\bar{z} \\
= \\
= \\
= \\
= \\
=\end{array}$ & $\begin{array}{r}2792 \\
-\quad 264 \\
-266 \\
-943 \\
312 \\
-252 \\
-\quad 39 \\
240 \\
131\end{array}$ & 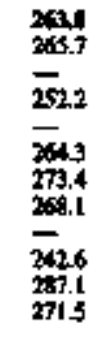 & $\begin{array}{l}16.5 \\
16.79 \\
16.10 \\
\overline{-16.94} \\
17.50 \\
17.11 \\
\overline{15.54} \\
18.15 \\
17.39\end{array}$ & $\begin{array}{l}1.91 \\
\frac{1.95}{207} \\
-.96 \\
.97 \\
.97 \\
2.05 \\
1.90 \\
1.45\end{array}$ & $\begin{array}{r}21,675 \\
1,711 \\
6,57 \\
-\quad 4,09 \\
-\quad, 416 \\
1,507 \\
2,67 \\
739 \\
679 \\
1,766\end{array}$ & $\begin{array}{l}281.0 \\
281.0 \\
2 * 1.0 \\
-281.0 \\
\overline{2} \\
281.0 \\
281.0 \\
281.0 \\
281.0 \\
281.0 \\
28.0\end{array}$ & $\begin{array}{l}2.93 \\
2.93 \\
2.95 \\
2.93 \\
\frac{2.95}{2.95} \\
2.93 \\
2.93 \\
2.95 \\
2.95\end{array}$ & $\begin{array}{l}\bar{z} \\
\bar{z} \\
\bar{z} \\
\bar{z} \\
=\end{array}$ & $\begin{array}{l}\frac{41}{45} \\
\frac{100}{100} \\
\frac{21}{51} \\
\frac{68}{70} \\
31\end{array}$ & $\begin{array}{r}8 \\
55 \\
100 \\
700 \\
\frac{79}{40} \\
100 \\
12 \\
30 \\
69\end{array}$ \\
\hline 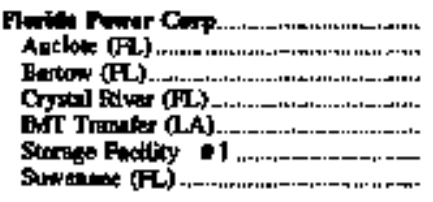 & $\begin{array}{l}\overline{-}^{519} \\
= \\
202\end{array}$ & $\begin{array}{l}173.4 \\
= \\
575.6 \\
= \\
=\end{array}$ & $\begin{array}{l}= \\
\overline{4} \\
457 \\
= \\
=\end{array}$ & $\begin{array}{l}-m \\
= \\
= \\
-6\end{array}$ & $\begin{array}{r}1,93 \\
-\quad 1 \\
-\quad 2 \\
950 \\
50\end{array}$ & 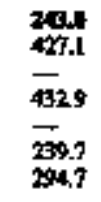 & $\begin{array}{l}1598 \\
24.76 \\
-1.10 \\
27.10 \\
\frac{13.69}{12.84}\end{array}$ & 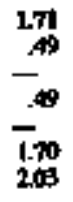 & $\begin{array}{l}-193 \\
=\end{array}$ & $\begin{array}{l}202 \\
304.7 \\
= \\
=\end{array}$ & $\begin{array}{l}\frac{3.3}{3.19} \\
\frac{3}{3.06}\end{array}$ & $\begin{array}{l}- \\
= \\
100 \\
= \\
=\end{array}$ & $\begin{array}{c}\frac{31}{100} \\
\frac{100}{100} \\
+0\end{array}$ & $\begin{array}{l}\frac{3}{100} \\
\frac{-}{60}\end{array}$ \\
\hline 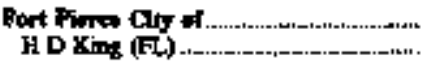 & $=$ & $m$ & $=$ & I & $\overline{-}$ & I & $\overline{-}$ & $=$ & 302 & $\begin{array}{l}302.3 \\
3023\end{array}$ & $\begin{array}{l}3.15 \\
3.15\end{array}$ & $=$ & $\overline{-}$ & 100 \\
\hline Frpoond Cony of of & $\frac{\pi}{22}$ & $\begin{array}{l}\text { 9.1.6 } \\
\text { 9i.6 }\end{array}$ & $\begin{array}{l}15.47 \\
15.87\end{array}$ & $\frac{4}{28}$ & $\overline{-}$ & $\overline{-}$ & $\overline{-}$ & $=$ & 7 & $\begin{array}{l}\text { at, } \\
\text { 211.0 }\end{array}$ & $\begin{array}{l}2.11 \\
2.11\end{array}$ & \$ & $\overline{-}$ & 2 \\
\hline 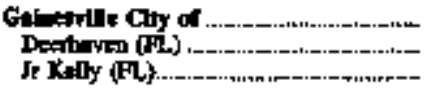 & -48 & $\begin{array}{l}1444 \\
1648 \\
-\end{array}$ & $\begin{array}{l}43.69 \\
-\end{array}$ & -56 & $\bar{z}$ & $\bar{z}$ & $\bar{z}$ & $=$ & $\begin{array}{l}335 \\
342 \\
193\end{array}$ & $\begin{array}{l}\operatorname{2os} 4 \\
285.4 \\
285,4\end{array}$ & $\begin{array}{l}297 \\
297\end{array}$ & -5 & $\vec{z}$ & $\begin{array}{r}51 \\
22 \\
100\end{array}$ \\
\hline 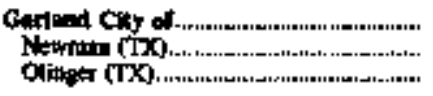 & $\bar{z}$ & $\bar{z}$ & 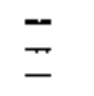 & 二 & $\bar{z}$ & $\bar{z}$ & $\vec{z}$ & $\bar{z}$ & $\begin{array}{l}1,61 \\
1,598\end{array}$ & $\underset{2 m .6}{2 m .6}$ & $\begin{array}{l}227 \\
237\end{array}$ & $\bar{z}$ & $\bar{z}$ & $\begin{array}{l}100 \\
100\end{array}$ \\
\hline 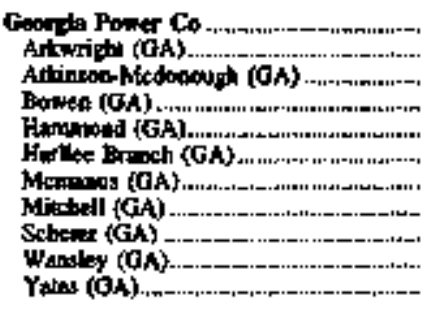 & $\begin{array}{r}2,375 \\
9 \\
86 \\
736 \\
106 \\
213 \\
-\quad 18 \\
735 \\
239 \\
147\end{array}$ & $\begin{array}{l}154.1 \\
184.9 \\
133.0 \\
140.1 \\
1485 \\
134.4 \\
-184.6 \\
176.3 \\
1850 \\
151.0\end{array}$ & $\begin{array}{l}37.48 \\
41.86 \\
36.26 \\
34.64 \\
38.52 \\
37.48 \\
\frac{15.69}{45.69} \\
35.80 \\
46.28 \\
3.95\end{array}$ & $\begin{array}{r}1.9 \\
1.97 \\
1.11 \\
.92 \\
1.95 \\
1.34 \\
1.23 \\
.90 \\
.78 \\
.90\end{array}$ & $\begin{array}{c}6 \\
-\quad 3 \\
1 \\
1 \\
41 \\
1 \\
1 \\
8 \\
1\end{array}$ & $\begin{array}{l}371.2 \\
\overline{7} \\
40.3 \\
438.6 \\
438.8 \\
330.3 \\
438.8 \\
438.3 \\
465.7 \\
432.6\end{array}$ & $\begin{array}{l}2145 \\
- \\
- \\
25.91 \\
15.51 \\
25.52 \\
20.20 \\
25.00 \\
25.50 \\
25.93 \\
25.16\end{array}$ & $\begin{array}{l}5 \\
- \\
-90 \\
90 \\
50 \\
.90 \\
50 \\
90 \\
50 \\
50\end{array}$ & $\begin{array}{l}\quad \text { sits } \\
= \\
= \\
= \\
= \\
= \\
=\end{array}$ & $\begin{array}{l}2060 \\
1868 \\
317.7 \\
= \\
= \\
= \\
= \\
=\end{array}$ & $\begin{array}{l}2.5 \\
1.91 \\
3.25 \\
= \\
= \\
= \\
= \\
= \\
=\end{array}$ & $\begin{array}{r}90 \\
62 \\
103 \\
100 \\
100 \\
100 \\
-\quad 88 \\
100 \\
99 \\
100\end{array}$ & $\begin{array}{c}-1 \\
= \\
\vdots \\
100 \\
12 \\
1\end{array}$ & $\begin{array}{l}15 \\
17 \\
17 \\
= \\
= \\
= \\
= \\
= \\
-\end{array}$ \\
\hline 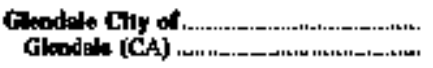 & $\overline{-}$ & $\ddot{-}$ & $=$ & $\overline{-}$ & $\overline{-}$ & $\overline{-}$ & $\overline{-}$ & $=$ & IIt: & 200 & $\begin{array}{l}253 \\
253\end{array}$ & $\Sigma$ & $\overline{-}$ & 100 \\
\hline 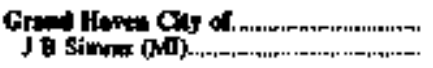 & 23 & $\begin{array}{l}1359 \\
135.9\end{array}$ & 34.35 & $\frac{2.26}{226}$ & $\bar{z}$ & $\overline{-}$ & $\overline{-}$ & $=$ & $*$ & $\mathbf{4 5 4}$ & 445 & itoo & $\bar{r}$ & * \\
\hline 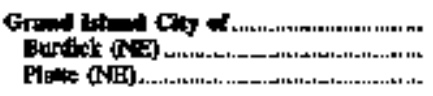 & -30 & $\frac{64}{69.8}$ & $\begin{array}{l}11.87 \\
\overrightarrow{11} .82\end{array}$ & $\underset{32}{313}$ & $\bar{z}$ & $\bar{z}$ & $\bar{z}$ & $\bar{z}$ & 3 & $\begin{array}{l}\mathbf{2 0 9} \\
\mathbf{2 0 4 9} \\
-\end{array}$ & $\begin{array}{l}2.39 \\
239 \\
-\end{array}$ & $\frac{97}{100}$ & $\bar{z}$ & 100 \\
\hline
\end{tabular}

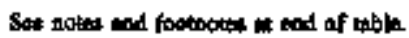


Table 57. Receipter Avernge Caet, and Qualty of Foesl Foels Delivered to U.S. Blectric Utilities by Company and Flant, July 1997 (Continued)

\begin{tabular}{|c|c|c|c|c|c|c|c|c|c|c|c|c|c|c|}
\hline \multirow{2}{*}{ 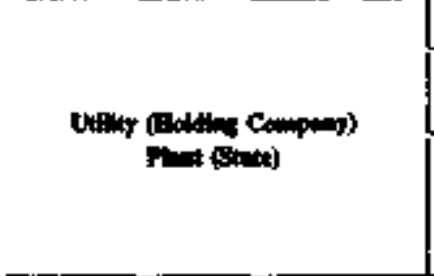 } & \multicolumn{4}{|c|}{ Conl } & \multicolumn{4}{|c|}{ 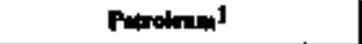 } & \multicolumn{3}{|c|}{$6=$} & \multicolumn{3}{|c|}{ s T Thall Bu } \\
\hline & Risula & \multicolumn{2}{|c|}{ Cant } & 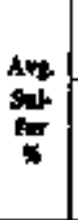 & (1.0.4) & \multicolumn{2}{|c|}{ Compits } & 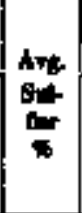 & 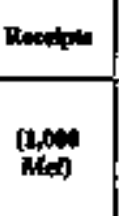 & \multicolumn{2}{|c|}{ Confl } & Conl & $\operatorname{tin}_{1=1}$ & Cat \\
\hline 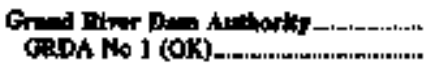 & 331 & $\sin$ & 154ts & $\begin{array}{r}\mathbf{4 8} \\
.88\end{array}$ & $\overline{-}$ & $\overline{-}$ & $\overline{-}$ & - & 19 & 24.1 & $\begin{array}{l}2.6 \\
2.48\end{array}$ & 100 & - & $\bullet$ \\
\hline 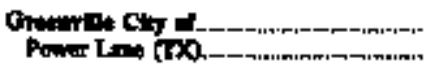 & $\overline{-}$ & - & $=$ & - & - & - & - & $\overline{-}$ & 8 & $\begin{array}{l}\text { 216i } \\
\text { 2160a }\end{array}$ & 236 & - & - & 100 \\
\hline 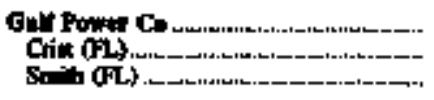 & $\begin{array}{r}211 \\
120 \\
91\end{array}$ & $\begin{array}{l}1869 \\
196.7 \\
1735\end{array}$ & $\begin{array}{r}45.12 \\
48.15 \\
41.13\end{array}$ & $\begin{array}{r}1.73 \\
.98 \\
2.72\end{array}$ & $\star$ & $\begin{array}{l}\min 7 \\
3928 \\
073\end{array}$ & $\begin{array}{l}25.42 \\
2285 \\
25.44\end{array}$ & $\begin{array}{r}45 \\
45\end{array}$ & $\begin{array}{r}36 \\
-39 \\
\end{array}$ & $\begin{array}{l}\mathbf{x u d} \\
\mathbf{x h o t}\end{array}$ & $\begin{array}{l}230 \\
230 \\
-\end{array}$ & $\begin{array}{r}98 \\
89 \\
100\end{array}$ & $\bullet$ & $\underline{11}$ \\
\hline 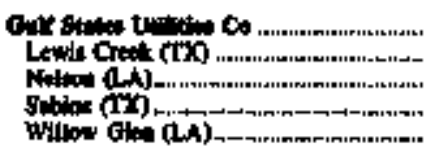 & $\underbrace{201}_{-}$ & $\begin{array}{l}1196 \\
1196 \\
-\end{array}$ & $\begin{array}{l}\min \\
\overrightarrow{20} \pi \\
=\end{array}$ & $\begin{array}{c}-46 \\
-\end{array}$ & $\begin{array}{l}- \\
z \\
z\end{array}$ & $\begin{array}{l}z \\
z \\
z\end{array}$ & $\begin{array}{l}z \\
\overline{-} \\
=\end{array}$ & $\begin{array}{l}- \\
-\end{array}$ & $\begin{array}{r}19,117 \\
2,583 \\
3,339 \\
6,362 \\
7,532\end{array}$ & $\begin{array}{l}271.1 \\
278.1 \\
227.1 \\
207.4 \\
2024\end{array}$ & $\begin{array}{l}2.39 \\
2.39 \\
2.34 \\
2.46 \\
2.35\end{array}$ & $\begin{array}{l}-5 \\
-5 \\
-\end{array}$ & $\begin{array}{l}z \\
- \\
- \\
-\end{array}$ & $\begin{array}{r}100 \\
50 \\
500 \\
100\end{array}$ \\
\hline & 20 & 1418 & 37.64 & .73 & - & $\overline{-}$ & - & 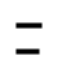 & 65 & 201.3 & $\begin{array}{l}2.94 \\
2.90\end{array}$ & 8 & - & 12 \\
\hline 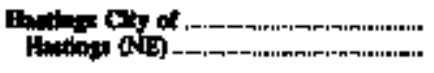 & 20 & 60.5 & 10.29 & $\frac{33}{39}$ & $\underline{-}$ & - & $\underline{-}$ & $\underline{-}$ & $\overline{-}$ & - & $\vec{z}$ & 100 & $\underline{-}$ & $\overline{-}$ \\
\hline 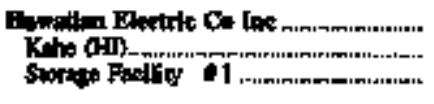 & $=$ & $\overline{-}$ & - & $\underline{-}$ & $\frac{6+3}{71}$ & $\begin{array}{l}30.3 \\
392.3 \\
329.0\end{array}$ & $\begin{array}{l}2.57 \\
20.91 \\
20.52\end{array}$ & A5 & $\underline{-}$ & $\frac{-}{-}$ & $\underline{z}$ & $=$ & $\begin{array}{l}100 \\
100 \\
100\end{array}$ & $\underline{-}$ \\
\hline 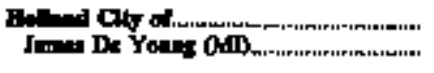 & $\mathbf{4}$ & $\begin{array}{l}1700 \\
1790\end{array}$ & 404 & tit & - & $\overline{-}$ & $\underline{-}$ & $\overline{-}$ & $\frac{25}{23}$ & 297.0 & 2.85 & 94 & $\overline{-}$ & 6 \\
\hline 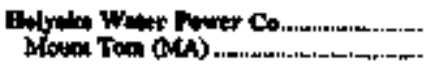 & 40 & $\begin{array}{l}\text { in13 } \\
\text { It1.3 }\end{array}$ & 47.4 & 92 & $\bullet$ & $\begin{array}{l}46.9 \\
436.9\end{array}$ & 2528 & 27 & $\vec{r}$ & - & $\vec{z}$ & 100 & 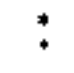 & $\underline{-}$ \\
\hline 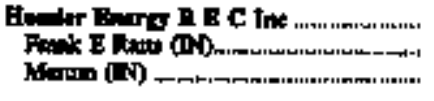 & $\begin{array}{r}50 \\
505\end{array}$ & $\begin{array}{l}17265 \\
1375 \\
119.9\end{array}$ & $\begin{array}{l}26.9 \\
30.72 \\
36.19\end{array}$ & $\begin{array}{l}2.4 \\
134 \\
3.16\end{array}$ & $\ddot{*}$ & $\begin{array}{l}1+4.4 \\
396.8\end{array}$ & 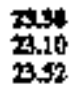 & $\begin{array}{l}- \\
-\end{array}$ & $\begin{array}{l}- \\
-\end{array}$ & $\begin{array}{l}- \\
-\end{array}$ & $=$ & $\begin{array}{l}100 \\
100 \\
100\end{array}$ & $\ddot{*}$ & $=$ \\
\hline 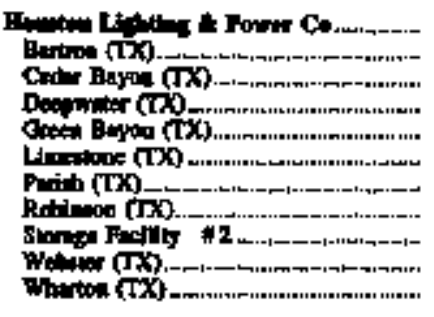 & $\begin{array}{l}= \\
= \\
= \\
= \\
= \\
=\end{array}$ & $\begin{array}{l}136.7 \\
= \\
= \\
= \\
1948 \\
= \\
= \\
=\end{array}$ & 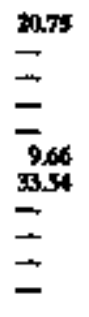 & $\begin{array}{l}5 \\
- \\
= \\
-6 \\
= \\
= \\
=\end{array}$ & $\begin{array}{l}- \\
= \\
= \\
= \\
= \\
-\end{array}$ & $\begin{array}{l}- \\
- \\
- \\
- \\
- \\
-\end{array}$ & $\begin{array}{l}= \\
= \\
= \\
= \\
= \\
= \\
=\end{array}$ & $\begin{array}{l}= \\
= \\
= \\
= \\
= \\
=\end{array}$ & 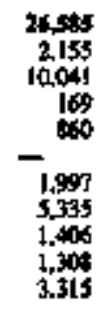 & $\begin{array}{l}2113 \\
2125 \\
201 \\
2215 \\
2221 \\
- \\
219.9 \\
221.7 \\
222.5 \\
222.2 \\
222.5\end{array}$ & $\begin{array}{l}226 \\
228 \\
225 \\
230 \\
229 \\
\frac{1}{224} \\
227 \\
227 \\
220 \\
227\end{array}$ & $\begin{array}{l}\text { - } \\
= \\
- \\
100 \\
86 \\
- \\
- \\
-\end{array}$ & $\begin{array}{l}= \\
= \\
= \\
= \\
= \\
= \\
=\end{array}$ & $\begin{array}{l}\$ 4 \\
100 \\
100 \\
100 \\
100 \\
-14 \\
100 \\
100 \\
100 \\
100\end{array}$ \\
\hline 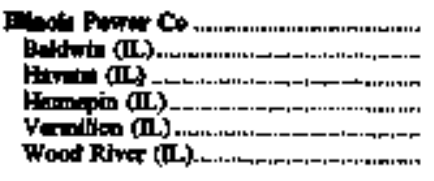 & $\begin{array}{r}3 \times 3 \\
221 \\
30 \\
72 \\
30 \\
8\end{array}$ & $\begin{array}{l}1143 \\
103.0 \\
138.3 \\
120.9 \\
1065 \\
120.5\end{array}$ & $\begin{array}{l}23.55 \\
22,31 \\
31,56 \\
25,13 \\
22,54 \\
30.55\end{array}$ & $\begin{array}{r}2.90 \\
2.90 \\
60 \\
285 \\
1.08 \\
1.04\end{array}$ & $\begin{array}{l}1 \\
2 \\
1 \\
1\end{array}$ & $\begin{array}{l}\text { M.t. } \\
40.7 \\
\frac{1}{40.9}\end{array}$ & $\begin{array}{l}175 \\
25.21 \\
25.04 \\
- \\
-\end{array}$ & $\begin{array}{l}30 \\
30 \\
-30 \\
-\end{array}$ & $-\begin{array}{r}35 \\
-15\end{array}$ & $\begin{array}{l}245.5 \\
\frac{106.5}{3} \\
\frac{620.2}{20.4}\end{array}$ & $\begin{array}{l}2.50 \\
\overline{3.07} \\
\frac{-}{6.41} \\
2.32\end{array}$ & $\begin{array}{r}94 \\
100 \\
99 \\
100 \\
95 \\
35\end{array}$ & $\begin{array}{r}\bullet \\
- \\
-\end{array}$ & $\frac{4}{4}$ \\
\hline 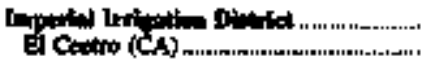 & $\overline{-}$ & - & $\underline{-}$ & 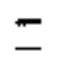 & $\overrightarrow{-}$ & $\overline{-}$ & $\overline{-}$ & $\overline{-}$ & 609 & 278.4 & $\begin{array}{l}2.11 \\
281\end{array}$ & $\overline{-}$ & $\overline{-}$ & 100 \\
\hline 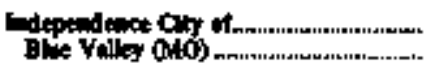 & 6 & $\operatorname{lot}_{1240}$ & 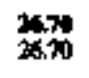 & 2.73 & 1 & 4238 & 24.45 & 30 & 74 & $\begin{array}{l}2556 \\
255.6\end{array}$ & $\begin{array}{l}2.36 \\
2.36\end{array}$ & 59 & 4 & 37 \\
\hline 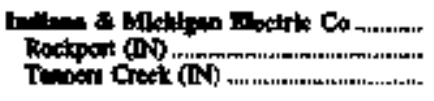 & $\begin{array}{r}10+7 \\
655 \\
162\end{array}$ & $\begin{array}{l}111.5 \\
106.5 \\
126.3\end{array}$ & $\begin{array}{l}\text { 24sts } \\
\text { 16.53 } \\
32.02\end{array}$ & 19 & $\begin{array}{r}3 \\
-\quad 3\end{array}$ & $\frac{1}{3804}$ & $\frac{1}{204}$ & $\overline{-}$ & $\underline{-}$ & $\overline{-}$ & $\underline{-}$ & $\begin{array}{l}\text { tot } \\
100 \\
100\end{array}$ & $\stackrel{-}{*}$ & $\underline{-}$ \\
\hline
\end{tabular}

Sen now ind foomotes at and of talle. 
Tabie 57. Receipts, Average Coot, and Qeality of Fowsll Fuels Detivered to US. Dlectric Utilities by Compony and Flant, Joing 1997 (Conthoed)

\begin{tabular}{|c|c|c|c|c|c|c|c|c|c|c|c|c|c|c|}
\hline \multirow{3}{*}{ 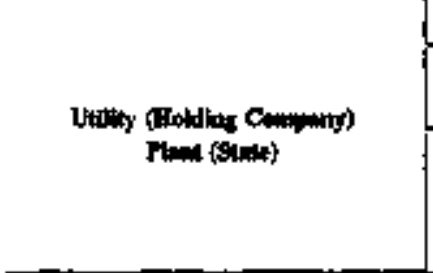 } & \multicolumn{4}{|c|}{ Conl } & \multicolumn{4}{|c|}{ | } & \multicolumn{3}{|c|}{$\theta$} & \multicolumn{3}{|c|}{ \% of Totat Dit } \\
\hline & \multirow{2}{*}{ 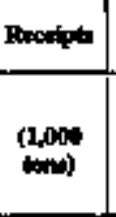 } & \multicolumn{2}{|c|}{$\cos$} & \multirow{2}{*}{ 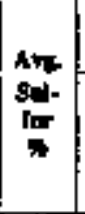 } & \multirow{2}{*}{ Racelpe } & \multicolumn{2}{|c|}{ Anerats } & \multirow{2}{*}{$\mid \begin{array}{c}\text { Avt } \\
\text { sot } \\
\text { tor } \\
4\end{array}$} & \multirow{2}{*}{ Bpolpity } & \multicolumn{2}{|c|}{ Avats } & \multirow[b]{2}{*}{ Ceal } & \multirow[b]{2}{*}{$\mid$} & \multirow[b]{2}{*}{ Ges } \\
\hline & & Pants & 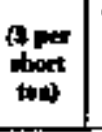 & & & $\underset{\text { Pat }}{\text { par }}$ & per & & & Conda & imer & & & \\
\hline 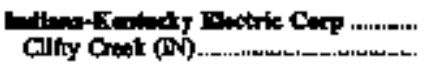 & 360 & II48 & $\frac{129}{23.50}$ & 96 & 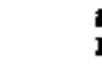 & $\mathbf{4 0 . 3}$ & $\begin{array}{l}\mathbf{2 5 . 3 4} \\
25.32\end{array}$ & 30 & $\ddot{z}$ & $\underline{-}$ & $\overline{-}$ & $\begin{array}{l}100 \\
100\end{array}$ & : & $\ddot{z}$ \\
\hline 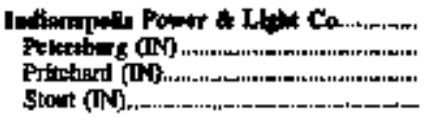 & $\begin{array}{c}616 \\
451 \\
58 \\
107\end{array}$ & $\begin{array}{r}\mathbf{4} 4 \\
09.7 \\
1007 \\
1127\end{array}$ & $\begin{array}{l}21.11 \\
20,01 \\
22.21 \\
25.12\end{array}$ & $\begin{array}{r}2.26 \\
2.646 \\
1.26\end{array}$ & $-\mathbf{s}$ & $\frac{\operatorname{cic}}{\min }$ & 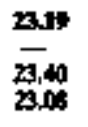 & $\begin{array}{c}-14 \\
-04 \\
04\end{array}$ & $\begin{array}{l}E \\
E\end{array}$ & $\begin{array}{l}= \\
- \\
-\end{array}$ & $\begin{array}{l}\overline{-} \\
\bar{z}\end{array}$ & $\begin{array}{r}99 \\
100 \\
98 \\
98\end{array}$ & $\begin{array}{r}\mathbf{1} \\
-2 \\
2\end{array}$ & $=$ \\
\hline 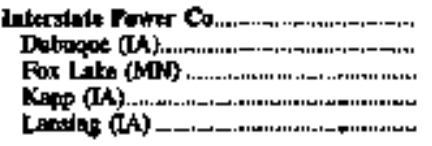 & $\begin{array}{r}141 \\
-\quad 5 \\
52 \\
.84\end{array}$ & $\begin{array}{l}1647 \\
\$ 09.6 \\
\frac{135.2}{191.6}\end{array}$ & $\begin{array}{l}32.34 \\
24.07 \\
\overrightarrow{30.62} \\
33.66\end{array}$ & $\begin{array}{r}.69 \\
2.27 \\
-5.5 \\
.61\end{array}$ & $\begin{array}{l}\ddot{z} \\
\dot{z}\end{array}$ & $\begin{array}{l}\text { Pos. } \\
398 \% \\
= \\
=\end{array}$ & $\begin{array}{l}20,46 \\
= \\
=\end{array}$ & $\begin{array}{l}= \\
=\end{array}$ & $\begin{array}{r}315 \\
313 \\
-\quad 2\end{array}$ & $\begin{array}{l}2015 \\
400.0 \\
237.5 \\
3618 \\
-\end{array}$ & $\begin{array}{l}2.39 \\
4.00 \\
2.7 \\
3.71 \\
7\end{array}$ & $\begin{array}{c}90 \\
99 \\
100 \\
100\end{array}$ & $\frac{1}{=}$ & $\underset{100}{10}$ \\
\hline 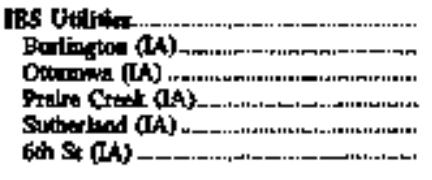 & $\begin{array}{l}29 \\
41 \\
51 \\
72 \\
47 \\
13\end{array}$ & $\begin{array}{r}16.3 \\
104.0 \\
89.9 \\
98.3 \\
7.0 \\
156.9\end{array}$ & $\begin{array}{l}164 \\
18.7 \\
15.04 \\
16.99 \\
13.20 \\
31.93\end{array}$ & $\begin{array}{l}41 \\
.72 \\
.34 \\
34 \\
34 \\
53\end{array}$ & $\begin{array}{l}= \\
= \\
=\end{array}$ & $\begin{array}{l}= \\
= \\
= \\
=\end{array}$ & $\begin{array}{l}\bar{z} \\
\bar{z}\end{array}$ & $\begin{array}{l}= \\
= \\
=\end{array}$ & $=$ & $\begin{array}{l}253.4 \\
= \\
270.0 \\
309.3 \\
2007\end{array}$ & 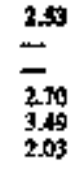 & $\begin{array}{r}90 \\
100 \\
100 \\
98 \\
93 \\
67\end{array}$ & $\begin{array}{l}= \\
= \\
=\end{array}$ & $\frac{5}{-}$ \\
\hline 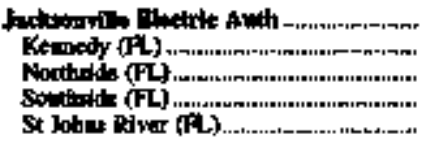 & $\begin{array}{l}z^{205} \\
295\end{array}$ & $\begin{array}{l}\text { Es1.6 } \\
\bar{z} \\
161.6\end{array}$ & $\begin{array}{l}\frac{10 \pi}{-} \\
\frac{\pi}{40,4 \pi}\end{array}$ & $\begin{array}{l}1.4 \\
= \\
\frac{1}{1.44}\end{array}$ & $\begin{array}{l}16 \\
- \\
-\quad 63\end{array}$ & $\begin{array}{l}\frac{165}{262.3} \\
\frac{1}{417.3}\end{array}$ & $\frac{16.5}{16.60}$ & $\begin{array}{c}1.2 \\
1.32 \\
-35\end{array}$ & $\begin{array}{r}1,30 \\
1.99 \\
-\quad 49 \\
+99\end{array}$ & $\begin{array}{l}2743 \\
2760 \\
265.0 \\
276.0 \\
-\end{array}$ & $\begin{array}{l}2.19 \\
2.91 \\
2.97 \\
2.91 \\
=\end{array}$ & $\begin{array}{l}-73 \\
=\end{array}$ & $\frac{13}{62}$ & $\begin{array}{r}14 \\
100 \\
30 \\
100 \\
-\end{array}$ \\
\hline 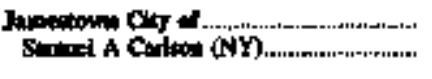 & It & $\begin{array}{l}132.9 \\
132.9\end{array}$ & $\begin{array}{l}\text { 32.71 } \\
\text { 33.71 }\end{array}$ & 1.98 & $\bar{z}$ & $\overline{-}$ & $\bar{z}$ & $=$ & $\overline{-}$ & $\bar{z}$ & $\overline{-}$ & 100 & $\overline{-}$ & $\overline{-}$ \\
\hline 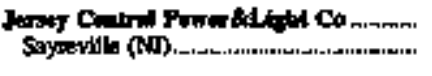 & $\bar{z}$ & $\overline{-}$ & $\overline{-}$ & $\overline{-}$ & 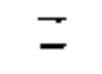 & $\overrightarrow{-}$ & $\overline{-}$ & $=$ & 93 & $\begin{array}{l}39,3 \\
299.3\end{array}$ & $\begin{array}{l}216 \\
216\end{array}$ & $\overline{-}$ & $\bar{z}$ & 100 \\
\hline 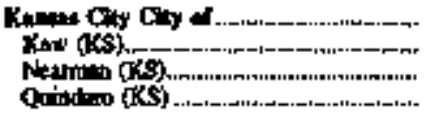 & $\begin{array}{r}13 \\
6 \\
46\end{array}$ & $\begin{array}{l}12.0 \\
127.4 \\
\text { II]. }\end{array}$ & $\begin{array}{l}18,24 \\
27,09 \\
13,66 \\
26.28\end{array}$ & $\frac{\$ 15}{100}$ & $-^{13}$ & $\begin{array}{l}\frac{2 x .5}{4} \\
\frac{\pi}{42}\end{array}$ & $\frac{247}{\overline{24}, 7}$ & $\stackrel{-}{=}$ & $\begin{array}{r}12 \\
-\quad 1 \\
11\end{array}$ & $\begin{array}{l}227.4 \\
236.6 \\
\frac{125.2}{25}\end{array}$ & $\frac{27}{2.7}$ & $\begin{array}{c}96 \\
99 \\
100 \\
92\end{array}$ & $\frac{3}{7}$ & $\frac{1}{1}$ \\
\hline 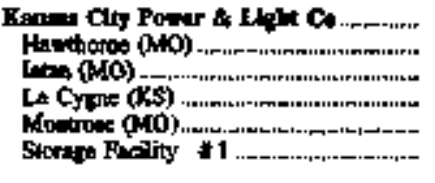 & $\begin{array}{l}125 \\
215 \\
313 \\
156 \\
-\end{array}$ & $\begin{array}{l}76.1 \\
65.0 \\
81.2 \\
65.0 \\
97.7 \\
-\end{array}$ & $\begin{array}{l}14.28 \\
11.95 \\
14.28 \\
11.25 \\
16.89 \\
-\end{array}$ & $\begin{array}{l}30 \\
34 \\
.34 \\
27 \\
-2\end{array}$ & ${ }^{-}$ & $\begin{array}{l}\frac{46.1}{\overline{40.9}} \\
\frac{-}{412.0}\end{array}$ & 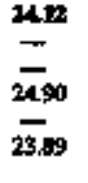 & $\begin{array}{l}.16 \\
-.15 \\
-16\end{array}$ & $=$ & $\begin{array}{l}254 \\
= \\
= \\
=\end{array}$ & $\begin{array}{l}2.56 \\
2.56 \\
= \\
=\end{array}$ & $\begin{array}{r}97 \\
99 \\
900 \\
99 \\
109 \\
-\end{array}$ & $\frac{2}{-3}$ & $\begin{array}{r}\quad \\
\quad \\
- \\
- \\
= \\
=\end{array}$ \\
\hline 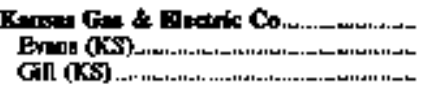 & $\bar{z}$ & $\bar{z}$ & $\bar{z}$ & $\bar{z}$ & $\bar{z}$ & $\bar{z}$ & $\bar{z}$ & $\Xi$ & $\begin{array}{l}2,69 \\
1,090 \\
919\end{array}$ & $\begin{array}{l}207 \\
207 \\
207\end{array}$ & $\begin{array}{l}20 \% \\
210\end{array}$ & $\bar{z}$ & $\bar{z}$ & $\begin{array}{l}160 \\
100 \\
100\end{array}$ \\
\hline 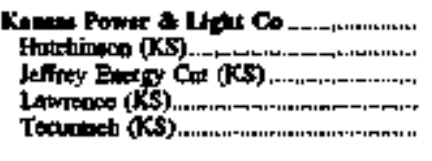 & $-\infty$ & $\begin{array}{l}1149 \\
113.5 \\
121.1 \\
121.1\end{array}$ & $\begin{array}{l}\overline{18.3} \\
\overline{18.64} \\
25.05 \\
25.03\end{array}$ & $\begin{array}{c}34 \\
.38 \\
.38 \\
.43\end{array}$ & $\overline{-}$ & $\begin{array}{l}2856 \\
1999 \\
499.2 \\
=\end{array}$ & $\begin{array}{l}1671 \\
19.60 \\
26.04 \\
=\end{array}$ & $\begin{array}{l}1,27 \\
1.35 \\
1.00 \\
=\end{array}$ & $=$ & $\begin{array}{l}\frac{212}{258.6} \\
\mathbf{2 5 . 1}\end{array}$ & $\begin{array}{l}\frac{237}{7} \\
\frac{-}{239} \\
235\end{array}$ & $\begin{array}{c}9 \\
100 \\
99\end{array}$ & $\begin{array}{l}100 \\
\stackrel{+}{-}\end{array}$ & $\bar{z}$ \\
\hline 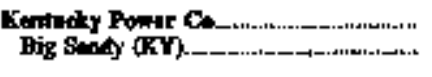 & $\begin{array}{l}\text { 21t } \\
211\end{array}$ & to6.9 & $\begin{array}{l}24.10 \\
24.10\end{array}$ & 1.26 & & $\operatorname{sing}$ & $\begin{array}{l}\mathbf{2 3 . 9 1} \\
23.91\end{array}$ & $\overline{-}$ & $\bar{z}$ & $\bar{z}$ & $\overline{-}$ & $\begin{array}{l}99 \\
99\end{array}$ & $\begin{array}{l}1 \\
1\end{array}$ & $\overline{-}$ \\
\hline 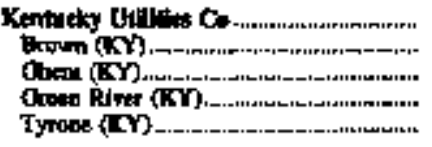 & $\begin{array}{l}\mathrm{m3} \\
117 \\
425 \\
23 \\
6\end{array}$ & $\begin{array}{l}1143 \\
119.4 \\
1135 \\
1003 \\
115.4\end{array}$ & $\begin{array}{l}27.98 \\
25.06 \\
27.81 \\
21.90 \\
30.00\end{array}$ & $\begin{array}{l}1.56 \\
1.25 \\
1.60 \\
2.49 \\
84\end{array}$ & - & $\begin{array}{l}50.2 \\
502.2 \\
\frac{40.1}{500.8}\end{array}$ & $\begin{array}{l}234.41 \\
29.53 \\
29.17 \\
-19.45\end{array}$ & $\begin{array}{r}40 \\
40 \\
-40\end{array}$ & $\begin{array}{l}= \\
z \\
=\end{array}$ & $\begin{array}{l}\bar{z} \\
\bar{z}\end{array}$ & $\begin{array}{l}\bar{z} \\
\bar{z} \\
=\end{array}$ & $\begin{array}{r}\mathbf{7 0} \\
900 \\
100 \\
91\end{array}$ & $\begin{array}{c}\dot{1} \\
\dot{0} \\
\end{array}$ & $\begin{array}{l}\bar{z} \\
\bar{z} \\
=\end{array}$ \\
\hline
\end{tabular}

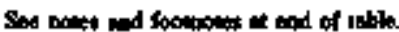


Table 57. Recelpts, Averoge Cont, and Quality of Fosil Foelo Delivered to U.S. Electric Utiflies by Company and Plant, Jwy 1997 (Conthued)

\begin{tabular}{|c|c|c|c|c|c|c|c|c|c|c|c|c|c|c|}
\hline \multirow{3}{*}{ 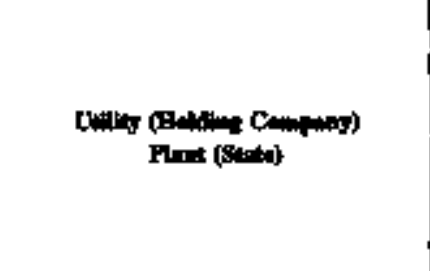 } & \multicolumn{4}{|c|}{ Cnes } & \multicolumn{4}{|c|}{ 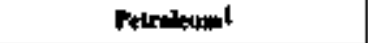 } & \multicolumn{3}{|c|}{$\mathbf{G m}$} & \multicolumn{3}{|c|}{ F a Telal Bt } \\
\hline & \multirow{2}{*}{ Rocinin } & \multicolumn{2}{|c|}{ Ansto } & \multirow{2}{*}{$\mid$} & \multirow{2}{*}{ 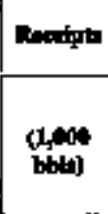 } & \multicolumn{2}{|c|}{$\cos \cos ^{2}$} & \multirow{2}{*}{ 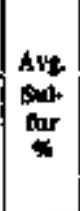 } & \multirow{2}{*}{ Recipts } & \multicolumn{2}{|c|}{ Averasos } & \multirow[b]{2}{*}{ | } & \multirow[b]{2}{*}{$\lim _{\lim }^{\text {Pro }}$} & \multirow[b]{2}{*}{ Ge: } \\
\hline & & (c) & $\begin{array}{c}\text { it par } \\
\text { thort } \\
\text { toal }\end{array}$ & & & 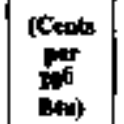 & $\$$ & & & $\begin{array}{l}\text { (Centu } \\
\text { par } \\
\text { 106) }\end{array}$ & Mat & & & \\
\hline 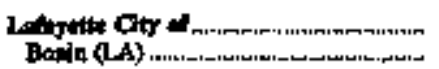 & $\bar{z}$ & $\bar{z}$ & $=$ & $=$ & $\overline{-}$ & $\mp$ & $=$ & $z$ & $\begin{array}{l}\text { 911 } \\
\text { 9]f }\end{array}$ & $\begin{array}{l}2354 \\
235.0\end{array}$ & $\begin{array}{l}2.47 \\
2.47\end{array}$ & - & $=$ & $\begin{array}{l}100 \\
100\end{array}$ \\
\hline 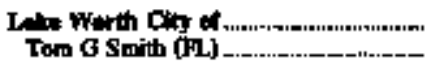 & $\bar{z}$ & - & $\ddot{-}$ & $=$ & & 3730 & $\begin{array}{l}21.87 \\
21.87\end{array}$ & 0.14 & $\begin{array}{l}269 \\
269\end{array}$ & $306 \%$ & $\begin{array}{l}3.19 \\
3.19\end{array}$ & $=$ & $\begin{array}{l}2 \\
2\end{array}$ & 94 \\
\hline 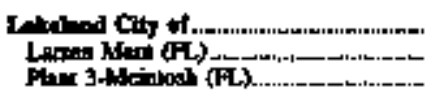 & -16 & $\frac{175,7}{173.7}$ & $\frac{45 \pi}{4507}$ & $\frac{122}{1.22}$ & $\begin{array}{l}31 \\
18 \\
13\end{array}$ & $\begin{array}{l}2900 \\
2050 \\
3000\end{array}$ & $\begin{array}{l}1023 \\
1822 \\
1847\end{array}$ & $\begin{array}{l}231 \\
245 \\
211\end{array}$ & $\begin{array}{r}1,64 \\
609\end{array}$ & $\begin{array}{l}3060 \\
306.0 \\
3060\end{array}$ & $\begin{array}{l}3.20 \\
3.20 \\
3.20\end{array}$ & -5 & $\begin{array}{r}6 \\
30 \\
3\end{array}$ & $\begin{array}{l}36 \\
80 \\
26\end{array}$ \\
\hline 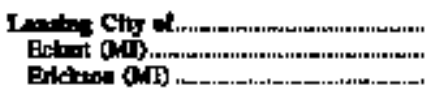 & $\underset{\mathbf{2}}{\mathbf{0}}$ & 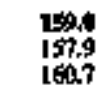 & $\begin{array}{l}37.89 \\
\text { 35.11 } \\
4.55\end{array}$ & $\begin{array}{l}.7 \\
.66 \\
.99\end{array}$ & +1 & $\begin{array}{l}421.4 \\
421.0 \\
421.0\end{array}$ & $\begin{array}{l}24,40 \\
24,40 \\
24.40\end{array}$ & $\begin{array}{l}30 \\
30 \\
30\end{array}$ & $\bar{z}$ & ㄴ & $\bar{z}$ & $\begin{array}{l}100 \\
100 \\
100\end{array}$ & : & $\begin{array}{l}\bar{z} \\
-\end{array}$ \\
\hline 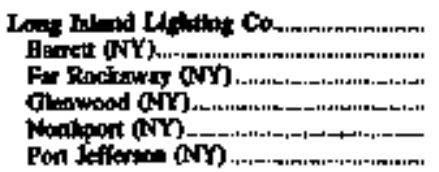 & $\begin{array}{l}= \\
= \\
=\end{array}$ & $\begin{array}{l}z \\
z \\
z\end{array}$ & $\begin{array}{l}z \\
= \\
=\end{array}$ & $\begin{array}{l}z \\
= \\
=\end{array}$ & $\begin{array}{l}= \\
=\end{array}$ & $\begin{array}{l}2567 \\
= \\
=\end{array}$ & $\begin{array}{l}163 \\
\bar{z} \\
\overline{16,33}\end{array}$ & $\begin{array}{l}n \\
= \\
=\end{array}$ & $\begin{array}{r}9,455 \\
2,045 \\
07 \\
1,010 \\
4,1,20 \\
1,208\end{array}$ & $\begin{array}{l}27.0 \\
240.9 \\
218.9 \\
2521 \\
254.9 \\
249.9\end{array}$ & $\begin{array}{l}2.5 \\
2.49 \\
2.37 \\
261 \\
237 \\
2.43\end{array}$ & $\begin{array}{l}= \\
= \\
= \\
=\end{array}$ & $\begin{array}{l}z^{4} \\
= \\
=\end{array}$ & $\begin{array}{r}90 \\
100 \\
100 \\
100 \\
91 \\
100\end{array}$ \\
\hline 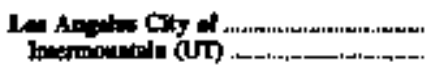 & 415 & 1403 & $\mathbf{3 1 2 . 2 2}$ & .59 & $\bar{z}$ & $\overline{-}$ & $=$ & $\bar{z}$ & - & $\overline{-}$ & $=$ & $\begin{array}{l}100 \\
100\end{array}$ & $\overrightarrow{-}$ & $\underline{-}$ \\
\hline 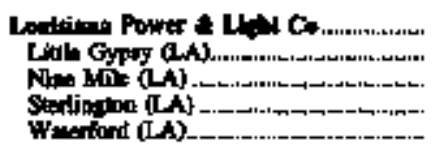 & $\begin{array}{l}- \\
\bar{z} \\
\bar{z}\end{array}$ & $\begin{array}{l}\bar{z} \\
\bar{z} \\
-\end{array}$ & $\begin{array}{l}\bar{z} \\
\overline{-}\end{array}$ & $\begin{array}{l}\bar{z} \\
\overline{-}\end{array}$ & $z_{2}^{2}$ & $\begin{array}{l}\operatorname{man} \\
\overline{-} \\
293.8\end{array}$ & $\begin{array}{l}\text { 15.6 } \\
\bar{z} \\
19.06\end{array}$ & $\begin{array}{l}\frac{1.00}{7} \\
\overline{1} .00\end{array}$ & $\begin{array}{r}16,238 \\
4,196 \\
7,001 \\
3,375 \\
3,235\end{array}$ & $\begin{array}{l}244.3 \\
246.1 \\
242.4 \\
259.1 \\
256.3\end{array}$ & $\begin{array}{l}2.53 \\
2.48 \\
2.51 \\
237 \\
2.66\end{array}$ & $\begin{array}{l}= \\
=\end{array}$ & $\begin{array}{l}-1 \\
- \\
4\end{array}$ & $\begin{array}{c}99 \\
100 \\
100 \\
100 \\
96\end{array}$ \\
\hline 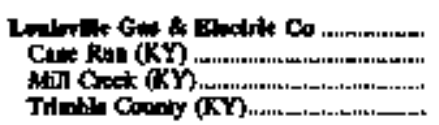 & $\begin{array}{l}40 \\
140 \\
160\end{array}$ & $\begin{array}{l}\$, 5 \\
9.2 \\
9.49 \\
9.9\end{array}$ & $\begin{array}{l}21.70 \\
22.34 \\
21.74 \\
21.31\end{array}$ & $\begin{array}{l}3.3 \\
3.24 \\
3.09 \\
403\end{array}$ & $-{ }^{2}$ & $\frac{-}{480}$ & $\frac{2896}{28.69}$ & $\frac{25}{-3}$ & $\begin{array}{r}38 \\
36 \\
-\quad 2 \\
\end{array}$ & $\begin{array}{l}275.5 \\
275.5 \\
2755 \\
-\end{array}$ & $\begin{array}{l}2.82 \\
2.82 \\
2.82 \\
-\end{array}$ & $\begin{array}{r}91 \\
98 \\
98 \\
100\end{array}$ & $-\frac{1}{2}$ & $\stackrel{*}{*}$ \\
\hline 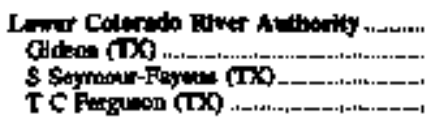 & $-\infty$ & $\begin{array}{l}\frac{96}{96.6} \\
-\end{array}$ & $\frac{16,69}{16,47}$ & $\begin{array}{c}34 \\
-34\end{array}$ & $\begin{array}{l}= \\
=\end{array}$ & $\begin{array}{l}= \\
=\end{array}$ & $\begin{array}{l}\bar{z} \\
\bar{z}\end{array}$ & $\begin{array}{l}= \\
=\end{array}$ & $\begin{array}{r}3,862 \\
2,474 \\
-1,988\end{array}$ & $\begin{array}{r}211.1 \\
209.5 \\
2140\end{array}$ & $\begin{array}{l}2.14 \\
2.13 \\
- \\
2.13\end{array}$ & $\frac{4}{100}$ & $\begin{array}{l}= \\
\bar{z}\end{array}$ & $\begin{array}{l}35 \\
100 \\
100\end{array}$ \\
\hline 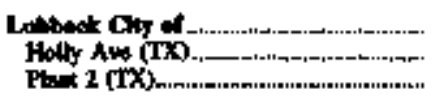 & $\bar{z}$ & $\bar{z}$ & $\bar{z}$ & $\bar{z}$ & $\bar{z}$ & $\bar{z}$ & $\bar{z}$ & $\bar{z}$ & $\begin{array}{r}158 \\
765 \\
66\end{array}$ & $\begin{array}{l}235.1 \\
218.9 \\
430.0\end{array}$ & $\begin{array}{l}2.25 \\
2.19 \\
4.25\end{array}$ & $\bar{z}$ & $\bar{z}$ & $\begin{array}{l}100 \\
100 \\
100\end{array}$ \\
\hline 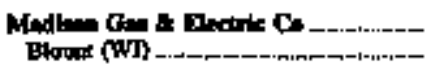 & : & $\begin{array}{ll}133.5 \\
133.5\end{array}$ & $\begin{array}{l}24.51 \\
28.51\end{array}$ & 1.72 & $\overline{-}$ & $\overline{-}$ & $\overline{-}$ & $\overline{-}$ & $\begin{array}{l}213 \\
213\end{array}$ & 2555 & 260 & 4 & $\bar{z}$ & $\begin{array}{l}56 \\
56\end{array}$ \\
\hline 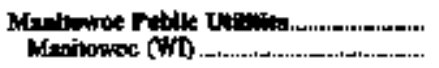 & $\stackrel{5}{5}$ & 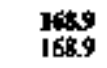 & 42.47 & s9 & $=$ & $\bar{z}$ & $\overline{-}$ & $\bar{z}$ & 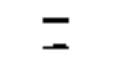 & $\overline{-}$ & $\overline{-}$ & $\begin{array}{l}106 \\
100\end{array}$ & 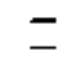 & $\overline{-}$ \\
\hline 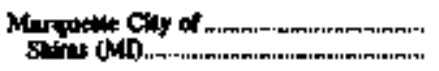 & $\overline{-}$ & $\overline{-}$ & - & - & 2 & $\begin{array}{lll}4 \% 0.1 \\
4 \% 0.1\end{array}$ & 25.51 & 二 & $\bar{z}$ & 二 & $\overline{-}$ & $=$ & 100 & $\overline{-}$ \\
\hline 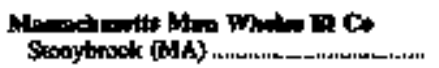 & 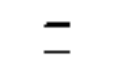 & - & $\overline{-}$ & $=$ & $=$ & $=$ & $\bar{z}$ & $=$ & $\begin{array}{l}745 \\
758\end{array}$ & $\begin{array}{l}271.0 \\
271.0\end{array}$ & $\begin{array}{l}276 \\
276\end{array}$ & $=$ & $\overline{-}$ & 100 \\
\hline 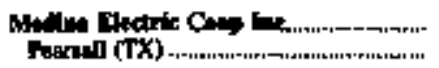 & $\overline{-}$ & $=$ & $\overline{-}$ & $=$ & $\overline{-}$ & $\overline{-}$ & $\overline{-}$ & $\overline{-}$ & $\begin{array}{l}36 \\
36\end{array}$ & ${ }_{240.0}^{240.6}$ & $\begin{array}{l}2.59 \\
295\end{array}$ & $=$ & $\bar{z}$ & $\operatorname{mon}_{100}$ \\
\hline 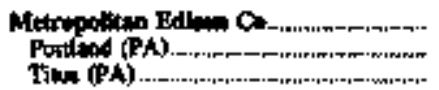 & $\begin{array}{l}\frac{6}{36} \\
46\end{array}$ & $\begin{array}{l}134.3 \\
135.9 \\
140.2\end{array}$ & $\begin{array}{l}36.47 \\
35.69 \\
36.94\end{array}$ & $\begin{array}{l}1.56 \\
1.58 \\
154\end{array}$ & - & $\begin{array}{l}\text { stes } \\
424.6\end{array}$ & $\frac{24.35}{24.25}$ & $\frac{3}{30}$ & $\bar{z}$ & $\bar{z}$ & $\bar{z}$ & $\begin{array}{l}100 \\
100 \\
100\end{array}$ & $\frac{}{7}$ & $=$ \\
\hline 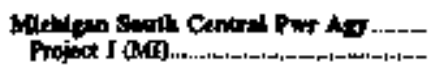 & 3 & $\begin{array}{l}\mathbf{1 6 1 0} \\
\mathbf{1 6 1 . 0}\end{array}$ & 39.99 & 3.72 & 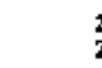 & $\begin{array}{l}3569 \\
38 \times 6.9\end{array}$ & 2291 & $\begin{array}{l}30 \\
30\end{array}$ & $\bar{z}$ & $\bar{z}$ & $\overline{-}$ & $\log _{63}$ & $\begin{array}{l}19 \\
17\end{array}$ & $\overline{-}$ \\
\hline
\end{tabular}

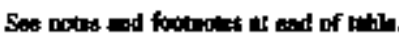


Table 57. Recaipts, Averaye Cost, and Qually of Fostl Fuets Dellvered to U.S. Flectric Utilities by Company and Plant, July 1997 (Continued)

\begin{tabular}{|c|c|c|c|c|c|c|c|c|c|c|c|c|c|c|}
\hline \multirow{3}{*}{ 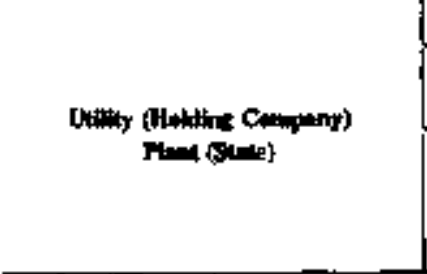 } & \multicolumn{4}{|c|}{ Cond } & \multicolumn{4}{|c|}{ 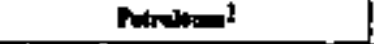 } & \multicolumn{3}{|c|}{ tor } & \multicolumn{3}{|c|}{ S of Treth Den } \\
\hline & \multirow{2}{*}{ 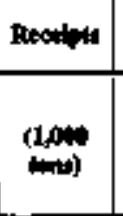 } & \multicolumn{2}{|c|}{ ATEr:Ga } & \multirow{2}{*}{$\begin{array}{c}\text { swo } \\
\text { sut } \\
6\end{array}$} & \multirow{2}{*}{ 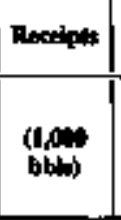 } & \multicolumn{2}{|c|}{ Arerey } & \multirow{2}{*}{$\begin{array}{c}\text { Avg. } \\
\text { S-1 } \\
\text { hir } \\
5\end{array}$} & \multirow{2}{*}{ (1) } & \multicolumn{2}{|c|}{ Conty } & \multirow[b]{2}{*}{ Cen } & \multirow{2}{*}{$\begin{array}{l}\text { Prop } \\
\operatorname{len}\end{array}$} & \multirow[b]{2}{*}{ 6as: } \\
\hline & & (Centi & $\underset{\text { tonis }}{(5 \mathrm{per})}$ & & & $\begin{array}{l}\text { (Cunts } \\
\text { par } \\
\text { pot } \\
\text { Btin) }\end{array}$ & & & & 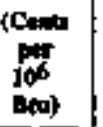 & & & & \\
\hline 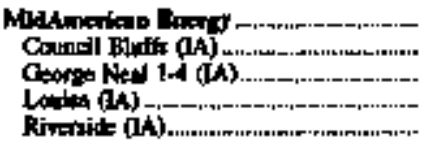 & $\begin{array}{r}138 \\
262 \\
106 \\
126 \\
44\end{array}$ & $\begin{array}{r}1.9 \\
79.7 \\
75.7 \\
106.9 \\
101.7\end{array}$ & $\begin{array}{l}14.17 \\
13.26 \\
13.25 \\
17.94 \\
17.37\end{array}$ & $\begin{array}{l}035 \\
35 \\
37 \\
37 \\
28\end{array}$ & $\begin{array}{l}- \\
- \\
-\end{array}$ & $\begin{array}{l}\overrightarrow{-} \\
\vec{m} \\
-\end{array}$ & $\begin{array}{l}- \\
-\end{array}$ & $\begin{array}{l}\vec{m} \\
\vec{m}\end{array}$ & $\begin{array}{r}34 \\
4 \\
2 \\
5 \\
3\end{array}$ & $\begin{array}{l}364.1 \\
346.2 \\
373.6 \\
153.9 \\
359.0\end{array}$ & $\begin{array}{l}3.54 \\
3.40 \\
3.78 \\
3.63 \\
3.63\end{array}$ & $\begin{array}{l}100 \\
100 \\
100 \\
100 \\
100\end{array}$ & $\begin{array}{l}- \\
- \\
-\end{array}$ & $\begin{array}{l}+ \\
+ \\
* \\
*\end{array}$ \\
\hline 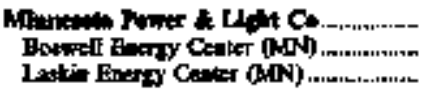 & $\mathbf{3 4 5}$ & $\begin{array}{l}111.6 \\
111.1 \\
114.7\end{array}$ & $\begin{array}{l}21.46 \\
20.53 \\
21.44\end{array}$ & $\begin{array}{l}4 \\
49 \\
39\end{array}$ & 3 & 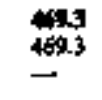 & $\begin{array}{l}27.00 \\
27.60 \\
-\end{array}$ & $\underset{20}{20}$ & $\frac{-}{-}$ & $\frac{-}{-}$ & $\frac{-}{-}$ & $\begin{array}{l}100 \\
100\end{array}$ & $\stackrel{\bullet}{-}$ & $\underline{-}$ \\
\hline 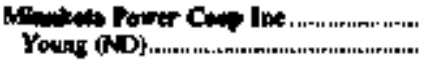 & 35 & 50.3 & $\begin{array}{l}7,18 \\
7,88\end{array}$ & .87 & $\frac{14}{12}$ & $\begin{array}{r}45.7 \\
435.7\end{array}$ & 25.4 & .40 & - & $\underline{-}$ & - & 99 & $\mathbf{1}$ & - \\
\hline 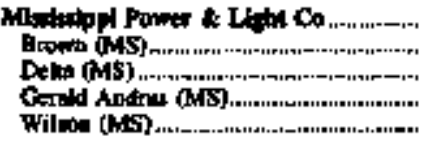 & $\begin{array}{l}- \\
z \\
-\end{array}$ & $\begin{array}{l}- \\
z \\
z\end{array}$ & $\vec{m}$ & $\begin{array}{l}z \\
z\end{array}$ & $\begin{array}{r}106 \\
106\end{array}$ & $\begin{array}{l}2716 \\
460.5 \\
2746 \\
269.4\end{array}$ & $\begin{array}{l}17.1 \\
27.17 \\
18.18 \\
17.73\end{array}$ & $\begin{array}{l}1.72 \\
30 \\
- \\
30 \\
251\end{array}$ & $\begin{array}{r}5,204 \\
826 \\
689 \\
2,192 \\
3,107\end{array}$ & $\begin{array}{l}239.4 \\
23.3 \\
245.2 \\
244.5 \\
237.6\end{array}$ & $\begin{array}{l}2.41 \\
24 \% \\
2.56 \\
2.54 \\
2.45\end{array}$ & $\begin{array}{l}- \\
- \\
-\end{array}$ & $\begin{array}{c}11 \\
15 \\
12\end{array}$ & $\begin{array}{r}83 \\
103 \\
100 \\
85 \\
80\end{array}$ \\
\hline 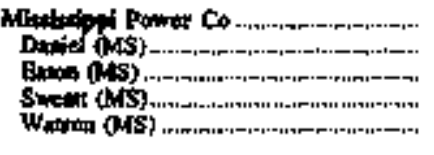 & $=\frac{206}{265}$ & $\begin{array}{l}1+6,9 \\
1+83 \\
-\end{array}$ & $\begin{array}{l}27.74 \\
27.97 \\
-32.65\end{array}$ & $\begin{array}{l}\frac{4}{39} \\
-9\end{array}$ & $\bar{E}$ & $\begin{array}{l}393 \\
3903 \\
= \\
-\end{array}$ & $\begin{array}{l}2200 \\
- \\
-\end{array}$ & $\begin{array}{l}= \\
= \\
=\end{array}$ & $\begin{array}{r}2,401 \\
-445 \\
549 \\
1,205\end{array}$ & $\begin{array}{l}234.3 \\
236.1 \\
247.4 \\
27.7\end{array}$ & $\begin{array}{l}2.43 \\
-2.46 \\
2.53 \\
2.37\end{array}$ & $\begin{array}{c}74 \\
-100 \\
-74\end{array}$ & $\begin{array}{c}\bullet \\
- \\
-\end{array}$ & $\begin{array}{c}21 \\
\frac{21}{100} \\
100 \\
26\end{array}$ \\
\hline 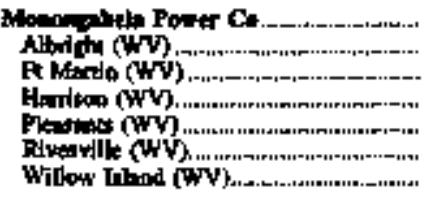 & $\begin{array}{r}771 \\
18 \\
145 \\
329 \\
270 \\
3 \\
6\end{array}$ & $\begin{array}{l}13.1 \\
1060 \\
115.5 \\
110.1 \\
96.7 \\
133,6 \\
318.8\end{array}$ & $\begin{array}{l}25.64 \\
27.19 \\
27.39 \\
27.64 \\
21.67 \\
34.61 \\
32.38\end{array}$ & $\begin{array}{l}3.44 \\
1.73 \\
1.44 \\
3.28 \\
3.84 \\
1.99\end{array}$ & $: 3$ & $\begin{array}{l}46.6 \\
45.9 \\
41.3 \\
40.0 \\
46.2 \\
449.1 \\
561.3\end{array}$ & $\begin{array}{l}26.13 \\
27.18 \\
26.13 \\
27.24 \\
28.79 \\
26.60 \\
33.24\end{array}$ & $\begin{array}{l}30 \\
30 \\
30 \\
30 \\
30 \\
30 \\
30\end{array}$ & $\begin{array}{cr} & 1 \\
- & \\
& 10 \\
& 1\end{array}$ & $\begin{array}{l}37.78 \\
- \\
476.5 \\
300.6 \\
\overrightarrow{312.9}\end{array}$ & $\begin{array}{l}3.79 \\
- \\
\overline{4.76} \\
3.01 \\
- \\
3.13\end{array}$ & $\begin{array}{c}140 \\
99 \\
99 \\
100 \\
900 \\
99\end{array}$ & $\begin{array}{l}* \\
1 \\
1 \\
+ \\
1 \\
1\end{array}$ & $\begin{array}{l}- \\
\overline{-} \\
\bar{*}\end{array}$ \\
\hline 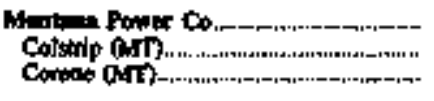 & $\begin{array}{l}77 \\
709\end{array}$ & $\begin{array}{r}r .9 \\
T 1.1 \\
14.4\end{array}$ & $\begin{array}{l}12.44 \\
12.04 \\
24.65\end{array}$ & $\begin{array}{l}+73 \\
.74 \\
21\end{array}$ & $\begin{array}{l}3 \\
3\end{array}$ & $\begin{array}{l}5104 \\
5104 \\
+\end{array}$ & 30.2 & م- & $\begin{array}{r}15 \\
15\end{array}$ & $\frac{12.5}{121.8}$ & $\frac{1.77}{1.27}$ & $\begin{array}{l}160 \\
95\end{array}$ & $\begin{array}{l}* \\
-\end{array}$ & $\frac{*}{5}$ \\
\hline 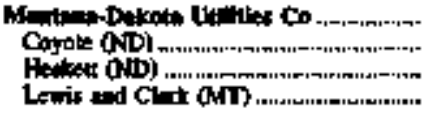 & $\begin{array}{r}194 \\
146 \\
13\end{array}$ & $\begin{array}{r}94.1 \\
85.6 \\
109.6 \\
94.4\end{array}$ & $\begin{array}{l}12.54 \\
11.84 \\
15.47 \\
12.84\end{array}$ & $\begin{array}{r}\$ 3 \\
1.07 \\
.55\end{array}$ & $\begin{array}{c}- \\
-\end{array}$ & $\begin{array}{l}- \\
-\end{array}$ & $\begin{array}{l}- \\
- \\
-\end{array}$ & $\begin{array}{l}- \\
-\end{array}$ & $\stackrel{\bullet}{\bullet}$ & 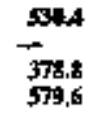 & $\frac{604}{6.70}$ & $\begin{array}{l}\mathbf{r} \\
100 \\
100\end{array}$ & $\begin{array}{l}- \\
- \\
-\end{array}$ & $\stackrel{*}{*}$ \\
\hline 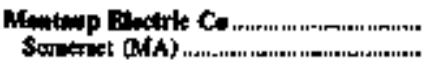 & $\frac{2}{29}$ & $\begin{array}{l}1716 \\
178.6\end{array}$ & 45.55 & .74 & $=$ & $\overrightarrow{-}$ & 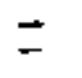 & $\overline{-}$ & - & $\overline{-}$ & $\overline{-}$ & 10 & - & $\because$ \\
\hline 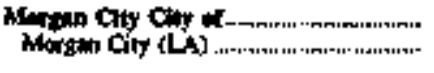 & - & - & 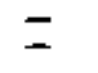 & $\overline{-}$ & $\overline{-}$ & $\stackrel{+}{-}$ & $\underline{-}$ & $=$ & 115 & 200 & 233 & $\underline{-}$ & 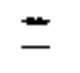 & IO0 \\
\hline 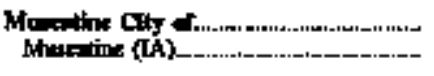 & 104 & 690 & $\begin{array}{l}\text { 15.4. } \\
\text { 15.84 }\end{array}$ & 1.11 & - & $\overline{-}$ & $\overline{-}$ & $\overrightarrow{-}$ & $\mathbf{1}$ & 350 & 3,5 & 100 & $\overline{-}$ & - \\
\hline 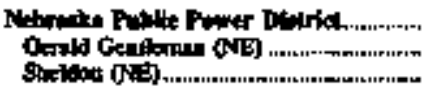 & $\begin{array}{r}\$ 34 \\
466 \\
68\end{array}$ & $\begin{array}{l}4.1 \\
4 \pi .4 \\
60.7\end{array}$ & $\begin{array}{r}\text { 8.16 } \\
10.63\end{array}$ & $\begin{array}{l}21 \\
.25 \\
.23\end{array}$ & $\stackrel{+}{+}$ & $\begin{array}{l}415.5 \\
165.5 \\
-\end{array}$ & $\begin{array}{c}27.01 \\
27.01 \\
-\end{array}$ & $\underline{-}$ & $\begin{array}{r}11 \\
10 \\
1\end{array}$ & $\begin{array}{l}160.4 \\
159.9\end{array}$ & $\begin{array}{l}1.90 \\
164 \\
4.64\end{array}$ & $\begin{array}{l}100 \\
100 \\
100\end{array}$ & $\ddot{*}$ & 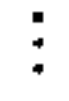 \\
\hline 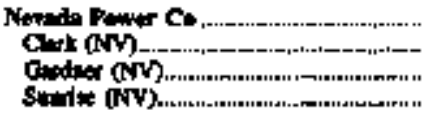 & -7 & $\frac{1+2}{120}$ & $\begin{array}{l}29.34 \\
29.34\end{array}$ & $\stackrel{4}{-}$ & 5 & $\frac{\cos 3}{403}$ & $\overline{25.25}$ & $\begin{array}{l}3 \\
-30 \\
-\end{array}$ & $\underbrace{3,07}_{405}$ & $\begin{array}{l}196.0 \\
196.6\end{array}$ & $\frac{2.00}{2.102}$ & $\underbrace{39}$ & $\begin{array}{c}- \\
-\end{array}$ & $\frac{\infty}{100}$ \\
\hline 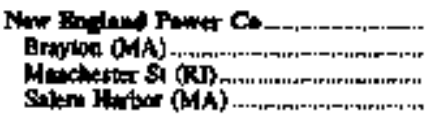 & 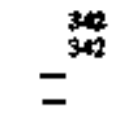 & $\begin{array}{l}15: 0 \\
15+0 \\
-\end{array}$ & $\begin{array}{l}39.27 \\
\$ 9.27 \\
=\end{array}$ & $\frac{4}{-6}$ & $\overline{-}^{m 2}$ & $\begin{array}{l}\frac{2927}{252,7} \\
\frac{-}{75}\end{array}$ & $\frac{-16.17}{16.17}$ & $\begin{array}{l}1.29 \\
\overline{1.29}\end{array}$ & $\begin{array}{r}2150 \\
133 \\
2006 \\
-\end{array}$ & $\begin{array}{l}20.4 \\
261.7 \\
290.3 \\
-\quad\end{array}$ & $\begin{array}{l}24 \\
2.68 \\
2.98 \\
-\end{array}$ & 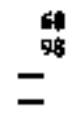 & $\frac{11}{10}$ & $\begin{array}{r}10 \\
2 \\
100 \\
-\end{array}$ \\
\hline
\end{tabular}

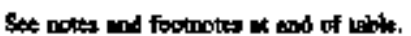


Table 57. Reteipss, Averaye Cost, and Quality of Fostil Fueb Delivened to U.S. Electric Utilities by Company and Plant, Juby 1997 (Conthined)

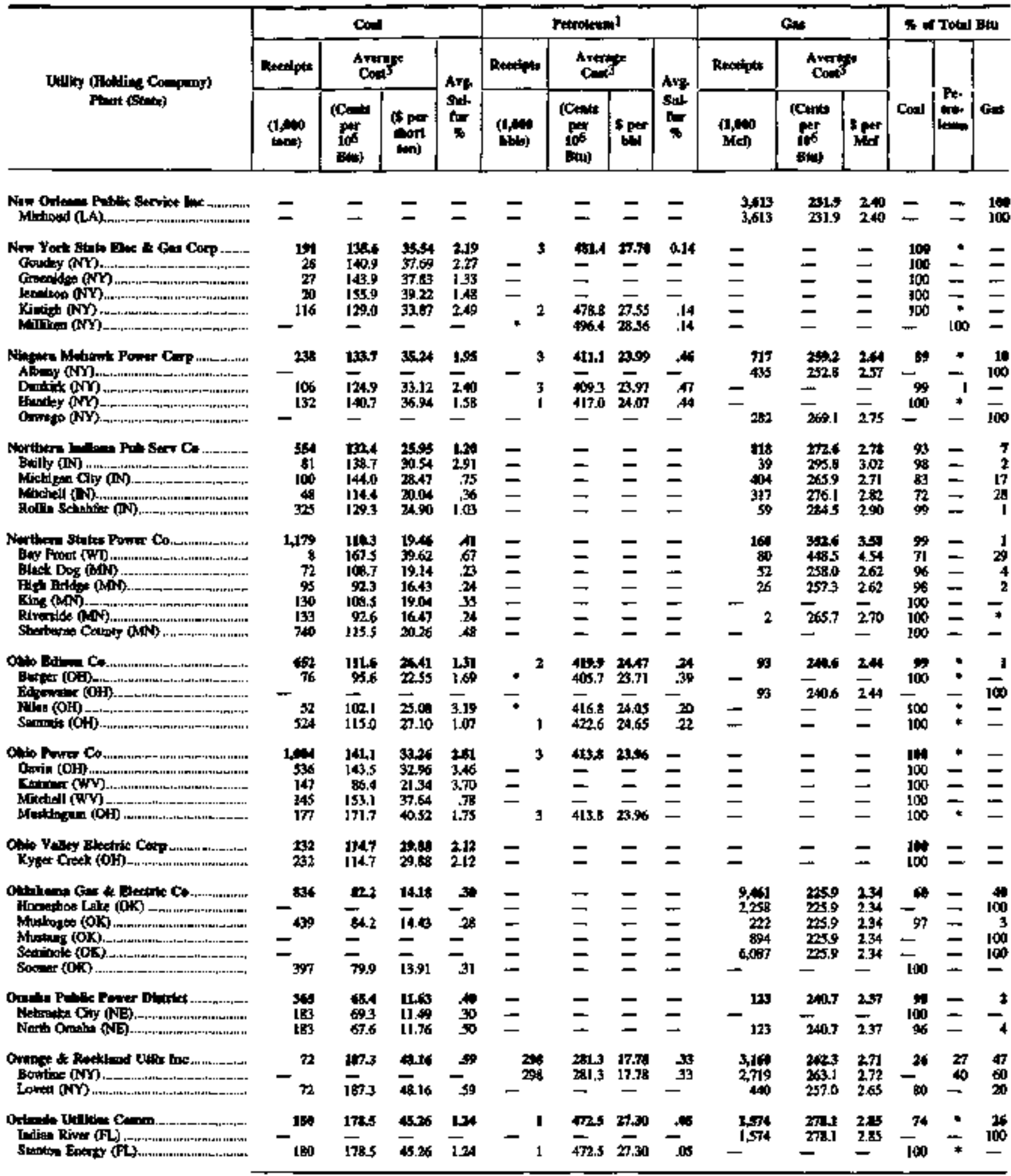

See nowes and footoctes a tod of ubth. 
Table 57. Receipts, Average Cost, and Quality of Fossil Foele Delivered to U.S. Electsic Utilitios by Cosmpany and Plent, July 1997 (Contmed)

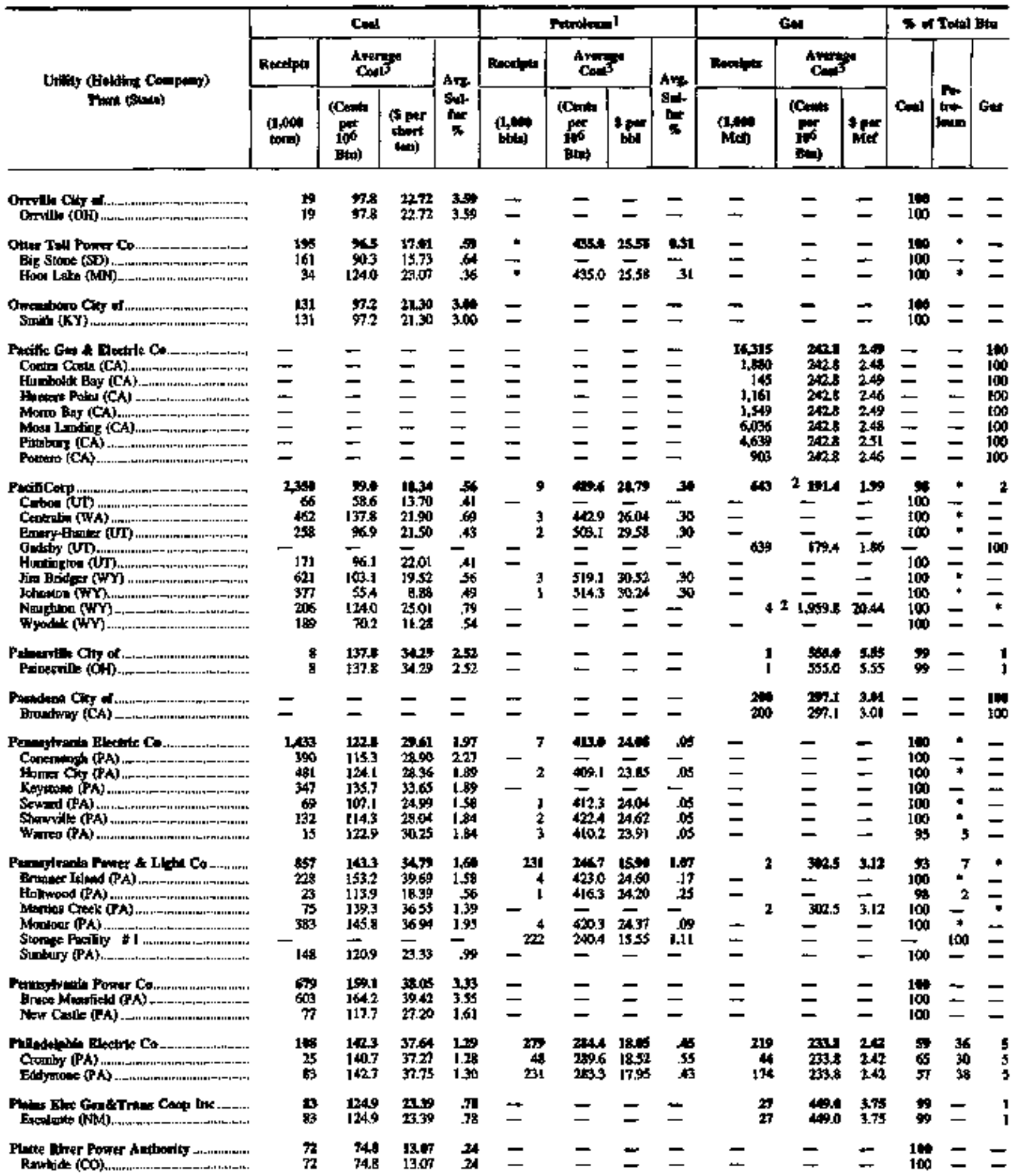

Ses notes and foomotes or end of twole 
Table 57. Receipts, A werage Cost, and Qualts of Fosen Foets Delivered to U.S Flectrie Utilties by Compeng and Fian, Ju'y 1997 (Continued)

\begin{tabular}{|c|c|c|c|c|c|c|c|c|c|c|c|c|c|c|}
\hline \multirow{3}{*}{ 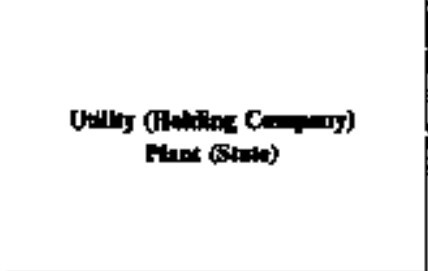 } & \multicolumn{4}{|c|}{ con } & \multicolumn{4}{|c|}{ Petroltane] } & \multicolumn{3}{|c|}{ cen } & \multicolumn{3}{|c|}{ 6 d Total Bup } \\
\hline & \multirow{2}{*}{ (1, tint } & \multicolumn{2}{|c|}{ Anipip } & \multirow{2}{*}{$\begin{array}{c}\text { An } \\
\text { sed. } \\
\text { nit } \\
0\end{array}$} & \multirow{2}{*}{ 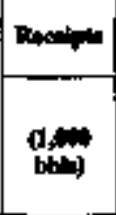 } & \multicolumn{2}{|c|}{ Ansors } & \multirow{2}{*}{ Ave } & \multirow{2}{*}{ Reciplip } & \multicolumn{2}{|c|}{ Artoty } & \multirow[b]{2}{*}{ con } & \multirow[b]{2}{*}{ tom } & \multirow[b]{2}{*}{ Gea } \\
\hline & & (Ew) & intint & & & 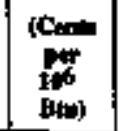 & 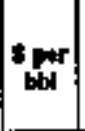 & & & 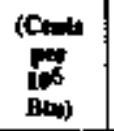 & Mef & & & \\
\hline 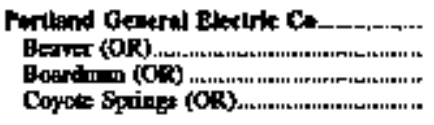 & -23 & $\frac{116.2}{116.2}$ & $\begin{array}{l}2639 \\
\overline{20.29} \\
-\end{array}$ & $\frac{136}{-36}$ & - & $\frac{4}{445.7}$ & $\begin{array}{l}2 \times 1 \\
\overline{20.21}\end{array}$ & $\begin{array}{l}\text { ass } \\
-\end{array}$ & $\begin{array}{r}115 \\
-\quad 100 \\
15\end{array}$ & $\begin{array}{l}133.4 \\
|3| .4 \\
\overline{1} \\
146.6\end{array}$ & $\frac{1.35}{1.48}$ & -7 & $-\mathbf{1}$ & $\frac{12}{100}$ \\
\hline 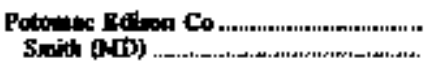 & it & $\begin{array}{l}1334.6 \\
333.6\end{array}$ & 33.27 & $\operatorname{lin}_{1,02}$ & : & \$as. & 24.21 & 30 & $=$ & \pm & $\bar{z}$ & 9 & $\mathbf{i}$ & 二 \\
\hline 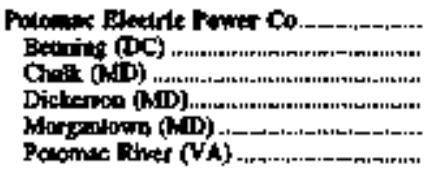 & $-\underset{45}{45}$ & $\begin{array}{l}1525 \\
\frac{-}{167.0} \\
100.3 \\
155.7 \\
191.5\end{array}$ & $\begin{array}{l}\mathbf{4 0 4} \\
466 \\
369.4 \\
40.64 \\
39.32\end{array}$ & $\begin{array}{l}1.34 \\
\overline{1.34} \\
1.45 \\
1.59 \\
.69\end{array}$ & $\begin{array}{c}206 \\
127 \\
127 \\
3 \\
3\end{array}$ & $\begin{array}{l}39.2 \\
347.2 \\
263.6 \\
403.9 \\
379.7 \\
395.9\end{array}$ & $\begin{array}{l}1,13 \\
21.17 \\
16,81 \\
23.02 \\
22.20 \\
23.19\end{array}$ & $\begin{array}{l}10 \\
98 \\
.70 \\
30 \\
30 \\
30\end{array}$ & $\begin{array}{l}{ }_{1,303} \\
= \\
=\end{array}$ & $\begin{array}{l}2101 \\
= \\
=\end{array}$ & $\begin{array}{l}235 \\
\frac{-}{235} \\
= \\
-\end{array}$ & $\begin{array}{l}79 \\
- \\
59 \\
99 \\
99 \\
99\end{array}$ & $\begin{array}{r}12 \\
100 \\
16 \\
1 \\
1\end{array}$ & $\begin{array}{l}16 \\
= \\
=\end{array}$ \\
\hline 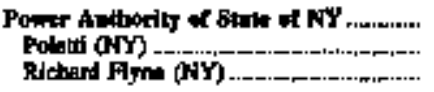 & $\bar{z}$ & $\overline{-}$ & $\bar{z}$ & $\overline{-}$ & $\bar{z}$ & $\bar{z}$ & $\ddot{z}$ & $\bar{z}$ & $\begin{array}{l}3,697 \\
3,897 \\
753\end{array}$ & $\begin{array}{l}20,1 \\
240.3 \\
4620\end{array}$ & $\begin{array}{l}2,4 \\
249 \\
4.57\end{array}$ & $\bar{z}$ & $\bar{z}$ & $\begin{array}{l}100 \\
100\end{array}$ \\
\hline 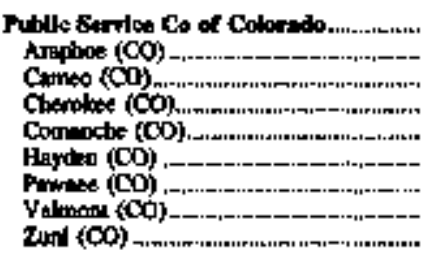 & $\begin{array}{r}143 \\
60 \\
21 \\
141 \\
264 \\
145 \\
119 \\
-\quad 32\end{array}$ & 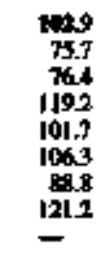 & $\begin{array}{l}19.7 \\
13.27 \\
16.52 \\
26.63 \\
13.54 \\
22.61 \\
14.76 \\
26.81 \\
-\end{array}$ & $\begin{array}{l}34 \\
31 \\
60 \\
.48 \\
.25 \\
.39 \\
.40 \\
.0\end{array}$ & $\begin{array}{l}= \\
= \\
= \\
=\end{array}$ & $\begin{array}{l}\bar{z} \\
\bar{z} \\
\bar{z} \\
\bar{z}\end{array}$ & $\begin{array}{l}\bar{z} \\
\bar{z} \\
\bar{z} \\
=\end{array}$ & $\begin{array}{l}\bar{z} \\
\bar{z} \\
\bar{z}\end{array}$ & $\begin{array}{r}21 \\
136 \\
2 \\
12 \\
16 \\
26 \\
1 \\
33 \\
45\end{array}$ & $\begin{array}{r}2+465 \\
205.0 \\
700.9 \\
2065.5 \\
265.9 \\
2017.2 \\
907.1 \\
275.4 \\
260.3\end{array}$ & $\begin{array}{r}4.15 \\
2.01 \\
7.01 \\
2.00 \\
2.64 \\
22.69 \\
9.57 \\
270 \\
2.58\end{array}$ & $\begin{array}{c}97 \\
87 \\
100 \\
100 \\
900 \\
97 \\
100 \\
-\end{array}$ & $\begin{array}{l}\bar{z} \\
\bar{z} \\
\bar{z} \\
\bar{z}\end{array}$ & $\begin{array}{r}13 \\
13 \\
1 \\
1 \\
1 \\
4\end{array}$ \\
\hline 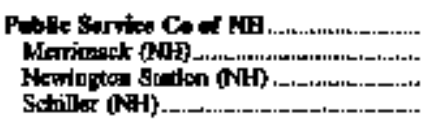 & $-\quad 4$ & $\frac{1075}{177.4}$ & $\frac{4.46}{34.15}$ & $\frac{117}{1.43}$ & - & $\begin{array}{l}415 \% \\
528,0 \\
299.3 \\
-\end{array}$ & $\begin{array}{l}2101 \\
30.56 \\
15.60 \\
+\end{array}$ & ${ }_{-}^{n}$ & -3 & $\frac{203}{2092}$ & $\frac{2.74}{274}$ & $\begin{array}{l}\text { ya } \\
100 \\
\frac{1}{100}\end{array}$ & $\begin{array}{l}1 \\
-\end{array}$ & $\frac{1}{86}$ \\
\hline 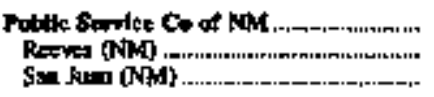 & -6 & $\frac{141}{158.1}$ & $\frac{29.16}{29.26}$ & $\frac{14}{94}$ & 一 & $\frac{561.4}{561.0}$ & $\frac{1+4}{3204}$ & $\frac{1}{100}$ & -114 & $\begin{array}{l}317.3 \\
317.2\end{array}$ & $\begin{array}{l}3.25 \\
3.25 \\
-\end{array}$ & $\frac{1}{100}$ & $\stackrel{-}{\bullet}$ & 100 \\
\hline 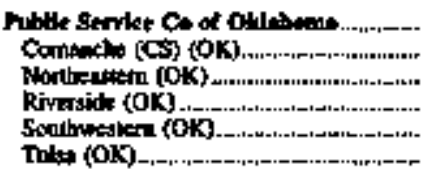 & $\begin{array}{l}-^{357} \\
- \\
-\end{array}$ & $\begin{array}{l}91.9 \\
\overline{91.9} \\
= \\
-\end{array}$ & $\begin{array}{l}\frac{1632}{1632} \\
= \\
=\end{array}$ & $\begin{array}{l}23 \\
-3 \\
=\end{array}$ & $\begin{array}{l}= \\
= \\
=\end{array}$ & $\begin{array}{l}= \\
= \\
=\end{array}$ & $\begin{array}{l}= \\
\bar{z} \\
=\end{array}$ & $\begin{array}{l}= \\
= \\
=\end{array}$ & $\begin{array}{r}1,45 \\
1,397 \\
2,397 \\
4,020 \\
1,526 \\
095\end{array}$ & $\begin{array}{l}2 \% 1 \\
237.1 \\
237.1 \\
237.2 \\
237.1 \\
237.0\end{array}$ & $\begin{array}{l}243 \\
249 \\
243 \\
242 \\
246 \\
240\end{array}$ & $\begin{array}{l}38 \\
= \\
-\end{array}$ & $\begin{array}{l}= \\
= \\
=\end{array}$ & $\begin{array}{r}40 \\
100 \\
200 \\
100 \\
100\end{array}$ \\
\hline 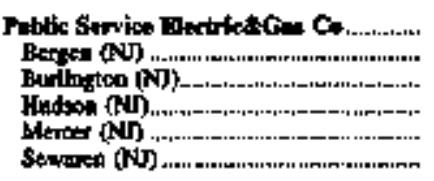 & $\begin{array}{ll} & 6 \\
- & \\
- & 6\end{array}$ & $\begin{array}{l}17.1 \\
= \\
\overline{17} .1\end{array}$ & $\begin{array}{l}\frac{791}{\pi} \\
= \\
=\end{array}$ & $\begin{array}{l} \pm \\
- \\
-\end{array}$ & $=^{30}$ & $\begin{array}{l}\frac{44.4}{40.4} \\
= \\
=\end{array}$ & $\begin{array}{l}25 \% \\
\overline{2} \text {.nn } \\
= \\
=\end{array}$ & $\begin{array}{l}-4 \\
- \\
-\end{array}$ & $\begin{array}{r}3,793 \\
1,79 \\
624 \\
336 \\
643\end{array}$ & $\begin{array}{l}264 \\
269.4 \\
269.4 \\
269.4 \\
269.4\end{array}$ & $\begin{array}{l}281 \\
284 \\
279 \\
277 \\
279 \\
279\end{array}$ & $\overline{-}^{4}$ & $\begin{array}{l}-4 \\
= \\
=\end{array}$ & $\begin{array}{r}n \\
100 \\
73 \\
100 \\
88 \\
100\end{array}$ \\
\hline 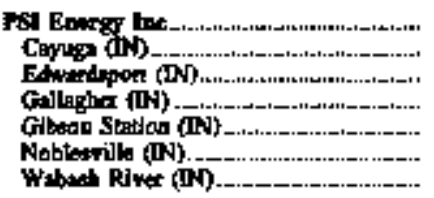 & $\begin{array}{r}1,161 \\
239 \\
36 \\
69 \\
674 \\
7 \\
136\end{array}$ & $\begin{array}{l}114.0 \\
113.6 \\
110.8 \\
104.4 \\
114.4 \\
113.4 \\
113.9\end{array}$ & $\begin{array}{l}25.53 \\
25.84 \\
24.91 \\
27.11 \\
25.40 \\
2.51 \\
25.17\end{array}$ & $\begin{array}{l}1.53 \\
1.75 \\
.75 \\
2.38 \\
1.90 \\
1.97 \\
1.62\end{array}$ & $-^{19}$ & $\begin{array}{l}\frac{1}{2} \\
\overline{403.9} \\
\overline{21.2}\end{array}$ & $\begin{array}{l}346 \\
\overline{-} \\
234 \\
2447 \\
\overline{2434}\end{array}$ & $\begin{array}{l} \pm \\
-30 \\
-30 \\
-30\end{array}$ & $\begin{array}{l}z \\
= \\
= \\
=\end{array}$ & $\begin{array}{l}\bar{z} \\
\bar{z} \\
=\end{array}$ & $\begin{array}{l}\bar{z} \\
\bar{z} \\
\bar{z}\end{array}$ & $\begin{array}{c}100 \\
100 \\
100 \\
99 \\
100 \\
100 \\
90\end{array}$ & $\frac{\overrightarrow{-}}{-1}$ & $\begin{array}{l}\bar{z} \\
\bar{z} \\
=\end{array}$ \\
\hline 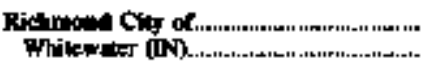 & $\begin{array}{l}18 \\
18\end{array}$ & $\begin{array}{ll}1509 \\
1509\end{array}$ & $\begin{array}{l}\text { 36.14 } \\
\mathbf{3 5 . 1 4}\end{array}$ & 2010 & 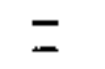 & $=$ & $\overline{-}$ & $\overline{-}$ & 二 & $=$ & $\overline{-}$ & in & $\bar{z}$ & 二 \\
\hline 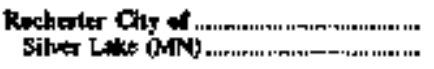 & II & 199,4 & 3t.5s & $\begin{array}{l}t, 61 \\
1,61\end{array}$ & $\bar{z}$ & $\overline{-}$ & $\bar{z}$ & $\bar{z}$ & 13 & $\frac{2 \times 3.2}{263.2}$ & $\begin{array}{l}246 \\
265\end{array}$ & $\begin{array}{l}95 \\
95\end{array}$ & $\overline{-}$ & $\begin{array}{l}5 \\
5\end{array}$ \\
\hline
\end{tabular}

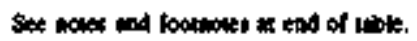


Table 57. Receipts, A werage Cost, and Quality of Fos:ll Fuets Delivered to U.S. Electric Utilities by Company and Plont, July $19 \%$ (Contimed)

\begin{tabular}{|c|c|c|c|c|c|c|c|c|c|c|c|c|c|c|}
\hline \multirow{3}{*}{ 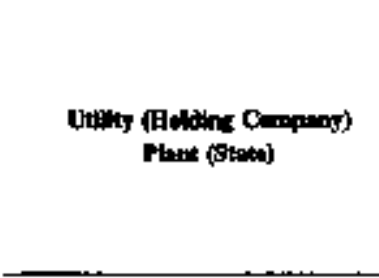 } & \multicolumn{4}{|c|}{ Cod } & \multicolumn{4}{|c|}{ Petrolen-1 } & \multicolumn{3}{|c|}{ Gu } & \multicolumn{3}{|c|}{ 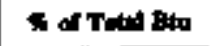 } \\
\hline & \multirow{2}{*}{$\mid$\begin{tabular}{|c|} 
Dectipts \\
(2000)
\end{tabular}} & \multicolumn{2}{|c|}{ Avenge } & \multirow{2}{*}{ 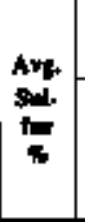 } & \multirow{2}{*}{ 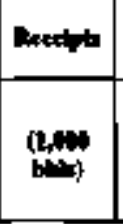 } & \multicolumn{2}{|c|}{ Avernge } & \multirow{2}{*}{ in. } & \multirow{2}{*}{ (14) } & \multicolumn{2}{|c|}{ Amatis } & \multirow[b]{2}{*}{ Conl } & \multirow[b]{2}{*}{$\operatorname{tim}_{\lim }$} & \multirow[b]{2}{*}{ Eev } \\
\hline & & $\begin{array}{c}\text { (C)wld } \\
106 \\
\text { Btoul } \\
\end{array}$ & $\underset{\text { toot }}{\text { tomer }}$ & & & 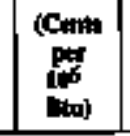 & sper & & & $\underset{\text { Bars }}{\text { torts }}$ & $\sec$ & & & \\
\hline 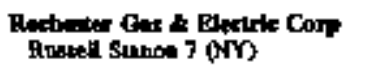 & 21 & $\begin{array}{l}1399 \\
1399\end{array}$ & $\begin{array}{l}37.25 \\
3725\end{array}$ & $\begin{array}{l}235 \\
235\end{array}$ & $\bar{z}$ & $\bar{z}$ & $\overline{-}$ & $\bar{z}$ & $\bar{z}$ & $\bar{z}$ & $\bar{z}$ & 100 & $\overline{-}$ & $\bar{Z}$ \\
\hline 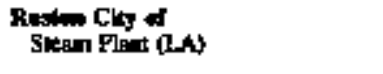 & $\bar{z}$ & $\bar{z}$ & $\overline{-}$ & $\bar{z}$ & $\overline{-}$ & $\bar{z}$ & $\overline{-}$ & $\bar{z}$ & 230 & $\begin{array}{l}2198 \\
2199\end{array}$ & 20 & $\bar{z}$ & $\overline{=}$ & 100 \\
\hline 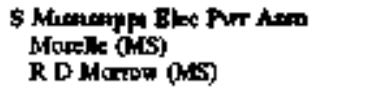 & $-\stackrel{n}{n}$ & $\frac{211.1}{211} 1$ & $\begin{array}{l}\sin 26 \\
5226\end{array}$ & $\stackrel{4}{-4}$ & $\bar{z}$ & $\bar{z}$ & $\bar{z}$ & $\bar{z}$ & $\begin{aligned} 789 \\
-\end{aligned}$ & $\begin{array}{l}231.1 \\
2311 \\
-\end{array}$ & $\begin{array}{l}239 \\
239 \\
-\end{array}$ & $\bar{T}_{100}^{72}$ & $\bar{z}$ & $\frac{100}{100}$ \\
\hline 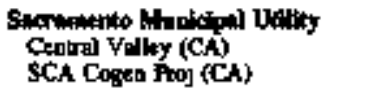 & $\bar{z}$ & $\bar{z}$ & $\bar{z}$ & $\bar{z}$ & $\bar{z}$ & $\bar{z}$ & $\bar{z}$ & $\bar{z}$ & $\begin{array}{l}806 \\
301 \\
325\end{array}$ & $\begin{array}{l}2154 \\
2984 \\
2384\end{array}$ & $\begin{array}{l}2.14 \\
218 \\
218\end{array}$ & $\overline{-}$ & $=$ & $\begin{array}{l}100 \\
100\end{array}$ \\
\hline 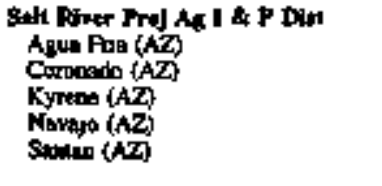 & $-^{135}$ & $\begin{array}{l}\frac{1375}{2672} \\
\frac{1138}{-}\end{array}$ & $\begin{array}{l}29,46 \\
\overline{5075} \\
\overline{2497} \\
=\end{array}$ & $\begin{array}{l}-56 \\
-42 \\
-58 \\
-\end{array}$ & $\begin{array}{l}- \\
- \\
-\end{array}$ & $\begin{array}{l}545 \\
\overline{523} 6 \\
\overline{s e n} \\
-\end{array}$ & $\begin{array}{l}31.55 \\
\overline{30} 29 \\
\overline{34} 61 \\
=\end{array}$ & $\begin{array}{l}-37 \\
- \\
-\end{array}$ & $\begin{array}{r}1,236 \\
-\quad 6 \% \\
-\quad 9 \\
541\end{array}$ & $\begin{array}{l}244 \\
2452 \\
\frac{3}{3321} \\
\overline{2} \\
2414\end{array}$ & $\begin{array}{l}267 \\
\frac{249}{336} \\
\frac{-}{243}\end{array}$ & $\frac{{ }^{93}}{100}$ & $\begin{array}{l}\ddot{-} \\
\dot{*}\end{array}$ & $\frac{100}{100}$ \\
\hline 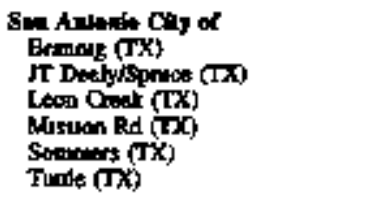 & $\begin{array}{l}-450 \\
= \\
=\end{array}$ & $\begin{array}{l}91 \% \\
= \\
= \\
=\end{array}$ & $\begin{array}{l}\text { 15.35 } \\
\overrightarrow{1535} \\
= \\
= \\
=\end{array}$ & $\begin{array}{l}-3 \\
= \\
= \\
=\end{array}$ & $\begin{array}{l}\bar{z} \\
\bar{z} \\
=\end{array}$ & $\begin{array}{l}= \\
= \\
= \\
=\end{array}$ & $\begin{array}{l}= \\
= \\
= \\
=\end{array}$ & $\begin{array}{l}= \\
= \\
= \\
=\end{array}$ & $\begin{array}{r}6,461 \\
2,691 \\
1 \\
25 \\
57 \\
3,665 \\
412\end{array}$ & $\begin{array}{l}2014 \\
2314 \\
231 \\
2314 \\
2314 \\
2314 \\
2314\end{array}$ & $\begin{array}{l}2.35 \\
235 \\
235 \\
233 \\
234 \\
235 \\
234\end{array}$ & $\begin{array}{l}5 \\
= \\
= \\
= \\
=\end{array}$ & $\begin{array}{l}\bar{z} \\
\bar{z} \\
\bar{z}\end{array}$ & $\begin{array}{l}400 \\
100 \\
100 \\
100 \\
100 \\
100\end{array}$ \\
\hline 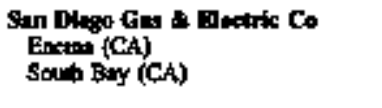 & $\bar{z}$ & $\bar{z}$ & $\bar{z}$ & $\bar{z}$ & $\bar{z}$ & $\ddot{m}$ & $\bar{z}$ & $\bar{z}$ & $\begin{array}{l}5,347 \\
28,358 \\
2,909\end{array}$ & $\begin{array}{l}2502 \\
2506 \\
2541\end{array}$ & $\begin{array}{l}2.55 \\
253 \\
257\end{array}$ & $\bar{z}$ & $\bar{E}$ & $\begin{array}{l}100 \\
100\end{array}$ \\
\hline $\begin{array}{l}\text { San Migol Betinc Coep Int } \\
\text { Sen Mupul (TX) }\end{array}$ & 280 & 60 & 627 & $\operatorname{lm}$ & $=$ & $\bar{z}$ & $=$ & $\overline{-}$ & $\overline{-}$ & $\overline{-}$ & $\overline{-}$ & 100 & $\overline{-}$ & $\vec{z}$ \\
\hline 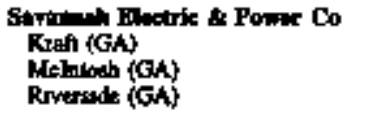 & $\begin{array}{r}64 \\
-\quad 39 \\
\end{array}$ & $\begin{array}{l}146 \\
1424 \\
1392 \\
-\end{array}$ & $\begin{array}{l}y 054 \\
3116 \\
285 \\
-7\end{array}$ & $\begin{array}{r}1.18 \\
\pm 72 \\
81 \\
-\end{array}$ & $\stackrel{-}{-}$ & $\frac{48.1}{4181}$ & $\begin{array}{l}2697 \\
2597\end{array}$ & ${ }_{-\infty}^{5}$ & $-\begin{array}{r}360 \\
-36\end{array}$ & $\begin{array}{l}2140 \\
2142 \\
\frac{3}{3115}\end{array}$ & $\frac{224}{319}$ & $\begin{array}{c}73 \\
100 \\
-\end{array}$ & $\stackrel{\div}{-}$ & $\frac{37}{37}$ \\
\hline 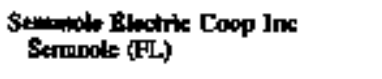 & 331 & 1746 & $\underset{243}{243}$ & 276 & & 4241 & $\frac{247}{2478}$ & $\frac{22}{22}$ & $\overline{-}$ & $=$ & $=$ & $\mathbf{m}$ & : & $\overline{-}$ \\
\hline 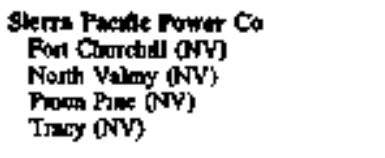 & $-^{61}$ & $\begin{array}{l}\text { 1tass } \\
\text { 18s } \\
=\end{array}$ & $\begin{array}{l}\frac{145}{245} \\
=\end{array}$ & $\begin{array}{l}3 \\
- \\
-\end{array}$ & $\begin{array}{l}- \\
=\end{array}$ & $\begin{array}{l}\frac{457}{493} \\
\frac{-}{-}\end{array}$ & $\begin{array}{l}253 \\
2873 \\
= \\
-\end{array}$ & $\begin{array}{l}\bar{z} \\
\bar{z}\end{array}$ & $\begin{array}{r}3,400 \\
-1,168 \\
1.248\end{array}$ & $\begin{array}{l}1854 \\
1855 \\
-1855 \\
1855\end{array}$ & $\begin{array}{l}191 \\
191 \\
\frac{1}{192} \\
192\end{array}$ & $\begin{array}{l}\overline{100}^{32} \\
-\end{array}$ & $\ddot{\div}$ & $\frac{60}{1000}$ \\
\hline Sineten Chy of & $\frac{82}{82}$ & 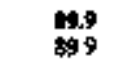 & $\begin{array}{l}1946 \\
19 \$ 6\end{array}$ & 299 & 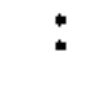 & 4135 & 244 & 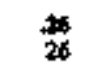 & $\overline{-}$ & $\bar{z}$ & $=$ & 100 & : & $=$ \\
\hline 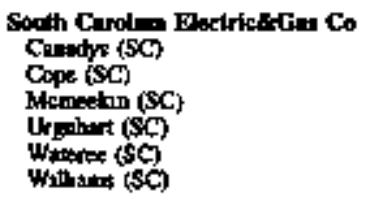 & $\begin{array}{r}462 \\
62 \\
72 \\
53 \\
34 \\
162 \\
80\end{array}$ & $\begin{array}{l}1919 \\
1516 \\
1480 \\
1 \$ 94 \\
1512 \\
1495 \\
1622\end{array}$ & 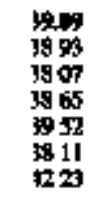 & $\begin{array}{l}131 \\
144 \\
133 \\
136 \\
134 \\
13 \\
\text { Bi }\end{array}$ & $\begin{array}{r}19 \\
9 \\
1 \\
-\quad 2 \\
6 \\
1\end{array}$ & $\begin{array}{l}722 \\
4310 \\
1363 \\
4383 \\
\overline{438} \\
4312\end{array}$ & $\begin{array}{l}247 \\
249 \\
790 \\
2540 \\
2010 \\
2499\end{array}$ & $\begin{array}{l}20 \\
20 \\
20 \\
20 \\
-20 \\
20 \\
20\end{array}$ & $\begin{array}{r}\mathbf{4} \\
-\quad 28 \\
-\quad \begin{array}{r}2 \\
-\end{array}\end{array}$ & $\begin{array}{l}48.4 \\
4257 \\
4254 \\
4232 \\
=\end{array}$ & $\begin{array}{l}45 \\
436 \\
436 \\
433 \\
=\end{array}$ & $\begin{array}{r}99 \\
99 \\
100 \\
99 \\
99 \\
99 \\
100\end{array}$ & $\begin{array}{r}1 \\
3 \\
-1 \\
-1\end{array}$ & $\frac{2}{*}$ \\
\hline $\begin{array}{l}\text { Som Curnthe Pul Bart Anil } \\
\text { Croes (SC) } \\
\text { Jeffents (SC) } \\
\text { Whyt (SC) }\end{array}$ & $\begin{array}{r}419 \\
166 \\
39 \\
213\end{array}$ & $\begin{array}{l}1326 \\
1313 \\
1286 \\
134\end{array}$ & $\begin{array}{l}\text { 4his } \\
3391 \\
3391 \\
3451\end{array}$ & $\begin{array}{l}1.16 \\
111 \\
156 \\
111\end{array}$ & $\begin{array}{l}z \\
-\end{array}$ & $\begin{array}{l}- \\
- \\
-\end{array}$ & $\begin{array}{l}z \\
-\end{array}$ & $\begin{array}{l}\bar{z} \\
\bar{z}\end{array}$ & $\begin{array}{l}\overline{-} \\
\overline{-} \\
-\end{array}$ & $\begin{array}{l}z \\
z\end{array}$ & $\frac{z}{-}$ & $\begin{array}{l}100 \\
100 \\
100 \\
100\end{array}$ & $\begin{array}{l}= \\
=\end{array}$ & $=$ \\
\hline
\end{tabular}

Soe nowes and toonopss at and of uble 
Toble 57. Recelpts, Awernge Cost, and Qualiky of Foosti Fuds Dellvered to U.S. Electric Utllites by Company and Fent, Jaly 1997 (Continoed)

\begin{tabular}{|c|c|c|c|c|c|c|c|c|c|c|c|c|c|c|}
\hline \multirow{3}{*}{ 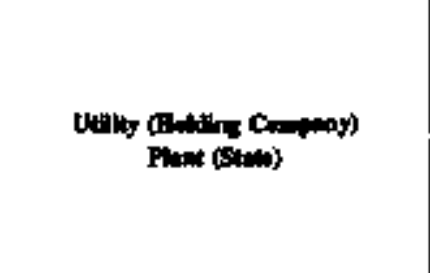 } & \multicolumn{4}{|c|}{ Coid } & \multicolumn{4}{|c|}{ Finlend } & \multicolumn{3}{|c|}{ Gt: } & \multicolumn{3}{|c|}{ क of T: Bt? } \\
\hline & \multirow{2}{*}{ 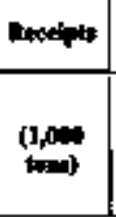 } & \multicolumn{2}{|c|}{ Aresto } & \multirow{2}{*}{ 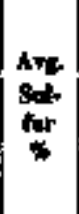 } & \multirow{2}{*}{ Receipa } & \multicolumn{2}{|c|}{ conts } & \multirow{2}{*}{$\mid \begin{array}{l}\text { Avg } \\
\text { sot. } \\
\text { Arr } \\
4\end{array}$} & \multirow{2}{*}{$\begin{array}{l}\text { Rosifipta } \\
\text { (1,10) }\end{array}$} & \multicolumn{2}{|c|}{ Axerste } & \multirow[b]{2}{*}{ Cool } & \multirow[b]{2}{*}{ Por } & \multirow[b]{2}{*}{ Ges: } \\
\hline & & 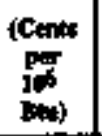 & $\underset{\tan }{\tan }$ & & & 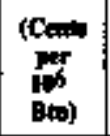 & 5 mar & & & 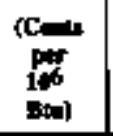 & Spto & & & \\
\hline 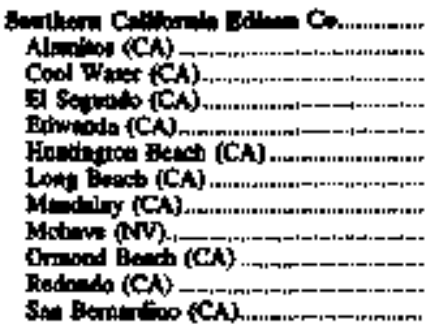 & $\begin{array}{l}z^{30} \\
= \\
= \\
= \\
=\end{array}$ & $\begin{array}{l}\frac{1340}{=} \\
= \\
= \\
= \\
= \\
=\end{array}$ & $\begin{array}{l}= \\
= \\
= \\
= \\
= \\
= \\
=\end{array}$ & $\begin{array}{l}= \\
= \\
= \\
= \\
= \\
= \\
=\end{array}$ & $\begin{array}{l}\bar{z} \\
\bar{z} \\
\bar{z} \\
\bar{z} \\
\bar{z}\end{array}$ & $\begin{array}{l}\bar{z} \\
\bar{z} \\
\bar{z} \\
\bar{z} \\
\bar{z}\end{array}$ & $\begin{array}{l}\bar{z} \\
\bar{z} \\
\bar{z} \\
\bar{z} \\
\bar{z}\end{array}$ & $\begin{array}{l}\bar{z} \\
\bar{z} \\
\bar{z} \\
\bar{z} \\
\bar{z}\end{array}$ & $\begin{array}{r}18,203 \\
5,400 \\
1,011 \\
1,014 \\
1,201 \\
1,050 \\
1,75 \\
1,242 \\
54 \\
3,535 \\
3,411 \\
.40\end{array}$ & 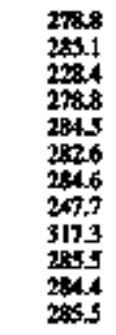 & $\begin{array}{l}284 \\
2897 \\
2.95 \\
2.87 \\
2.87 \\
287 \\
287 \\
2.58 \\
3.28 \\
298 \\
289 \\
2.89\end{array}$ & $\begin{array}{l}28 \\
= \\
= \\
= \\
= \\
= \\
= \\
=\end{array}$ & $\begin{array}{l}= \\
= \\
= \\
= \\
= \\
= \\
=\end{array}$ & $\begin{array}{c}72 \\
100 \\
100 \\
100 \\
100 \\
100 \\
100 \\
100 \\
1 \\
100 \\
100 \\
100\end{array}$ \\
\hline 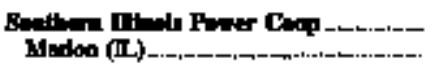 & s.0 & 737 & 1416 & $\begin{array}{l}255 \\
255\end{array}$ & & 4344, & $\begin{array}{l}24.75 \\
24.75\end{array}$ & $\bar{z}$ & $=$ & $\bar{z}$ & $=$ & $\begin{array}{l}\text { 16i } \\
100\end{array}$ & 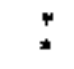 & $\overline{-}$ \\
\hline 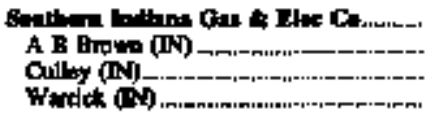 & $\begin{array}{l}255 \\
125 \\
118 \\
12\end{array}$ & $\begin{array}{l}\mathbf{1 3 . 1} \\
\$ 0.9 \\
\$ \$ 2.2 \\
\mathbf{9 0 . 5}\end{array}$ & $\begin{array}{l}21.4 \\
21.04 \\
21.64 \\
21.21\end{array}$ & $\begin{array}{l}\frac{3.35}{3.75} \\
2.94 \\
2.66\end{array}$ & $\begin{array}{l}\bar{z} \\
=\end{array}$ & $\begin{array}{l}\bar{z} \\
\end{array}$ & $\bar{z}$ & $\bar{z}$ & $\begin{array}{r}14 \\
12 \\
+\quad 2\end{array}$ & $\begin{array}{l}3060 \\
300.5 \\
330.2 \\
403\end{array}$ & $\begin{array}{l}3.14 \\
3.11 \\
3.41 \\
5.01\end{array}$ & $\begin{array}{l}100 \\
100 \\
100 \\
100\end{array}$ & $\begin{array}{l}\bar{z} \\
\bar{z}\end{array}$ & $:$ \\
\hline 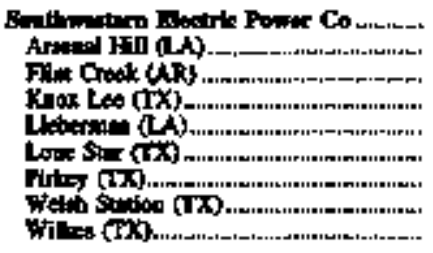 & $\begin{array}{l}-90 \\
= \\
- \\
-492\end{array}$ & $\begin{array}{l}\frac{146.7}{190.4} \\
= \\
= \\
\frac{5.9}{16.9}\end{array}$ & $\begin{array}{l}\frac{22.09}{32.03} \\
= \\
\overline{11.79} \\
=\end{array}$ & $\begin{array}{l}-76 \\
-.40 \\
= \\
\overline{1.40} \\
-38\end{array}$ & $\begin{array}{l}= \\
= \\
= \\
= \\
=\end{array}$ & $\begin{array}{l}\bar{z} \\
\bar{z} \\
\bar{z} \\
=\end{array}$ & $\begin{array}{l}\bar{z} \\
\bar{z} \\
\bar{z} \\
\bar{z}\end{array}$ & $\begin{array}{l}\bar{z} \\
\bar{z} \\
= \\
\bar{z}\end{array}$ & 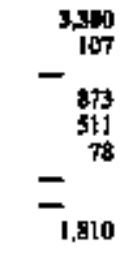 & $\begin{array}{l}217.5 \\
227.6 \\
\overline{2}+9.7 \\
211.3 \\
221.0 \\
\overline{2}\end{array}$ & $\begin{array}{l}2.34 \\
2.38 \\
\frac{2.38}{2.37} \\
2.21 \\
\frac{-}{2.36}\end{array}$ & $\begin{array}{l}- \\
\frac{100}{-} \\
= \\
= \\
100 \\
-\end{array}$ & $\begin{array}{l}= \\
= \\
= \\
=\end{array}$ & $\begin{array}{l}\frac{20}{100} \\
\frac{1000}{100} \\
\frac{100}{100} \\
\frac{7}{100}\end{array}$ \\
\hline 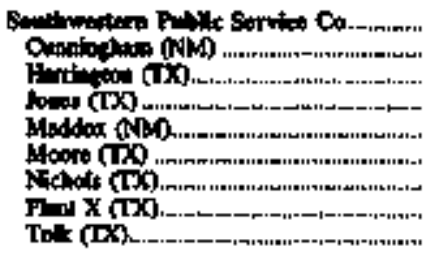 & $\begin{array}{l}-815 \\
-413 \\
= \\
= \\
402\end{array}$ & $\begin{array}{l}1052 \\
\overline{1050} \\
= \\
= \\
\overline{2060}\end{array}$ & $\begin{array}{l}\frac{32.35}{27.83} \\
= \\
= \\
= \\
= \\
35.91\end{array}$ & $\begin{array}{l}3 \\
37 \\
= \\
= \\
=\end{array}$ & $\begin{array}{l}= \\
= \\
= \\
= \\
=\end{array}$ & $\begin{array}{l}\bar{z} \\
\bar{z} \\
\bar{z} \\
\bar{z}\end{array}$ & $\begin{array}{l}\bar{z} \\
\bar{z} \\
\bar{z} \\
\bar{z}\end{array}$ & $\begin{array}{l}= \\
\bar{z} \\
= \\
= \\
=\end{array}$ & $\begin{array}{r}9,344 \\
1,501 \\
17 \\
2,576 \\
926 \\
275 \\
2,063 \\
1.728 \\
257\end{array}$ & $\begin{array}{l}237.4 \\
226.5 \\
260.0 \\
232.3 \\
224.5 \\
236.5 \\
219.9 \\
225.1 \\
259.9\end{array}$ & $\begin{array}{l}2.34 \\
2.65 \\
2.60 \\
2.32 \\
2.25 \\
2.57 \\
2.20 \\
2.25 \\
2.60\end{array}$ & $\begin{array}{l}- \\
= \\
= \\
= \\
= \\
=\end{array}$ & $\begin{array}{l}= \\
= \\
= \\
= \\
=\end{array}$ & $\begin{array}{r}100 \\
100 \\
000 \\
100 \\
100 \\
100 \\
100 \\
100 \\
4\end{array}$ \\
\hline 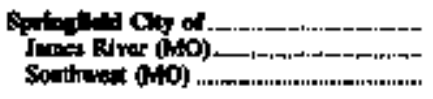 & $\begin{array}{l}y \\
54 \\
43\end{array}$ & $\begin{array}{l}1170 \\
1220 \\
110.2\end{array}$ & $\begin{array}{l}21.71 \\
23.40 \\
19.60\end{array}$ & $\begin{array}{l}.31 \\
.37\end{array}$ & $\bar{z}$ & $\bar{z}$ & $\bar{z}$ & $\bar{z}$ & $\begin{array}{r}29 \\
278 \\
20\end{array}$ & $\begin{array}{l}2034 \\
220.4 \\
2034\end{array}$ & $\begin{array}{l}226 \\
226 \\
2.27\end{array}$ & $\begin{array}{l}36 \\
97\end{array}$ & $\bar{z}$ & $\begin{array}{r}16 \\
21 \\
3\end{array}$ \\
\hline 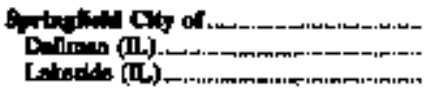 & $\begin{array}{c}101 \\
119 \\
18\end{array}$ & $\begin{array}{l}1120 \\
1+8.0 \\
1120\end{array}$ & $\begin{array}{l}24.73 \\
24.73 \\
24.73\end{array}$ & $\frac{3.22}{3.22}$ & $\bar{z}$ & $\frac{-}{-}$ & $\bar{Z}$ & $\bar{z}$ & 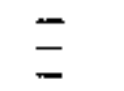 & $\bar{z}$ & $\ddot{-}$ & $\begin{array}{l}106 \\
100 \\
\text { too }\end{array}$ & $\bar{z}$ & $\bar{z}$ \\
\hline 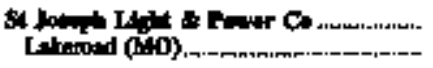 & 4 & $\begin{array}{l}\text { 9.1 } \\
95.1\end{array}$ & $\begin{array}{l}18.78 \\
18.78\end{array}$ & 153 & $\begin{array}{l}2 \\
2\end{array}$ & $\begin{array}{l}2128 \\
2128\end{array}$ & $\begin{array}{l}1404 \\
14.00\end{array}$ & $\mathbf{1 . 7 5}$ & 137 & $\begin{array}{l}246.6 \\
246.6\end{array}$ & $\begin{array}{l}2.45 \\
2.45\end{array}$ & 85 & $\mathbf{1}$ & 13 \\
\hline 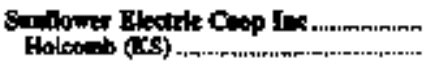 & $\mathbf{1 0 9}$ & $\begin{array}{l}1160 \\
1160\end{array}$ & 19,57 & $\begin{array}{l}31 \\
.31\end{array}$ & $\bar{z}$ & $=$ & $\bar{z}$ & $\bar{z}$ & 4 & 2920 & 2.86 & 100 & 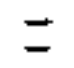 & : \\
\hline 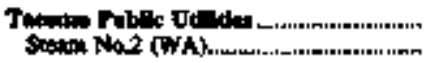 & $\bullet$ & $\begin{array}{l}\text { 121.6 } \\
121.0\end{array}$ & 5.30 & 43 & $\dot{*}$ & 40 & $\begin{array}{l}26,49 \\
26,49\end{array}$ & .50 & $*$ & $\underset{4<\geq 0}{4 \rightarrow 20}$ & 4,53 & $\frac{27}{22}$ & $\frac{25}{25}$ & $\frac{5}{5}$ \\
\hline 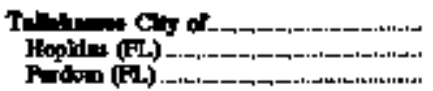 & $\bar{z}$ & $\bar{E}$ & $\bar{z}$ & $\overline{-}$ & $\begin{array}{l}- \\
z\end{array}$ & $\ddot{-}$ & $\bar{z}$ & $\bar{z}$ & $\begin{array}{r}1,59 \\
1,466 \\
493\end{array}$ & $\begin{array}{l}2+2.0 \\
288,0 \\
304.0\end{array}$ & $\begin{array}{l}3.05 \\
2.99 \\
3.10\end{array}$ & $\bar{z}$ & $\bar{z}$ & $\begin{array}{l}\mathbf{1} 00 \\
100 \\
100\end{array}$ \\
\hline 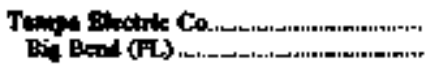 & $--^{\mathbf{m 3}}$ & 19ts & 36.37 & $\underline{1.7}$ & .19 & 424.7 & 2406 & 50 & $=$ & $\bar{z}$ & $\bar{z}$ & - & $\begin{array}{r}7 \\
100\end{array}$ & - \\
\hline
\end{tabular}

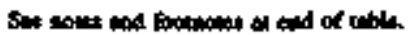


Table 57. Receipts, Average Cont, and Qualify of Eowtil Futs Delivered to US. Electric Uutities by Compuny and Plont, Jaly $19 \%$ (Continow)

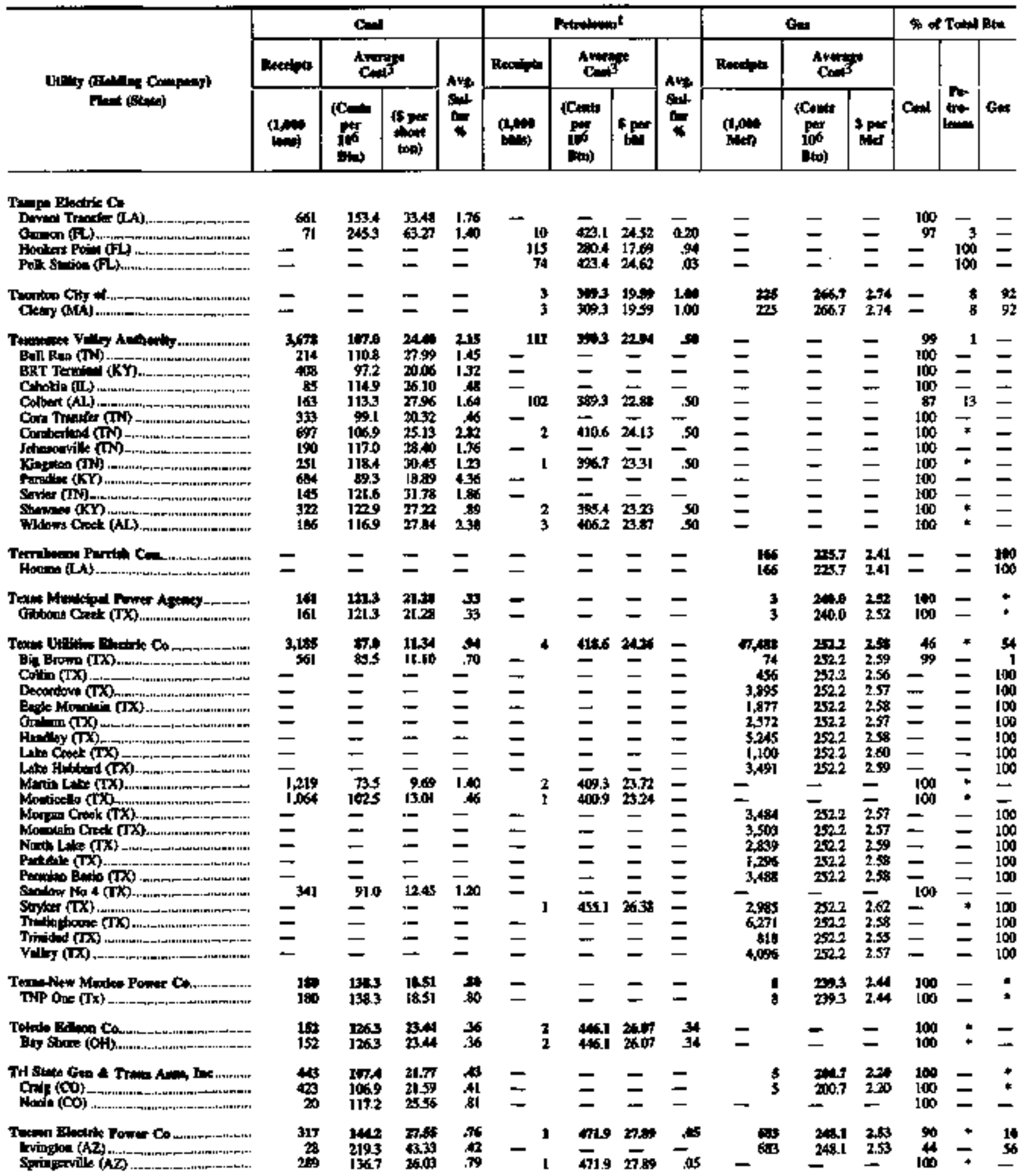

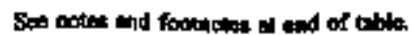




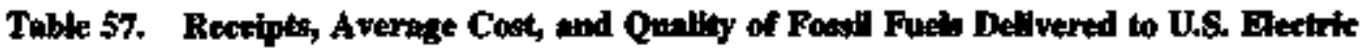
Utilities by Company and Pinnt, Joly 19 'n (Contineed)

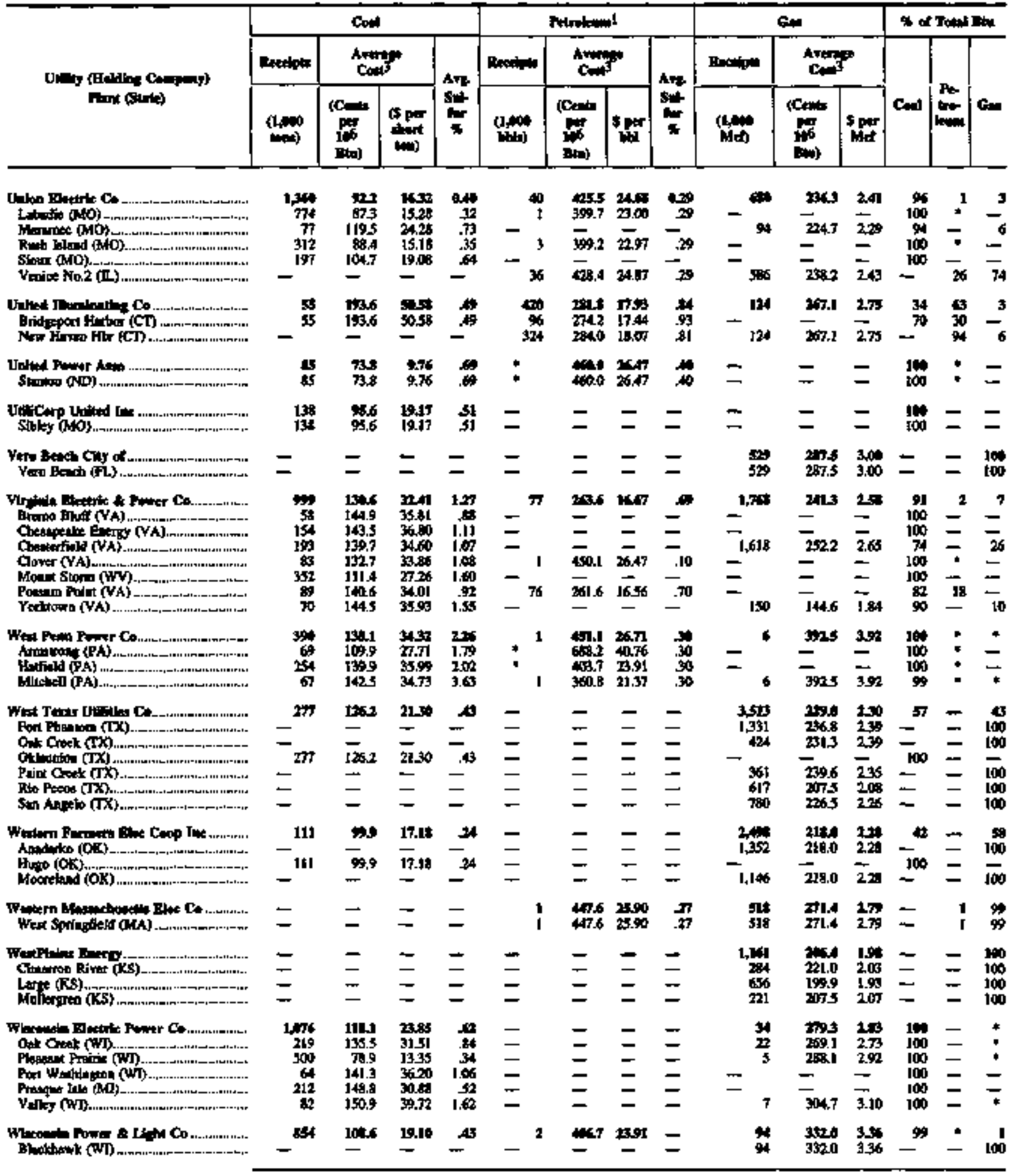

Sec notes and foothoces an and of tolble. 


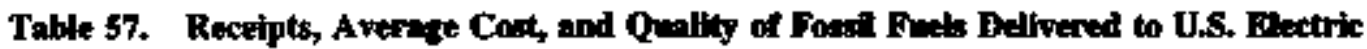
Utilities by Compony and Plane, Jubly 1997 (Coatinened)

\begin{tabular}{|c|c|c|c|c|c|c|c|c|c|c|c|c|c|c|}
\hline \multirow{3}{*}{ 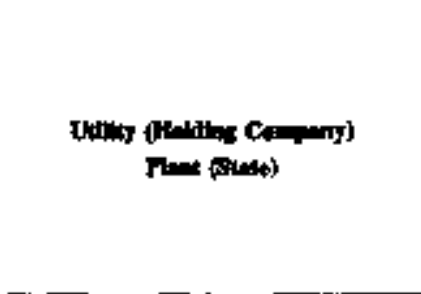 } & \multicolumn{4}{|c|}{ sid } & \multicolumn{4}{|c|}{ Jalding } & \multicolumn{3}{|c|}{ Ga: } & \multicolumn{3}{|c|}{ W of thet the } \\
\hline & 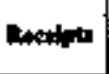 & \multicolumn{2}{|c|}{ 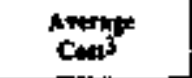 } & \multirow{2}{*}{ 사안 } & \multirow{2}{*}{ 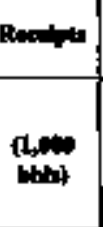 } & \multicolumn{2}{|c|}{ Awation } & \multirow{2}{*}{$\begin{array}{l}\text { Awt } \\
\text { swit } \\
\text { twit }\end{array}$} & \multirow[t]{2}{*}{ Decolpts } & \multicolumn{2}{|c|}{ Aration } & \multirow[b]{2}{*}{ Cad } & & \multirow[b]{2}{*}{ Ena } \\
\hline & (1)aris & 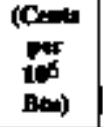 & 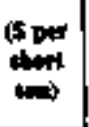 & & & 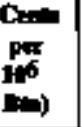 & & & & 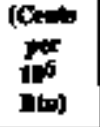 & ind & & & \\
\hline 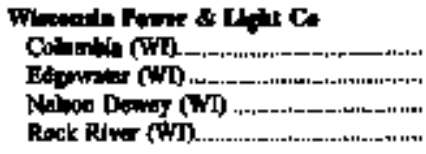 & $\begin{array}{r}414 \\
261 \\
119 \\
31\end{array}$ & $\begin{array}{r}972 \\
120.9 \\
130.2 \\
116.4\end{array}$ & $\begin{array}{l}16.49 \\
21,47 \\
2741 \\
20.45\end{array}$ & $\begin{array}{r}0.44 \\
40 \\
48 \\
.39\end{array}$ & $\begin{array}{l}-1 \\
-\quad 1\end{array}$ & $\begin{array}{l}\overline{4012} \\
\overline{411.9}\end{array}$ & $\begin{array}{l}\overline{23.77} \\
\overline{2422}\end{array}$ & $\begin{array}{l}- \\
- \\
-\end{array}$ & $\begin{array}{l}- \\
- \\
-\end{array}$ & $\frac{-}{-}$ & $\begin{array}{l}- \\
-\end{array}$ & $\begin{array}{r}100 \\
100 \\
100 \\
99\end{array}$ & $\begin{array}{r}- \\
-\end{array}$ & $\begin{array}{l}- \\
- \\
-\end{array}$ \\
\hline 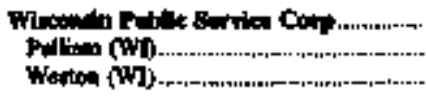 & $\begin{array}{r}215 \\
617\end{array}$ & $\begin{array}{l}10.45 \\
103.1 \\
105.0\end{array}$ & $\begin{array}{l}184 \\
1834 \\
184\end{array}$ & $\frac{36}{26}$ & - & $\overline{-}$ & $\underset{-}{-}$ & $\underline{-}$ & $\begin{array}{r}48 \\
38\end{array}$ & $\begin{array}{l}25 r 5 \\
2780 \\
278.5\end{array}$ & $\begin{array}{l}282 \\
282 \\
282\end{array}$ & $\begin{array}{c}99 \\
90 \\
\text { 100 }\end{array}$ & - & $\begin{array}{r}1 \\
+\end{array}$ \\
\hline 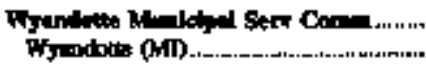 & 15 & $\begin{array}{l}1342 \\
134.2\end{array}$ & $\begin{array}{l}3601 \\
3601\end{array}$ & 238 & - & 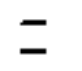 & $\overline{-}$ & $\underline{-}$ & $\ddot{n}$ & - & - & $\log _{100}$ & - & - \\
\hline DS, Thinl & 71406 & J12: & $25, x$ & $H$ & 11) & and & 17.75 & 1,4 & 373,4 & 21486 & 249 & $\boldsymbol{7}$ & 4 & t5 \\
\hline
\end{tabular}

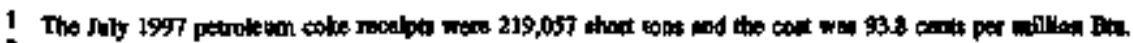

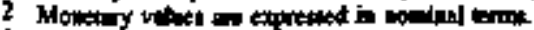

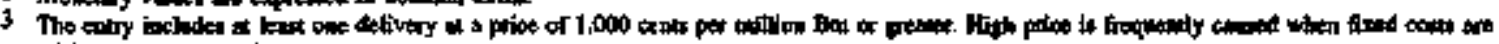

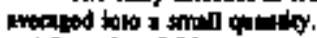

* Iesa dim 0.0S.

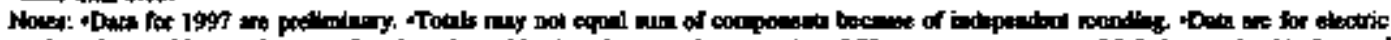

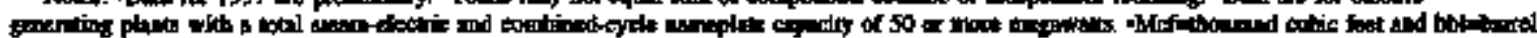

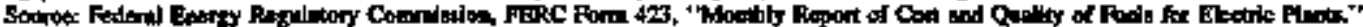




\section{Appendix A}

\section{General Information}

\section{Articles}

Feature articles on electric power eneroy-related subjects are frequently included in this publication. The following articles and special focus items bave appeared in previous issues.

June 1990. . . . . . . . . . Potrolent Fati-S witehing Capability in the Electric Utility Industry

Aptil $1991 \ldots \ldots \ldots \ldots$. . . . W. Wholesale Electricity Transactions

Apríl $1992 \ldots \ldots \ldots \ldots$ Electric Utility Demand-Side Mabagement

April $1992 \ldots \ldots \ldots \ldots \ldots$ Nonutility Power Poducers

August 1992. . . . . . . Performence Optimization and Repowering of Generating Uaits

February $1993 . \ldots \ldots \ldots$. Improvement in Nuclear Power Plant Capacity Factors

Oetober $1993 \ldots \ldots \ldots$ Municipal Solid Waste in the U.S. Energy Supply

November 1993. . . . . . . Electric Utiljty Demand-Sjde Management and Regulatory Effects

November 1994. . . . . . . The Impact of Flow Control and Tax Reform on Ownesship and Growth in the U.S. Waste-to-Energy Industry

July 1995. . . . . . . . . Nonutility Electric Generation; Industrial Power Production

August 1995. . . . . . . . . Steam Generator Degradation and Its Impact on Continned Operation of Pressurized Water Regctors in the United States

September $1995 \ldots \ldots \ldots$. . . . . Sources of Nuclear Fuel

November 1995. . . . . . . Relicensing and Environmental Issues Affecting Hydropower

May 1996 . . . . . . . . U.S. Electric Utility Demand-\$ide Management: Trends and Analysis

June $1996 \ldots \ldots \ldots \ldots \ldots$. Upgrading Tramsmission Capacity for Wholesale Electric Power Trade

For additional information or questions regarding availability of article reprints, please contact the National Energy Information Center, at (202)586-8800 or by FAX at (202)586-0727. 
Eleatric Power Monthly Data Guide

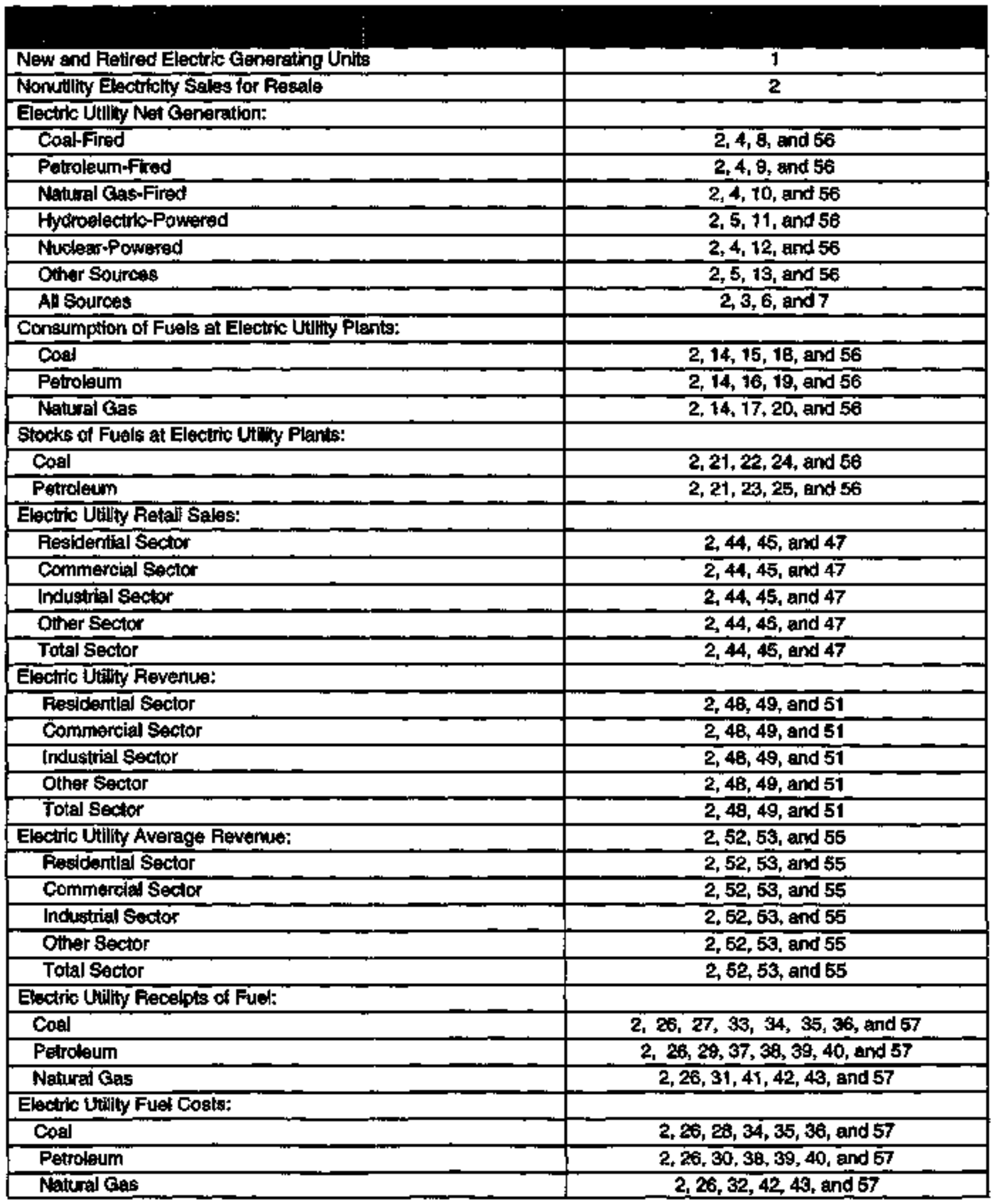




\section{Blbliography}

1. Energy Information Administration, Office of Cosl, Nuclear, Electric and Alternate Fuels, Itoentory of Power Plants in the United States, DOE/EIA-0095(93) (Washington DC, 1994), pp. 247-248.

2. Energy Information Administration, Office of Statistical Standards, Ant Assessment of the Quality of Selected ELA Data Series. Electric Potwer Data, DOE/ELA-0292(B9) (Washington DC, 1989).

3. Kott, P.S, "Nonresponse in a Periodic Sample Survey," Joumul of Business and Economic Statistics, April 1987, Volume 5, Number 2, Pp. 287-293.

4. Kraub, J.R., Jr., "Ratio Estination and Approximate Optimutu Stratification in Electrk Power Surveys," Proceedings of the Section on Surdey Reserrch Methods, Ametican Statistlcal Assactation, 1989, pp. 849-853.

5. Kraub, J.R, Jr., "More Model Sampling and Aralyses Applied to Electric Fower Data," Procendings of the Section on Suroey Research Methods, Amertican Statistical Association, 1992, pp. 876-881.

6. Royall, RM. (1970), "On Finite Population Sampling Theory Under Certain Linear Regression Madels," Bionetrika, 57, 377-387.

7. Royall, RM., and W.G. Cumberland (1978), "Variance Estimation in Finite Population Sampling, Journal of the American Statistical Association, 73, 351-358.

8. Royall, R.M., and W.G. Cumberland (1981), "An Empitical Study of the Ratio Estimator and Estimators of Its Variance," Joumal of the Anterican Statistical Association, 76, 66-68.

9. Knaub, J.R., Jr., "Alternative to the Iterated Reweighted Least Squares Method: Apparent Heteroscedasticify and Lirear Regression Model Sampling," Procedings of the Intemational Conference on Establishment Surxeys, American Statistical Associatton, 1993, pp. 520-525.

10. Rao, P.S.R.S. (1992), Unpublished notes on model covariance.

11. Haruen, M.H., Hurwitz, W.N. and Madow, W.C., (1953), "Sample Survey Methods and Theory," Volume II, Theory, pp. 56-58.

12. Knaub, J,R, J $x_{+}$"Relative Standard Error for a Ratio of Variables at an Aggregate Level Under Model Sampling," in Proceedings of the Section on Suroey Research Methods, American Statistical Association, 1994, PP. 310-312.

13. Knaub, J.R., Ir., "Weighted Multiple Regression Estimation for Surveg Model Sampling," InterStat (http://interstat.stat.vt.edu), May 1996. 



\section{Appendix B}

\section{Technical Notes}

\section{Data Sources}

The Electric Porver Monthly (EPM) is prepared by the Coal and Electric Data and Renewables Division, Office of Coal, Nuclear, Electric and Alternate Fuels (CNEAF), Energy Information Administration (EIA), US. Department of Energy. Data published in the EPM are compiled from seven data sources. Those forms are: the Fom ELA-759, "Monthly Power Plant Report," the Form ELA-900, "Monthy Nonutility Sales for Resale Report," the FERC Form 423, "Monthly Report of Cost and Quality of Fuels for Electric Plants," the Form E\&A-826, "Monthly Electric Utility Sales and Reventze Report with State Distributlons," the Form ElA-861, "Arnual Electric Utility Report," the Form ElA-860, "Annual Electric Generator Report," and the Form EIA-867, "Annual Nonutility Power Producer Report."

\section{Form EIA-759}

The Form EIA-759 is a cutoff model sample of approximately 360 electric utilities drawn from the frame of all operators of electric utility plants (approximately 700 electric utilities) that gererate electric power for public use. Data will be collected on an annual basis from the recraining operators of electric utdity plants. The new monthly data collection is from all utilities with at least one plant with a nameplate capacity of 25 megawatts or more. (Note: includes all nuclear units). However, the few utilities that generate electricity using renewable fuel sources other than hydroelectric are all included in the sample. The Form EIA-759 is used to collect monthly data on net generation; consumption of coal, petroleum, and natural gas; and end-of-the-nonth stocks of coal and petroleum for each plant by fuel-type combination. Summary data from the Form EIA-759 are also contained in the Eletric Power Antual (EPA), Monthy Energy Review (MER), and the Annual Entrgy Revieto (AER). These reports present aggregate data estimates for electric utilities at the U.S., Census division, and North Amerionn Electric Reliability Council Region (NERC) levels.

Instrument and Dosign History. Prior to 1996, the Bureau of the Census and the U.S. Geological Survey collected, compiled, and published data on the electric power industry. In 1936, the Federal Power Commission (FPC) assumed ofl data collection and publication responsibilities for the electric power tndustry and implemented the FPC Form 4t The Federal Power Act,
Sections 311 and 312, and FPC Order 141 define the legislative authority to collect power production data. The Form EIA-759 replaced the FPC Form 4 in tanuary 1982. As of the January 1996 reporting period, the Form EIA-759 was changed to collect data from a cutoff model sample of plants with a nameplate capacity of 25 megawatts or more.

Data Procesalng The Form EIA-759, along with a return envelope, is mailed to respondents approximately 4 working days before the end of the month. The completed forms are to be returned to the EIA by the 10th day after the end of the reporting rwonth. After receipt, data from the completed forms are manually logged in and edited before being keypunched for automatic data processing. An edit program drecks the data for errors not found during manual editing. The electric ublities are telephoned to obtain data in cases of missing reports and to verify data when questions arise during editing. After all forms are received from the respondents, the final autumated edit is subritted. Following verification of the data, text and tables of aggregated data are produced for inclusion in the EPM. Following EIA approval of the $E P M$, the data are made avathable for public use, on a cost-recovery basis, through custom computer runs, data tapes, or in publications.

\section{FERC Form 423}

The Federal Energy Regulatory Comutission (FERC) Form 423 is a monthity record of delivered-turel purchases, submutted by approximately 230 electric utilities for each electric generating plant with a total skeam-electric and combined-cycle nameplate capactty of 50 or more megawatts. Summary date from the FERC Form 423 are also contained in the EPA, MER, and the Cost ard Quality of Fudls for Electric Utatify Plants - Annual. These reports present aggregated data on electric utilities at the U.S., Census division, and State levels.

Instrument and Dedgn History. On July 7, 1972, the FPC issued Oriter Number 453 enacting the New Code of Federal Regulations, Section 141.61, legally creating the FPC Fotm 423. Originally, the form was used to collect data only on fossil-steam plants, but was amended in 1974 to include data on internal combustion and combustion turbines. The FERC Form 423 replaced the FPC Form 423 in January 1983. The FERC Form 423 eliminated peaking units, which were previously 
collected on the FPC Form 423. In addition, the generator nameplate capacity threshold was changed from 25 megawatts to 50 megawatts. This reduction in coverage elimunated appronderately 50 utilities and 250 plants. Alt histotical FPC Forn 423 data in this publication were reviged to reflect the new generator nouneplate capacity threbild of 50 or more megawatts reported on the FERC Form 423. In January 1991, the collection of data on the FERRC Fonm 423 was extended to include combined-cycle units. Historical data have not been revised to include these units. Starting with the Jamuary 1993 data, the FERC began to collect the data directly from the respondents.

Deta Proceasing The FERC processes the data through edits and each month provides the EIA with a diskelte containing the data. The EIA reviews the data for accuracy. Beginning with May 1994 data, an additional quality check began in which coal data are compared with data prepared by Resource Data Intemational, Irc., of Boulder, Colorado. Following verification of the data, text and tables of aggregated data are produced for inctusion in the EPM. After the EPM is cleared by the EIA, the data become available for public use, on a cost-necovery basis, through custon computer runs or in publications.

\section{Form ElA-826}

The Form EIA-826 is a monttlly collection of data from approximately 260 of the largest pritnarily intesvorowned and publicty owned electric ublibes. A model is then applied to estimate for the entire universe of U.S. electric utilities. The electric power sales data are used by the Federal Reserve Board in their esonomic analyses.

Instrument and besign Hlatory. The collection of electric power sales, reventue, and income data began in the early 1940's and was established as FPC Form 5 by FPC Order 141 in 1947. In 1980, the report was revised with only selected income thems remaining and became the FERC Form 5. The Form ElA-826 replaced the FERC Form 5 in January 1983. In January 1987, the Form ElA-826 was changed to the "Monthly Electric Utility Sales and Revenue Report with Stabe Distributions. "It was formetly titled, "Electric Utility Company Monthly Statement." The Form FlA-826 was revised in January 1990, and some data elements were elitminated. In 1993, EIA for the first time used a model aample for the Form EIA-826. A stratfied-random sample, enploying auxiliary data, was used for each of the 4 previous years. (See previous isstes of this publication and (Kraub, 12) for details.) The current sample for the Form ElA-826, which was designed to obtain extimstes of elextrity sales and revente per kilowatthour at the State level by end-use sector, was chosen to be in effect for the January 1993 data.
Frame. The frame for the Form EIA-826 was originally based on the 1989 submission of the Form ElA-861 (Section 1.4), which consisted of approximately 3,250 electric utilities selling retall and/or sales for resale. Note that for the Form EIA-826, the EIA is orly interested in retail sales. Updates have been made to the frame to reflect rergers that affect data processing. Some electric utilities serve in more than ore State. Thus, the State-service area is actually the sampling unit. For each State served by each utility, there is a utility State-part, or "State-service area." Thus approach allows for an explicit calculation of estimates for sales, revenue, and revenue per kilowatthour by end-use sector (residential, commercial, industrial and other) at State, Census division, and the US. level. Regressor data came from the Form Ela-861. (Note that estimates at the "State level" are for sales for the entire State, and similarly for "Census division" and "U.S." levels.)

The preponderance of electric power sales to ultimate consumers in each Stabe are rade by a few large utilities. Ranking of electric utilities by retal sales on a State-by-State basis revealed a consistent pattem of dorrinance by a few electric utilities in nearly all $\mathbf{5 0}$ States and the Distriat of Columbia. These dominant electric. utilities were selected as a model sample. These electric utilitles constikute about 8 petcent of the population of U.S. electric utilities, but provide three-quarters of the total U.S. retail ejectricity sales. The procedures used to derive electricity sales, nevenue, revenue per kilowatthour, and associated coefficient of variation (CV) estimates are provided in the Form ElA-826 subsection of the Fonulas Data Section See (Knaub, 12) for a study of CV estimates for this survey.

Data Proces:ing. The forms are mailed each year to the electric utilities with State-parts selected in the sample. The completed form is to be rehurned to the EIA by the last calendar day of the month following the reporting month. Nonrespondents are teiephoned to obtain the dats. Imputation, in model sampling, is an impliót part of the ettration. That is, data that are not available, elither bacaluse it was not part of the sarnple or because the data are missing, are estimated using a model. The data are edited and entered into the computer where additionai shecks are completed. After all forms have been received from the respondents, the final automated edit is submitted. Following vertiftcation, tables and text of the aggregated data are produced for inclusion in the EPM. After the EPM recelves dearance from the FIA, the data are made available for public use through custom computer runs, data tapes, or in publications (EPA, AER) on a cost-recovery basis. 


\section{Form EIA-900}

The Form EIA-900, "Monthly Nonutility Sales for Resale Report, is a cutoff model sample drawn from the frame for the Form ELA-867, "Anmual Nonutility Power Producer Report." Members of the Form EIA-867 frame with nameplate capacity greater than or equal to 50 megawatts constitute the sample for the Form E1A-900. Unlike the Form EIA-867 which gatters dato on a number of topics, however, the Form EIA-900 currently is used to collect data on only one element, sales by nonutilities for resale through the power grid.

Intrumint and Doilgn Hitatory. The Form ElA-900 was umplemented to collect monthly data, starting with January 1996. The reason for its inception was to fill, in part, a "data gap" that exdsted on a monthly basis when comparing utility sales to end users (from the Form EIA-826) with utility generation (from the Form ElA-759). This data gap occurred because utility sales data include electricity punchased from nonutilities and because of other factors such as transmission losses and inports/exports. In light of sampling and nonsampling errot, a more complete description of events may be gleaned by induding results based on the Form ELA-900.

Data Processing. The Form EIA-900 is mailed to all operating Form ELA-867 respondent facilities with more than 50 megawatts of total operating capadity. In 1996 , there were approximately 380 respondents for the Form EIA-900. Data stibmissilon is allowed by internet e-mail, postal mail, telepthote or tacsimile (FAX) transcuission. In the near future, the EIA plans to allow touchtorte data entry. At first subrission, the number for the one datum element collected is compared to a previously submitted number, through the use of an interactive edit. Later, batch edits are applied. One edit is used to compare total sales, generation, line losses and inports/exports to determine if the results are reasonable. Another edit is applied on an individual, anuual basis, to compare 12 month totals for the Form EJA-900 submissions to the corresponding Form EIA-867 submissions.

\section{Form ElA-861}

The Form ElA-861 is a mandatory census of electric utilities in the United States. The survey is used to collect information on power production and sales data from approximateiy 3,250 electric utilities. The data collected are used to maintain and update the EIA's electrtc utility frame data base. This daka base suyports queries from the Excecutive Branch, Congress, other public agencies, and the general public. Sumunary data from the Form EIA-861 are also contained in the Electric Sales and Ranerue; the Eletric Pouter Annteat; the Financial Statistics of Selected Publichy Owned Electric Utilities; the Finential
Statistics of Selected Inoestor-Owned Electric Utilities; the $A E R$; and, the Arrourl Ontloct for U.S. Electric Power. These reports present aggregate totals for electric utilities on a national level, by State, and by ownership type.

Instrument and Dealgn History. The Form ELA-861 was implemented in January 1985 to collect data as of year-end 1984. The Federal Administration Act of 1974 (Public Law 93-275) defines the legislative authority to collect these data.

Data Processing. The Form EIA-861 is mailed to the respondents in February of each year to collect data as of the end of the preceding calendar year. The data are manually editad before being entered into the interactive on-line system. Intemal edit dhecks are performed to verify that current data total across and between schedules, and are comparable to data reported the previous year. Edit thecks are also performed to compare data reported on the Form EIA-861 and similar data reported on the Forms EIA-826; EIA-412, "Annual Report of Public Electric Utilities;" and FERC Form 1, "Annual Report of Major Electric Utikties, Licensees, and Others." Respondents are telephoned to obtain clarification of reported data and to obtain missing data.

\section{Form EIA-860}

The Form EIA-860 is a mandatory censtus of electric utilities in the United States that operate power plants or plan to operate a power plant within 10 years of the reporting year. The survey is used to collect data on electric utilities' existing power plants and their 10-year plans for constructing new plants, generating urit additions, modifications, and retirements in existing plants. Data on the arvey are collected at the gererating unit level. These data are then aggregated to provide totals by energy source (coal, petroleum, gas, water, nuclear, other) and geographic area (State, NERC region, Federal region, Census division). Additionaly, at the national level, data are aggregated to provide totals by prime mover. Date from the Form EIA-\$60 are also sumruarized in the Inventory of Power Plants in the United States and the EPA, and as input to publications (AER) and studies by other offices in the Department of Energy.

In:trument and Design History. The Form EIA-860 was implenented in January 1985 to collect data as of yearend 1984. The Federal Energy Adntinistration Act of 1974 (Public Law 93-275) defines the legislative authority to collect these data.

Dats Procaniting. The Form ERA-860 ts malled to approx. imately 900 respondents in November or December to collect data as of January 1 of the reporting year, where the reporting year is the calendar year in which the report 
was gled. Effective with the 1996 reporting year, respondents have the option of filing Form EJA-860 directly with the EIA of through an agent, such as the respondent's regional electric reliablitsy council. Data reported through the regional electric reliability councils are gubmitted to the FIA electronically from the North American Flectric Reliability Council (NERC). Data for each respondent are preprinted from the applicable data base. Respondents are irstructed to verify all preprinted data ard to supply missing data. The data are manually edited before being keypunched for automatic data processing- Computer programs containing additional edit checks are run. Respondents are telephoned to obtain correction or darification of reported data and to obtain missing data, as a restolt of the manual and automatic editing process.

\section{Form ElA-867}

The Form EIA-867 is a mandatory survey of all existing and planned nomutility electric generating facilities in the United States with a total generator nameplate capacity of 1 or more megawatts. In 1992, the reporting threshold of the Form EIA-B67 was lowered to include all facilities with a combined nameplate capacity of 1 or more megawatts. Previously, data were collected every 3 years from facilities with a nameplate capacity between 1 and 5 mezawatts. Flanued generators are defined as a proposal by a company to install electric generating equipment at an existing or planned facility. The proposal is based on the owner having obtained (1) all environmental and regulakxy approvals, (2) a contract tor the ejectric energy. or (3) financial cosore on the facility. The Form consists of Schedules I, "Identification and Certification;" Schedule II, "Facility Information"; Schedule III, "Standard Industrial Classification Code Designation"; Schedule IVA, "Facility Fuel Information"; Schedule IVB, "Facility Thennal and Generation Information"; Schedule V, "Facility Exvirormental Information"; and Schedule VL "Electric Generator buformation."

Submission of the Form ElA-\$67 is required from all facilities that have a combined facility nameplate capacty of 1 megawatt or more. Schedule V. "Facility Environmental Grformation" is only required of those facilities of 25 megawatts or more.

The form is used to collect data on the installed capacity, energy consumption, generation, and electric energy sales to electric utilities and other nonutilities by facillty. Additionally, the form is used to collect dita on the quality of fuels burned and the types of environmental equipment used by the respondent. These data are aggregated to provide geographic totals for seleoted States and at the Census division and national levels. Stroe the Form ELA-867 data are considered confidential, suppression of some data is nesessary to protect the confidentiality of the individual respondent data. See "Confidentiality of the Data" in this section for further inforration.

Instrument and Design History. The Form ElA-867 was implemented in December 1989 to collect data as of yearend 1989. The Federal Energy Administration Act of 1984 (Public Law 93-275) defines the legislative authority to collect these data.

Data Processing. The Form ELA-867 is mailed to the regpondents it January to collect data as of the end of the preosting calendar year. Static data for each respondent are preprinted from the previous year, and the respondents are instructed to verify all preprinted information and to supply the missing data. The correleted fortus are to be returned to the ELA by April 30. The response rate fcr all facilities for which addresses were conffrmed was 100 percent. The data are manually edited before being keyed for automatic data processing. Computer programs containing additional edit chexks are run. Respondents are telephored to obtain corrections of clarifications of reported data and to obtain missing data as a result of the manual and automated editing.

\section{Formulaendethodologles}

The following formuta is ased to calculate percent differences.

Percent Difference $=\left(\frac{x\left(t_{2}\right)-x\left(t_{1}\right)}{x\left(t_{1}\right)}\right) \times 100$.

where $x\left(t_{1}\right)$ and $x\left(t_{2}\right)$ denote the quantity at year $\xi_{\xi}$ and subsequent year $t_{2}$.

\section{Form ElA-826}

The Form EIA-626 data are collected at the utility level by sector and State. When a utillity hes sales in more than one State, the State data that may be required are dependent upon the sample selection that was done for each State independendy. Data from the Form ElA-826 are used to determine stimates by sector at the State, Census division, and national level for the entire corresponding State, Census division, or national category. Form ELA-861 data were used as the frame from which the sample was selected, and also as regressor data.

The sample consists of approsimately 260 electric utilities. This inctirdes a somewhat larger number of Stale-service aneas for electric utilities. Estimation procedures include inputation to account for notresponse. Nonsampling error tutsk also be considered. The nonsampling enror is 
not estimated directly, although attempts are made to minimize it.

State-level ales and revenue estimates are calculated. Also, a ratio estimation procedure is tused for estimation of revenue per kilowatthour at the State level. These estimates are accumulated separately to produce the Cenous division and U.S. level estimates.

The coefficient of variatican (CV) statistic, usually gdven as a percent, describes the magnitude of sampling error that might reasonably be incurred. The $\mathrm{CV}$, cometimes referred to as the relative standard error, is the square root of the estimated variance, divided by the variable of interest. The vaiable of interest may be the ratio of two variables (for exanple, revenue per kilowatthour), or a single variable (for example, sales).

The sampling error may be less than the nonsampling error. Nonsampling errors may be attributed to many sources, inctuding the response errors, definitional difficulities, differences in the interpretation of questions, mistakes in recording or cading data obtained, and other errors of collection, response, or coverage. These nonsampling errors also occur in complete censuses. In a complete census, this problem may become ummanageable. One indicator of the magritude of possible nonsampling error tray be gleaned by examining the history of revisions to data for a survey (Table B2).

Coefficients of variation are indicators of error due to sampling. (CVs do not account for nonsampling errors, such as errors of misclassification or transposed digits. However, estimates of Cvs, although not designed to measure nonsampling error, are affected by them). In fact, large CV estimates found in preliminary work with these data have often indicated nonsampling errors, which were then identified and corrected. Using the Central Linit Theoren, which applies to sums and means such as are applicable here, there is approximrately a 68-percent chance that the true sampling error is less than the corresponding $C V$. Note that reported $C V$ 's are always estimates, themselves, and are usually, as here, reported as percents. As an example, suppose that a revenue-per-kilowatthour value is estimated to be $B .13$ cents per kilowatthour with an estimated $C V$ of 1.6 percent. This means that, ignoring any nonsarnpling error, there is approximately a 68-percent chance that the true average revenue per kilowatthour is within approximately 1.6 percent of 5.13 cents per killowatthour (that is, between 5.05 and 5.21 cents per kilowatthour). Thene is approximately a 95-percent chance of a true sampling etror being 2 CVs or less.

The basic approach used is shown in (Royall, 6) with additicenal discussion of varlance estimation in (Royall and Cumberland, 7), (Royall and Cumberland, 8), and (Knaub,5). From (Royall, 6), for sales or revenue for any sector at the State level, if we let $x$ represent an observation from the Form EIA-861, $y$ represents an obeevation from the Form ElA-826, and 9 represents an estimated value for data not collected, then

$$
\begin{aligned}
& y_{i}=b x_{i}+x_{i}^{\gamma} e_{\sigma_{j}^{2}} \\
& \dot{y}_{i}=b x_{i} \\
& \hat{b}(\gamma)=\left[\sum_{k=1}^{n} x_{k}^{1-\lambda y} y_{k}\right] /\left[\sum_{k=1}^{n} x_{k}^{2-\lambda y}\right]
\end{aligned}
$$

Here, $\mathbf{n}$ is the Form BJA-826 sample size for that State, and $b$ is the factor ('slope') relating $x$ to $y$ in the linear regression. $Y$ is taken to be 17 (see (Knaub, 5)), although more reseanch (Kraub, 9) could refine this. For the Form EIA-826, $\gamma=$ th has certainly been shown to be adequate (see (Knaub, 5), page 878, Table 1). The variance formula for $V$, found in (Royall and Cumberland, 7 and 8 ) performs well for sales and for revenue. For revenue per kilowathour, the model covartance comes from notes provided by Profeseor Podurl S.R.S. Rao (Rao, 10) of the University of Rochester and the Energy Information Admintstration. Aggregate level CV estimates for reveruve per kibowatthonr are calculated as supported by (Haneen, Hurwitz and Madow, 11). Details are pubtished in (Knaub, 12).

As a final adjustment basext on our most complete data, use is made of final Form ELA-861 data, when available. The annual totals for Form ETA-826 data by State and end-use sector are companed to the corresponding Form EIA-861 values for sales and revenue. The ratio of these two values in each case is then used to adjust each corresponding monthly value.

Additional information or dariflcation can be addressed to the Energy Irformation Admindatration as indicated in the "Contacts" section of this publication.

\section{Form EIA-900}

The Form ElA-900 data are collected at the facility level, which is roughly the nonutility equivalent of plant level. Like the Form EIA-826, cutoff model sampling and estimation are employed, however, the estimation formula ane moxified by use of a second regressor. It was found that more variability occurred under the single regressor model than was generally found in the case of the Form EIA-826, but that through the use of nameplate capacty as a second regressor, results were greatly improved. Increasing variance as regressor values increase (heteroscedasticity), a phenomenon which 
caused us to use a value for garnma greater than zero in the case of the Fom EIA-826, is at least as important a consideration here, and further study to increase effictency may be performed. A paper, "Weighted Multiple Regression Estimation for Survey Model Sampling," has been accepted for publication in the Intemet statistics journal, Interstat at http://interstatstat.vtedu/intersta.htm. Thts paper explains a great deal of the background and methodology involved in providing a satisfactory estimator in this case. It appears at the Web tite given above, under May 1996 (Kraub, 13).

\section{Form ElA-769}

Data for the Form EIA-759 are collected at the plant level. Estimates are then provided for geographic levels. Consumption of fuel(s) is converted from quantities (in short tons, barrels, or thrusand cubic feet) to Btw at the plank level. End-of-month fuel stocks for a sirgle generating plant may not equal beginning-of-the-month stocks plus receipts less consumption, for many reasors, inchuding the fact that several plants may sture the same fiel stock.

Like the Form ELA-900, cutoff model sampling ard estimation are employed, using the same multiple regression model. Once again, as described under the corresponding subsection on the Form EIA-900, details of the estirnation of totals and varlances of totals are published on the Internet in a paper entitled "Wetghted Multiple Regression Estimation fo. Survey Model Sampling (Knaub, 13). ${ }^{\sigma}$

At the fuel and State level (i.e., lowest aggregate level), there are a number of cases where the minimal sample gize of three is not met, when using a $25 \mathrm{MW}$ culoff. Imputation of historic values for the grallett plarks is used to supplement actual values for the largest ones. However, at the NERC level, this is not necessary. Data element totals for each NERC region, by fuel type, are estimated using model sampling. These samples are composed solely of data reported for the plants actually in the sample. The national level estimate from this is then considered our best estimate, and all other estimates are apportioned accordingly.

\section{FEAC Form 420}

Data for the FERC Rorm 423 are collected at the plant level. These data are then used in the following formulas toproduce aggregates and averages for each fule type at the State, Censtus tivision, and US. level. For these formulas, receipts and average heat content are at the plant level. For each geographic reglon, the summation
$\Sigma$ represents the sum of atl plants in that geographtc region. Additlonally,

- For coal, units for neceipts (R) are in tons, units for average heat content $(A)$ tre in Btu per pound, and the turit converstion (US) is 2,000 pounds per ton;

- For petroleum, units for receipts (R) are in barrels, units for average heat content $(A)$ are in Btu per gatlon, and the unit conversion (US) is 42 gallons per barrel;

- For gas, units for receipts $(R)$ are in thousand cubic feet (Mcf), average heat content (A) are in But per cabic foot, and the unit comversion (U) is 1,000 cubic feet per Mcf.

Total Btu a $\sum_{i}\left(R_{i} \times A_{i} \times U\right)$

where $I$ denotes a plant; $R_{1}=$ receipts for plant $\mathfrak{l}$;

$A_{f}=$ average heat content for recelpts at plant $\mathrm{I}$; and,

$\mathrm{U}=$ unit conversion;

Weighted Average $B t u=\frac{\sum_{i}\left(R_{i} \times A_{j}\right)}{\sum_{f} R_{i}}$,

where $I$ denotes a plant; $R_{1}=$ receipts for plant $I ;$ and, $A_{1}=$ average heat content for receipts at plant $I$.

The wejghted average cost in cents per million Bhu is calculated using the following formula:

Weighted Average Cost $=\frac{\sum_{i}\left(R_{i} \times A_{i} \times C_{i}\right)}{\sum_{i}\left(R_{i} \times A_{i}\right)}$,

where I denotes a plant, $R_{1}=$ receipts for plant I; $A_{\text {i }}$ average heat content for receipts at plant $I$; and $C_{i}=$ cost in cents per million Bhu for plant $I$.

The weighted average cost in dollars per unit is calculated using the following formula:

Weighted Average Cost $=\frac{U \sum_{i}\left(R_{t} \times A_{i} \times C_{l}\right)}{10^{3} \sum R_{i}}$

where $I$ denotes a plant; $R_{i}=$ receipts for plant $I$; $A_{i}=$ average heat content for receipts at plant $I_{;}$

$U=$ unit conversion; and, $C_{i}=$ cost in cents per million Bhu for plant $I$. 


\section{Form E14-861}

Dath for the Form EIA-861 are collected at the utility level from all electric utilities in the United States, its tersitories, and Puerto Rico. Form EIA-861 data in this publication are for the United States only. These data ane then aggregated to provide geographic totals at the State, NERC region, Census division, and rational level. Sources and disposition of data are also provided by utility class of ownership and retail consumer class of servlce Average revenue (nominal foillara) per kilowatthour of electricity sold is calculated by dividirg total arunual retail zevenue (nominal dollars) by the total anrual retail sales of electricity.

Average revenue per kilowatthour is defined as the cost per unit of electricity sold and is calculated by dividing retail electric revenue by the corresponding sales of electricity. The average revenue per kilowathour is calculated for all consumers and for each sector (restdential, commercial, industrial, and other sales).

Electric utilities typically exroloy a number of rate schedules within a single sector. These alternative rate schedules reflect the varying corsurnption levels and pattens of consurners and their associated impact on the wosts to the elactric utility for providing electrical service. The average revestue per kilowatthour reported in this publication by sector represents a weighted average of monsumer revemue and sales within that sector and across sectors for all consumers,

The electric revenue used to derive the average revenue per kilowatthour is the operating revenue reported by the electric utility. Operating revenue includes energy charges, demand charges, contumer service charges, environmental strchanges, fuel adjustments, and ofher miscellaneous charges.

Electric utility operating revenues cover, among other costs of servike, State and Federal income taxes and taxes other than income taxes paid by the utlity. The Federal compconent of these taxes are, for the most part, "payroll" taxes. State and local authorities tax the value of plant (property taxes), the amount of revenues (gross receipts (exes), purctases of materials and services (sales ard use (axes), and a potentially long list of other items that vary extensively by taxing authority. Taxes deducted from employees' pay (such as Federal income taxes and einployes' share of social security taxes) are not a part of the vitity's "tax costs," but are paid to the taxing authorities in the rame of the employees. These taxes are included in the utility's cost of service (for example, reverue requirements) and are included in the amounts recovered from consumers in rates and reported in operating revenues.
Electric uttlities, like many other business enterptises, are requined by various taxing authorities to collect and remit taxes assessed on their consumers. In this regard, the electric utility serves as an agent for the taxing authority. Taxes assessed on the consumer, such as a gross receipts tax or sales tax, are called "pass through" taxes. These taxes do not represent a cost to the utitity and are not recorded in the operating reverues of the utility. However, taxing authurities differ as to whether a specific tax is assessed on the utility or the consumer-which, in turn, determines whether or not the tax is included in the operating revenue of the electric utility.

\section{Form EIA-860}

Data from the Form EIA-660 are submitted at the geverating unit level and are then aggregated to provide total capacity by energy source and geographic area. In addition, at the national level, data are aggregated by prime mover.

Estimated values for net summer and net winter capability for electric generating uribs were developed by use of a regression fomula. The formula is used to estimate values for existing units where data are missing and for projected units. It was found that a zero-intercept linear regression works very well for estimating capability based on nameplate capacity. The only parameter then is the slope $(b)$ that is used to relate capacity to capability as follows: $\hat{y}=6 x$, where $\hat{y}$ is the estimated capability, and $\boldsymbol{x}$ is the known nameplate capacity. There will be difterent value for 6 for different prime movers and for summer and winter capabilities and it will also depend upon the age of the generator. For more details see the Inzentory of Potoer Plants.

\section{Form ElA-867}

Gross electricity generation data from the Fom KlA-867, repotted by geverator, are aggregated to provide totals by energy source and geographic area. Nonutility power producers report gross electricity generated on the Form ElA-867, unlike electric utilithes that report net generation on various BIA and FERC forms. Norutilities generally do not measure and recond electrical consumption used solely for the production of electricity. Nonutility generators and associated auxiliary equipment are often an integral part of a manufacturing or other industrial process and individual watthour meters are not generally installed on auxiliary equipment.

Estimated values for net geveration from nonutlity power producers were developed by EIA using gross generation, prime trover, fuels, and type of air pollution control data reported on the Form BLA-867. The difference between gross and net generation is the electricity 
constumed by atuxiliary equipment and environmental control devices such as pumps, tans, coal pulverizers, particulate collectors, and fure gas desulfurization (FGD) units. The differeme between gross and net gereration is sometimes called parasitic load. In smaller power plants rotating auxiliaties ase almost always electric motors. In large power plants that produce stean, rotating ouxiliviries can be powered by elther steam turbines or electric notors and sometimes both because of cold startup requirements.

Thts methodology for estimatimg net generation from gross generation is based on determiring typical energy consumption for auxiliary electricai equipment associated with electrical generators. For instance, wind turbines have none of the auxiliaries common to a coal-buming power plant such as a coal pulverizers, fans, and enission controls. On the other hand, windfarms do consume electricity sirno automatic, computer-based control systerns are used to control blade pitch and speed thereby affecting generator electricity output.

Shown below are the ocnversion factors used to estimatert net generation by nonutility generators. The factors are typical of a modern electric power plant but contld vary significantly between individual plants. Net generation is calculated by multiplying the appropriate conversion factor by the reported gross electrical generation.

\begin{tabular}{|c|c|}
\hline Pille & 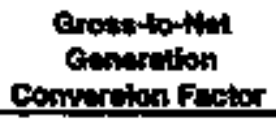 \\
\hline Cas (Conbustion) Tubine) & 98 \\
\hline untemal Combustion $, \ldots+\cdots, \ldots+\cdots+\cdots+$ & 90 \\
\hline 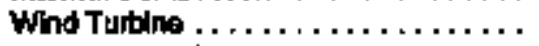 & 90 \\
\hline Boler-Photowaluatc $\ldots \ldots \ldots \ldots \ldots \ldots$ & 99 \\
\hline Hydredulte Tubine $\ldots \ldots \ldots \ldots \ldots$. & 99 \\
\hline Fund Can $\ldots \ldots \ldots \ldots \ldots \ldots \ldots \ldots$ & 99 \\
\hline Othit $\ldots \ldots \ldots \ldots \ldots \ldots \ldots \ldots$ & 97 \\
\hline
\end{tabular}

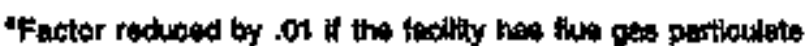

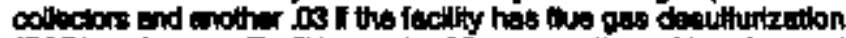
(FCD) ecpipminl. Facintes under 25 megawats and buming toal

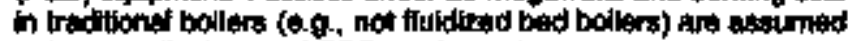
to have perticutato and FaD equipment.

These conversion factors were estimated by the staff of the Ofifice of Coal, Nudear, Electric and Alternate Fuels, Energy Information Admiristration. The primary reference tabed in developing the conversion factors was Stenn, Its Gerneration and Use, 40th Edition, Babcock \& Wicox, Barberton, Ohio.

\section{Average Heat Content}

Heat content values (Table B1) collected on the FERC Fonm 423 were used to convert the consumption data from the Form EHA-759 into Btu. Respondents to FERC Form 423 represent a subset of all generating plants (steam plants with a capacity of 50 megawatts or larger), while Form EIA-759 respondents generaily represent gereatimg plants with a combined capactly of 25 or mone megawatts. The results, therefore, may not be completely representative.

\section{Oualiky of Data}

The CNEAF office is resporsible for routine data improvement and quality assurance activitles. All operations in this office are done in accordance with formal stardards extablished by the EIA. These standards are the measuring rod necessary for quality statistics. Data improvervent efforts include verification of data-keyed input by automatlc computerized methods, editing by subject matter specialists, and follow-up on nonrespordents. The CNEAF office supports the quality assurance efforts of the data collectors by providing advisory reviews of the structure of information requirements, and of proposed designs for new and revised data collection. forms and systems. Once inplemented, the actual performance of wodking data collection systems is also validated Computerized respondent data flles are checked to identify those who fatl to tespond to the survey. By law, nonrespondents may be fined or atherwise penalized for not filing a mandatory ELA data form. Before invoking the law, the ELA tries to obtain the required information by encouraging cooperation of nonrespondents.

Completed forms neceived by the CNEAF office are sorted, screened for completeness of reported information, and keyed onto computer tapes for storage and transfer to random access data bases for computer proceseing. The information coded on the computer tapes is manuatly spot-checked against the forms to certify accuracy of the tapes. To ensure the quality standards esablished by the EIA, formulas that use the past history of data values in the data base have been designed and inplemented to theck data input for errors automatically. Data values that fall outside the ranges prescribed in the formulas are verified by telephoning respordents to resolve any discrepancies.

Conceptual problems aftecting the qualtity of data are discussed in the report, $A n$ Assessment of the Quality of Selected ELA Data Series: Electric Power Data. This report is published by the Energy Irformation Administration (Office of Statistical Standards). See them 2 in Appendix A.

\section{Data Proctation}

Monthly sample survey data have both sampling and nonsampling errors. Sampling errors may be expected 
since all data are not collected and, therefore, must be mathemationlly estimated. (Note that the aruntual series for a monthly sample is not subject to sampling enror because it is a census). Nonsampling errors are the result of incomect allocation of data (for example, transcriptions or misclassifications) and can be difficult to control and estizutate. A study of coefficients of variance and data revisiors was conducted so that the appropriate levels of precieion, based on the accuracy and completeness of the datz from which the entimates are derived, is provlded in this report for average revenue per kilowatthour of elertrictty sold. It was judged that three sigriffcart digits are justlfied for average reverue per kilowattour of electhity sold at the U.S. level except for monthly data prior to 1990 where two sigrificant digits are more approprate.

\section{Data Imputation}

It may become necessary (as in March and April 1996 FERC Form 423 data) to impute for some data, even if a 100-percent cerstus is rorinally collected without incident. In such cases, a modeling approach, similar to what is dore for the Form ELA-826, can be implemented. The stimation methodologies for model sampling and model imputation are identical.

\section{Dots Euting Syotem}

Data from the form stirveys are edited on a monthly basis using automated systems. The edit includes both deterministic checks, in which records are checked for the presence of required fields and their validity; and statistical checks, in which estimation techriques are used to validate data according to their behavior in the past and in comparison to other cturrent fields. When all data have passed the edit process, the system builds monthly master files, which are used as input to the EPM.

\section{Conitidentially of the Doth}

In general, the data collected on the forms used for input to this report are not contidential. However, data fron the Form ElA-900, "Monthly Sales for Resale," and from the Form EIA-867, "Arunual Nonutility Power Prothuers," are considered confidential and must adhere to EIA's "Policy on the Disclosure of Individually Identifiable Energy Information in the Possession of the BA" (45Federal Register 59812 (1980)).

\section{Rounding Rules for Data}

Given a number with $\mathbf{r}$ digits to the left of the decimal and $d+t$ digits in the fraction part, with $d$ being the place to which the number is to be rounded and ibeing the remaining digits which will be truncaked, this number is rotended to $r+d$ digits by adding 5 to the $(r+d+1)$ th digit when the rumber is positive or by subtracting 5 when the number is negative. The $t$ digits are then truncated at the $(r+d+1)$ th digit. The symbol for a rounded number truncaled to zero is $\left({ }^{*}\right)$.

\section{Data Corration Procedure}

The Office of Coal, Nudear, Electric and Altemate Fuejs has adopted the following policy with respect to the revision and correction of recurrent data in energy publications:

1. Annual survey data collected by this office are published either as preliminary or final when first appearing in a data report. Data initially released as preliminary will be so moted in the report. These dafa will be nevised, If necessary, and declared final in the next publication of the data.

2. Al monthly and quarterly survey data collected by this office are published as preltminary. These data are revised only after the completion of the 12-month cycle of the data. No cuvisions are made to the published data before this.

3. The magnitudes of changes due to revistons experienced in the past will be included in the data reports, so that the teader can assess the accuracy of the data.

4. After data are published as final, corrections will be mude ordy in the event of a greater than one percent difference at the national level. Corrections for differences that are less than the beforementioned threshold are left to the discretion of the Office Director. Note that in this discussion, changes of revisions are referred to as "errors."

In acoocdaroe with policy statement rumber 3, the mean value (unweighted average) for the absolute values of the 12 monthly revisions of each itent are provided at the U.S. level for the past 4 years (Table B2). For example, the mean of the 12 monthly absolute errors (absolute differences between prelindinary and final monthly data) for mol-fired generation in 1995 was 49 . That is, on average, the absolute valute of the change made each month to coal-fired generation was 49 million kilowatthours.

The US. total net summer capability, updated monthly in the EPM (Table 1), is based solely on new etectric generating units and retirements which come to the altention of the EIA during the year through telephone calls with electric utilities and on the Form EIA-759, "Monthly Power Plant Report," and may not include all activity for the month. Data on net summer capability, induding 
new electric generating units, are collected armually on the Form EIA-860, "Arnual Electric Generator Report." Preliminary data for net summer capsibility are published in the Electric Powter Anmual (EPA). Final data are published in the troxentory of Poover Plonts. With respect to net summer capability published in the EPM, the EIA examines the accuracy of that data by comparing the annual total vahue with the final annual total vajue published in the IFP.

\section{NERC Agoregation}

Beginning in January 1986, NERC region totals for the Form EIA-759 are aggregates based on membership of the tudividual electric utilities in NERC. Prior to January 1986, NERRC region totals were aggregates defined by the physical location of the power plants generating electritcity.

\section{Ues of the Gloceary}

The terms in the glossary have been defined for general use. Restrictions on the defirutions as used in these data collextion systems are included in each definition when necessary to define the terms as they are used in this report. 
Table B1. Average Hent Cantent of FosstrFuel Recejpts, Jub 1997

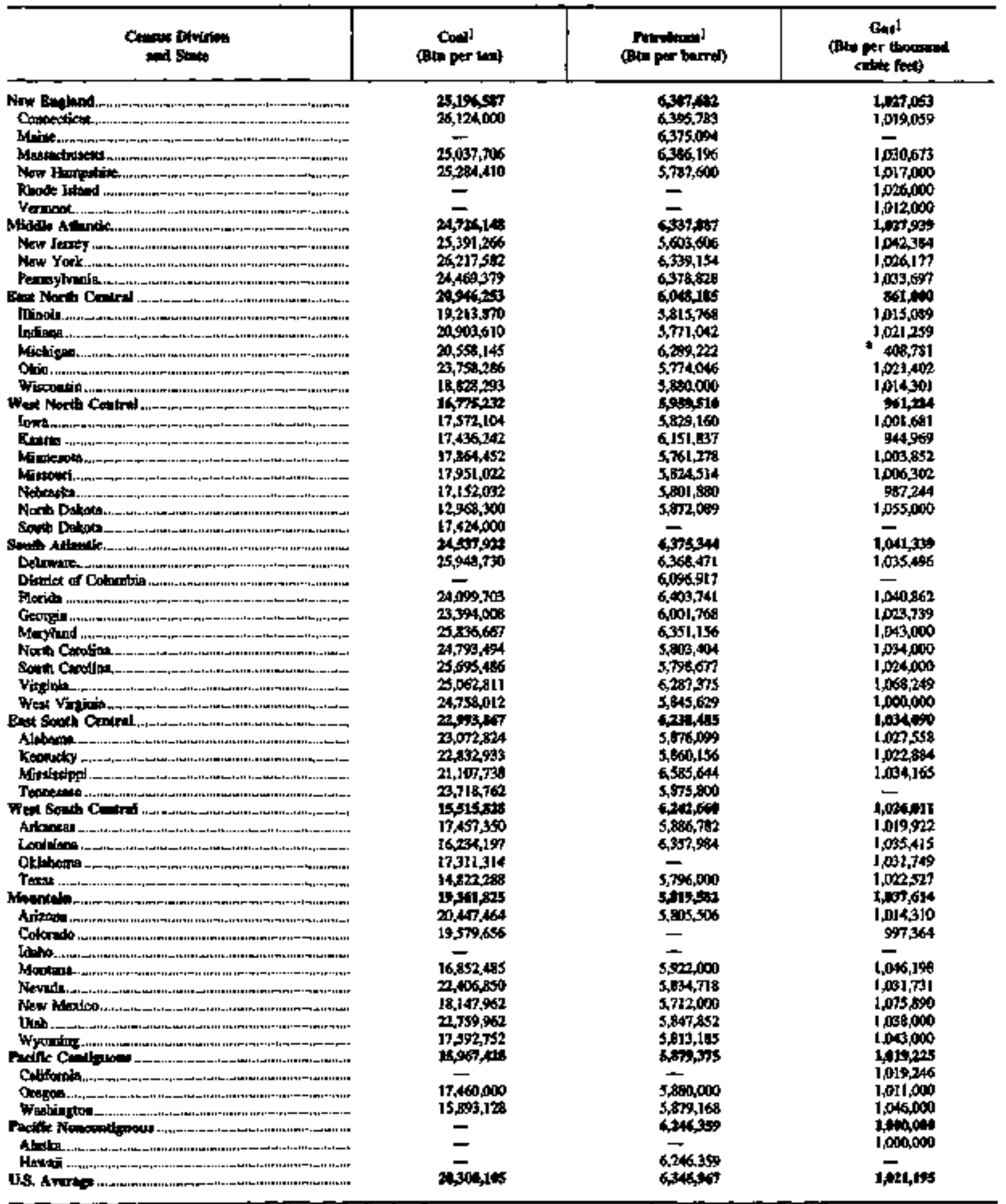

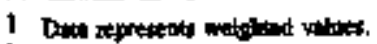

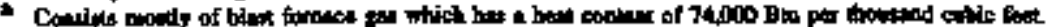

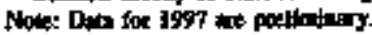

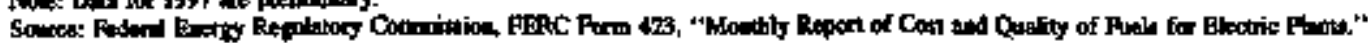


Tabte B2. Comparison of Preliminary Versus Find Pholisthed Data at the U.S. . Level, 1993 Through 1996

\begin{tabular}{|c|c|c|c|c|}
\hline \multirow{2}{*}{ bee } & \multicolumn{4}{|c|}{ 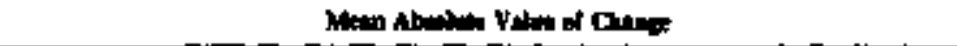 } \\
\hline & 19s & 194 & 10:3 & Lows \\
\hline \multirow{2}{*}{\multicolumn{5}{|c|}{ 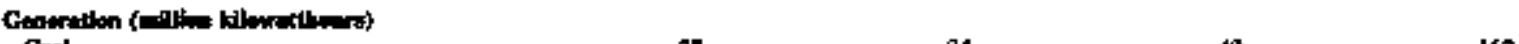 }} \\
\hline 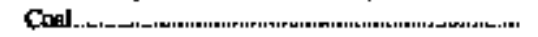 & 28 & & & \\
\hline 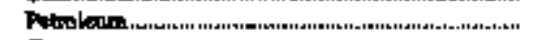 & 3 & 25 & 6 & 64 \\
\hline 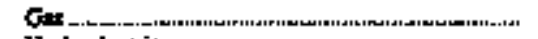 & 19 & 29 & 38 & 84 \\
\hline 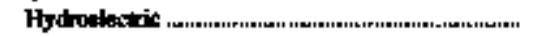 & 10 & 6 & 6 & 298 \\
\hline 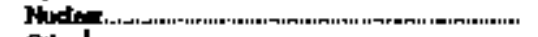 & 0 & \% & 0 & 4 \\
\hline Ctherl & 0 & 1 & 0 & 0 \\
\hline 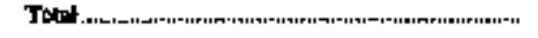 & 26 & 113 & 11 & 462 \\
\hline \multicolumn{5}{|l|}{ Comariptice } \\
\hline 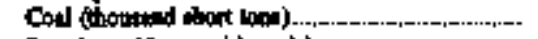 & $\$ 3$ & $\mathbf{0}$ & 27 & 105 \\
\hline 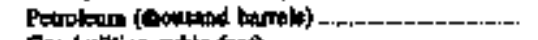 & 10 & 13 & 1 & 94 \\
\hline 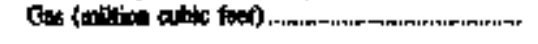 & 327 & 470 & 300 & 89 \\
\hline \multicolumn{5}{|l|}{ Sinchent } \\
\hline 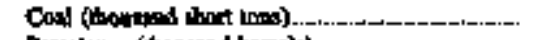 & 208 & 124 & 310 & 233 \\
\hline 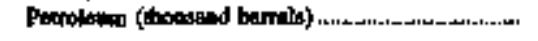 & 203 & $\mathbf{E 1}$ & 239 & 201 \\
\hline \multicolumn{5}{|l|}{ 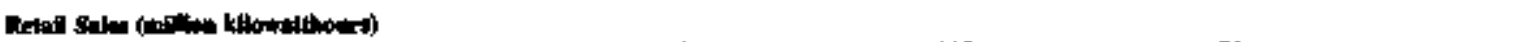 } \\
\hline 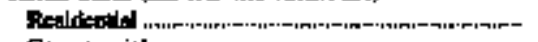 & \$1 & 115 & 79 & $\rightarrow$ \\
\hline 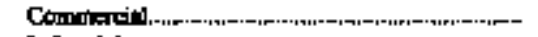 & 59 & 397 & 780 & $\hookrightarrow$ \\
\hline 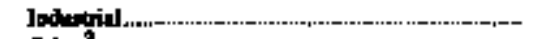 & 175 & 805 & 141 & $\rightarrow$ \\
\hline Oubg' & 96 & 24 & 167 & - \\
\hline 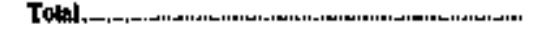 & 219 & 602 & 694 & - \\
\hline \multicolumn{5}{|l|}{ 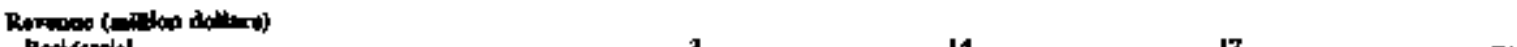 } \\
\hline Rethondd & 3 & 14 & 17 & - \\
\hline 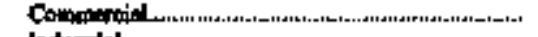 & 3 & 31 & s) & - \\
\hline | & 7 & $\$ 1$ & 23 & $\rightarrow$ \\
\hline 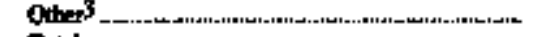 & 5 & 4 & 5 & $\rightarrow$ \\
\hline 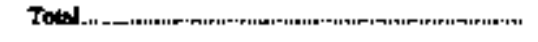 & 11 & 49 & $\mathbf{2 2}$ & $\rightarrow$ \\
\hline \multicolumn{5}{|l|}{ 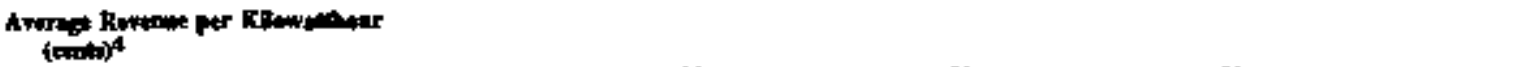 } \\
\hline 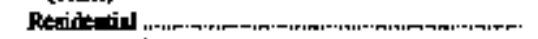 & .05 & .0I & .41 & - \\
\hline 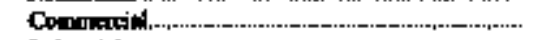 & 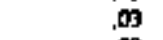 & $.0 \mathrm{I}$ & .01 & - \\
\hline 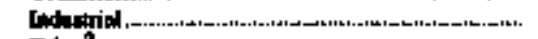 & .63 & .02 & 03 & $\rightarrow$ \\
\hline Dther & .0S & .04 & 20 & - \\
\hline Jard & .03 & 01 & .01 & - \\
\hline \multicolumn{5}{|l|}{ 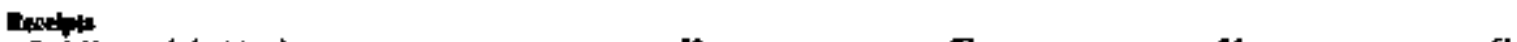 } \\
\hline 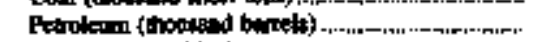 & 13 & 28 & 2 & 7 \\
\hline 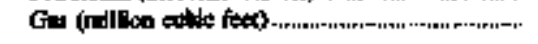 & 315 & 211 & $\boldsymbol{2} \boldsymbol{\gamma}$ & 566 \\
\hline \multicolumn{5}{|l|}{ 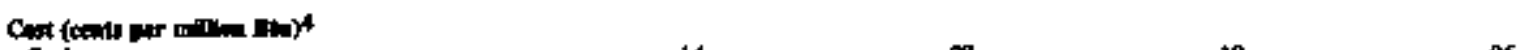 } \\
\hline Codi...-- & .14 & \% & 10 & 06 \\
\hline 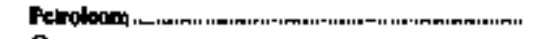 & 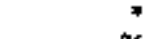 & \$1 & , At & A1 \\
\hline 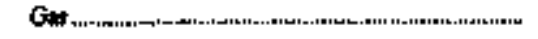 & .06 & 어 & .15 & $\boldsymbol{9 7}$ \\
\hline
\end{tabular}

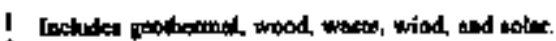

2 Stochs are ent of noon vilues.

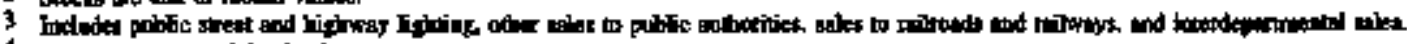

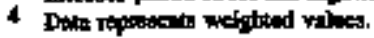

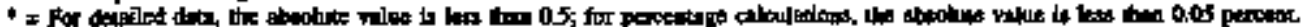

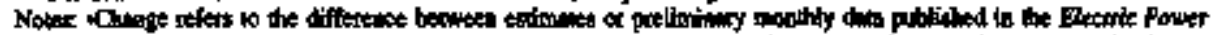

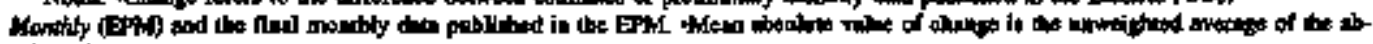
10late shicion.

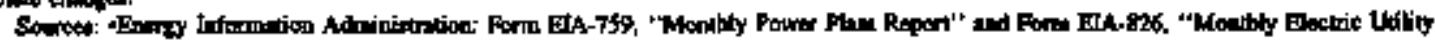

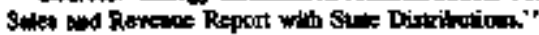




\begin{tabular}{|c|c|}
\hline Unit & Equatimul \\
\hline 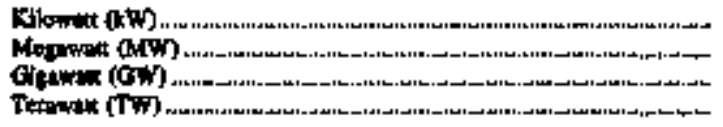 & 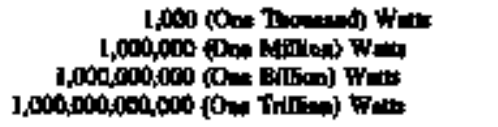 \\
\hline 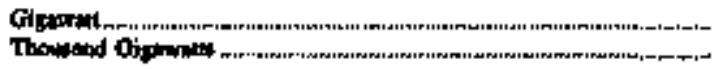 & 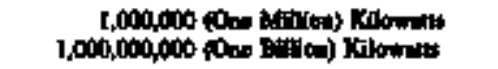 \\
\hline 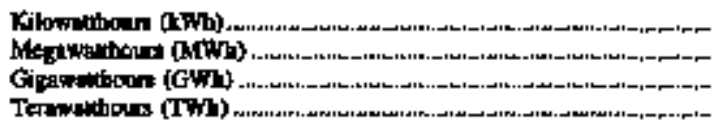 & 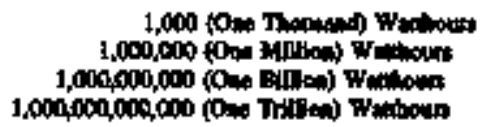 \\
\hline 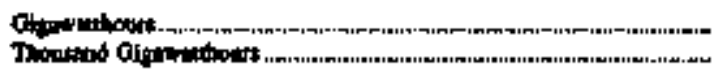 & 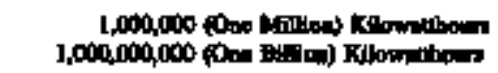 \\
\hline
\end{tabular}

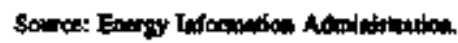

Table B4. Comparion of Sample Veraus Census Publithed Data at the U.S. Lerel, $19 \%$ and 1996

\begin{tabular}{|c|c|c|c|c|c|c|}
\hline \multirow[b]{2}{*}{ Itan } & \multicolumn{3}{|c|}{ wes } & \multicolumn{3}{|c|}{ 196 } \\
\hline & sent & Conis & & Banim & Ca:cen & 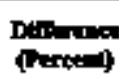 \\
\hline \multicolumn{7}{|l|}{ 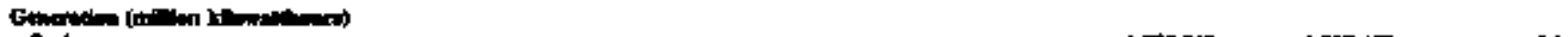 } \\
\hline 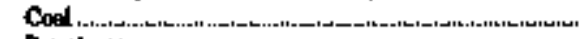 & $\rightarrow$ & $\rightarrow$ & - & $1, T 55,043$ & $1,737,453$ & a.l \\
\hline 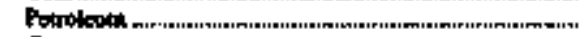 & - & $\longrightarrow$ & $\rightarrow$ & $66,26 \mathrm{I}$ & as,69d & -9 \\
\hline 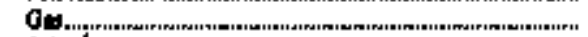 & - & - & - & 263,262 & 262,750 & -2 \\
\hline 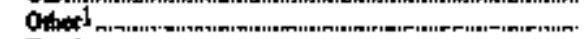 & - & $\sim$ & - & $1,012,75$ & $1011, \$ 4$ & -1 \\
\hline 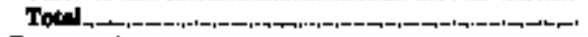 & - & $\Rightarrow$ & - & 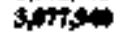 & 3 strim & 4 \\
\hline \multicolumn{7}{|l|}{ Contoonpeles } \\
\hline Cod (i,000 then thes) & $=$ & $\rightarrow$ & - & 873491 & E74,61 &. $\mathbf{1}$ \\
\hline 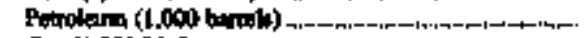 & $\Rightarrow$ & $\rightarrow$ & $\rightarrow$ & $114,78:$ & 113,274 & -13 \\
\hline G:t $(1,000 \mathrm{Mch})$ & - & $=$ & - & $2.73 \times 552$ & $2,72,109$ & -1 \\
\hline \multicolumn{7}{|l|}{ Bteche 2} \\
\hline 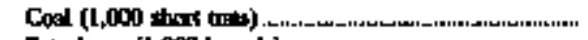 & $\rightarrow$ & $\rightarrow$ & - & 114,623 & 114,003 & \% \\
\hline 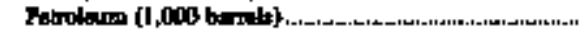 & - & - & - & 47,547 & $\$, 600$ & 4 \\
\hline \multicolumn{7}{|l|}{ 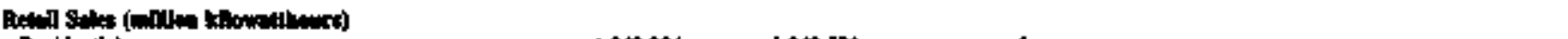 } \\
\hline Roddowitid & $1,049,304$ & $1,000,501$ & -1 & $\longleftarrow$ & $\rightarrow$ & - \\
\hline 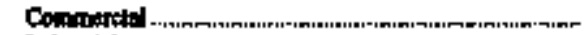 & 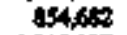 & o.0.685 & 9 & 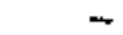 & $\rightarrow$ & - \\
\hline IMdoftedel & $1,013,107$ & 1.012693 & 4 & $\longrightarrow$ & 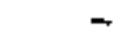 & - \\
\hline 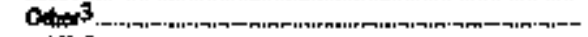 & thin & $93 \mathrm{MO}$ & -2.2 & $\longleftarrow$ & - & - \\
\hline All Sederd & 34.44) & $3,13,2 \pi$ & $\boldsymbol{m}$ & $\rightarrow$ & $\neg$ & - \\
\hline \multicolumn{7}{|l|}{ 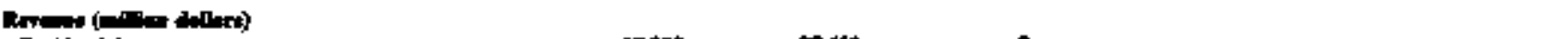 } \\
\hline 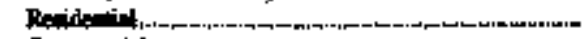 & 8T, & 81,610 & -2 & 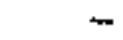 & $\rightarrow$ & $\longleftarrow$ \\
\hline 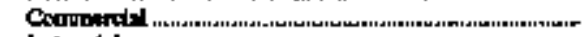 & $6 \$$ & 6:3035 & 8 & - & - & $\hookrightarrow$ \\
\hline budutriall. & 7, & Q9,175 & -.7 & $\rightarrow$ & $\rightarrow$ & - \\
\hline 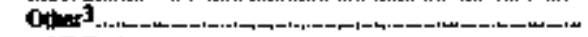 & 6534 & 6,597 & 5 & - & - & - \\
\hline 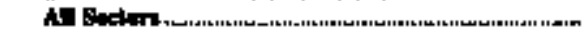 & 2nsth & 2,717 & $*$ & $\longleftarrow$ & $m$ & $\infty$ \\
\hline \multicolumn{7}{|l|}{ 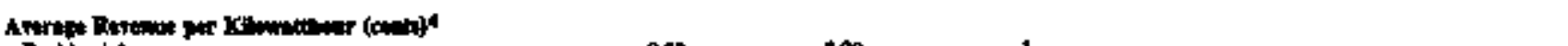 } \\
\hline 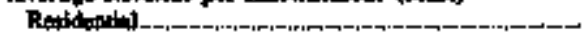 & 800 & 100 & -11 & - & - & - \\
\hline 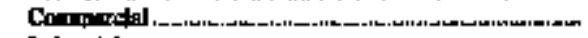 & 800 & $\mathbf{6 0 0}$ & -1 & 2 & $\rightarrow$ & 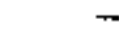 \\
\hline 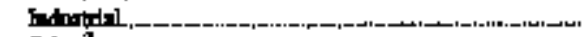 & 5.00 & 5.00 & -7 & $\rightarrow$ & 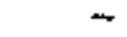 & 4 \\
\hline Chor ${ }_{1}$ & 7.00 & 700 & 27 & $\rightarrow$ & - & 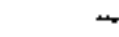 \\
\hline Al Sected & 7 it & 7 th & -56 & - & - & - \\
\hline
\end{tabular}

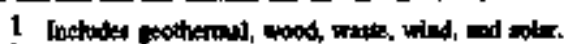

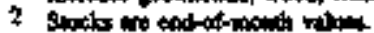

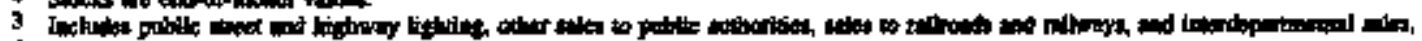

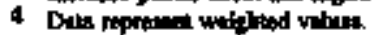

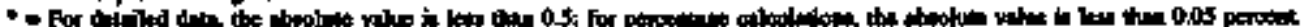

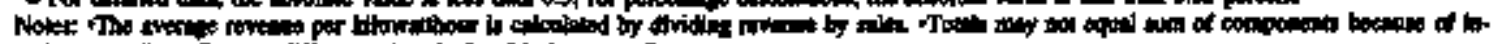

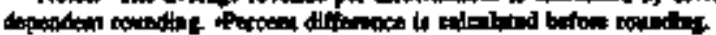

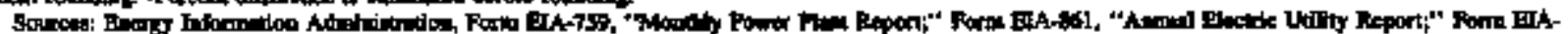

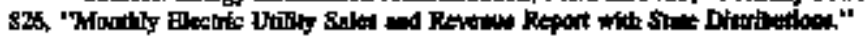


Figut B1. North Amorlean Electric ReliabiHy Council Regione for the Contlguous United States and Naska

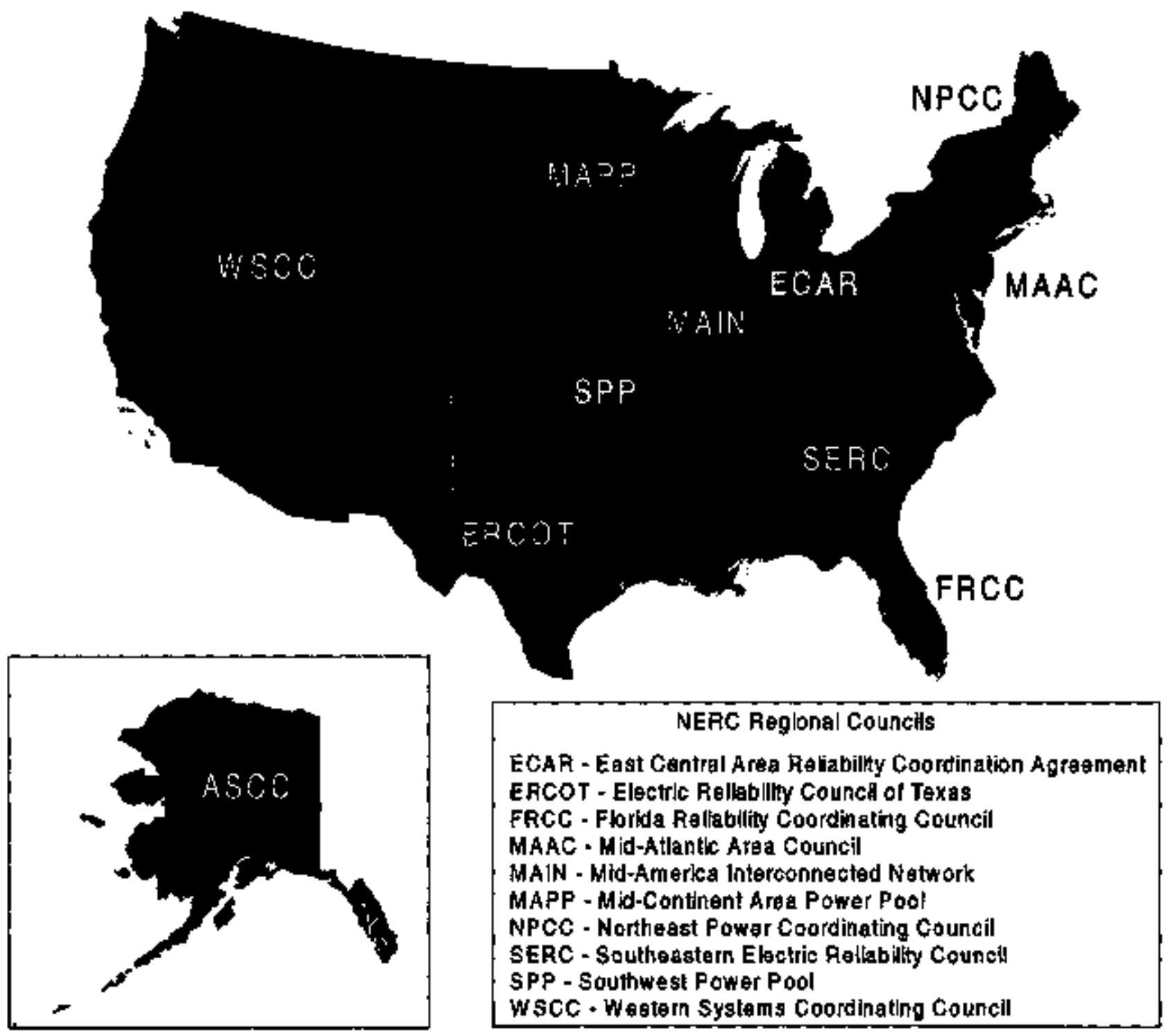

Note: The Alaske System8 Coordinating Council (ASCC) is an affiliate NERC member.

Sovrce: Energy information Adma histratlon, Office of Coal, Nuclear, Elactric and Allernate Fuels. 


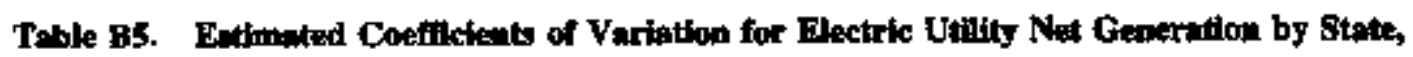
Angost 1997

(Percent)

\begin{tabular}{|c|c|c|c|c|c|c|}
\hline Sectit & Cial & 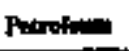 & Can & Hydedentitit & Mection & Oarr? \\
\hline 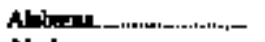 & 0.0 & 0.0 & 0.0 & 00 & 0.0 & - \\
\hline Abukta .............................. & 0 & 17.0 & 5 & 5.9 & - & - \\
\hline Arizon & 0 & . & 0 & 0 & 0 & - \\
\hline Alknt: & 0 & 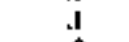 & .2 & 0 & .0 & - \\
\hline Culifortalan..........,......, & - & 0 & 0 & .1 & .0 & 0.0 \\
\hline Colocido & 0 & 1.7 & 8 & l & - & .0 \\
\hline 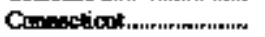 & 0 & 3 & .0 & 1.5 & 0 & 0 \\
\hline Delentrone & A & 0 & 0 & - & - & - \\
\hline Distrut of Collnoth ..... & - & .0 & - & - & - & - \\
\hline Flocidd & -0 & 0 & , & .6 & , & - \\
\hline Coxpia ...... & .4 & 0 & 2 & 4 & . & - \\
\hline 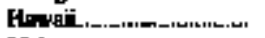 & - & .0 & - & .0 & - & - \\
\hline 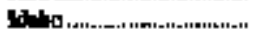 & - & .0 & 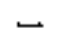 & 1 & - & - \\
\hline 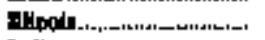 & .0 & 4 & 3 & 0 & .0 & 0 \\
\hline 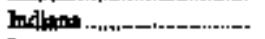 & 0 & 0 & 2 & 0 & - & - \\
\hline Fond ._._._-_............... & .0 & 84 & $1 . \overline{7}$ & 5 & 0 & 0 \\
\hline 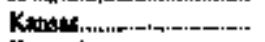 & 0 & 13.6 & 21 & - & 0 & - \\
\hline Koulucky................... & 0 & 0 & D & 9 & - & $\rightarrow$ \\
\hline 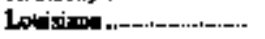 & .0 & 0 & $\hat{0}$ & - & .0 & - \\
\hline 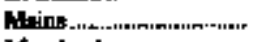 & - & 1 & 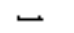 & g & .0 & 0 \\
\hline Mtrylad......-- & $\mathbf{0}$ & .1 & .0 & $\theta$ & .0 & $\rightarrow$ \\
\hline 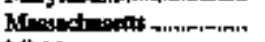 & .0 & , & 1 & .0 & 0 & - \\
\hline 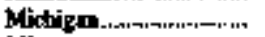 & 0 & 5 & 14 & 4.9 & 0 & - \\
\hline MHtuatodx , & 0 & $\vec{J}$ & 22 & 94 & 0 & 0 \\
\hline Mhtofitippi & 0 & $\Delta$ & 0 & - & 0 & - \\
\hline Minsuit..............._-.... & (1) & 6 & I.J & .3 & .0 & 0 \\
\hline 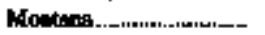 & 0 & 0 & . & 0 & - & - \\
\hline Netersh................tu-- & . & 21.4 & 35 & .0 & $\theta$ & 0 \\
\hline 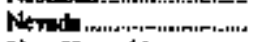 & 0 & b & , 0 & 0 & $\rightarrow$ & - \\
\hline 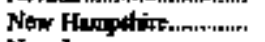 & 0 & D & $D$ & 0 & .0 & - \\
\hline 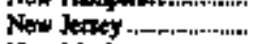 & .0 & 0 & 0 & 0 & 0 & - \\
\hline New Moles & .1 & 0 & .0 & 0 & - & - \\
\hline NEW Yoek ......-_.........., & 0 & .1 & 0 & .0 & .0 & 0 \\
\hline 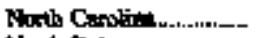 & 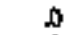 & $\mathbf{0}$ & 0 & 2 & 0 & - \\
\hline 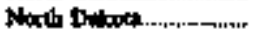 & 0 & 0 & 0 & 0 & - & - \\
\hline Ohin____....................... & A & : 1 r & , 1 & 0 & 0 & - \\
\hline Otk:hoth & 0 & 2.3 & .1 & 至 & - & - \\
\hline 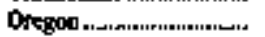 & 0 & 0 & 0 & 0 & - & $\mathbf{0}$ \\
\hline Petangytypi & 0 & .0 & 0 & 1,2 & 0 & - \\
\hline Rhode lalend & 0 & 0 & 0 & - & - & - \\
\hline Sord Caroling............... & 0 & 0 & 0 & 1.8 & 0 & - \\
\hline Sotw DuTnte................. & 0 & 0 & 0 & 0 & - & - \\
\hline 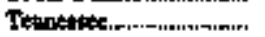 & 0 & 0 & 0 & $\boldsymbol{0}$ & 0 & - \\
\hline 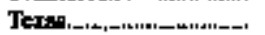 & .0 & 0 & 0 &. & 0 & 0 \\
\hline & , I & 15 & II.5 & 3.3 & - &, 0 \\
\hline 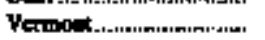 & - & 234 & $D$ & 3.7 & 0 &, 0 \\
\hline 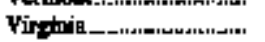 & .0 & 0 & 0 & .3 & 9 & 0 \\
\hline 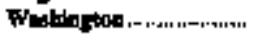 & 0 & 0 & 0 & 0 & 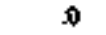 & -1) \\
\hline Weat Vurginit ............... & .0 & 0 & 0 & $\boldsymbol{0}$ & - & - \\
\hline 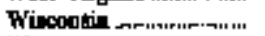 & 0 & 4 & 3 & 8 & 0 & 0 \\
\hline Wyomits ..................... & .0 & 0 & 0 & 2 & $\rightarrow$ & - \\
\hline
\end{tabular}

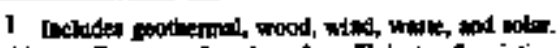

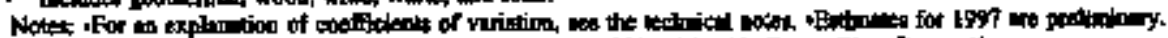

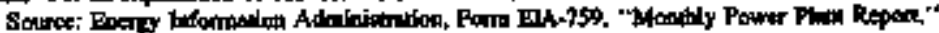


Table B6. Intimated Coefiicients of Variotion for Electric Utality Fuel Comsomption and Stocles by States August 199

(Pencent)

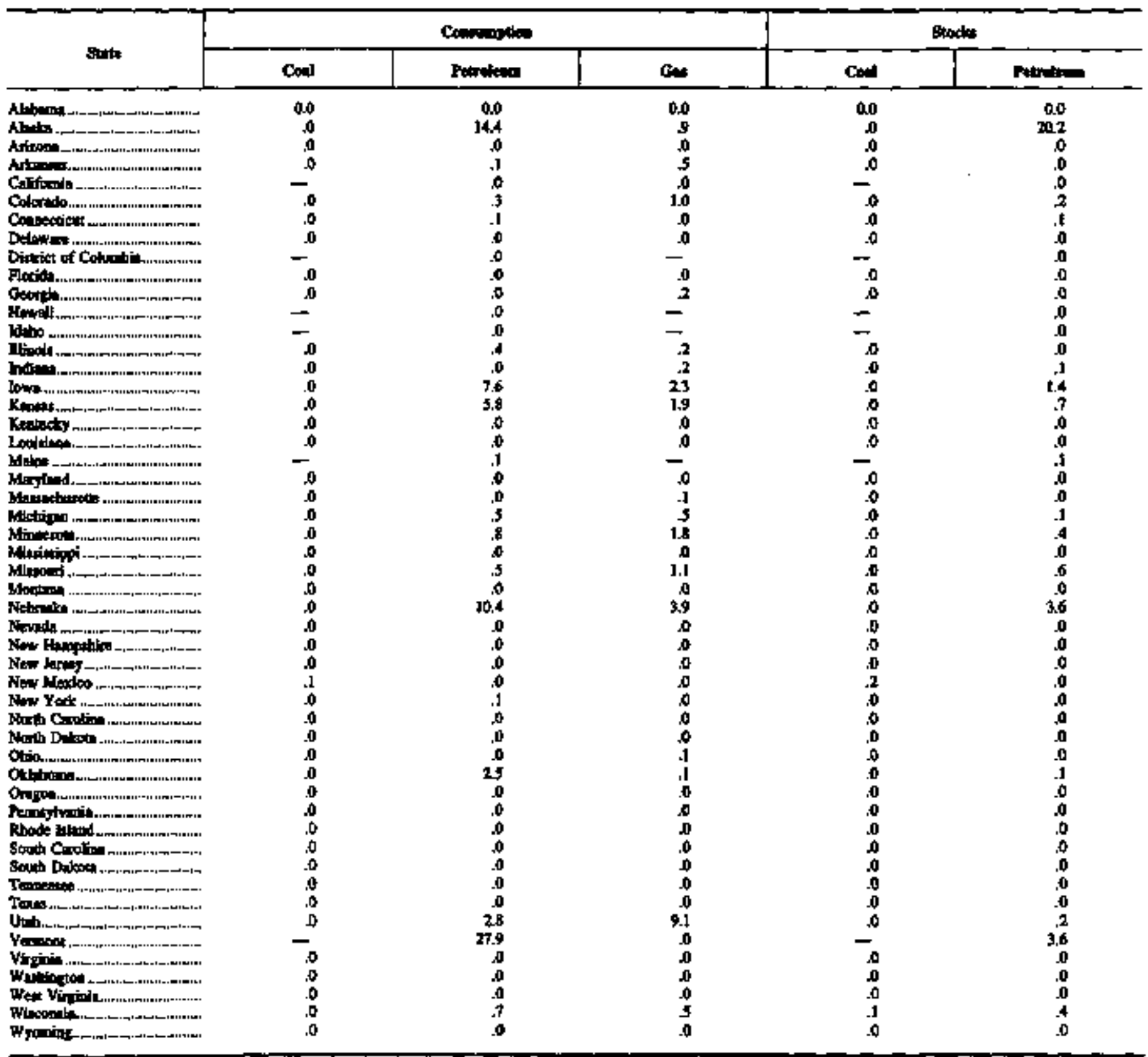

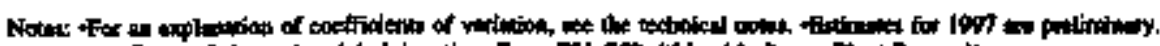

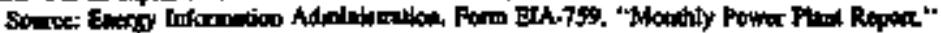




\section{Glossary}

Ampere The urit of measurement of electrical current produced in a cincuit by 1 volt actíng tivrough a resistance of 1 ohur.

Anthracite: A hard, black lustrots coal, often referred to as hard coal, containing a high percentage of fixed carbon and a low percentage of volatile matter. Comprises three groups classified according to the following ASTM Specification Do8-84, on a dry mineral-matter-free basis:

$\begin{array}{llllr} & \begin{array}{l}\text { Fixed } \\ \text { Carbon } \\ \text { Limits }\end{array} & \begin{array}{l}\text { Volatile } \\ \text { Matter }\end{array} \\ & \text { GE } & \text { LT } & \text { GT } & \text { IE } \\ \text { Meta-Anthracite } & \mathbf{9 8} & - & - & 2 \\ \text { Anthracite } & 92 & 98 & 2 & 8 \\ \text { Semianthracite } & \mathbf{8 6} & \mathbf{9 2} & \mathbf{8} & \mathbf{1 4}\end{array}$

Average Revente per Kllowatthour. The average revenue per kilowathour of electricity sold by sector (residential, commercial, industrial, or other) and geographic area (State, Census division, and national), is calculated by dividing the total monthly reventre by the corresponding total monthly sales for each sector and geographic area.

Barrel: A volumetric untt of measure for crude oil and petzoleim products equivakent to $42 \mathrm{U} . S$. galknos.

Baselpad: The minimum amount of electric power delivered or required over a given period of time at a steady rate.

Baseload Capacity: The genterating equipment normally operated to serve hoads on an around-the-dock basis.

Baseload Plant: A plant, usually housing high-efficiency steam-electric units, which is normally operated to take all or part of the minimum load of a system, and which consequently produces electricity at an essentially $\mathrm{com}$ stant rate and runs continuously. These units are operated to maxirize system mechanical and thermal efficiency and minimize system operating costs.

\section{Bcf: The abbreviation for 1 billion cubic feet.}

Bituminous Coal: The trost common coal. It is dense and black (often with well-defined bants of bright and dull material). Its moisture content ustally is less than 20 percent. It is used for genetating electricity, making coke, and space heating. Comprise five groups classified according to the following ASTM Specification D388-84, on a dry mineral-matter-free (mm) basis for fixed-carbon and volatile matter and a moist munf basis for calorific value.

$\begin{array}{lll}\text { Fixed } & \text { Volatile } & \text { Calorific } \\ \text { Carbon } & \text { Matter } & \text { Value } \\ \text { Limitis } & \text { Limits } & \text { Limits } \\ & & \text { Btu/Db }\end{array}$

$\begin{array}{lllllll} & \text { GE } & \text { LT } & \text { GT } & \text { LT } & \text { GE } & \text { LE } \\ \text { LV } & 78 & 86 & 14 & 22 & - & - \\ \text { MV } & 69 & 78 & 22 & 31 & - & - \\ \text { HVA } & - & 69 & 31 & - & 14000 & - \\ \text { HVB } & - & - & - & - & 13000 & 14000 \\ \text { HVC } & - & - & - & - & 10500 & 13000\end{array}$

Boiler A device for generating steam for power, processing, or heating purposes or for producing hot water for heating purposes or hot water supply. Heat from an external combustion source is transmitted to a fluid contained within the tubes in the boiler shell. This fiuid is delivered to an end-use at a desired pressure, temperature, and cuality.

Bta (British Thermal Unit): A standard unit for measuring the quantity of heat energy equal to the quantity of heat required to raise the temperature of 1 pound of water by 1 degree Fahrenheit.

Capability: The maximum load that a generating unit, generating station, or other electrical apparatus cari carry under specified conditions for a given period of time without exceeding approved limits of temperature and stress.

Capacity: The full-load continuous rating of a generabor, prime mover, cr other electric equipment under specified conditions as desigrated by the manufacturer. It is usually indicated on a mamesplate attached to the equipment.

Capacity (Purchased): The amount of energy and capacity available for punchase from outside the system. 
Censta Divisions: The nine geographic divisions of the United States established by the Bureau of the Census, U.S. Deparment of Comnerce, for the purpose of statistical analysis. The boundaries of Census divisions coincide with State boundaries. The Pacific Division is subdivided into the Pacific Contiguous and Patifx Noncontiguous areas.

Circuit: A conductor or a system of conductors through which electric current flows.

Coal; A black or brownish-black solid combustible substance formed by the partial decomposition of vegetable matter without access to air. The rank of coal, which inchudes anthracite, bittuminous coal, subbttuminous coal. and lignite, is based on fixed carbon, volatile matter, and heating value. Coal rank indicates the progressive alteration from ligrite to anthractte. Lignite contatins approximately 9 to 17 mitilion Btu per ton. The contents of subbituminous and bituminous coal range from 16 to 24 million Btu per ton and from 19 to 30 million Btu per ton, respectively. Anthracite contains approximately 22 to 28 nuillion Btu per ton.

Coincidental Demandt The sum of two or more demands that oxcur in the same time interval.

Coincidental Peak Load: The sum of two or more peak loads that oxcur in the same time interval.

Coke (Petroletm): A residue high in carbon content ard low in hydrogen that is the final product of thermal decomposition in the condensation process in cracking. This product is reported as marketable coke or catalyst coke. The converstion factor is 5 barrels ( 42 U.S. gallons each) per short ton.

Combined Pumped-Storage Plant: A pumped-storage hydroelectric power plant that uses both pumped water and natural streamflow to produce electricity.

Commercial Operation: Cornuencial operation begins when cuntrol of the loading of the generator is turned over to the system dispatcter.

Compressor A pump or other type of machine using a turbine to compress a gas by reductng the volume.

Consumption (Fuel): The amount of fuel used for gross generation, providing standby service, start-up and/or flame stabilization.

Coutract Recelpts: Purchases based on a negotiated agreement that generally covers a period of $I$ or more years.

Cost: The amount paid to acquire resouktes, such as plant and equipment, fuel, or labor services.
Crude Oil (including Leape Condensate): A mixture of hydrocarbans that existed in liquild phase in underground reservoirs and that remsins liquid at atmospheric pressure after passing through surface separating facilfies. Included are lease condensate and bquid hydrocarbons produced from tar sands, gilscrite, and shale oil. Drip gases are also inctuded, but topped crude oil (residual oil) and other urfinished oils are exciuded tiquids produced at natural gas processing plants and mixed with crude oil are likewise exctuded where identifiable.

Current (Electric): A flow of electrons in an electrical conductor. The strength or rate of movement of the electricity is measured in amperes.

Demand (Electric): The rate at which electric energy is delivered to or by a system, part of a system, or piece of equipment, at a given tristant or averaged over any designated period of time

Demind Interval: The time period during which flow of electricity is measured (usually in 15-, 30, or 60-mtinute increments.)

Electric Plant (Physical): A facility containing prime movers, electric generators, and auxiliary equipment for converting mechanical, chemical, and/or fissinn energy into electric energy.

Electric Uthity: An enterpsise that is engrged in the generation, transmission, or distribution of electric energy primarlity for use by the public and that is the major power supplier wthin a designated service area. Electric utilities inchude investor-owned, publicty owned, cacperatively owned, and government-owned (muricipals, Federal agencles, State projects, and public power diatricts) systems.

Enerby: The cepacify for doing work as measured by the capability of doing work (potential energy) or the conversion of this capability to motion (kinetic energy). Energy has several forms, some of which are easily convertible and can be changed to another form useful for work. Most of the world's convertifle energy comes from fossil fuels that are burned to produce heat that is then used as a transfer medium to mechanical or other means in order to tocomplish tasks. Electrical energy is usually measured in kilowathorus, while heat energy is usually measured in British thermal units.

Energy Deliveriea: Energy generated by one electric utility system and delivered to another system through one or more transmission lines.

Energy Receipts: Ervergy generated by one electric utility bystem and received by another system through one or more transmilstion lines. 
Energy Sounce: The primary source that prowides the power that is corverted to elestricity through chemical, mechanical, or other means. Energy sources inctude coal, petroleum and petroleum products, gas, water, uranium, wind, sunlight, geothermal, and other sources.

Fahrenheit: A temperature scale on which the boiling point of water is at 212 degrees above zero on the scale and the freezing point is at 32 degrees above zero at standard atmospheric pressure.

Fallure or Harard: Any electric power supply equipment or faclity failure or other event that, in the judgment of the reporting entity, constitutes a hazard to maintaining the continuity of the bulk electric power supply system such that a load reduction action may become necessary and a reportable outage may occur. The imposition of a special operatting procedure, the extended purchase of emergency power, other bulk power system actions that may be caused by a ratural disester, a mafor equipment failure that would inpact the bulk power supply, and an envirormental and/or regulatory action requiring equip. ment outages are types of abnormal conditions that should be reported.

Firm Ga: Gas sold on a continuous and generally long-term contract.

Foesil Fuel: Any naturally occurring organic fuel, such as petroteum, coal, and natural gas.

Fonsil-fuel Etant: A plant using coal, petroleum, or gas as its sounce of energy.

Fuel: Any substance that can be burned to produce heat; also, materials that can be fissioned in a chain reaction to proderce heat.

Fuel Bmergencies: An emergency that exists when sup. plies of fuels or hydroelectric storage for generation are at a level or estimated to be at a level that would threaten the reliability or adequacy of bulk electric power supply. The following factors should be taken into account to determine that a fuel emergency exists: (1) Fuel stock or hydroelectric project water storage levels are 50 percenk or less of normal for that particular time of the year and a continued downward trend in fuel stock or hydroelectric project water storage level are estimated; or (2) Unscheduled dispatch or emergency generation is causing an abnomal use of a particular fuel type, such that the future supply or stocks of that fuel could reach a level which threatens the reliability or adequacy of bulk electric power supply.

Gas: A fuel bumed under boilers and by intemal combustion engines for electric generation. These include natural, manuffactured and waste gas.
Generation (Flectrtcity): The process of producing electric energy by transforming other forms of energy; also, the amount of electric energy produced, expressed in watthours (Wh).

Gross Gentration: The total amount of electric energy produced by the generating units at a generating station or stations, measured at the gerterator terminals.

Net Generation: Gross generation less the electric energy consumed at the generating station for station use.

Generator: A madhine that converts mecharical energy into electrical energy.

Gentrator Nameplote Capacily: The full-load continuous rating of a generator, prime mover, or other electric power production equipment under specific conditions as designated by the manufacturer. Installed generator nameplate rating is usually indicated on a natneplate physically attached to the generator.

Geothermal Plant: A plant in which the prime mover is a steam nubine. The turbine is driven ejther by steam protuced from hot water or by natural steam that derives its energy from heat found in rocks or fluids at various depths bereath the surface of the earth. The energy is extracted by drilling and/or pumping.

\section{Gigawatt (GW): One billion watts.}

\section{Gigawatthour (GWh): One billion watthours.}

Gross Generation: The total amount of electric energy produced by a gererating facility, as measured at the generator terminals.

Heavy Oil: The fuel ofls remaining after the lighter oils have been distilled off during the refining process. Except for start-up and flame stabilization, virtually all petroleum used in steam plants is heavy oil.

Horsepower. A unit for measuring the rate of work (or power) equivalent to 33,000 foot-pounds per minute or 746 watts.

Hydroelectric Plant: A plant in which the hurbine generators are diviven by falling water.

Inatantaneous Peak Demand: The maximum dernard at the instant of greatest load.

Integrated Demand: The summation of the continuously varying instantaneous demand averaged over a specified interval of time. The information is usually determined by examining a demand meter. 
Intemal Combustion Plant: A plant in which the prime mover is an internal combustion engine. An internal conbustion engine has one or more cylinders in which the process of cornhustion takes place, converting energy released from the rapid burning of a fulel-air mixture into mechanical energy. Diesel or gas-fired engines are the principal types used in electric planks. The plant is wsually operated during periods of high demand for electriclty.

Intemruptible Gast Cas sold to customers with a provision that perrits curtailment or cessation of service at the discretion of the distributing company under certain circumstances, as specified in the service contract.

Kilowath (kW): One thousand watts.

Kilowatthour (kWh)! One thousand watthours.

Light Oil: Lighter fuet oils dishilled off during the refining process. Virtually all petroleum used in internal comr bustion and gat-burtine engines is light oil.

Lignite: A brownish-black cal of low rank with high inherent moishure and volatile matter (used almost exclasively for electrir power generation). It is also referred to as brown coal. Comprises two groups classified according to the following ASTM Specification D388-84 for calorific values on a moist material-matter-free basis:

Limits Btu/lb.

$\begin{array}{lcc} & \text { GE } & \text { LT } \\ \text { Lignite A } & 6300 & 8300 \\ \text { Ligrite B } & - & 6300\end{array}$

Maxtmum Demand: The greatest of all demands of the load that has occurred within a specified period of time.

Mcf: One thousand cubic feet.

Megawatt (MW): One million watts.

Megawatthour (MWh): One million watthours.

Mef: One mullion cubtc feet.

Natural Gas: A naturally occurring mixture of hydrocarbon and nonhydrocarbon gases found in porous geological formations beneath the earth's surface, often in assoclation with petroleum. The princlpal constituent is methane.

Net Energy for Load: Net generation of main gererating units that are system-owned or system-operated plus energy receipts minus energy deliveries.

Net Generation: Gross generation minus plant use from all electric utility owned plants. The energy required for pumping at a pumped-storage plant is regarded as plank use and must be deducted from the gross gernerabion.

Net Summer Capability: The steady hourly output, which generating equipment is expected to supply to system losd exchusive of auxiliary power, as demonstrated by tests at the time of sumuner peak demand.

Noncoincidental Peak Load: The sum of two or more peak loads on individual systems that do not occur in the same time interval. Meaningful only when considering loads within a limited period of time, such as a day, weak, month, a heating or cooling season, and usually for not more than 1 year.

North Ameriean Electric Reliability Council (NERC): A council formed in 1968 by the electrle utility industry to promote the reliability and adequacy of bulk power supply in the electric utility systems of North America. The NERC Regions are:
ASCC - Alaskan System Coordination Council
BCAR - East Central Area Reliability Cootdmation Agreement
ERCOT - Electric Reliability Council of Texas
FRCC - Florida Reliability Coordinating Council
MAIN - Mid-America Interconnected Network
MAAC - Mid-Atlantic Area Courcil
MAPP - Mid-Continent Area Power Pool
NPCC - Northeast Power Coordinating Council
SERC - Southeastem Electric Reliabiliky Conncil
SPP - Southwest Power Pool
WSCC - Westetn Systents Coordinating CounciI

Nuclear Fuel: Fissionable uraterials that have been enriched to such a composition that, when placed in a nuclear reactor, will support a self-sustaining fission chatn reaction, producing heat in a controlled manner for process use.

Nuclear Power Plant: A facility in which heat produced in a reactor by the fissioning of nuclear fuel is used to drive a steam turbine.

Off-Peak Gaak Gas that is to be delivered and taken on demand when demand is not at its peak.

Ohm: The unit of meastmement of electrical resistance. The resistance of a circuit in which a potential difference of 1 volt produces a curtent of 1 ampere.

Operible Nuclear Unit: A nuclear urit is "operable" after it completes low-power testing and is granted authortzation to operate at full power. This occurs when it recetves its full power amendment to its operating license from the Nuckear Regulatory Commission.

Other Gas: Includes manufactured gas, coke-oven gas, blast-furnace gas, and refinery gas. Manufactured gas is 
obtatned by cilstillation of coal, by the thermal decomposition of at, or by the reaction of steam passing through a bed of heated coal or woke.

Other Generation: Electricity originating from these sounces; bionsass, fuel cells, geothermal heat, solar power, waste, wind, and wood.

Other Unavailable Capability: Net capability of main generating urits that are unavailable for load for reasons other than full-forced cutrage or scheduled maintenance. Legal restrictions or other causes make these units unavailable.

Peak Demand: The maximum load during a specified period of time

Peak Load Plant: A plant usually housing old, low-efficiency steam units; gas turbines; diesels; or pumped-storage hydroelectric equipment normally used during the peak-ioad periods.

Peaking Capacity: Capacity of generating equipment normally reserved for operation during the hours of highest daily, weekly, or seasonal loads. Some generating equipment may be operated at certain times as peaking capacity and at other times to serve loads on an around-the-dock basis.

Percent Difference: The relative change in a quantity over a specified time period. It is calculated as follows: the current value has the previous value subtracted from it; this new number is divided by the absolute value of the previous value; then this new number is multiplied by 100.

Petroleum: A mixture of hydrocarbons extsting in the liquid state found in natural underground reservoirs, ofter associated with gas. Petroleum includes fuel oil No. 2, No 4, No, 5, No. 6; kopped crude; Kerosente; and jet fuel.

Petroletum Coke: See Coke (Petroleum).

Petroleum (Crude Oil): A maturally occurring, oily, flanumable liquid composed principally of hyctrocarbons. Crutie oil is occasionaliy found in spings or poots but usually is drflled from wells beneath the earth's surface.

Plank A faility at which are located prime movers, electric generators, and atuxiliary equipment for converting mecharical, chemical, and/or nuclear enengy into electric energy. A plant may contatn mone than one type of prime mover. Flectric utility plents exclude facitities that satisfy the definition of a qualifying facillty under the Public Utility Regulatory Policies Act of 1978 .

Plant Use: The electric energy used in the operation of a plant. Included in this definition bs the energy required for pumping at pumped-storage plants.
Plant-Use Electricity: The electric energy used in the operation of a plant. This energy total is subtracted from the gross energy production of the plant; for reporting purposes the plant erergy production is then reported as a net figure. The energy required for pumping at pumped-storage plants is, by definition, subtracted, and fhe eneryy production for these plants is then reported as a net figure.

Power. The rate at which energy is transferred. Elextrical energy is usually measured in watts. Also used for a measurement of capacity.

Price: The amount of money or consideration-in-kind for which a service is bought, sold, or offered for sale.

Prime Mover: The motive force that drives an electric generator (e.g., steam engine, turbine, or water wheel).

Production (Electric): Act or process of producing electric energy from other forms of energy; also, the amount of electric energy expressed in watthours (Wh).

Pumped-Storage Hydroelectric Flant A plant that usually generates electric energy during peak-load periods by ustirs water previously pumped into an elevated storage reservoir during off-peak periods when excess generating capacity is available to do so. When additional generating capacity is needed, the water can be released from the reservoir through a conduit to turbine generators located in a power plant at a lower level.

Pare Punped-Storage Hydroelectric Flant: A plant that produces power only from water that has previousty been pumped to an upper reservoir.

Qualifying Facility (QF): This is a cogenerator of small power produce that meets certain ownership, operating and efficiency criteria established by the Federal Energy Regulatory Commussion (FERC) pursuant to the PURPA, and has filed with the FERC for $\mathrm{QF}$ status or has self-cetified. For additional infortration, see the Code of Federal Regulation, Title 18, Part 292.

Roiltoad and Railway Electric Sarvice: Electrictty supplied to rallroads and interubat and street ratiways, for general railroad use, thcluding the propulsion of cars or focomotives, where such electricity is supplied under separate ard distinct rate schedules.

Receipto: Purchases of fuel.

Reserve Margin (Operating): The amount of unused avaitable capsbility of an electric power system at peak load for a utility system as a percentage of total capability.

Restoration Tina: The time when the major portion of the interrupted load has been restored and the emergency is 
considered to be ended. However, some of the loads internupted may not have been restored due to local problems.

Restricted-Universe Census: This is the complete enumeration of data from a specifically defined subset of entities including, for example, those that exceed a given level of sales or generator naineplate capacity.

Retail: Sales covering electrical energy stpplied tor residertial, commercial, and industrial end-use purposes. Other small dasses, such as agriculture and steet lighting, also are induded in this category.

Running and Quick-Start Capability: The net capability of generating units that carry load or have quick-btart capability. In general, quick-start capability refers to generating units that can be available for load within a 30-miniute period.

Salex The amount of kilowatthors sold in a given period of time; usually grouped by classes of secvire, such as residential, commencial, industrial, and other. Other sales indude public street and highway lighting, other sales to public authorities and railways, and interdepartmental sales.

Sales for Resele: Energy supplied to other electric uttlities, cooperatives, municipalities, and Federal and State electric agencies for resale to ulfminate consumers.

Scheduled Outnge: The shutdown of a generating unit, transmission itne, or other facility, for inspection or maintenance, in accortance with an advance schedule.

Short Ton: A unit of weight equal to 2,000 pounds.

Spot Purchases: A single shipment of fuel or volumes of tuel, purchased for delivery within 1 year. Spot purchases are often made by a user to fulfill a certain portion of energy bequirements, to meet unanticipated energy needs, or to take advantage of low-fued prices.

Stendby Focllity: A factity that supports a utility system and is gemerally ruming under no-load. It is available to replace or stipplement a facility normaliy in service.

Standby Service: Support service that is available, as needed, to supplement a consumer, a utility system, or to another utility if a schedule or an agreement authorizes the transaction. The service is not regularly used.

Steam-Electric Plant (Conventional): A plant in which the prime mover is a steam turbine. The steam used to dwve the untine is pooduced in a boiler where fossil fuels are burned.
StodksA supply of fuel accumulated for future use. This includes coal and fuel oil stocks at the plant site, in coal cars, tanks, or barges at the plant site, ot at separate storage sites.

Subbituminous Coal: Subbituninous coal, or black lignite, is dull black and generally contains 20 to 30 percent moisture. The heat content of subbituminous coal ranges from 16 to $24 \mathrm{million}$ Btu per ton as received and averages about 18 million Btu per ton. Subbiturninous coal, mined in the western cosal fields, is used for gererating electricity and space heaking.

Stwbstation: Facility equipment that switches, changes, or regulates electric voltage.

Sulfurt One of the elements present in varying quantities in coal which onntributes to environmental degradation when coal is burned. In terms of sulfur content by weight, coal is generally classified as low (iess than or equal to 1 percent), medium (greater than 1 percent and less thar or equal to 3 percent), and high (greater than 3 percent). Sulfur cantent is measured as a percent by weight of coal on an "as received" or a "dry" (moisture-free, usually part of a laboratory analysis) basis.

Switching Station: Facility equipment used to tie together two or more electric cincuits through swithes. The switches are selectively arranged to permit a circuit to be disconnected, or to change the electric connection between the circuits.

Syatem (Electric): Physically connected generation, trarsmission, and distribution faclities operated as an integrated unit wader one central tranagernent, or cperating supervision.

Transformer: An electrical device for changing the voltage of altemating current.

Transmisaion: The movement or transfer of electric energy over an interconnected group of lines and associated equipment between points of supply and points at which it is transformed for delivery to cht sumers, or is delitvered to other electric systems. Transmission is considered to end when the energy is transformed for distribution to the consumer.

Tran:mission System (Electric); An interconnected group of electric transmiscion lines and associated equipment for moving or transferring electric energy in bulk between points of supply and points at which it is transformed for delivery ofer the distribution system lines to consumers, or is delivered to other electric systems.

Turbine: A machine for generating rotary mecharical power from the energy of a stream of fluid (such as water, sheam, or hot gas). Tutolnes convert the kinetic energy of 
fluids to mechanical energy through the principles of inpulse and reaction, or a mixture of the two.

Watt: The electrical unit of power. The rate of energy tansfer equivalent to 1 ampere flowing tuder a pressure of 1 volt at urity power factor.

Watthour (Wh): An electrical energy unit of measure equal to 1 watt of power supplied to, or taken from, an electric dircuit steadily for 1 hour.
Wheeling Service: The movement of electricity from one system to another over transmission facilities of intervening systems. Wheeling service contracts can be established between two or more systems.

Yexr to Date: The cumulative sum of each month's value starting with Janteary and enting with the current month of the data. 


\section{The Energy Information Administration's latest Energy InfoDisc CD-ROM now has internet connectivity, the exciting feature that links you to ElA's World Wide Web Site.}

The most comprehensive collection of energy information and analysis tools available today

Featuring special topics like the chenging structure of the electric power industry

information from more than 200 of ElA's current pertodicals, analytical publications, and articles

Speed and portability of CD-ROM, plus access to frequent updates and new products on ElA's Web slite

\section{Advanced search capability}

Instant access to ElA's wide range of national energy database applications

A must for anyone needing current or historical energy information.

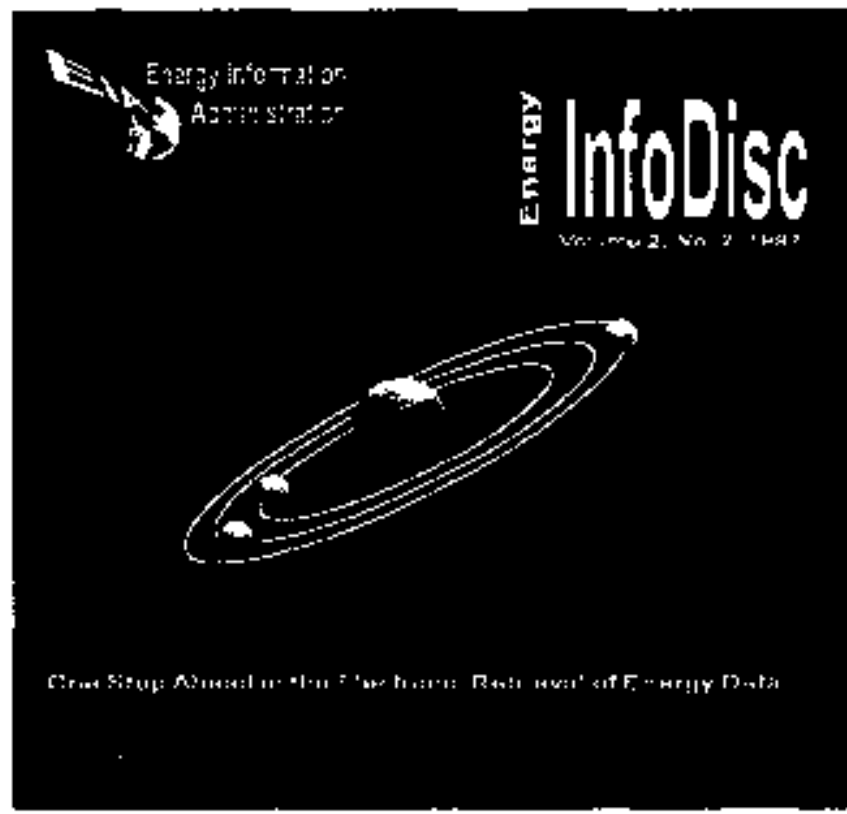

NOTE: Dooumente an be excened using elther Nkrovoit Whidowe or Mecintoeh System 7 operailng aystems.

Detabeat and application con only be acceresed using Mleronofl Windowr.

\section{Don't be without this important resource. SubscribeToday!}

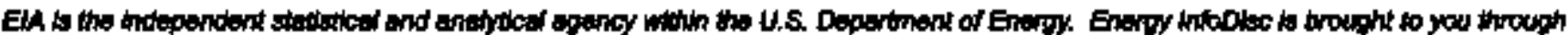

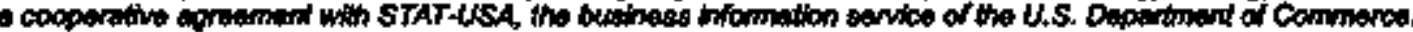

\section{STAT-USA Order Form}

Yos, enter my order for the Enengy IntoDlac Co-row: Number of copias:

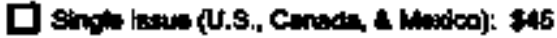

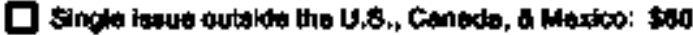

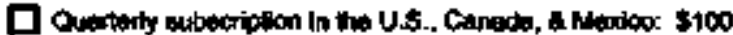

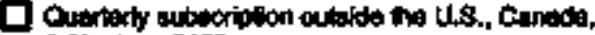

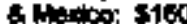

\begin{tabular}{|c|c|}
\hline 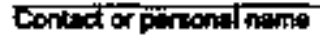 & 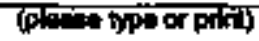 \\
\hline
\end{tabular}

Siract adotoss

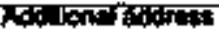

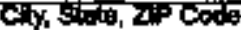

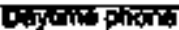

Finger

\section{Charge yourorder $\overline{\mathrm{O}} \mathrm{F}$}

To tax your order: (202) 482-2164

To phone your orders $(24,2) 402-1456$

Toll Fin: 1- (tose) othtuta

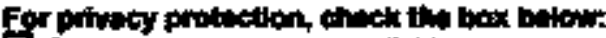

Do not mulce my nerite antileble to requesters.

Check mithod of paymont:

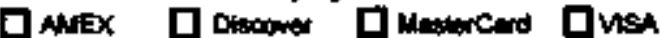
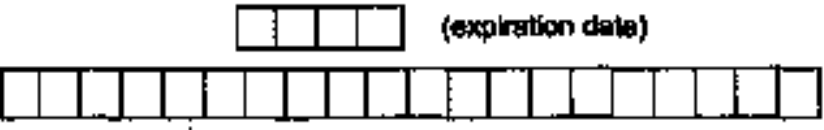

Thank you for your ordent

Authataing atrolume

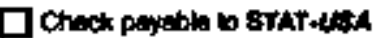

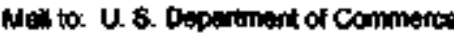

STAT-USA, Room HAgals

Watingan, DC 20030 